\title{
Purification, Characterisation and Synthesis of Glycerolipids Extracted from L. plantarum and B. longum subsp. infantis
}

by

Janelle Sauvageau

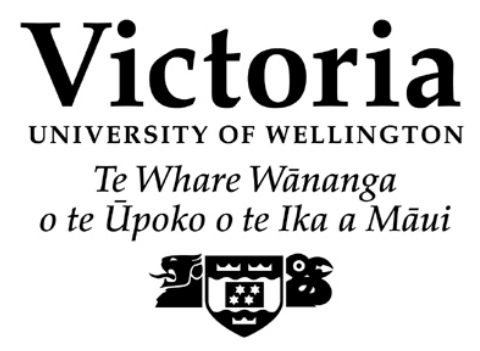

\begin{abstract}
A thesis
submitted to the Victoria University of Wellington in fulfilment of the requirements for the degree of Doctor of Philosophy
\end{abstract}

Victoria University of Wellington 2013 



\section{Abstract}

Glycolipids from the cell wall of Gram-positive bacteria have been the topic of my $\mathrm{PhD}$. It is well known that many bacterial glycolipids (e.g. LPS, TDMs and PIMs) have profound immunological effects, and therefore the characterisation, biological testing and synthesis of gram-positive bacterial glycolipids is of interest. The first part of this thesis includes a description of the extraction and characterisation of glycolipids from gut bacteria including Bifidobacterium and Lactobacillus genus and the second part focussed on the chemical synthesis of Streptococcus sp. DSM 8747 glycolipids and lipoteichoic acid analogues (LTA).

Members of the genus Lactobacillus are common in the gut microbiota and are often used as probiotics. As lactobacilli are known to have benefits to human health, compounds on its surface are of high interest. To date, the structures of the glycolipids from L. plantarum have not been conclusively assigned. Thus, for the first time, the full characterisation of the four principal glycolipids of the L. plantarum cell wall was reported using sugar, linkage and FAME analysis, as well as ESI-MS/MS and 1D- and 2D-NMR spectroscopy. The major glycolipids were identified as: $\alpha$-D-Glc $p$-diglyceride, $\alpha$-D-Gal $p$ - $(1 \rightarrow 2)-\alpha$-D-Glcp-diglyceride, $\quad \beta$-D-Glcp-( $1 \rightarrow 6)-\alpha$-D-Gal $p$ - $(1 \rightarrow 2)-6-O$-acyl- $\alpha$ D-Glcp-diglyceride and $\beta$-D-Glcp-( $1 \rightarrow 6)-\alpha-\mathrm{D}-\mathrm{Gal} p-(1 \rightarrow 2)-\alpha-\mathrm{D}-\mathrm{Glc} p$-diglyceride. These glycolipids showed weak activation of murine bone marrow macrophages in an initial biological screen.

After having identified the structures of the glycolipids from L. plantarum, the glycolipids from Bifidobacterium, a dominant member of the gut microbiota in infants, were extracted. Bifidobacteria are considered to be important in the development of a healthy immune system and they are believed to exhibit anticancerous properties, alleviate the symptoms of irritable bowel syndrome, and are thought to reduce atopic disease. Despite this, the chemical nature of immunomodulatory compounds on the surface of bifidobacteria has not been well documented. Thus, glycolipids were extracted from B. longum subsp. infantis, fractionated chromatographically and 
analyzed using NMR spectroscopy, constituent sugar and linkage analysis, and fatty acid analysis. These analyzes revealed a novel glycolipid, containing an unprecedented mixed acetal moiety and a galactofuranose moiety as a head group. However, like $L$. plantarum glycolipids, bifidobacterial glycolipids were shown only to induce little macrophage activity when tested.

Having successfully characterised a novel glycolipid present in bifidobacteria, analogues of this glycolipid as well as poly(glycerophosphate) lipotechoic acids analogues (LTAs) were then synthesised. Much debate still remains about the role of LTAs during Gram-positive bacterial infection. This is partly due to differences in the biological activities of extracted versus synthesised LTAs and highlights the need for structurally defined non-contaminated LTAs when investigating the effect of these glycolipids on the innate immune response. An efficient synthesis of the core lipoteichoic acid (LTA) anchor of the Streptococcus species DSM 8747, and derivatives thereof, was achieved. These Streptococcus glycolipids contain a galactofuranose moiety and thus have similarities to the novel glycolipid that was found in bifidobacteria. The syntheses, which commence with readily available D-galactose, are short (7-9 steps), convergent, and high-yielding (33-37\% overall yield). In total 11 different targets were synthesised. The biological activity of these compounds was also investigated, with several analogues (particularly the sn-1,2-di-acylglycerol LTA anchors) found to induce macrophage activation. 


\section{Acknowledgements}

"So long, and thanks for all the fish"

-Douglas Adams

This $\mathrm{PhD}$ has been an adventure that I could not have finished without the help of many people.

The most important person sadly does not want to be acknowledged, but he had me crying on his shoulder more often than I care to remember and repeatedly convinced me to keep going. He also cleaned the flat, cooked for me, picked me up at crazy hours, brought me my lunch, brought me my computer and supported me in so many other ways. Thank you so much, you are the best.

I would like to thank both my supervisors Ian Sims and Mattie Timmer. I would like also to acknowledge all the people that took part in writing my our published papers (Chapter 2 and 4) as well as Chapter 3 that will hopefully be published soon; Jason Ryan, Kirill Lagutin, Ian Sims, Bridget Stocker, Mattie Timmer, Amy Foster, Ashna Khan, and Stephanie Chee.

Then, I am also very grateful to everyone at IRL, in particular Richard Furnaux and Ian Sims. I consider the time I have worked there as one of the best I have had in my life. Without all this support, I don't think I would have even started my PhD. Once I had started, Ian Sims supervision was invaluable. Thanks also to Kirill Lagutin and Mikhail Vyssotski who advised me in all lipid analysis related issues.

A special thanks to Ashna Khan and Stephanie Chee who tested my multiple samples in a macrophage assay, to Jason Ryan who grew the bacterial biomass, to Mali Camberti who conducted the allerginicity assays and to Odette Shaw and Jacqui Harper who tested some compounds on DCs. Thanks as well to Mary Roberts who helped me with English related questions and thanks to Richard Tilley and John Spencer who helped me in a time of need. 
Finally I would like to thank warmly the immunoglycomics group members. Thanks to Amy Foster and Jessie Bird for making me laugh so hard and to Janice Cheng and Hilary Corkran for their companionship and a great time in Madrid. Of course, I would not dare to forget Stefan Munneke, Gert-Jan Moggre, Alexander Hunt-Painter, Kristiana Santoso, Selma Eising, Anna Win-Mason, and again Stephanie Chee and Ashna Khan. 
To Mahé

Looking forward to seeing you 


\section{Table of Contents}

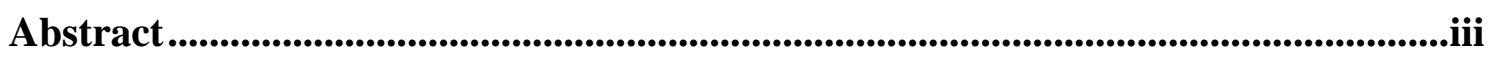

Acknowledgements..........................................................................................................................v

Table of Contents ............................................................................................................................ix

List of Figures................................................................................................................................xiii

List of Schemes ..........................................................................................................................

List of Tables .............................................................................................................................

Glossary ........................................................................................................................

Introduction ............................................................................................................................................

1.1 Gram-Positive and Gram-Negative Bacteria ……...................................... 1

1.2 Gram-Negative Bacteria........................................................................

1.3 Gram-Positive Bacteria …………………………..............................

1.4 Gram-Positive Cell Wall Components and Biosynthetic Pathways ............... 3

1.4.1 Capsular Polysaccharides and Exopolysaccharides ............................. 3

1.4.2 Wall Teichoic Acids...........................................................................

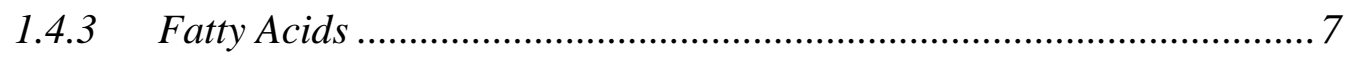

1.4.4 Glycolipids and Phospholipids ................................................... 10

1.5 Chemical Synthesis and Biological Activity of Gram-Positive Cell Wall

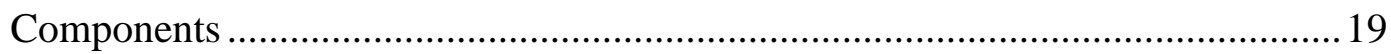

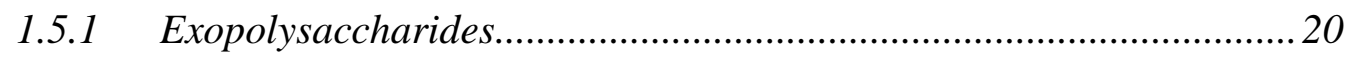

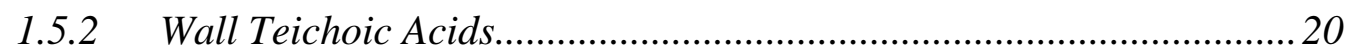

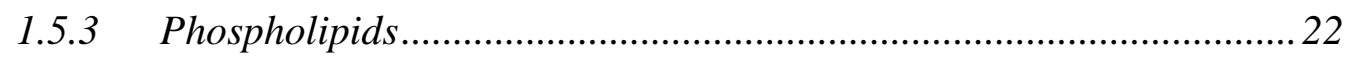

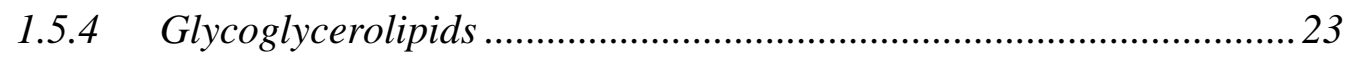

1.5.5 Lipoteichoic Acids......................................................................... 25 


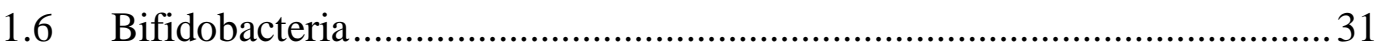

1.6.1 Cell Wall Components (CPS and WTA)............................................ 33

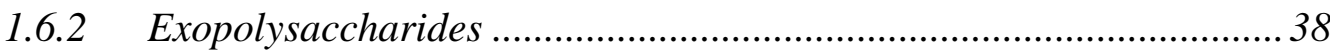

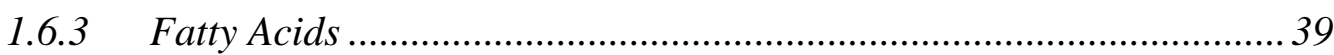

1.6.4 Phosphoglycerolipids............................................................... 41

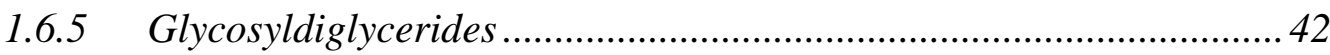

1.6.6 Phosphated Galactolipids and Lipoteichoic Acid............................. 44

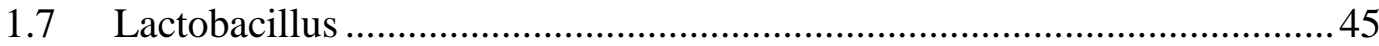

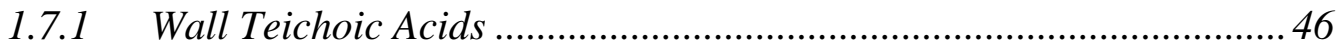

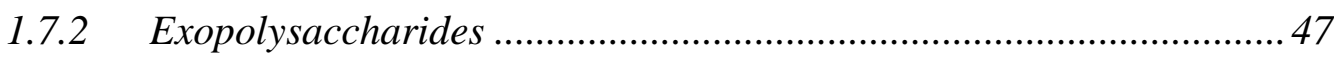

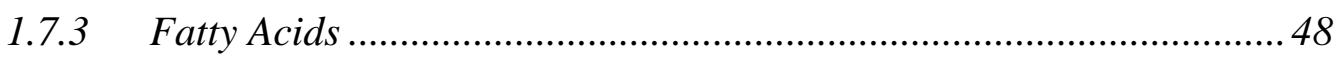

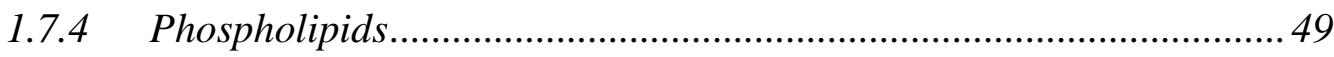

1.7.5 Glycosyldiglycerides and Lipoteichoic Acids ................................... 50

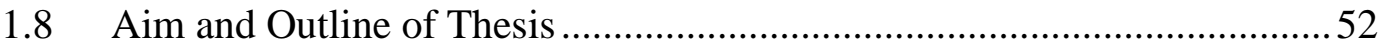

\section{Isolation and Structural Characterisation of the Major Glycoglycerolipids from}

Lactobacillus plantarum ............................................................................................................53

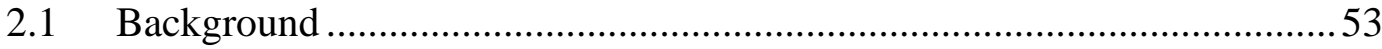

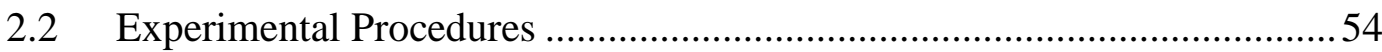

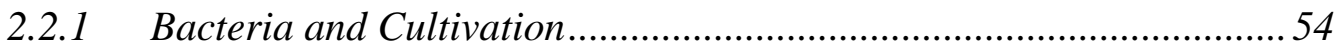

2.2.2 Isolation and Purification ............................................................... 55

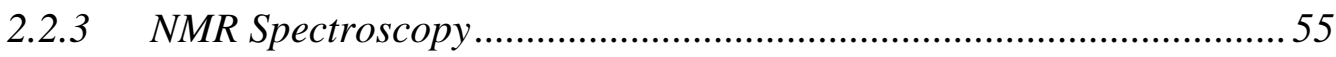

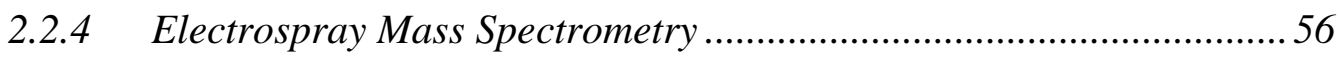

2.2.5 General Analyzes ........................................................................... 56

2.2.6 Constituent Sugar Analysis .............................................................57

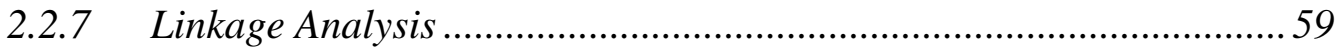

2.2.8 Sugar Absolute Configuration ............................................................ 61

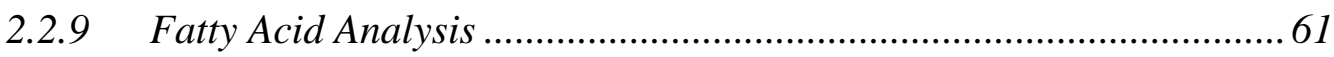

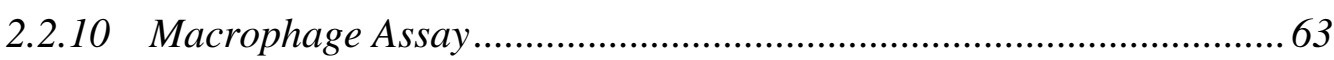

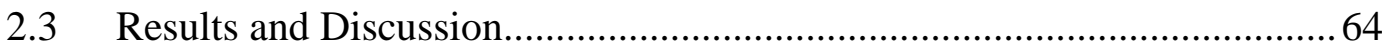

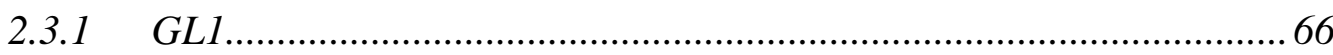

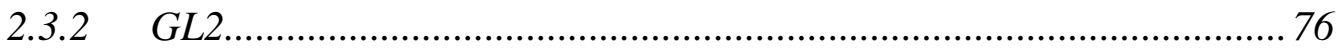

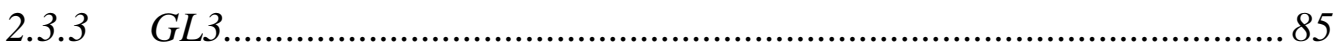


2.3.4 Bioactivity of L. plantarum Glycoglycerolipids .............................. 88

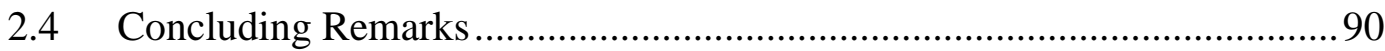

2.5 Supplementary data-NMR, MS spectra and Compound Characterisation.. 91

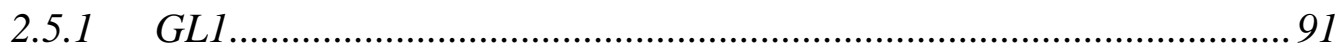

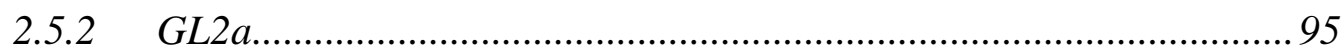

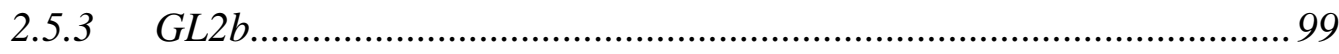

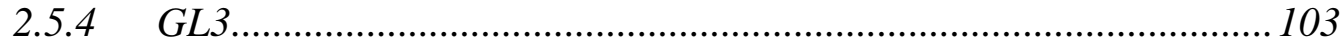

Discovery of a Novel Glycolipid from B. longum subs. infantis (ATCC 15702T) .107

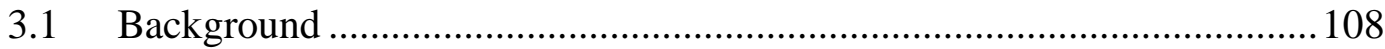

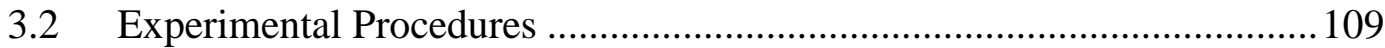

3.2.1 Bacteria and Cultivation ................................................................ 109

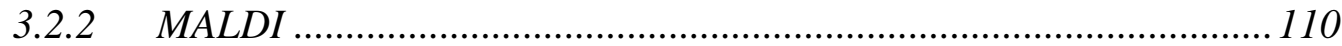

3.2.3 Thin layer chromatography $($ TLC) ............................................. 110

3.2.4 Extraction and purification of glycolipids ..................................... 111

3.2.5 NMR spectroscopy …............................................................... 111

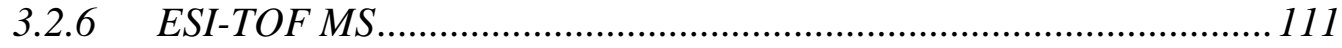

3.2.7 Alditol Acetates Method ............................................................... 111

3.2.8 Glycosyl Linkage Analysis .......................................................... 112

3.2.9 Sugar Absolute Configuration ..................................................... 112

3.2.10 Fatty Acid Analysis ...................................................................... 112

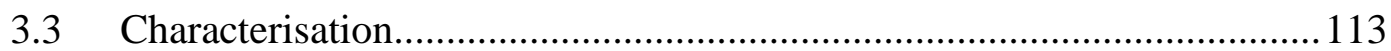

3.3.1 Whole Cell MALDI Analysis ......................................................... 113

3.3.2 Glycolipid Isolation and Structural Characterisation ...................... 114

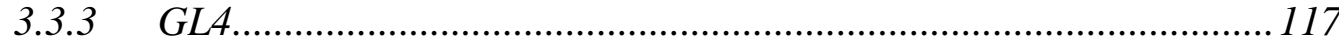

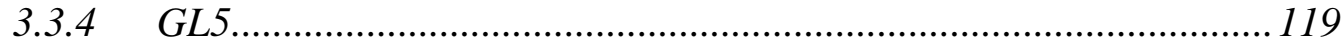

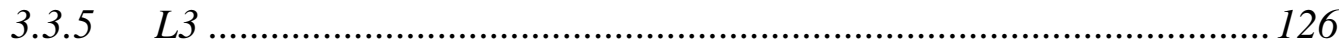

3.4 Biological Screening of Extracted Compounds ....................................... 129

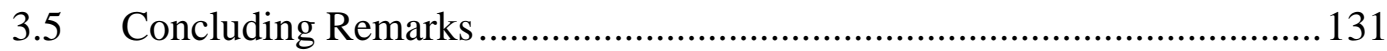

3.6 Supplementary Data-NMR and MS Spectra and Compound

Characterisation

3.6.1 GLA 


3.6.2 GL5

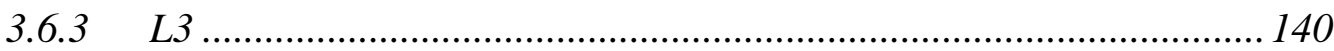

\section{Synthesis and Biological Activity of the Lipoteichoic Acid Anchor from}

Streptococcus sp. DSM 8747 ...................................................................................... 143

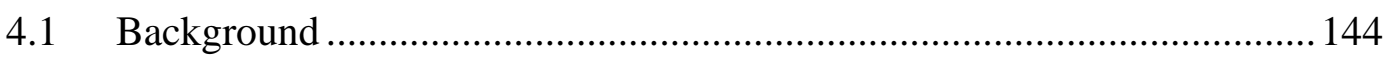

4.2 Results and Discussion................................................................... 145

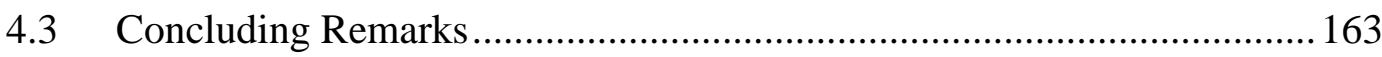

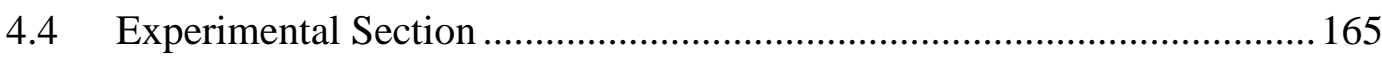

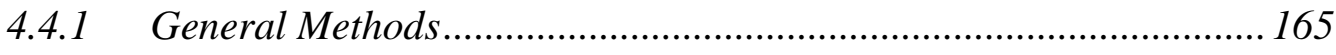

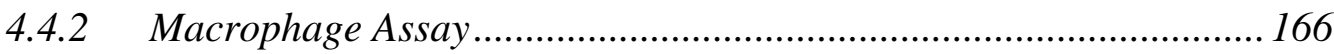

4.4.3 Compound Characterisation............................................................ 167

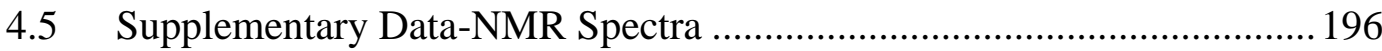

Future Prospects and Conclusion............................................................................... 237

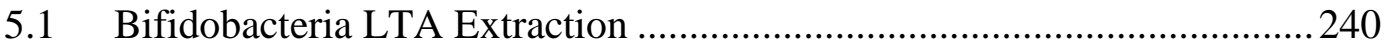

5.2 Stereochemistry of Novel Glycolipid (GL5) ...................................... 243

5.3 Synthesis of Novel Glycolipid (GL5) …............................................. 253

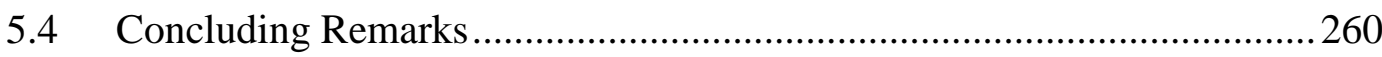

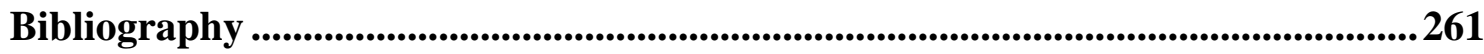




\section{List of Figures}

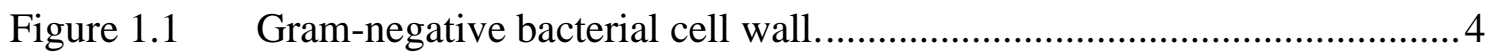

Figure $1.2 \quad$ Gram-positive bacterial cell wall....................................................... 4

Figure 1.3 Repeating unit of an L. Rhamnosus exopolysaccharide. ${ }^{20}$.......................6

Figure 1.4 Teichoic acid from Streptococcus pneumonia ${ }^{24}{ }^{2}$..................................... 7

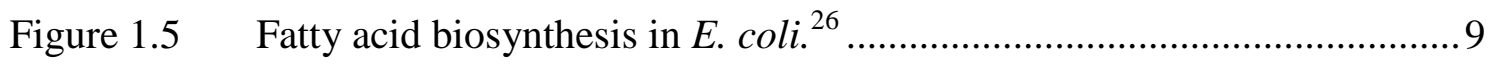

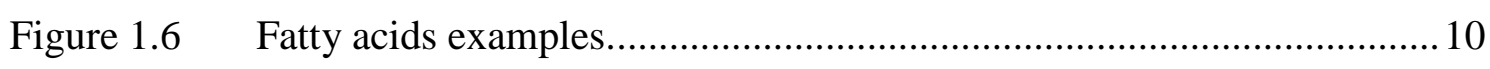

Figure 1.7 LTA biosynthetic pathway for $S$. aureus, from glycerol to phosphatidyl glycerol $14 .^{30,32}$

Figure 1.8 LTA biosynthetic pathway for Staphylococcus aureus, from

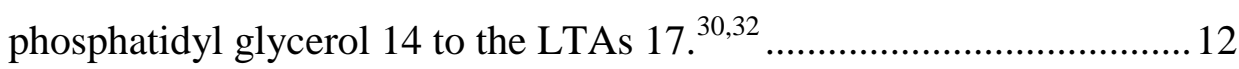

Figure 1.9 Phosphatidylethanolamine (PE).................................................... 13

Figure 1.10 Examples of MGDG, DGDG and TGDGs found in bacteria, as

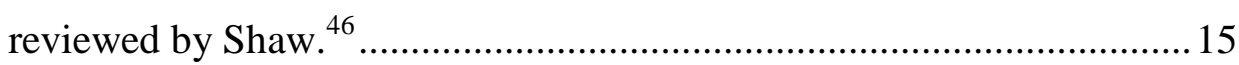

Figure 1.11 Glycolipids found in; a. P. propionicum, ${ }^{51}$ b. D. radiodurans, ${ }^{52}$ c. A. scleromae and A. globiformis. ${ }^{53}$ 16

Figure 1.12 a. S. pneumonia lipoteichoic acid, ${ }^{24}$ b. S. aureus lipoteichoic acid, ${ }^{60}$ c. B. bifidum var. pennsylvanicus lipoteichoic acid. ${ }^{62}$ 19

Figure 1.13 Galactosyldiglycerides synthesised and tested by Kinjo et al. ${ }^{82}$............24

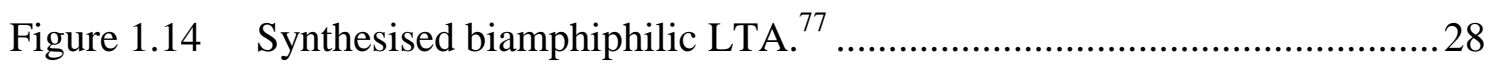

Figure 1.15 Photomicrograph of Bifidobacterium longum subs. infantis ATCC 15702 using a scanning electron microscope measured by David Flinn.

Figure 1.16 Proposed structures of CPS from a. B. breve YIT $4010,{ }^{106}$ b. $B$. adolescentis $^{107}$, c. B. longum YIT4028 $8^{108}$, d. B. catunelatum, $^{109}$ e. B. infantis, ${ }^{105}$ f. B. bifidum BIM B-465. ${ }^{110}$

Figure 1.17 Proposed mannitol teichoic acid from B. bifidum var. pennsylvanicus. $^{114}$ 38

Figure 1.18 EPS repeating unit of a. B. longum JBL05 ${ }^{121}$ and b. B. animalis EPS. ${ }^{119}$

Figure 1.19 Example of a whole bacterial gas chromatogram of fatty acid methyl esters. 
Figure 1.20 Example of fatty acids found in bifidobacteria. 41

Figure 1.21 MS fragmentation spectrum of a. 18:1 DMA ${ }^{131}$ and b. Methyl palmitate. ${ }^{130}$ 43

Figure 1.22 Glycosyldiglycerides identified in B. bifidum var. pensylvanicus. ${ }^{44}$.......44

Figure 1.23 LTA fragment structure proposed for Bifidobacterium bifidum YIT 4007 and YIT 4010, described by Iwasaki. ${ }^{62}$ 45

Figure 1.24 WTA structure from L. plantarum N.I.R.D. C106. ${ }^{164}$............................. 46

Figure 1.25 Ribitol and glycerol phosphate backbones. ${ }^{166}$.......................................47

Figure 1.26 L. plantarum glycolipids isolated by Shaw et al. ${ }^{46}$..................................50

Figure 1.27 L. plantarum LTA fragments isolated by a. Fischer ${ }^{190}$ and b. Jang et $a l .^{57}$

Figure 2.1 GC-MS chromatogram of GL1 alditol acetates showing the glucitol hexaacetate signal. .58

Figure 2.2 Glucitol hexaacetate electron impact spectrum taken from the signal at 29.3 minutes in GL1 chromatogram (Figure 2.1). .58

Figure 2.3 GC-MS chromatogram of GL1 PMAA showing the glucitol PMAA signal.

Figure 2.4 Glucitol PMMA electron impact spectrum taken from the signal at 18.7 minutes in GL1 chromatogram (Figure 2.3).

Figure 2.5 GC-MS traces of (2-hydroxypropylamino)alditol derivatives of Dand L-galactose, its mixture and the crude L. plantarum glycolipid extract showing the presence of D-galactose in the glycolipids.

Figure 2.6 TLC of glycolipid and purified fractions from L. plantarum IRL560. Silica gel TLC, eluted with $\mathrm{CHCl}_{3} / \mathrm{MeOH}(6 / 1$, v/v) and developed with a cerium molybdate stain.

Figure 2.7 GC-MS chromatogram of silylated L2 FAME showing the silylated hydroxyoctadecanoic FAME signal.

Figure 2.8 Silylated hydroxyoctadecanoic FAME electron impact spectrum taken at 56 minutes in L2 chromatogram (Figure 2.7). 68

Figure 2.9 GC-MS chromatogram of L2 FAME showing the unsilylated hydroxyoctadecanoic FAME signal.

Figure 2.10 Unsilylated hydroxyoctadecanoic FAME electron impact spectrum taken from the signal at 56 minutes in L2 chromatogram (Figure 2.9) 
Figure 2.11 Proton NMR spectrum of GL1 showing typical signals for dihydrosterculic acid, ${ }^{1} \mathrm{H}$ spectrum $\mathrm{CD}_{3} \mathrm{OD} / \mathrm{CDCl}_{3}(1 / 2$, v/v), 500 $\mathrm{MHz}$

Figure 2.12 GC-MS chromatogram of opened cyclopropane ring FAME showing the signal for the branched FAME.

Figure 2.13 Electron impact spectrum of the signal at $42.8 \mathrm{~min}$ in the GC-MS spectrum in Figure 2.12. .73

Figure 2.14 Proton to proton correlations showing the correlation (in blue) between GL1 H-2 and $\mathrm{H}-1 / \mathrm{H}-3$ in the ${ }^{1} \mathrm{H},{ }^{1} \mathrm{H}$ COSY spectrum $\mathrm{CD}_{3} \mathrm{OD} / \mathrm{CDCl}_{3}(1 / 2, \mathrm{v} / \mathrm{v}), 500 \mathrm{MHz}$

Figure 2.15 HMBC correlations proving the connections $\mathrm{C}-\mathrm{1}^{\prime}$ to $\mathrm{C}-3$ and $\mathrm{C}-1$ to $\mathrm{C}(\mathrm{O})$ in GL1. 75

Figure 2.16 ESI MS/MS of GL1 in positive ion mode. 76

Figure 2.17 TLC of glycolipid and purified fractions from L. plantarum IRL560. Silica gel, eluted with $\mathrm{CHCl}_{3} / \mathrm{MeOH}(6 / 1, \mathrm{v} / \mathrm{v})$ and developed with sulfuric acid stain

Figure 2.18 HMBC correlations proving the connections $\mathrm{C}-1^{\prime}$ to $\mathrm{C}-3, \mathrm{C}-2$ to $\mathrm{C}(\mathrm{O}), \mathrm{C}-1$ to $\mathrm{C}(\mathrm{O})$ and $\mathrm{C}-1^{\prime \prime}$ to $\mathrm{C}-2^{\prime}$.

Figure 2.19 ESI-MS/MS of GL2a in positive ion mode 80

Figure 2.20 Proton to proton correlations showing the correlation between shielded $\mathrm{H}-1^{\prime \prime \prime}$ and $\mathrm{H}-2^{\prime \prime \prime}$ in the ${ }^{1} \mathrm{H},{ }^{1} \mathrm{H}$ COSY spectrum of GL2b $\mathrm{CD}_{3} \mathrm{OD} / \mathrm{CDCl}_{3}(1 / 2, \mathrm{v} / \mathrm{v}), 600 \mathrm{MHz}$

Figure 2.21 HMBC correlations proving the connections of $\mathrm{C}-1^{\prime}$ to $\mathrm{C}-3, \mathrm{C}-2$ to $\mathrm{C}(\mathrm{O}), \mathrm{C}-1$ to $\mathrm{C}(\mathrm{O}), \mathrm{C}-1^{\prime \prime}$ to $\mathrm{C}-2^{\prime}, \mathrm{C}-1^{\prime \prime \prime}$ to $\mathrm{C}-6^{\prime \prime}$ and $\mathrm{C}-6^{\prime}$ to $\mathrm{C}(\mathrm{O})$ in GL2b 83

Figure 2.22 ESI-MS/MS of GL2b in positive ion mode 85

Figure 2.23 ESI MS/MS of GL3 in positive ion mode. 88

Figure 2.24 Glycoglycerolipids NO production by BMMs. BMMs were stimulated with glycoglycerolipid (GL1, GL2a, GL2b and GL3) (20 $\mu \mathrm{g} / \mathrm{mL}$ and $40 \mu \mathrm{g} / \mathrm{mL})$, or LPS $(100 \mathrm{ng} / \mathrm{mL})$ and the supernatants were analyzed for NO after $48 \mathrm{~h}$ using the Griess assay. Mean and SD of triplicate samples from a representative experiment of two are indicated. 
Figure 3.1 MALDI spectrum of heat-killed whole cells of B. longum subs. infantis ATCC15702T.

Figure 3.2 TLC of purified glycolipids from Bifidobacteria. B. $l$. crude lipid extract, L1-3, GL4 and GL5 extracted from B. longum subs. infantis. Silica gel, eluted with $\mathrm{CHCl}_{3} / \mathrm{MeOH}(6 / 1, \mathrm{v} / \mathrm{v})$ and developed with Hannessian's stain.

Figure 3.3 ESI MS/MS of GL4 in positive ion mode.

Figure 3.4 Proton to proton correlations showing that the acetal signal correlated to the fatty acid chains in the ${ }^{1} \mathrm{H},{ }^{1} \mathrm{H}$ COSY spectrum of GL5, $\mathrm{CD}_{3} \mathrm{OD} / \mathrm{CDCl}_{3}(1 / 2, \mathrm{v} / \mathrm{v}), 600 \mathrm{MHz}$.

Figure 3.5 Proton to carbon correlations showing the presence of a furanose moiety and the connections between the different parts of the molecule in the ${ }^{1} \mathrm{H},{ }^{13} \mathrm{C}$ HMBC of GL5, $\mathrm{CD}_{3} \mathrm{OD} / \mathrm{CDCl}_{3}(1 / 2, \mathrm{v} / \mathrm{v})$, $600 \mathrm{MHz}$.

Figure 3.6 MS/MS of GL4 in a. positive ion mode and b. negative ion mode. 124

Figure 3.7 Plasmalogalactosylalkylglycerol glycolipid extracted from equine brain. ${ }^{255,256}$

Figure 3.8 Glyceroplasmalopsycosine extracted from bovine brain. ${ }^{257}$ 125

Figure 3.9 Proposed mechanism showing coupling between plasmalogen lipids and DAG. ${ }^{261}$ 125

Figure 3.10 MSMS of L3a in negative ion mode. 127

Figure 3.11 MSMS of L3b in negative ion mode. 127

Figure 4.1 Lipoteichoic acid from Streptococcus sp. DSM 8747 145

Figure 4.2 $\quad{ }^{13} \mathrm{C}$ NMR spectrum of compounds 20 1., sn-3-20 2. and sn-1-20 3. showing the anomeric and acetal quaternary carbon region.

Figure 4.3 Magnification of the COSY NMR spectrum of $s n-3-7$ a showing the correlation between H-6' and the hydroxyl proton.

Figure 4.4 Magnification of sn-3-22a HMBC spectrum showing the correlations between $\mathrm{H}-6 \mathbf{6}^{\prime} \mathrm{a}, \mathrm{H} 6^{\prime} \mathrm{b}, 1$ and 2 to their respective carbonyl carbons.

Figure 4.5 $\quad{ }^{1} \mathrm{H},{ }^{31} \mathrm{P}$ HMBC of $s n-3-23 \mathrm{a}$ showing the correlation between H6'a,

H6'b and the phosphorous. 156 
Figure 4.6 Magnification of ${ }^{13} \mathrm{C}$ NMR spectrum from $s n-3-33$ a showing separate signals for the two phosphotriesters and coupling with phosphorous (e.g. Two signals for C-2", one broad signal for C-6').....161

Figure 4.7 BMM were stimulated with LTA-derivatives (2a-d, 3a, 4a, 5a) (20 $\mu \mathrm{g} / \mathrm{mL}$ and $40 \mu \mathrm{g} / \mathrm{mL})$, or LPS (100 ng/mL), or $s n-3-$ galactofuranosyl-glycerol (GalfGro, $20 \mu \mathrm{g} / \mathrm{mL}$ and $40 \mu \mathrm{g} / \mathrm{mL}$ ) and the supernatants analyzed for NO after $48 \mathrm{~h}$ using the Griess assay. Mean and SD of triplicate samples from a representative experiment of two are indicated.

Figure 5.1 Glycoglycerolipids NO production by BMMs. BMMs were stimulated with glycoglycerolipids (GL1, GL2a, GL2b, GL3, GL4 and GL5) $(20 \mu \mathrm{g} / \mathrm{mL}$ and $40 \mu \mathrm{g} / \mathrm{mL})$, or LPS (100 $\mathrm{ng} / \mathrm{mL})$ and the supernatants were analyzed for NO after $48 \mathrm{~h}$ using the Griess assay. Mean and SD of triplicate samples from a representative experiment of two performed are indicated

Figure $5.2 s n-3-2 \mathrm{~b}$ activates BMM.

Figure 5.3 BMM were stimulated with a LTA extract from Bifidobacteria, (20 $\mu \mathrm{g} / \mathrm{mL}$ and $40 \mu \mathrm{g} / \mathrm{mL})$, or LPS (100 $\mathrm{ng} / \mathrm{mL})$ and the supernatants analyzed for NO using the Griess assay after $48 \mathrm{~h}$. Mean and SD of triplicate samples from a representative experiment of two performed are indicated.

Figure 5.4 Structure of GL5 showing the 3 unknown stereocenters.

Figure 5.5 GC-MS chromatogram of derivatised glycerol 21.

Figure 5.6 $\quad{ }^{13} \mathrm{C}$ NMR spectrum expansion of derivatised glycerol 21 showing the presence of diastreomers. 
xviii 


\section{List of Schemes}

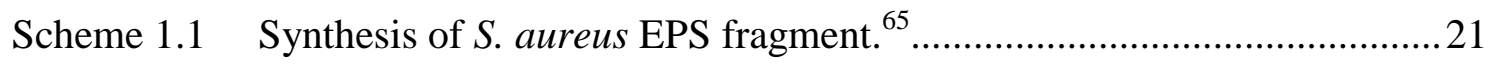

Scheme 1.2 Retrosynthesis of a Enterococcus faecalis fragment. ${ }^{70}$...........................22

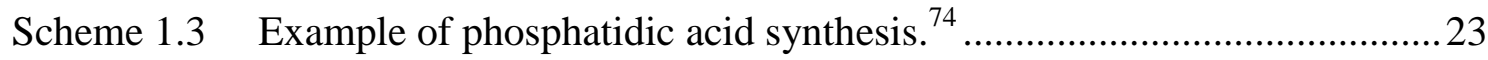

Scheme 1.4 Synthesis of 1,2-di-O-octanoyl-3-O- $\beta$-D-galactopyranosyl-snglycerol. ${ }^{84}$

Scheme 1.5 Synthesis of 1-O-palmitoyl-2-O-oleoyl-3- $O$ - $\alpha$-D-galactopyranosylsn-glycerol. ${ }^{85}$

Scheme 1.6 Retrosynthetic scheme of Staphylococcus aureus LTA adapted from Stadelmaier et al. ${ }^{77}$

Scheme 1.7 Synthesis of galactofuranose building block for Streptococcus

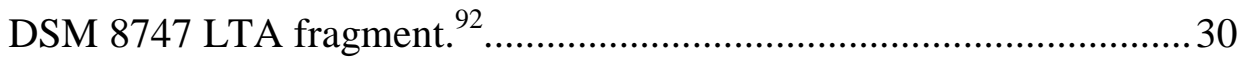

Scheme 1.8 Synthesis of Streptococcus pneumonia LTA fragment. ${ }^{92}$...................... 31

Scheme 1.9 Synthesis of B. catunelatum cell wall polysaccharide fragment. ${ }^{113}$........37

Scheme 1.10 Dimethyl acetal formation under acidic conditions. ${ }^{131}$........................... 41

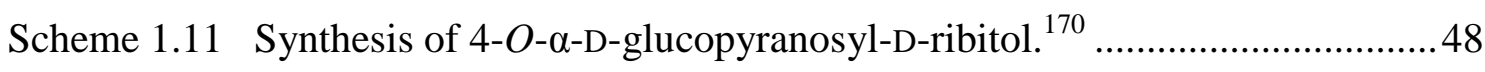

Scheme 1.12 Biomechanism of cyclopropane fatty acid formation. ${ }^{180}$.........................49

Scheme 2.1 Phenol-sulfuric acid reaction with glucose...........................................56

Scheme 2.2 Constituent sugar analysis forming glucitol hexaacetate from GL1.......57

Scheme 2.3 Glucosyl linkage analysis method forming a PMAA from GL1.............59

Scheme 2.4 Derivatisation of D-galactose into a (2-hydroxypropylamino)alditol using sodium cyanoborohydride and $R$-(-)-1-amino-2-propanol.

Scheme 2.5 Cyclopropane ring opening via hydrogenation in acetic acid using

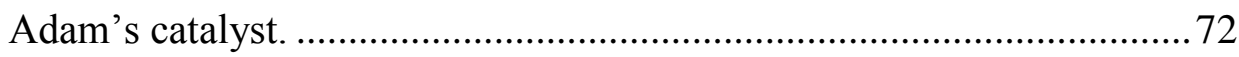

Scheme 3.1 Plasmalogen biosynthetic pathway in animals. ${ }^{263}$............................... 128

Scheme 4.1 Retrosynthesis of the LTA glycolipid anchor and lipidated

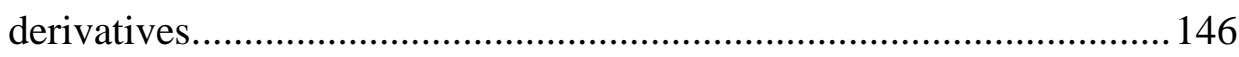

Scheme 4.2 $R$-(-)-Solketal ((-)-10) synthesis from the method of Hirth et al ${ }^{281} \ldots . . .147$

Scheme $4.3 \quad R$-(-)-Solketal ((-)-10) synthesis from the method of Lok et al. ${ }^{283} \ldots \ldots . . .148$

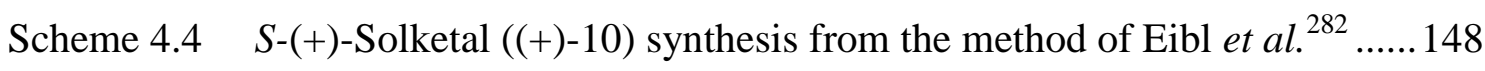

Scheme 4.5 Synthesis of core glycolipid anchor 2. ............................................. 149

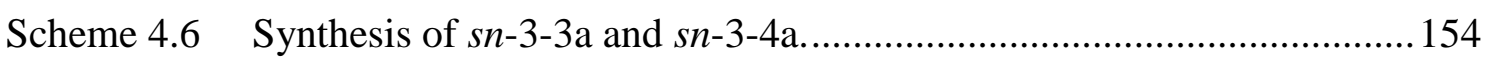


Scheme 4.7 Attempt to synthesise phosphoramidite 26.

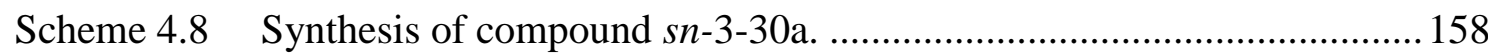

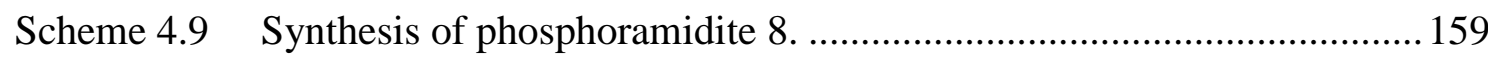

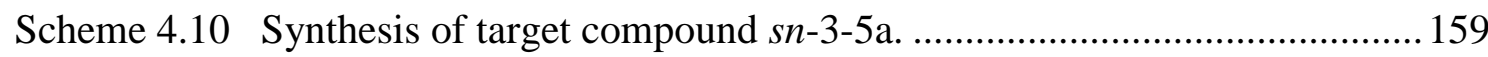

Scheme 5.1 Degradation method for absolute configuration determination by

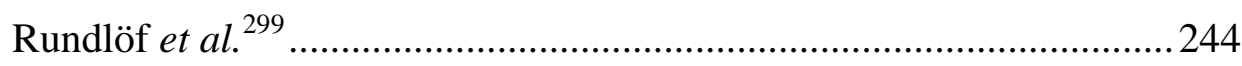

Scheme 5.2 Derivatisation methods adapted from Rundlöf et al ............................2246

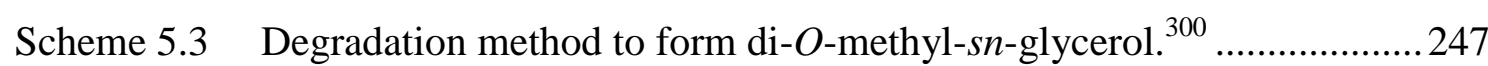

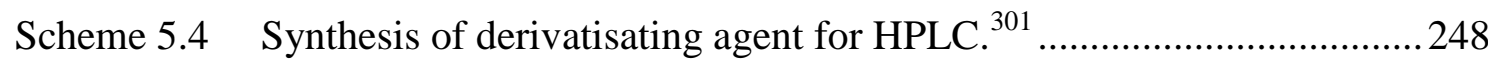

Scheme 5.5 Attempt to synthesise enantiomerically pure 24................................ 248

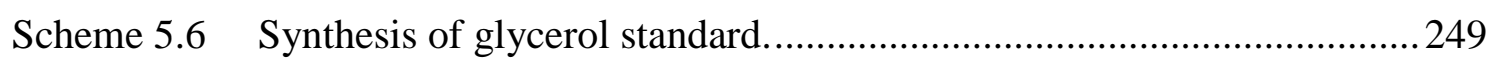

Scheme 5.7 Degradation method to form glycerol derivatives.............................. 252

Scheme 5.8 Retrosynthetic pathway for four different diastereomers of GL5 ........ 255

Scheme 5.9 Analogues for varying the stereochemistry of stereocenter 2 and 3....255

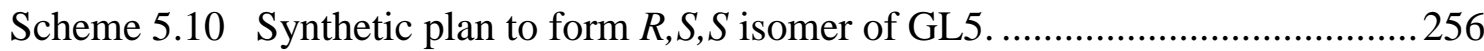

Scheme 5.11 Attempts to synthesise building block 34 to obtain a $S, S$ configuration for GL5. ............................................................. 257

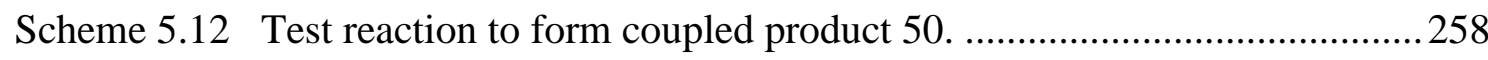

Scheme 5.13 Attempted coupling to form coupled product 52.............................258

Scheme 5.14 Plan for synthesising intermediate 39............................................259 


\section{List of Tables}

Table 2.1 Chemical characteristics of isolated compounds.................................... 70

Table 2.2 NMR spectroscopic data for GL1 .................................................... 74

Table 2.3 NMR spectroscopic data for GL2a.................................................... 79

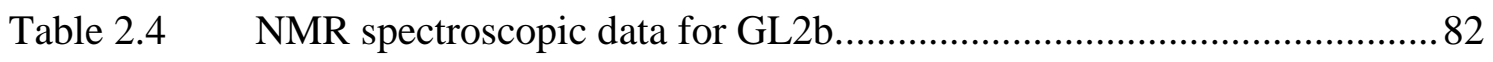

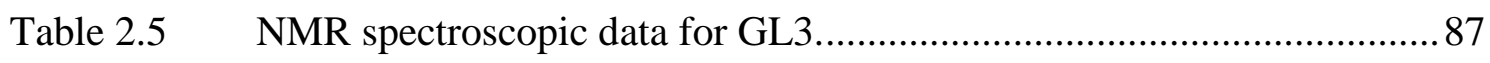

Table 3.1 Chemical characteristics of isolated compounds................................... 116

Table 3.2 NMR spectroscopic data for GL4 .................................................. 118

Table 3.3 NMR spectroscopic data for GL5 f..................................................... 121 


\section{Glossary}

\begin{tabular}{|c|c|}
\hline $\mathbf{A B C}$ & ATP-binding cassette transporter \\
\hline $\mathrm{ACP}$ & Acyl-carrier protein \\
\hline ADP & Adenosine diphosphate \\
\hline AMP & D-Alanine-D-alanyl carrier protein ligase \\
\hline ATCC & American Type Culture Collection \\
\hline ATP & Adenosine triphosphate \\
\hline BAIB & bis(acetoxyiodo)benzene \\
\hline BMM & Bone marrow macrophage \\
\hline CAN & Ceric ammonium nitrate \\
\hline CDP-Gro & Cytidine diphosphate glycerol \\
\hline $\operatorname{CoA}$ & Coenzyme A \\
\hline CDP & Cytidine diphosphate \\
\hline CID & Collision induced dissociation \\
\hline CL & Cardiolipin \\
\hline COSY & Correlation spectroscopy \\
\hline CPS & Capsular polysaccharide \\
\hline CSA & Camphorsulfonic acid \\
\hline CTP & Cytidine triphosphate \\
\hline DcP & D-alanyl-carrier protein \\
\hline DAG & Diacylglycerol \\
\hline DC & Dendritic cell \\
\hline DCC & N,N'-Dicyclohexylcarbodiimide \\
\hline DCE & Dichloroethane \\
\hline DCM & Dichloromethane \\
\hline DBU & 1,8-Diazabicyclo[5.4.0]undec-7-ene \\
\hline DDQ & 2,3-Dichloro-5,6-dicyano-1,4-benzoquinone \\
\hline DEAE & Diethylaminoethyl cellulose \\
\hline DGDG & Diglycosyldiglyceride \\
\hline DHB & 2,5-Dihydroxybenzoic acid \\
\hline DIPA & Diisopropylamine \\
\hline DIPEA & N,N-Diisopropylethylamine \\
\hline
\end{tabular}




\begin{tabular}{|c|c|}
\hline DMA & Dimethyl acetal \\
\hline DMAP & 4-Dimethylaminopyridine \\
\hline DMF & Dimethylformamide \\
\hline DMP & Dimethoxypropane \\
\hline DMSO & Dimethyl sulfoxide \\
\hline DPG & Diphosphatidyl glycerol \\
\hline DSM & $\begin{array}{l}\text { Deutsche Sammlung von Mikroorganismen (German collection of } \\
\text { microorganisms) }\end{array}$ \\
\hline ECL & Equivalent chain length \\
\hline EDCI & 1-Ethyl-3-(3-dimethylaminopropyl)carbodiimide \\
\hline EPS & Exopolysaccharide \\
\hline FAME & Fatty acid methyl ester \\
\hline FID & Flame ionisation detector \\
\hline GC-MS & Gas chromatograph-mass spectrometer \\
\hline GTF & Glycosyl-1-phosphate transferase \\
\hline HSQC & Heteronuclear single-quantum correlation \\
\hline НМBC & Heteronuclear multiple-bond correlation \\
\hline ICR & Imprinting control region \\
\hline IL & Interleukin \\
\hline LAB & Lactic acid bacteria \\
\hline LAL & Limulus amebocyte lysate \\
\hline LPS & Lipopolysaccharide \\
\hline LTA & Lipoteichoic acid \\
\hline MALDI & Matrix-assisted laser desorption spectrometry \\
\hline MGDG & Monoglycosyldiglyceride \\
\hline MIDI & Microbial identification system database \\
\hline MRS & Man-Rogosa-Sharp \\
\hline MSD & Mass selective detector \\
\hline NAD & Nicotinamide adenine dinucleotide \\
\hline NADP & Nicotinamide adenine dinucleotide phosphate \\
\hline NAG & N-acetylglucosamine \\
\hline NAM & $\mathrm{N}$-acetylmuramic acid \\
\hline NIS & N-Iodosuccinimide \\
\hline NO & Nitric oxide \\
\hline
\end{tabular}




\begin{tabular}{|c|c|}
\hline PA & Phosphatidic acid \\
\hline PAMPS & Pathogen -associated molecular patterns \\
\hline PE & Phosphatidyl ethanolamine \\
\hline PBS & Phosphate buffered saline \\
\hline PG & Peptidoglycan \\
\hline PMAA & Partially methylated alditol acetates \\
\hline Prenol-P & Poly(prenylphosphate) \\
\hline PRRs & Pathogen-recognizing receptors \\
\hline PS & Phosphatidyl serine \\
\hline PTSA & p-Toluenesulfonic acid \\
\hline PyBOP & Benzotriazol-1-yl-oxytripyrrolidinophosphonium hexafluorophosphate \\
\hline Pyr & Pyridine \\
\hline quant. & Quantitative \\
\hline $\mathbf{R C M}$ & Refine clostridium media \\
\hline rt & Room temperature \\
\hline RT & Retention time \\
\hline SEM & Scanning electron microscope \\
\hline SPE & Solid-phase extraction \\
\hline TBAF & Tetra- $n$-butylammonium fluoride \\
\hline TBAI & Tetrabutylammonium iodide \\
\hline TCA & Trichloroacetic acid \\
\hline TEA & Triethylamine \\
\hline TEMPO & $(2,2,6,6$-Tetramethylpiperidin-1-yl)oxyl \\
\hline TFA & Trifluoroacetic acid \\
\hline TGDG & Triglycosyldiglyceride \\
\hline THF & Tetrahydrofuran \\
\hline TLC & Thin layer chromatography \\
\hline TLRs & Toll like receptors \\
\hline TMM & Trimethoxymethane \\
\hline TMS & Trimethylsilyl \\
\hline UDP & Uridine diphosphate \\
\hline WTA & Wall teichoic acid \\
\hline
\end{tabular}




\section{Glycoglycerolipid nomenclature ${ }^{\mathrm{a}}$}

The $s n$ (for stereospecifically numbered) notation system will be used in this thesis in order to simplify glycerol nomenclature. As glycerol is prochiral, depending on the substitution on the primary hydroxyls, the $S$ or $R$ prefix used in the Cahn-Ingold-Prelog designation often changes, causing confusion. ${ }^{1}$

In order to determine the stereospecific numbering, the following convention is used: the glycerol unit is drawn in the Fischer projection with the secondary hydroxyl to the left. IUPAC has defined the $s n$ numbering as explained in the subsequent quote.

"Esters, ethers and glucose derivatives of glycerol are designated by a prefix, denoting the substituent, preceded by a locant. The carbon atoms of glycerol are numbered stereospecifically, with carbon atom 1 at the top of the formula shown below. To differentiate this numbering system from others that have been used, the glycerol is always accompanied by the prefix "sn" in systematic and abbreviated names."b

$$
{ }_{3}-{ }_{3}^{2}-\mathrm{OH}
$$

\section{Glycerol}

\section{Example:}<smiles>[R]C(=O)OCC(COC([R])=O)OC(O)C(O)C(O)O</smiles>

\section{1,2-di-O-acyl-3-O-ß-D-galactosyl-sn-glycerol}

\footnotetext{
${ }^{a}$ Adapted from 1.Nomenclature of Lipids. In Biochemical Nomenclature and Related Documents, 2nd Edition, Liébecq, C., Ed. Portland Press: London, 1992; p 10.

${ }^{\mathrm{b}}$ Quoted from: http://www.chem.qmul.ac.uk/iupac/misc/glylp.html
} 


\section{Chapter 1}

\section{Introduction}

\subsection{Gram-Positive and Gram-Negative Bacteria}

Bacteria are prokaryotic organisms populating most environments on earth. They need to survive a large range of environments (high or low temperature, $\mathrm{pH}$ etc.) and thus their cell walls need to be equipped to tolerate harsh exterior conditions. ${ }^{2}$ Some bacteria are pathogenic while others are beneficial and the study of their surface components is important in order to understand the mechanisms involved in their beneficial/pathogenic effects. ${ }^{3,4}$ The surface components of bacteria can be classified using Gram staining. ${ }^{5}$ Gram-positive bacteria possess a thick peptidoglycan (PG) layer which stains a dark violet colour, whereas Gram-negative bacteria stain pink due to the counterstain (saffranin). Both Gram-positive and Gram-negative bacteria can have beneficial and pathogenic effects that can be related to the surface components. The introduction will focus on Gram-positive bacteria and their beneficial effects. In Section 1.2 and 1.3 the Gram-negative and Gram-positive cell walls will be examined. Then, the specific Grampositive structural components and the biosynthesis of these components will be 
considered. Finally, published chemical synthese of structural components and the biological activity of some structures will be discussed.

\subsection{Gram-Negative Bacteria}

The Gram-negative cell wall consists of two phospholipid membranes sandwiching a thin PG layer (Figure 1.1). ${ }^{6}$ The latter usually contains alternating repeating units of $N$ acetylmuramic acid (NAM) and $N$-acetylglucosamine (NAG). PG and smaller molecules such as amino acids, peptides, mono- and oligosaccharides, are situated in the periplasmic place (between both membranes). ${ }^{7}$ The two membranes are composed of phospholipids and proteins. The cell wall components are synthesised in the inner membrane or cytoplasmic membrane. ${ }^{6}$ Small molecules such as small saccharides diffuse through the outer membrane, while bigger molecules such as proteins are blocked out. ${ }^{8}$ The outer membrane also anchors lipopolysaccharides (LPS), antigenic endotoxins, that help solidify the outer membrane and protect it against bile salts and other noxious compounds. ${ }^{6,7}$ This membrane is covalently attached to the PG layer with a lipoprotein. Often, the outer membrane is covered by a proteineous capsular layer (Alayer). ${ }^{9}$

\subsection{Gram-Positive Bacteria}

The Gram-positive cell wall is distinguished from the Gram-negative cell wall by having only a single phospholipid membrane and a PG layer that is much thicker (Figure 1.2). The thick PG strengthens the cell structure as a major constituent of the cell wall and protects the cell against osmotic pressure. ${ }^{10}$ The amino sugars present in the peptidoglycan layer are highly conserved between bacteria. Differences between the peptide compositions are observed between strains and species. In contrast to Gramnegative bacteria containing LPS, Gram-positive bacteria usually possess lipoteichoic acids (LTAs) that are anchored in the phospholipid layer. Some Gram-positive bacteria also hold wall teichoic acids (WTAs), polymers of ribitol phosphates and/or glycerol phosphates, which are not present in Gram-negative bacteria. These WTAs are 
covalently attached to the NAM and are interwoven in the PG layer. LTA is believed to promote autolysis at the septum during binary fission while WTA would be involved in autolysis inhibition in the rest of the cell. ${ }^{11,12}$ Capsular polysaccharides (CPS) are attached to different surface structures on the PG such as the peptides or NAG. They are believed to play a role in the structural stability of the cell. ${ }^{11}$ In contrast to CPS, exopolysaccharides (EPS) can also be produced in some bacteria as a slime, and are believed to have a protective function against the environment such as preventing dessication and phage attack, and to be involved in cell adhesion. ${ }^{13}$ Finally, the phospholipid bilayer, containing proteins and glycolipids, holds the cytoplasm from the exterior of the cell, keeping ions and other molecules from diffusing. ${ }^{14}$

\subsection{Gram-Positive Cell Wall Components and Biosynthetic Pathways}

The structure of Gram-positive cell wall components is often strain/species specific. ${ }^{14}$ In the next section, a general review of the component structures excluding proteins and the peptidoglycan layer will be presented. As the literature on Gram-positive bacteria is abundant (SciFinder ${ }^{\circledR}$ ca. 25727 hits), only examples of the components, extracted in specific bacteria, will be given. Also, the sections on EPS/CPS, WTA and phosphoglycerolipids are important in order to understand how all the components are related and why the decision of choosing glycolipids and LTAs for extraction and synthesis was made. However, as the other components are beyond the scope of this work, the overview will be less extensive.

\subsubsection{Capsular Polysaccharides and Exopolysaccharides}

CPS and EPS are two types of glycan repeating units found in Gram-positive bacteria. These polysaccharides have an organised structure, and they can be very diverse. For example, 93 different CPS were detected for S. pneumonia. ${ }^{15}$ Although these polysaccharides are very diverse, each individual polymer has an organised structure of distinct repeating units. CPS can be liberated after phosphodiester cleavage from the 
cell wall, whereas EPS are released as a slime, therefore the distinction between these polysaccharides is not always apparent during isolation. ${ }^{16,17}$ Furthermore, the CPS and EPS biosynthetic pathways have been found to be similar, adding to the confusion. ${ }^{15}$

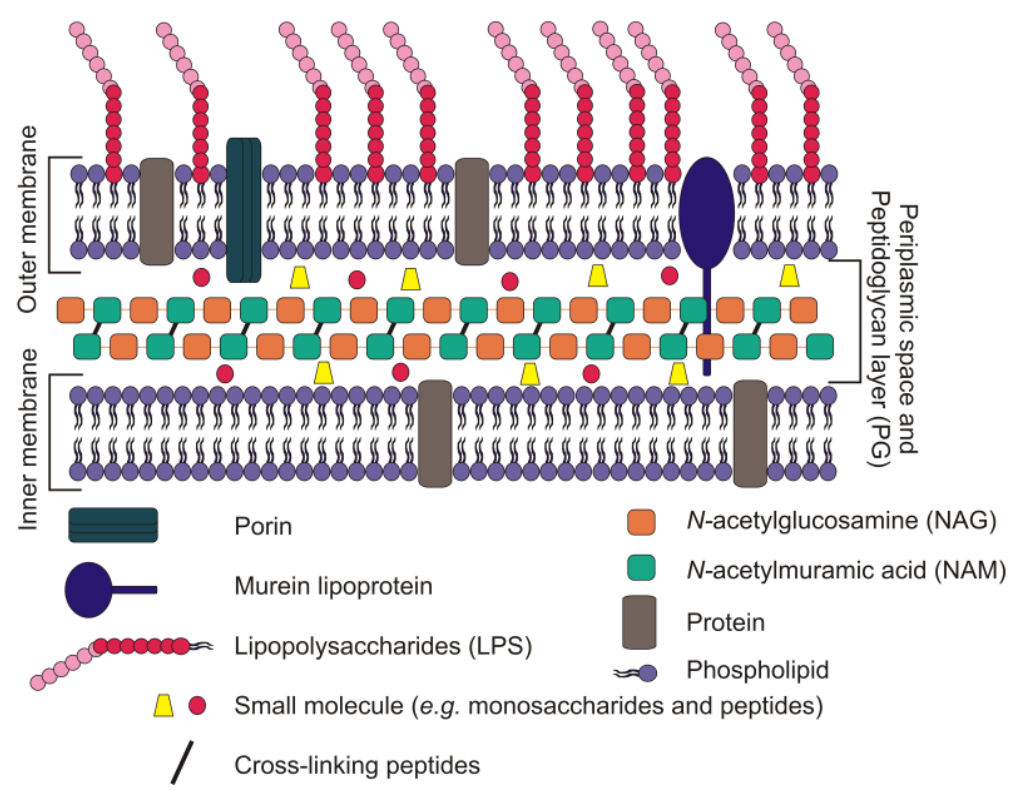

Figure 1.1 Gram-negative bacterial cell wall.

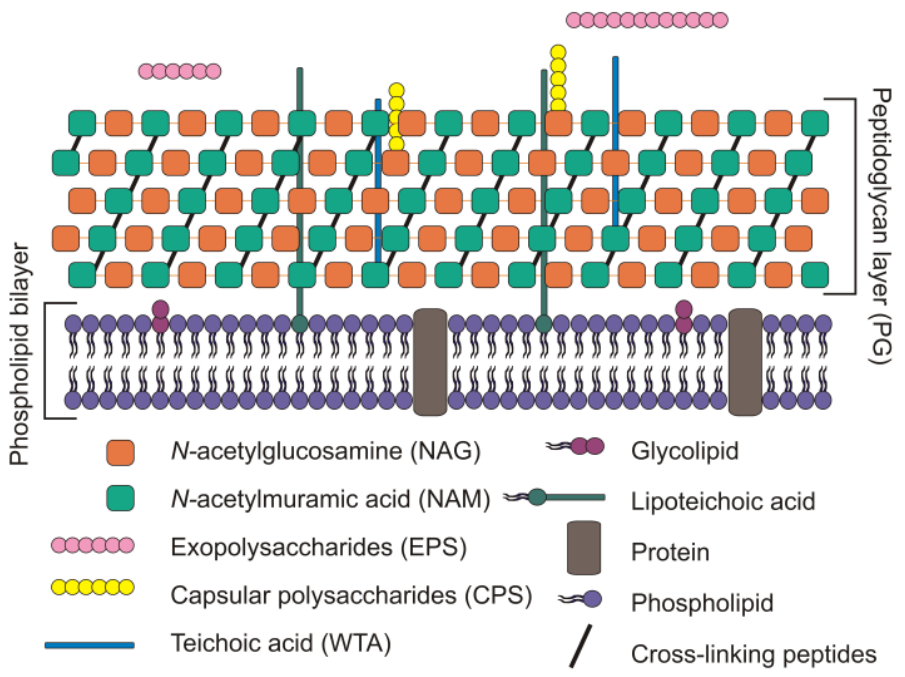

Figure 1.2 Gram-positive bacterial cell wall. 
Both CPS and EPS fall into two general classes of polysaccharides: homo- and heteropolysaccharides. Homopolysaccharides, consisting of a single type of sugar residue are usually synthesised extracellularly with glucansucrases or fuctansucrases. ${ }^{18}$ In contrast, heteropolysaccharides, that comprise several different sugar residues, are synthesised intra- and extracellularly. First, the repeating unit is synthesised intracellularly and transferred to undecaprenol, in the cell membrane, by a glycosyl-1-phosphate transferase (GTF). Additional monosaccharides are added to this structure with specific GTFs and the oligosaccharides are transported outside the cell with a flipase to be subsequently polymerised with a polymerase. ${ }^{15,18}$ EPS are then released while CPS are covalently attached to the PG.

\subsubsection{Extraction and Analysis}

As both polysaccharides have different locations, they are obtained for analysis or biological testing in different ways. EPS are usually precipitated in $60 \%$ ethanol from the bacterial culture supernatant while CPS first needs to be released from the cell wall with pepsin, trypsin and $\mathrm{N}$-acetylmuramidase before extraction with trichloroacetic acid (TCA). The analysis of both polysacchararides is similar and involves constituent sugar and glycosyl linkage analysis, NMR and MS. Previous analyzes showed that the EPS composition as well as the amount of EPS differ depending on the strain, species and media. ${ }^{19}$ Figure 1.3 shows an example of an EPS precipitated with ethanol from $L$. rhamnosus growth medium. ${ }^{20}$

\subsubsection{Wall Teichoic Acids}

WTAs are polymers of poly(alditol phosphates) and/or poly(saccharides phosphates) that are covalently bound to the PG layer. In this document, the teichoic acids (WTAs and LTAs) are segregated according to their differences in structure (The glycerol stereochemistry differs between WTAs and LTAs), anchoring (LTAs are anchored in the membrane while WTAs are covalently bound to the cell surface) and biosynthetic pathway (the WTA pathway utilises undecaprenol as membrane anchor before transfer 
while the LTA pathway doesn't utilise undecaprenol). Interestingly, WTA is considered a virulence factor, as it was found to be essential for colonisation. ${ }^{21,22}$

In the first step of the biosynthetic pathway of $S$. pneumonia WTA, poly(prenyl phosphate) (Prenol-P), anchored in the cell membrane, is coupled successively to NAG and $N$-acetylmannosamine (ManNAc). ${ }^{15}$ Then $s n$-glycerol 3-phosphate is polymerised on ManNAc and the glycerol moieties are partially glucosylated. Alanines are also partially transferred from the LTA to the glycerol catalysed by an ATP-binding cassette transporter $(\mathrm{ABC}){ }^{21,23}$ The WTA is finally covalently coupled to the PG layer on the NAM moiety $(O-6){ }^{14}$

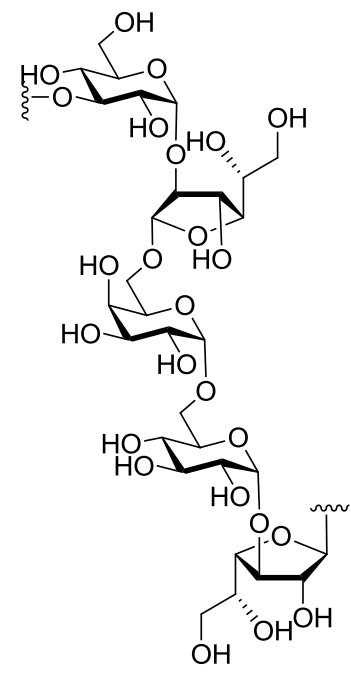

Figure 1.3 Repeating unit of an L. Rhamnosus exopolysaccharide. ${ }^{20}$

\subsubsection{Extraction and Analysis}

WTAs are usually extracted after LTA extraction. Here, the pellet remaining from previous LTA extraction can be re-extracted using TCA/water $(10 \%$, v/v). Further purification steps are then required to obtain pure WTAs (e.g. anion exchange chromatography with DEAE-sephadex). ${ }^{24}$ In Figure 1.4, Streptococcus pneumonia WTA is represented as an example. ${ }^{24}$ 


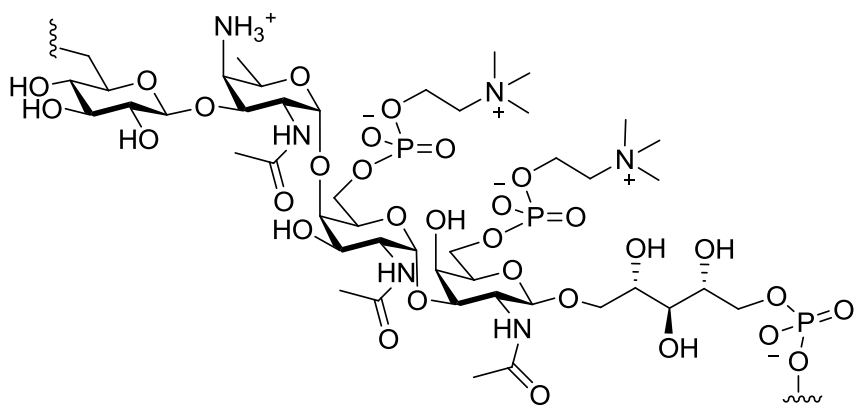

Figure 1.4 Teichoic acid from Streptococcus pneumonia. ${ }^{24}$

\subsubsection{Fatty Acids}

Fatty acids are carboxylic acids containing long aliphatic chains and they are often attached to phospholipids, glycoglycerolipids or LTAs as part of the membrane and thus are usually not found as free fatty acids in the cell. Although most bacteria are known to incorporate exogenous fatty acids in their membranes, ${ }^{25}$ bacteria also utilise fatty acids which are de novo biosynthesised, even though these fatty acids require much more energy for their synthesis. The fatty acid biosynthetic pathway adapted from Chan and Vogel for the Gram-negative bacteria Escherichia coli is believed to be similar to Gram-positive bacteria (Figure 1.5). ${ }^{26}$ First, Apo-Acyl carrier protein (Apo-ACP) is converted into an acceptor thiol (holo-ACP) through ACP synthase. The coupling of $\mathrm{HCO}_{3}{ }^{-} \mathbf{1}$ and acetyl-CoA 2 is catalysed by acetyl-CoA carboxylase and malonyl-CoA 3 is obtained. Holo-APC is then transferred to malonyl-CoA 3 by a Malonyl-CoA-ACP transacetylase (FabD). The next step involves $\beta$-oxoacyl synthase III (FabH) for the combination of malonyl-ACP 4 and acetyl-CoA 2 to form $\beta$-oxobutyryl-ACP 5 in a first step designed as "a" (pink pathway). Step " $b$ " represents the reduction of ketone $\mathbf{5}$ into alcohol $\mathbf{6}$ and is made by the $\beta$-oxoacyl reductase (FabG) with NADPH. Alkene 7 is obtained by dehydration with a $\beta$-hydroxyacetyl dehydratase (FabZ, step c). Here, cisproduct 9 is obtained if FabA is present (step f, blue pathway). However, when FabZ is present, trans-product 7 results (black pathway). In the blue pathway, the hydrogenation step is omitted as the enzyme doesn't hydrogenate the cis products. Then elongated compound $\mathbf{1 0}$ is formed as the molecule is further elongated with FabB and acid $\mathbf{4}$. After this, elongated $\mathbf{1 0}$ re-enters the black cycle (step h). Finally, hydrogenation of 
alkene 7 with NADH and an enoyl-reductase (FabI) gives hydrogenated product 8 (step d). This intermediate can then be extended with malonyl-ACP 4 and $\beta$-oxoacyl synthase (FabF). The four last steps (elongation, reduction, deshydratation and hydrogenation) can be repeated until the chain reaches a proper length for being introduced into the membrane with acyltransferases. This length is controlled by enzymes present in the different bacteria. Here, a range of fatty acid chain lengths are introduced into the lipids. The vast range of fatty acids in the microbiome is due to the effect of the enzyme's substrate specificity in each species. ${ }^{27}$

\subsubsection{Extraction and Analysis}

Usually, fatty acid analysis involves a simple transesterification from the cell biomass using sodium methoxide and methanol, and then treatment with $\mathrm{HCl}$ in $\mathrm{MeOH}$ before injection in the gas chromatograph (GC) and gas-chromatograph mass-spectrometer $(\mathrm{GC}-\mathrm{MS}){ }^{28}$ In bacteria, fatty acid analysis usually shows a mixture of different fatty acid chain lengths between $\mathrm{C} 12$ to $\mathrm{C} 20$, saturated or monounsaturated. ${ }^{29}$ Examples of fatty acids found in bacteria are presented in Figure 1.6. 

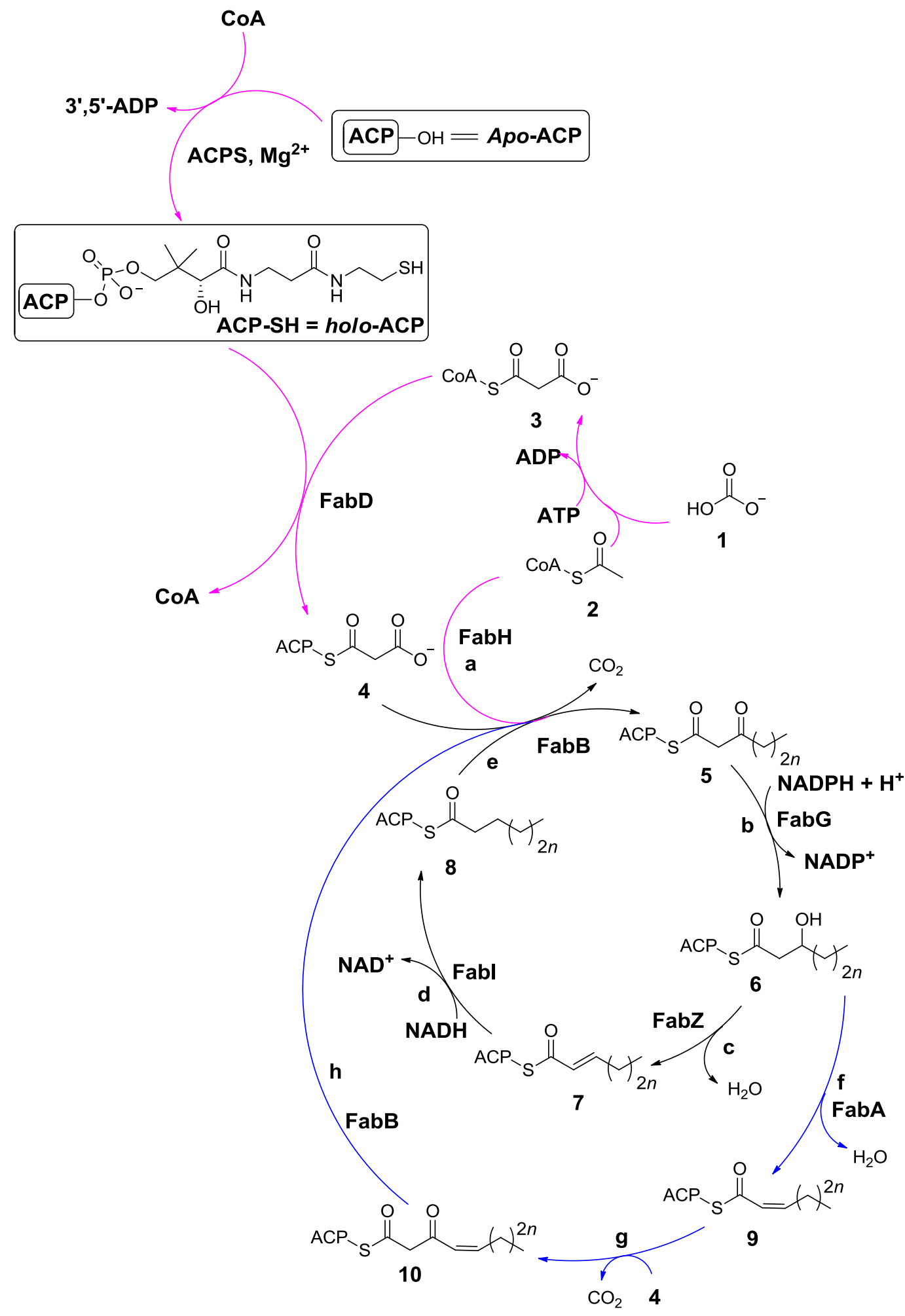

Figure 1.5 Fatty acid biosynthesis in E. coli. ${ }^{26}$ 


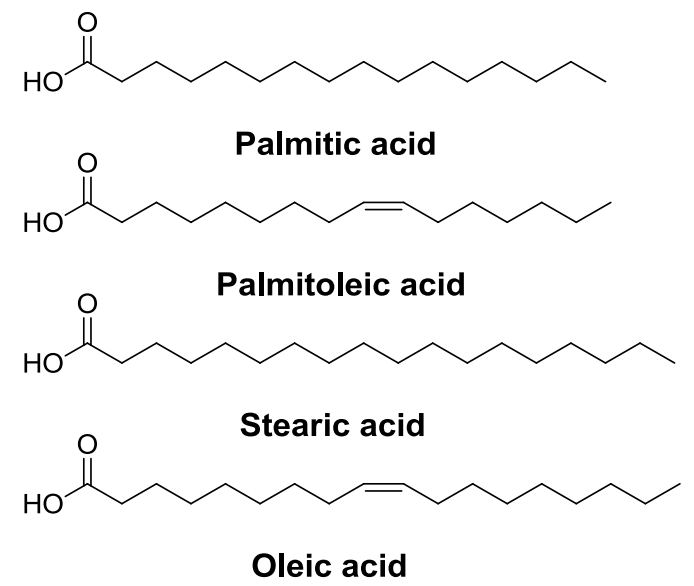

Figure 1.6 Fatty acids examples.

\subsubsection{Glycolipids and Phospholipids}

Glycoglycerolipids are neutral lipids with single sugars or short oligosaccharides. They are related to phospholipids and LTAs through their biosynthetic pathway. The Staphylococcus aureus biosynthetic pathway is similar to most Gram-positive bacteria pathways (Figure 1.7). ${ }^{30-32}$

In the first biosynthetic step, glycerol is selectively phosphated by the action of a glycerol kinase Figure 1.7. The phosphate is transferred from adenosine triphosphate (ATP) to the C-3 of the glycerol to give sn-glycerol 3-phosphate $\mathbf{1 1}$ and release adenosine diphosphate (ADP) to be recycled. Subsequently, two acyl groups are transferred from an acylated acyl carrier protein (Acyl-ACP) to sn-glycerol 3-phosphate with acyltransferases. Following this step, the coenzyme cytidine triphosphate (CTP) reacts with 3-phosphatidic acid (PA) 12 to form cytidine diphosphate diacylglycerol (CDP-DAG). In a first instance, CDP-DAG can be the precursor for phosphatidylserine (PS) and phosphatidylethanolamine (PE) after the elimination of $\mathrm{CO}_{2}$. Also, phosphatidyl glycerophosphate $\mathbf{1 3}$ can be formed following the reaction of CDP-DAG with sn-glycerol 3-phosphate via the action of phosphatidylglycerolphosphate synthase. Phosphatidyl glycerol $\mathbf{1 4}$ is obtained after the elimination of a phosphate via the action of phosphatidylglycerolphosphate phosphatase, and used to either build cardiolipin 
(Figure 1.7) or to introduce sn-glycerol 1-phosphate to the glycosyldiglyceride (Figure 1.8).

Monoglucosyldiglyceride $\mathbf{1 5}$ is generated via YpfP enzyme which adds a first glucose residue to DAG via an activated form of glucose (UDP-Glc) and UDP is released (Figure 1.8). This step is repeated to form a diglucosyldiglyceride 16, which then enters the membrane to act as an anchor for LTA. The condensation of diglucosyldiglyceride 16 with phosphatidyl glycerol 14 to release DAG, is catalysed via LTA polymerase (LtaS) and thus form the lipoteichoic acid $17 .^{33}$ The LTA phosphoglycerol chain is then further elongated with multiple units of $s n$-glycerol 1-phosphate from phosphatidylglycerol $14 .{ }^{34}$ From there the LTA can be alanylated which requires a D-alanine-Dalanyl carrier protein ligase (AMP) and a D-alanyl-carrier protein (Dcp). ${ }^{35}$

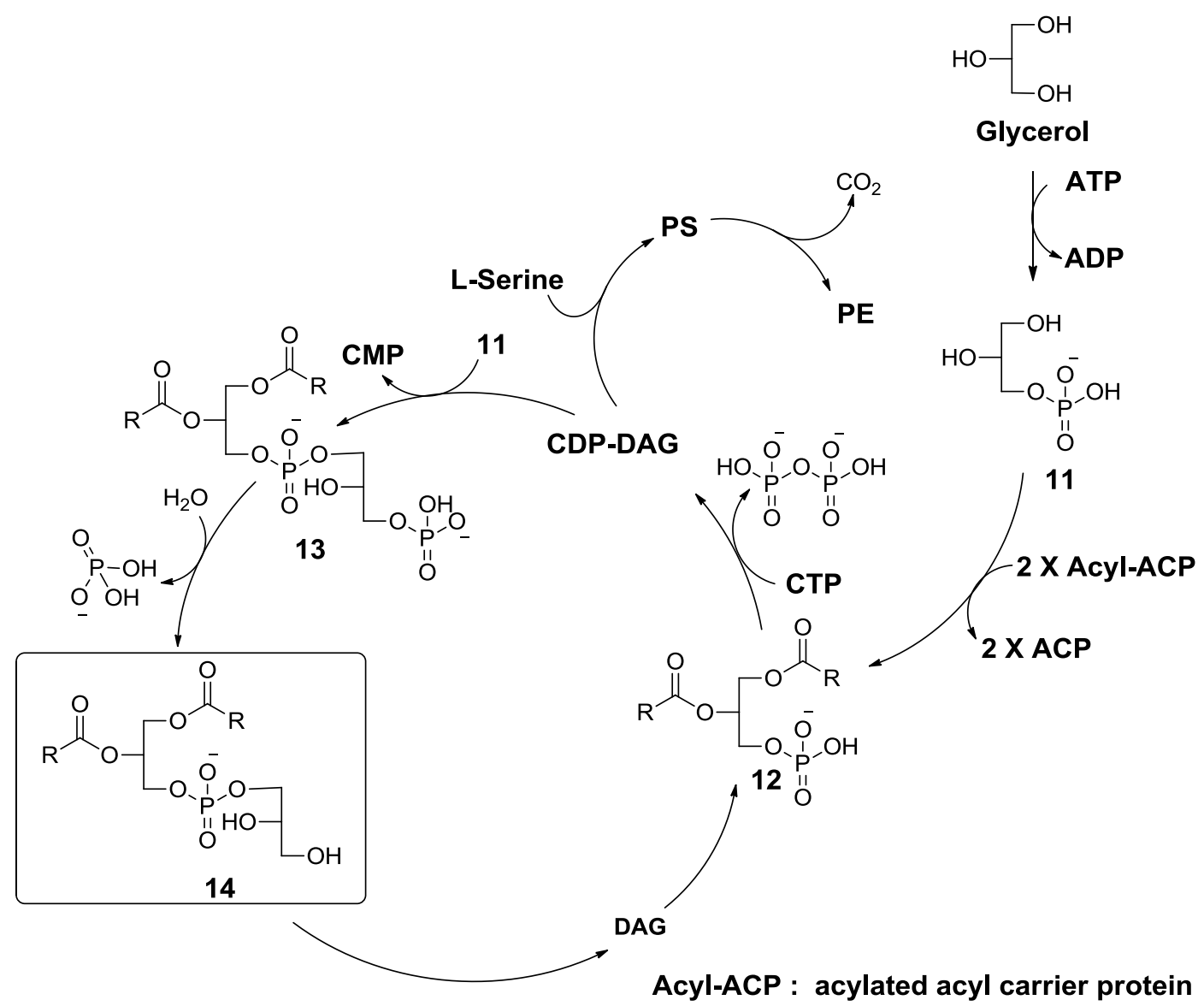

Figure 1.7 LTA biosynthetic pathway for S. aureus, from glycerol to phosphatidyl glycerol $14 .{ }^{30,32}$ 


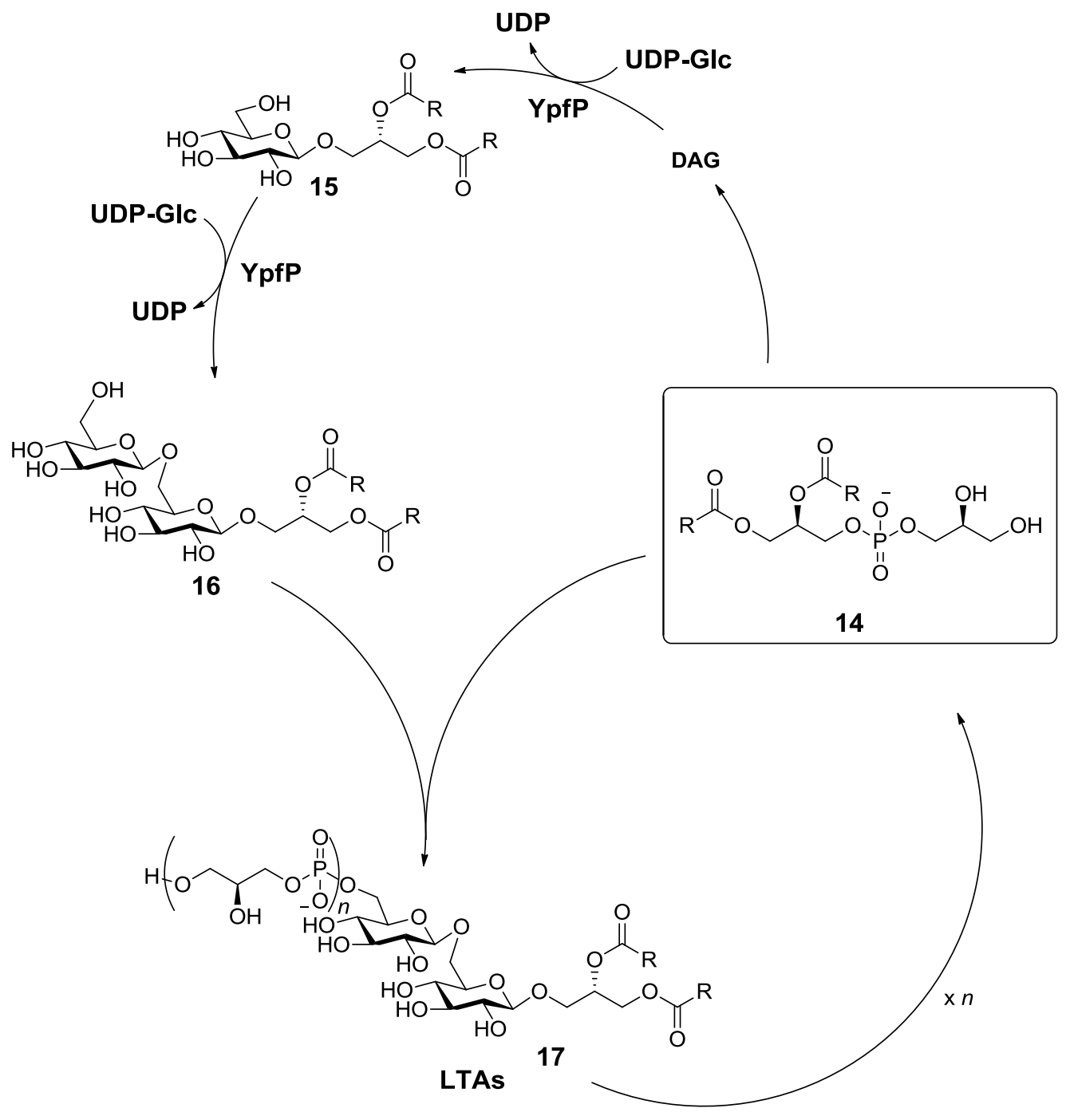

Figure 1.8 LTA biosynthetic pathway for Staphylococcus aureus, from phosphatidyl glycerol $\mathbf{1 4}$ to the LTAs $\mathbf{1 7} .^{30,32}$

\subsubsection{Phosphoglycerolipids}

Most of the research on lipids has focussed on phosphoglycerolipids ${ }^{36}$ as the phosphoglycerolipids are a major part of bacterial membranes (phospholipid layer). The major phospholipids found in Gram-positive bacterial membranes are phosphatidyl glycerol and phosphatidylethanolamine (PE). Although PE (Figure 1.9) is not found in all Gram-positive bacteria, it can form a significant part of the cell wall and cardiolipin (CL) can also be present. ${ }^{29,37}$ An example of phosphoglycerolipid cell wall composition 
was published by Golfine et al. ${ }^{38}$ They detected PE (35\%), phosphatidyl glycerol (48\%), CL (11\%) and glucosaminyl phosphatidylglycerol (6\%) in the membrane of Bacillus megaterium.

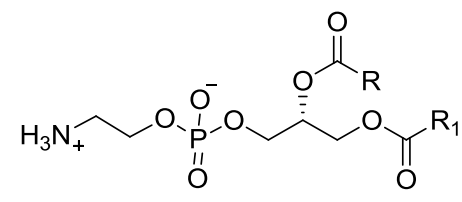

Figure 1.9 Phosphatidylethanolamine (PE).

\subsection{Extraction and Analysis}

For analysis purposes, phospholipids are extracted with $\mathrm{CHCl}_{3} / \mathrm{MeOH} / \mathrm{H}_{2} \mathrm{O}$ and purified using silica gel chromatography. Usually, their analysis involves the comparison of standards with LC/MS spectra. ${ }^{39}$

\subsubsection{Glycoglycerolipids}

Bacterial glycoglycerolipids have been widely studied. ${ }^{40}$ They are usually composed of carbohydrate residues for which the anomeric center is glycosidically bound to the 3position of sn-1,2-diglycerides. To determine the complete structure of such compounds, the nature of the constituent sugars, the linkage between the residues, the linkage between the saccharide and the glycerol, the location and identification of the fatty acids and the stereochemistry of the sugar must be known. There are major differences in how the chemical analyzes are performed today compared to how they were conducted in the past. This is discussed in more detail in the next section. Glycoglycerolipids are neutral lipids such as monoglycosyldiglyceride (MGDG) and diglycosyldiglyceride (DGDG), and are mostly found in bacteria lacking PE. As glycoglycerolipids usually don't form bilayer structures, they are believed to provide nonleaky discontinuity in the membrane. Other biological functions such as membrane 
fusion for cell division and the integration of non-lipid components into the membrane can be assisted by these glycoglycerolipids. ${ }^{37}$

\subsection{Extraction and Analysis}

As glycolipids are not covalently attached to the cell wall, they are readily extracted using $\mathrm{CHCl}_{3} / \mathrm{MeOH} / \mathrm{H}_{2} \mathrm{O}$ mixtures from fresh or lyophilised cells. ${ }^{41,42}$ In the past, glycoglycerolipids were extracted and analyzed using different staining methods, enzymatic reactions, paper chromatography with or without sodium periodate oxidation and sometimes silylation followed by GC. ${ }^{43-45}$ These analyzes led to the discovery that DGDGs are the major glycosyldiglyceride type found in bacteria and that MGDG do not usually accumulate in significant amounts even though they are biosynthetic precursors of diglycosyldiglycerides (Figure 1.8). Five major types of diglycosyl diglycerides were found in different bacteria as reviewed by Shaw. ${ }^{46}$ These consisted of $\alpha$-Gal- $\alpha$-Glc-diglyceride, $\alpha$-Glc- $\alpha$-Glc-diglyceride, $\beta$-Glc- $\beta$-Glc-diglyceride, $\alpha$-Man- $\alpha$ Man-diglyceride and $\beta-$ Gal- $\beta$-Gal-diglyceride. Also, triglycosyldiglycerides (TGDG) and tetraglycosyldiglycerides have been extracted in lower amounts in some bacteria. Figure 1.10 depicts examples of some of these different glycolipids found prior to 1970. As shown in this Figure, the major sugars detected were glucose, galactose and mannose. In a few cases, other glycolipids containing different sugars such as glucuronic and galacturonic acid were found in few cases. Also, an additional acylation at the 6-position of terminal sugars was believed to be due to an extraction artefact consisting of acyl migration, however shortly later, it was found that acyl transferases can also acylate glycolipids at this position. ${ }^{47,48}$ 


\section{MGDG}<smiles>[R]C(=O)OC[C@@H](COC(O)C1OC(CO)C(O)C(O)C1O)OC([R])=O</smiles>

M. Iaidlawii

\section{DGDG}

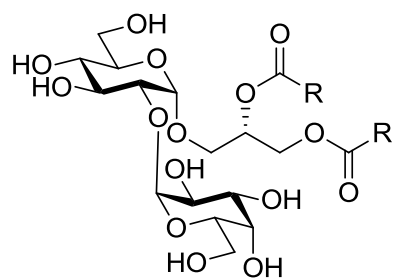

L. casei

L. buchneri

L. plantarum

L. helveticus

L. acidophilus

L. fermenti

TGDG

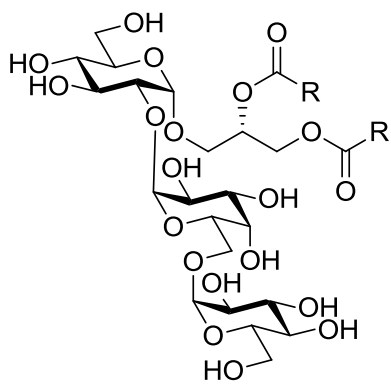

L. casei

L. helveticus

L. acidophilus<smiles>[R]C(=O)OCC(COC(O)C1(O)C(O)C(O)C(O)C1(O)O)OC([R])=O</smiles>

A. globiformis

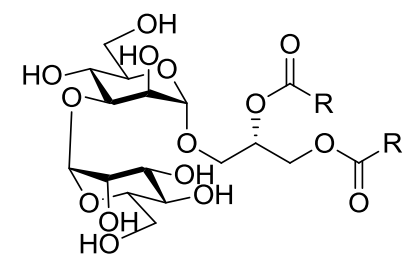

M. lactitum

A. globiformis

A. pascens

A. crystallopoites

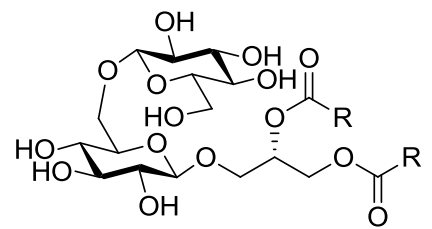

S. lactis

S. saprophyticus

S. aureus

B. subtillis

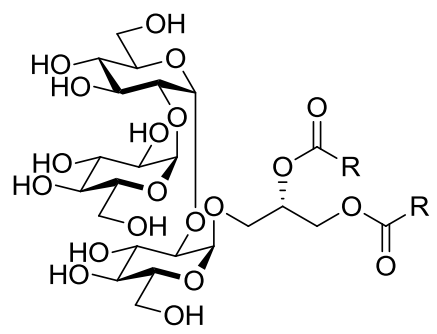

L. acidophilus

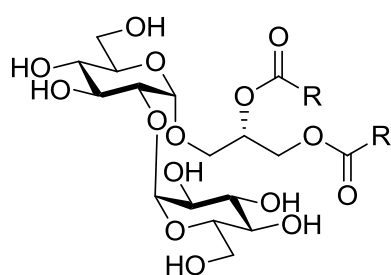

M. Iaidlawii

S. hemolyticus

S. lactis

S. pyrogenes

S. faecalis

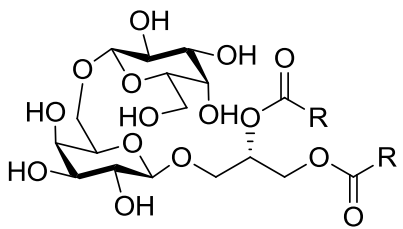

A. globiformis

A. pascens

A. crystallopoites

Figure 1.10 Examples of MGDG, DGDG and TGDGs found in bacteria, as reviewed by Shaw. ${ }^{46}$ 
Today, a few groups have started to revisit the field of bacterial glycoglycerolipids using constituent sugar analysis, ${ }^{49}$ glycosyl linkage analysis, ${ }^{50}$ NMR and MS. ${ }^{51-53}$ From these studies new structures were sometimes found. Examples of glyceroglycolipids found using modern techniques in Propionibacterium propionicum (Figure 1.11a), ${ }^{51}$ Deinococcus radiodurans (Figure 1.11b), ${ }^{52}$ c. Arthrobacter scleromae and Arthrobacter globiformis (Figure 1.11c) ${ }^{53}$ are given in Figure 1.11. Even though the glycolipids found in $D$. radiodurans and A. scleromae are typical glycoglycerolipids, a novel type of glycolipid containing an ether lipid was detected in P. propionicum. This finding shows that to revisit the field in glycolipid analysis might shed a new light on structures that were not detected before due to the limitations of previous analytic methods.

a

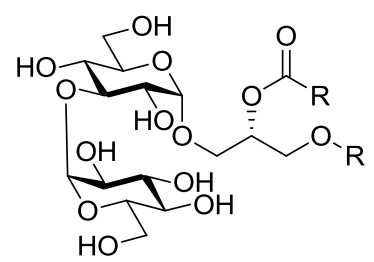

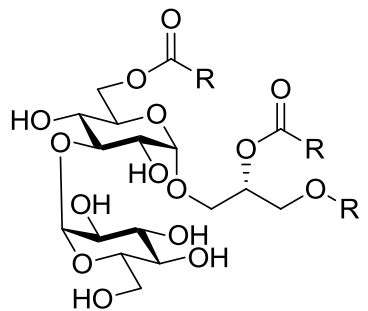

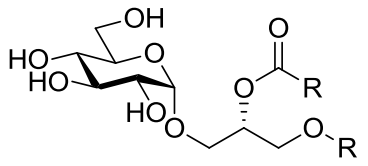

b<smiles>[R]C(=O)OC[C@@H](COC(O)C1(CO)COCC1(O)CO)OC([R])=O</smiles>

C

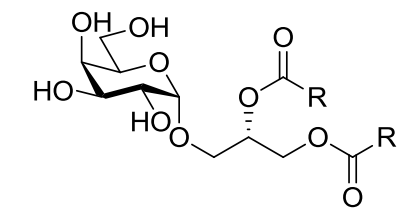

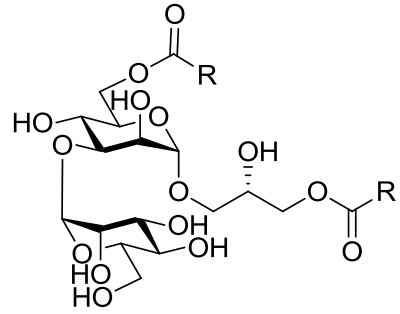

Figure 1.11 Glycolipids found in; a. P. propionicum, ${ }^{51}$ b. D. radiodurans, ${ }^{52}$ c. A. scleromae and A. globiformis. ${ }^{53}$ 


\subsubsection{Lipoteichoic Acids (LTA) and Lipoglycans}

LTAs are repeating units of alditol phosphates (e.g. glycerol phosphate) anchored to the cell membrane by a lipid anchor and they are believed to have roles such as being involed in autolysins regulation. ${ }^{54}$ LTAs are present mostly in firmicutes (low guanine and cytosine content) while lipoglycans are mostly found in Actinobacteria and Tenericutes (high in guanine and cytosine content) making these molecules phylum specific. ${ }^{30}$ Lipoglycans, in comparison to LTAs, contain a sugar repeating unit instead of an alditol. ${ }^{54}$ The immunological properties of LTAs have given conflicting results, in the past, due to contamination of the extracts with other cell wall components. ${ }^{55}$

\subsection{Extraction and Analysis}

LTAs and lipoglycans are usually extracted using hot phenol/water. However, these extractions were found to produce less active LTAs ${ }^{56}$ and a milder extraction method involving butanol is now usually used. ${ }^{57}$ The purification is difficult and involves multiple subsequent columns. By example, Morath et al. used at first a hydrophobic interaction chromatography system on an octyl-sepharose column with an increasing linear gradient of $n$-propanol in an ammonium acetate buffer (descending salt gradient). Then, an anion exchange chromatographic system with an increasing linear gradient of ammonium chloride was utilised with a DEAE-sephacel column. These types of chromatographic steps are often slow, even though sample collectors are available. In the specific case of the DEAE system, the elution speed is about $25 \mathrm{~mL} / \mathrm{h}$ while collecting usually $6 \mathrm{~mL}$ fractions. ${ }^{58}$ Morath et al. collected 65 fractions over a total of $15 \mathrm{~h}$ for one column. Also, detection of these LTAs is problematic as proteins are usually detected using a UV-Vis detector. As often LTAs contain also alanines visible in the UV-Vis spectrum, other types of detection are required as the ultimate goal is to purify LTAs from active lipoproteins. Thus Morath et al. used a phosphate determination assay where each individual fraction is determined for its phosphate content. ${ }^{56}$ The NMR spectrum measured from such LTAs show broad signals and for further analysis the LTAs must be degraded to obtain additional information via NMR (such as acetic acid treatment to obtain the glycolipid anchor). Then MS, constituent 
sugar and linkage analysis as well as fatty acid analysis can be used to gain more information. $^{57}$

Bacterial LTAs are categorised into 4 types (I-IV). Type I is bound covalently to the membrane via a glycoglycerolipid (anchor). It consists of a 1,3-linked polyglycerophosphate chain. In type II, sugar residues are inserted between the glycerophosphate moieties. In contrast to type II, type III contains positively charged sugar residues (glucosamine) linked on $s n-2$ of the glycerol moieties present on the chain. Finally, type IV, does not contain a typical polyglycerophosphate chain, but it holds choline residues that compensate the negative charges on the phosphates. ${ }^{59}$ Figure 1.12 shows two types of LTAs found in low G-C content bacteria (Streptococcus pneumonia in Figure 1.12a, and S. aureus in Figure 1.12b $)^{24,60,61}$ and a lipoglycan found in high G-C content bacteria (Bifidobacteria bifidum var. pennsilvanicus, Figure 1.12c). ${ }^{62}$ From those different types of LTAs, type $I$ is the most widespread (Figure $1.12 b){ }^{59}$ 
a.
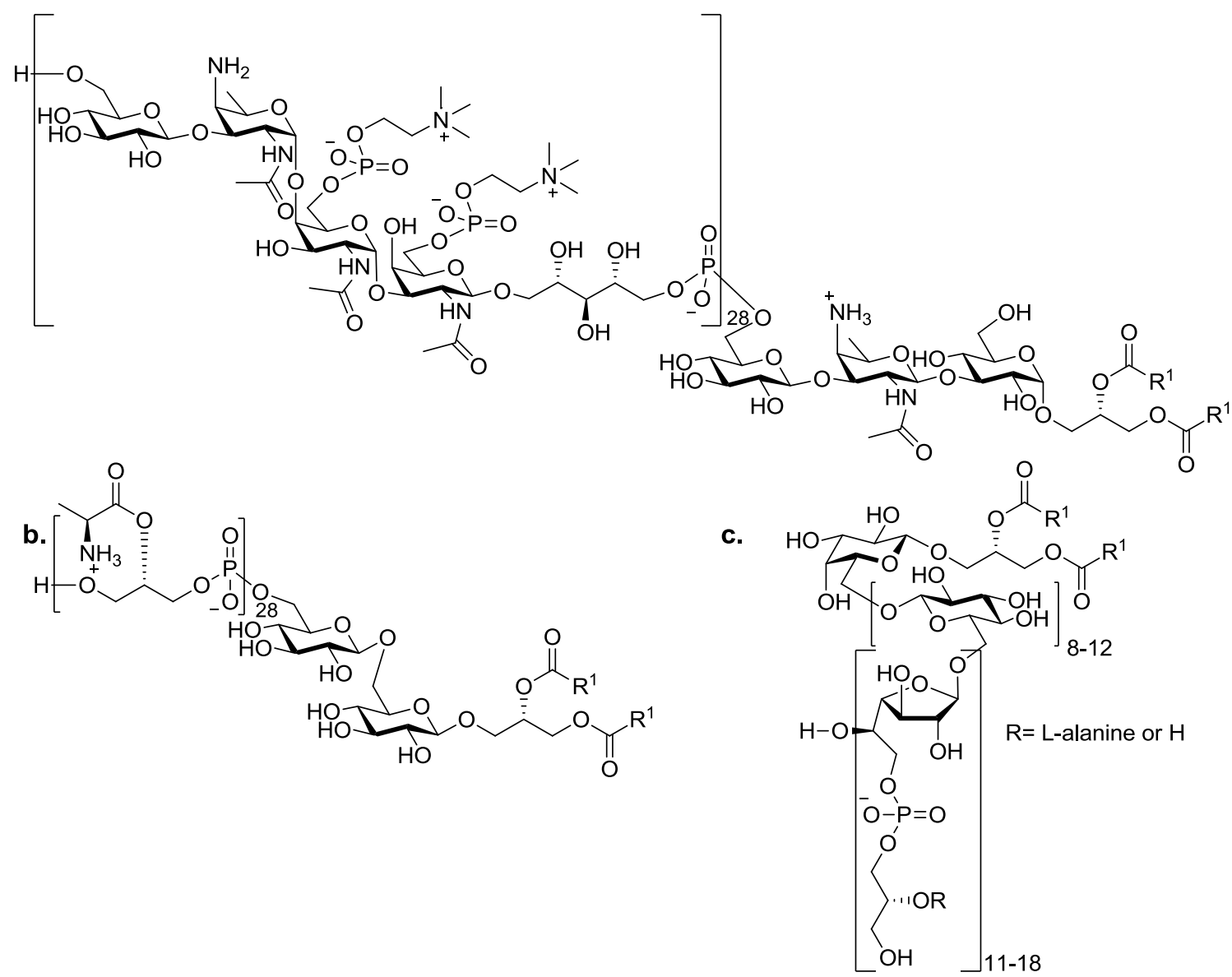

Figure 1.12 a. S. pneumonia lipoteichoic acid, ${ }^{24}$ b. S. aureus lipoteichoic acid, ${ }^{60}$ c. $B$. bifidum var. pennsylvanicus lipoteichoic acid. ${ }^{62}$

\subsection{Chemical Synthesis and Biological Activity of Gram-Positive Cell Wall Components}

In this section, the synthesis and biological activity of different cell wall components will be discussed. Fatty acids, however, will be excluded from the discussion because pure fatty acids are usually bought directly from the supplier in synthesis and the literature on the immunological properties of fatty acids focuses mostly on polyunsaturated fatty acids and rarely discusses other free fatty acids. ${ }^{63,64}$ Even though 
phosphoglycerolipids, WTAs, CPS and EPS can be interesting targets for further research concerning their biological properties, the glycolipids and lipoteichoic acids will be described in more detail as their synthesised structures were reported to be biologically active.

\subsubsection{Exopolysaccharides}

The immunological properties of EPS have been thoroughly reviewed by Ruas-Madiedo et $a l .{ }^{18}$ In order to assess structure activity relationships in EPS, synthesis is necessary. Like PG, EPS are composed of long chains and the synthesis of EPS fragments is usually made. $^{65,66}$ Scheme 1.1 shows the reported synthesis of a $S$. aureus EPS fragment 65. First, glucosamine 23 was selectively protected at the 6-position with a trityl group. This step was followed by an improved benzoylation to form benzoylated compound $\mathbf{2 4}$ as the $\beta$-anomer which is required for the oxazoline formation. The trityl group was then selectively removed and the 6-position was protected with an acetate to form acetylated compound 25. To obtain oxazoline 26, $\mathrm{FeCl}_{2}$ was utilised and subsequent coupling with thioglycoside 27 gave coupled product 28. After this, the selective deprotection of the acetate and the resulting deprotected compound coupling with bromide $\mathbf{2 9}$ afforded trisaccharide 30. Next, the coupling of trisaccharide $\mathbf{3 0}$ with glycosyl acceptor $\mathbf{3 1}$ and global deprotection gave EPS fragment 32. ${ }^{65}$

\subsubsection{Wall Teichoic Acids}

The study of WTAs immunological properties is of interest as they are believed to be a virulence factor. ${ }^{67-69}$ Like EPS, only fragments of WTAs are usually synthesised due to their high lengths, yet longer chains might show higher immunological properties. Scheme 1.2 shows the retrosynthesis of E. faecalis WTA fragment. Here, Hogendorf et al. predicted that target $\mathbf{3 3}$ could be assembled from benzylated glycerol $\mathbf{3 4}$ and phosphoramidites $\mathbf{3 5}, \mathbf{3 6}$ and $\mathbf{3 7}$. They successfully synthesised fragment $\mathbf{3 3}$ in 14 linear steps and $17 \%$ overall yield using a solution-phase synthetic strategy. ${ }^{70}$ This strategy aims to use, in the future, automated solid phase synthesis to achieve the synthesis of longer, hopefully active, polymers. 

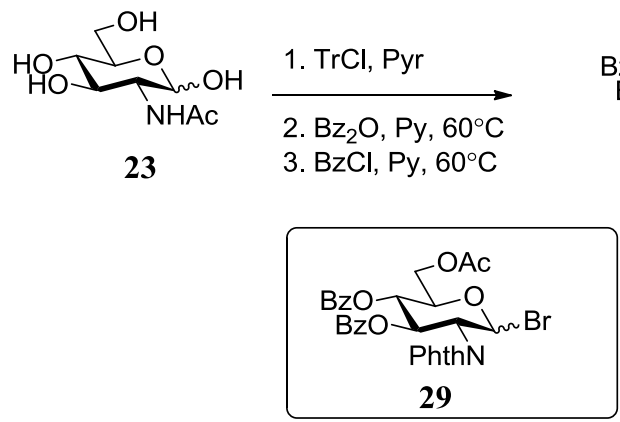

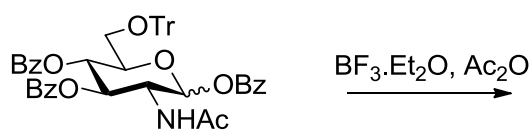

24

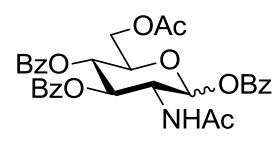

25

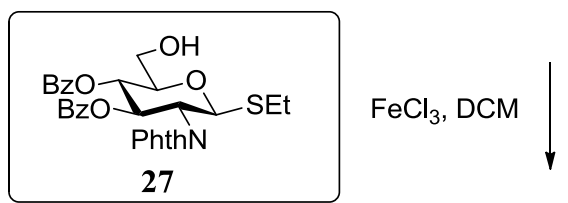

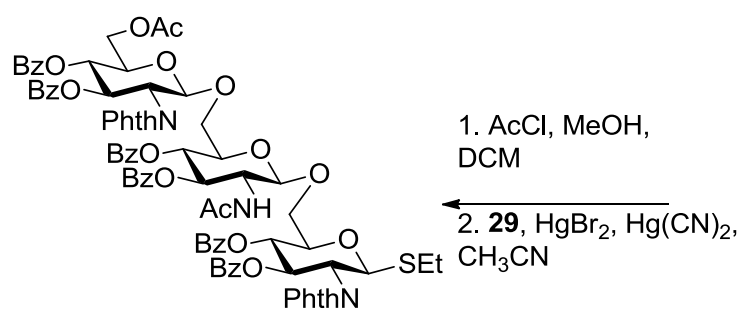

30

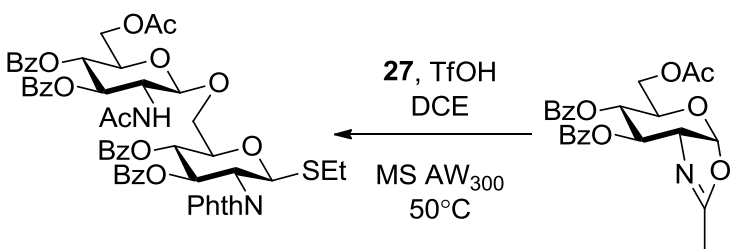

28

26

1. 31, NIS, TfOH, MS $4 \AA$

2. $\mathrm{AcCl}, \mathrm{MeOH}, \mathrm{DCM}$

3. $\mathrm{H}_{2}, \mathrm{Pd}(\mathrm{OH})_{2} / \mathrm{C}, \mathrm{MeOH}-\mathrm{THF}-\mathrm{H}_{2} \mathrm{O}$

4. $\mathrm{Ac}_{2} \mathrm{O}, \mathrm{Pyr}$

5. $\mathrm{N}_{2} \mathrm{H}_{4}, \mathrm{H}_{2} \mathrm{O}, \mathrm{EtOH}$

$\checkmark$
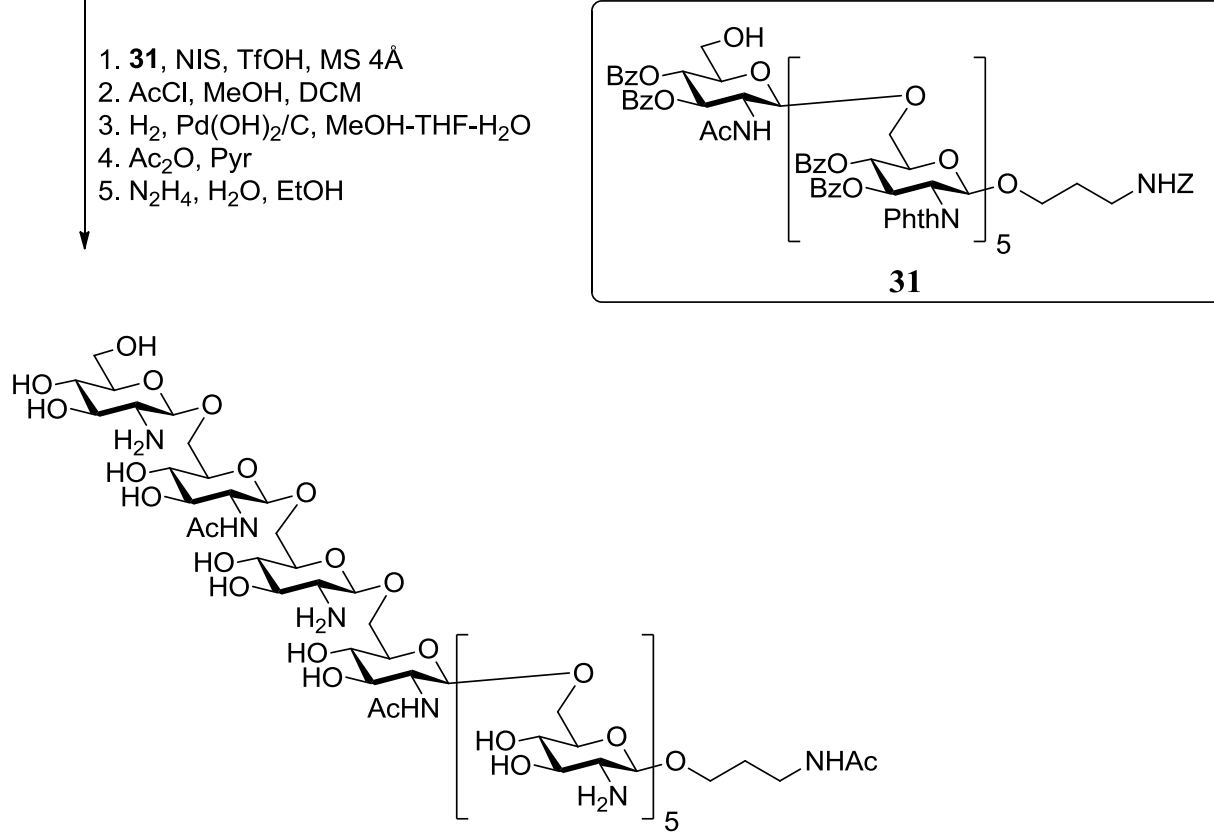

32

Scheme 1.1 Synthesis of $S$. aureus EPS fragment. ${ }^{65}$ 

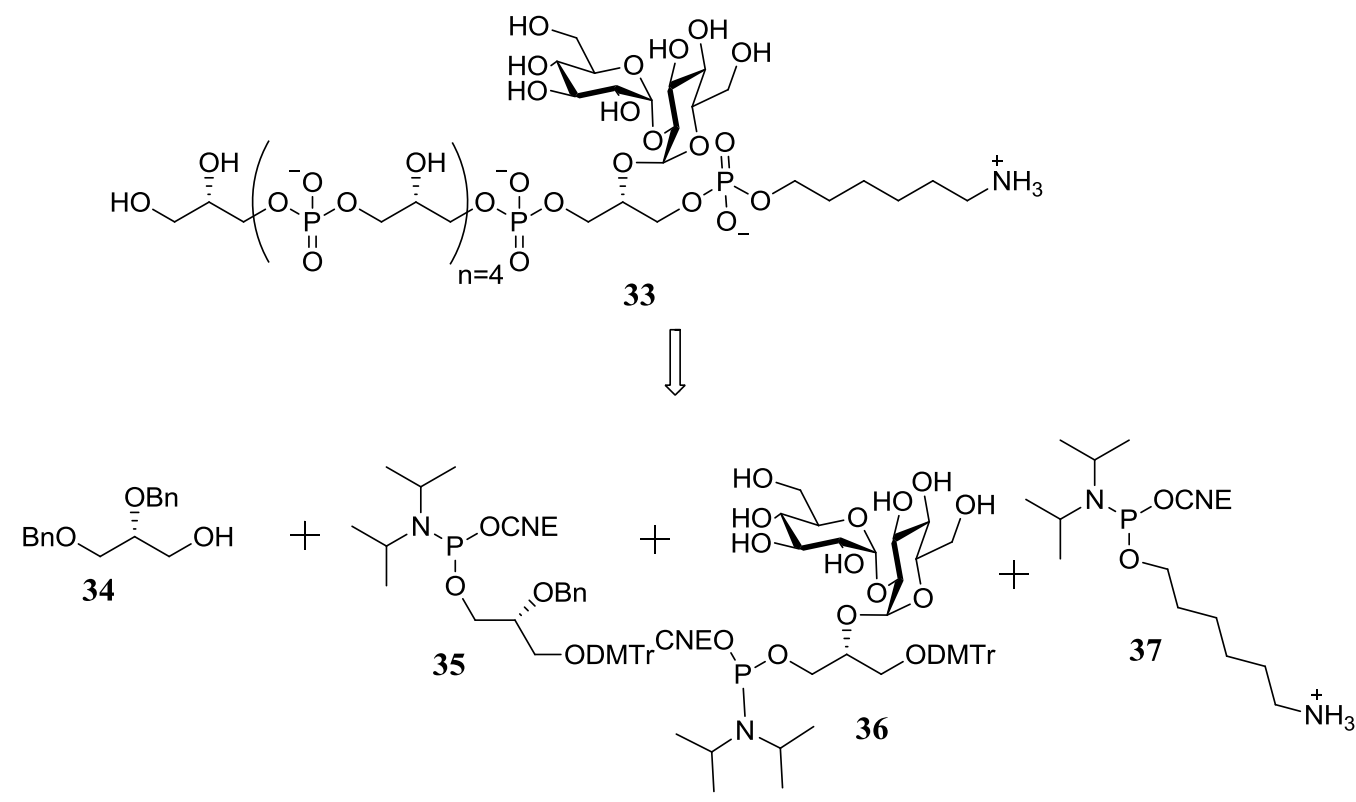

Scheme 1.2 Retrosynthesis of a Enterococcus faecalis fragment. ${ }^{70}$

\subsubsection{Phospholipids}

Few articles and reviews discuss bacterial phospholipid immunoproperties and those that do focus mostly on cardiolipin. ${ }^{71,72}$ Indeed, phospholipids containing usual saturated and monounsaturated fatty acids might be used as drug carriers as they are biocompatible. $^{73}$ In contrast, the synthesis of phospholipids has been widely covered. ${ }^{74}$ Scheme 1.3 depicts an example of PA synthesis. ${ }^{74}$ Here 2,3-O-isopropylidene-sn-glycerol (-)-38 is esterified with R-COOH using DCC and DMAP, which is then deprotected with dimethylboronbromide to form ester 39. Tritylated DAG $\mathbf{4 0}$ is then obtained following tritylation of the primary alcohol and esterification of the secondary alcohol with a different fatty acid $\left(\mathrm{R}^{1}-\mathrm{COOH}\right)$. Deprotection of the trityl-group with $\mathrm{BF}_{3} \cdot \mathrm{OEt}_{2}$ gave DAG 41, which was subsequently phosphorylated with phosphoryl chloride. The final PA 42 was obtained after hydrolysis of the chloride. 


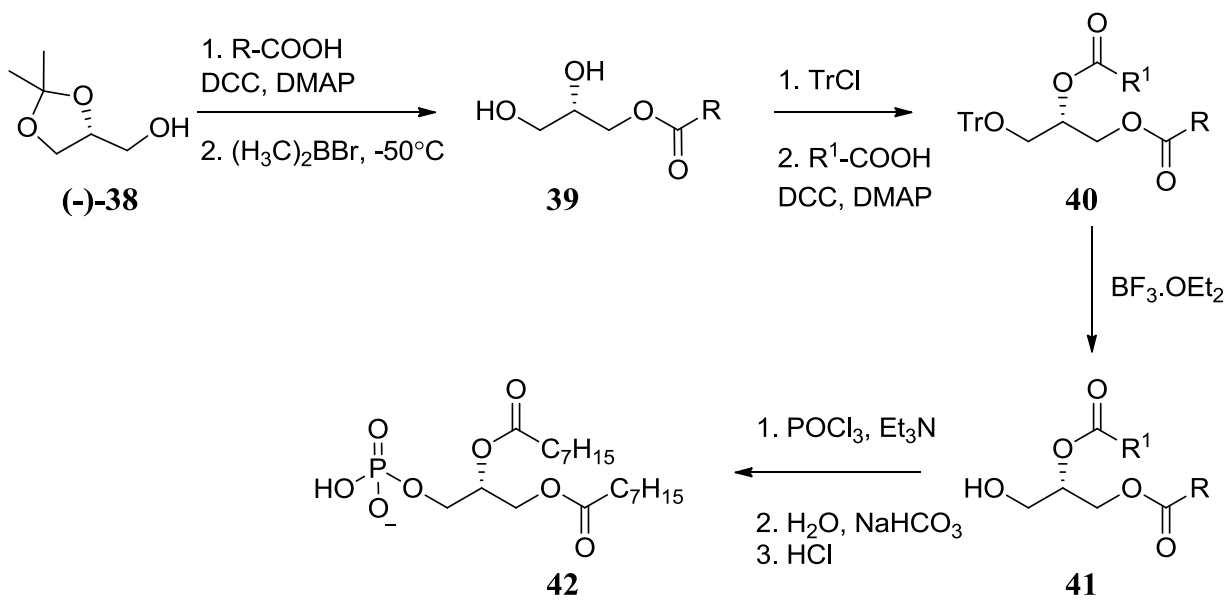

Scheme 1.3 Example of phosphatidic acid synthesis. ${ }^{74}$

\subsubsection{Glycoglycerolipids}

Many glycoglycerolipids have been found to be biologically active where the fatty acid chain has an influence on the nature of the activity. ${ }^{63,75,76}$ Glycoglycerolipids extracted from natural sources are mixtures of fatty acids with varying chain length and thus to gain a comprehensive understanding of structure/function it is necessary to synthesise analogues with clearly defined fatty acid chains. ${ }^{63,77-79}$ In this section the immunological properties of glycoglycerolipids will be summarised. In an early publication, Naito et al. evaluated the activation of mouse macrophage by synthetic glycerolipid liposomes. They reported that 3-O-( $\beta$-D-cellobiosyl)-1,2-di- $O$-palmitoyl-sn-glycerol, 3- $O$-( $\beta$-Dmaltosyl)-1,2-di- $O$-palmitoyl-sn-glycerol and 3-O-( $\beta$-D-lactosyl)-1,2-di- $O$-palmitoyl-snglycerol strengthened the immune system against transplantable tumor cells in imprinting control region (ICR) mice and that macrophage seemed to be activated by glycoglycerolipids. ${ }^{80}$ Later, Deininger et al. observed the induction of cytokine release in whole blood cells by a specific LTA and the LTA anchor 3-O-( $\beta$-D-gentiobiosyl)-1,2di- $O$-myristoyl-sn-glycerol. In this case, the LTA derivatives showed a higher activity than the glycolipid anchor. ${ }^{81}$ Thereafter, Borrelia burgdorferi glycolipid analogues were synthesised and induced IL-2 production in mouse and human $i$ NKT cells. ${ }^{82}$ The 
glycolipid tested by Kinjo consisted of synthesised galactosyldiglycerides (Figure 1.13). Here they found that the position and length of the lipid mattered for $i$ NKT cells activation and that BbGL-11c induced more IL-2 production than the other glycolipids tested.
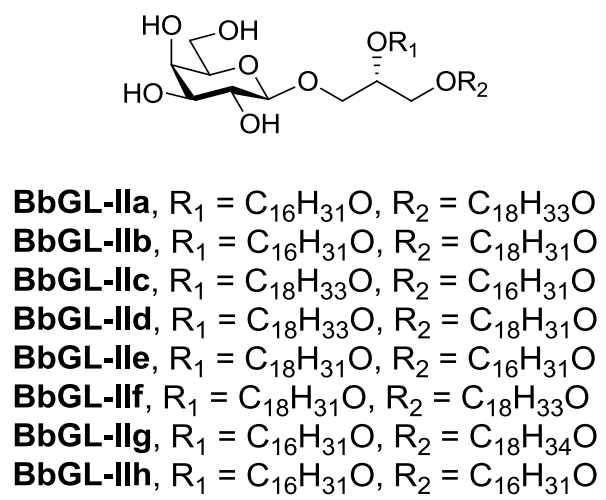

Figure 1.13 Galactosyldiglycerides synthesised and tested by Kinjo et al. ${ }^{82}$

Furthermore, Kinjo et al. measured the secretion of IFN- $\gamma$ by $i$ NKT cells from two Streptococcus pneumonia purified glycolipids and five different synthesised analogues of $\alpha$-glucosyldiacyldiglyceride. Here, the synthesised analogues had different fatty acid chain lengths and fatty acids positions on the glycerol moiety ( $s n-1$ or $s n-2)$. They found that the purified glycolipids were more active than the synthesised ones. In addition, only the glucosyldiglyceride analogue containing palmitic acid at the $s n-1$ position and cis-vaccenic acid at the $s n-2$ position of the glycerol showed significant activity. ${ }^{78}$ The same group later compared synthesised analogues of glucosyldiglycerides and galactosyldiglycerides. The glucose derivatives induced a higher IL-2 production in $i$ NKT cells compared to the galactose derivatives. Also, they demonstrated that in addition to the sugar, the type and position of the fatty acid chain influenced IL-2 production. Indeed, the galactose derivative with palmitic acid on $s n-2$ and cis-vaccenic acid on $s n-1$ of the glycerol moiety was more active than the galactose derivative with cis-vaccenic acid on $s n-2$ and palmitic acid on $s n-1$ of the glycerol moiety. ${ }^{83}$ 
Syntheses of glycosyldiglycerides have been widely reported. ${ }^{74}$ This usually involves coupling of a protected sugar moiety to isopropylidene glycerol, deprotection of the acetonide, esterification and final deprotection. An example of a forward synthesis is shown in Scheme 1.4. ${ }^{84}$ Here per- $O$-acetylated galactose imidate $\mathbf{4 3}$ was first coupled to chiral isopropylidene glycerol (+)-38 to form coupled product 44 . Then the acetates were cleaved and the hydroxyls benzylated to afford benzylated compound $\mathbf{4 5}$. Then acetonide deprotection gave diol 46 and to obtain diglyceride 47 subsequent esterification using octanoyl chloride was undertaken. A final hydrogenation provided 1,2-di-O-octanoyl-3-O- $\beta$-D-galactopyranosyl-sn-glycerol 48.

In an attempt to simplify the synthesis of glycolipids, Schombs achieved a one pot synthesis of 1-O-palmitoyl-2-O-oleoyl-3-O- $\alpha$-D-galactopyranosyl-sn-glycerol. ${ }^{85}$ Their method (Scheme 1.5) involves the iodination of per- $O$-trimethylsilylated glucoside 49, followed by the addition of diacylglycerol acceptor $\mathbf{5 0}$ in the presence of TBAI and DIPEA. After deprotection of the silyl groups they obtained glycosyldiglyceride $\mathbf{5 1}$ using acidic resin in an overall yield of $72 \%$.

\subsubsection{Lipoteichoic Acids}

In the past, it has been assumed that LTA is an immunostimulatory component of Gram-positive bacteria together with PG and lipopeptides. Indeed, pathogen-pattern recognizing receptors (PRRs) such as toll-like receptors (TLRs) recognize pathogenassociated molecular patterns (PAMPS) on the surface of bacteria and activate innate immunity. ${ }^{86}$ In particular, TLR2 is activated by bacterial cell-wall glycopolymers and lately, it has been shown that the glycolipid anchor activity is amplified by the LTA poly(glycerophosphate) backbone. ${ }^{81}$ However, the study of the immunological properties of natural LTAs has been hampered because of the harsh extraction and purification conditions resulting in the isolation of artefactual compounds formed from LTA breakdown while using the hot phenol extraction method. The analysis can thus be misleading. Also, it is known that LTA derivatives extracted from bacteria contain active lipoproteins. In order to retain the structural integrity of natural LTAs, improved extraction methods using milder solvent (butanol) have been used rather than the hot- 
phenol extraction. ${ }^{55}$ Even though the problems surrounding the chemical characterisation of the LTAs seemed to be solved, the issues related to the purification of the LTAs are still remaining. In the past, many reports were published on lipoteichoic acid activity, ${ }^{59,87,88}$ yet today most of the LTA activity has actually been assigned to lipoprotein contamination. ${ }^{12}$ Hence, the need for clean samples, free of active contaminants for biological testing, has pushed forward the synthesis of LTA and LTA derivatives. $^{86}$

A first example of a retrosynthetic strategy is shown in Scheme 1.6. Here, to synthesise a fragment of $S$. aureus LTA, Stadelmaier et al. envisioned that target $\mathbf{5 2}$ could be made from the ligation of alcohol $\mathbf{5 3}$ and phosphoramidite 54. The synthesis of compound $\mathbf{5 3}$ was then anticipated from the coupling of glycerol derivative $\mathbf{3 4}$ and phosphoramidites $\mathbf{5 5}$ and 56. Then it was anticipated that regioselectively protected gentiobiosyl-DAG $\mathbf{5 7}$ could form phosphoramidite $\mathbf{5 4}$ after protecting group manipulation, esterification and reaction with benzyloxybis(diisopropylamino)phosphine. From this strategy, they produced both D-alanine and L-alanine derivatives. These monoamphiphilic LTAs were then tested on human whole blood cells and found both to stimulate TNF- $\alpha$ production. However, differences were observed in the activity where the L-alanine derivative had a similar activity compared to the natural purified extract, while D-alanine derivative showed a 10 to 100 fold less activity. ${ }^{89,90}$ Later, Stadelmaier et al. also synthesised a bisamphiphilic moiety (Figure 1.14) and tested the compounds in the same assay. The bisamphiphilic moiety was twice as active as the monoamphiphilic moiety. ${ }^{77}$ As a significant effort is required for the synthesis of such derivatives, only few LTA fragments were synthesised and tested until now. ${ }^{86}$

An additional, very interesting example of another synthesis of a specific LTA derivative is depicted in Schemes 1.7 and 1.8. The LTA derivative synthesised was based on a unique LTA, extracted primarily from Streptococcus sp. DSM 8747, which had a structure that differed from $S$. pneumonia LTA. $^{91}$ This LTA also differed from other LTAs due to its single galactofuranose moiety. In most LTAs, the sugar residues are usually present in the pyranose form. Due to its peculiar characteristics, a fragment of this LTA was synthesised by Qiao et al..$^{92}$ The first part of the chemical synthesis, the 
synthesis of the galactofuranose building block, is shown in Scheme 1.7 while the reminder of the synthesis is depicted in Scheme 1.8.

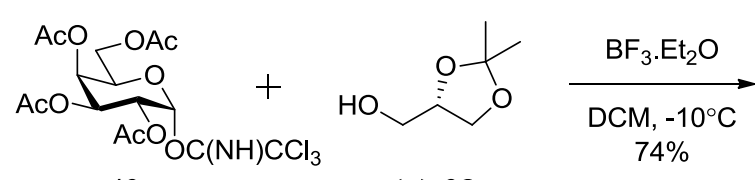

43

$(+)-38$<smiles>CC(=O)OCC(OC(C)=O)C(OC(C)=O)C(O)OCC1COC(C)(C)O1</smiles>

44

1) $\mathrm{MeONa}, \mathrm{MeOH}$

2) $\mathrm{NaH}, \mathrm{BnBr}, \mathrm{DMF}$ $87 \%$

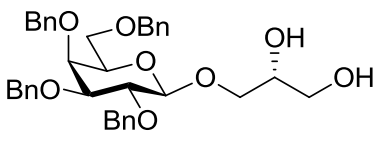

$$
\frac{\mathrm{Zn}\left(\mathrm{NO}_{3}\right)_{2} \cdot 6 \mathrm{H}_{2} \mathrm{O}}{\mathrm{CH}_{3} \mathrm{CN}, 50^{\circ} \mathrm{C}}
$$
$90 \%$

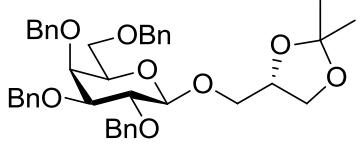

45 $\mid \begin{aligned} & \mathrm{Cl}(\mathrm{CO}) \mathrm{C}_{7} \mathrm{H}_{15}, \mathrm{Pyr} \\ & \mathrm{DCM} \\ & 95 \%\end{aligned}$

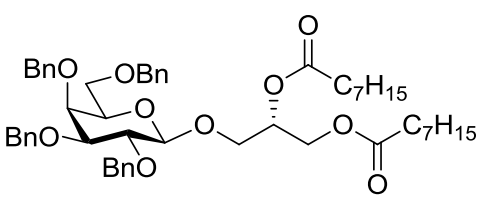

47

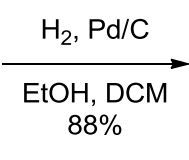
$88 \%$

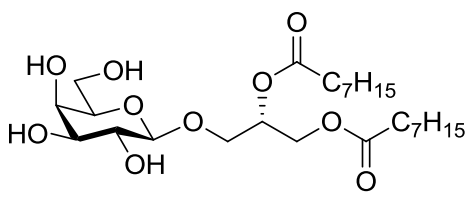

48

Scheme 1.4 Synthesis of 1,2-di-O-octanoyl-3-O- $\beta$-D-galactopyranosyl-sn-glycerol. ${ }^{84}$

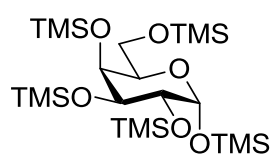

49

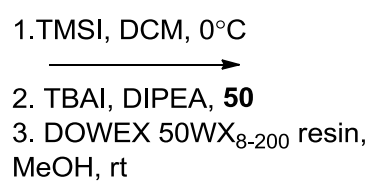

$\mathrm{OH}, \mathrm{r}$

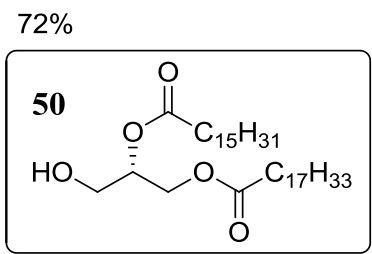

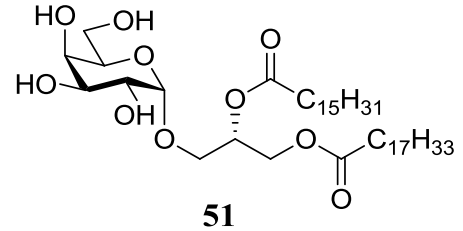

51

Scheme 1.5 Synthesis of 1-O-palmitoyl-2- $O$-oleoyl-3- $O$ - $\alpha$-D-galactopyranosyl-snglycerol. ${ }^{85}$ 


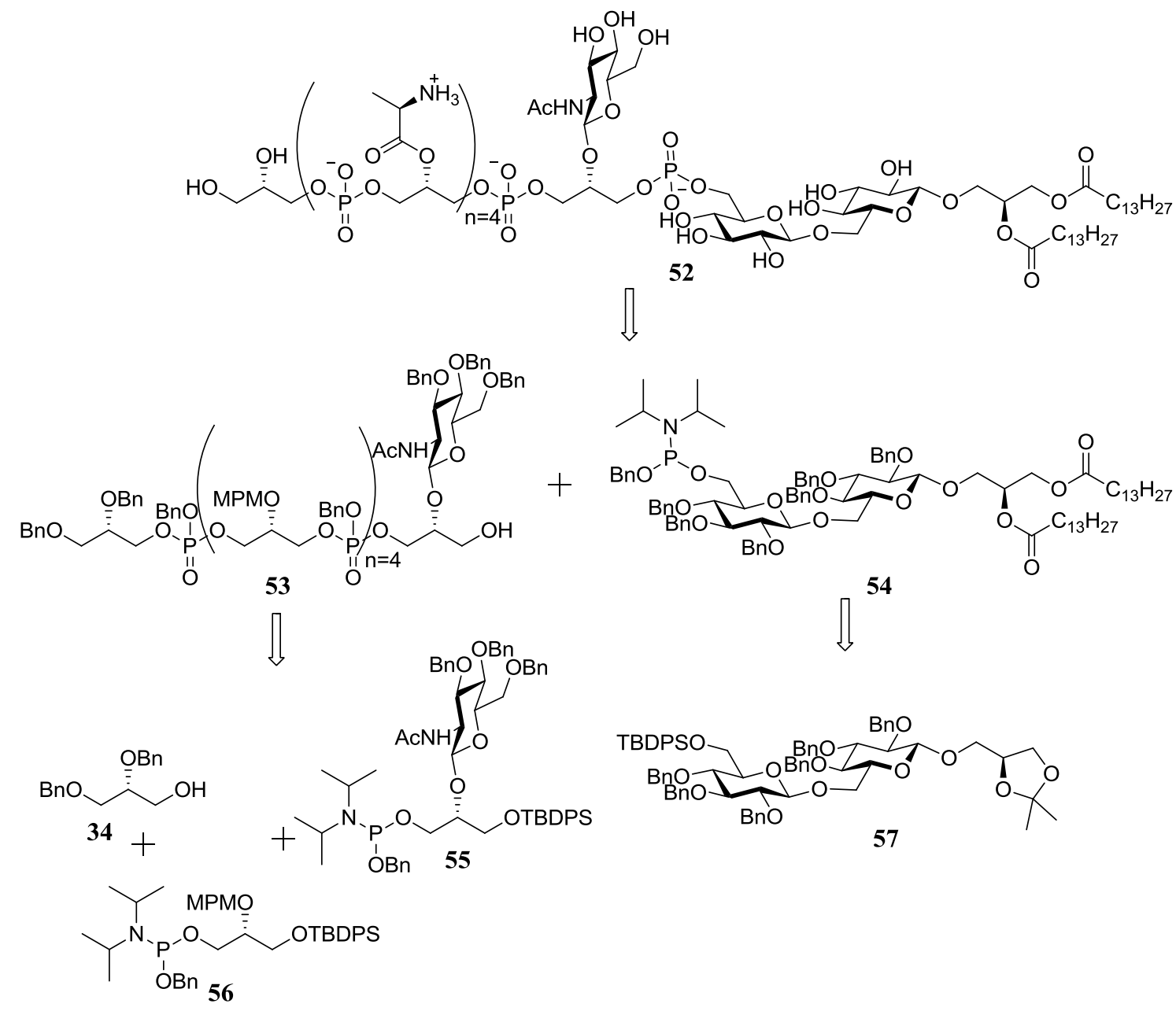

Scheme 1.6 Retrosynthetic scheme of Staphylococcus aureus LTA adapted from Stadelmaier et al. $^{77}$

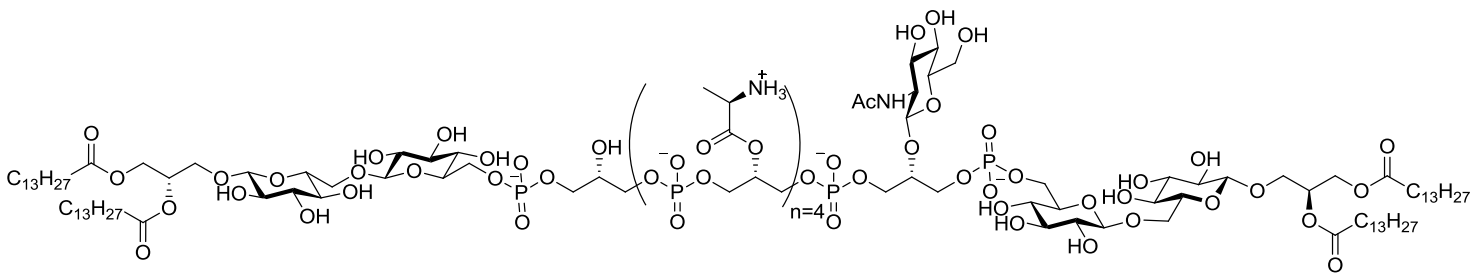

Figure 1.14 Synthesised biamphiphilic LTA. ${ }^{77}$

In Scheme 1.7, alkyl glycoside $\mathbf{5 9}$ is first formed from galactose 58. Then perbenzoylated compound $\mathbf{6 0}$ is obtained after treatment of galactofuranose $\mathbf{5 9}$ with benzoyl chloride in pyridine. Acetate formation of the anomeric position using acetic 
anhydride and sulfuric acid gave acetylated compound $\mathbf{6 1}$ and the acetate was subsequently removed to form benzoylated compound 62. Treatment of the anomerically deprotected 62 with trichloroacetonitrille and DBU provided imidate 63 which was next coupled with glycerol hexylidene derivative $\mathbf{6 4}$ to form coupled product 65. The removal of the benzoyl groups and benzylation afforded galactofuranoside derivative 66. A final removal of the diol protecting group with $p$-toluenesulfonic acid gave precursor 67. In Scheme 1.8, diol 67 is esterified to yield to ester 68 then the benzyl-groups are removed to give deprotected 69. To obtain compound 70 the selective protection at $O-6$ using $\mathrm{TBSCl}$ was required. Then subsequent benzylation gave protected anchor 71. Phosphoramidite 73 was obtained after desilylation using TBAF to form alcohol 72 and phosphytilation. Finally, target compound 75 was formed after coupling with phosphate glycerol derivative 74, oxidation, MPM cleavage, partial esterification with alanine and final deprotection. Despite this successful synthetic route, the authors did not assess the biological activity of Gal $f$ LTA derivatives and no references to any biological evaluation of this molecule can be found in the literature. 


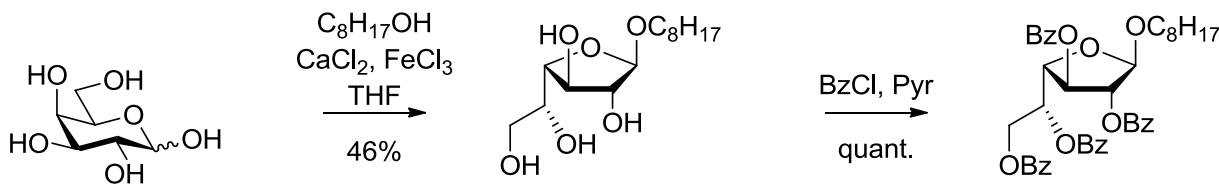

58

59

60

$$
\underset{0^{\circ} \mathrm{C}}{\mathrm{Ac}_{2} \mathrm{O}, \mathrm{H}_{2} \mathrm{SO}_{4}} \downarrow
$$

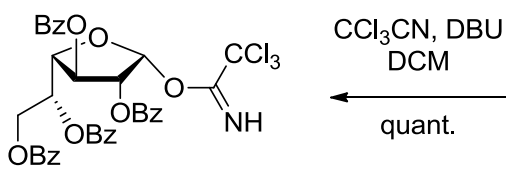

63

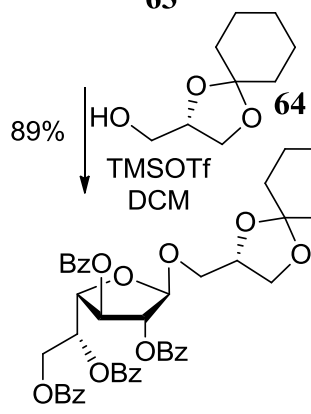

$\underset{96 \%}{\text { i. } \mathrm{NaOMe}, \mathrm{MeOH}}$
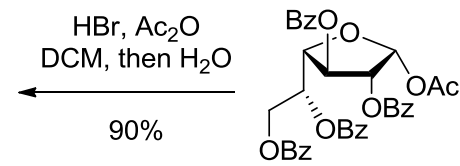

62

61

65

66

67

Scheme 1.7 Synthesis of galactofuranose building block for Streptococcus DSM 8747 LTA fragment. $^{92}$ 
<smiles>OC[C@H](O)CCOC(OCc1ccccc1)C(OBr)C(Br)COc1ccccc1</smiles>

67

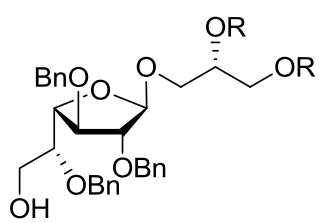

72

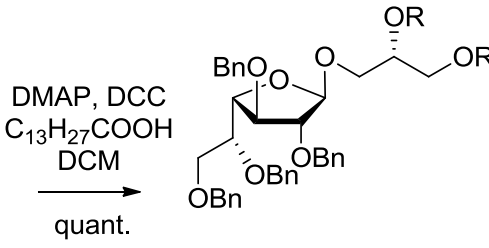

68
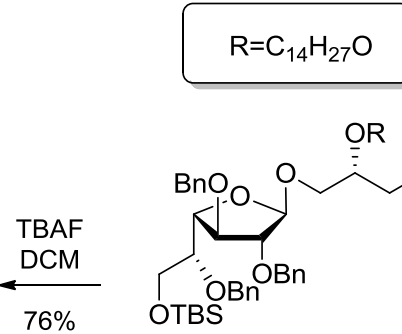

$76 \%$

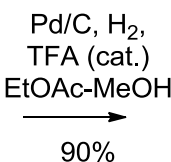<smiles>[R]OC[C@H](CO[C@H](O)[C@@H](O)[C@H](O)[C@H](O)CO)O[R]</smiles>

69

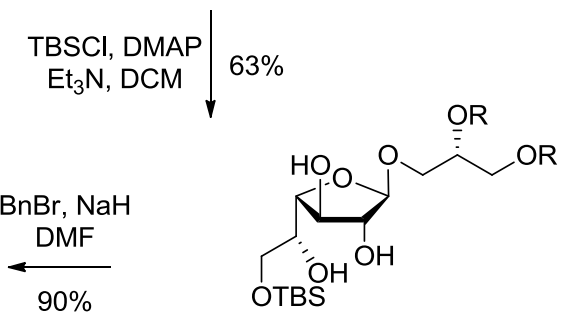

70<smiles>CC(C)NC(C)Cc1nnn[nH]1</smiles>

73

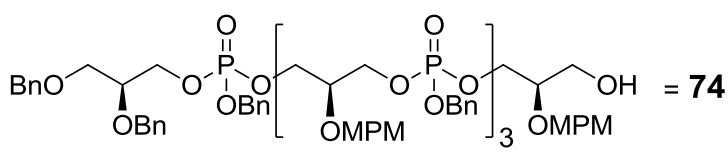

$\mathrm{R}^{1}=\mathrm{H}(\sim 50 \%)$ or<smiles>CC(=O)C(C)C(C)=O</smiles>

Scheme 1.8 Synthesis of Streptococcus pneumonia LTA fragment. ${ }^{92}$

\subsection{Bifidobacteria}

Bifidobacteria, first isolated from human faeces by Tissier as Bacillus bifidus ${ }^{93}$, then named L. bifidus, ${ }^{94}$ and later reclassified as the genus Bifidobacterium by Orla-Jensen, ${ }^{95}$ belong to the phylum Actinobacteria. Nowadays, the Bifidobacterium genus includes over 30 species that can be isolated from various sources, including human faeces and 
the female human urogenitary tract. ${ }^{96}$ Bifidobacteria are non-motile, Gram-positive, anaerobic and fermentative bacteria. ${ }^{97}$ Many reviews have been published on the properties and structure of bifidobacteria and their application and role as probiotics. ${ }^{97,98}$ Also, the subject of whole bacterial immunological properties has been profusely covered. ${ }^{99,100}$ Depending on the species, bifidobacteria shape can vary greatly from a $\mathrm{Y}$ structure to branched rods measuring between 2 and $3 \mu \mathrm{m} .{ }^{101}$ A SEM photomicrograph of Bifidobacteria infantis ATCC 15702 is shown in Figure 1.15.

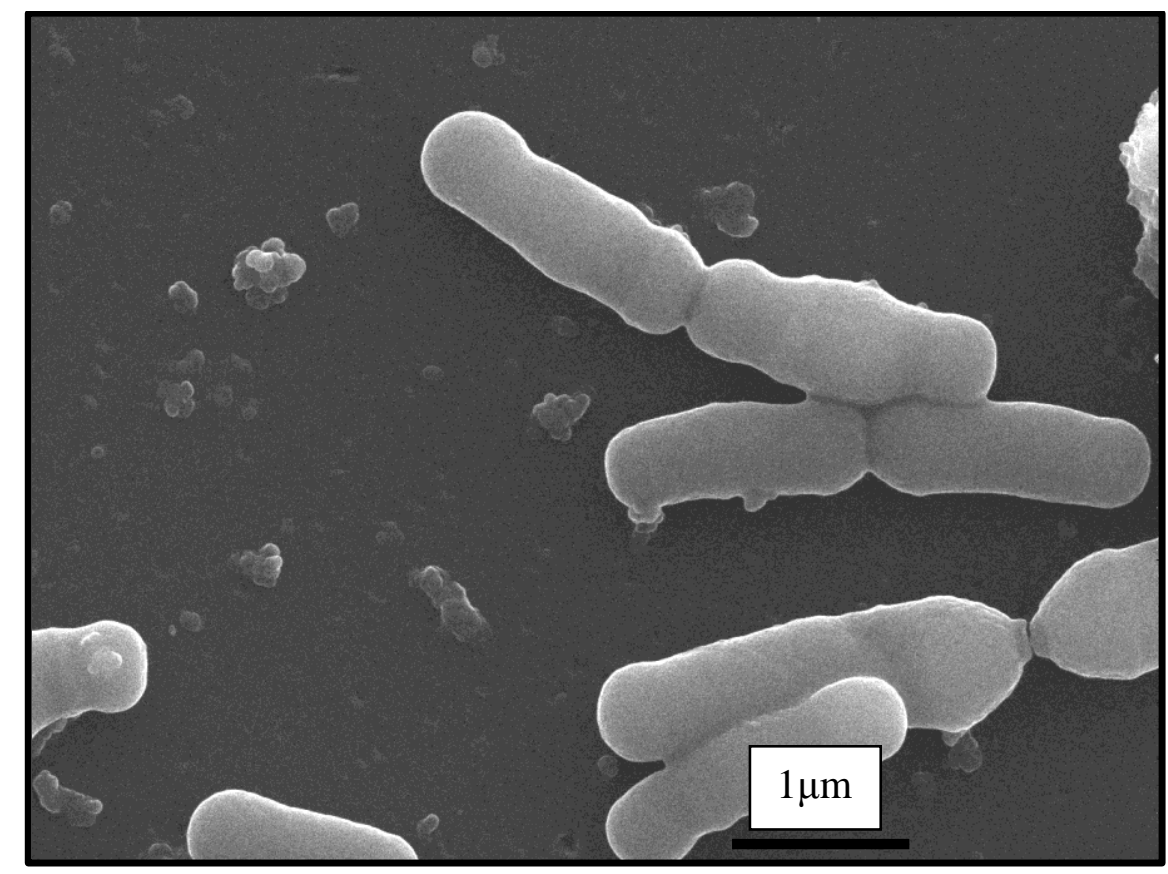

Figure 1.15 Photomicrograph of Bifidobacterium longum subs. infantis ATCC 15702 using a scanning electron microscope measured by David Flinn.

Bifidobacteria are generally considered beneficial members of the human intestinal tract and their effects include tumour inhibition, immunity stimulation and alleviation of the symptoms of irritable bowel syndrome. ${ }^{98}$ Yet, the compounds responsible for these beneficial effects are actually unknown. Research on the structural components present in the cell-wall of Bifidobacterium has shown that components such as lipoteichoic acid, ${ }^{102}$ exopolysaccharides, ${ }^{103}$ and glycolipids are biologically active. ${ }^{104}$ However, the precise structure and purity of these biologically active components is often lacking due to a paucity of structural data. In this section, the bifidobacteria cell wall components 
will be explored, their chemical synthesis explained and the immunogenicity data described.

\subsubsection{Cell Wall Components (CPS and WTA)}

The cell wall components, such as CPS and WTA, are often obtained using digestion with RNAase, DNAase, trypsin, pepsin, and pronase. ${ }^{105}$ However, this extraction method often leads to mixtures of PG, CPS, and WTA. Furthermore, due to CPS and WTA high molecular weight, their structural characterisation is difficult. Typical analyzes of CPS and WTA consists of glycosyl linkage analysis, NMR, amino acid analysis, and comparative analysis using paper chromatography and post hydrolysis paper electrophoresis. ${ }^{105,106}$

\subsubsection{Cell Wall Polysaccharides}

CPS from a number of bifidobacterial species and strains have been extracted and analyzed. Fragments of proposed CPS structures show that these CPS from different species vary greatly (Figure 1.16). ${ }^{105-110}$ An early publication by Habu et al. described the CPS from 4 different strains of bifidobacteria. Not surprisingly, every strain showed different sugar composition and structure. An example of the structure proposed for $B$. breve YIT 4010 is shown in Figure 1.16a. ${ }^{106}$ Here, a repeating tetrasaccharide unit composed of $[\rightarrow 3) \beta-\operatorname{Glc}(1 \rightarrow 3) \beta-\operatorname{Gal}(1 \rightarrow 4) \alpha-\operatorname{Gal}(1 \rightarrow 2) \alpha-\operatorname{Glc}(1 \rightarrow]$ was branched on $O$ 6 of the $\alpha$-Gal residue.

In 1988, the same group detected an entirely different structure in the cell wall of $B$. adolescentis (Figure 1.16b). ${ }^{107}$ This structure consisted of a repeating unit of $[\rightarrow 2) \alpha-$ $6 \mathrm{dTal}(1 \rightarrow 3) \beta-6 \mathrm{dTal}(1 \rightarrow 3) \beta-6 \mathrm{dTal}(1 \rightarrow 3) \beta-6 \mathrm{dTal}(1 \rightarrow 2) \alpha-6 \mathrm{dTal}(1 \rightarrow 2) \alpha-6 \mathrm{dTal}(1 \rightarrow]$ with glucose residues branches on $O-3$ of the $\alpha$ residues. Later, they also purified a structure containing L-rhamnose and galactose from B. longum YIT3028 (Figure 1.16c). ${ }^{108}$ An additional paper from the same group described a polymer composed exclusively of galactose in B. catunelatum YIT4016 (Figure 1.16d). ${ }^{109}$ Here, the core 
consisted of a galactofuranose repeating unit, branched with galactofuranose residues. As with the research done by Nagaoka et al., the analysis performed by ToneShimokawa et al. revealed galactofuranose and galactopyranose in B. infantis CPS (Figure 1.16e). ${ }^{105}$ However, in this case, glucopyranose was detected as part of the branching units. A final example, where galactofuranose and galactopyranose were found, is depicted in Figure 1.16f. Here, in contrast to Tone-Shimokawa et al., Zdorovenko et al. described in B. bifidum BIM B-465 a repeating unit containing not only galactofuranose and galactopyranose, but also glucopyranose. ${ }^{110}$ Galactofuranose and galactopyranose were also found in Bifidobacteria adolescentis M101-4 and in $B$. pseudocatenulatum MBL8320 CPS. These CPS were shown to strongly stimulate murine splenocytes in vitro. ${ }^{111,112}$

\subsection{Synthesis of a CPS Fragment from B. catunelatum}

To test the immunological properties of compounds, it is important to know the level of purity of these compounds. As discussed earlier, different strains of bifidobacteria contain different CPS structures. To understand the immunologic properties of these compounds, knowledge of the structure analyzed is valuable. CPSs that have been isolated and analyzed are commonly contaminated with cell wall components. Thus, in order to test the immune function of these molecules, chemical synthesis is required to obtain pure compounds. ${ }^{86}$ However, as CPSs are often of high molecular weight (MW), only the synthesis of fragments is feasible. Scheme 1.9 shows the synthesis of a fragment from B. catunelatum. ${ }^{113}$ Here, diacetonide $\mathbf{7 6}$ is benzylated and a regioselective deprotection of the acetonide is achieved to form diol 77. Coupling of glycosyl acceptor $\mathbf{7 7}$ and glycosyl donor $\mathbf{7 8}$ with TMSOTf and NIS gave coupled product 79 in poor yield, as separation of the $\alpha$ and $\beta$ anomers was only possible after acetylation. Deprotection of the acetate with a saturated solution of ammonia in $\mathrm{DCM} / \mathrm{MeOH}$ followed by coupling of glycosyl acceptor $\mathbf{8 0}$ and imidate $\mathbf{8 1}$ gave coupled product 82. Then after subsequent protecting group manipulations and formation of the trichloroacetimidate, glycosyl donor $\mathbf{8 3}$ is treated with dodecanol to form trisaccharide 84. Selective acetate deprotection of the Galf moiety gave glycosyl 
acceptor 85. The last steps were the coupling of glycosyl acceptor $\mathbf{8 5}$ with imidate $\mathbf{8 3}$ and global deprotection to obtain final product $\mathbf{8 6}$.

\subsubsection{Wall Teichoic Acids}

Publications on the detection of WTA from bifidobacteria are scarce. Veerkamp et al. found a mannitol teichoic acid in B. bifidum var. pennsylvanicus. ${ }^{114}$ In Figure 1.17, the sugar portion is linked via a mannitol phosphate and contains L-rhamnose and glucose. Further research on the immunological properties of WTAs is sparse. 
a.

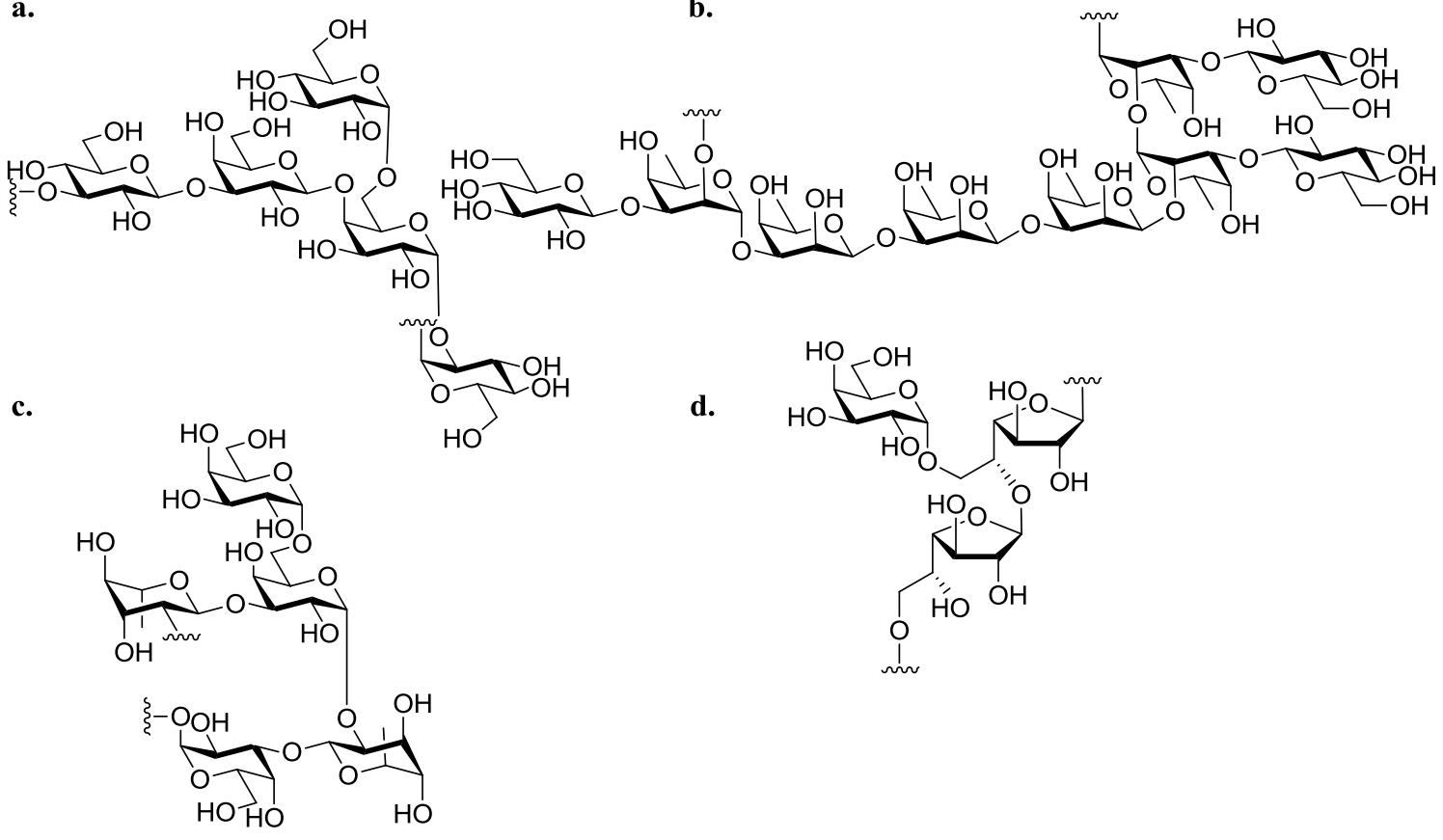

e.

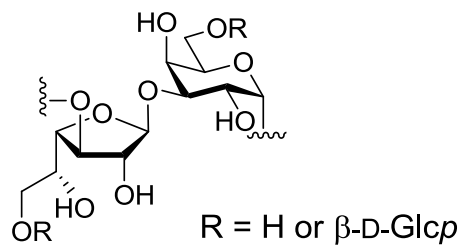

b.

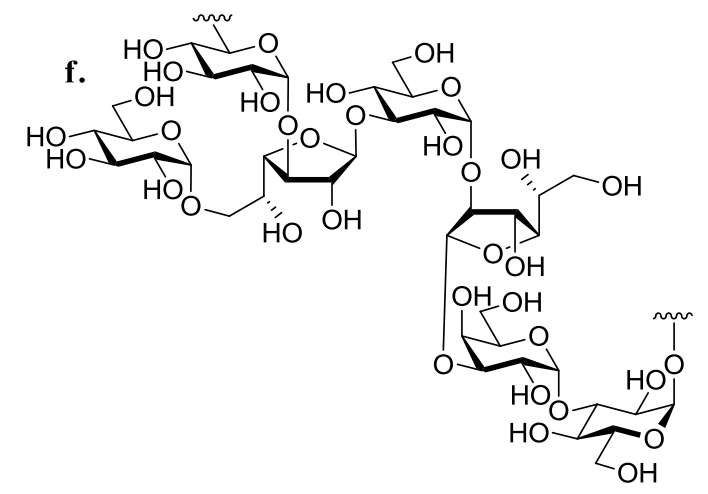

Figure 1.16 Proposed structures of CPS from a. B. breve YIT $4010,{ }^{106}$ b. $B$. adolescentis $^{107}$, c. B. longum YIT4028 ${ }^{108}$, d. B. catunelatum, ${ }^{109}$ e. B. infantis, ${ }^{105}$ f. $B$. bifidum BIM B-465. ${ }^{110}$ 


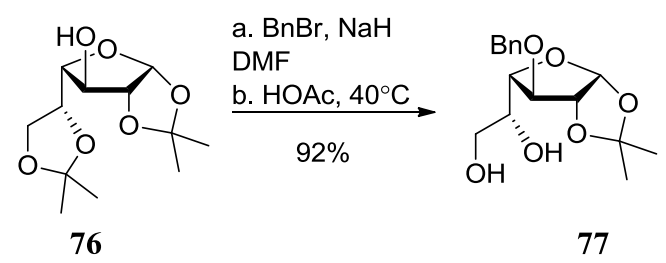

a. 78 , TMSOTf (cat.)

NIS, DCM

b. $\mathrm{Ac}_{2} \mathrm{O}$, Pyridine

$27 \%$
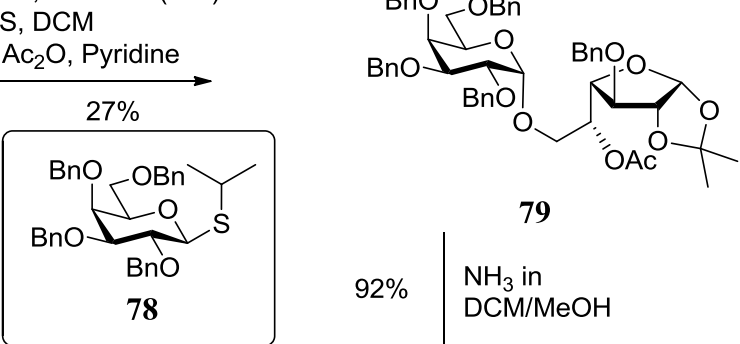

79

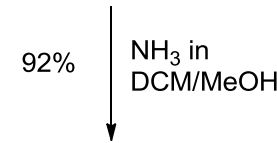

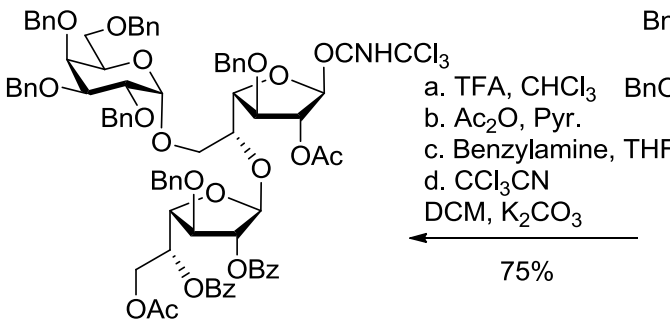

$\mathrm{BnO}-\mathrm{OBn}$

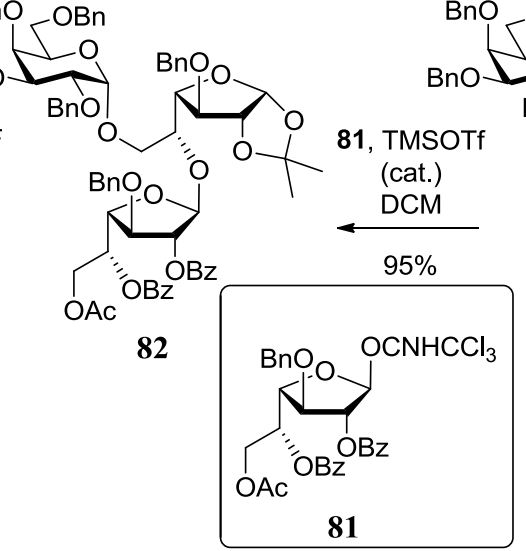

80

$97 \% \downarrow \begin{aligned} & \mathrm{C}_{12} \mathrm{H}_{25} \mathrm{OH}, \\ & \text { TMSOTf (cat.), DCM }\end{aligned}$

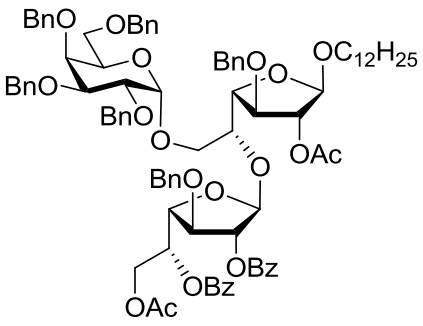

84

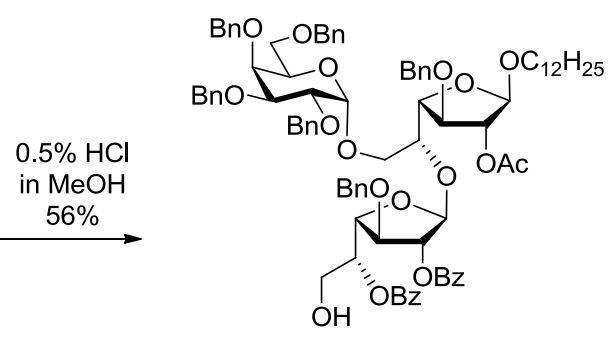

85

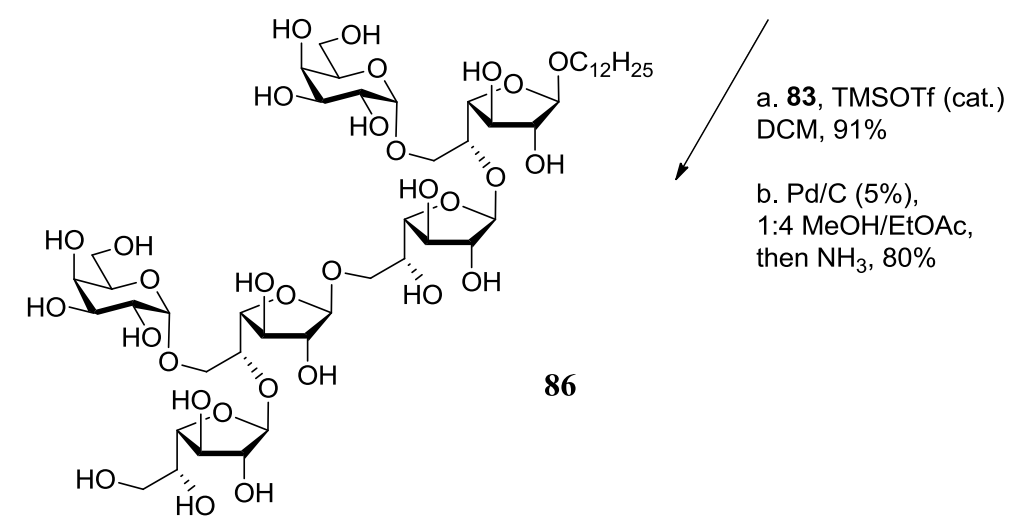

Scheme 1.9 Synthesis of B. catunelatum cell wall polysaccharide fragment. ${ }^{113}$ 


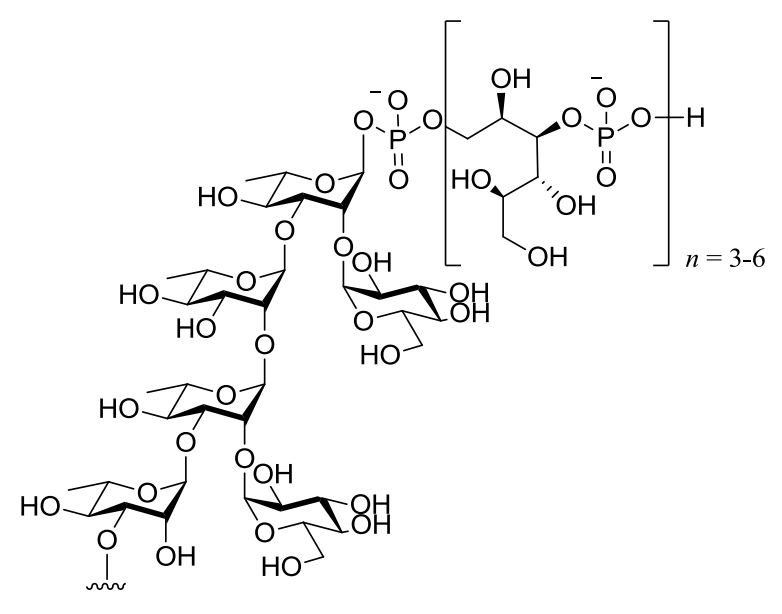

Figure 1.17 Proposed mannitol teichoic acid from B. bifidum var. pennsylvanicus. ${ }^{114}$

\subsubsection{Exopolysaccharides}

As the EPS is often strain or species specific, there is wide interest in the immunological properties of bifidobacteria EPS. ${ }^{115,116}$ EPS, released as a slime that surrounds the cell, is usually precipitated out of the growth medium using cold ethanol. The amount of EPS as well as the length and the level of branching obtained from this process vary between different species which is also observed for CPS. ${ }^{117}$ Impurities, such as proteins, are known to precipitate with the EPS when using the cold ethanol precipitation method. In order to test specifically EPS immunological properties, it is of importance to know the structure and purity of the mixture. ${ }^{118}$ Sugars contained in EPS are identified via different methods including enzymatic assays, ${ }^{118}$ GC-MS after hydrolysis and derivatisation, ${ }^{119}$ HPLC after hydrolysis, ${ }^{115,120}$ and NMR. ${ }^{119}$ Figure 1.18 shows examples of proposed structures for B. longum (a.) and B. animalis (b.). In B. longum, the repeating unit reported was $[\rightarrow 4) \alpha-\mathrm{D}-\mathrm{Gal}(1 \rightarrow 4) \alpha-\mathrm{D}-\mathrm{Gal}(1 \rightarrow 4) \beta-\mathrm{D}$ $\operatorname{Glc}(1 \rightarrow 3) \alpha-\mathrm{D}-\mathrm{Gal}(1 \rightarrow 3) \beta$-L-Rha $(1 \rightarrow]$ with branched glucose and galactose residues. ${ }^{121}$ Rhamnose was also detected in the EPS from B. animalis subsp. lactis, but in contrast with Kohno et al. findings, galactofuranose was identified in the structure. ${ }^{119}$ 
a.

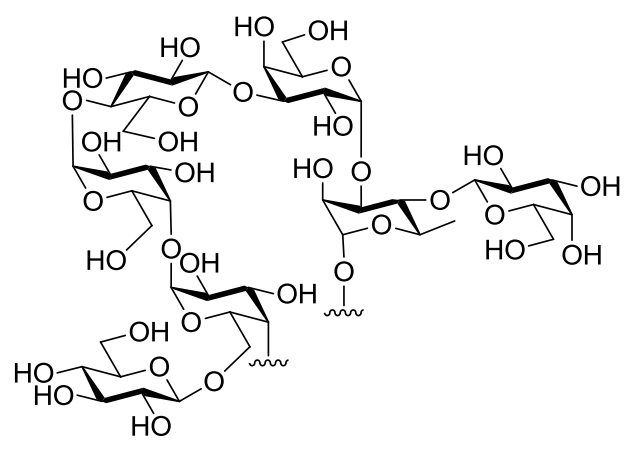

b.

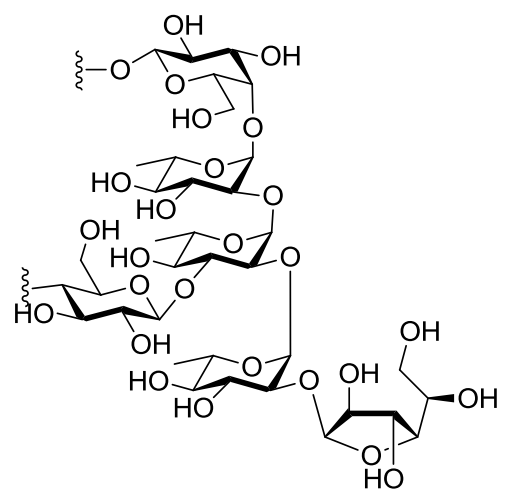

Figure 1.18 EPS repeating unit of a. B. longum JBL05 ${ }^{121}$ and b. B. animalis EPS. ${ }^{119}$

\subsubsection{Fatty Acids}

Usually, free fatty acids are found only in minor amounts in the bacteria and are commonly attached to phospholipids, glycolipids or waxes. ${ }^{122}$ For this reason, while analysing the whole cell fatty acids, a mixture from all the components in the cell is obtained. Initial studies used GC to determine what fatty acids were present. The fatty acids were derivatised by methanolysis using hexane and $\mathrm{BF}_{3}$-methanol at $100{ }^{\circ} \mathrm{C}$ for 15 minutes and, after injection in the GC, their retention times (RTs) were compared to standards. ${ }^{123-125}$ The disadvantage of this method is that the RT of varying FAMEs are similar, leading to ambiguous results (Figure 1.19). 


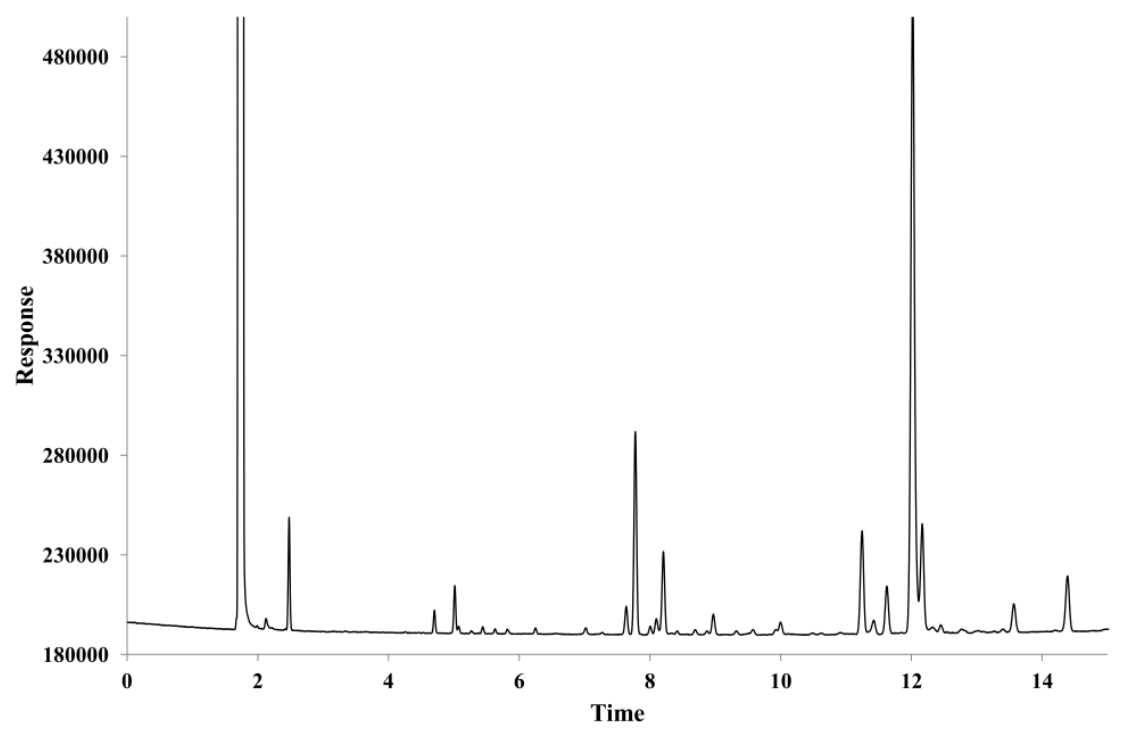

Figure 1.19 Example of a whole bacterial gas chromatogram of fatty acid methyl esters.

The fatty acids from Bifidobacterium bifidum var. pennsylvanicus were studied thoroughly by Veerkamp and Fischer between 1970 and 1987. ${ }^{123,124,126}$ Here, the fatty acids obtained were saturated, unsaturated and branched (e.g. Iso-18:0 and Anteiso19:0) some of which contained a cyclopropane ring (e.g. Cyclo-19:0). In 1998, other groups started to identify FAMEs using the microbial identification system database (MIDI). This MIDI database uses actual GC FAME RTs for bacterial identification. However, as before, the comparison between the RTs can lead to ambiguous results due to overlapping signals. After methyl esterification, it was found using MIDI that FAME and dimethyl acetals (DMA) were present. ${ }^{127-129}$ Figure 1.20 shows examples of fatty acids extracted from bifidobacteria. The most accepted method used today to confirm the identity of compounds such as DMA is GC-MS. The DMA fragmentation pattern is very characteristic (strong fragment signal at $m / z=75$ for $\mathrm{C}_{3} \mathrm{H}_{7} \mathrm{O}_{2}{ }^{+}$, Figure 1.21a) and differs greatly from saturated FAME fragmentation patterns (Figure 1.21b). ${ }^{130,131}$ DMAs (89), in contrast to FAMEs, are mainly formed from plasmalogen (87) under acidic conditions (Scheme 1.10). Indeed, the aldehyde $\mathbf{8 8}$ obtained from acid hydrolysis of 
plasmalogen 87 reacts with methanol to form DMA 89. The presence of DMA in bifidobacteria was later confirmed by Oberg et al. who found these derivatives in $B$. animalis subs. lactis using GC-MS after methylation. ${ }^{131}$

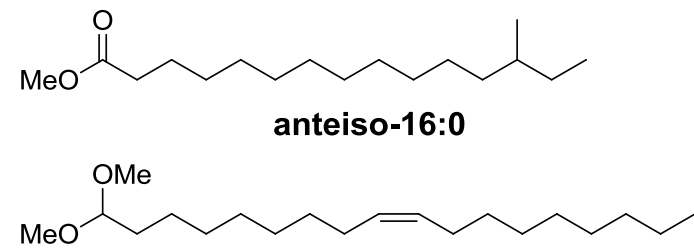

DMA-18:1

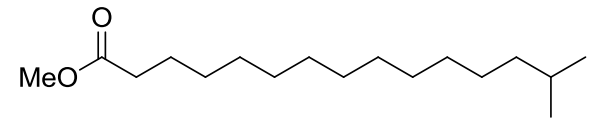

iso-16:0

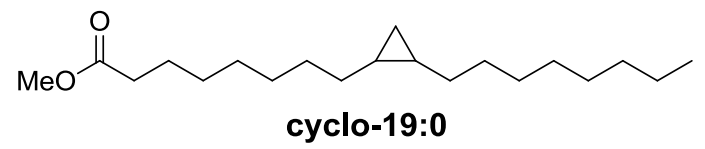

Figure 1.20 Example of fatty acids found in bifidobacteria.

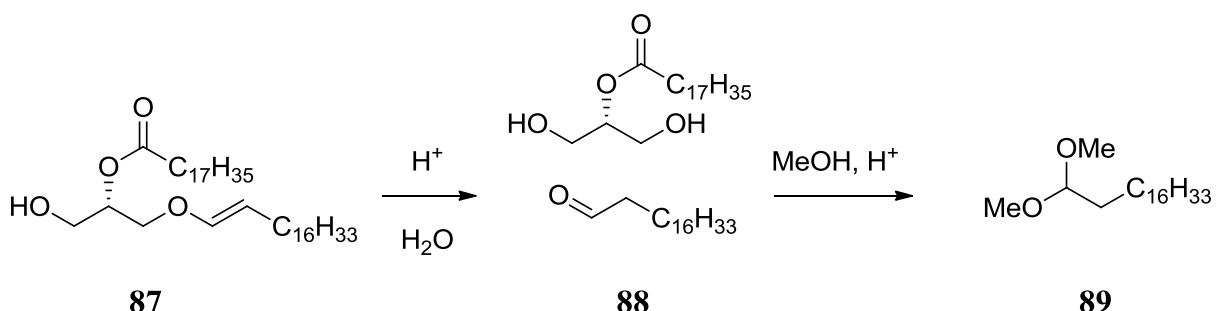

Scheme 1.10 Dimethyl acetal formation under acidic conditions. ${ }^{131}$

\subsubsection{Phosphoglycerolipids}

As most of the phospholipids are considered biocompatible, a very limited amount of groups studied bifidobacterial phospholipids. In an early paper Exterkate and Veerkamp extracted the cytoplasmic lipids from bifidobacteria using the method of Blight and Dyer ${ }^{42}$ that uses $\mathrm{CHCl}_{3} / \mathrm{MeOH} / \mathrm{H}_{2} \mathrm{O}$ extractions. The isolated lipids were purified on a silicic acid column using $\mathrm{CHCl}_{3} /\left(\mathrm{CH}_{3}\right)_{2} \mathrm{CO} /\left(\mathrm{CHCl}_{3} / \mathrm{MeOH} / \mathrm{H}_{2} \mathrm{O}[10 / 10 / 1\right.$, v/v/v] $)$. The resulting phospholipids were analyzed using 2D thin-layer chromatography (TLC) and 
against standards. The major phospholipids identified were phosphatidyl- and diphosphatidylglycerol, phosphatidyl glycerol. Additionally, minor amounts of phosphatidic, alanyl phosphatidyl glycerol and mono-, di- and tri-acyl-bis(glycerophosphoryl)glycerol were also detected. ${ }^{132}$ Exterkate et al. also described the phospholipids composition of Bifidobacterium and more recently Lactobacillus strains. The most significant difference reported here was the presence of alanyl phosphatidylglycerol in bifidobacteria and its absence in lactobacilli. ${ }^{133}$ More recently, Novik et al. extracted the lipids from bifidobacteria using $\mathrm{CHCl}_{3} / \mathrm{MeOH}$ and purified them by silica gel column chromatography. After comparison of standards to the compounds using 2D TLC, they found the major phospholipid components to be diphosphatidylglycerol, phosphatidylglycerol, phosphatidylethanolamine and phosphatidylcholine with minor constituents such as phosphatidylinositol and lysophosphatidylcholine. ${ }^{134,135}$

\subsubsection{Glycosyldiglycerides}

Veerkamp and co-workers first extracted glycolipids from bifidobacteria in 1969 using $\mathrm{CHCl}_{3} / \mathrm{MeOH} /$ acetate buffer mixtures. After silicic acid column chromatography, they obtained only galactolipids (diacyl-monogalactosyldiglyceride, monoacylmonogalactosyldiglyceride, monogalactosyldiglyceride, monoacyldigalactosyldiglyceride, monogalactosylmonoglyceride and digalactosyldiglyceride). ${ }^{132}$ Following work in 1971 by Exterkate and Veerkamp studied the effect of cell-wall inhibition on the composition of lipids. After a bifidobacteria growth without human milk, they found a decrease in galactose glycolipid content and also observed an additional lipid (trigalactosyldiglyceride). ${ }^{136}$ Later, in 1972, Veerkamp published the structure of the galactosyldiglyceride in B. bifidum var. pennsylvanicus. The glycolipids were extracted and purified by preparative TLC and mono-, di- and trigalactosyldiglyceride-containing acyl derivatives were identified as the major glycolipids (Figure 1.22). ${ }^{44}$ These investigations, however, were limited by the analytical techniques available at the time and only partially described the structure of the galactolipids. For instance, fatty acid analysis (identification of the fatty acids using 
GC-MS following methyl esterification of the fatty acids) of the glycosyldiglycerides was not reported, nor were full NMR and mass data.

a.

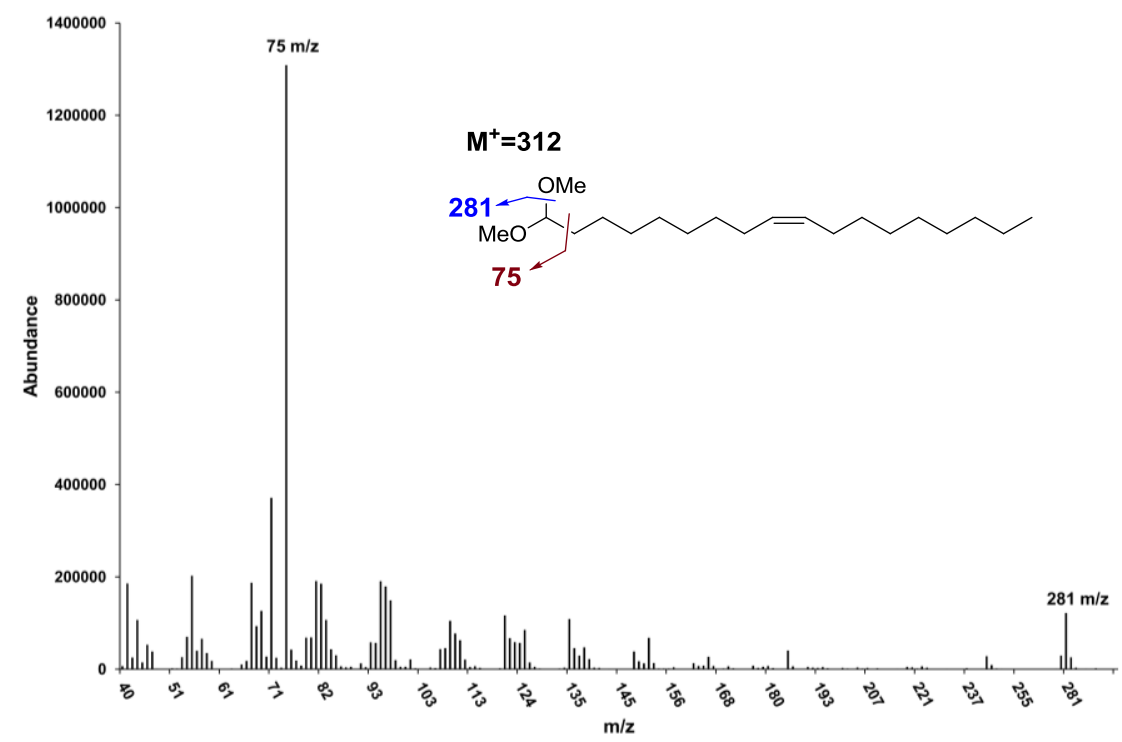

b.

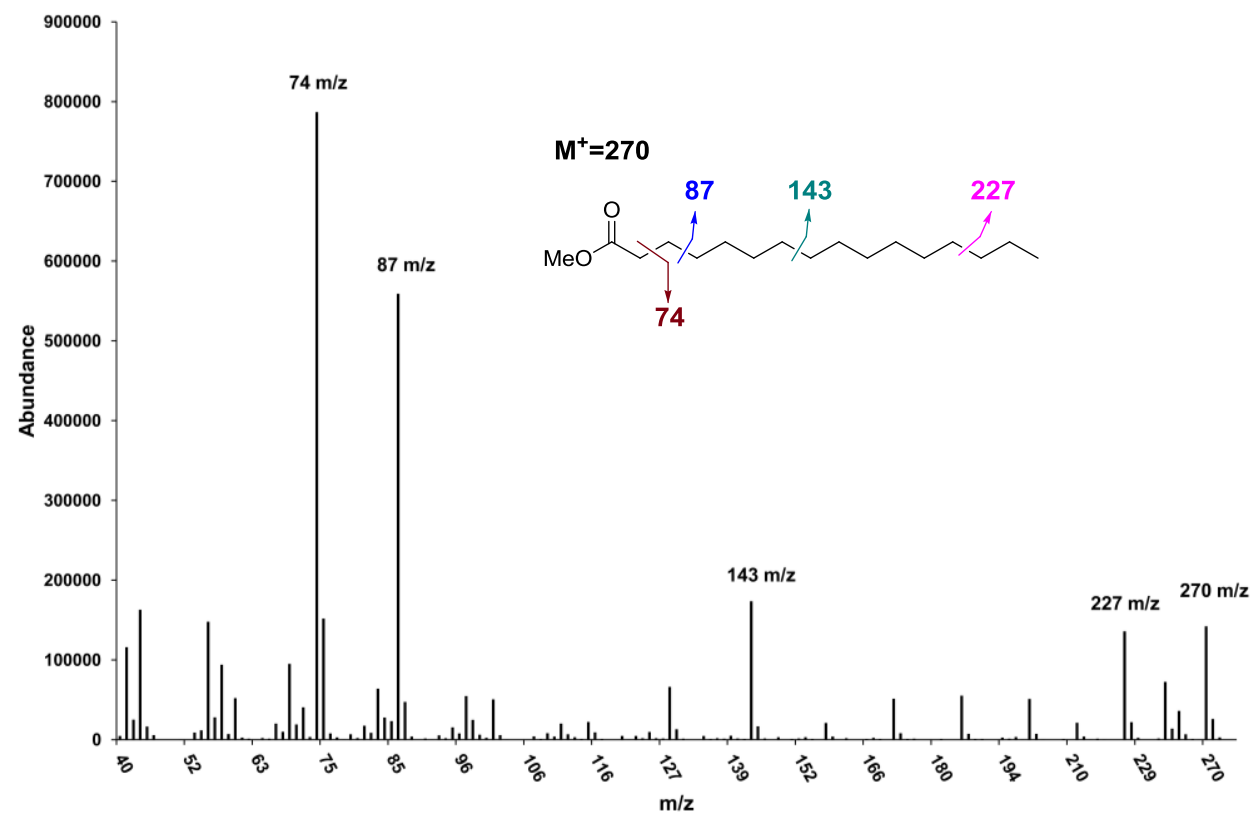

Figure 1.21 MS fragmentation spectrum of a. 18:1 DMA ${ }^{131}$ and b. Methyl palmitate. $^{130}$ 
More recently, Novik and et al. ${ }^{104,134,137,138}$ have isolated and analyzed by TLC the principal galactolipids from a number of bifidobacterial species and strains, although again no detail of their structure was given in their publication and the compounds were not purified. Novik also described that some glycolipids had different mobility on TLC than that described for other bacteria, suggesting unusual structures as the reason. These glycolipids have only been investigated via TLC or sugar analysis. As glycosyldiglycerides were found to be biologically active (Section 1.5.4) and as Novik et al. also found some activity in bifidobacterial extracts ${ }^{104}$ it is important that the identity of these compounds is established for further investigations concerning their synthesis and biological activity. Therefore, the data generated using NMR, ESI-MS, mass-spectrometry fatty acid analysis, and sugar analysis after purification would be a contribution to the literature on bifidobacterial glycoglycerolipids.
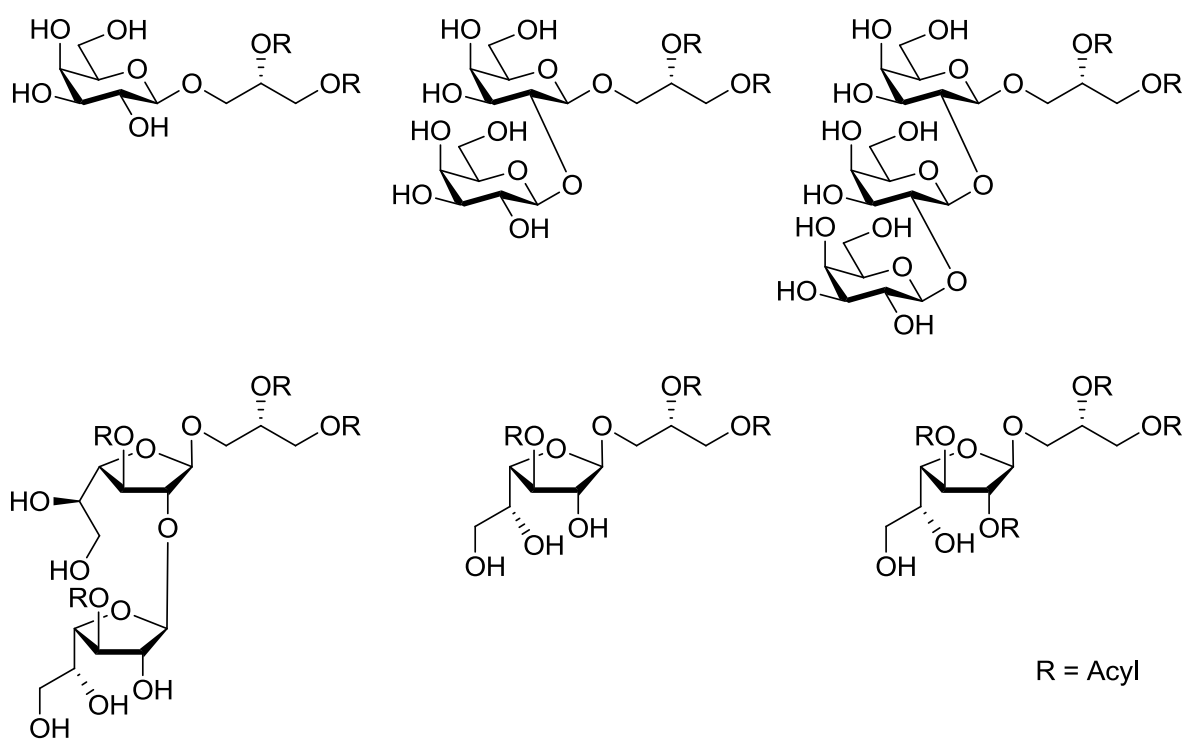
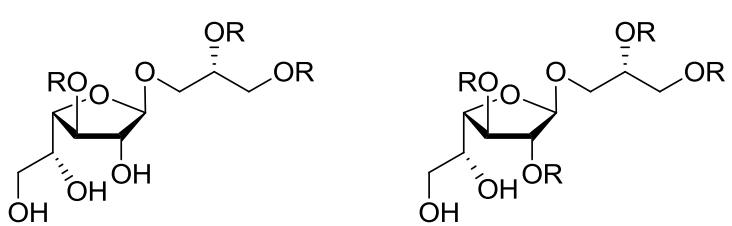

$\mathrm{R}=\mathrm{Acyl}$

Figure 1.22 Glycosyldiglycerides identified in B. bifidum var. pensylvanicus. ${ }^{44}$

\subsubsection{Phosphated Galactolipids and Lipoteichoic Acid}

Bifidobacterial LTAs were shown to be biologically active and thus implicit knowledge of LTA structures is important. ${ }^{102,139-143}$ The structure of LTA extracted from B. bifidum was first proposed by Op Den Camp, then corrected by Fischer et al., and finally Fischer's findings were confirmed by Iwasaki in $1990 .^{62,124,144-146}$ The structure 
confirmed by Iwasaki (Figure 1.23) which was extracted using hot phenol, contains a galactosyldiglyceride anchor, linked to a $\beta$-glucose polysaccharide. The last glucose is attached to a galactofuranose-glycerophosphate repeating unit.

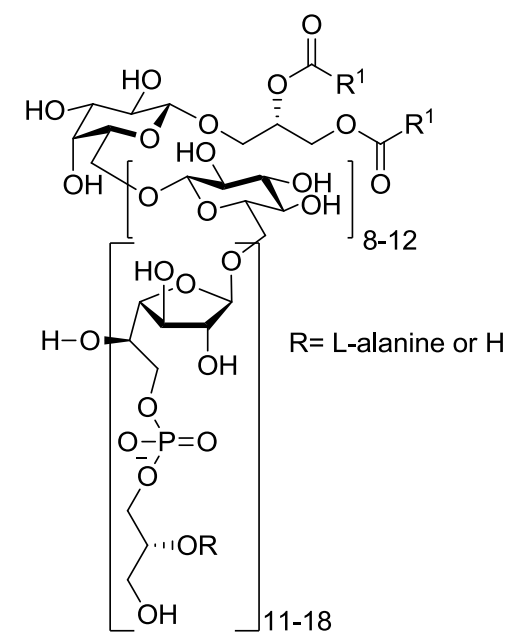

Figure 1.23 LTA fragment structure proposed for Bifidobacterium bifidum YIT 4007 and YIT 4010, described by Iwasaki. ${ }^{62}$

\section{$1.7 \quad$ Lactobacillus}

Lactobacilli, as with bifidobacteria, are used as a probiotic and are also believed to be beneficial. ${ }^{147}$ Both bacteria are found in the human gut and are often discussed together as they are both part of the lactic acid bacteria group (LAB). ${ }^{148,149}$ Other similarities include the fact that they are also Gram-positive, fermentative bacteria and can be rod shaped. However, contrarily to bifidobacteria, lactobacilli belong to the Firmicutes genus while bifidobacteria belong to the Actinomycetes genus. In contrast to bifidobacteria, lactobacilli are ubiquitous, as they can be found in all environments containing carbohydrates such as milk products, food, beverages and the human intestinal tract. ${ }^{150}$ Due to lactobacilli omnipresence and the many different species ( $c a$ 106), ${ }^{150}$ literature regarding lactobacilli is profuse (SciFinder ${ }^{\circledR} c a .64159$ hits) and many reviews describe lactic acid bacteria and Lactobacillus cell wall. ${ }^{151,152}$ The study of the cell wall components from L. plantarum, previously named L. arabinosus, ${ }^{153}$ is one of the most investigated LABs because of its probiotic properties, is important. ${ }^{154}$ As this 
research focuses on L. plantarum, only the publications discussing the compounds on the surface of $L$. plantarum will be discussed. In order to understand $L$. plantarum whole cells immunological properties, it is importand to determine structurally their surface components. ${ }^{155-157}$

\subsubsection{Wall Teichoic Acids}

The presence of ribitol phosphate and/or glycerol phosphate type polysaccharides was detected during initial studies of the immunologically active L. plantarum WTAs. ${ }^{158,159}$ D-Ala or D-glucose was detected in a strain dependent manner on the ribitol phosphate and /or glycerol phosphate. ${ }^{158,160-162}$ After hydrolysis, the analysis revealed fragments of the structure that implied the presence of a polymer consisting of a long poly(ribitolphosphate) chain containing $\alpha$-D-glucose residues on the 2 and 3 positions of the chain. Adams et al. found the same structural components, however the glucose residue was linked with phosphodiester bonds to form the backbone of the WTA. ${ }^{163}$ This finding was later confirmed by Archibald et al. and the repeating units of glucosylglycerol linked with phosphodiester bonds was also described (Figure 1.24). ${ }^{164}$

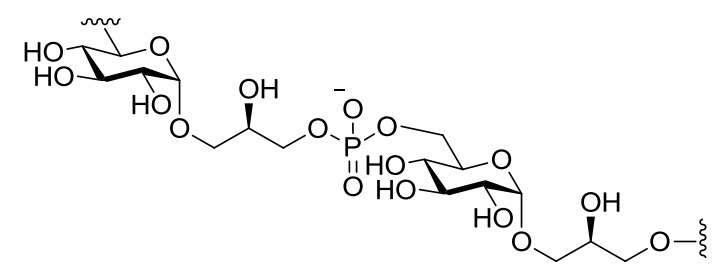

Figure 1.24 WTA structure from L. plantarum N.I.R.D. C106. ${ }^{164}$

Later, Tomita et al. isolated monomeric units of L. plantarum WTA and found 3,4- $\alpha-\mathrm{D}-$ diglucosyl-2-phosphoryl ribitol and 3,4- $\alpha$-D-diglucosyl-1-phosphoryl ribitol. ${ }^{165}$ They also compared the WTA from different strains of L. plantarum and found two principal backbones depending on the strain (Figure 1.25). The first backbone consisted of 6linked units of 1-O-( $\alpha$-D-glucosyl)-3-O-phosphoryl-sn-glycerol while the second backbone was formed with ribitol phosphate. Also, depending on the strain, the glucose residues were linked at different positions of the ribitol and contained either 
monoglucosyl or diglucosyl moieties. ${ }^{166}$ Phosphorylated rhamnose-containing polysaccharides were also found in some strains of L. plantarum. ${ }^{167-169}$

a.

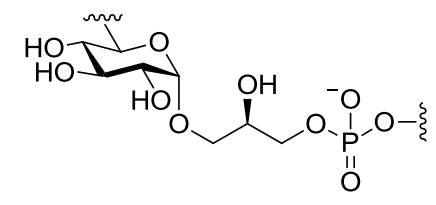

b.<smiles>COCC(O)[C@@H](O)C(O)COP(C)(=O)O</smiles>

Figure 1.25 Ribitol and glycerol phosphate backbones. ${ }^{166}$

\subsubsection{Chemical Synthesis of Wall Teichoic Acids}

Sargent and Baddiley synthesised the 4- $O$ - $\alpha$-D-glucopyranosyl-D-ribitol 93 described by Archibald et al. (Section 1.7.1). As shown in Scheme 1.11 a selective protection step using benzoyl chloride and starting from partially protected ribitol $\mathbf{9 0}$ gave benzoylated precursor 91. Subsequent condensation of alcohol 91 with Brigl's anhydride 92 and global deprotection afforded target compound $\mathbf{9 3}{ }^{170}$

\subsubsection{Exopolysaccharides}

The structure of the immunologically active ${ }^{171}$ EPS from L. plantarum has been studied by Tallon et al. Here, two different types of EPS have been described. The first type had a lower molecular weight $\left(4 \times 10^{4} \mathrm{Da}\right)$ and a ratio of $1 / 1 / 3$, galactose/rhamnose/glucose, and contained smaller amounts of phosphate and glycerol. In the second type, a higher molecular weight component $\left(8 \times 10^{4} \mathrm{Da}\right)$ and a ratio of $1 / 1 / 3$, galactose $/ N$ acetylgalactosamine/glucose, was found. ${ }^{154}$ Later, Ismail and Nampoothiri purified and characterised the EPS from L. plantarum. In this case, $\alpha$-D-mannose, $\alpha$-D-glucose and $\beta$ D-glucose were found using NMR, however, no additional information was given on the structure. ${ }^{172}$ In the same year, Wang et al. analyzed EPS produced by L. plantarum isolated from kefir. They found a ratio of 1/5/7 of mannose/glucose/galactose. Galactose and glucose were also exclusively detected in the EPS of L. plantarum in a medium 
containing $13 \%$ whey and supplemented with yeast extract and glucose. ${ }^{173}$ In 2011 , Liu et al. analyzed the EPS from different strains of lactobacilli. It was reported that arabinose, galactose, glucose, mannose, fructose and maltose were identified in EPS after hydrolysis. This EPS also induced macrophage activation. ${ }^{174}$

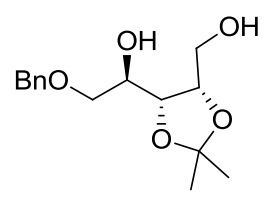

90

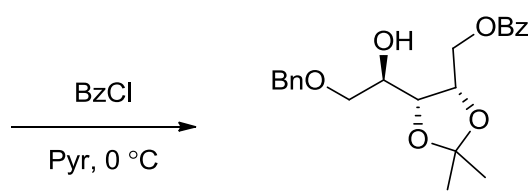

91

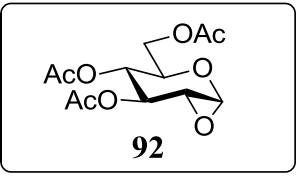

1) $92,100^{\circ} \mathrm{C}$

2) $\mathrm{NaOMe}, \mathrm{MeOH}$

3) $\mathrm{H}_{2}, \mathrm{Pd} / \mathrm{C}$

4) $\mathrm{H}_{2} \mathrm{SO}_{4} / \mathrm{EtOH}(5 \%, v / v), 80^{\circ} \mathrm{C}$

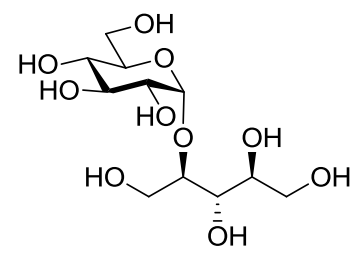

93

Scheme 1.11 Synthesis of 4- $O$ - $\alpha$-D-glucopyranosyl-D-ribitol. ${ }^{170}$

\subsubsection{Fatty Acids}

The cell wall of $L$. plantarum contains saturated and monounsaturated fatty acids commonly found in most bacteria (C12 to $\mathrm{C} 18) .{ }^{175,176} \mathrm{~A}$ fatty acid usually found in lactobacilli and is also present in L. plantarum is lactobacillic acid. ${ }^{177-179}$ In Scheme 1.12, lactobacillic acid is depicted at the sn-2-position of the glycerol in phosphatidylethanolamine 95. As implied in Scheme 1.12 monounsaturated fatty acids are required for the synthesis of cyclopropane fatty acid (CFA). In this case, an extra step in the biosynthetic pathway is required, and is catalysed by a CFA synthase. Here, the double bond of the monounsaturated fatty acid, typically present on phospholipid 94 or glycolipid, attacks the methyl group on the $S$-adenosine-methionine (AdoMet) and after elimination of a methyl proton, the cycle would be formed to give cyclic fatty acid 95. Intermediates such as branched fatty acids can also be formed in this process. ${ }^{180,181}$ 
Further studies on the subject determined the absolute configuration to be $11 R$ and $12 S .^{182}$

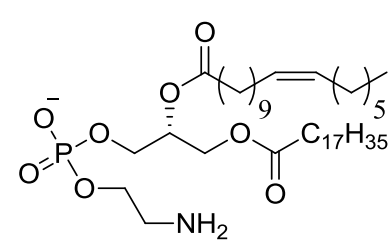

94

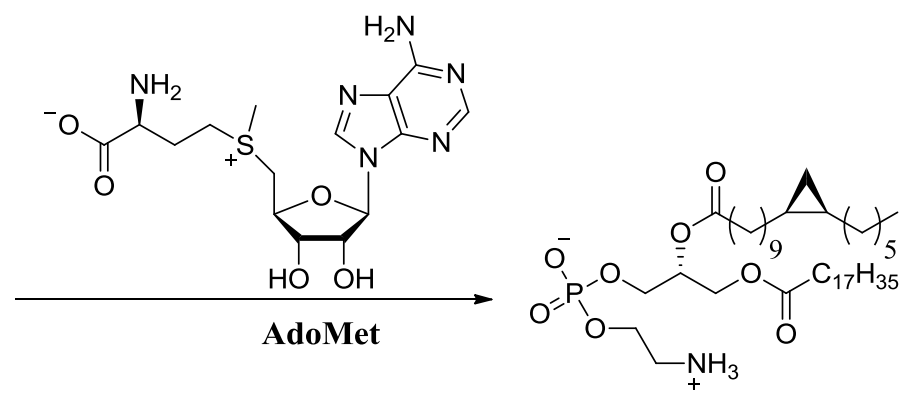

95

Scheme 1.12 Biomechanism of cyclopropane fatty acid formation. ${ }^{180}$

\subsubsection{Phospholipids}

Only a few groups studied the phospholipids from L. plantarum using TLC and MALDI. ${ }^{183,184}$ The major phospholipids found by Exterkate et al. were diphosphatidylglycerol (DPG), phosphatidylglycerol, lysyl-phosophatidylglycerol and PA. ${ }^{133}$ Here, no mention of CL was made, and this phospholipid was later observed by Arbogast et al. in L. plantarum. ${ }^{185}$ The synthesis of CL from phosphatidylglycerol via a CL synthetase bound to the membrane in $L$. plantarum was subsequently studied by Buritt et al., who found that the amount of CL synthesised diminished with time. ${ }^{186}$ Finally, the use of mass spectrometry to compare the phospholipids in different bacteria/strains allowed a putative assignment. This type of mass-spectrometric comparison was made by Drucker et al. who identified phosphatidylglycerol and PS ${ }^{183}$ while Calvano et al., with similar analyzes, detected mainly phosphatidylglycerol and CL. ${ }^{184}$ 


\subsubsection{Glycosyldiglycerides and Lipoteichoic Acids}

The literature regarding L. plantarum LTA and glycosyldiglycerides is very confusing. Though many groups have extracted and characterised LTA and glycosyldiglycerides, the reported configuration of the anomeric carbon on the glucose moiety at the nonreducing end of the TGDG has been inconsistent. An early publication by Ikawa compared the lipids from different lactic acid bacteria including L. plantarum and determined that some lipids contained glycerol, glucose and galatose. ${ }^{187}$ Brundish et al. also analyzed the glycolipids from different lactobacilli species, identifying partially the structures of $L$. plantarum to be $\beta$-glucosylgalactosyl- $\beta$-glucosyl-( $1 \rightarrow 1)$-glycerol. ${ }^{43,46}$ In contrast with the results published by Brundish et al., Shaw et al described glycoglycerolipids with $\alpha$-glucose at the non-reducing end instead of $\beta$-glucose (Figure 1.26). ${ }^{46}$ Nakano and Fischer also compared the glycolipids present in L. casei with the ones from L. plantarum using 2D TLC and suggested that the glycolipids from both species were similar. ${ }^{188}$ All reports of L. plantarum have not conclusively determined the glycoglycerolipids present in L. plantarum.
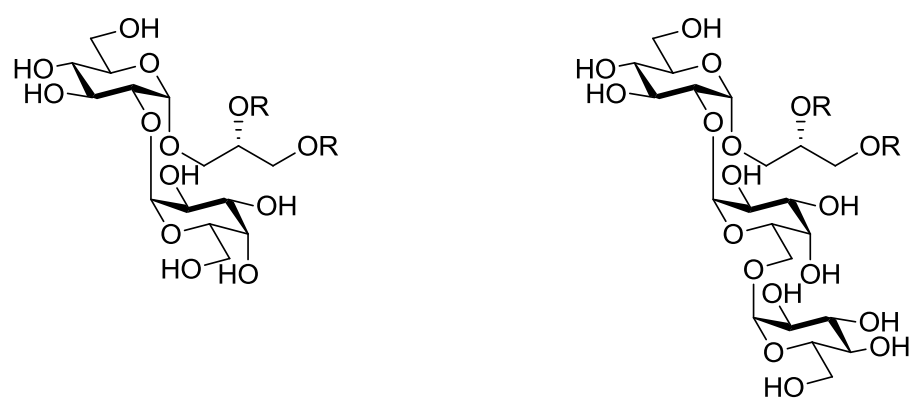

Figure 1.26 L. plantarum glycolipids isolated by Shaw et al. ${ }^{46}$

As LTAs are derived from glycosyldiglycerides (Section 1.4.4), the study of LTAs also shed light on the glycolipids present in L. plantarum. However, as with glycosyldiglycerides, the configuration of the residues was not conclusively determined. Early publications on L. plantarum LTAs described a glycolipid anchor containing a $\beta$ glucose at the non-reducing end of TGDG (Figure 1.27a). ${ }^{189,190}$ Later, Jang et al. extracted the lipoteichoic acids from L. plantarum. Here, instead of a $\alpha$-glucose at the 
reducing end of TGDG, the structures proposed by Jang et al. suggested a $\beta$-glucose moiety (Figure 1.27b). ${ }^{57}$ Regarding the immunomodulatory properties of these glycolipids, many L. plantarum crude LTA extracts were found to be active. Additionally, it was anticipated that the immune response could be modulated by the Dalanine content. ${ }^{191-193}$ Indeed, when cells of a Dlt ${ }^{-}$mutant were tested for cytokine production in PBMCs, there was a significant reduction in proinflamatory cytokines (e.g. IL-12, $\mathrm{TNF} \alpha$ ), compared to cells of a L. plantarum wild type. These results suggested that the D-alanines are responsible for the biological activity observed in the wild type as the Dlt mutant cells do not contain D-alanines in their LTAs. This fact is interesting, however, none of these publications described the immunological properties of fully characterised purified compounds.

a.
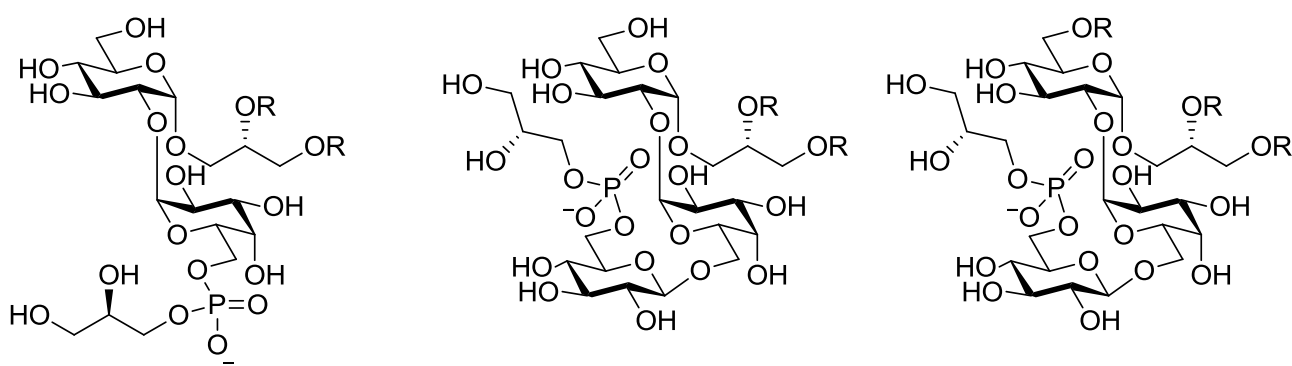

b.
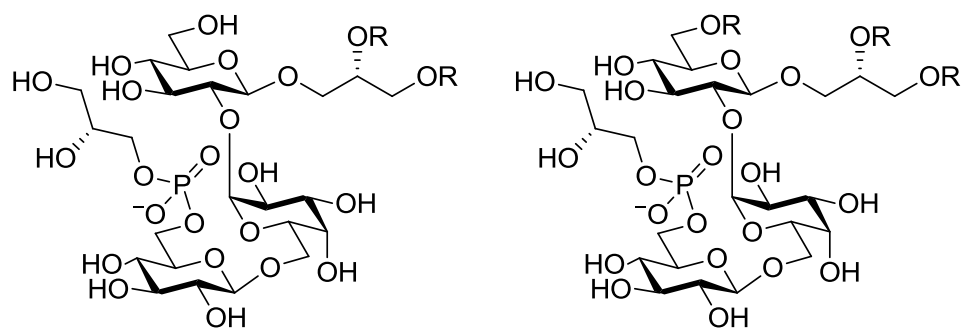

Figure 1.27 L. plantarum LTA fragments isolated by a. Fischer ${ }^{190}$ and b. Jang et al. ${ }^{57}$ 


\subsection{Aim and Outline of Thesis}

Due to the lack of characterisation data such as NMR and MS for the components present in lactobacilli and bifidobacteria and the absence of immunological testing on fully characterised pure compounds, from their cell wall it is important to fully characterise and assess their biological activity. Thus, this research aims to extract, characterise, synthesise and evaluate the immunological properties of bacterial commensal glycoglycerolipids. After purification, the glycolipids were analyzed using constituent sugar and glycosyl linkage analysis, fatty acid analysis, NMR and ESI/MS. In Chapter 2, the glycoglycerolipids from L. plantarum are described and tested in a macrophage activation assay. Chapter 3 reports a novel glycolipid isolated from $B$. longum subs. infantis and its immunological properties are also evaluated.

Furthermore, due to contamination caused while extracting LTAs, the synthesis of such pure compounds for biological testing is of interest. The synthesis of eleven analogues of GL5 was achieved and the analogues were tested for their ability to induce macrophage activation as described in Chapter 4. Finally, Chapter 5 summarizes priliminary results and describes future prospects, and conclusions of the project. Additionally this chapter will discuss necessary steps to determine the stereochemistry of GL5. 


\section{Chapter 2}

\section{Isolation and Structural Characterisation of the Major Glycoglycerolipids from Lactobacillus plantarum ${ }^{194}$}

To date, the structures of the glycoglycerolipids from Lactobacillus plantarum, a commonly used beneficial probiotic, have not been conclusively assigned. In this chapter, for the first time, the full characterisation of the four principal glycoglycerolipids of the L. plantarum cell wall is presented using sugar, linkage and FAME analysis, as well as ESI-MS/MS and 1D- and 2D-NMR spectroscopy. The major glycoglycerolipids are assigned as being: $\alpha$-D-Glcp-diglyceride, $\alpha$-D-Galp-(1 $\rightarrow 2)-\alpha-D-$

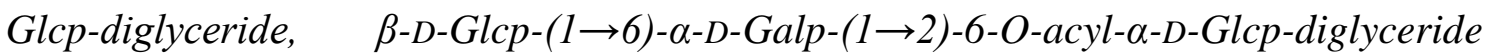
and $\beta$-D-Glcp- $(1 \rightarrow 6)-\alpha-D$-Galp- $(1 \rightarrow 2)-\alpha-D$-Glcp-diglyceride.

\subsection{Background}

Lactobacillus plantarum is a widespread member of the genus Lactobacillus and is commonly found in fermented food products and anaerobic plant matter. ${ }^{195}$ It is also a major coloniser of the human gastrointestinal tract and the mucosa of the mouth and nose. ${ }^{196}$ Due to the potential health benefits of lactobacilli, there has been wide interest 
in the immunomodulatory properties of this commensal. ${ }^{197}$ It is well known that many bacterial glycoglycerolipids (e.g. LPS, TDMs and PIMs) have profound immunological effects, ${ }^{63,76,198-202}$ and for this reason, characterising the major glycoglycerolipids in $L$. plantarum is of interest. In the late 1960s, Shaw et al. reported on the isolation and structural characterisation of glycosyldiglycerides from L. plantarum $^{43}$ and Lactobacillus casei, ${ }^{203}$ and found a glucosylgalactosylglucosyl-glycerol in L. plantarum, but were unable to deduce the glycosidic linkages and anomeric configurations. ${ }^{46}$ In 1977, Nakano and Fischer elucidated the structures of mono-, di-, tri- and tetraglycosyldiglycerides from L. casei using enzymatic methods, TLC and gas-liquid chromatography. Through TLC analysis, Nakano and Fischer concluded that the membrane glycoglycerolipids from $L$. casei and $L$. plantarum were very similar. ${ }^{188}$ In addition, Fischer et al. studied the relationship of phosphoglycolipids and lipoteichoic acids of several Gram-positive bacteria and saw similar phosphoglycolipids present in both L. casei and L. plantarum. ${ }^{189,190}$ From the aforementioned studies, a number of glycoglycerolipids have been inferred to be present in L. plantarum, ${ }^{43,46,57,188-190,203}$ however, no conclusive spectral information has been provided. This is an important omission that needs to be addressed if a better understanding of the taxonomy and immunomodulatory properties of $L$. plantarum is to be achieved. ${ }^{204}$ Herein, the structures of the four major glycoglycerolipids, isolated from L. plantarum IRL-560 using modern analytical techniques, are unequivocally established.

\subsection{Experimental Procedures}

\subsubsection{Bacteria and Cultivation}

\section{[The bacterial growth was conducted by Jason Ryan, IRL]}

Cultures of L. plantarum IRL-560 cells were grown plated onto Refined Clostridium Media (RCM) agar and incubated anaerobically at $37{ }^{\circ} \mathrm{C}$ for $24 \mathrm{~h}$. Glycolipid production was conducted anaerobically in a $18 \mathrm{~L}$ fermenter using a carbohydrate-free modified MRS (Man-Rogosa-Sharp) media in a 20 L Braun bioreactor (Sartorius- 
Stedim, Aubagne Cedex, France). The culture was then centrifuged at $3000 \mathrm{~g}$ for $10 \mathrm{~min}$ to yield a watery paste and the cell-mass was freeze dried. Next, the dried cell-mass was washed three times with PBS buffer ( $\mathrm{pH} 7.3$ ) and centrifuged (15 min, $31186 \mathrm{~g}, 4{ }^{\circ} \mathrm{C}$ ) or until the amount of carbohydrate detected in the washes by colorimetric assay was under $1 \%(\mathrm{w} / \mathrm{v})$. To remove the PBS buffer, the cells were washed with deionised water and centrifuged, then freeze-dried and weighed.

\subsubsection{Isolation and Purification}

The dried cell-mass $(8 \mathrm{~g})$ was extracted twice $\left(400 \mathrm{~mL}, 18 \mathrm{~h}, 37{ }^{\circ} \mathrm{C}\right)$ with $\mathrm{CHCl}_{3} / \mathrm{MeOH} / \mathrm{H}_{2} \mathrm{O}(10 / 5 / 1, \mathrm{v} / \mathrm{v} / \mathrm{v})$. After filtration, the combined extracts were evaporated to dryness ( $\mathrm{ca} .0 .9 \mathrm{~g}$ ), suspended in $\mathrm{CHCl}_{3} / \mathrm{MeOH} / \mathrm{H}_{2} \mathrm{O}(100 \mathrm{~mL}, 5 / 5 / 4.5$, $\mathrm{v} / \mathrm{v} / \mathrm{v})$ and rested overnight $\left(16 \mathrm{~h}, 4{ }^{\circ} \mathrm{C}\right)$ to remove polar impurities. The organic fraction was evaporated to dryness and analyzed by TLC. The crude lipid extracts were preabsorbed onto silica gel and fractionated by silica gel column chromatography. Glycoglycerolipids were eluted successively with a step-wise gradient of $\mathrm{MeOH}$ in $\mathrm{CHCl}_{3}$ and further purified, if needed, using preparative TLC. GL2a and GL2b were separated from each other using size-exclusion chromatography on a Sephadex LH-20 column eluting with $\mathrm{DCM} / \mathrm{MeOH}(1 / 1, \mathrm{v} / \mathrm{v})$.

\subsubsection{NMR Spectroscopy}

Purified glycoglycerolipids were dissolved in $0.7 \mathrm{~mL} \mathrm{CDCl}_{3} / \mathrm{CD}_{3} \mathrm{OD}(2 / 1, \mathrm{v} / \mathrm{v})$. Samples were analyzed at $22{ }^{\circ} \mathrm{C}$ and the chemical shift measured relative to chloroform and TMS. The fatty acid methyl esters were dissolved in $\mathrm{CDCl}_{3}$. NMR spectra were recorded on a Bruker Advance III $500 \mathrm{MHz}$ spectrometer or a Varian DirectDrive 600 (600 MHz for ${ }^{1} \mathrm{H}, 150 \mathrm{MHz}$ for ${ }^{13} \mathrm{C}$ ) spectrometer. 


\subsubsection{Electrospray Mass Spectrometry}

Electrospray mass spectrometry (or accurate mass measurement) was performed in the positive (or negative) ion mode on a Micromass Q-Tof Premier instrument. All MS/MS experiments were performed on a Q-Tof Premier mass spectrometer (Micromass, UK).

\subsubsection{General Analyzes}

Total carbohydrate was estimated colourimetrically by the phenol-sulfuric acid method using glucose $(0-80 \mu \mathrm{g})$ as the standard. ${ }^{205}$ For example, in Scheme 2.1, glucose is dehydrated to form 5-(hydroxymethyl)furfural using sulfuric acid. Following this, the furfural was condensed with two molecules of phenol to form a product that is measured at $490 \mathrm{~nm}$ on a spectrometer.

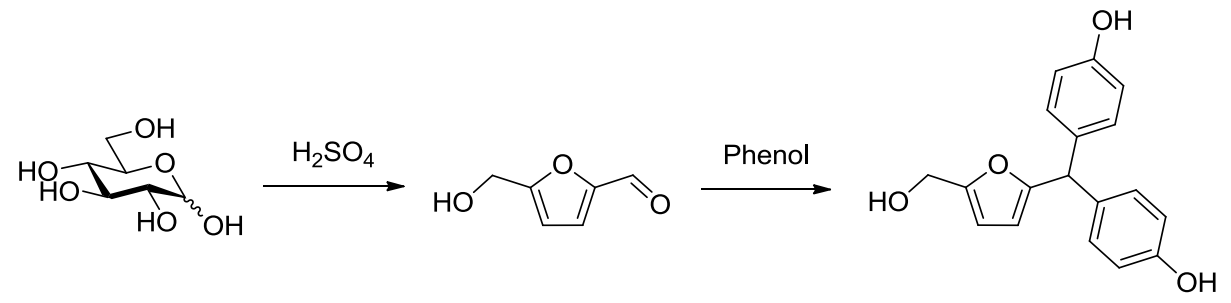

Scheme 2.1 Phenol-sulfuric acid reaction with glucose.

For TLC analysis, glycolipid samples were dissolved in $\mathrm{CHCl}_{3} / \mathrm{MeOH}(2 / 1$, v/v) and applied to dried silica pre-coated TLC plates. The glycoglycerolipids were eluted with $\mathrm{CHCl}_{3} / \mathrm{MeOH}(6 / 1, \mathrm{v} / \mathrm{v})$, sprayed and developed with a cerium molybdate (Hanessian's) stain, with a molybdate stain for the detection of phosphorous and a sulfuric acid stain. $^{206,207}$ 


\subsubsection{Constituent Sugar Analysis}

The constituent neutral sugar composition was determined by GC-MS following acidic hydrolysis and derivatisation to alditol acetates. ${ }^{49}$ Briefly, samples were hydrolysed with $2 \mathrm{M}$ TFA $\left(400 \mu \mathrm{L}, 1 \mathrm{~h}, 120{ }^{\circ} \mathrm{C}\right)$, neutralised with $2 \mathrm{M} \mathrm{NH} \mathrm{NH}_{4} \mathrm{OH}(0.4 \mathrm{~mL})$, using $50 \mu \mathrm{g}$ myo-inositol as an internal standard, reduced with $2 \%(\mathrm{w} / \mathrm{v}) \mathrm{NaBD}_{4}$ in water, and then acetylated with acetic anhydride $(200 \mu \mathrm{L})$ in pyridine (Scheme 2.2). Alditol acetates were quantified by GC on a SGE BPX90 capillary column (30 m x $0.25 \mathrm{~mm}$ i.d., $0.25 \mu \mathrm{m}$ film thickness) with the $\mathrm{GC}$ oven programmed from $80^{\circ} \mathrm{C}$ (held for $1 \mathrm{~min}$ ) to $150{ }^{\circ} \mathrm{C}$ at a rate of $25^{\circ} \mathrm{C} / \mathrm{min}$, and then to $250{ }^{\circ} \mathrm{C}$ at a rate of $4{ }^{\circ} \mathrm{C} / \mathrm{min}$ and analyzed by MS using an Agilent 5973 Inert MSD. Identifications were based on peak retention times and by comparison of electron impact spectra with standard spectra (Figure 2.1 and 2.2). Weight calibration constants were determined from standard sugar mixes (derivatised at the same time as the samples) following the TAPPI standard method T249 cm-85 (1995). ${ }^{\mathrm{c}}$

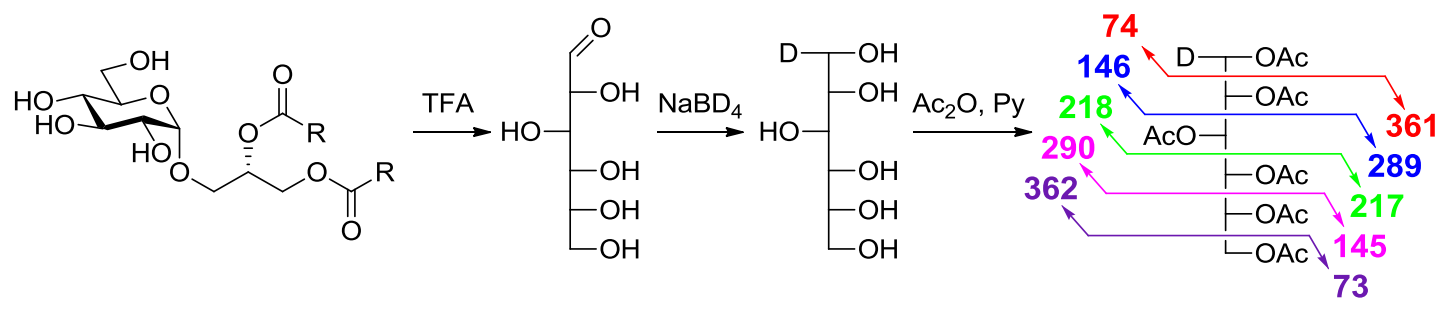

Scheme 2.2 Constituent sugar analysis forming glucitol hexaacetate from GL1.

\footnotetext{
c TAPPI, Technical Association of the Pulp and Paper Industry. In Carbohydrate Composition of Extractive-free Wood and Pulp by Gas-Liquid Chromatography; TAPPI Test Methods, T249 cm-85, TAPPI, Atlanta, GA, 1985.
} 


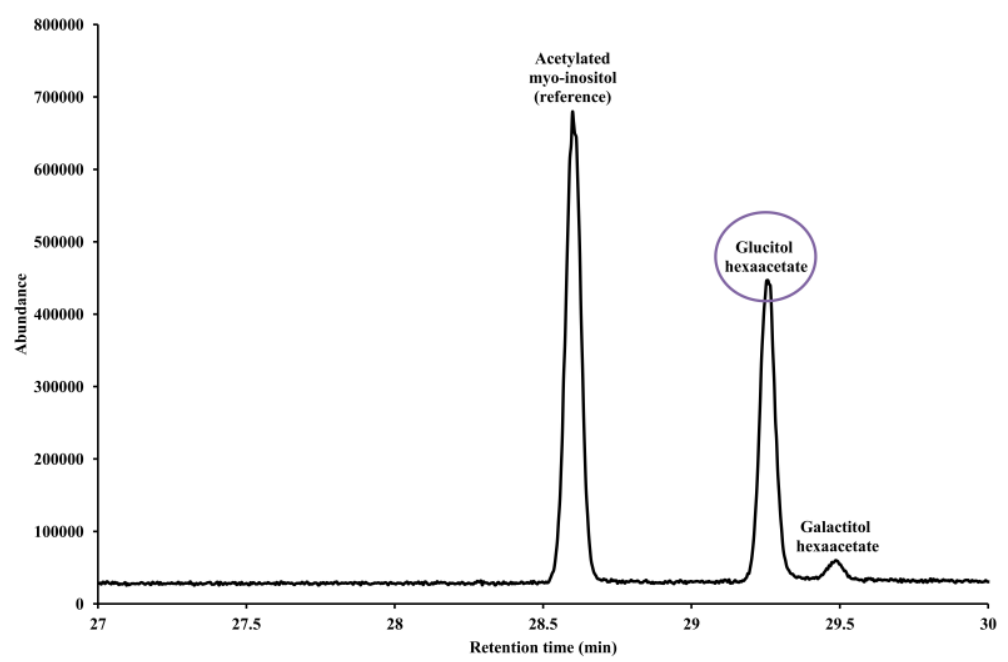

Figure 2.1 GC-MS chromatogram of GL1 alditol acetates showing the glucitol hexaacetate signal.

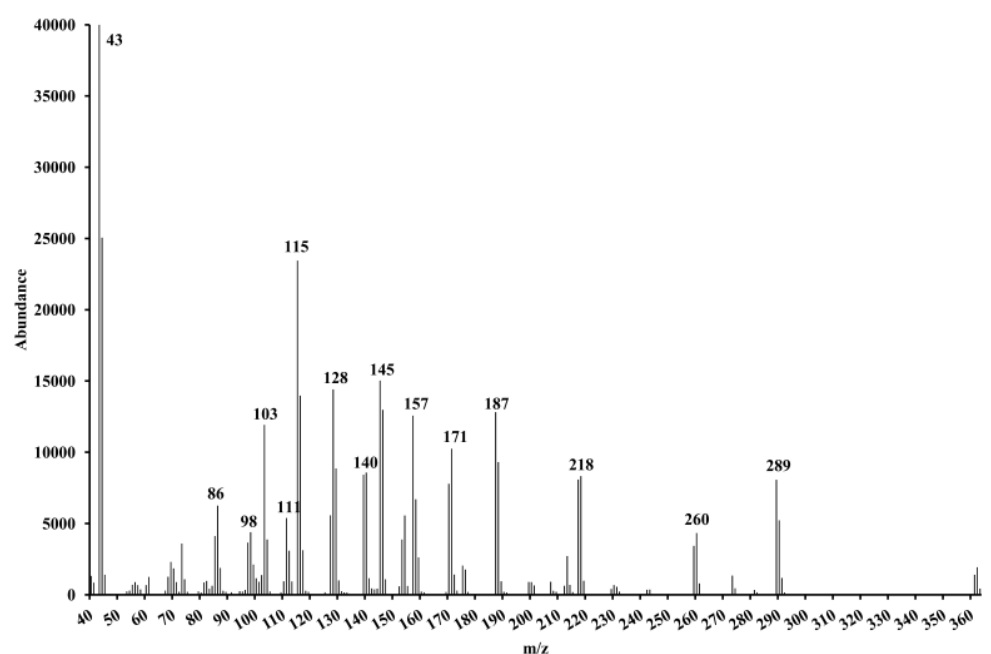

Figure 2.2 Glucitol hexaacetate electron impact spectrum taken from the signal at 29.3 minutes in GL1 chromatogram (Figure 2.1). 


\subsubsection{Linkage Analysis}

Samples (ca. $0.5 \mathrm{mg}$ ) were methylated with methyl iodide and sodium hydroxide as described by Ciucanu and Kerek, ${ }^{50}$ with the exception that samples were dispersed in DMSO $(0.5 \mathrm{~mL})$ and heated $\left(50{ }^{\circ} \mathrm{C}\right)$ overnight under argon. After extraction into chloroform, the methylated polysaccharides were hydrolysed with TFA, reduced and then acetylated to form partially methylated alditol acetates (PMAA) before analysis by GC-MS as described above (Scheme 2.3). Identifications were based on peak retention times and by comparison of electron impact spectra with the spectra obtained from reference standards (Figure 2.3 and 2.4). Here, the partially methylated alditol acetate standards were prepared following the method of Doares et al. ${ }^{208}$

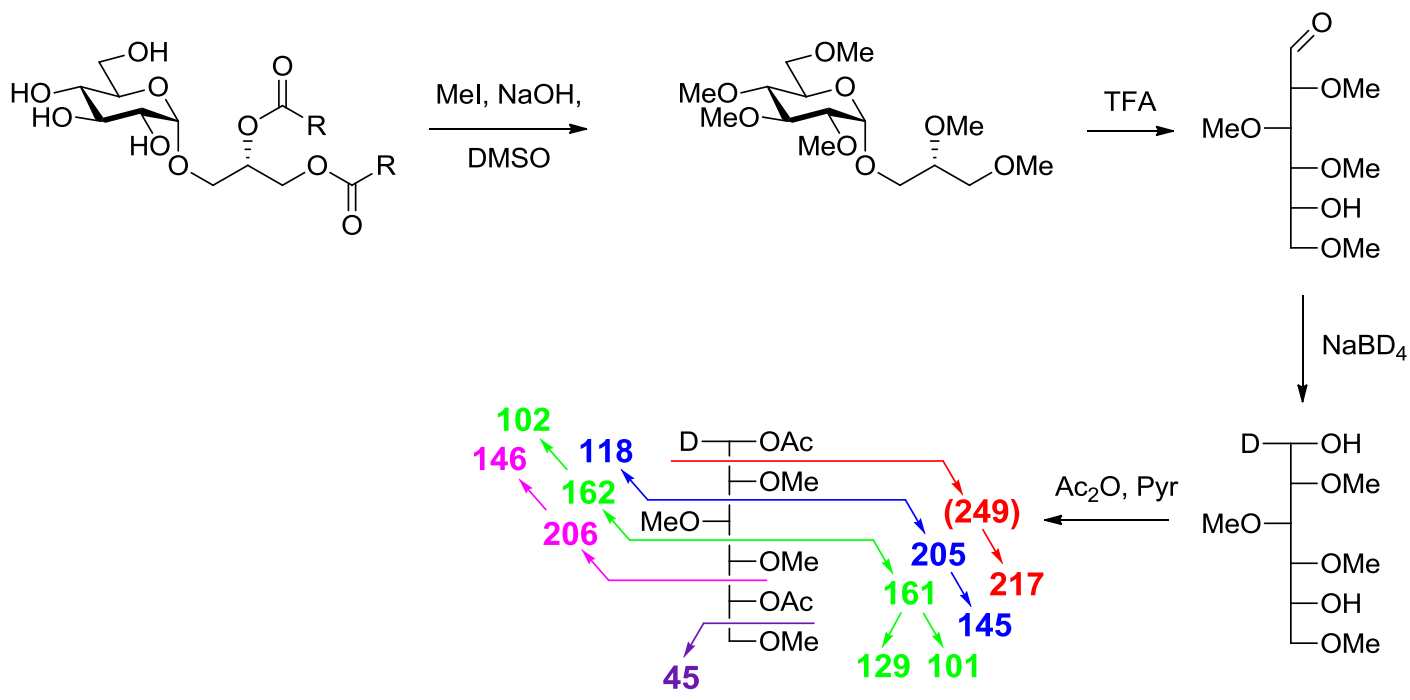

Scheme 2.3 Glucosyl linkage analysis method forming a PMAA from GL1. 


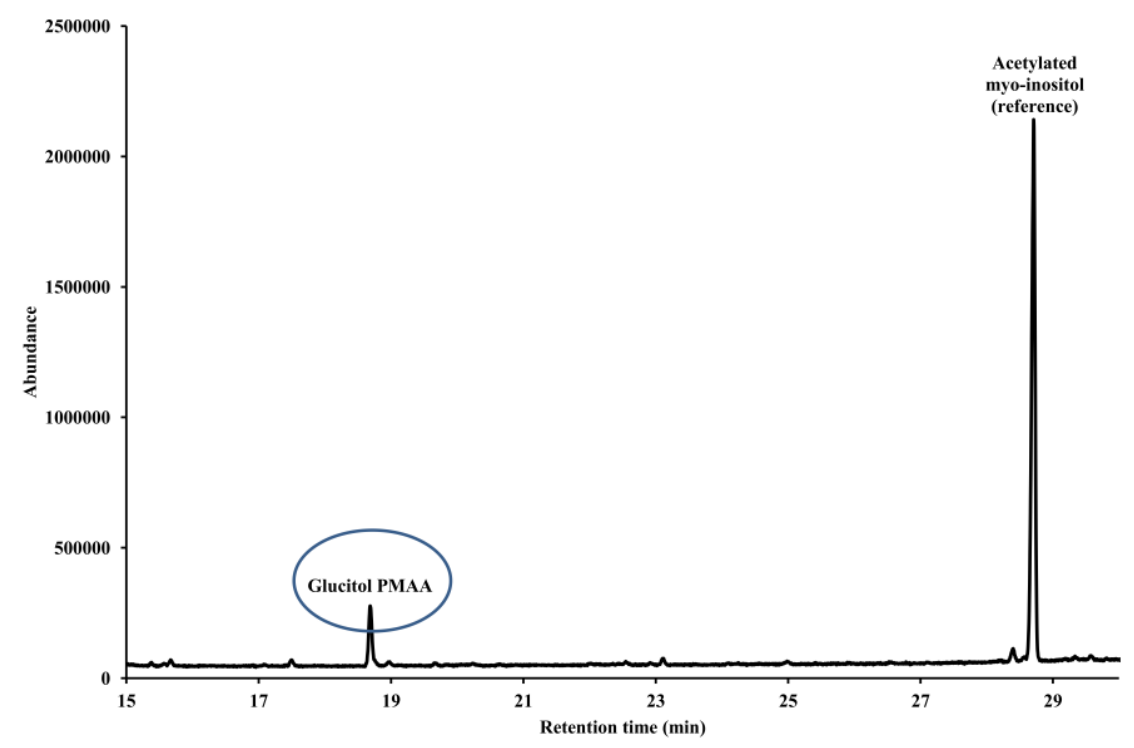

Figure 2.3 GC-MS chromatogram of GL1 PMAA showing the glucitol PMAA signal.

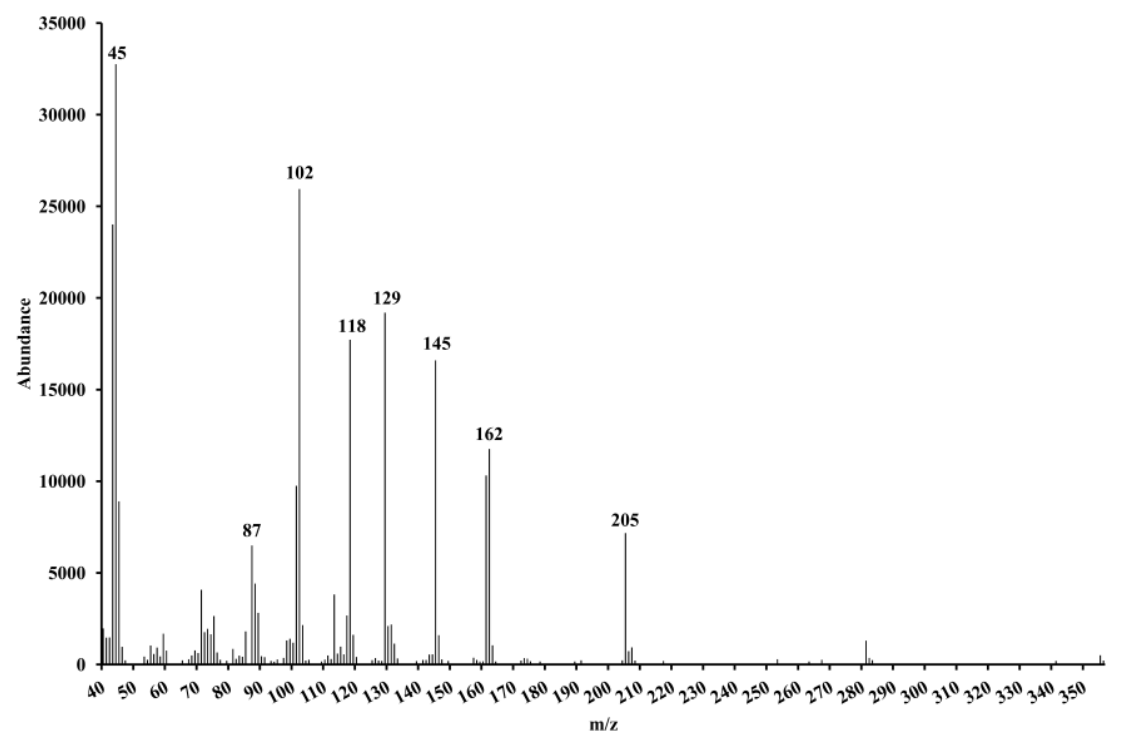

Figure 2.4 Glucitol PMMA electron impact spectrum taken from the signal at 18.7 minutes in GL1 chromatogram (Figure 2.3). 


\subsubsection{Sugar Absolute Configuration}

Crude glycolipid extracts $(1 \mathrm{mg})$ were hydrolysed with $2 \mathrm{M}$ TFA $\left(1 \mathrm{~h}, 120{ }^{\circ} \mathrm{C}\right)$, then dried. To this $R$-(-)-1-amino-2-propanol in methanol $(20 \mu \mathrm{L}, 1.6 \mathrm{M})$, acetic acid $(17 \mu \mathrm{L}$, $3.5 \mathrm{M})$ and sodium cyanoborohydride in methanol $(13 \mu \mathrm{L}, 3 \% \mathrm{w} / \mathrm{v})$ were added and heated to $65{ }^{\circ} \mathrm{C}$ for $1 \mathrm{~h}$ (Scheme 2.4). After drying the samples, the residues were acetylated as mentioned above, dissolved in acetone and then injected onto a GC-MS using a non-polar column (HP-5ms, $30 \mathrm{~m}, 0.32 \mathrm{~mm}$ i.d.). Here, the temperature programme started at $180{ }^{\circ} \mathrm{C}$ (held for $1 \mathrm{~min}$ ) rose to $220^{\circ} \mathrm{C}$ at a rate of $2{ }^{\circ} \mathrm{C} / \mathrm{min}$ (held for $2 \mathrm{~min}$ ), and then rose again to $250{ }^{\circ} \mathrm{C}$ at a rate of $1{ }^{\circ} \mathrm{C} / \mathrm{min}$ (held $5 \mathrm{~min}$ ). Crude samples were compared with derivatives of $\mathrm{D}^{-}$and L-glucose and galactose references. $^{209}$

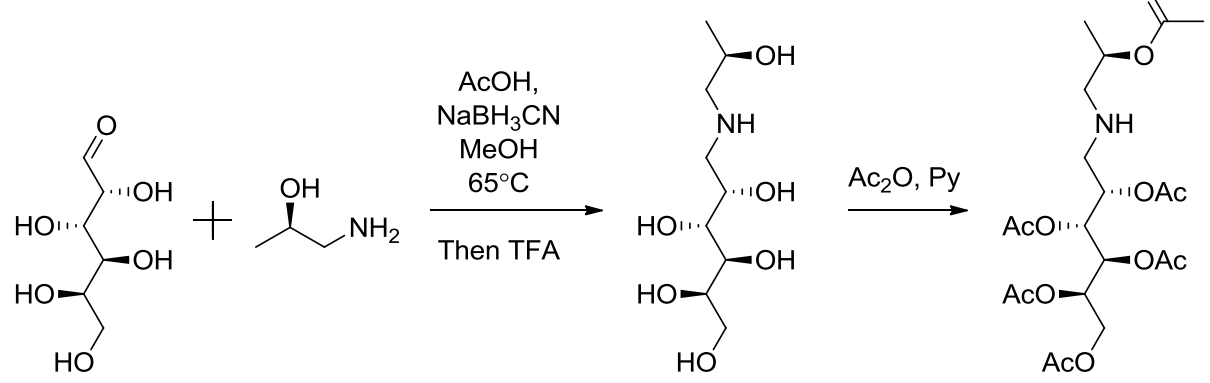

Scheme 2.4 Derivatisation of D-galactose into a (2-hydroxypropylamino)alditol using sodium cyanoborohydride and $R$-(-)-1-amino-2-propanol.

\subsubsection{Fatty Acid Analysis}

Fatty acid methyl esters (FAME) were prepared by direct transesterification from samples of biomass (ca. $0.5 \mathrm{mg}$ ) without extraction, as described by Svetashev et al. ${ }^{28}$ In brief, samples were dissolved in $1 \%$ sodium methoxide in dry methanol (30 min, $80{ }^{\circ} \mathrm{C}$ ), and then treated with $5 \%$ anhydrous $\mathrm{HCl}$ in methanol $\left(30 \mathrm{~min}, 80{ }^{\circ} \mathrm{C}\right)$. The resulting FAMEs and DMA were taken up in hexane and analyzed by GC and GCMS. ${ }^{210}$ One of the possible methods, when using GC, is to compare equivalent chain 
lengths (ECL). Here, as mentioned in Chapter 1 (Section 1.6.3) the fatty acid retention times (RT) are compared with standard RTs. ${ }^{210}$ Indeed, the relationship between the logarithm of the retention time and the chain length is linear if homologous standard straight-chain fatty acids are measured under identical isothermal conditions. The identity of the unknown fatty acids can then be read from the curve drawn of the standard fatty acids retention time logarithm against their chain length (semi-log plot). ${ }^{211}$ However, even if this method is useful for a fast determination of the fatty acids characterisation, it does not confirm without question, the exact identity of the fatty acids. Furthermore, as it has been shown that glycolipids can be active depending on their fatty acid content, ${ }^{63,78}$ it is of importance to know exactly what type of fatty acids are present in the glycolipid mixtures. Therefore, the identity of the fatty acids is confirmed using GC-MS and additional derivatisation methods.

The ECL method was used in this study for preliminary results, and the fatty acids identity was confirmed using further analyzes. The hydroxyl fatty acids were converted into their TMS derivatives to locate the hydroxyl on the fatty acid. ${ }^{212 c}$ The position of the cyclopropane group was elucidated after separation of the cyclopropanated fatty acids from the saturated fatty acids by means of silver ion SPE (Supelco). ${ }^{\mathrm{c}}$ This separation step was followed by hydrogenation in acetic acid over Adam's catalyst to open the cyclopropane ring. ${ }^{213 \mathrm{~d}}$ The resulting fatty acids were converted into pyrrolidides using pyrrolidine/HOAc $(10 / 1, \mathrm{v} / \mathrm{v})^{214}$ and analyzed by GC-MS. The position of the double bond in the monounsaturated fatty acids and DMAs was located by GC-MS of the dimethyl disulfide adducts. ${ }^{214 c}$ The analysis was performed on an Agilent 5890N gas chromatograph, equipped with a flame ionisation detector (FID) and a polar column (SolgelWax, $30 \mathrm{~m}, 0.32 \mathrm{~mm}$ i.d.). Hydrogen was used as the carrier gas with a split ratio of 1:30 and a separation temperature of $195^{\circ} \mathrm{C}$. GC-MS analyzes were performed on an Agilent 5890N gas chromatograph, equipped with a 5973 Inert MSD and non-polar column (HP-5ms, $30 \mathrm{~m}, 0.32 \mathrm{~mm}$ i.d.). ${ }^{215}$ Helium was used as the carrier gas. The programme started at $100{ }^{\circ} \mathrm{C}$, with a temperature gradient of $5{ }^{\circ} \mathrm{C} / \mathrm{min}$ to 160 ${ }^{\circ} \mathrm{C}$, then $1{ }^{\circ} \mathrm{C} / \mathrm{min}$ to $240{ }^{\circ} \mathrm{C}$ before being left at $240{ }^{\circ} \mathrm{C}$ for $25 \mathrm{~min}$.

\footnotetext{
d Assay conducted by Kirill Lagutin, IRL
} 


\subsubsection{Macrophage Assay}

\section{[Conducted by Ashna Khan and Stephanie Chee]}

\subsubsection{Materials and Methods}

C57BL/6 male mice were bred and housed in a conventional animal facility at the Malaghan Institute of Medical Research, Wellington, New Zealand. All animals used for the experiments were aged between 8-10 weeks. All experimental procedures were approved by the Victoria University Animal Ethics Committee in accordance with their guidelines for the care of animals. Extracted glycolipids were tested to be endotoxinfree $(\leq 0.125 \mathrm{EU} / \mathrm{mL})$ with an endotoxin kit (Pyrotell, LAL test, Associates of Cape Cod Inc., E. Falmouth, MA, USA).

\subsubsection{Generation of Bone Marrow Derived Macrophages (BMMs)}

Bone marrow cells were collected from the tibia and femur of C57BL/6 mice and were cultured (250000 cells per $\mathrm{mL})$ in Iscove's Modified Dulbecco's Medium (IMDM, Gibco) supplemented with 5\% FBS (Gibco), 1\% Penicillin streptomycin (Gibco) and 2mercaptoethanol (55 mm, Invitrogen) containing $10 \mathrm{ng} / \mathrm{mL}$ GM-CSF (clone X63/GMCSF murine cells). Cells were incubated at $37{ }^{\circ} \mathrm{C}(5 \% \mathrm{CO} 2)$ and the medium was changed on days 2, 5 and 7. On day 10, the medium was removed, and the BMMs were primed with IFN- $\gamma(10 \mathrm{ng} / \mathrm{mL}$, Peprotech, Rocky Hill, NJ, USA) for $3 \mathrm{~h}$ prior to the addition of the compounds.

\subsubsection{BMM Assays}

Glycolipid stock solutions were prepared $(2.5 \mathrm{mg} / \mathrm{mL}$ in PBS containing 2\% DMSO), vortexed and warmed to $50{ }^{\circ} \mathrm{C}$ for $30 \mathrm{~min}$ (x 3) to ensure suspension of the compounds prior to administration to BMM cultures. BMM cultures were then treated with 20 or 40 
$\mu \mathrm{g} / \mathrm{mL}$ of glycolipid anchors, with LPS (100 $\mathrm{ng} / \mathrm{mL})$ as a positive control and medium only for the times indicated. Supernatants were collected and tested immediately for NO levels using the Griess assay. ${ }^{216}$

\subsection{Results and Discussion}

A large scale culture of L. plantarum yielded $8 \mathrm{~g}$ of washed, freeze-dried cells, from which $370 \mathrm{mg}$ of crude lipid extract was isolated through extraction using $\mathrm{CHCl}_{3} / \mathrm{MeOH} / \mathrm{H}_{2} \mathrm{O}$.

The configuration of the sugars present in the unpurified glycolipid extract was determined (Figure 2.5) after (2-hydroxypropylamino)alditol derivatives were formed from D-glucose and D-galactose. These residues were the sole constituent sugars present in the crude glycolipid mixture. Using the electron impact spectrum taken from the GCMS chromatogram (Figure 2.5), the (2-hydroxypropylamino)alditol signals were identified. The combined D- and L-galactose derivatives clearly show peak separation. Therefore, the differing retention times enabled the D- and L-galactose derivatives to be qualitatively determined. Finally, the sugar in the crude glycolipid mixture from $L$. plantarum was identified as D-galactose from its retention time. Glucose was similarly identified as D-glucose. This configuration is consistent with most other reports, which is not surprising as the D-configuration is predominant in nature.

Thin-layer chromatography of the crude lipid extract (Figure 2.6) revealed the presence of two highly mobile lipids, designated L1 and L2, and three distinct glycoglycerolipids (GL1-3). Comparison with known standards suggested that GL1 is a monoglycosyldiglyceride, while the more polar GL2 appeared to be a diglycosyldiglyceride. GL3, which had a particularly low $R_{f}$, was deemed to be a glycolipid containing more than two sugar units. In order to fully identify the extracted components, the five individual compounds were then fractionated by silica gel column chromatography (L1, L2 and GL1-GL3, Figure 2.6) and analyzed using ESI-MS and NMR, in addition to lipid, sugar and linkage analysis. 


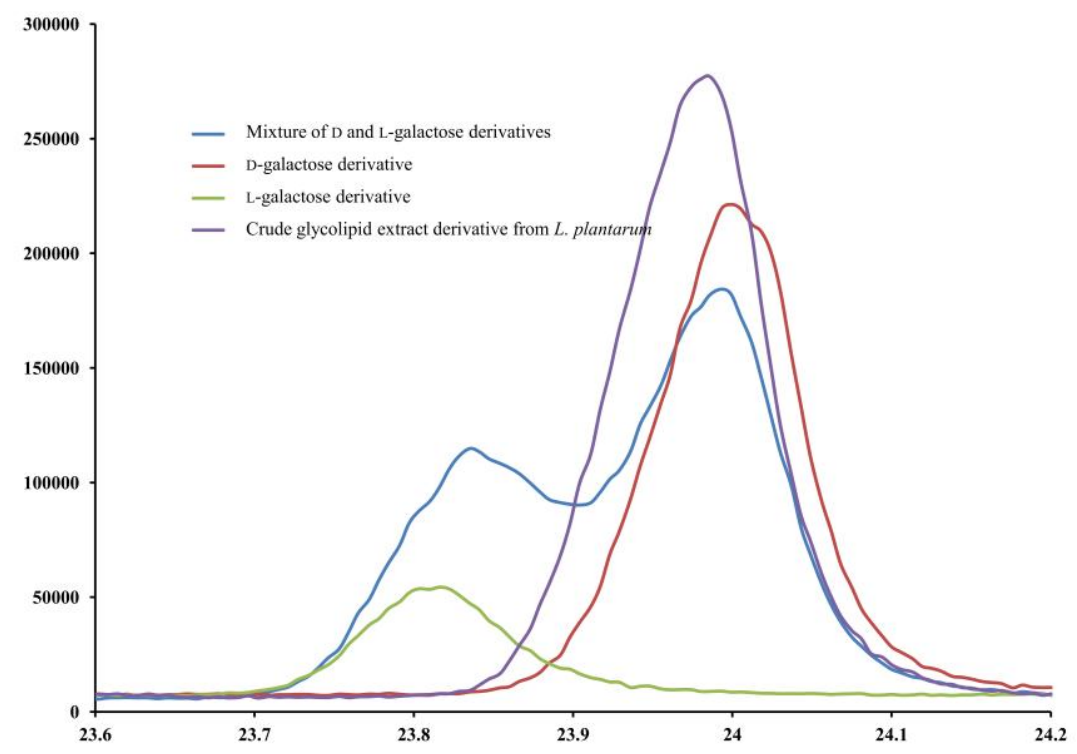

Figure 2.5 GC-MS traces of (2-hydroxypropylamino)alditol derivatives of D- and Lgalactose, its mixture and the crude $L$. plantarum glycolipid extract showing the presence of D-galactose in the glycolipids.

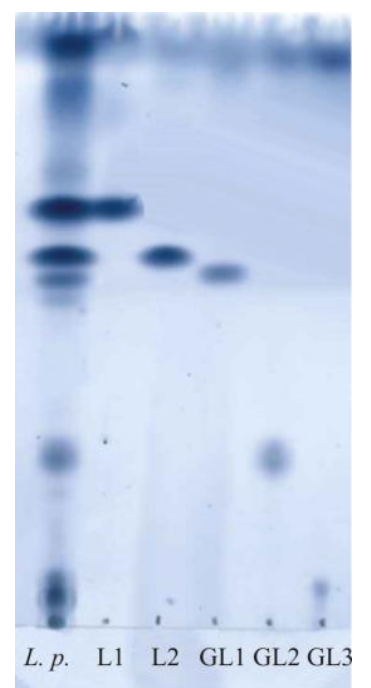

Figure 2.6 TLC of glycolipid and purified fractions from L. plantarum IRL-560. Silica gel TLC, eluted with $\mathrm{CHCl}_{3} / \mathrm{MeOH}(6 / 1, \mathrm{v} / \mathrm{v})$ and developed with a cerium molybdate stain. 
The NMR and ESI-MS analysis enabled L1 to be readily identified as oleic acid (HRMS calc. for $\left[\mathrm{C}_{18} \mathrm{H}_{33} \mathrm{O}_{2}\right]^{-}=281.2486$, found: 281.2484). Lipid L2, slightly more polar than oleic acid, was identified as hydroxyoctadecanoic acid (HRMS calc. for $\left[\mathrm{C}_{18} \mathrm{H}_{35} \mathrm{O}_{3}\right]^{-}=299.2592$, found: 299.2579 ). Both of these structures matched previously published data, ${ }^{217-219}$ although the position of the hydroxyl group of the hydroxyoctadecanoic acid still needed to be determined. This was easily determined using GC-MS by comparing the fragmentation pattern of FAME TMS derivatives with the unsilylated FAME. Figure 2.7 depicts the GC of the silylated FAME. In the chromatogram, one major signal (FAME-O-TMS) can be observed as well as plasticizer and the standard. The plasticizer is an inescapable artefact often observed due to the use of plastic in pipettes and caps. The electron impact spectrum of the peak at 56 min (Figure 2.8) shows two major fragments that were identified as the cleavage of the chain after $(\mathrm{m} / z=273)$ or before $(\mathrm{m} / z=215)$ the -OTMS group. The presence of 10 hydroxyoctadecanoic acid was also confirmed by the electron impact spectrum of the non-derivatised FAME (Figure 2.9 and 2.10) that showed a weak fragment at $\mathrm{m} / z=201$ corresponding to a FAME cleaved after the hydroxyl group.

As anticipated, the structural elucidation of the purified glycoglycerolipids GL1-GL3 was more involved, though full assignments were possible following consideration of fatty acid, sugar and linkage analysis, $\mathrm{R}_{f}$ values, yields and high-resolution mass spectrometry (HRMS) data (Table 2.1).

\subsubsection{GL1}

The fatty acid analysis for GL1 showed high amounts of palmitic (16:0), oleic (18:1n-9) and $c i s$-vaccenic acid (18:1n-7) and the cyclopropane containing dihydrosterculic $(9,10$ Mt 18:0) acid (Table 2.1). The presence of dihydrosterculic acid could also be confirmed via NMR, where a typically shielded signal was observed at $-0.33 \mathrm{ppm}$ (Figure 2.11) and was consistent with literature data and the fatty analysis data showed also the intermediate branched fatty acid (Section 1.7.3). Additionally, the cyclopropane ring was confirmed as being in the cis configuration as it showed a characteristic upfield signal at -0.33 . $^{220}$ The GC-MS spectrum of the FAME could not identify the position of 
the cyclopropane ring as the electron impact spectrum of the cyclopropane FAME undergoes rearrangement to form double bonds with an additional carbon. This spectrum thus provided no information to determine the position of the cyclopropane ring and so other derivatisation methods were required. ${ }^{221}$

At first, the cyclopropane ring was opened via hydrogenation in acetic acid using Adam's catalyst (Scheme 2.5). Here, three products are usually obtained, a straight chain ester and the two possible branched chain isomers. The two branched chain isomers eluted at the same retention time in the GC-MS (Figure 2.12) and thus a mixture of fragmentation patterns was obtained for these derivatives. The signal at 42.8 min (Figure 2.12 and 2.13), shows clearly the molecular ion for methyl hydroxyoctadecanoate $(312 \mathrm{~m} / z)$ indicating that the ring opening worked (the molecular ion for methyl dihydrosterculic acid is at $310 \mathrm{~m} / \mathrm{z}$ ). Weak signals at 157 and $185 \mathrm{~m} / \mathrm{z}$ represent the fragmentation of methyl 8-methyl-octadecanoate before and after the branching methyl, while the signals at 171 and $199 \mathrm{~m} / \mathrm{z}$ represent the fragmentation of methyl 9-methyl-octadecanoate. In order to confirm the signals obtained from these data, the pyrrolidide derivatives were obtained using pyrrolidine/HOAc $(10 / 1, \mathrm{v} / \mathrm{v})$. The fragmentation patterns corresponding to the same type of branching was also observed. This is not surprising as the major fatty acid obtained was oleic acid. Indeed, as seen in Chapter 1 (Section 1.7.3) the cyclopropane rings require an additional step in the biosynthetic pathway and are formed with AdoMet of unsaturated fatty acids. 


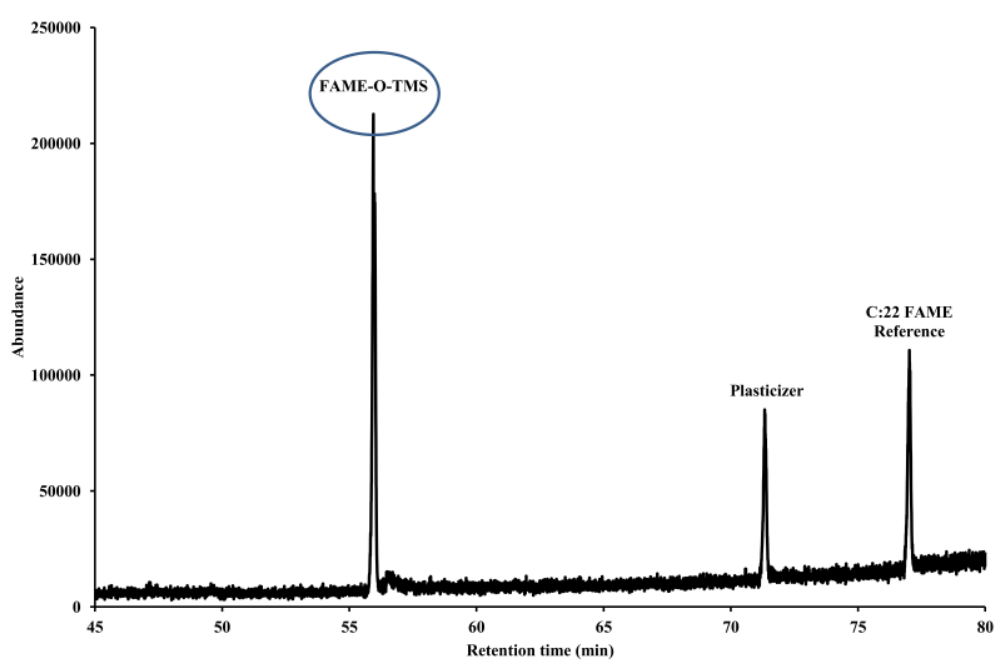

Figure 2.7 GC-MS chromatogram of silylated L2 FAME showing the silylated hydroxyoctadecanoic FAME signal.

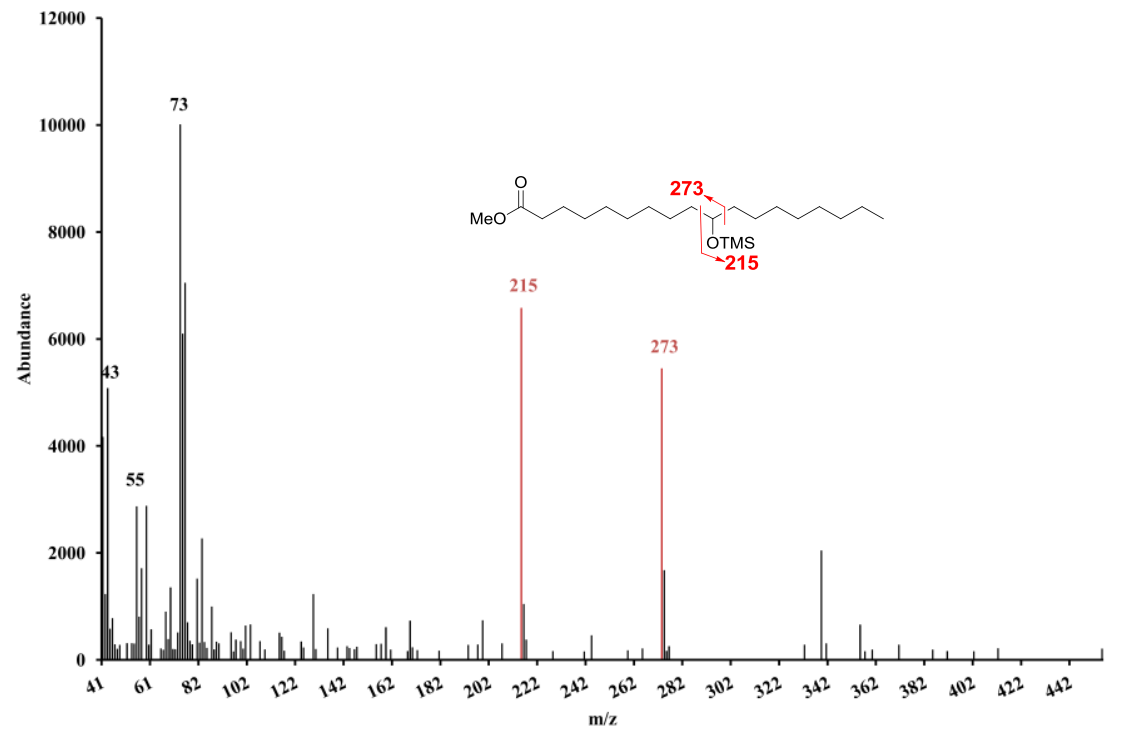

Figure 2.8 Silylated hydroxyoctadecanoic FAME electron impact spectrum taken at 56 minutes in L2 chromatogram (Figure 2.7). 


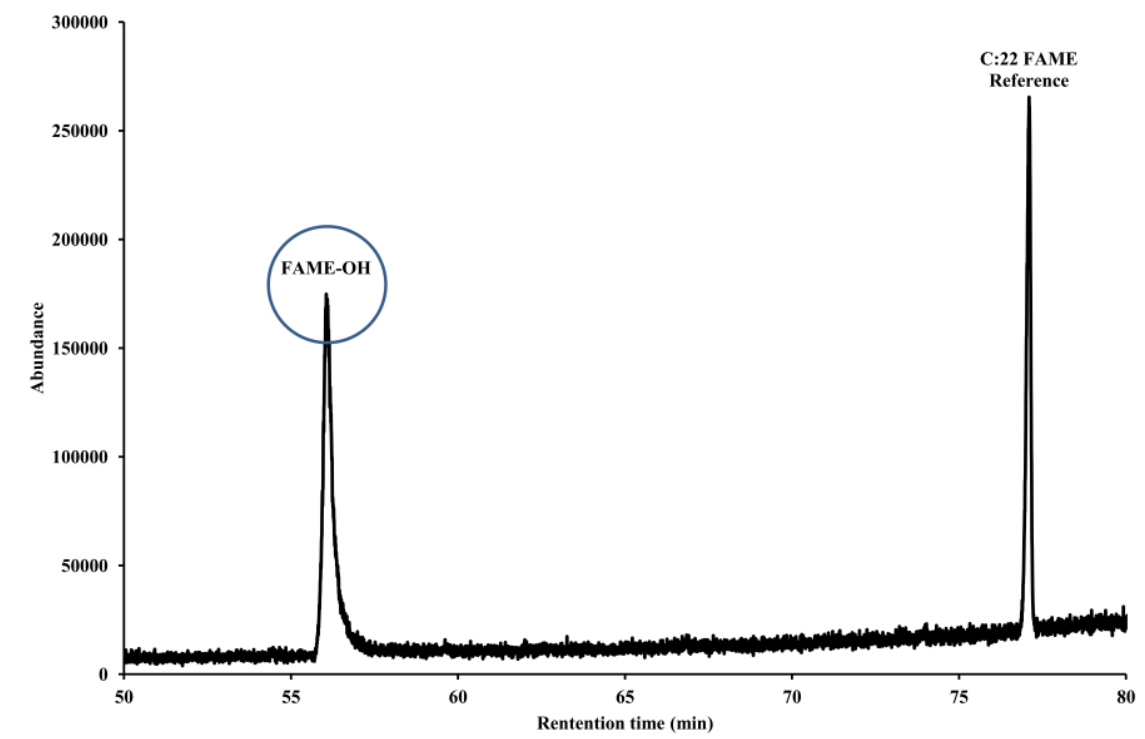

Figure 2.9 GC-MS chromatogram of L2 FAME showing the unsilylated hydroxyoctadecanoic FAME signal.

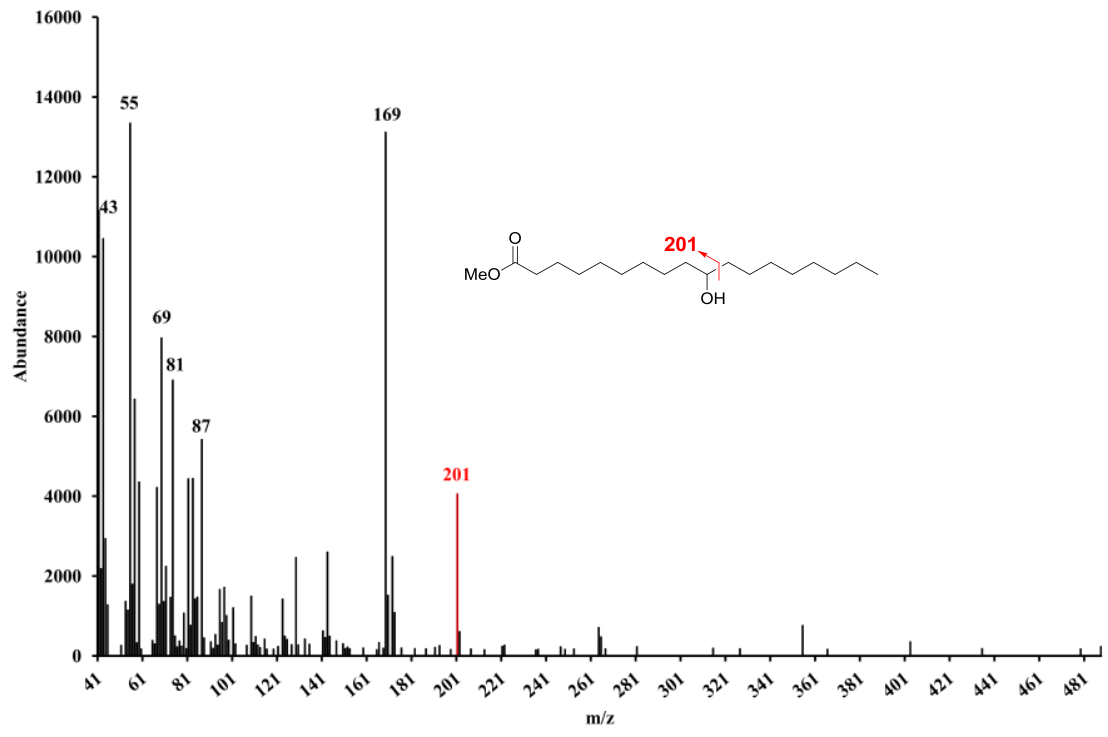

Figure 2.10 Unsilylated hydroxyoctadecanoic FAME electron impact spectrum taken from the signal at 56 minutes in L2 chromatogram (Figure 2.9). 
Table 2.1 Chemical characteristics of isolated compounds.

\begin{tabular}{|c|c|c|c|}
\hline & GL1 & GL2 & GL3 \\
\hline $\mathrm{R}_{f}$ & 0.56 & 0.26 & 0.06 \\
\hline Yield $^{\mathrm{a}}$ & 1.0 & 0.5 & 0.1 \\
\hline \multicolumn{4}{|l|}{ Neutral sugars ${ }^{\mathrm{a}}$} \\
\hline D-Gal & 2.5 & 3.8 & 0.8 \\
\hline D-Glc & 25.7 & 6.9 & 1.0 \\
\hline Total & 28.1 & 10.6 & 1.8 \\
\hline \multicolumn{4}{|l|}{ Linkage analysis ${ }^{\mathrm{b}}$} \\
\hline $\mathrm{t}-\mathrm{Gal} p$ & 0.1 & 0.2 & - \\
\hline 1,6-Galp & - & 0.2 & 0.4 \\
\hline $\mathrm{t}-\mathrm{Glc} p$ & 0.9 & 0.2 & 0.3 \\
\hline 1,2-Glcp & - & 0.4 & 0.3 \\
\hline Fatty acid content ${ }^{\mathrm{a}}$ & 25.5 & 23.0 & 2.6 \\
\hline \multicolumn{4}{|l|}{ Fatty acids ${ }^{\mathrm{a}, \mathrm{c}}$} \\
\hline $14: 0$ & 2.5 & 2.1 & - \\
\hline $16: 0$ & 15.7 & 15.7 & 9.2 \\
\hline $16: 1 n-7$ & 5.7 & 6.5 & 4.0 \\
\hline DMA 18:1n-9 & - & 1.8 & 6.8 \\
\hline 18:0 & 3.3 & 4.3 & 14.9 \\
\hline $18: 1 n-9$ & 28.8 & 20.9 & 48.4 \\
\hline $18: 1 n-7$ & 14.4 & 18.0 & 4.4 \\
\hline 19:0br $n-9$ & 3.9 & 3.5 & - \\
\hline 9,10-Mt 18:0 & 23.8 & 29.8 & 7.8 \\
\hline $\mathrm{HRMS}^{\mathrm{d}}$ & 819.5950 & $\begin{array}{l}981.6482 \\
1393.9287\end{array}$ & 1143.7009 \\
\hline
\end{tabular}

\footnotetext{
${ }^{a}$ Weight $\%$

${ }^{\mathrm{b}}$ Molar ratio

${ }^{\mathrm{c}}$ Fatty acids present under $1 \%$ are not shown

${ }^{\mathrm{d}}$ Major species.
} 


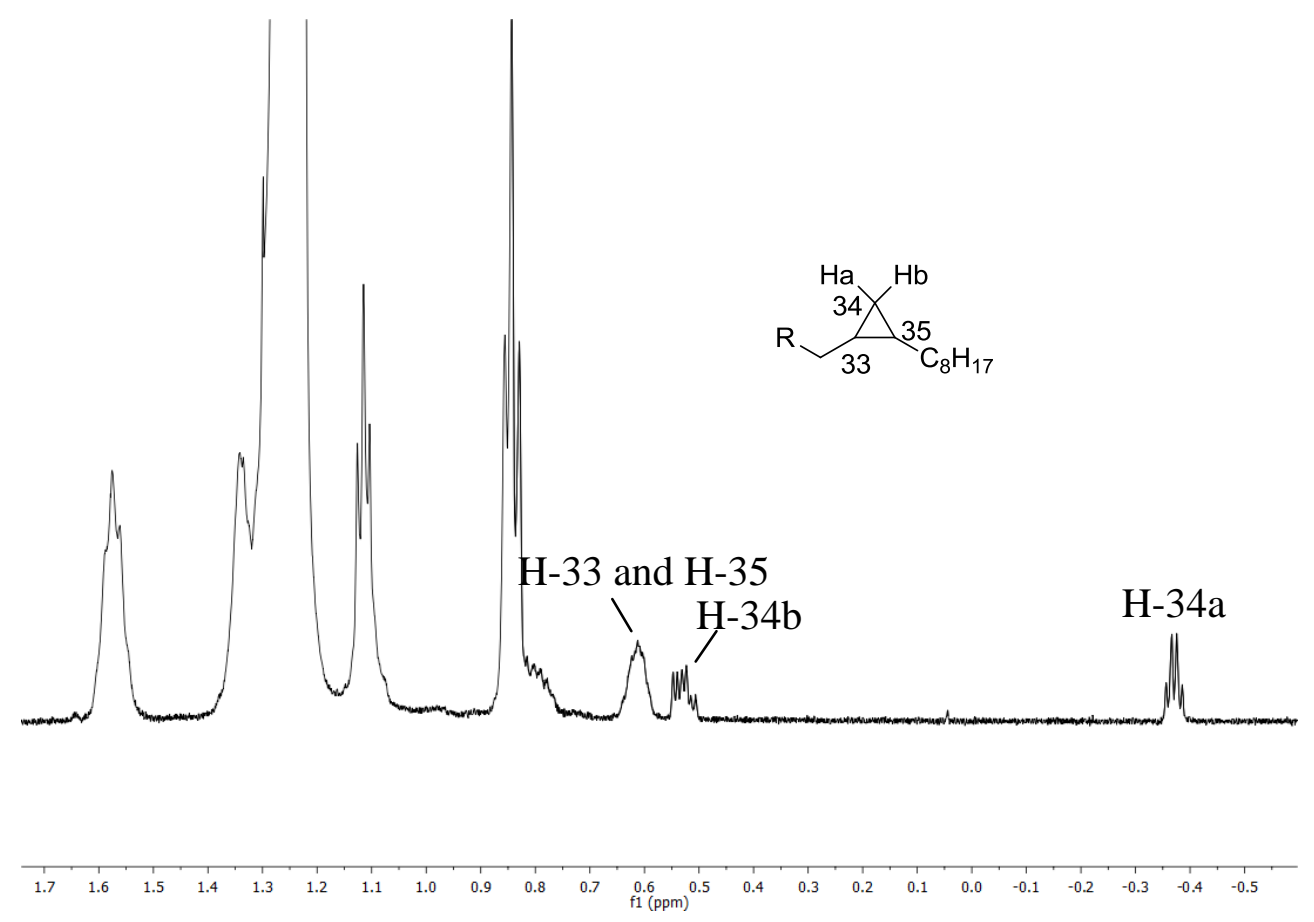

Figure 2.11 Proton NMR spectrum of GL1 showing typical signals for dihydrosterculic acid, ${ }^{1} \mathrm{H}$ spectrum $\mathrm{CD}_{3} \mathrm{OD} / \mathrm{CDCl}_{3}(1 / 2$, v/v), $500 \mathrm{MHz}$.

Additional information was obtained using neutral sugar analysis and showed D-glucose and D-galactose in a 10/1 ratio, which was confirmed by linkage analysis. Only terminal glucopyranose (t-Glcp) was observed as the linkage analysis indicated that the glucose residue was unmodified at any of its hydroxyls. In accordance with the TLC analysis and HRMS (HRMS calc. for $\left[\mathrm{C}_{46} \mathrm{H}_{84} \mathrm{NaO}_{10}\right]^{+}=819.5957$, found: 819.5950) and comparison with standards, this indicated that GL1 was a monoglucosyldiglyceride. NMR was then used to determine the anomeric configuration of the glucose residue (the galactose residue could not be observed using NMR) (Table 2.2), with resonances of the anomeric centre $\left(\delta_{\mathrm{H}-1^{\prime}}=4.83 \mathrm{ppm}, \mathrm{d}, J_{\mathrm{H}-1^{\prime}, \mathrm{H}-2^{\prime}}=3.8 \mathrm{~Hz}\right.$ and $\left.\delta_{\mathrm{C}-1^{\prime}}=99.8 \mathrm{ppm}\right)$ clearly revealing an $\alpha$-linked terminal-glucopyranose ( $\alpha$-t-Glcp). The $\alpha$-linkage was identified comparing the NMR chemical shift of the anomeric signal with the literature as well as considering a low vicinal coupling constant for $J_{\mathrm{H}-1^{\prime}, \mathrm{H}-2^{\prime}}(3.8 \mathrm{~Hz})$. Indeed, such as coupling constant is observed with a low dihedral angle between $\mathrm{H}-1^{\prime}$ and $\mathrm{H}-2^{\prime}$ indication of an $\alpha$-linkage. In addition, the presence of a diglyceride was apparent from a distinct low field proton resonance at $5.29 \mathrm{ppm}(\mathrm{H}-2)$, which showed COSY signals with two $\mathrm{ABX}$ systems upfield $\left(\delta_{\mathrm{H}-1}=4.18\right.$ and $4.44 \mathrm{ppm}, \delta_{\mathrm{H}-3}=3.62$ and $\left.3.83 \mathrm{ppm}\right)$ 
(Figure 2.14). HMBC between the glycerol H-1 and the carbonyl carbon $\left(\delta_{\mathrm{C}-7}=174.6\right.$ ppm) confirmed the presence of an acyl group at $s n-1$. An HMBC between the H-3 glycerol protons and the anomeric carbon $\left(\mathrm{C}-1^{\prime}\right)$, and between the anomeric proton $(\mathrm{H}-$ 1) and glycerol carbon (C-3) confirmed the attachment of the sugar to the glycerol (Figure 2.15). All proton and carbon NMR signals were in accordance with literature data, ${ }^{52,85}$ which also allowed for the assignment of the glycerol $(2 S)$ configuration (Table 2.2).

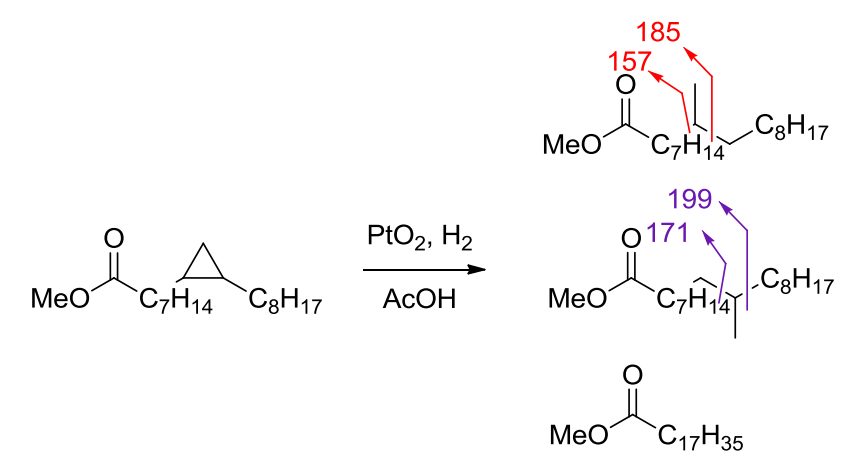

Scheme 2.5 Cyclopropane ring opening via hydrogenation in acetic acid using Adam's catalyst.

Using collision induced dissociation (CID), in positive ion mode, the connectivity between the structural components of GL1 was confirmed. ESI-MS/MS of the sodiated molecular ion (HRMS $m / z=819.5950$ for $\left[\mathrm{C}_{46} \mathrm{H}_{84} \mathrm{O}_{10}+\mathrm{Na}\right]^{+}$) showed first the loss of oleic acid and dihydrosterculic acid $(\mathrm{m} / \mathrm{z}=537.3$ and 525.3, respectively) (Figure 2.16). Here, the larger intensity of the $m / z=523.3$ fragment indicates that the dihydrosterculic acid is esterified at the $s n-1$ position in the glyceride as the loss of the fatty acid at this position creates a more stable ion. ${ }^{222}$ This regioselectivity was also observed for GL2a, GL2b and GL3. Further fragmentation of the ion at $m / z, 523.0$ to give a fragment ion of $\mathrm{m} / \mathrm{z} 375.3$ was consistent with the loss of hexose. The fragment obtained for the hexose itself $(m / z=185.0)$ was also readily observed. As such, GL1 was fully assigned as $\alpha$-Dglucopyranosyldiglyceride. This compound has not been previously identified in $L$. plantarum, although its presence in L. casei $^{203}$ and other bacteria such as Deinococcus radiodurans ${ }^{52}$ has been noted. 


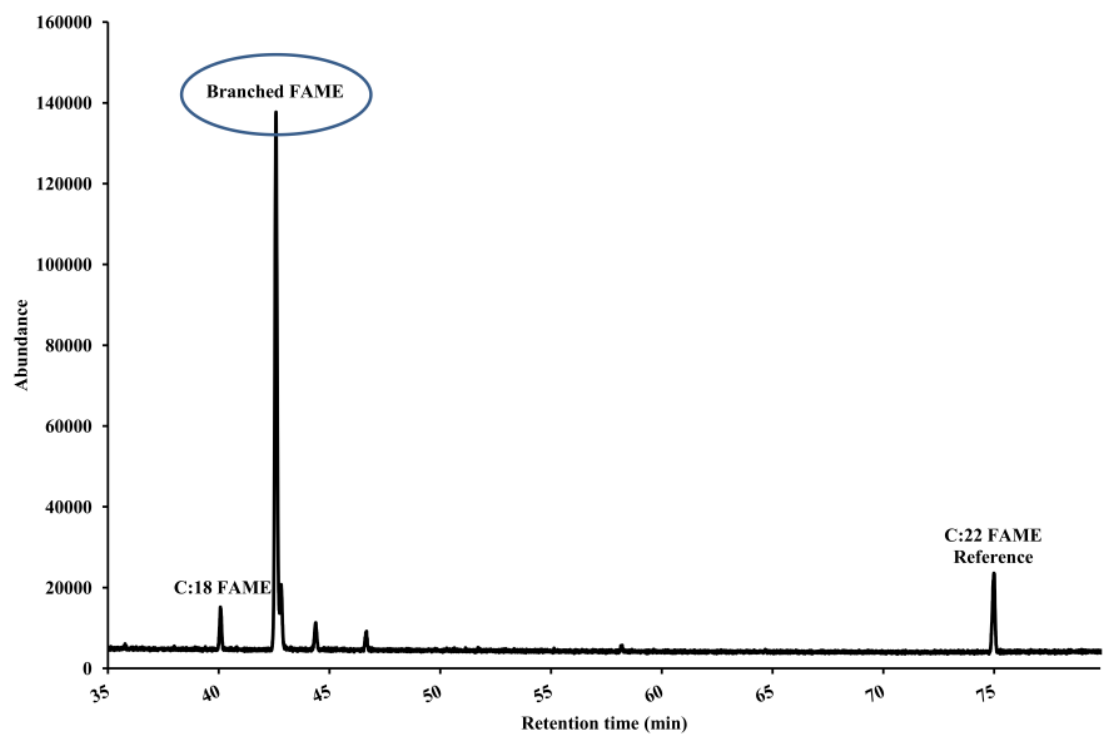

Figure 2.12 GC-MS chromatogram of opened cyclopropane ring FAME showing the signal for the branched FAME.

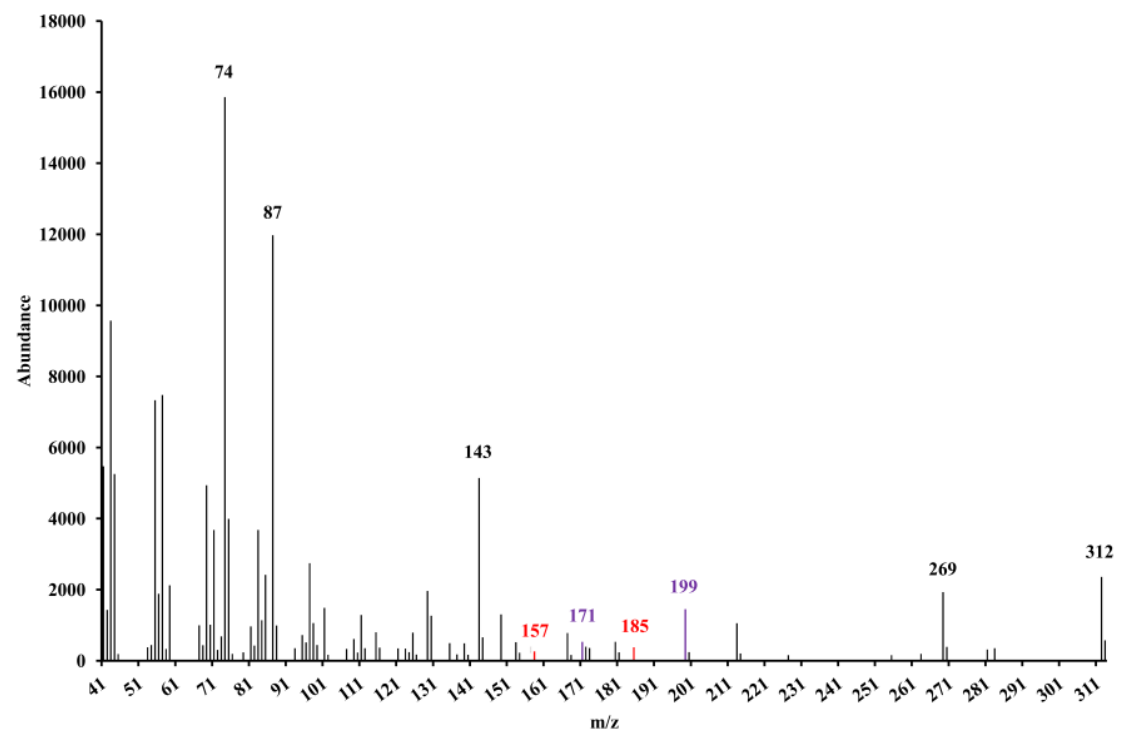

Figure 2.13 Electron impact spectrum of the signal at $42.8 \mathrm{~min}$ in the GC-MS spectrum in Figure 2.12. 
Table 2.2 NMR spectroscopic data for GL1.

\begin{tabular}{|c|c|c|c|c|c|c|c|c|}
\hline \multirow[b]{2}{*}{ Position } & \multirow{2}{*}{$\begin{array}{c}\delta_{1 \mathrm{H}} \\
(\mathrm{ppm})\end{array}$} & \multirow{2}{*}{$\begin{array}{c}\delta_{13 \mathrm{C}} \\
(\mathrm{ppm})\end{array}$} & \multirow{2}{*}{\multicolumn{2}{|c|}{$\begin{array}{l}\text { COSY } \\
(\mathrm{H}, \mathrm{H})\end{array}$}} & \multirow{2}{*}{\multicolumn{2}{|c|}{$\begin{array}{l}\text { HMBC } \\
(\mathrm{H}, \mathrm{C})\end{array}$}} & \multicolumn{2}{|c|}{ Literature } \\
\hline & & & & & & & $\delta_{1 \mathrm{H}}(\mathrm{ppm})^{85}$ & $\delta_{13 \mathrm{C}}(\mathrm{ppm})^{52}$ \\
\hline $1^{\prime}$ & $\begin{array}{l}4.83 \\
(\mathrm{~d}, 3.8)\end{array}$ & 99.8 & $2^{\prime}$ & & $3,3^{\prime}, 5^{\prime}$ & & 4.86 & 99.1 \\
\hline $2^{\prime}$ & $\begin{array}{l}3.43 \\
(\mathrm{dd}, 3.7,9.4)\end{array}$ & 72.4 & $1^{\prime}, 3^{\prime}$ & & - & & 3.49 & 72.0 \\
\hline $3^{\prime}$ & 3.65 & 74.3 & $2^{\prime}, 4^{\prime}$ & & 2 ' & & 3.72 & 73.9 \\
\hline $4^{\prime}$ & 3.40 & 70.6 & $5^{\prime}, 3^{\prime}$ & & $3^{\prime}, 5^{\prime}, 6^{\prime}$ & & $3.56-3.60$ & 70.1 \\
\hline $5^{\prime}$ & 3.56 & 72.3 & $6^{\prime}$ & & - & & $3.56-3.60$ & 71.8 \\
\hline $\begin{array}{l}6 a^{\prime} \\
6 b^{\prime}\end{array}$ & $\begin{array}{l}3.75 \\
(\mathrm{ABX}, 2.9,11.9) \\
3.72 \\
(\mathrm{ABX}, 2.9,11.9)\end{array}$ & 62.0 & $5^{\prime}$ & & - & & $\begin{array}{l}3.82 \\
\text { (bs) } \\
3.82 \\
\text { (bs) }\end{array}$ & 61.7 \\
\hline $3 a$ & $\begin{array}{l}3.62 \\
(\mathrm{ABX}, 6.2,10.7)\end{array}$ & & $2,3 b$ & & $1^{\prime}, 2,1$ & & $3.56-3.60$ & \\
\hline $3 b$ & $\begin{array}{l}3.83 \\
(\mathrm{ABX}, 5.4,10.8)\end{array}$ & 66.6 & $2,3 \mathrm{a}$ & & $1^{\prime}, 2,1$ & & $\begin{array}{l}3.82 \\
\text { (bs) }\end{array}$ & 66.2 \\
\hline 2 & $5.29(\mathrm{~m})$ & 69.7 & 1,3 & & - & & 5.25 & 69.8 \\
\hline $1 \mathrm{a}$ & $\begin{array}{l}4.18 \\
(\mathrm{ABX}, 6.1,12.3)\end{array}$ & & $2,1 b$ & & $3,2,25$ & & 4.15 & \\
\hline $1 b$ & $\begin{array}{l}4.44 \\
(\mathrm{ABX}, 3.4,12.1)\end{array}$ & 62.5 & $2,1 \mathrm{a}$ & & - & & 4.38 & 62.3 \\
\hline $\begin{array}{l}7 \\
25\end{array}$ & - & $\begin{array}{l}174.6 \\
174.1\end{array}$ & & & - & & & \\
\hline 8,26 & $2.34(\mathrm{t}, 7.5)$ & 34.7 & & 27 & $7.9,10$ & $25,27,28$ & & \\
\hline 9,27 & $1.61(\mathrm{~m})$ & 25.3 & 8,10 & 26,28 & 8,11 & 26,29 & & \\
\hline 15,16 & $5.33(\mathrm{~m})$ & & 14 & 17 & & 14 & & \\
\hline 14,17 & $2.03(\mathrm{~m})$ & 27.6 & 15 & 16 & 12,16 & 15,17 & & \\
\hline $\begin{array}{l}* 10-13, \\
18-23 \\
28-32 \\
36-42\end{array}$ & $1.25-1.35(\mathrm{~m})$ & $28-32$ & & & - & & - & - \\
\hline $34 a$ & $-0.33(\mathrm{q}, 4.3)$ & 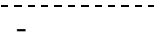 & & & - & & & \\
\hline $34 b$ & $0.58(q, 4.2)$ & - & 33,35 & & - & & & \\
\hline 33,35 & $0.67(q, 4.0)$ & 16.2 & $\begin{array}{l}34 b \\
-\end{array}$ & & - & & & \\
\hline 24,43 & $0.89(\mathrm{t}, 7.0)$ & 14.2 & 23 & 43 & 22 & 41 & & \\
\hline
\end{tabular}

* Overlapping signals

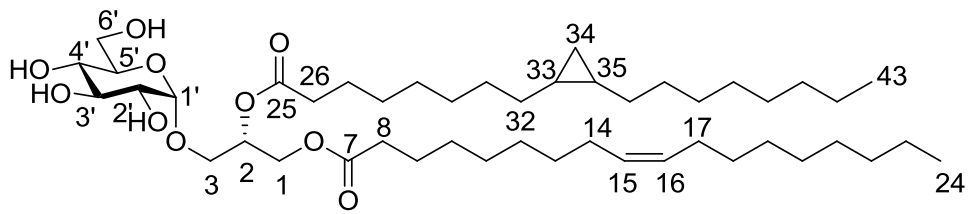




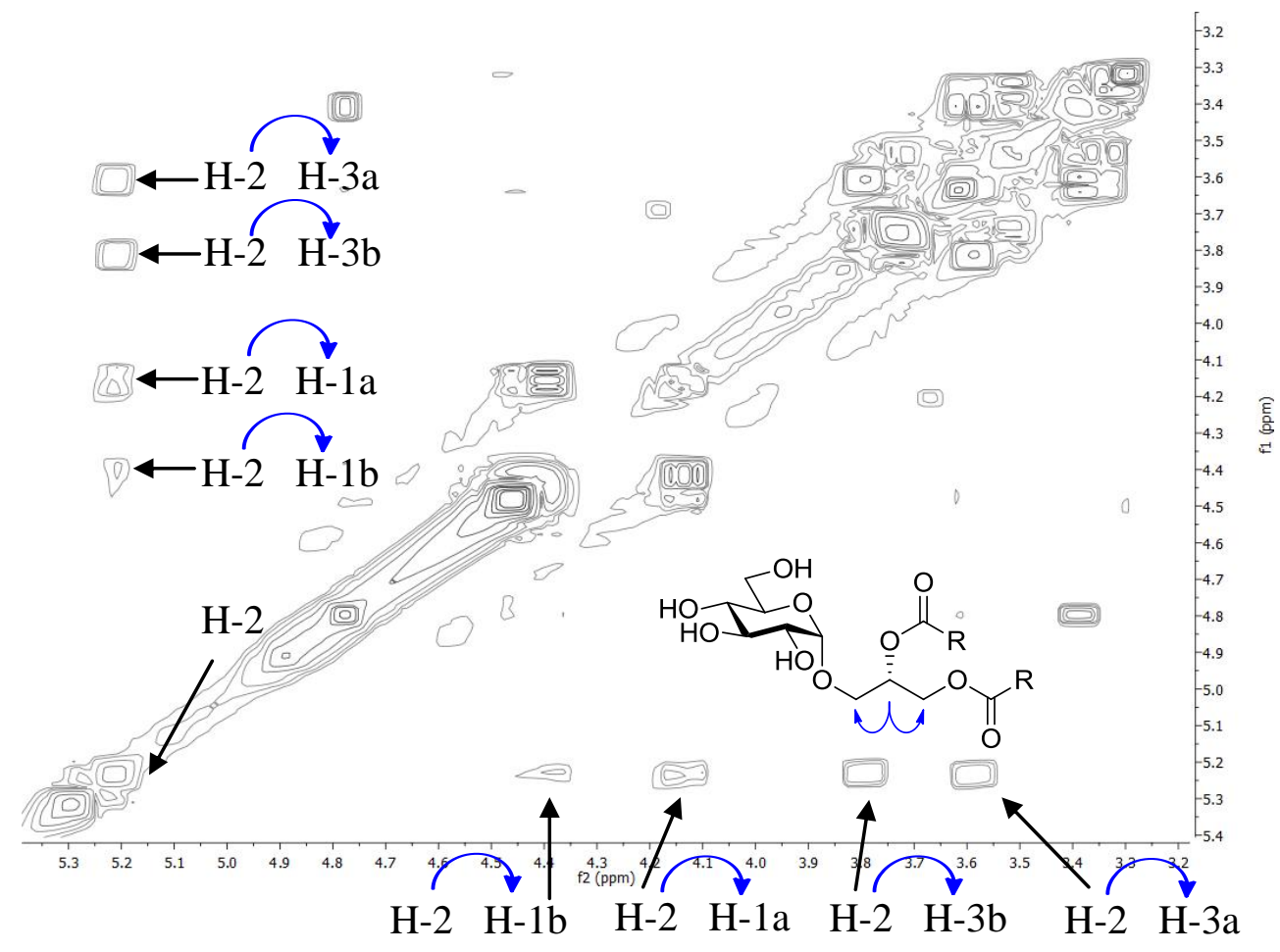

Figure 2.14 Proton to proton correlations showing the correlation (in blue) between GL1 $\mathrm{H}-2$ and $\mathrm{H}-1 / \mathrm{H}-3$ in the ${ }^{1} \mathrm{H},{ }^{1} \mathrm{H}$ COSY spectrum $\mathrm{CD}_{3} \mathrm{OD} / \mathrm{CDCl}_{3}(1 / 2, \mathrm{v} / \mathrm{v}), 500$ $\mathrm{MHz}$.

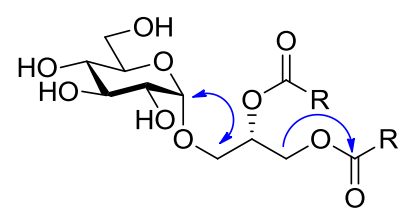

Figure 2.15 HMBC correlations proving the connections $\mathrm{C}-1^{\prime}$ to $\mathrm{C}-3$ and $\mathrm{C}-1$ to $\mathrm{C}(\mathrm{O})$ in GL1. 


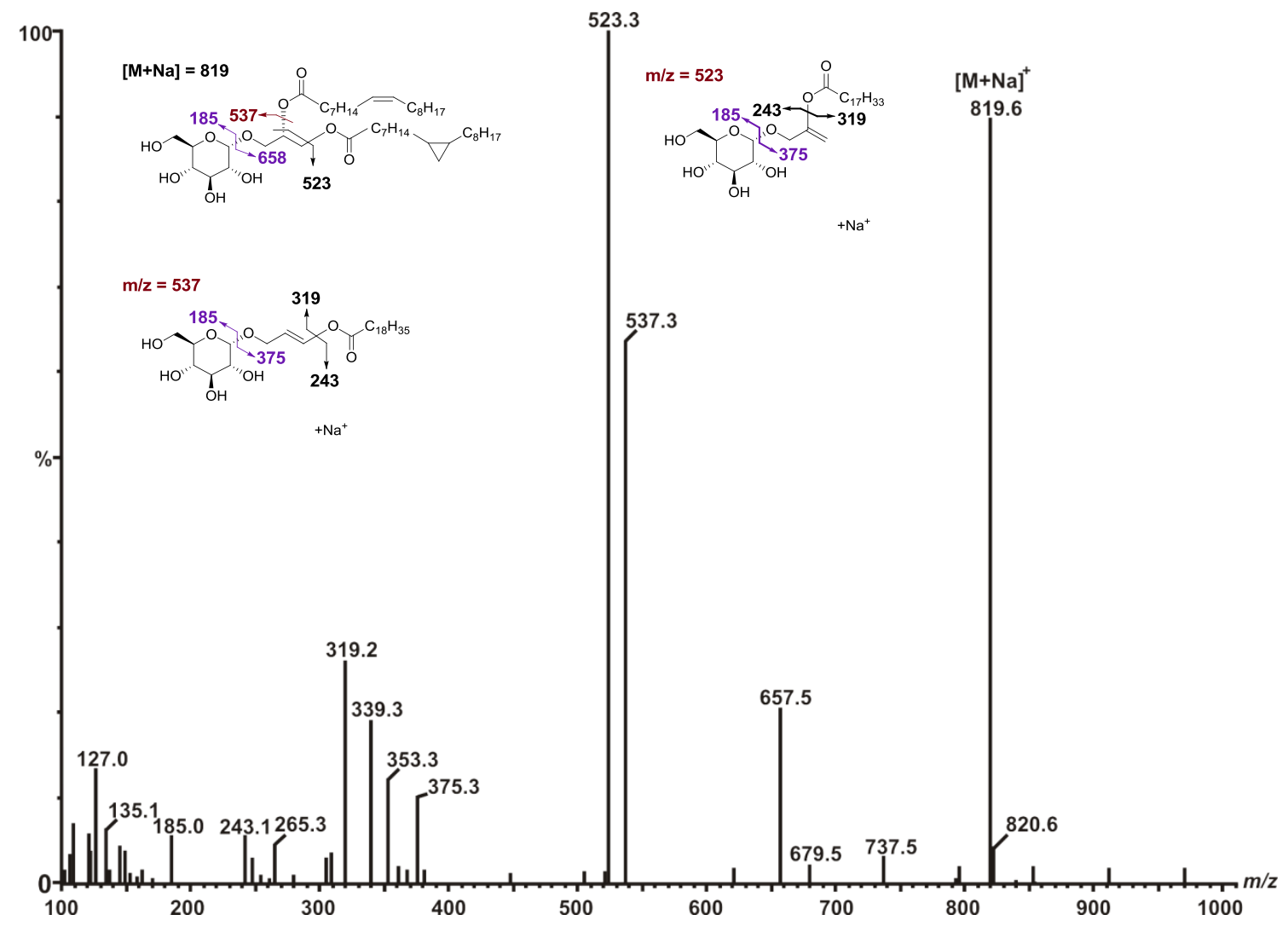

Figure 2.16 ESI MS/MS of GL1 in positive ion mode.

\subsubsection{GL2}

Fatty acid analysis of GL2 showed almost the same lipid profile as for GL1, with palmitic, oleic and dihydrosterculic acid esters being the predominant lipids (Table 2.1). GL2 contained both D-glucose and D-galactose in a 1.8/1 ratio, with linkage analysis revealing the presence of both terminal and 1,6-linked galactose ( $\mathrm{t}-$ and 1,6-Galp), and terminal and 1,2-linked glucose ( $\mathrm{t}-$ and 1,2-Glcp). This suggested that GL2 might contain more than one compound, although the TLC analysis showed only one spot. Indeed, two signals were observed in the HRMS spectrum-a major peak at $\mathrm{m} / \mathrm{z}=$ 981.6482 for a diglycosyldiglyceride (GL2a), and a minor peak at $\mathrm{m} / \mathrm{z}=1407.9382$ for $\left[\mathrm{C}_{76} \mathrm{H}_{136} \mathrm{O}_{21}+\mathrm{Na}\right]^{+}$corresponding to an acylated triglycosyldiglyceride (GL2b). The NMR spectrum of this glycolipid mixture also clearly showed two different glycerides, with the major product (GL2a) containing two anomeric signals, and the minor component (GL2b) containing three. Altough GL2a and GL2b had similar mobilities on 
silica, their appreciable differences in molecular weight indicated that they should separate on a size-exclusion column. Indeed, gel filtration using Sephadex LH-20, eluting with $\mathrm{MeOH} / \mathrm{DCM}(1 / 1, \mathrm{v} / \mathrm{v})$, resulted in a clean separation of the glycolipid mixture into homogenous GL2a and GL2b (Figure 2.17), with GL2b having a slightly higher mobility compared to GL2a.

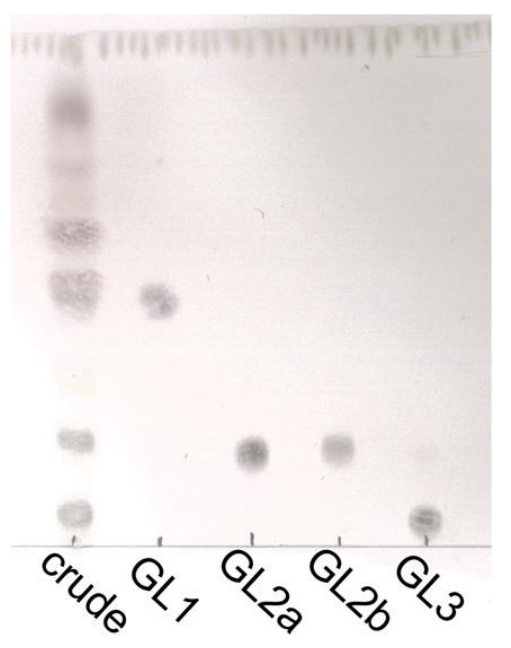

Figure 2.17 TLC of glycolipid and purified fractions from L. plantarum IRL-560. Silica gel, eluted with $\mathrm{CHCl}_{3} / \mathrm{MeOH}(6 / 1, \mathrm{v} / \mathrm{v})$ and developed with sulfuric acid stain.

For GL2a (Table 2.3), the chemical shifts for the anomeric signal $\left(\delta_{\mathrm{H}-1^{\prime}}=5.00 \mathrm{ppm}, \delta_{\mathrm{C}-}\right.$ $\left.1^{\prime}=96.8 \mathrm{ppm}\right)$ were reminiscent of an $\alpha$-glucopyranoside residue. This assignment was further supported by the coupling constants $J_{\mathrm{H}-1^{\prime}}, \mathrm{H}-2^{\prime}=2.5 \mathrm{~Hz}$ and ${ }^{1} J_{\mathrm{C}-1^{\prime}, \mathrm{H}-1^{\prime}}=171 \mathrm{~Hz}$ with the large coupling constants $J_{\mathrm{H}-2^{\prime}, \mathrm{H}-3^{\prime}}=11.3 \mathrm{~Hz}$ and $J_{\mathrm{H}-3^{\prime}, \mathrm{H}-4^{\prime}}=8.6 \mathrm{~Hz}$, and the $\mathrm{H}-$ $2^{\prime}$ proton chemical shift $\left(\delta_{\mathrm{H}-2^{\prime}}=3.60 \mathrm{ppm}\right)$ also confirming the glucose configuration. ${ }^{223,224}$ A second resonance in the anomeric region of the HSQC spectrum $\left(\delta_{\mathrm{H}-1}{ }^{\prime \prime} 5.00 \mathrm{ppm}, \delta_{\mathrm{C}-1^{\prime}}, 97.4 \mathrm{ppm}\right)$ had coupling constants $J_{\mathrm{H}-1^{\prime \prime}}, \mathrm{H}-2^{\prime \prime}=2.5 \mathrm{~Hz}$ and $J_{\mathrm{C}-1^{\prime \prime}}, \mathrm{H}-$ $1^{\prime \prime}=171 \mathrm{~Hz}$, indicating an $\alpha$-configuration. A large $J_{\mathrm{H}-2^{\prime \prime}}, \mathrm{H}-3^{\prime \prime}=11.9 \mathrm{~Hz}$ and very small $J_{\mathrm{H}-3^{\prime \prime}, \mathrm{H}-4^{\prime \prime}} \approx 1 \mathrm{~Hz}$, resulting from a coupling between an axial and an equatorial proton, revealed that the second (terminal) sugar corresponded to $\alpha$-D-Galp. ${ }^{225}$ Finally, HMBCs between the $\alpha-\mathrm{D}-\mathrm{Glc} p \mathrm{H}-2^{\prime}$ and the $\alpha-\mathrm{D}-\mathrm{Gal} p$ C-1"', and between the $\alpha-\mathrm{D}-\mathrm{Glc} p \mathrm{H}-1^{\prime}$ and the glycerol C-3 $\left(\delta_{\mathrm{C}-3} 66.1 \mathrm{ppm}\right)$, showed the linkage between the two sugar residues to be Gal-(1 $\rightarrow 2)$-Glc (Figure 2.18). This was in accordance with the linkage analysis and 
confirmed the structure of GL2a to be $\alpha$-D-galactopyranosyl-(1 $\rightarrow 2)-\alpha-D-$ glucopyranosyldiglyceride.

The fragmentation pattern for GL2a was used to confirm the assignment. In the same way as GL1, ESI-MS/MS was performed on the sodiated molecular ion (HRMS $\mathrm{m} / z=$ 981.6482 for $\left.\left[\mathrm{C}_{52} \mathrm{H}_{94} \mathrm{O}_{15}+\mathrm{Na}\right]^{+}\right)$using $\mathrm{CID}$, in positive ion mode. The observed fragmentation spectrum (Figure 2.19) revealed two major fragment ion peaks which were attributed to the loss of oleic acid and dihydrosterculic acid $(\mathrm{m} / \mathrm{z}=699.4$ and 685.4, respectively). Signals for the fragment ions $(\mathrm{m} / \mathrm{z}=819.6)$ and $(\mathrm{m} / \mathrm{z}=657.6)$ representing MGDG and DAG respectively were also readily identified, as was a disaccharide fragment ion $(\mathrm{m} / \mathrm{z}=347.1)$. Multiple subsequent fragmentations led to yet another series of ions, as identified by the loss of both fatty acids $(\mathrm{m} / z=405.5)$, and the loss of a monosaccharide and either fatty acid $(\mathrm{m} / \mathrm{z}=523.3$ and $\mathrm{m} / \mathrm{z}=537.3)$. These fragments confirm the connectivity of the structural components of GL2a and, in combination with the NMR analysis discussed above, unequivocally establish the structure of GL2a.

Historically, there has been ambiguity about the reducing end anomeric configuration in Lactobacillus glycoglycerolipids. On the basis of enzymatic hydrolysis studies, Brundish et $a l .{ }^{43}$ concluded that the monoglucosyldiglyceride was linked in a $\beta$-fashion with the glycerol, while Shaw ${ }^{46}$ later reported an $\alpha$-linkage, though no experimental data was provided for this assignment. These studies confirm an $\alpha$-D-glucopyranosyl linkage, which is also in accordance with Fischer's studies on the glycerophospholipids from $L$. plantarum, ${ }^{189,190}$ and the structure found in L. casei by Shaw et al. ${ }^{203}$ 
Table 2.3 NMR spectroscopic data for GL2a.

\begin{tabular}{|c|c|c|c|c|c|c|}
\hline Position & $\delta_{1 \mathrm{H}}(\mathrm{ppm})$ & $\delta_{13 \mathrm{C}}(\mathrm{ppm})$ & COSY $(\mathrm{H}-\mathrm{H})$ & & HMBC $(\mathrm{H}-\mathrm{C})$ & \\
\hline$\overline{1^{\prime}}$ & $5.00(\mathrm{~d}, 2.5)$ & 96.8 & $2^{\prime}$ & & $3,2^{\prime}, 5^{\prime}$ & \\
\hline $2^{\prime}$ & $3.60(\mathrm{~d}, 11.3)$ & 77.0 & $1^{\prime}, 3^{\prime}$ & & $1^{\prime \prime}, 3^{\prime}$ & \\
\hline $3^{\prime}$ & $3.77(\mathrm{t}, 11.4)$ & 72.1 & $2^{\prime}, 4^{\prime}$ & & $2^{\prime}, 4^{\prime}$ & \\
\hline $4^{\prime}$ & $3.44(\mathrm{t}, 8.6)$ & 70.2 & $3^{\prime}, 5^{\prime}$ & & $5^{\prime}, 6^{\prime}$ & \\
\hline $5^{\prime}$ & $3.57(\mathrm{~m})$ & 72.3 & \multicolumn{2}{|l|}{$4^{\prime}$} & \multicolumn{2}{|l|}{4} \\
\hline $6 a^{\prime}$ & $3.75(\mathrm{~m})$ & \multirow{2}{*}{61.6} & \multirow{2}{*}{\multicolumn{2}{|c|}{-}} & \multirow{2}{*}{\multicolumn{2}{|c|}{ - }} \\
\hline $6 b^{\prime}$ & $3.73(\mathrm{~m})$ & & & & & \\
\hline $1 "$ & $5.00(\mathrm{~d}, 2.5)$ & 97.4 & \multicolumn{2}{|l|}{$2 "$} & \multicolumn{2}{|l|}{$2^{\prime \prime}, 3^{\prime \prime}, 5^{\prime \prime}$} \\
\hline $2^{\prime \prime}$ & $3.80(\mathrm{~d}, 11.9)$ & 69.2 & \multicolumn{2}{|l|}{$1^{\prime \prime}$} & \multicolumn{2}{|l|}{$1^{\prime}$} \\
\hline $3^{\prime \prime}$ & $3.80(\mathrm{~m})$ & 70.4 & \multicolumn{2}{|l|}{$4^{\prime \prime}$} & \multicolumn{2}{|l|}{$2^{\prime \prime}, 5^{\prime \prime}$} \\
\hline $4 "$ & $3.95(\mathrm{~m})$ & 70.0 & \multicolumn{2}{|l|}{$3^{\prime \prime}, 5^{\prime \prime}$} & \multicolumn{2}{|l|}{$2^{\prime \prime}, 3^{\prime \prime}$} \\
\hline $5^{\prime \prime}$ & $4.09(\mathrm{~m})$ & 71.3 & \multicolumn{2}{|l|}{$4^{\prime \prime}, 6 a^{\prime \prime}, 6 b^{\prime \prime}$} & \multicolumn{2}{|l|}{$4^{\prime \prime}, 6^{\prime \prime}$} \\
\hline $6 a^{\prime \prime}$ & $3.74(\mathrm{~m})$ & & \multicolumn{2}{|l|}{$5^{\prime \prime}, 6 b^{\prime \prime}$} & \multicolumn{2}{|l|}{-} \\
\hline $6 b^{\prime \prime}$ & $3.79(\mathrm{~m})$ & 62.2 & \multicolumn{2}{|l|}{$5^{\prime \prime}, 6 a^{\prime \prime}$} & \\
\hline $3 \mathrm{a}$ & $3.64(\mathrm{~m})$ & & \multicolumn{2}{|l|}{$3 \mathrm{~b}, 2$} & \multicolumn{2}{|l|}{$1,2,1$} \\
\hline $3 b$ & $3.83(\mathrm{~m})$ & 66.1 & \multicolumn{2}{|l|}{$3 a, 2$} & \multicolumn{2}{|l|}{$1^{\prime}, 2,1$} \\
\hline 2 & $5.24(\mathrm{~m})$ & 70.3 & \multicolumn{2}{|l|}{1,3} & \multicolumn{2}{|l|}{25} \\
\hline $1 \mathrm{a}$ & $4.20(\mathrm{dd}, 6.0)$ & \multirow{2}{*}{63.0} & \multicolumn{2}{|l|}{$2,1 \mathrm{~b}$} & \multicolumn{2}{|l|}{$3,2,7$} \\
\hline $1 \mathrm{~b}$ & $4.43(\mathrm{dd}, 3.0)$ & & $2,1 \mathrm{a}$ & & \multicolumn{2}{|l|}{3,7} \\
\hline 7 & (1) & 174.6 & $\cdots$ & & & \\
\hline 25 & - & 174.1 & - & & & \\
\hline 8,26 & $2.34(t, 7.5)$ & 34.4 & 9 & 27 & $7,9,10$ & $25,27,28$ \\
\hline 9,27 & $1.62(\mathrm{~m})$ & 25.1 & 8,10 & 26,28 & $7,8,11$ & $25,26,29$ \\
\hline 15,16 & $5.35(\mathrm{~m})$ & 130.1 & 14 & 17 & 13,17 & 14,18 \\
\hline 14,17 & $2.03(\mathrm{~m})$ & 27.4 & 13,15 & 16,18 & 12,15 & 16,19 \\
\hline $10-13^{*}$ & & & & & & \\
\hline $18-23^{*}$ & $1.10-1.35(\mathrm{~m})$ & $28-32$ & & & - & \\
\hline $\begin{array}{l}28-32^{*} \\
36-42^{*}\end{array}$ & & & & & & \\
\hline$\frac{36-42^{*}}{34 a}$ & $-0.33(\mathrm{q}, 5.0)$ & - & $34 \mathrm{~b}, 33,35$ & & $33,35,36$ & \\
\hline $34 \mathrm{~b}$ & $0.57(\mathrm{q}, 4.2)$ & - & $34 a, 33,35$ & & $32,33,35$ & \\
\hline 33,35 & $0.65(\mathrm{q}, 4.0)$ & 16.2 & $34 \mathrm{a}, 34 \mathrm{~b}$ & & - & \\
\hline 24,43 & $0.89(\mathrm{t}, 6.2)$ & 14.2 & 23 & 42 & 22,23 & 42 \\
\hline
\end{tabular}

* Overlapping signals

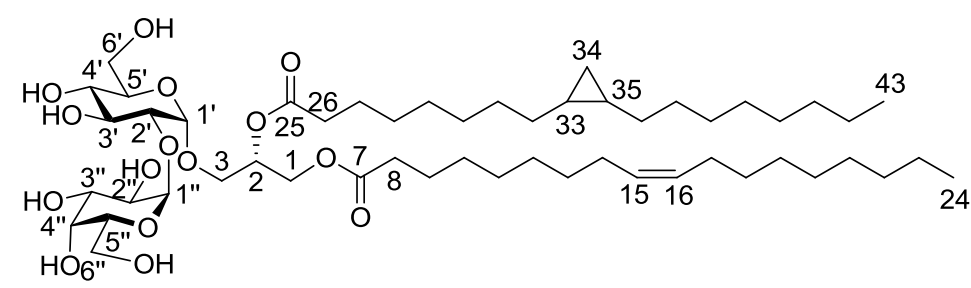




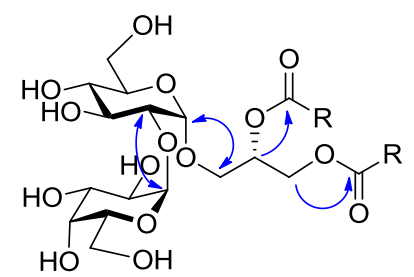

Figure 2.18 HMBC correlations proving the connections $\mathrm{C}^{-1} 1^{\prime}$ to $\mathrm{C}-3, \mathrm{C}-2$ to $\mathrm{C}(\mathrm{O})$, $\mathrm{C}-1$ to $\mathrm{C}(\mathrm{O})$ and $\mathrm{C}-1^{\prime \prime}$ to $\mathrm{C}-2^{\prime}$.

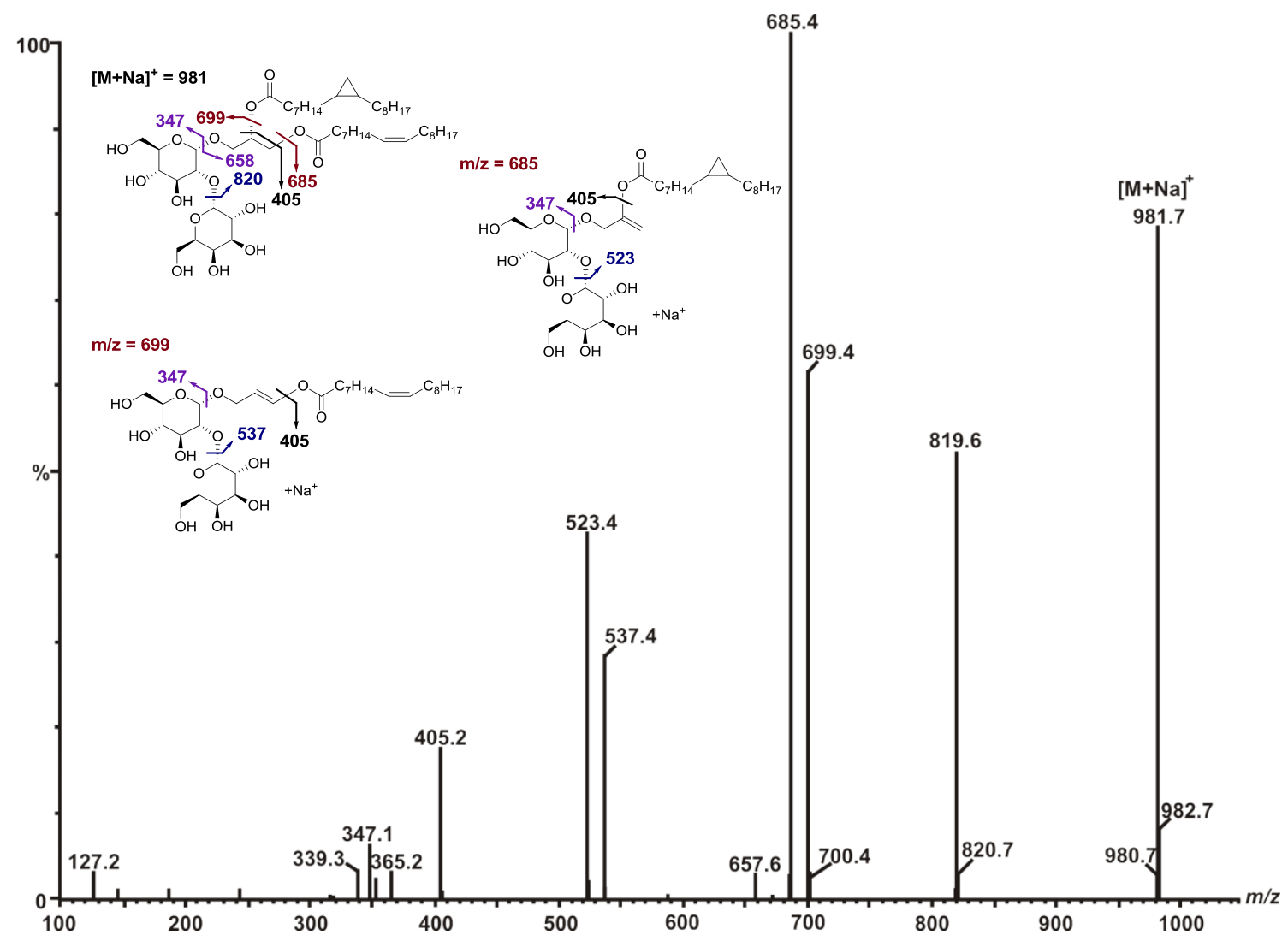

Figure 2.19 ESI-MS/MS of GL2a in positive ion mode.

NMR analysis of GL2b (Table 2.4) also revealed the presence of the $(1 \rightarrow 2)$-linked $\alpha$ Glc $p$ and $\alpha-G a l p$ residues observed for GL2a, however, the $\alpha-\mathrm{D}-\mathrm{Glc} p \mathrm{H}-6 \mathrm{a}^{\prime}$ and H-6b' were markedly shifted downfield $\left(\delta_{\mathrm{H}-6 \mathrm{a}^{\prime}}=4.26, \delta_{\mathrm{H}-6 \mathrm{~b}^{\prime}}=4.37 \mathrm{ppm}\right)$ and showed an HMBC to a carbonyl carbon $\left(\delta_{\mathrm{C}} 174.5 \mathrm{ppm}\right)$. This confirmed the presence of an 
additional fatty acid ester at the $\alpha$-D-Glc $p 6^{\prime}$-position, as predicted by HRMS $(\mathrm{m} / z=$ 1393.9287). An additional sugar unit was easily identified in the HSQC of GL2b, which showed a third residue with a distinct anomeric signal at $\delta_{\mathrm{H}-1^{\prime \prime \prime}}=4.37 \mathrm{ppm}$ and $\delta_{\mathrm{C}-1{ }^{\prime \prime}}=$ $103.5 \mathrm{ppm}$ and coupling constants $J_{\mathrm{H}-1{ }^{\prime \prime \prime}, \mathrm{H}-2^{\prime \prime \prime}}=10.2 \mathrm{~Hz}, J_{\mathrm{H}-2^{\prime \prime \prime}, \mathrm{H}-3^{\prime \prime \prime}}=9.7 \mathrm{~Hz}$ and $J_{\mathrm{H}-3^{\prime \prime \prime}}$, $\mathrm{H}-4^{\prime \prime \prime}=10.0 \mathrm{~Hz}$. This data, in combination with the typical upfield chemical shift of $\mathrm{H}-$ $2^{\prime \prime \prime}\left(\delta_{\mathrm{H}-2{ }^{\prime \prime}}=3.30 \mathrm{ppm}\right)$, confirmed the presence of a $\beta$-D-Glcp residue. ${ }^{146}$ This was confirmed in the COSY spectrum where $\mathrm{H}-1^{\prime \prime \prime}$ correlated with $\mathrm{H}-2^{\prime \prime \prime}$ in the $\beta$-glucose moiety (Figure 2.20). In addition to the HMBCs between $\alpha$-D-Gal $p, \alpha-\mathrm{D}-\mathrm{Glc} p$ and glycerol observed in GL2a, GL2b showed a correlation between the $\alpha-\mathrm{D}-\mathrm{Gal} p \mathrm{CH}_{2}-6$ " $\left(\delta_{\mathrm{H}-6 \mathrm{a}^{\prime \prime}}=3.82\right.$ and $\left.\delta_{\mathrm{H}-6 \mathrm{~b}^{\prime \prime}}=4.00 \mathrm{ppm}\right)$ and the $\beta-\mathrm{D}-\mathrm{Glcp} \mathrm{C}-1^{\prime \prime \prime}\left(\delta_{\mathrm{C}-1^{\prime \prime}}=103.5 \mathrm{ppm}\right)$, thus confirming the presence of the 1,6-Galp linkage detected in the linkage analysis (Figure 2.21).

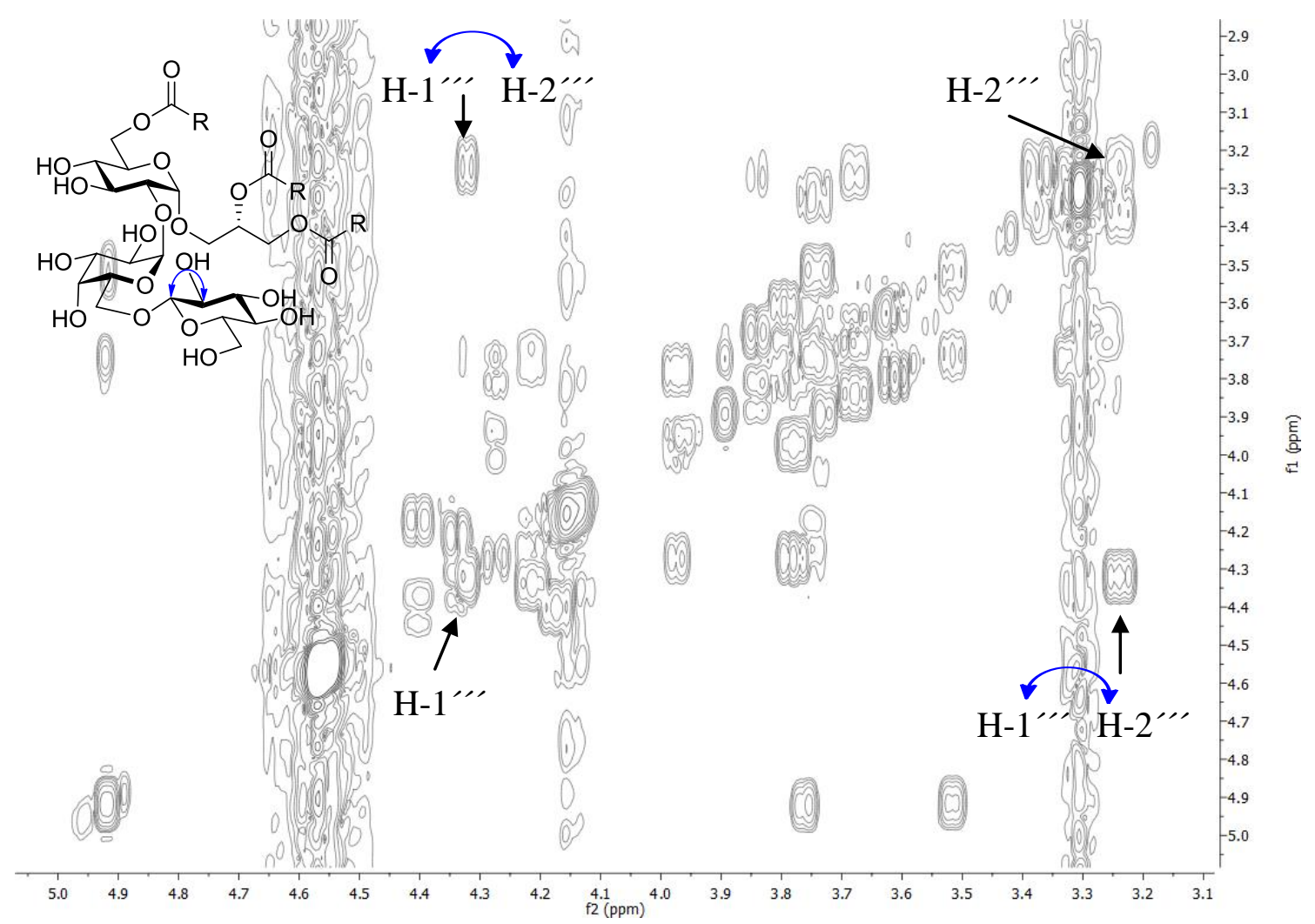

Figure 2.20 Proton to proton correlations showing the correlation between shielded $\mathrm{H}-1^{\prime \prime \prime}$ and $\mathrm{H}-2$ "' in the ${ }^{1} \mathrm{H},{ }^{1} \mathrm{H}$ COSY spectrum of $\mathrm{GL} 2 \mathrm{~b} \mathrm{CD} \mathrm{CD}_{3} \mathrm{OD} / \mathrm{CDCl}_{3}(1 / 2, \mathrm{v} / \mathrm{v}), 600$ MHz. 
Table 2.4 NMR spectroscopic data for GL2b.

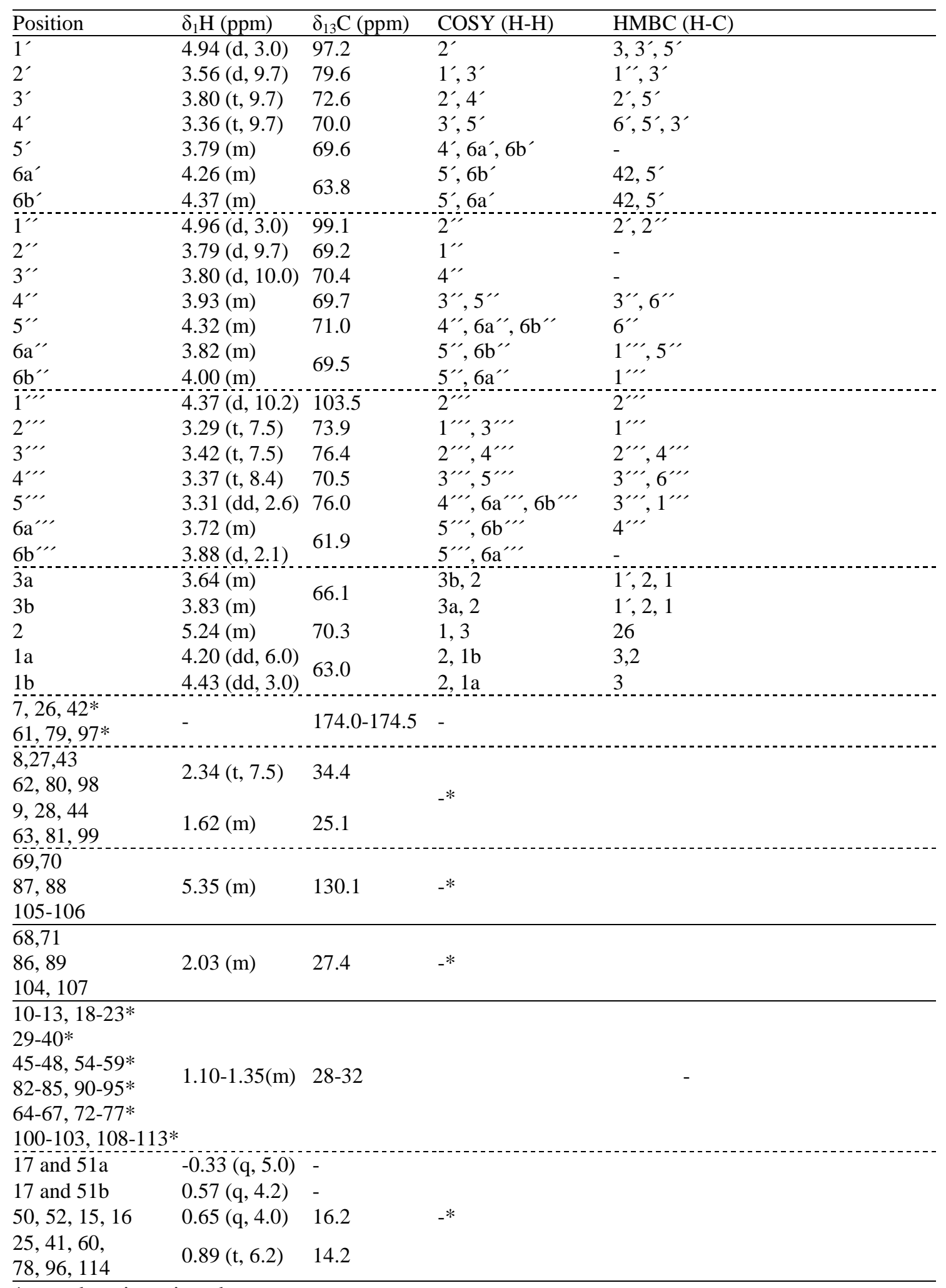

\footnotetext{
* overlapping signals
} 
Species 1:

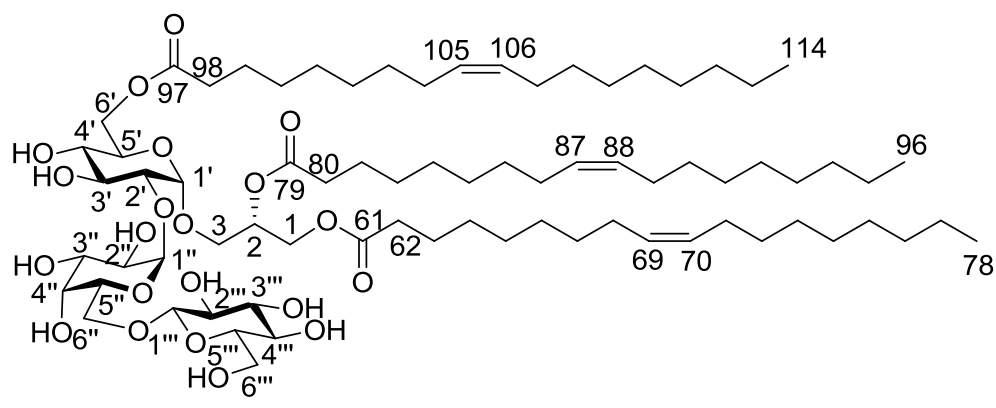

Species 2:
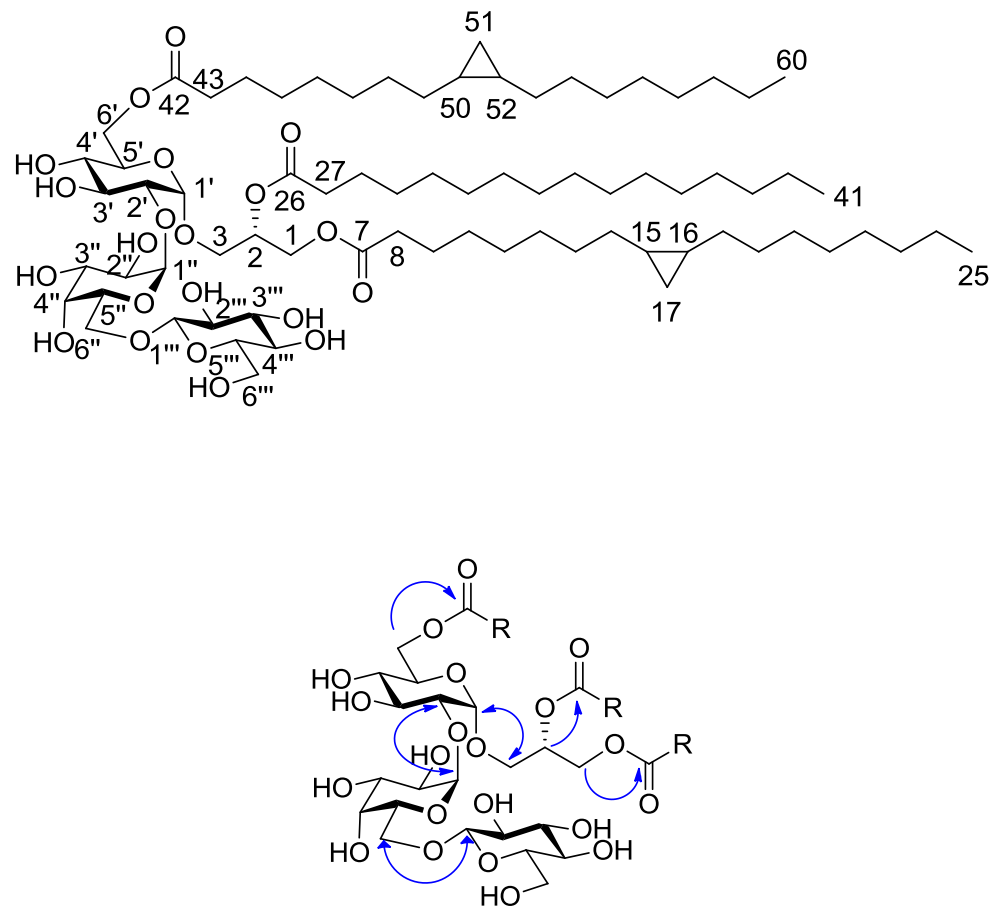

Figure 2.21 HMBC correlations proving the connections of $\mathrm{C}^{-1} \mathbf{1}^{\prime}$ to $\mathrm{C}-3, \mathrm{C}-2$ to $\mathrm{C}(\mathrm{O})$, $\mathrm{C}-1$ to $\mathrm{C}(\mathrm{O}), \mathrm{C}-1^{\prime \prime}$ to $\mathrm{C}-2^{\prime}, \mathrm{C}-1^{\prime \prime \prime}$ to $\mathrm{C}-6^{\prime \prime}$ and $\mathrm{C}-6^{\prime}$ to $\mathrm{C}(\mathrm{O})$ in GL2b.

The fragmentation pattern of GL2b was more complex than that observed for GL2a. At first, the fragmentation of the major ion in positive ion mode (HRMS $m / z=1393.9287$ for $\left[\mathrm{C}_{76} \mathrm{H}_{136} \mathrm{O}_{21}+\mathrm{Na}\right]^{+}$) using collision induced dissociation (CID), gave three major ions $(\mathrm{m} / \mathrm{z}=1111,1139$ and 1097) (Figure 2.22). If it is assumed that the molecular ion contains three oleic acids, $1111 \mathrm{~m} / \mathrm{z}$ corresponds to the loss of oleic acid and $1069 \mathrm{~m} / \mathrm{z}$ to the loss of a disaccharide. However, the presence of the two other fragments can be 
explained only if another species containing the same mass as $1393 \mathrm{~m} / \mathrm{z}$ is also present in the mixture. Indeed, the same mass can be obtained if two dihydrosterculic acids and one palmitic acid are present on the residue. Then the loss of palmitic acid and dihydrosterculic acid leads to the formation of two other major fragments $(\mathrm{m} / \mathrm{z}=1139$ and $m / z=1097$, respectively). The loss of a disaccharide on fragments 1139, 1111 and 1097 could also be observed $(\mathrm{m} / \mathrm{z}=815, \mathrm{~m} / \mathrm{z}=787$ and $\mathrm{m} / \mathrm{z}=773$ respectively). Also, a fragment corresponding to the disaccharide $(\mathrm{m} / \mathrm{z}=347.1)$ itself was observed. Two additional ions were identified as a monoacylated-containing oleic acid-trisaccharide $(\mathrm{m} / \mathrm{z}=831)$ and monoacylated-containing dihydrosterculic acid-trisaccharide $(\mathrm{m} / \mathrm{z}=$ $845)$. These fragments confirm the connectivity of the structural components of GL2b. Using NMR, the ratio of these two species is of approximately 3:7 (Species 1/Species $2)$. Indeed, this could be calculated using the integration of the cyclopropane protons ($0.33 \mathrm{ppm})$ and the double bond protons $(5.35 \mathrm{ppm})$.

Taken as a whole, this enabled GL2b to be conclusively identified as $\beta$-Dglucopyranosyl-( $1 \rightarrow 6)-\alpha$-D-galactopyranosyl-( $1 \rightarrow 2)-6-O$-acyl- $\alpha$-D-glucopyranosyldiglyceride, a glycolipid not previously observed in L. plantarum, although it has been observed in $L$. casei $^{188}$ and the corresponding phosphoglycolipid has been isolated from L. plantarum. ${ }^{189,190}$ 
Species 1:
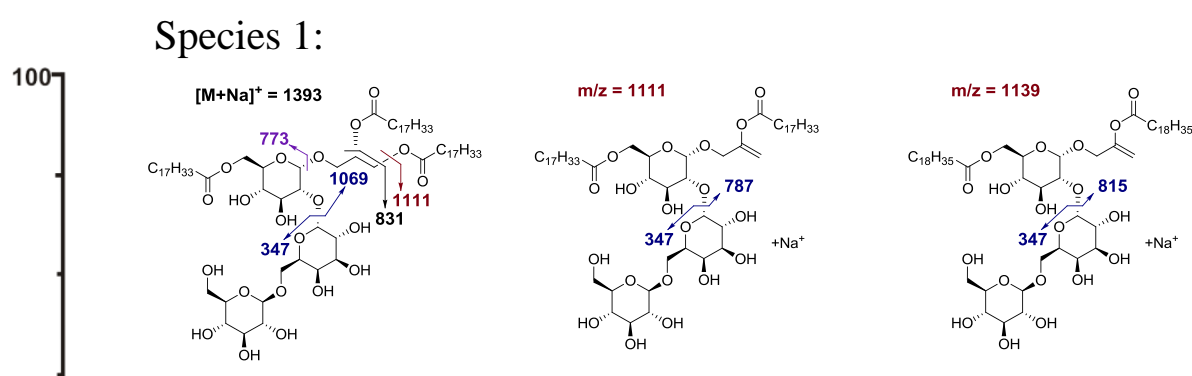

Species 2:

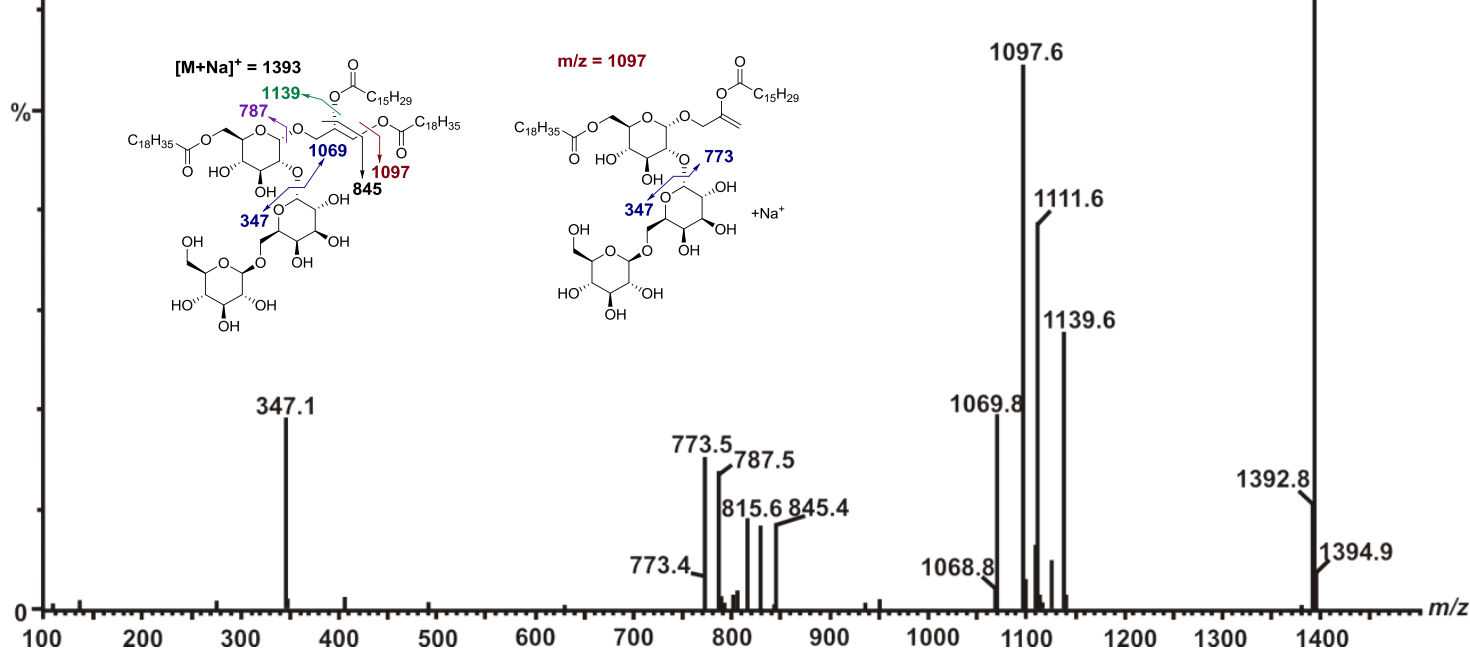

Figure 2.22 ESI-MS/MS of GL2b in positive ion mode.

\subsubsection{GL3}

In an analogous manner, the final glycolipid GL3 (Table 2.5) was found to contain tGlcp, 1,2-Glcp and 1,6-Gal $p$. NMR data exhibited a remarkable resemblance to that of GL2b, except for the shielded $\alpha-\mathrm{D}-\mathrm{Glc} p \mathrm{CH}_{2^{-}} 6^{\prime}\left(\delta_{\mathrm{H}-6 \mathrm{a}^{\prime}}=3.75 \mathrm{ppm}, \delta_{\mathrm{H}-6 \mathrm{~b}^{\prime}}=3.73 \mathrm{ppm}\right.$ and $\delta_{\mathrm{C}-6^{\prime}}=61.5 \mathrm{ppm}$ ), and the absence of an additional acyl group at this $6^{\prime}$-position. Using CID, in positive ion mode, the sodiated molecular ion (HRMS $\mathrm{m} / \mathrm{z}=1143.7009$ for $\left[\mathrm{C}_{58} \mathrm{H}_{104} \mathrm{O}_{20}+\mathrm{Na}\right]^{+}$) was fragmented to confirm the assignment of GL3. The observed fragmentation spectrum (Figure 2.23) revealed two major fragment ion peaks which were attributed to the loss of oleic acid and dihydrosterculic acid $(\mathrm{m} / \mathrm{z}=861.5$ and 847.5, respectively). Signals for DGDG $(m / z=981.7)$, MGDG $(m / z=819.6)$ and DAG $(\mathrm{m} / \mathrm{z}=657.5)$ were also readily identified, as were the disaccharide $(\mathrm{m} / \mathrm{z}=347.1)$ and trisaccharides $(\mathrm{m} / \mathrm{z}=509.2)$ fragments themselves. Multiple fragmentations led to yet 
another series of ions, as identified by the loss of both fatty acids $(m / z=567.2)$, the loss of a saccharide and either fatty acid $(\mathrm{m} / \mathrm{z}=685.4$ and $\mathrm{m} / \mathrm{z}=699.4)$ and the loss of a disaccharide and either fatty acid $(\mathrm{m} / z=523.3$ and $\mathrm{m} / \mathrm{z}=537.3)$. These fragments confirm the connectivity of the structural components of GL3 and, in combination with the NMR analysis discussed above, unequivocally establish the structure of GL3 to be $\beta$-D-glucopyranosyl$(1 \rightarrow 6)-\alpha-D-$ galactopyranosyl- $(1 \rightarrow 2)-\alpha-D-$ glucopyranosyldiglyceride. Here it should also be noted that Shaw identified a glucosylgalactosylglucosyldiglyceride from L. plantarum, although no information on the configuration of the anomeric centres was provided, nor was any experimental data given. $^{46}$ 
Table 2.5 NMR spectroscopic data for GL3.

\begin{tabular}{|c|c|c|c|c|}
\hline Position & $\delta_{1 \mathrm{H}}(\mathrm{ppm})$ & $\delta_{13 \mathrm{C}}(\mathrm{ppm})$ & COSY (H-H) & HMBC (H-C) \\
\hline $1^{\prime}$ & $4.97(\mathrm{~d}, 3.8)$ & 97.4 & $2^{\prime}$ & $3,2^{\prime}, 3^{\prime}$ \\
\hline $2^{\prime}$ & $3.58(\mathrm{dd}, 4.0,5.7)$ & 79.1 & $1^{\prime}, 3^{\prime}$ & $3^{\prime}, 1^{\prime \prime}$ \\
\hline $3^{\prime}$ & $3.78(t, 10.0)$ & 72.5 & $2^{\prime}, 4^{\prime}$ & $2^{\prime}, 4^{\prime}$ \\
\hline $4^{\prime}$ & $3.46(t, 9.8)$ & 69.8 & $3^{\prime}, 5^{\prime}$ & $3^{\prime}, 6^{\prime}$ \\
\hline $5^{\prime}$ & $3.58(\mathrm{~m})$ & 72.3 & $4^{\prime}, 6^{\prime}$ & $3^{\prime}, 6^{\prime}$ \\
\hline $6 a^{\prime}$ & $3.75(\mathrm{~m})$ & \multirow{2}{*}{61.5} & $5^{\prime}$ & \multirow{2}{*}{ - } \\
\hline $6 b^{\prime}$ & $3.73(\mathrm{~m})$ & & $5^{\prime}$ & \\
\hline $1 "$ & $4.96(\mathrm{~d}, 4.2)$ & 98.7 & 2 & $2,3,5$ \\
\hline $2^{\prime \prime}$ & $3.79(\mathrm{dd}, 4.7)$ & 69.2 & \multirow{2}{*}{$\begin{array}{l}1^{\prime \prime}, 3^{\prime \prime} \\
2^{\prime \prime}, 4^{\prime \prime}\end{array}$} & - \\
\hline $3^{\prime \prime}$ & $3.78(\mathrm{dd}, 3.3)$ & 70.4 & & - \\
\hline $4^{\prime \prime}$ & $3.93(\mathrm{~d}, 2.2)$ & 70.0 & $3^{\prime \prime}, 5^{\prime \prime}$ & $2^{\prime \prime}, 3^{\prime \prime}$ \\
\hline $5^{\prime \prime}$ & $4.34(\mathrm{dd}, 3.3)$ & 71.2 & $4^{\prime \prime}, 6 a^{\prime \prime}, 6 b^{\prime \prime}$ & \\
\hline $6 a^{\prime \prime}$ & $3.85(\mathrm{dd}, 2.5)$ & 69.6 & $5^{\prime \prime}, 6 b^{\prime \prime}$ & $1^{\prime \prime \prime}$ \\
\hline $6 b^{\prime \prime}$ & $4.00(\mathrm{dd}, 2.5)$ & & $5^{\prime \prime}, 6 a^{\prime \prime}$ & $1^{\prime \prime \prime}$ \\
\hline 1 & $4.39(\mathrm{~d}, 7.8)$ & 103.6 & $2 \cdots$ & $6^{\prime \prime}, 3$ \\
\hline $2^{\prime \prime \prime}$ & $3.28(\mathrm{t}, 9.0)$ & 74.1 & $1^{\prime \prime \prime}, 3^{\prime \prime \prime}$ & $1^{\prime \prime \prime}, 3^{\prime \prime \prime}$ or $5^{\prime \prime \prime}$ \\
\hline $3^{\prime \prime \prime}$ & $3.42(t, 9.7)$ & 76.6 & \multirow{2}{*}{$2^{\prime \prime \prime}$} & $1^{\prime \prime \prime}, 2^{\prime \prime \prime}, 4 " \prime$ \\
\hline $4^{\prime \prime \prime}$ & $3.37(\mathrm{t}, 9.8)$ & 70.5 & & $3^{\prime \prime \prime}$ or $5^{\prime \prime \prime}, 6^{\prime \prime \prime}$ \\
\hline $5^{\prime \prime \prime}$ & $3.30(\mathrm{dd}, 2.6)$ & 76.5 & $\begin{array}{l}- \\
6 a^{\prime \prime \prime}, 6 b^{\prime \prime \prime}\end{array}$ & \\
\hline $6 a^{\prime \prime \prime}$ & $3.75(\mathrm{dd}, 5.6)$ & & $5^{\prime \prime \prime}, 6 b^{\prime \prime \prime}$ & $5^{\prime \prime \prime}$ \\
\hline $6 b^{\prime \prime \prime}$ & $3.90(\mathrm{dd}, 5.6)$ & 61.9 & $5^{\prime \prime \prime}, 6 a^{\prime \prime \prime}$ & \\
\hline $3 a$ & $3.67(\mathrm{dd}, 6.4)$ & & $3 b, 2$ & $1^{\prime}, 2,1$ \\
\hline $3 b$ & $3.85(\mathrm{dd}, 6.4)$ & 06.0 & $3 a, 2$ & $1^{\prime}, 2,1$ \\
\hline 2 & $5.25(\mathrm{~m})$ & 70.3 & $1 \mathrm{a}, 1 \mathrm{~b}, 3 \mathrm{a}, 3 \mathrm{~b}$ & 25 \\
\hline $1 \mathrm{a}$ & $4.20(\mathrm{dd}, 6.0)$ & & $2,1 b$ & 2,7 \\
\hline $1 \mathrm{~b}$ & $4.44(\mathrm{dd}, 3.0)$ & 63.1 & $2,1 \mathrm{a}$ & 3,7 \\
\hline 7 & - & 174.6 & - & - \\
\hline 25 & - & 174.1 & - & - \\
\hline 8,26 & $2.34(t, 7.5)$ & 34.5 & 27 & $25,27,28$ \\
\hline 9,27 & $1.61(\mathrm{~m})$ & 25.2 & 26 & $25,27,29$ \\
\hline 15,16 & $5.35(\mathrm{~m})$ & 130.1 & 17 & 14,18 \\
\hline 14,17 & $2.03(\mathrm{~m})$ & 27.4 & 15 & 12,16 \\
\hline$* 10-13$ & & & & \\
\hline $\begin{array}{l}18-23 \\
28-32 \\
36-42\end{array}$ & $1.25-1.35(\mathrm{~m})$ & $28-30$ & & - \\
\hline $34 a^{3}$ & $-0.33(\mathrm{q}, 5.0)$ & - & $34 \mathrm{~b}, 33,35$ & $32,33,35,36$ \\
\hline $34 \mathrm{~b}$ & $0.58(\mathrm{q}, 4.2)$ & - & $34 a, 33,35$ & $32,33,35,36$ \\
\hline 33,35 & $0.67(\mathrm{q}, 4.0)$ & 16.1 & $34 a, 34 b$ & - \\
\hline 24,43 & $0.89(\mathrm{t}, 6.2)$ & 14.2 & 23 & 22 \\
\hline
\end{tabular}

* overlapping signals

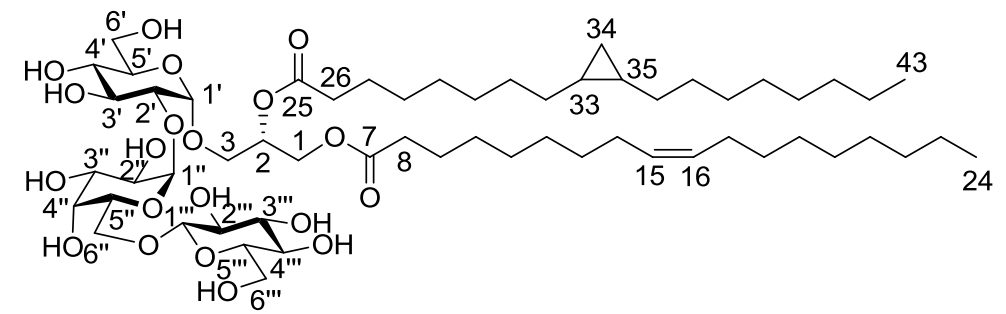




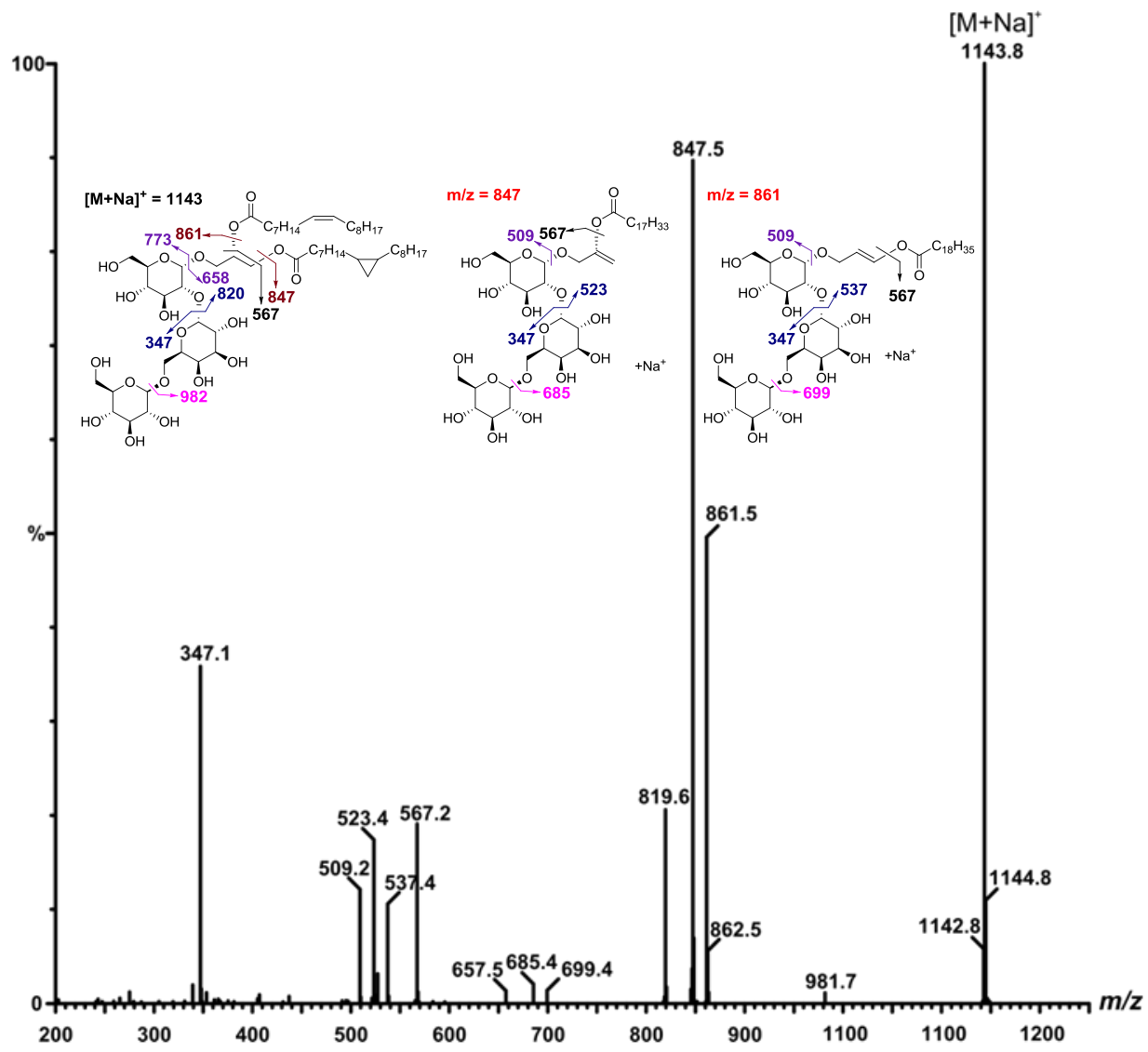

Figure 2.23 ESI MS/MS of GL3 in positive ion mode.

\subsubsection{Bioactivity of $L$. plantarum Glycoglycerolipids}

In order to provide an insight on the effects that L. plantarum glycolipids might have on the immune system, the purified compounds were tested in a macrophage activation assay. Macrophages are antigen presenting cells that play a crucial role in the first line of defence in an organism. When activated, macrophages produce nitric oxide (NO) which is known to be responsible for the cytotoxic effects of the cells. ${ }^{226,227}$ Although whole Lactobacillus cells were found to activate macrophages, ${ }^{99}$ biological data concerning the ability of $L$. plantarum glycolipids (incl. LTAs) to activate macrophages is scarce. ${ }^{193}$ Figure 2.24 shows that GL1 and GL2a activated bone marrow macrophages to a small extent, however as indicated by the error bars in the graph, this activation is not statistically significant. Additionally, GL2b and GL3 compared to LPS, did not show any activation. This data is not surprising as reports have shown that synthesised 
glycolipid anchors from $S$. aureus only induced minor IL-8 production when tested in human whole blood cells. ${ }^{81}$ Previous reports have described this cytokine as being proinflammatory and indicates macrophage activation. ${ }^{228}$ As whole blood cells also contain macrophages, such an assay results that those anchors only weakly activate mouse macrophages and human macrophages.

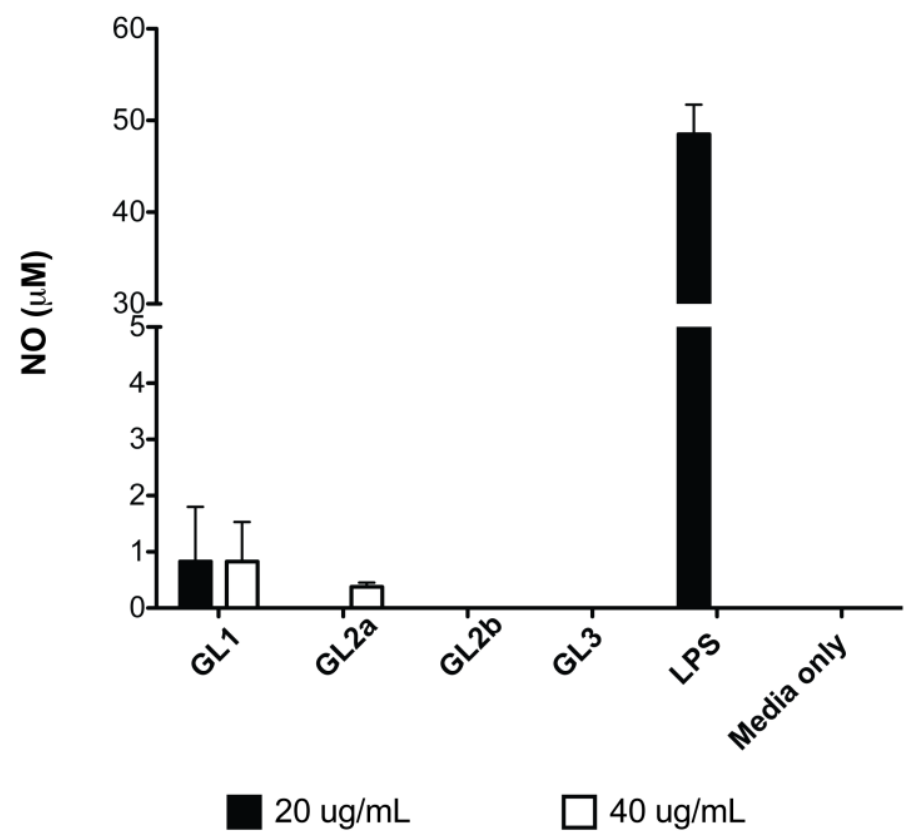

Figure 2.24 Glycoglycerolipids NO production by BMMs. BMMs were stimulated with glycoglycerolipid (GL1, GL2a, GL2b and GL3) $(20 \mu \mathrm{g} / \mathrm{mL}$ and $40 \mu \mathrm{g} / \mathrm{mL})$, or LPS $(100 \mathrm{ng} / \mathrm{mL})$ and the supernatants were analyzed for NO after $48 \mathrm{~h}$ using the Griess assay. Mean and SD of triplicate samples from a representative experiment of two are indicated. 


\subsection{Concluding Remarks}

In summary, four major glycoglycerolipids of L. plantarum IRL-560 were conclusively identified as $\alpha$-D-Glcp-diglyceride (GL1), $\alpha$-D-Gal $p-(1 \rightarrow 2) \quad \alpha$-D-Glcp-diglyceride (GL2a), $\beta$-D-Glcp-( $1 \rightarrow 6)$ - $\alpha$-D-Gal $p$-( $1 \rightarrow 2)-6$ - $O$-acyl- $\alpha$-D-Glc $p$-diglyceride (GL2b) and $\beta$-D-Glc $p-(1 \rightarrow 6)-\alpha-\mathrm{D}-\mathrm{Gal} p-(1 \rightarrow 2)-\alpha-\mathrm{D}-\mathrm{Glc} p$-diglyceride (GL3). Interestingly, these structures have also been found in L. casei, although there has been confusion about the anomeric configurations of similar glycoglycerolipids isolated from L. plantarum. By providing conclusive evidence for the structures of the isolated glycoglycerolipids, this will aid in correlating the biological activities of $L$. plantarum to its individual cell wall components. Moreover, the provision of detailed NMR and mass spectrometry data will be useful for the future identification of these types of compounds. Preliminary data on macrophage activation by $L$. plantarum glycolipids showed non-significant NO production. 


\subsection{Supplementary data-NMR, MS spectra and Compound Characterisation}

\subsubsection{GL1}

ESI-MS, positive ion mode

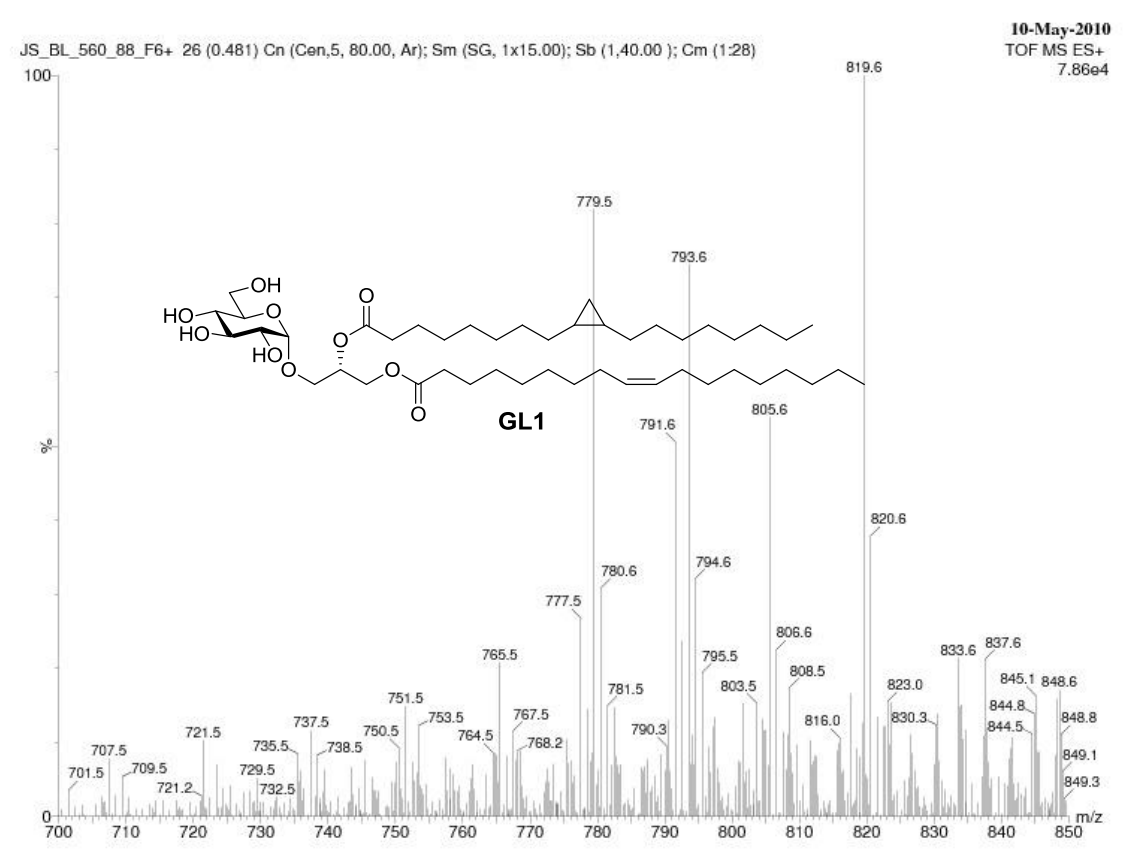

GL1: Colourless oil $(12.3 \mathrm{mg})[\alpha]_{\mathrm{D}}^{23}=49.1\left(c=0.5, \mathrm{CHCl}_{3} / \mathrm{MeOH}, 2 / 1, \mathrm{v} / \mathrm{v}\right)$; IR (film) $3382,2923,2853,1738,1456,1366,1228,1216,1151,1025,632,576 \mathrm{~cm}^{-1} ;{ }^{1} \mathrm{H},{ }^{13} \mathrm{C}$ NMR, HRMS and $\mathrm{R}_{f}$ data, see Table 2.1 and 2.2. 
${ }^{1} \mathrm{H} \mathrm{NMR}, \mathrm{CD}_{3} \mathrm{OD} / \mathrm{CDCl}_{3}(1 / 2, \mathrm{v} / \mathrm{v}), 500 \mathrm{MHz}$
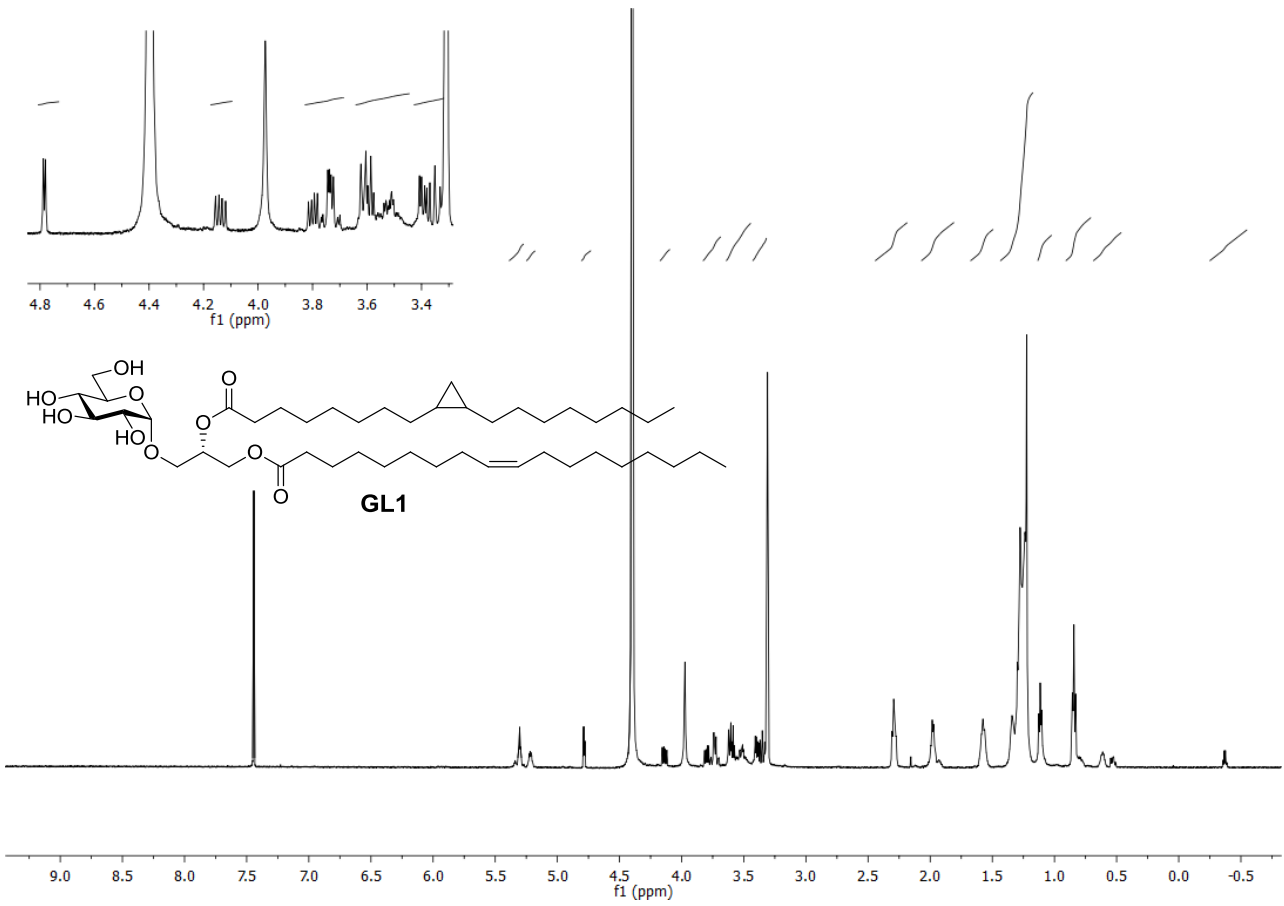

${ }^{13} \mathrm{C}_{\mathrm{NMR},} \mathrm{CD}_{3} \mathrm{OD} / \mathrm{CDCl}_{3}(1 / 2, \mathrm{v} / \mathrm{v}), 125 \mathrm{MHz}$
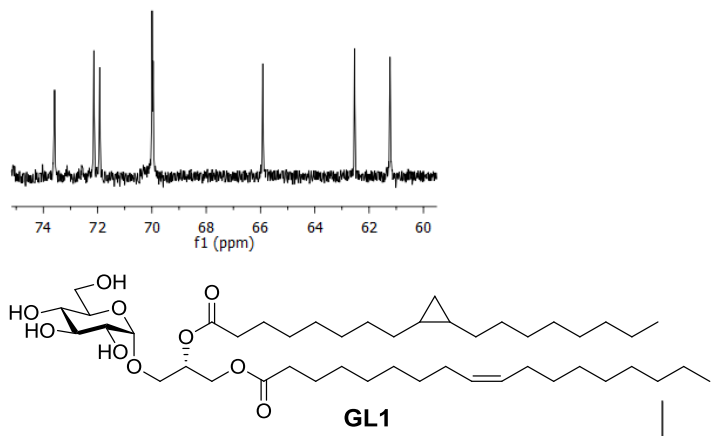

$\begin{array}{llllllllllllllllllllllllllll}130 & 220 & 210 & 200 & 190 & 180 & 170 & 160 & 150 & 140 & 130 & 120 & \begin{array}{l}110 \\ \mathrm{f} 1(\mathrm{ppm})\end{array} & 100 & 90 & 80 & 70 & 60 & 50 & 40 & 30 & 20 & 10 & 0 & -10\end{array}$ 
${ }^{1} \mathrm{H},{ }^{1} \mathrm{H} \operatorname{COSY}, \mathrm{CD}_{3} \mathrm{OD} / \mathrm{CDCl}_{3}(1 / 2, \mathrm{v} / \mathrm{v}), 500 \mathrm{MHz}$

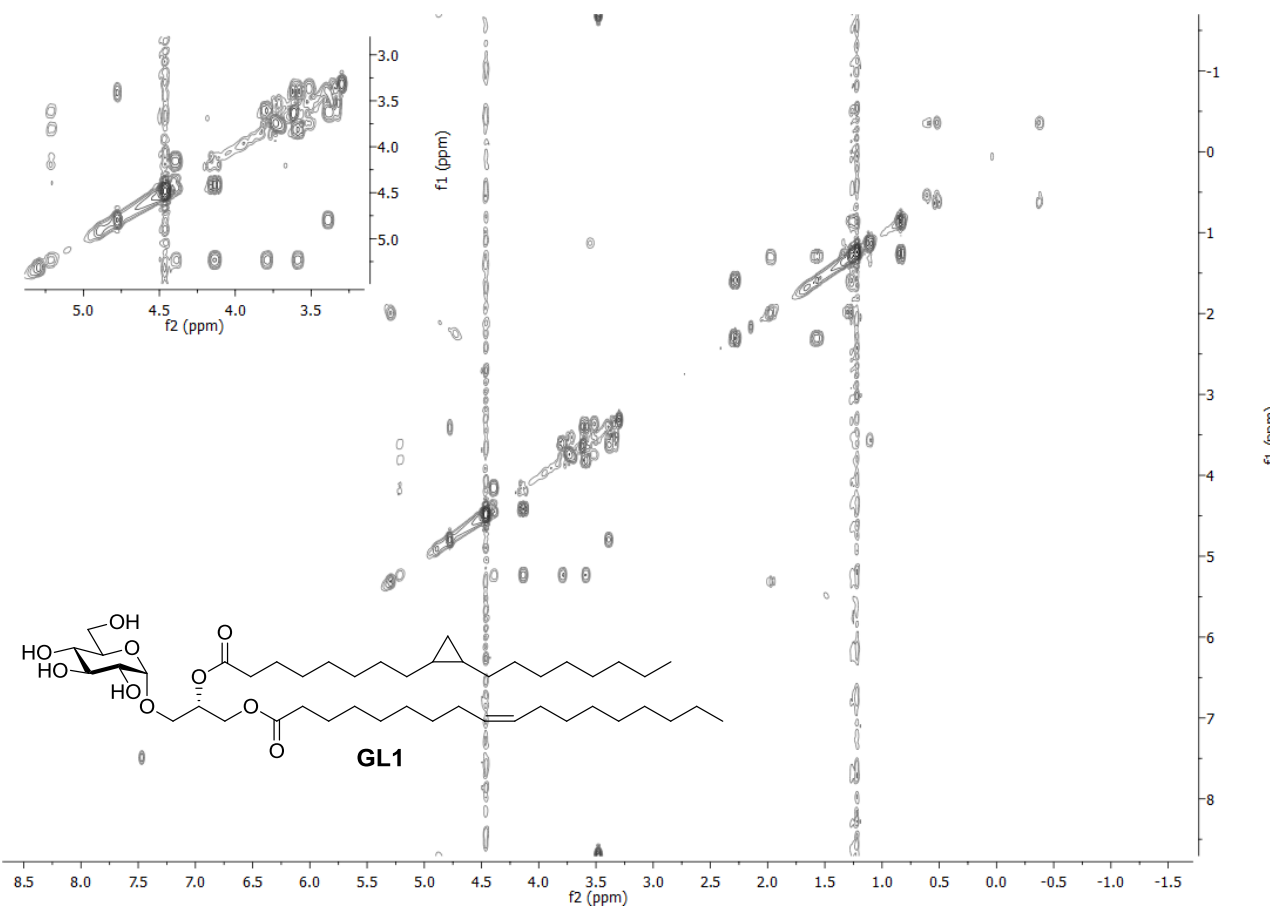

${ }^{1} \mathrm{H},{ }^{13} \mathrm{C}, \mathrm{HSQC}, \mathrm{CD}_{3} \mathrm{OD} / \mathrm{CDCl}_{3}(1 / 2, \mathrm{v} / \mathrm{v}), 500 \mathrm{MHz}$
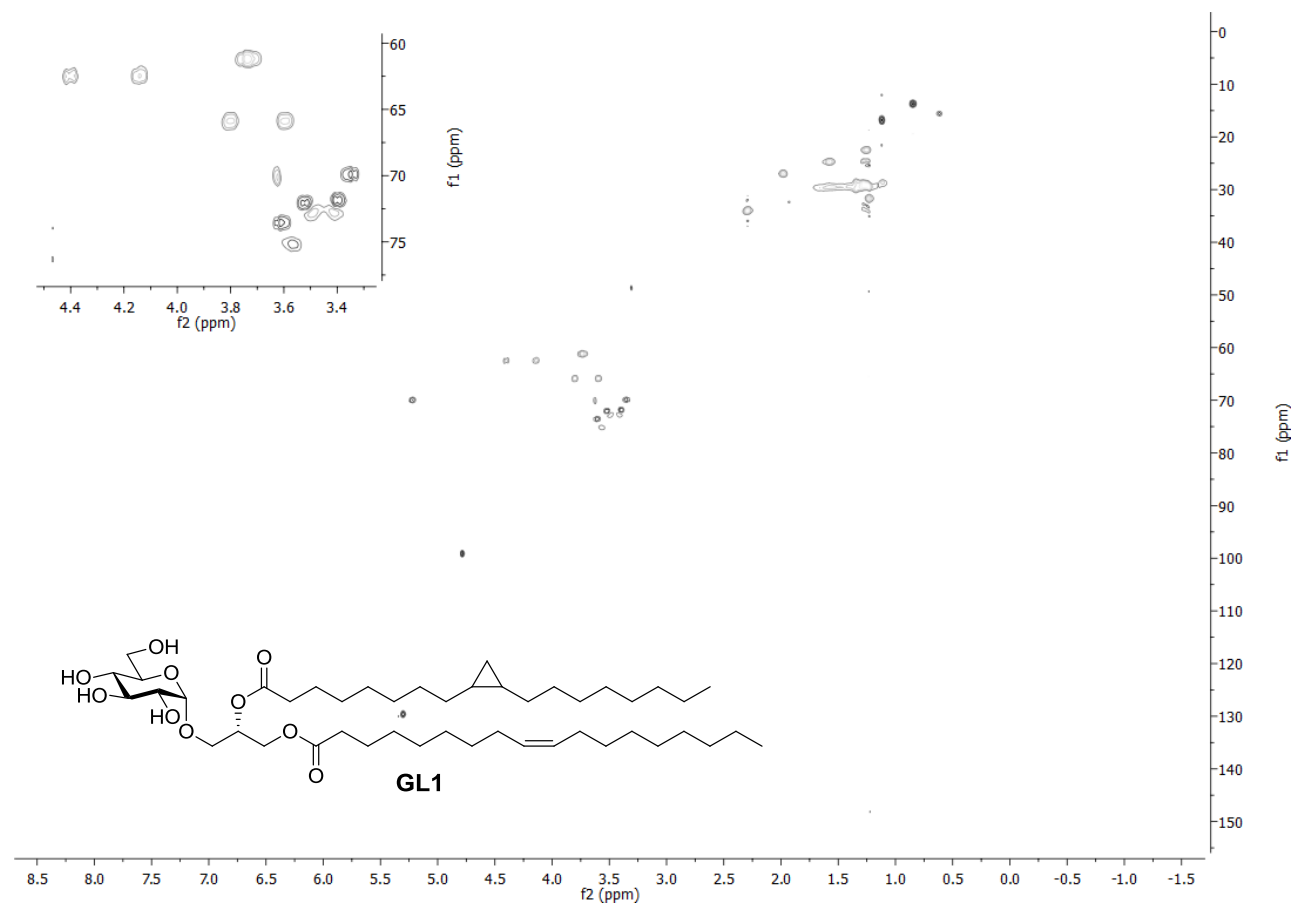
${ }^{1} \mathrm{H},{ }^{13} \mathrm{C}, \mathrm{HMBC}, \mathrm{CD}_{3} \mathrm{OD} / \mathrm{CDCl}_{3}(1 / 2, \mathrm{v} / \mathrm{v}), 500 \mathrm{MHz}$

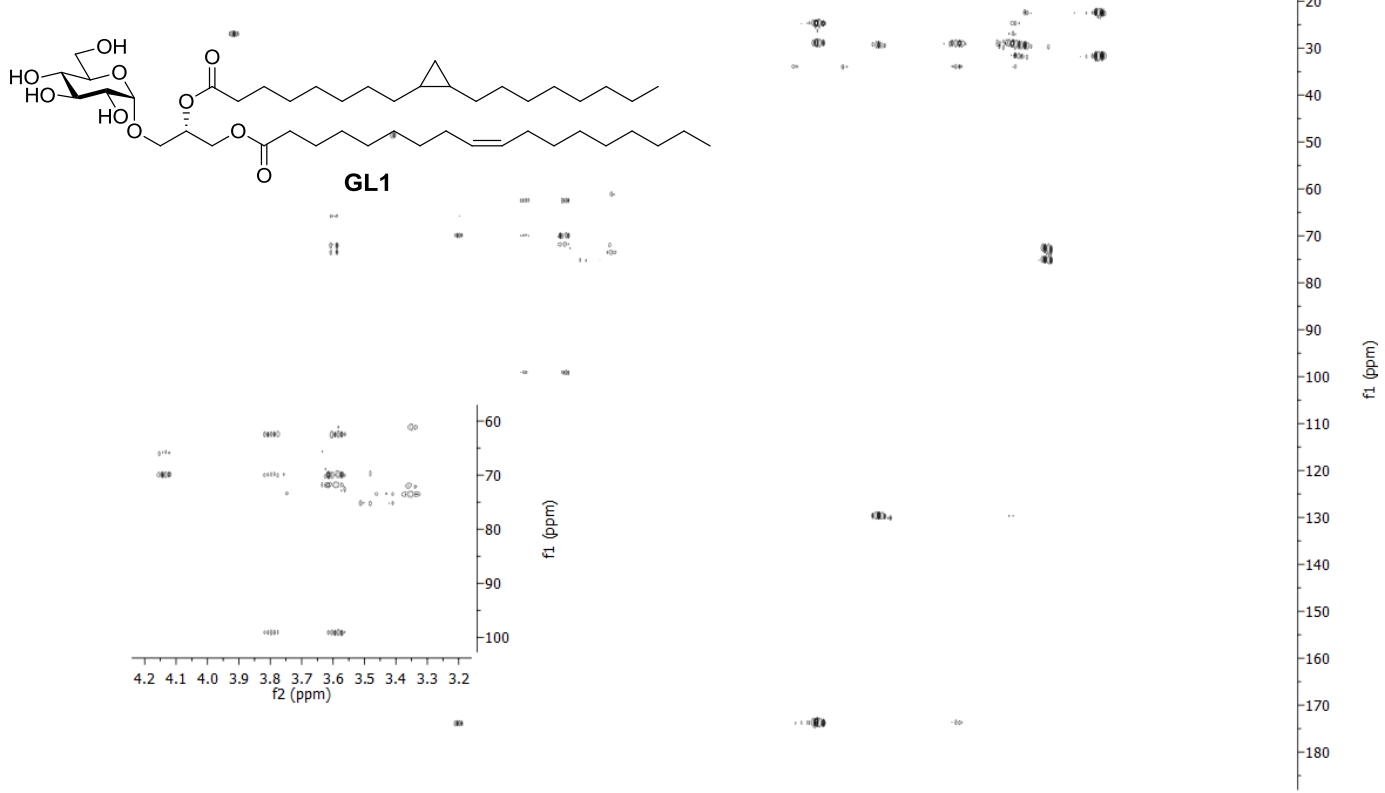

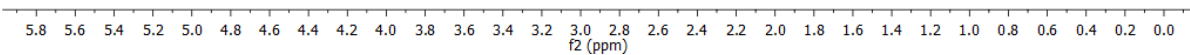




\subsubsection{GL2a}

ESI-MS, positive ion mode

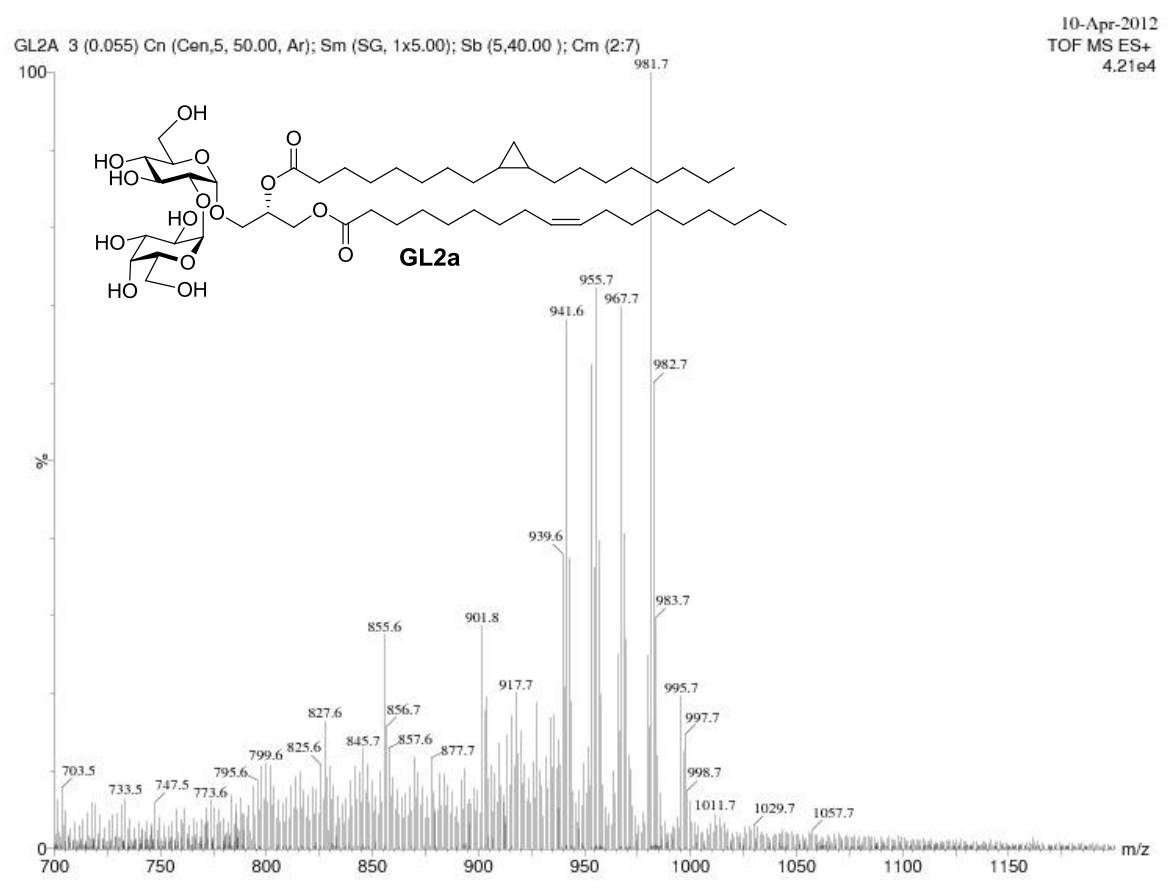

GL2a: Colourless oil $(0.7 \mathrm{mg})[\alpha]_{\mathrm{D}}{ }^{24}=-45.6\left(c=0.03, \mathrm{CHCl}_{3} / \mathrm{MeOH}, 2 / 1, \mathrm{v} / \mathrm{v}\right)$; IR (film) 3389, 2922, 2854, 1738, 1366, 1228, 1260, 1032, 632, $578 \mathrm{~cm}^{-1} ;{ }^{1} \mathrm{H},{ }^{13} \mathrm{C} \mathrm{NMR}$, HRMS and $\mathrm{R}_{f}$ data, see Table 2.1 and 2.3. 
GL2a, ${ }^{1} \mathrm{H}$ NMR, $\mathrm{CD}_{3} \mathrm{OD} / \mathrm{CDCl}_{3}(1 / 2, \mathrm{v} / \mathrm{v}), 600 \mathrm{MHz}$

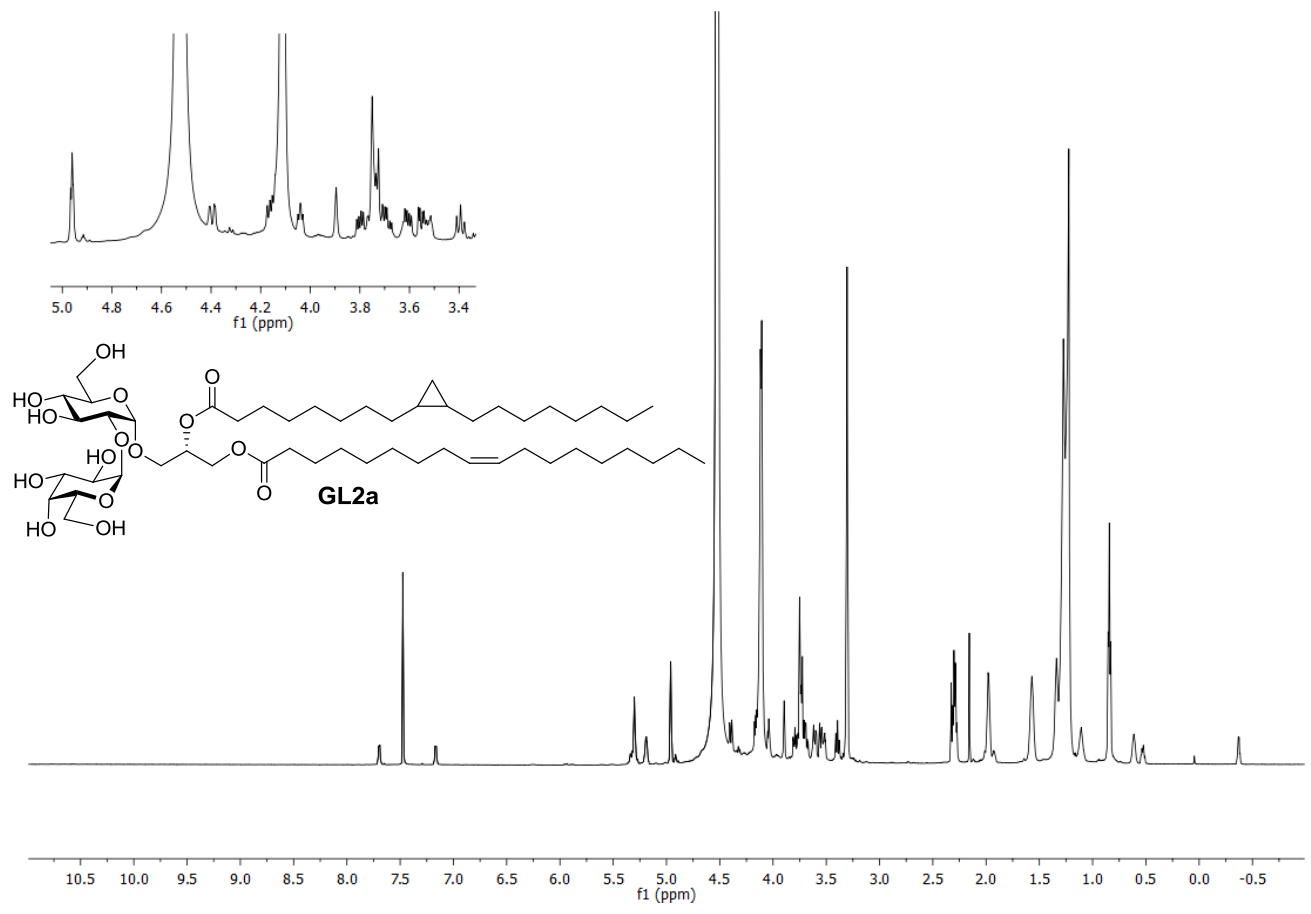

GL2a, ${ }^{13} \mathrm{C}$ NMR, $\mathrm{CD}_{3} \mathrm{OD} / \mathrm{CDCl}_{3}(1 / 2, \mathrm{v} / \mathrm{v}), 151 \mathrm{MHz}$
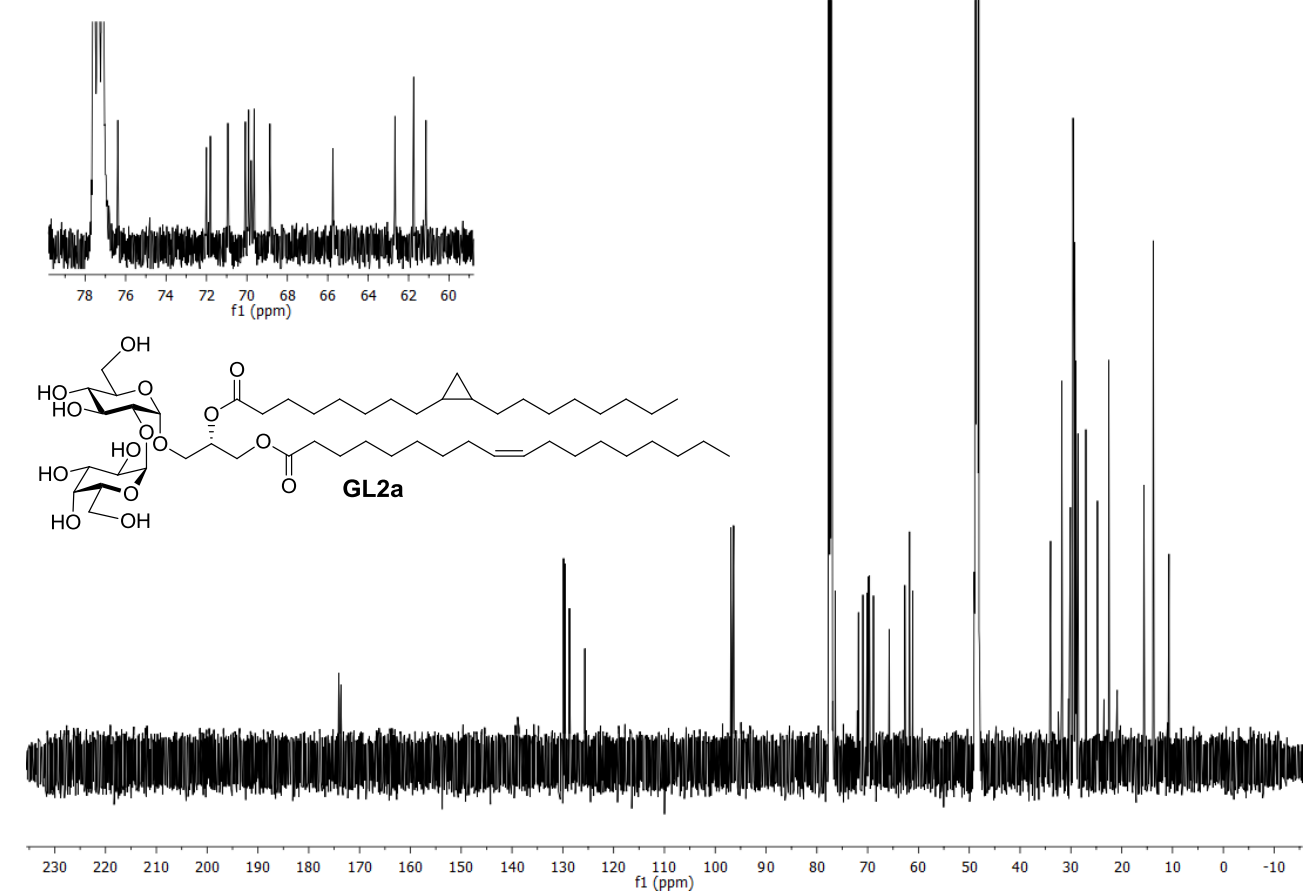
GL2a, ${ }^{1} \mathrm{H}^{1}{ }^{1} \mathrm{H} \mathrm{COSY}, \mathrm{CD}_{3} \mathrm{OD} / \mathrm{CDCl}_{3}(1 / 2, \mathrm{v} / \mathrm{v}), 600 \mathrm{MHz}$

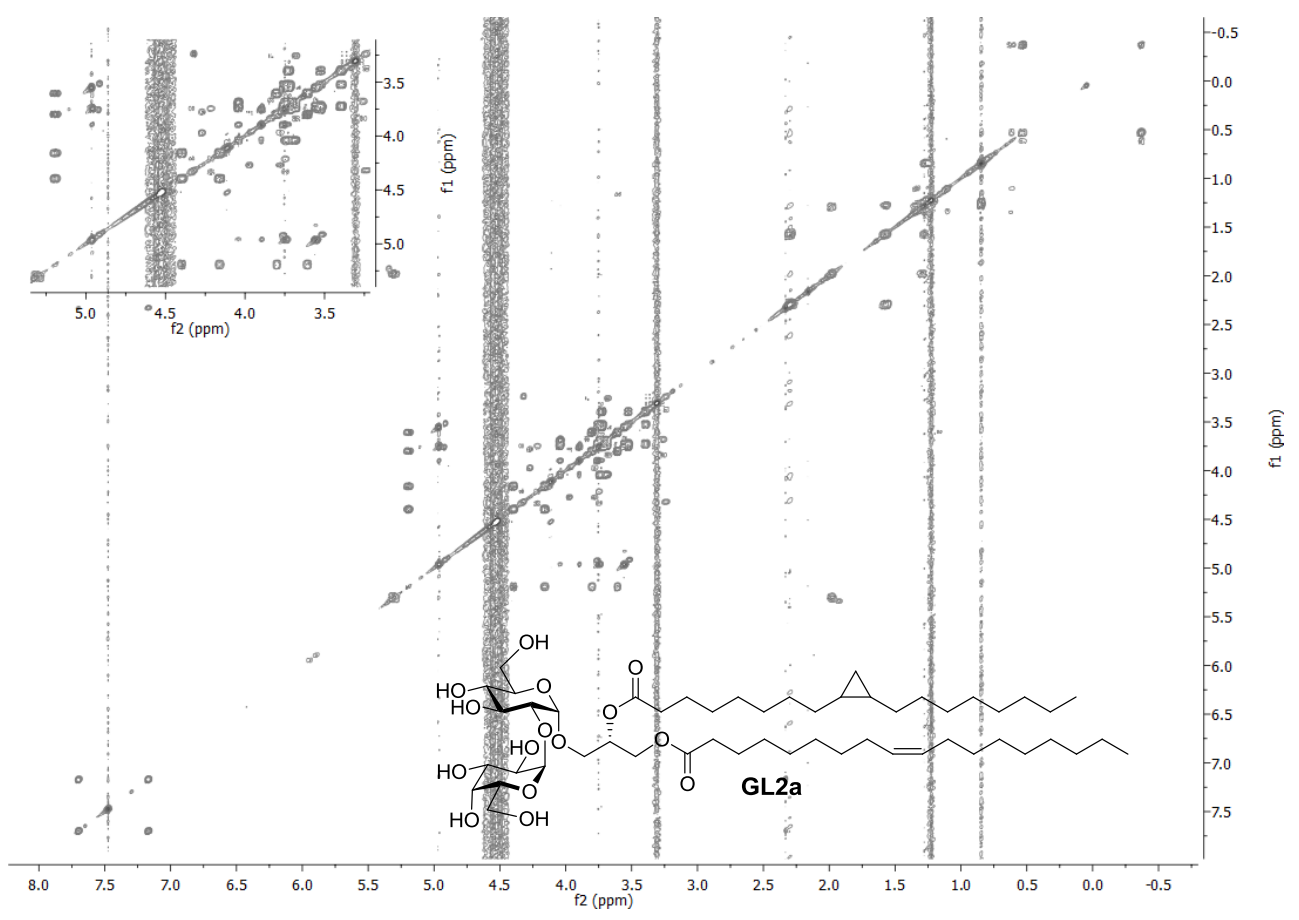

GL2a, ${ }^{1} \mathrm{H},{ }^{13} \mathrm{C}, \mathrm{HSQC}, \mathrm{CD}_{3} \mathrm{OD} / \mathrm{CDCl}_{3}(1 / 2, \mathrm{v} / \mathrm{v}), 600 \mathrm{MHz}$

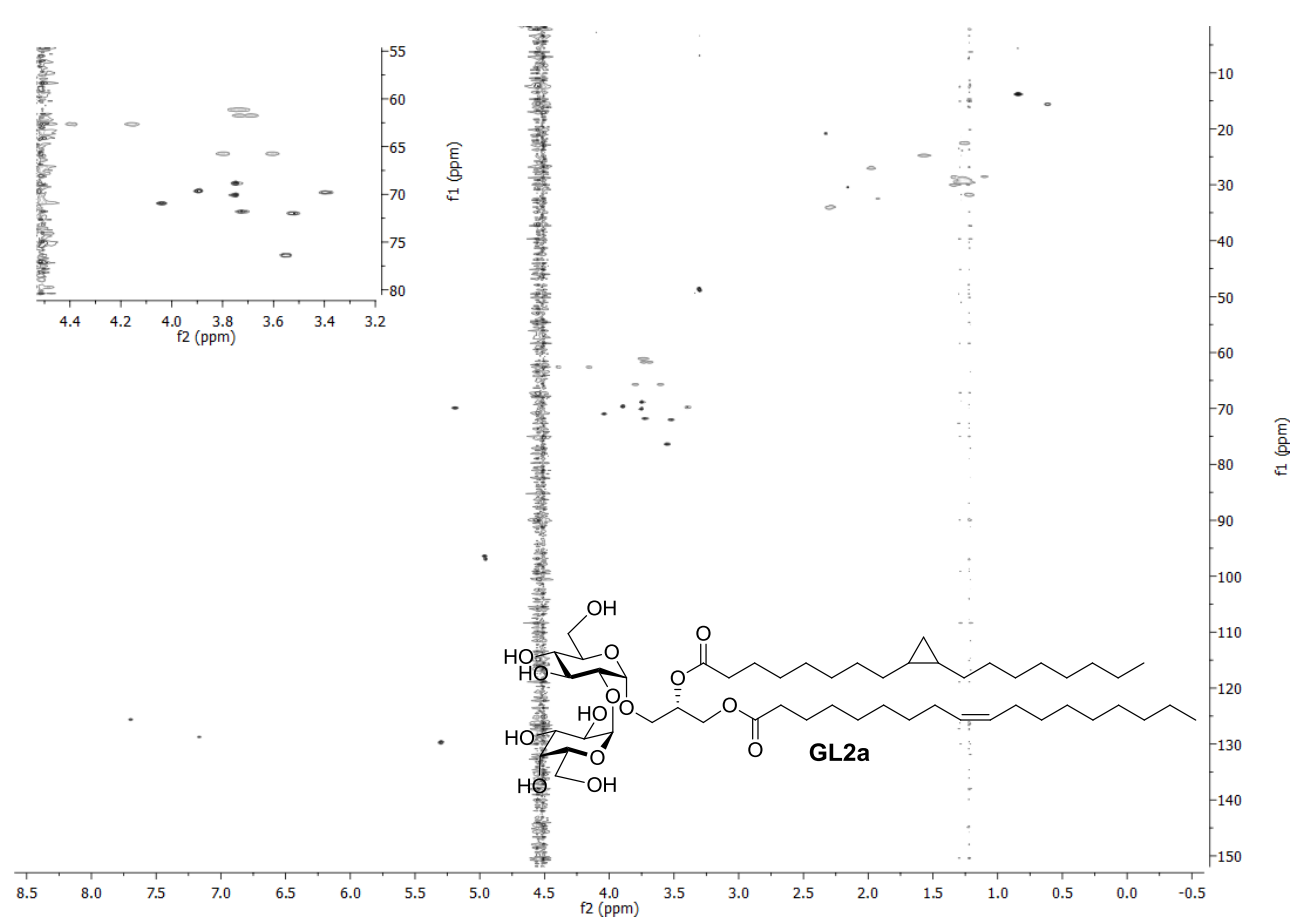


GL2a, ${ }^{1} \mathrm{H},{ }^{13} \mathrm{C}, \mathrm{HMBC}, \mathrm{CD}_{3} \mathrm{OD} / \mathrm{CDCl}_{3}(1 / 2, \mathrm{v} / \mathrm{v}), 600 \mathrm{MHz}$

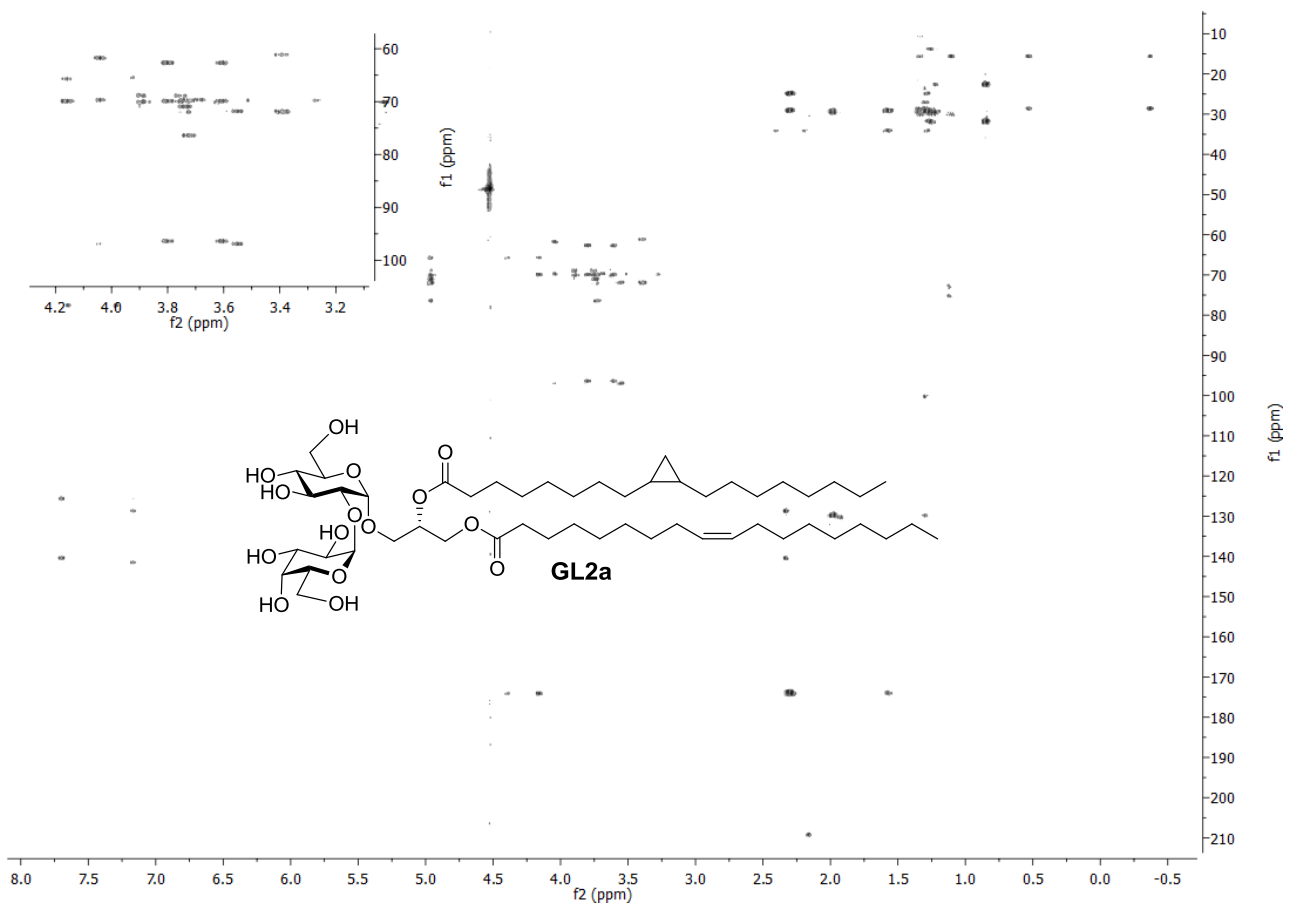

98 


\subsubsection{GL2b}

GL2b, ESI-MS, positive ion mode

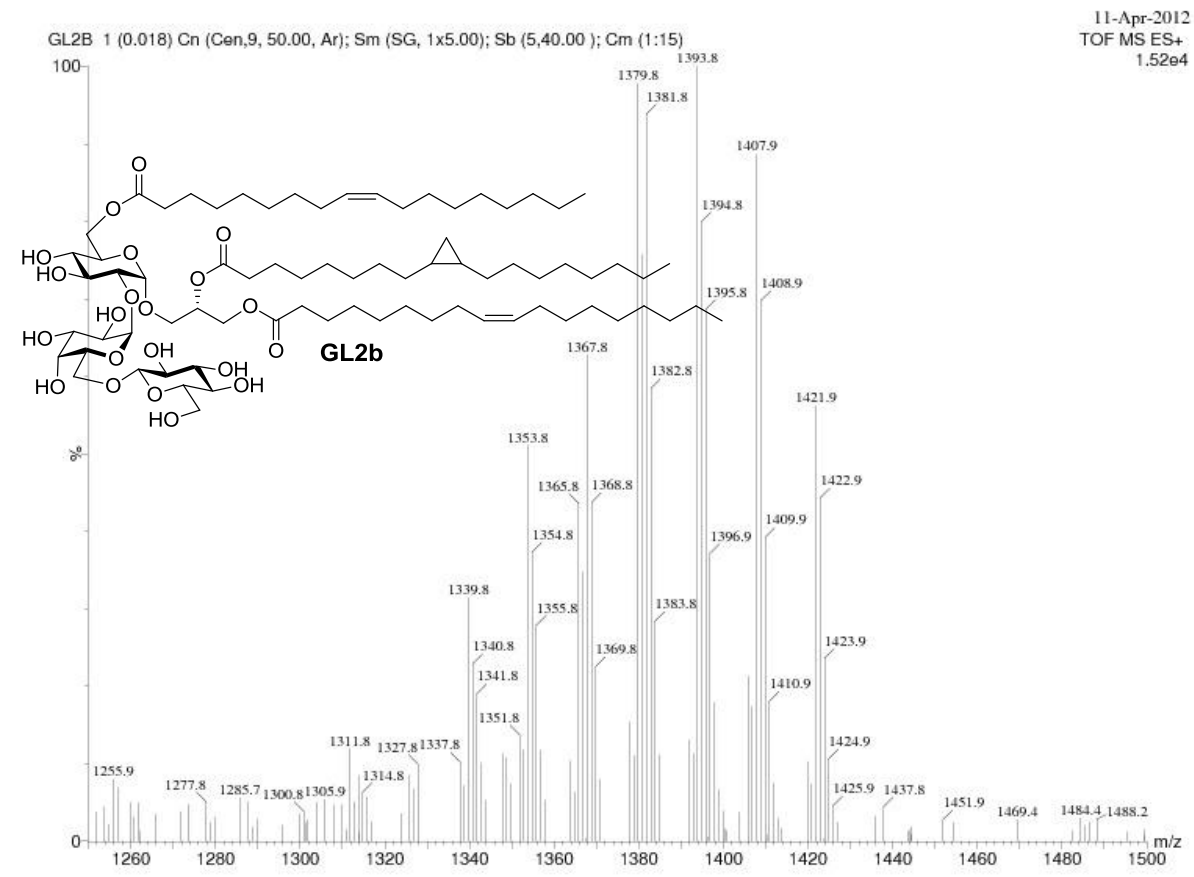

GL2b: Colourless oil $(0.9 \mathrm{mg})[\alpha]_{\mathrm{D}}^{20}=-31.3\left(c=0.15, \mathrm{CHCl}_{3} / \mathrm{MeOH}, 2 / 1, \mathrm{v} / \mathrm{v}\right)$; IR (film) 3382, 2922, 2851, 1738, 1366, 1216, 1054, 570, $569 \mathrm{~cm}^{-1} ;{ }^{1} \mathrm{H},{ }^{13} \mathrm{C}$ NMR, HRMS and $\mathrm{R}_{f}$ data, see Table 2.1and 2.4. 
GL2b, ${ }^{1} \mathrm{H}$ NMR, $\mathrm{CD}_{3} \mathrm{OD} / \mathrm{CDCl}_{3}(1 / 2, \mathrm{v} / \mathrm{v}), 600 \mathrm{MHz}$
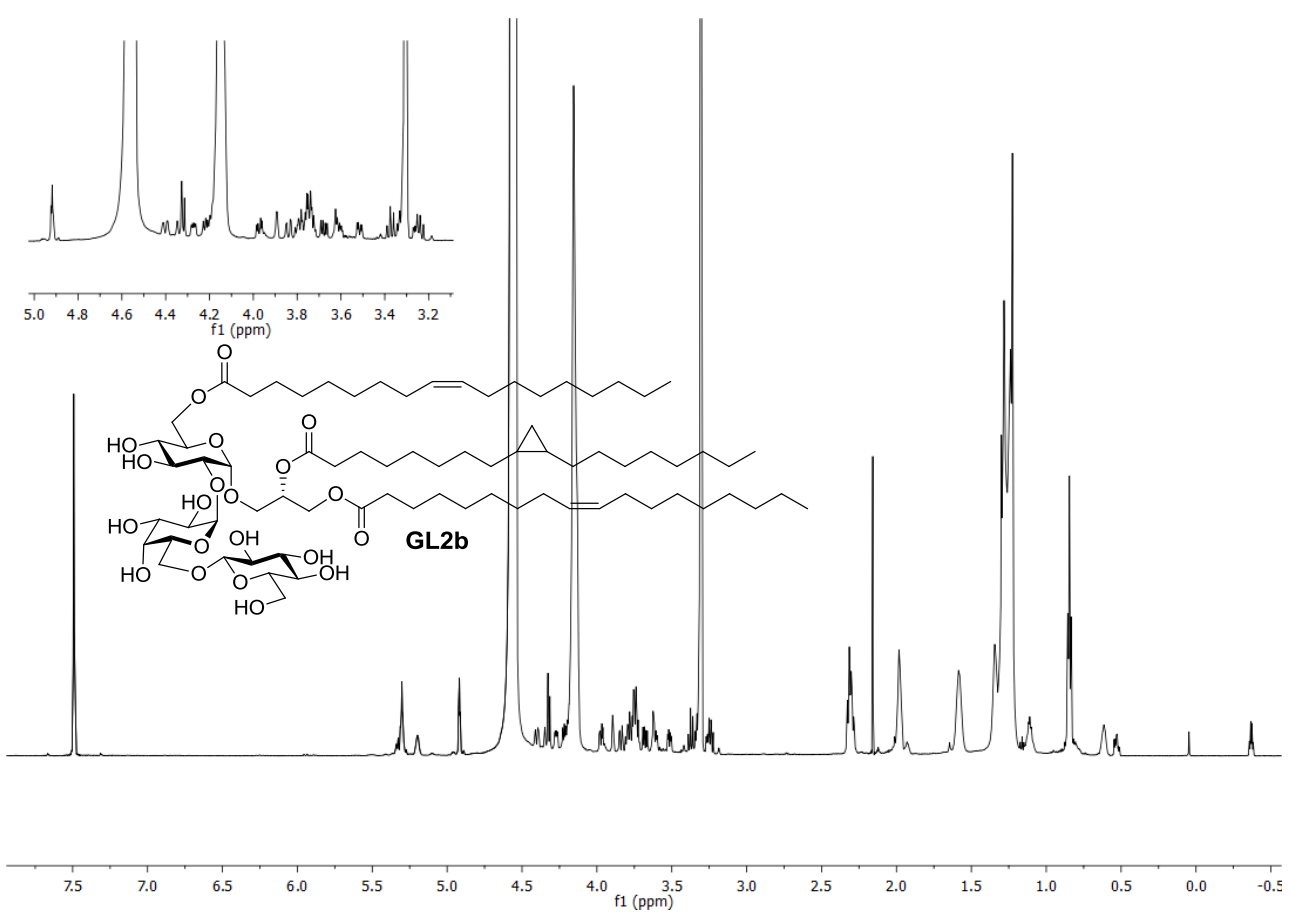

GL2b, ${ }^{13} \mathrm{C}$ NMR, $\mathrm{CD}_{3} \mathrm{OD} / \mathrm{CDCl}_{3}(1 / 2, \mathrm{v} / \mathrm{v}), 151 \mathrm{MHz}$

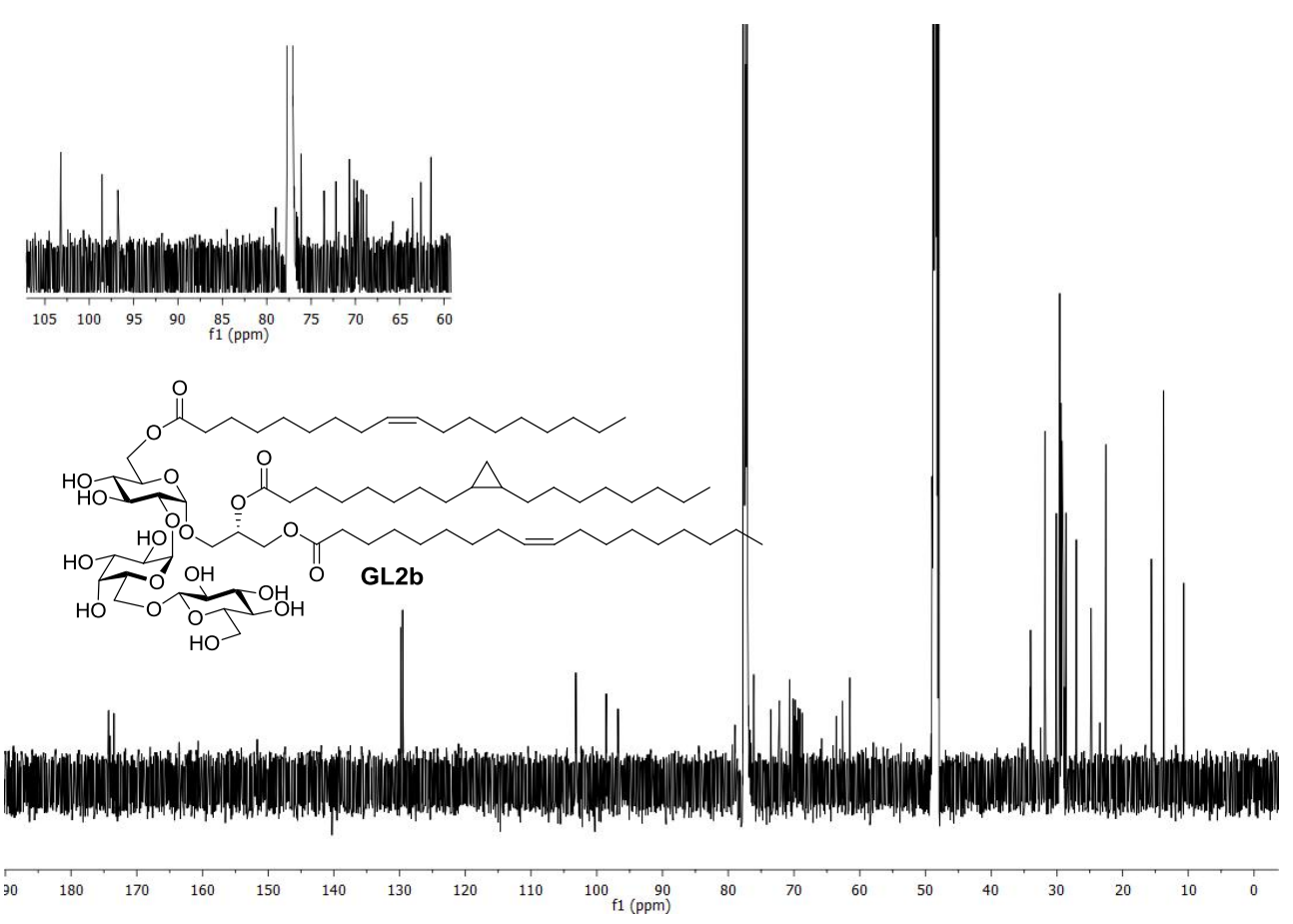


GL2b, ${ }^{1} \mathrm{H},{ }^{1} \mathrm{H}$ COSY, $\mathrm{CD}_{3} \mathrm{OD} / \mathrm{CDCl}_{3}(1 / 2, \mathrm{v} / \mathrm{v}), 600 \mathrm{MHz}$

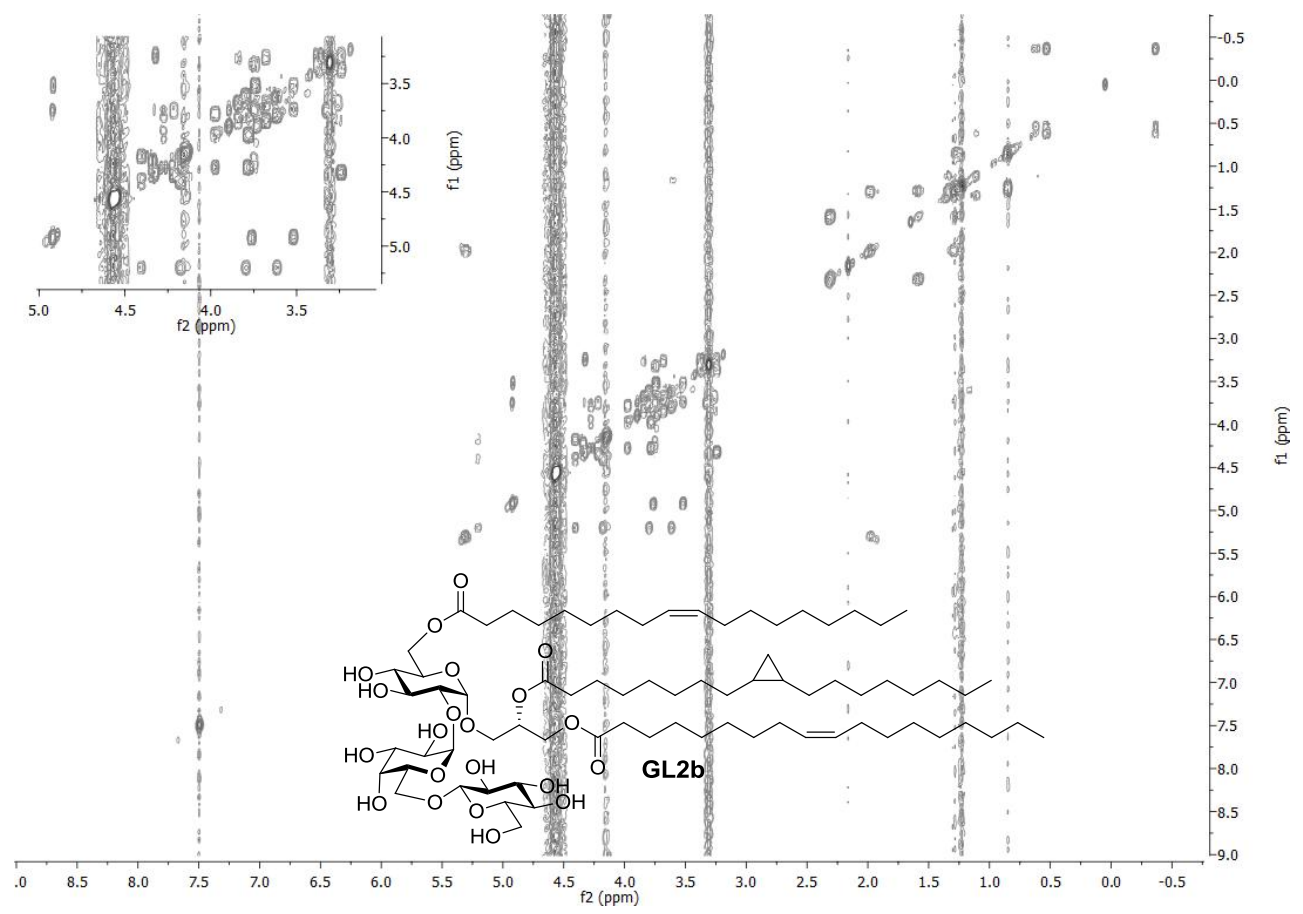

GL2b, ${ }^{1} \mathrm{H},{ }^{13} \mathrm{C}, \mathrm{HSQC}, \mathrm{CD}_{3} \mathrm{OD} / \mathrm{CDCl}_{3}(1 / 2, \mathrm{v} / \mathrm{v}), 600 \mathrm{MHz}$

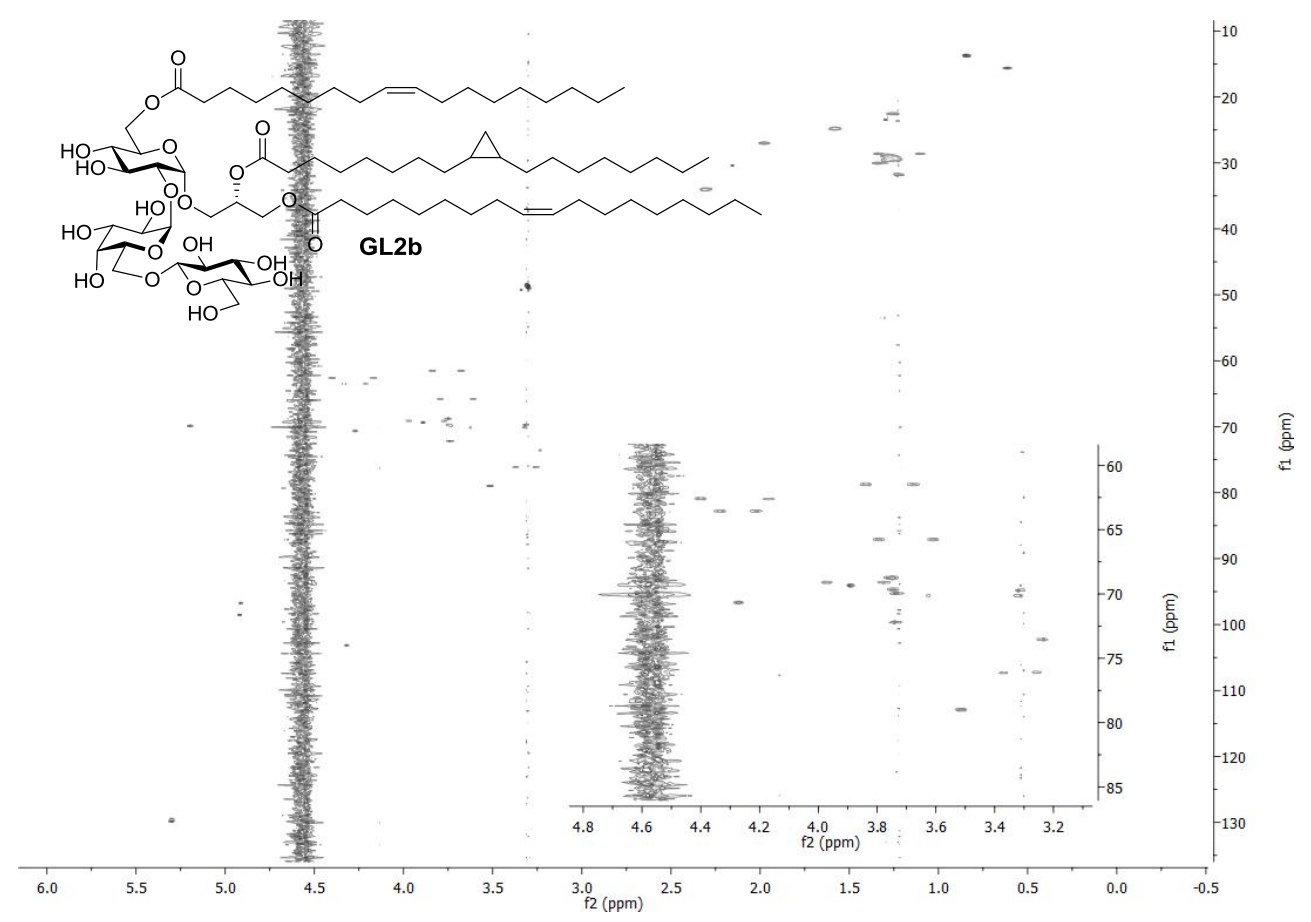


GL2b, ${ }^{1} \mathrm{H},{ }^{13} \mathrm{C}, \mathrm{HMBC}, \mathrm{CD}_{3} \mathrm{OD} / \mathrm{CDCl}_{3}(1 / 2, \mathrm{v} / \mathrm{v}), 600 \mathrm{MHz}$

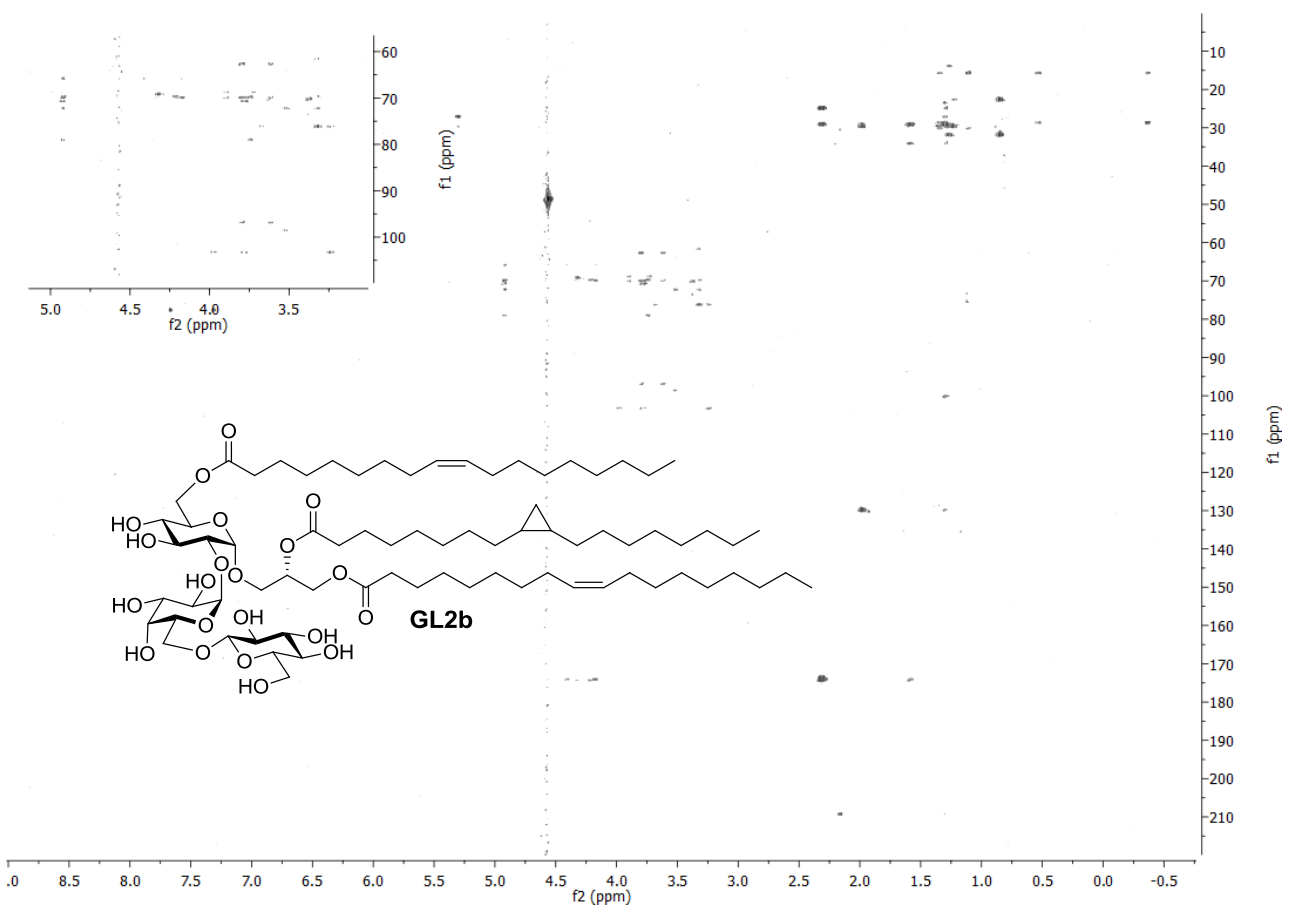




\subsubsection{GL3}

GL3, ESI-MS, positive ion mode

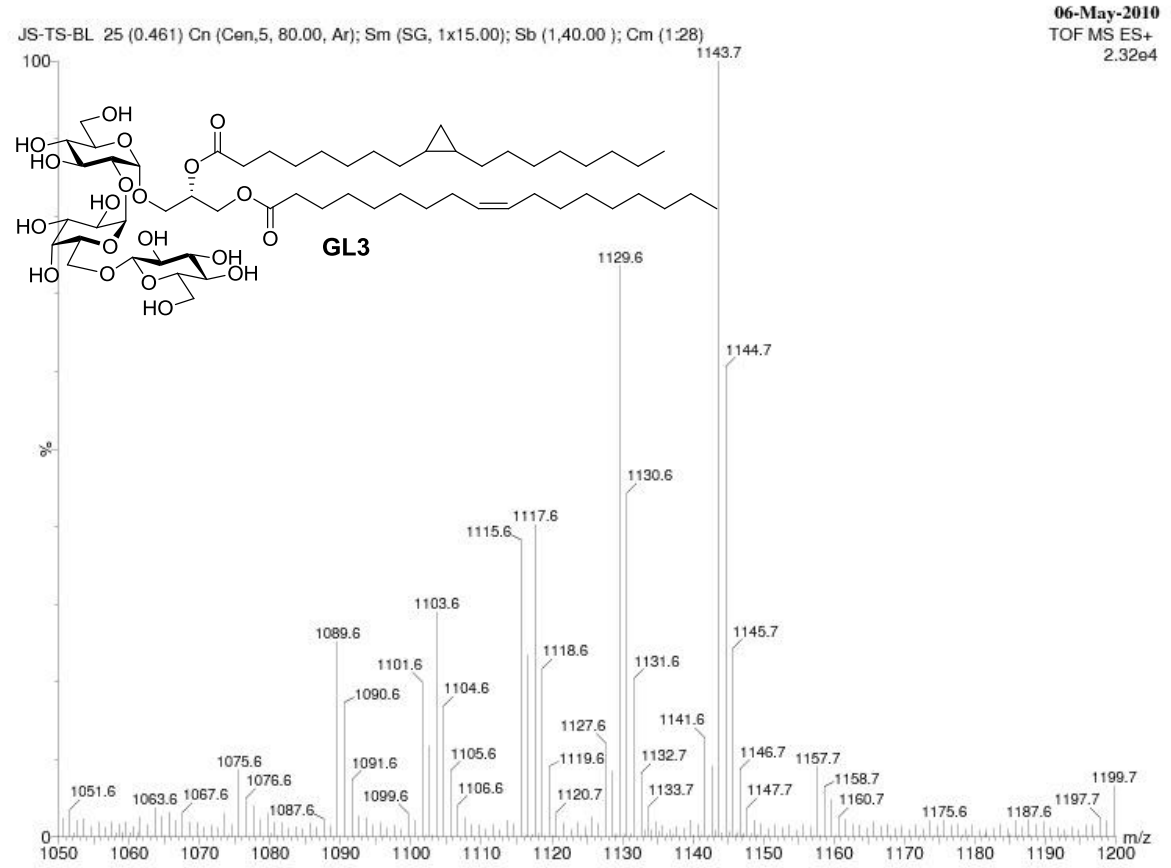

GL3: Colourless oil $(0.6 \mathrm{mg})[\alpha]_{\mathrm{D}}{ }^{25}=-59.3\left(c=0.03, \mathrm{CHCl}_{3} / \mathrm{MeOH}, 2 / 1, \mathrm{v} / \mathrm{v}\right)$; IR (film) $3409,2923,2853,1738,1456,1366,1245,1216,1154,632,569 \mathrm{~cm}^{-1} ;{ }^{1} \mathrm{H},{ }^{13} \mathrm{C}$ NMR, HRMS and $\mathrm{R}_{f}$ data, see Table 2.1 and 2.5. 
$\mathrm{GL}^{1}{ }^{1} \mathrm{H} \mathrm{NMR}, \mathrm{CD}_{3} \mathrm{OD} / \mathrm{CDCl}_{3}(1 / 2, \mathrm{v} / \mathrm{v}), 600 \mathrm{MHz}$

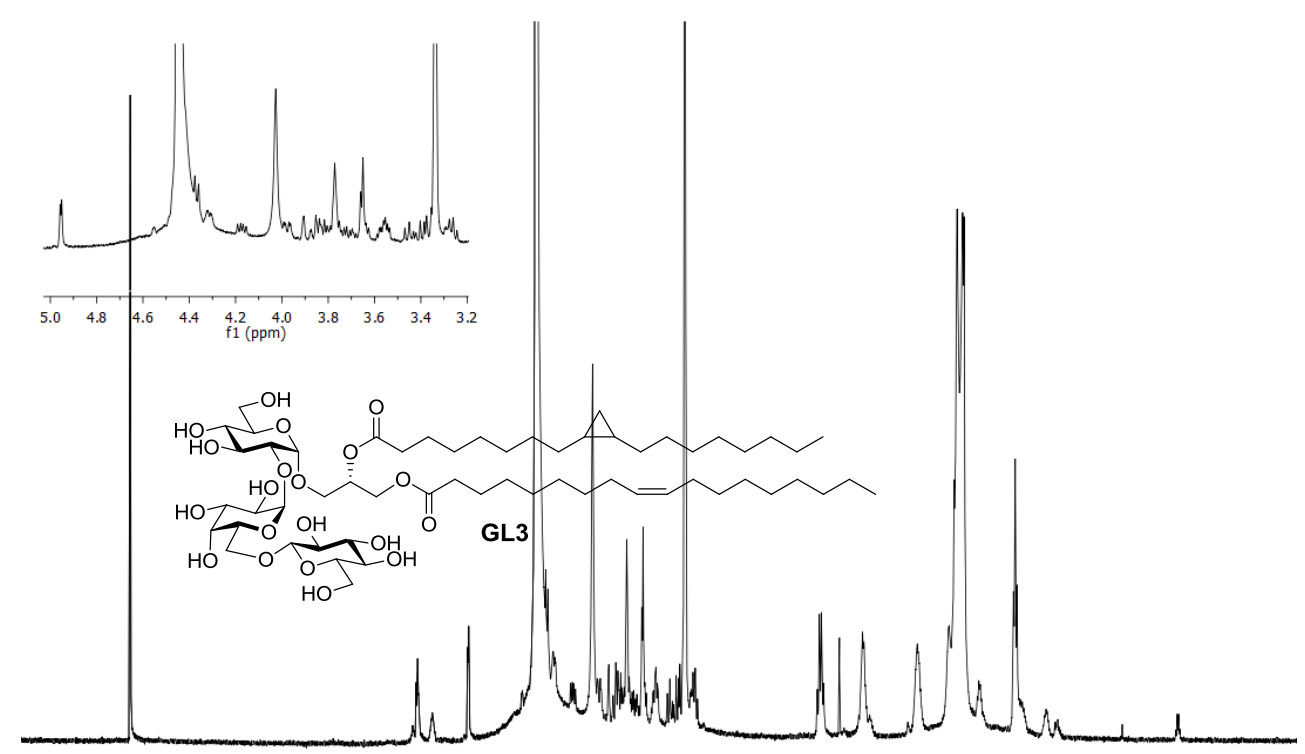

GL3, ${ }^{13} \mathrm{C}$ NMR, $\mathrm{CD}_{3} \mathrm{OD} / \mathrm{CDCl}_{3}(1 / 2, \mathrm{v} / \mathrm{v}), 151 \mathrm{MHz}$

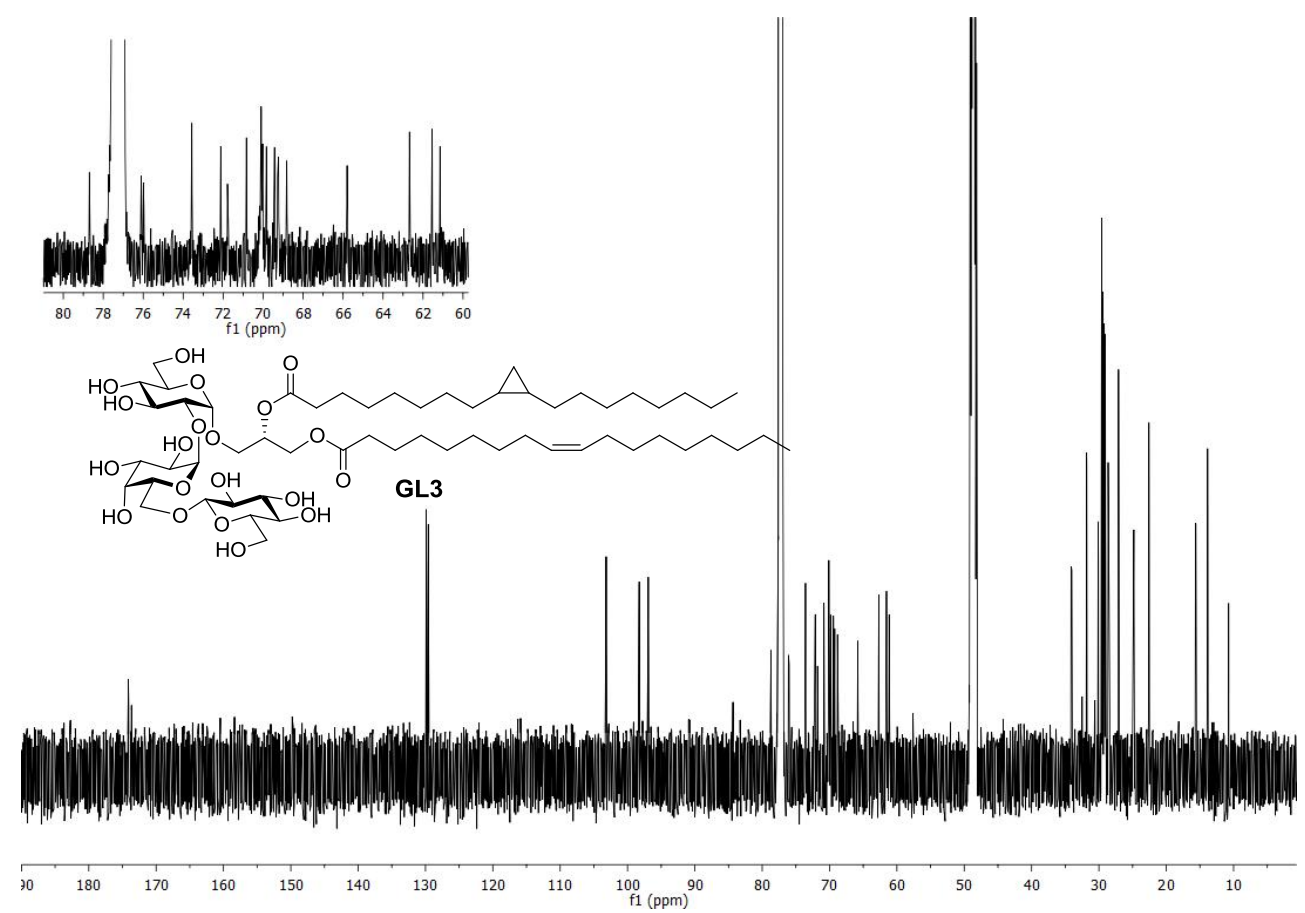


GL3, ${ }^{1} \mathrm{H},{ }^{1} \mathrm{H}$ COSY, $\mathrm{CD}_{3} \mathrm{OD} / \mathrm{CDCl}_{3}(1 / 2, \mathrm{v} / \mathrm{v}), 600 \mathrm{MHz}$

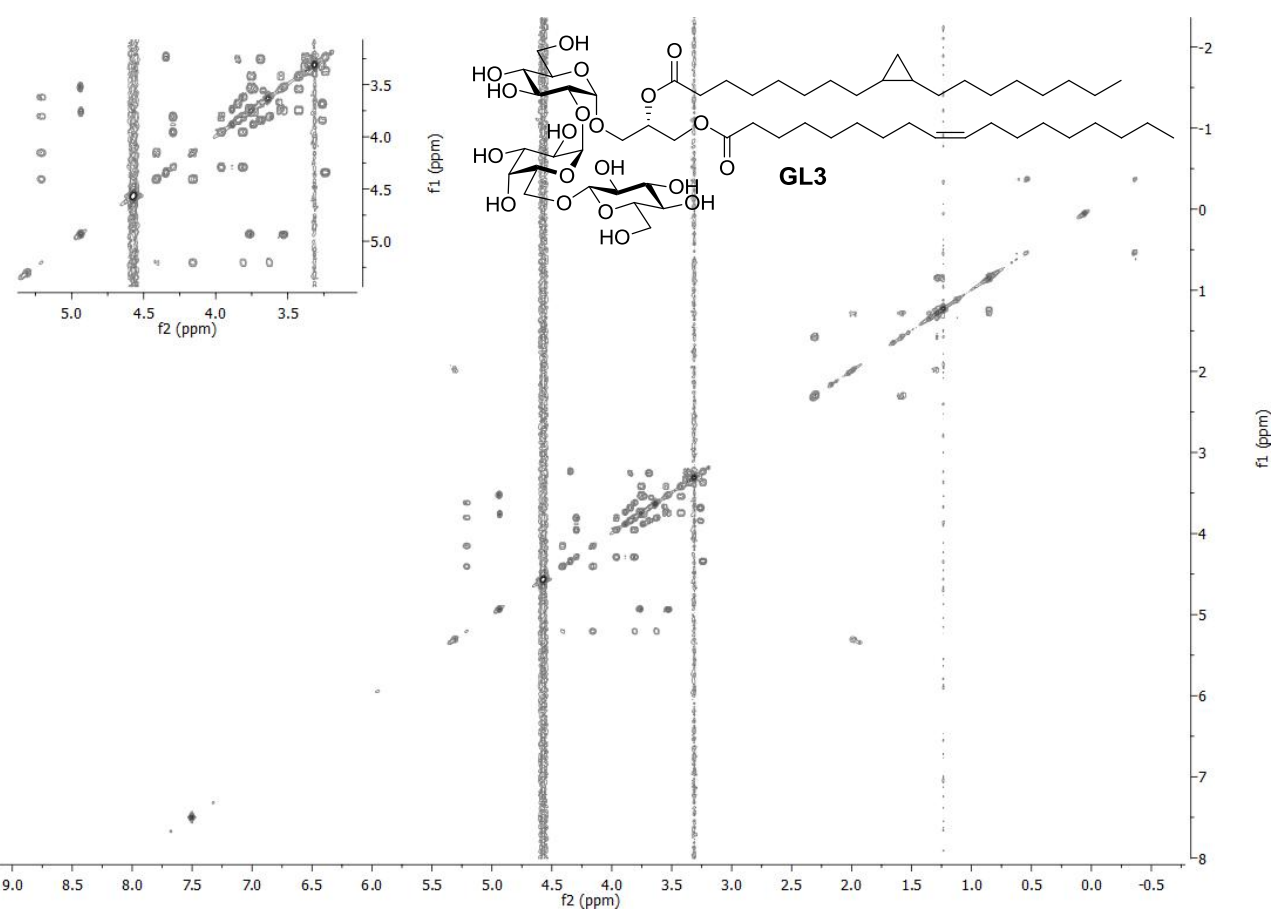

$\mathrm{GL} 3,{ }^{1} \mathrm{H},{ }^{13} \mathrm{C}, \mathrm{HSQC}, \mathrm{CD}_{3} \mathrm{OD} / \mathrm{CDCl}_{3}(1 / 2, \mathrm{v} / \mathrm{v}), 600 \mathrm{MHz}$

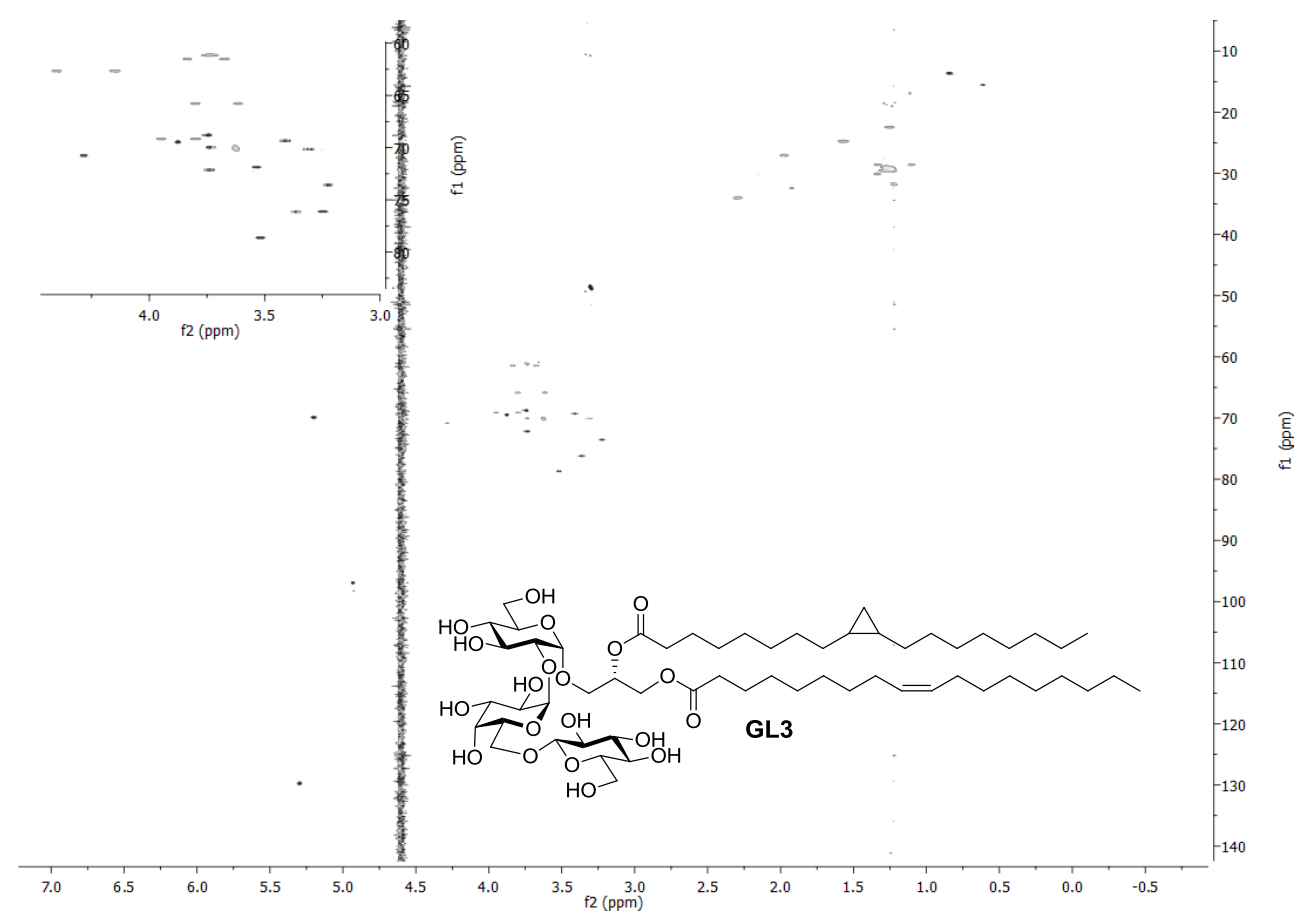


$\mathrm{GL} 3,{ }^{1} \mathrm{H},{ }^{13} \mathrm{C}, \mathrm{HMBC}, \mathrm{CD}_{3} \mathrm{OD} / \mathrm{CDCl}_{3}(1 / 2, \mathrm{v} / \mathrm{v}), 600 \mathrm{MHz}$

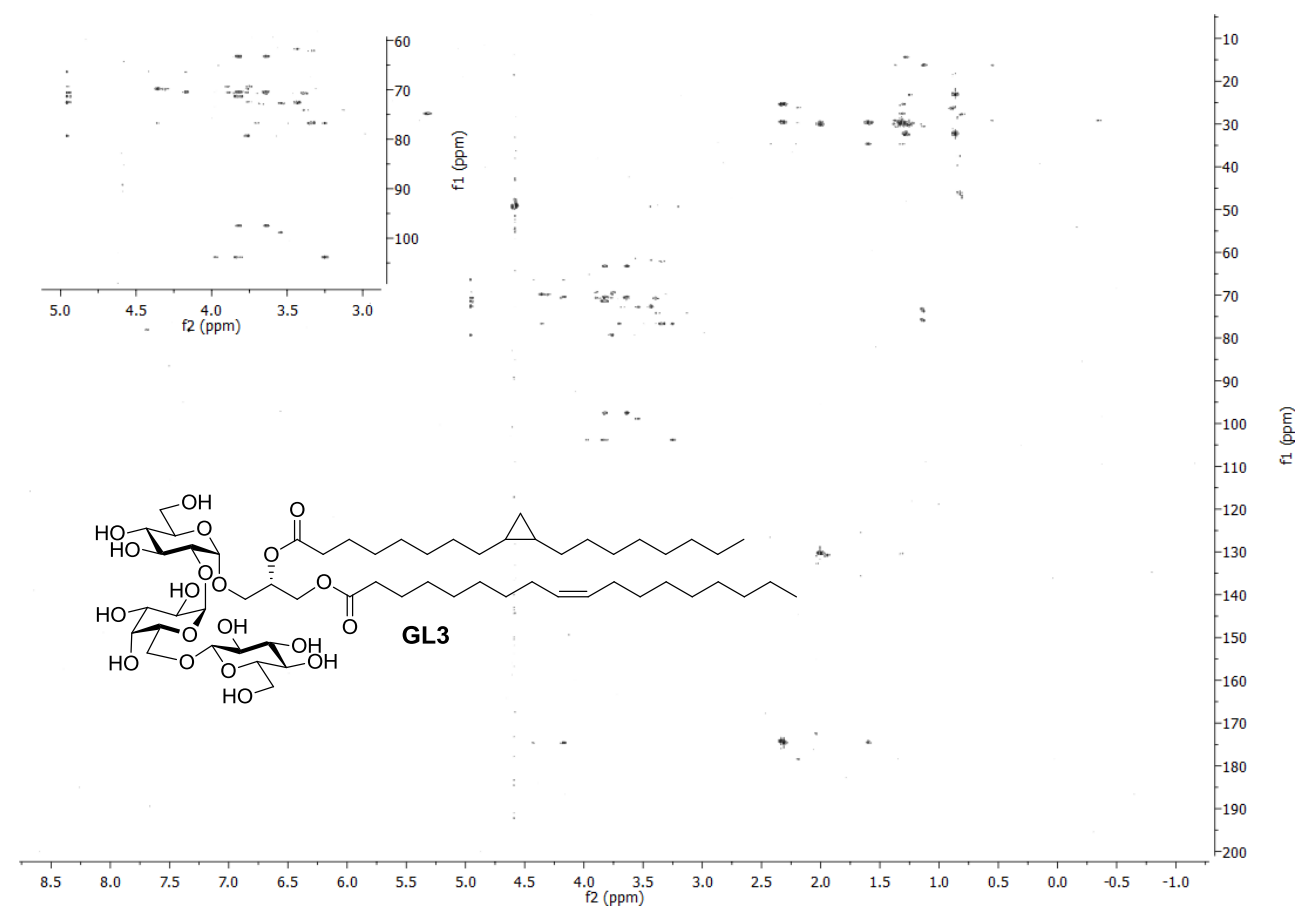




\section{Chapter 3}

\section{Discovery of a Novel Glycolipid from $B$. longum subs. infantis (ATCC 15702T)}

Bifidobacteria are dominant members of the microbial community in the intestinal tract. Studies have shown that glycolipids extracted from the cell surface of these bacteria elicit immune responses and as bifidobacteria are beneficial, the characterization of these compounds is of interest. Despite this, the chemical nature of such immunomodulatory compounds has not been well documented. Herein, is presented for the first time the isolation and full structural characterization of the glycolipids from Bifidobacterium longum, including a novel glycolipid structure comprising an unprecedented mixed acetal glycolipid and a rapid MALDI method is reported to analyze the whole-cell mass glycolipid spectra. 


\subsection{Background}

Bifidobacteria, dominant members of the microbial community in the intestinal tract of infants, are thought to be important in developing a healthy immune system. In adults, the presence of bifidobacteria is associated with reduced incidence of bowel cancer, is thought to alleviate the irritable bowel syndrome symptoms, and is believed to help reduce atopic disease. ${ }^{98,229}$ For this reason, Bifidobacterium longum has been used as probiotic in order to improve the consumers health. ${ }^{230}$

The chemical basis of these health benefits is not fully understood, but evidence suggests that it is, in part, due to the modification of the immune responses. As discussed in Chapter 1, the bifidobacterial cell wall consists of glycolipids, a peptidoglycan layer, lipoteichoic acids, wall teichoic acids, cell wall polysaccharides and exopolysaccharides. Although the immunomodulatory effects of glycolipids present in other bacterial cell walls are well understood, there is little published literature on the composition and structure of bifidobacterial glycolipids. ${ }^{231-233}$ Veerkamp have identified the presence of mono-, di- and tri-galactosyldiglyceride derivatives as the major constituents of B. bifidum var. pennsylvanicus. ${ }^{44}$ More recently, Novik et al., ${ }^{104,134,137,138}$ analyzed the principal glycolipids from a number of bifidobacterial species and strains by thin layer chromatography (TLC) and found an increased anti-glycolipid antibody response against glycolipid fractions. However, in both studies, only limited detail pertaining to glycolipid structure was presented but as seen in Chapter 1, the immunological properties of glycolipids seem to be related to their chain lengths and thus structural characterization would give important insights for future structure activity relationship studies. A method, such as Matrix-assisted laser desorption/ionization (MALDI) can thus be used to rapidly screen the structures of these glycolipids. ${ }^{234-237}$

The screening of bacterial samples using MALDI for lipid content is now a common occurrence in lipidomics. ${ }^{238-240}$ This technique consists of extracting the lipids or glycolipids and analyzing the crude mixture using MALDI. ${ }^{238}$ A faster and simpler method involves MALDI analysis of whole bacterial cells. ${ }^{241-245}$ Here, whole bacterial 
cells are crystallized with a matrix and subjected to the MALDI laser beam. MALDI analysis on whole cells has been used mostly for taxonomy purposes, while the specific analysis of glycolipids using this technique has not been much utilized. Calvano et al. analyzed whole Lactobacillus plantarum cells by MALDI and observed masses corresponding to glycolipids including diglycosyldiglycerides (DGDGs) and triglycosyldiglycerides (TGDGs), and other polar lipids. ${ }^{184}$ Shu et al. also attributed specific masses as DGDG in Bacillus subtilis using whole cell MALDI analysis. ${ }^{240}$ Glycolipids were attributed putatively and further structural analysis using NMR, ESIMS, sugar analysis and fatty acid analysis is required.

There is growing interest in the beneficial effects of bifidobacteria as probiotics, however there is a lack of information on the chemical composition of the bifidobacterial cell wall. In this chapter, the glycolipids of bifidobacteria that have been shown to induce differential immune response in immune assays in vitro are analyzed. ${ }^{104}$ Accordingly, the objective of this work was to highlight the structures of the key glycolipids found in a human biotype of B. longum subs. infantis (ATCC $15702 \mathrm{~T}$ ), and for the first time, provide full structural characterization of these glycolipids. This is a first step in gaining a better understanding of the structure/activity relationship providing the beneficial effects of bifidobacteria.

\subsection{Experimental Procedures}

For details on the experimental procedures see Chapter 2.

\subsubsection{Bacteria and Cultivation}

\section{[The bacterial growth was conducted by Jason Ryan, IRL]}

B. longum subs. infantis (ATCC 15702L) was obtained from the American Type Culture Collection (ATCC, Manassas, USA) and B. longum subs. longum (DSM 20219) was obtained from the German Collection of Microorganism and Cell Cultures (DSM, 
Braunschweig, Germany). The B. longum cultures were grown plated onto Refined Clostridium media (RCM) agar and incubated anaerobically at $37{ }^{\circ} \mathrm{C}$ for $24 \mathrm{~h}$. Glycolipid production was conducted anaerobically at $37{ }^{\circ} \mathrm{C}$ for $24 \mathrm{~h}$. Glycolipid production was conducted anaerobically in a $18 \mathrm{~L}$ fermenter using a Carbohydrate-free Man-Rogosa-Sharp (MRS) media in a 20 L Braun bioreactors (Sartorius-Stedim, Aubagne Cedex, France). The culture was then centrifuged at $3000 \mathrm{~g}$ for $10 \mathrm{~min}$ to yield a watery paste and the cell-mass was freeze-dried. Next, the dried cell-mass was washed three times with PBS buffer (pH 7.3) and centrifuged (15 min, $\left.31186 \mathrm{~g}, 4^{\circ} \mathrm{C}\right)$ or until the amount of carbohydrate detected in the washes by colorimetric assay was under $1 \%$ $(w / v)$. To remove the PBS buffer, the cells were washed with deionised water and centrifuged, then freeze-dried and weighed.

\subsubsection{MALDI}

Whole washed bacterial cells $(10 \mathrm{mg})$ were suspended in a mixture of $\mathrm{CHCl}_{3} / \mathrm{MeOH}$ $(1 / 1, \mathrm{v} / \mathrm{v}, 1 \mathrm{~mL})$ or MiliQ water $(1 \mathrm{~mL})$. The cell suspension $(1 \mu \mathrm{L})$ and MALDI matrix $(1 / 1, v / v)$ was deposited using the dry droplet method onto a stainless steel plate in sextuplicate. Reproducible spectra were obtained using a solution of 2,5dihydroxybenzoic acid (DHB) $(30 \mathrm{mg} / \mathrm{mL})$ in $\mathrm{CH}_{3} \mathrm{CN} / \mathrm{H}_{2} \mathrm{O}(7 / 3$, v/v) as a matrix. The MS/MS experiments were conducted on a AB SCIEX TOF/TOFTM 5800 system operating in reflectron positive ion mode. Mass calibration was performed externally using a peptide mixture containing des-arg ${ }^{1}$-bradykinin, angiotensin 1 , neurotensin and glu ${ }^{1}$-fibrinopeptide B.

\subsubsection{Thin layer chromatography (TLC)}

Bifidobacterial glycolipids samples were dissolved in $\mathrm{CHCl}_{3} / \mathrm{MeOH}(2 / 1$, v/v) and applied to dried silica pre-coated TLC plates. The bifidobacterial glycolipids were eluted with $\mathrm{CHCl}_{3} / \mathrm{MeOH}(6 / 1, \mathrm{v} / \mathrm{v})$, sprayed and developed with a cerium molybdate (Hanessian's) stain and molybdate stain to detect phosphorous.$^{206,207}$ 


\subsubsection{Extraction and purification of glycolipids}

Bifidobacterial dried cell-mass (6 g) was extracted twice $\left(400 \mathrm{~mL}, 18 \mathrm{~h}, 37^{\circ} \mathrm{C}\right)$ with $\mathrm{CHCl}_{3} / \mathrm{MeOH} / \mathrm{H}_{2} \mathrm{O}(10 / 5 / 1, \mathrm{v} / \mathrm{v} / \mathrm{v})$. After filtration, the combined extracts were then evaporated to dryness ( $c a .0 .8 \mathrm{~g}$ ) and suspended (100 $\mathrm{mL}$ ) in $\mathrm{CHCl}_{3} / \mathrm{MeOH} / \mathrm{H}_{2} \mathrm{O}$ $(5 / 5 / 4.5, \mathrm{v} / \mathrm{v} / \mathrm{v})$ and rested overnight $\left(16 \mathrm{~h}, 4{ }^{\circ} \mathrm{C}\right)$ to remove the polar material. The organic fraction was evaporated to dryness and analyzed by TLC. The crude lipid extracts were preabsorbed onto silica gel and fractionated on silica columns. Glycolipids were eluted successively with a step-wise gradient of $\mathrm{MeOH}$ in $\mathrm{CHCl}_{3}$ and further purified, if needed, using preparative TLC.

\subsubsection{NMR spectroscopy}

Purified glycolipids were dissolved in $0.7 \mathrm{~mL} \mathrm{CDCl}_{3} / \mathrm{CD}_{3} \mathrm{OD}(2 / 1$, v/v). Samples were analyzed at $30{ }^{\circ} \mathrm{C}$ and the chemical shift measured relative to TMS. The fatty acid methyl esters were dissolved in $\mathrm{CDCl}_{3}$. NMR spectra were recorded on a Bruker Advance III 500MHz spectrometer or a Varian DirectDrive $600\left(600 \mathrm{MHz}\right.$ for ${ }^{1} \mathrm{H}, 150$ $\mathrm{MHz}$ for ${ }^{13} \mathrm{C}$ ) spectrometer.

\subsubsection{ESI-TOF MS}

Electrospray mass spectrometry (or accurate mass measurement) was performed in the positive (or negative) ion mode on a Micromass Q-Tof Premier instrument. All MS-MS experiments were performed on a Q-Tof Premier mass spectrometer (Micromass, UK).

\subsubsection{Alditol Acetates Method}

The constituent sugar neutral composition was determined by GC-MS following TFA mediated acid hydrolysis and derivatisation to alditol acetates. ${ }^{49}$ 


\subsubsection{Glycosyl Linkage Analysis}

Bacterial glycolipids ( $c a .0 .5 \mathrm{mg}$ ) were methylated as described by Ciucanu and Kerek ${ }^{50}$ with the exception that samples were dispersed in DMSO $(0.5 \mathrm{~mL})$ and heated $\left(50{ }^{\circ} \mathrm{C}\right)$ overnight under argon. After extraction into chloroform, the methylated polysaccharides were hydrolysed with TFA, reduced, and then acetylated before analysis by GC-MS as described above. Identifications were based on peak retention times and by comparison of electron impact spectra with standard spectra. Here, the partially methylated alditol acetate standards were prepared following the method of Doares et al. ${ }^{208}$

\subsubsection{Sugar Absolute Configuration}

After hydrolysis, the sugar residues were subjected to a reductive amination with $R$-(-)1-amino-2-propanol and cyanoborohydride following the method of Cases et al. ${ }^{209}$

\subsubsection{Fatty Acid Analysis}

Fatty acid methyl esters (FAMEs) were prepared by direct transesterification from samples of biomass ( $c a .0 .5 \mathrm{mg}$ ) without extraction, as described by Svetashev et al. ${ }^{28}$ The resulting FAMEs and DMA were taken up in hexane and analyzed by GC and GCMS. ${ }^{210}$ The hydroxy fatty acids were derived to their TMS derivatives. ${ }^{212 \mathrm{e}}$ The position of the cyclopropane group was elucidated after separation of saturated fatty acids fraction by means of silver ion SPE. ${ }^{\text {ee }}$ This separation step was followed by hydrogenation in acetic acid over Adam's catalyst to open the cyclopropane ring. ${ }^{213 \mathrm{e}}$ The resulting fatty acids were converted to pyrrolidides ${ }^{214 e}$ and analyzed by GC-MS analyzes. The position of the double bond in the monounsaturated fatty acids and DMAs was located by GC-MS of dimethyldisulfide adducts. ${ }^{214 \mathrm{f}}$

\footnotetext{
${ }^{\mathrm{e}}$ Supelco. 2006. Discovery® Ag-Ion SPE for FAME fractionation and cis/trans separation (technical report).

http://www.sigmaaldrich.com/etc/medialib/docs/Supelco/Bulletin/t406062.Par.0001.File.tmp/t406062.pdf ${ }^{\mathrm{f}}$ Assay conducted by Kirill Lagutin, IRL
} 


\subsection{Characterisation}

\subsubsection{Whole Cell MALDI Analysis}

Heat-killed, whole cells of Bifidobacterium longum subsp. infantis $(10 \mathrm{mg} / \mathrm{mL})$ were suspended in either $\mathrm{CHCl}_{3} / \mathrm{MeOH}(1 / 1, \mathrm{v} / \mathrm{v})$ or water using $\mathrm{DHB}(30 \mathrm{mg} / \mathrm{mL})$ as a matrix and analyzed by MALDI mass spectrometry (Figure 3.1). The mass spectrum shows a number of molecular ions, with three $(\mathrm{m} / \mathrm{z} 779, \mathrm{~m} / \mathrm{z} 805$ and $\mathrm{m} / \mathrm{z}, 819)$ corresponding to a monoglycosyldiglyceride (MGDG) containing different fatty acid chains (16:0/18:1, 18:1/18:1 and 18:1/19:1). The most abundant molecular ion in the mass spectrum $(m / z, 881)$ did not correspond to any known glycolipid even though a series of molecular ions at $\mathrm{m} / \mathrm{z}, 855, \mathrm{~m} / \mathrm{z} 827$ and $\mathrm{m} / \mathrm{z} 881$ differed from each other by masses consistent with varying fatty acid chain lengths $\left(\Delta_{881-855}=26 \mathrm{~m} / \mathrm{z}, \mathrm{CH}=\mathrm{CH}\right.$ and $\Delta_{855-827}=28 \mathrm{~m} / \mathrm{z}, \mathrm{CH}_{2} \mathrm{CH}_{2}$ ). Further, MALDI mass spectrometry of B. longum subsb. longum DSM 20219 also showed this mass pattern. In order to determine the nature of the compound with this intriguing mass $(\mathrm{m} / \mathrm{z} 881)$, the glycolipids on the surface of $B$. longum subs. infantis were extracted and purified. 


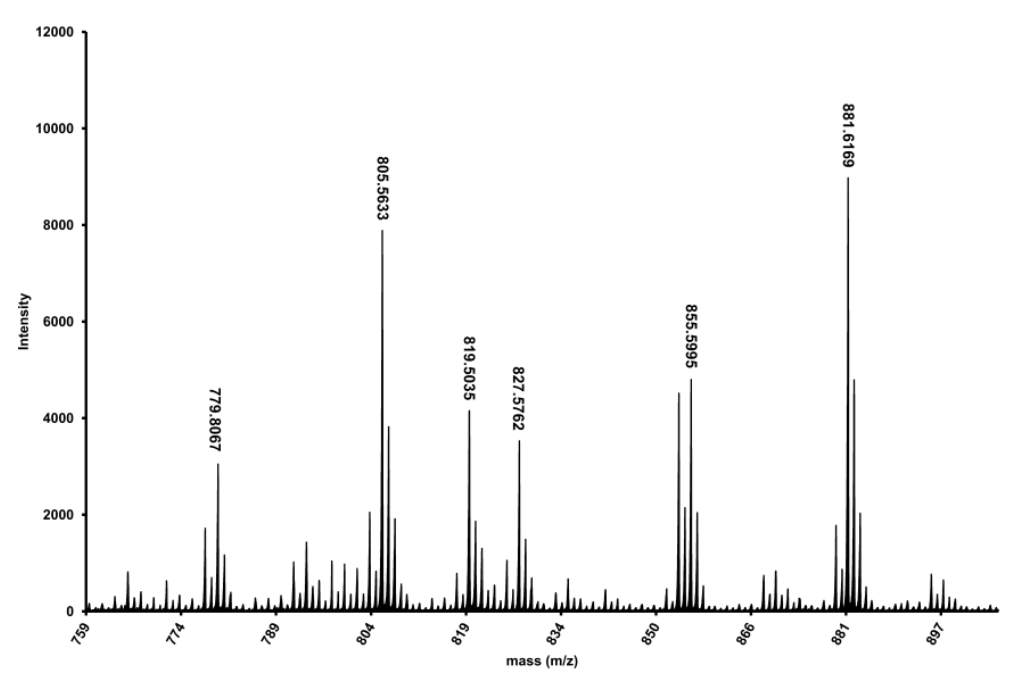

Figure 3.1 MALDI spectrum of heat-killed whole cells of B. longum subs. infantis ATCC15702T.

\subsubsection{Glycolipid Isolation and Structural Characterisation}

In order to isolate sufficient amounts of each compound observed by MALDI, cells were cultured in $18 \mathrm{~L}$ of medium, isolated by centrifugation and freeze-dried. Extraction of the resulting cell mass $(6 \mathrm{~g})$ using $\mathrm{CHCl}_{3} / \mathrm{MeOH} / \mathrm{H}_{2} \mathrm{O}$ yielded $480 \mathrm{mg}$ of crude lipid. Fractionation on silica gel of the extracts gave 5 individual fractions. These isolated compounds were analyzed using ESI-MS and NMR, in addition to fatty acid, sugar and linkage analysis. Two compounds with higher running spots on TLC $\left(\mathrm{R}_{f}=0.21\right.$ and $\mathrm{R}_{f}=$ 0.36, $\mathrm{CHCl}_{3} / \mathrm{MeOH}, 6 / 1$, v/v, Figure 3.2) were identified as lipids. Analysis of lipid 1 (L1) revealed the presence of oleic acid (HRMS calc. for $\left[\mathrm{C}_{18} \mathrm{H}_{33} \mathrm{O}_{2}\right]^{-}=281.2486$, found : 281.2484), and the slightly more polar lipid L2 was attributed to 10hydroxyoctadecanoic acid (HRMS calc. for $\left[\mathrm{C}_{18} \mathrm{H}_{35} \mathrm{O}_{3}\right]^{-}=299.2592$, found: 299.2579). Both structures matched previously published data. ${ }^{29,218,219}$ 


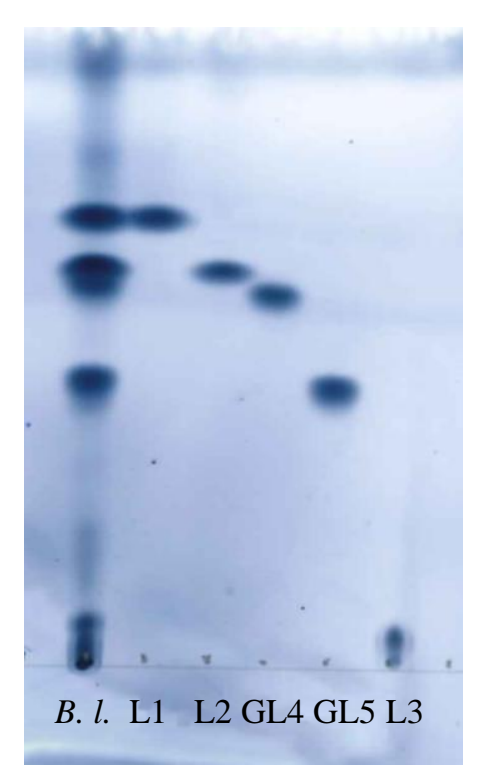

Figure 3.2 TLC of purified glycolipids from Bifidobacteria. $B$. . $l$. crude lipid extract, L1-3, GL4 and GL5 extracted from B. longum subs. infantis. Silica gel, eluted with $\mathrm{CHCl}_{3} / \mathrm{MeOH}(6 / 1, \mathrm{v} / \mathrm{v})$ and developed with Hannessian's stain.

Two compounds with an $\mathrm{R}_{f}$ on TLC of 0.56 and $0.41\left(\mathrm{CHCl}_{3} / \mathrm{MeOH}, 6 / 1\right.$, v/v, Figure 3.2) were next identified as glycolipids (GL4 and GL5). Again, fatty acid, sugar and linkage analysis, in addition to $\mathrm{R}_{f}$ values, yields and HRMS, were used to provide an overview of the chemical characteristics of the individual glycolipids GL4 and GL5 (Table 3.1). The glycolipids were then fully characterized using ${ }^{1} \mathrm{H}$ - and ${ }^{13} \mathrm{C}-\mathrm{NMR}$, and assignments were made using COSY, HSQC and HMBC experiments (Table 3.3 and 3.4). 
Table 3.1 Chemical characteristics of isolated compounds.

\begin{tabular}{|c|c|c|c|}
\hline & B. l. subs. & infantis & \\
\hline & L3 & GL4 & GL5 \\
\hline $\mathrm{R}_{f}$ & 0.10 & 0.56 & 0.41 \\
\hline Yield & $0.3 \%$ & $0.1 \%$ & $1.8 \%$ \\
\hline Neutral sugar (w\%) & & & \\
\hline D-Gal & - & 10.6 & 15.6 \\
\hline D-Glc & - & 0.8 & - \\
\hline Total & - & 11.4 & 15.6 \\
\hline Linkage analysis (mol ratio) & & & \\
\hline $\mathrm{t}-\mathrm{Gal} f$ & - & - & 1.0 \\
\hline $\mathrm{t}-\mathrm{Gal} p$ & - & 0.8 & - \\
\hline $\mathrm{t}-\mathrm{Glcp}$ & - & 0.2 & - \\
\hline $\begin{array}{llll}\text { Total fatty acid content } \\
(\mathrm{w} \%)\end{array}$ & 48 & 32.0 & 54.7 \\
\hline Fatty acids and DMA (w\%) & & & \\
\hline $14: 0$ & - & - & 1.7 \\
\hline DMA 16:1n-7 & 1.9 & - & 1.0 \\
\hline 16:0 & 7.4 & 6.5 & 13.3 \\
\hline $16: 1 n-7$ & 1.7 & 2.3 & 4.7 \\
\hline DMA $18: 1 n-9$ & 2.8 & - & 36.4 \\
\hline 18.00 & 7.4 & 4.3 & 3.1 \\
\hline 18:1n-9 & 35.4 & 41.6 & 23.4 \\
\hline $18: 1 n-7$ & 2.8 & 38.9 & 9.7 \\
\hline $18: 1 n-6$ & 1.2 & - & 3.1 \\
\hline 9,10-Mt-19:0 & - & 2.9 & - \\
\hline HRMS & & & \\
\hline Positive Ion Mode & & 805.5770 & 881.6349 \\
\hline Negative Ion Mode & 401.2466 & - & - \\
\hline & 417.2421 & - & - \\
\hline
\end{tabular}

* Fatty acids present under $1 \%$ are not shown. DMA = fatty aldehyde dimethyl acetal 


\subsubsection{GL4}

For GL4 (Table 3.2), sugar and linkage analysis revealed terminal galactopyranose (tGalp) as the major sugar present ( $0.8 \mathrm{~mol}$ ratio) as well as small amount ( 0.2 mol ratio) of terminal glucopyranose ( $\mathrm{t}-\mathrm{Gal} p)$. In addition, fatty acid analysis showed that oleic acid accounted for most of the lipid content (41.6\%) with cis-vaccenic acid in almost equal proportion (38.9\%). Other fatty acids present included palmitic acid, palmitoleic acid, stearic acid and dihydrosterculic acid. HRMS of GL4 (m/z 805.5770 for $\left[\mathrm{C}_{45} \mathrm{H}_{82} \mathrm{O}_{10}+\mathrm{Na}\right]^{+}$) was consistent with the presence of a monoglycosyldiglyceride, and NMR analysis confirmed the presence of a $\beta$-linked galactopyranose $\left(\delta_{\mathrm{H}-1^{\prime}}=4.24 \mathrm{ppm}\right.$, $J_{\mathrm{H}-1^{\prime}, \mathrm{H}-2^{\prime}}=7.8 \mathrm{~Hz}$, and $\delta_{\mathrm{C}-1^{\prime}}=109.2 \mathrm{ppm}$, and the small $\left.J_{\mathrm{H}-3^{\prime}, \mathrm{H}-4^{\prime}}=3.3 \mathrm{~Hz}\right){ }^{246} \mathrm{HMBC}$ between $4.2 \mathrm{ppm}(\mathrm{H}-1)$ with carbonyl carbon $\left(\delta_{\mathrm{C}(\mathrm{O})}=174 \mathrm{ppm}\right)$ confirmed the presence of an acyl group at this position. An HMBC between the 3.7/4.0 ppm glycerol protons and the anomeric carbon confirmed the attachment of the sugar to the glycerol.

The connectivity between the structural components of GL4 was confirmed using ESIMS/MS. The sodiated molecular ion (HRMS $m / z=805.5770$ for $\left[\mathrm{C}_{45} \mathrm{H}_{82} \mathrm{O}_{10}+\mathrm{Na}\right]^{+}$) was fragmented to give one major fragment corresponding to loss of a first oleic acid $(\mathrm{m} / \mathrm{z}=$ 525.3, Figure 3.3). Further fragmentation and loss of a second oleic acid gave another minor fragment $(\mathrm{m} / z=243)$. $\beta$-D-galactopyranosyl-diglyceride common in nature in both plants and bacteria. ${ }^{204,247}$ GL4 was identified as a $\beta$-D-galactopyranosyl-diglyceride as all proton and carbon NMR signals were in accordance with literature data (Table 3.2). ${ }^{53}$ Its presence has been observed previously in bifidobacteria, ${ }^{44}$ and has also been recorded in two opportunistic pathogens, found in soil: Arthrobacter globiformis and Artrobacter scleromae. ${ }^{53,248}$ 
Table 3.2 NMR spectroscopic data for GL4.

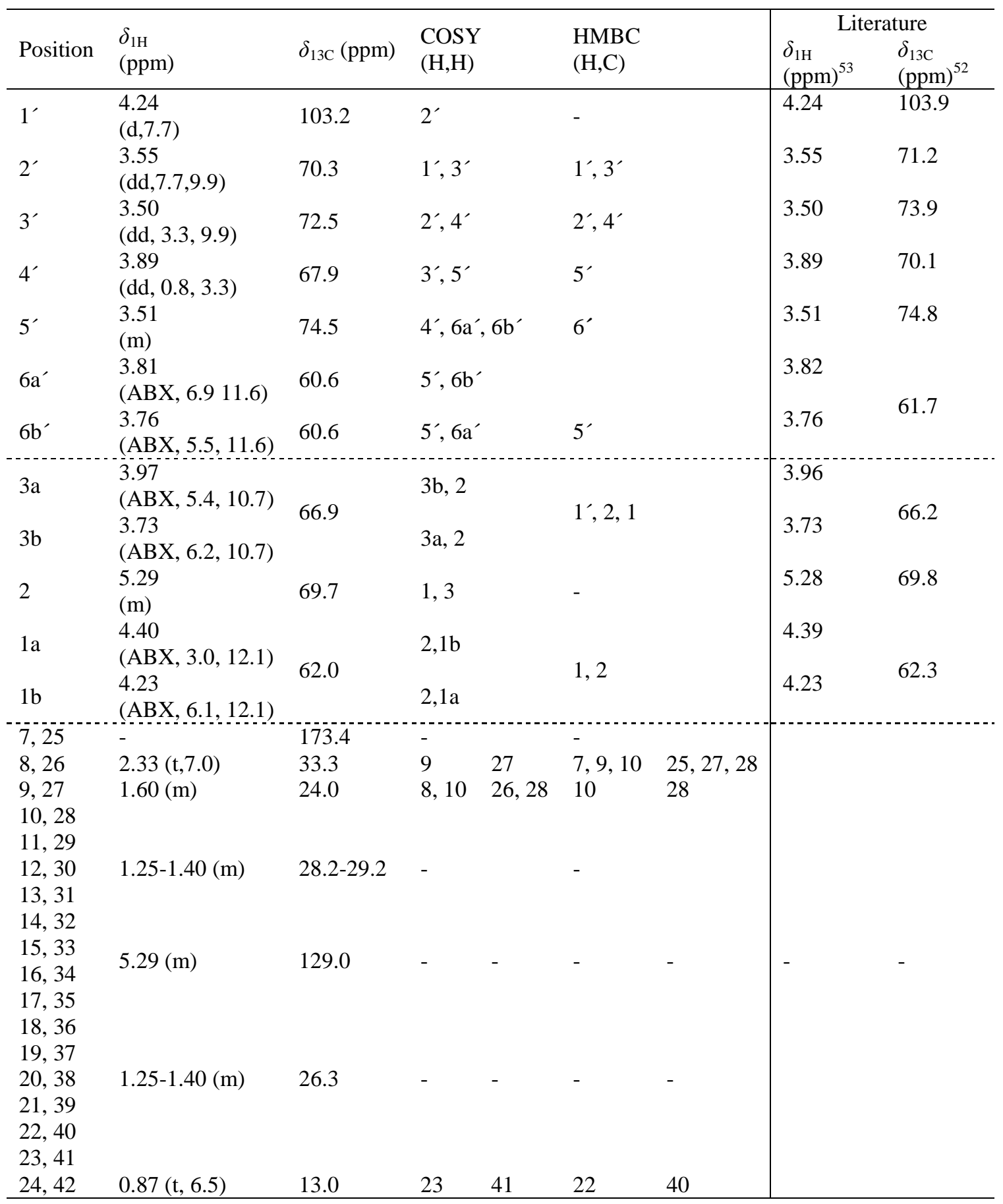

*Overlapping signals

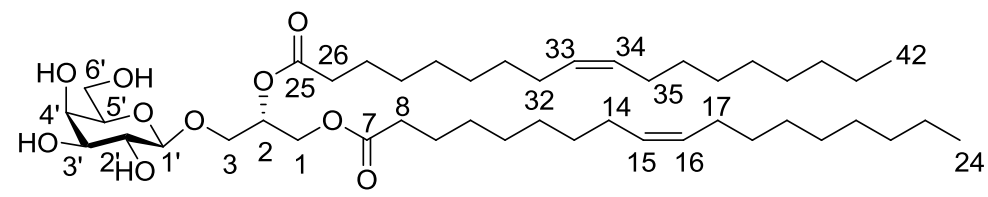




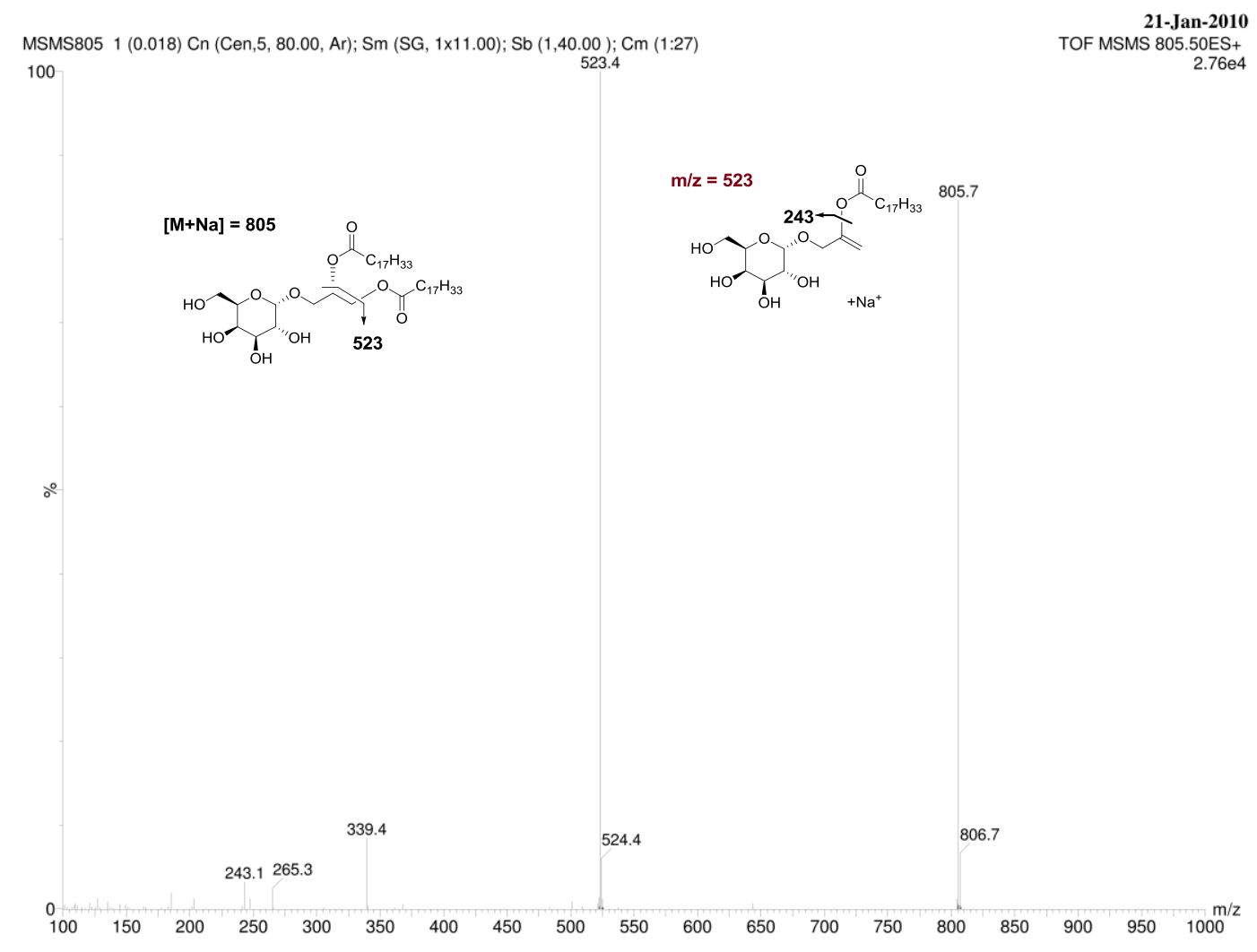

Figure 3.3 ESI MS/MS of GL4 in positive ion mode.

\subsubsection{GL5}

Glycolipid GL5 (Table 3.3) was of particular interest, as it showed unique mobility on TLC, intermediate to that of mono- and diglycosyldiglycerides (Figure 3.2). A compound with a similar mobility on TLC was also observed by Novik et al., ${ }^{104}$ however its structure was not elucidated. In contrast to GL4, constituent sugar and linkage analyzes of GL5 revealed the presence of terminal galactofuranose (t-Galf), which is a configuration previously observed in bifidobacterial glycolipids isolated by Veerkamp in the 1970s. ${ }^{44}$ Galactofuranoses are usually formed from UDP-Gal $p$ via a UDP-Gal $p$ mutase to lead to UDP-Galf. As seen in Chapter 1 (Section 1.4.4), a UDPsugar is usually bound to DAG via an YpfP enzyme to form the glycosyldiglyceride, and thus the presence of both sugars (Galp and Galf) can be explained. ${ }^{249}$ The presence of a t-Gal $f$ residue was readily confirmed by the ${ }^{1} \mathrm{H}-\mathrm{NMR}$ spectrum that showed an 
apparent singlet at $\delta 4.94$ ppm corresponding to $\mathrm{H}-1$ of a $\beta$-Gal $f$ moiety. The presence of a singlet was due to a small vicinal coupling constant $(<2 \mathrm{~Hz}){ }^{250}$ This assignment was confirmed by the ${ }^{13} \mathrm{C}$ chemical shift and the direct spin-spin coupling constant $\left({ }^{1} J_{\mathrm{C}-1^{\prime}}, \mathrm{H}-\right.$ $\left.1^{\prime}\right)$ of the anomeric carbon $\left(108.6 \mathrm{ppm}, J_{\mathrm{C}-1^{\prime}, \mathrm{H}-1^{\prime}}=172 \mathrm{~Hz}\right)^{251-253}$ and the HMBC correlation between the anomeric proton and $\mathrm{C}^{-4^{\prime}}(\delta 85.1 \mathrm{ppm})$. In a galactofuranose moiety, the configuration of the anomeric position cannot be distinguished using the coupling constant. Indeed, the dihedral angle between $\mathrm{H}^{-} \mathbf{1}^{\prime}$ and $\mathrm{H}-2^{\prime}$ is very similar for an $\alpha$ - or a $\beta$ - linkage resulting in similar coupling constants, thus indistinguishable. The use of the ${ }^{1} \mathrm{~J}_{\mathrm{CH}}$ coupling constant is commonly used to this effect. Due to stereoelectronic effects, an equatorial proton showed a ${ }^{1} J_{\mathrm{C}-1^{\prime}, \mathrm{H}-1}$ 'coupling constant of $\sim 170 \mathrm{~Hz}$, while a axial proton showed a ${ }^{1} J_{\mathrm{C}-1^{\prime}, \mathrm{H}-1^{\prime}}$ coupling constant of $\sim 160 \mathrm{~Hz} .{ }^{251-253}$ Other sugar resonances $\left({ }^{1} \mathrm{H}\right.$ and $\left.{ }^{13} \mathrm{C}\right)$, assigned on the basis of the COSY, HSQC and HMBC 2D NMR spectra, were all consistent with a $\beta$-t-Gal $f$ moiety. ${ }^{251,252}$

Perhaps the most unique characteristic of GL5, however, was the positive-ion HRMS $\left(m / z=881.6349\right.$ for $\left.\left[\mathrm{C}_{48} \mathrm{H}_{90} \mathrm{O}_{12}+\mathrm{Na}\right]^{+}\right)$, which was previously observed by MALDI mass spectrometry of the whole cells. Such a molecular formula is unexpectedly high in oxygen for a monoglycosyldiglyceride, although it could correspond to the condensation product of a hexose, two glycerols, a fatty acid, and a lipid ether with an additional degree of unsaturation. Indeed, this realization proved key in solving the unique structure of GL4. Further information on the nature of the lipid ether was obtained via lipid analysis (Table 3.1), which in addition to the anticipated palmitic and oleic acids, revealed high amounts of dimethyl acetals (DMAs). DMAs typically arise from enol ether type lipids (e.g. plasmalogens) following methanol/acid treatment (Chapter 1, Section 1.6.3). ${ }^{29,254}$ Furthermore, NMR spectroscopy of GL5 showed an unusual triplet at $\delta_{\mathrm{H}-13}=4.54 \mathrm{ppm}\left(J_{\mathrm{H}-13}, \mathrm{H}-14=5.9 \mathrm{~Hz}\right)$ with an associated $\delta_{\mathrm{C}-13}=109$ ppm. COSY and HMBC experiments then showed this proton to be part of a long chain acetal, thus explaining the observed DMAs in the fatty acid analysis (Figure 3.4 and $3.5)$.

The connectivity of the acetal centre was readily established by way of HMBC analysis, which revealed correlations between the acetal centre and two $\mathrm{CH}_{2}$-groups of two different glycerols. One glycerol was acylated at the 2-position $\left(\delta_{\mathrm{H}-8}=5.20 \mathrm{ppm}\right)$, as 
evidenced by an HMBC with a lipid carbonyl $\left(\delta_{\mathrm{C}-31}=174.2\right)$, while the 7-position showed an HMBC with the H-1 of the galactofuranose. The second glycerol did not show any further HMBCs and appeared unsubstituted. All fragments predicted by HRMS were now connected and allowed the structure of GL5 to be formulated as a 1- $\beta$ D-galactofuranosyl-3-glyceroplasmalo-monoglyceride.

Table 3.3 NMR spectroscopic data for GL5.

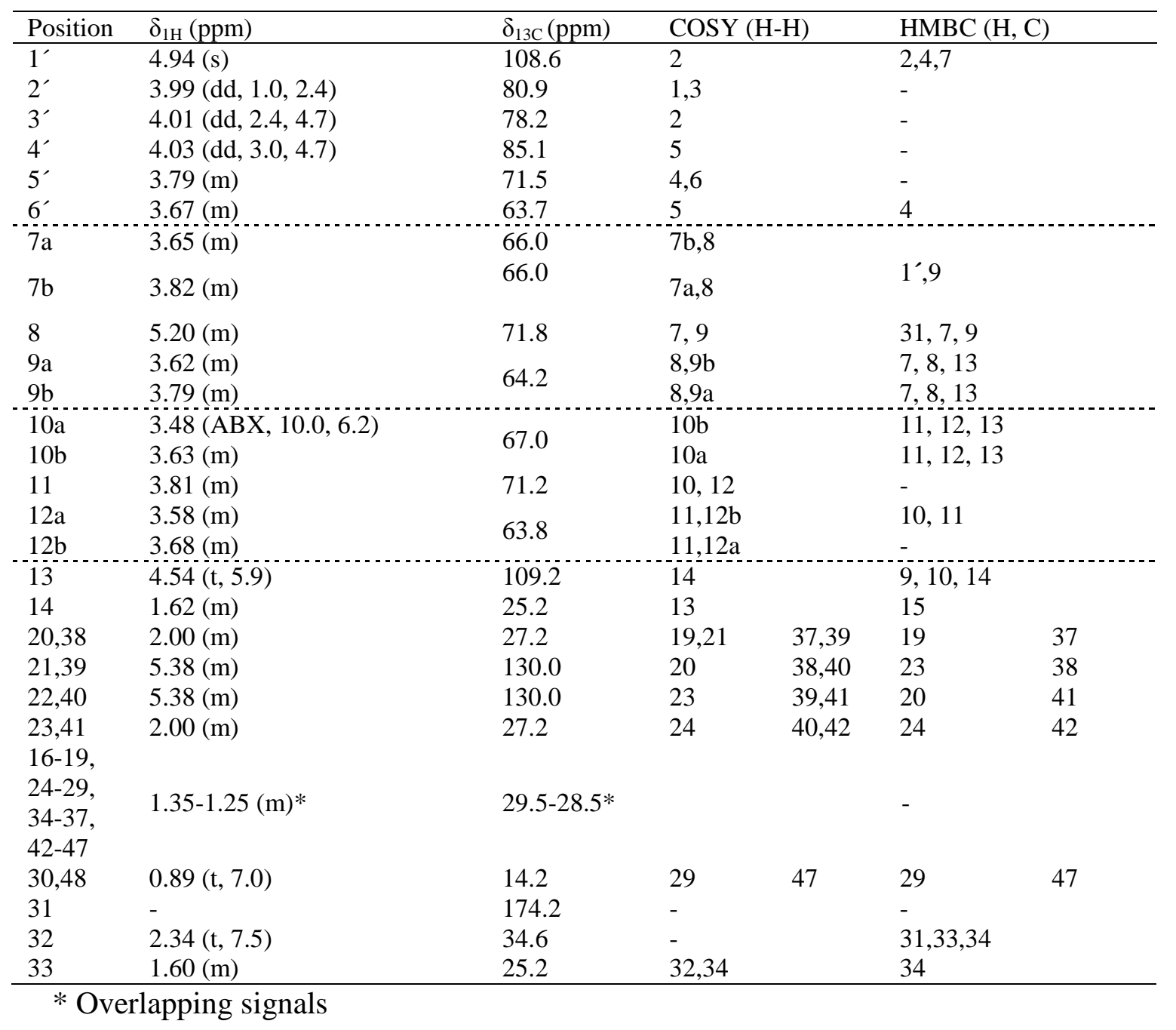

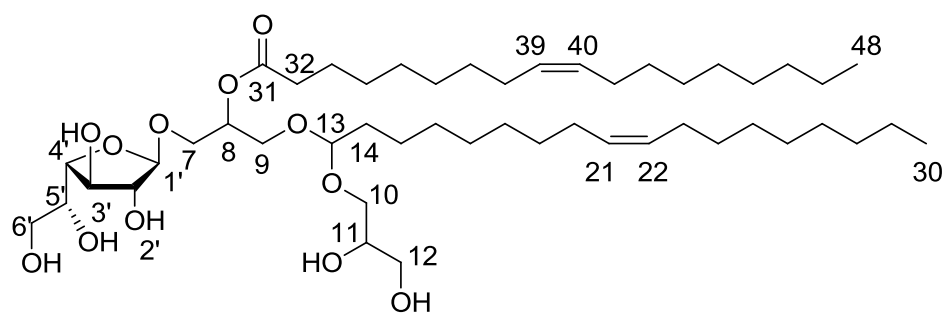




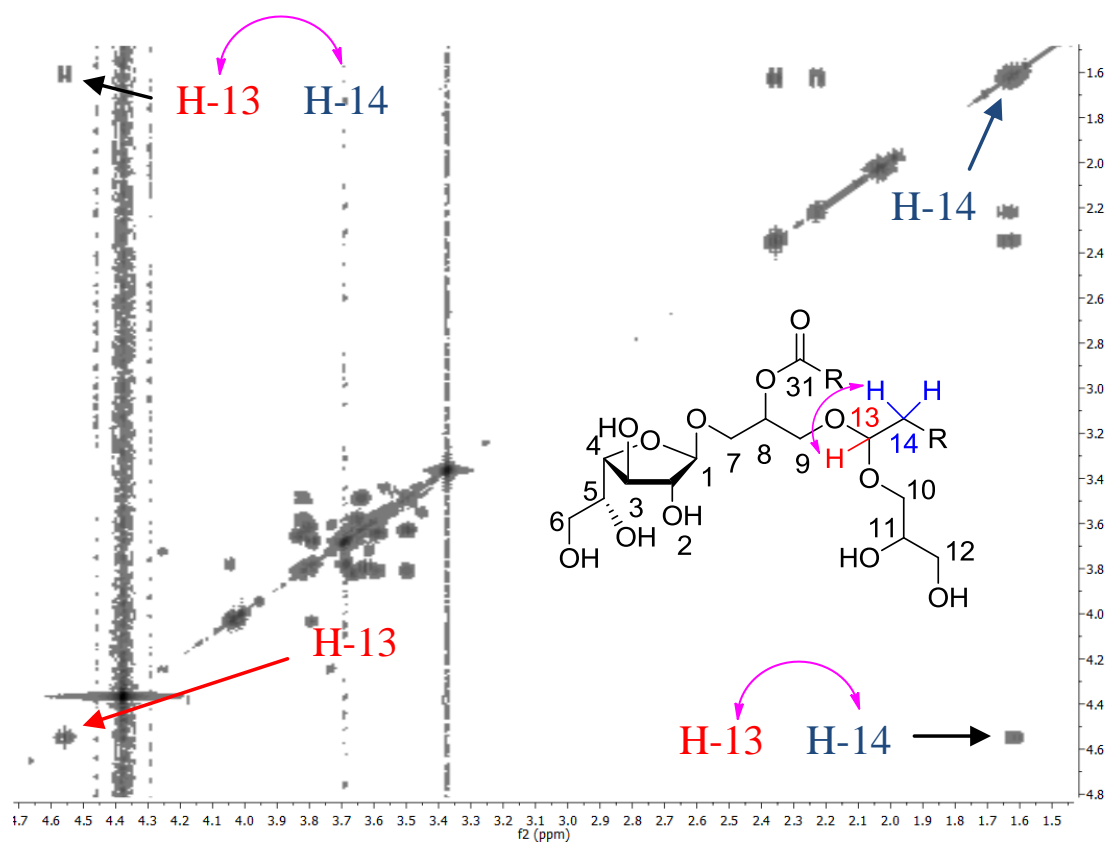

Figure 3.4 Proton to proton correlations showing that the acetal signal correlated to the fatty acid chains in the ${ }^{1} \mathrm{H},{ }^{1} \mathrm{H}$ COSY spectrum of GL5, $\mathrm{CD}_{3} \mathrm{OD} / \mathrm{CDCl}_{3}(1 / 2, \mathrm{v} / \mathrm{v})$, $600 \mathrm{MHz}$.

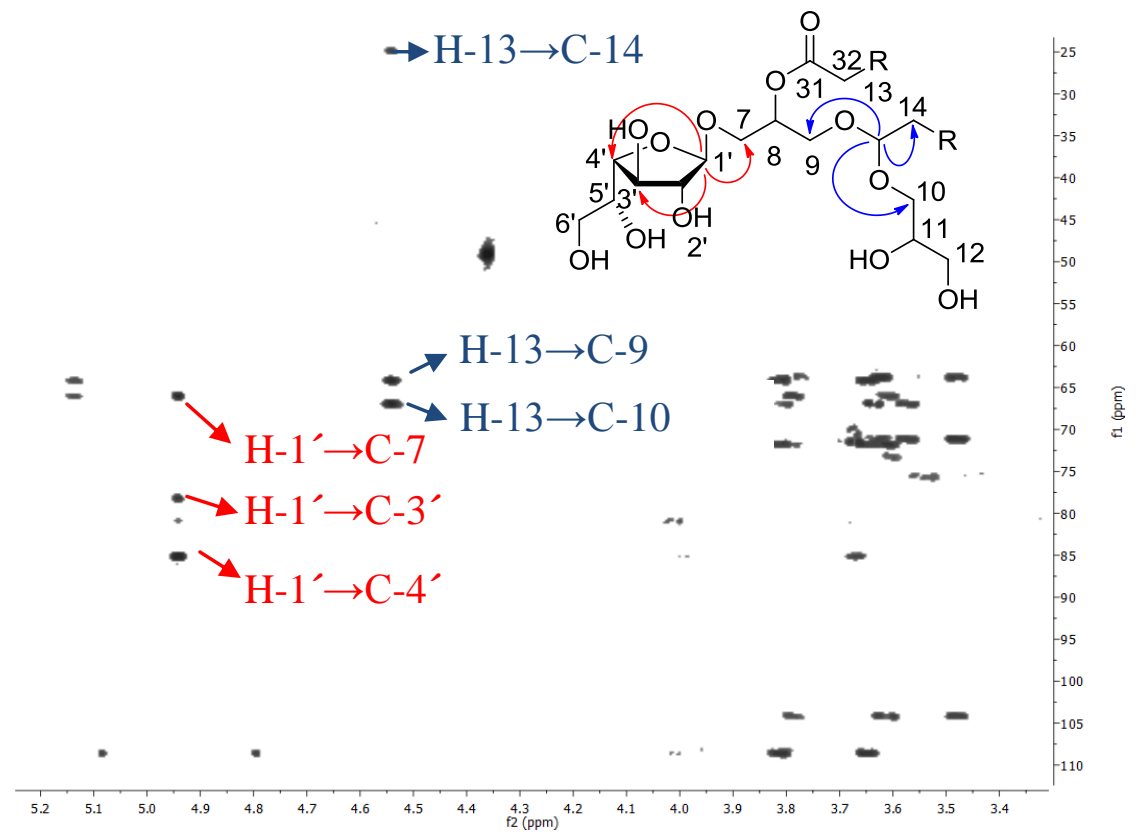

Figure 3.5 Proton to carbon correlations showing the presence of a furanose moiety and the connections between the different parts of the molecule in the ${ }^{1} \mathrm{H},{ }^{13} \mathrm{C}$ HMBC of $\mathrm{GL} 5, \mathrm{CD}_{3} \mathrm{OD} / \mathrm{CDCl}_{3}(1 / 2, \mathrm{v} / \mathrm{v}), 600 \mathrm{MHz}$. 
To confirm GL5 assignment, ESI-MS/MS was used and all fragments were accounted for. The major molecular ion peak in positive ion mode $(\mathrm{m} / \mathrm{z}=881.6)$ was fragmented using CID and the most abundant fragment ion $(\mathrm{m} / \mathrm{z}=541.3)$ was attributed to the loss of glycerol and aldehyde (Figure 3.6a). Additional fragments at $\mathrm{m} / \mathrm{z}=789.6,719.6$, 259.1 and 203.1 were also detected and could be explained by the loss of glycerol, galactose, fatty aldehyde and fatty acid, respectively (Figure 3.6a). In addition, MS/MS in negative ion mode (Figure 3.6b) showed the quasimolecular ion of $\mathrm{m} / \mathrm{z}=893.6$ $[\mathrm{M}+\mathrm{Cl}]^{-}$and loss of $\mathrm{HCl}\left(\mathrm{m} / z=857.6[\mathrm{M}-\mathrm{H}]^{-}\right)$. CID fragmentation of the $\mathrm{m} / z .893 .6$ $[\mathrm{M}+\mathrm{Cl}]^{-}$ion provided a major fragment at $\mathrm{m} / z=281.2$ corresponding to the oleate anion. Higher mass fragments consisted of $\mathrm{m} / z=431.3$ (loss of galactose and oleate), $m / z=575.4$ (loss of oleic acid) and $m / z=593.4$ (loss of oleyl). The loss of these fragments confirms the connectivity of the structural components and unequivocally corroborated the unusual structure of GL5.

Acetal containing glycolipids exist as plasmalogalactosylceramide and plasmalogalactosylalkylglycerol (Figure 3.7), ${ }^{255,256}$ glyceroplasmalopsychosine (Figure $3.8),{ }^{257}$ aldehydogenic ethanolamine phospholipid, ${ }^{258}$ and glycerol acetal of plasmalogen. ${ }^{259,260}$ These acetal type glycolipids have also been found in Sarcina ventriculi $^{261}$, with the acetal moiety believed to be the result of a head-to-head coupling defense mechanism from the bacteria after a perturbating event (heat shock, $\mathrm{pH}$, antibiotics). The mechanism would involve a nucleophilic attack of a hydroxyl group on the enol ether moiety of a plasmalogen under acidic conditions (Figure 3.9). This type of defense mechanism or even the occurrence of an acetal containing glycolipids remains unprecedented in bifidobacteria. 
a. Positive ion mode

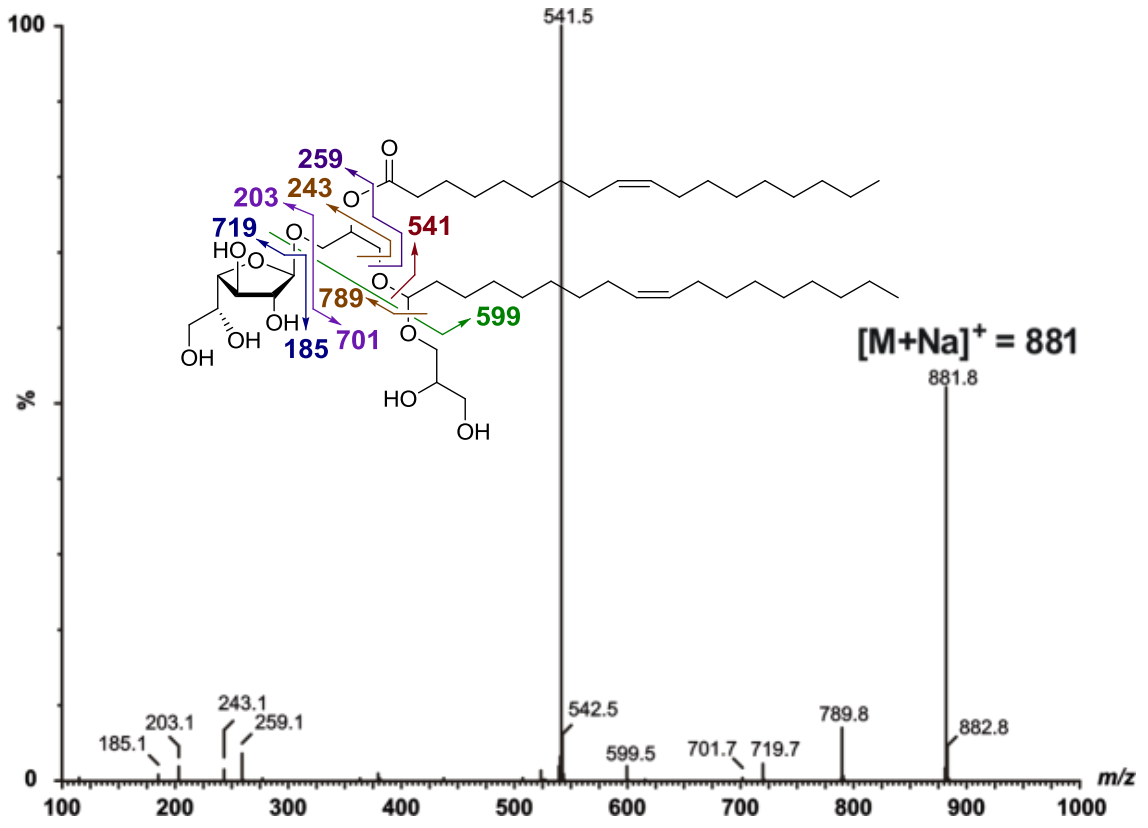

b. Negative ion mode

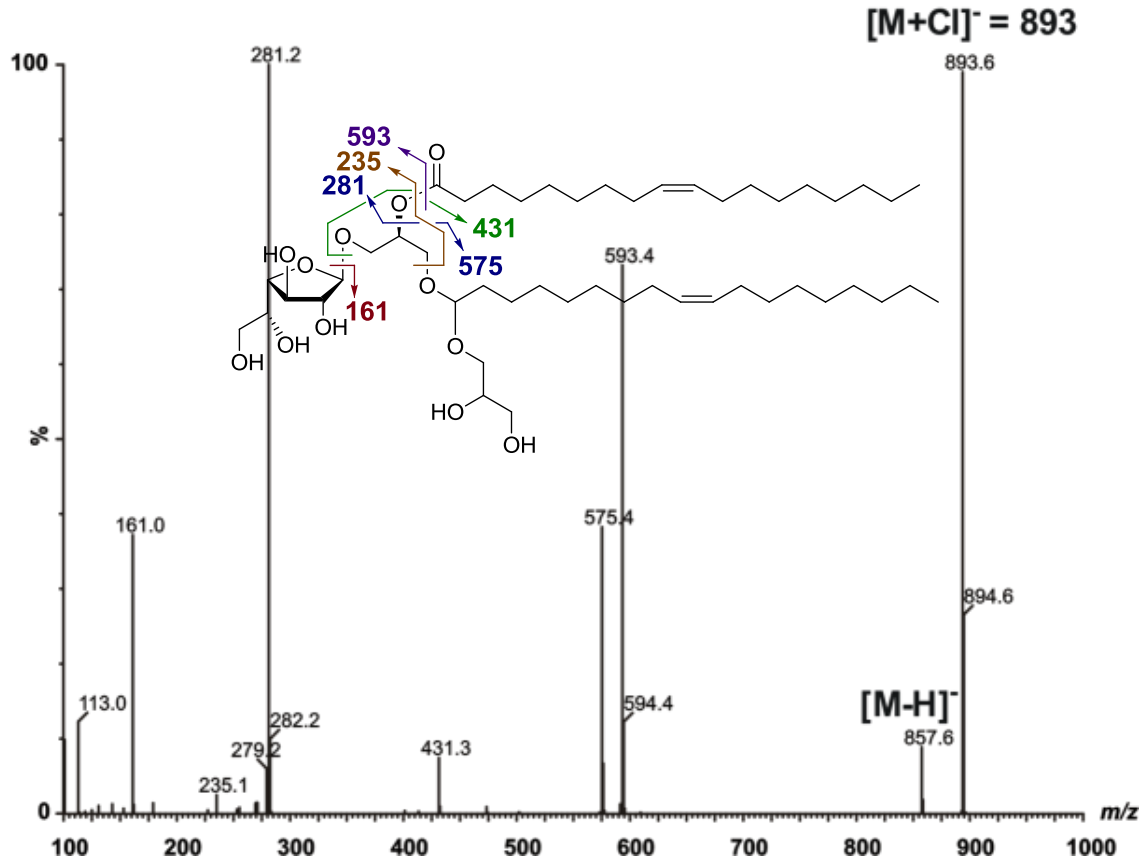

Figure 3.6 MS/MS of GL4 in a. positive ion mode and b. negative ion mode. 


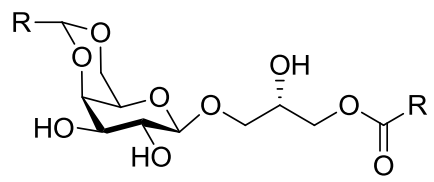

Figure 3.7 Plasmalogalactosylalkylglycerol glycolipid extracted from equine brain. ${ }^{255,256}$

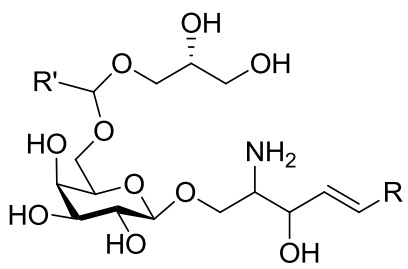

Figure 3.8 Glyceroplasmalopsycosine extracted from bovine brain. ${ }^{257}$
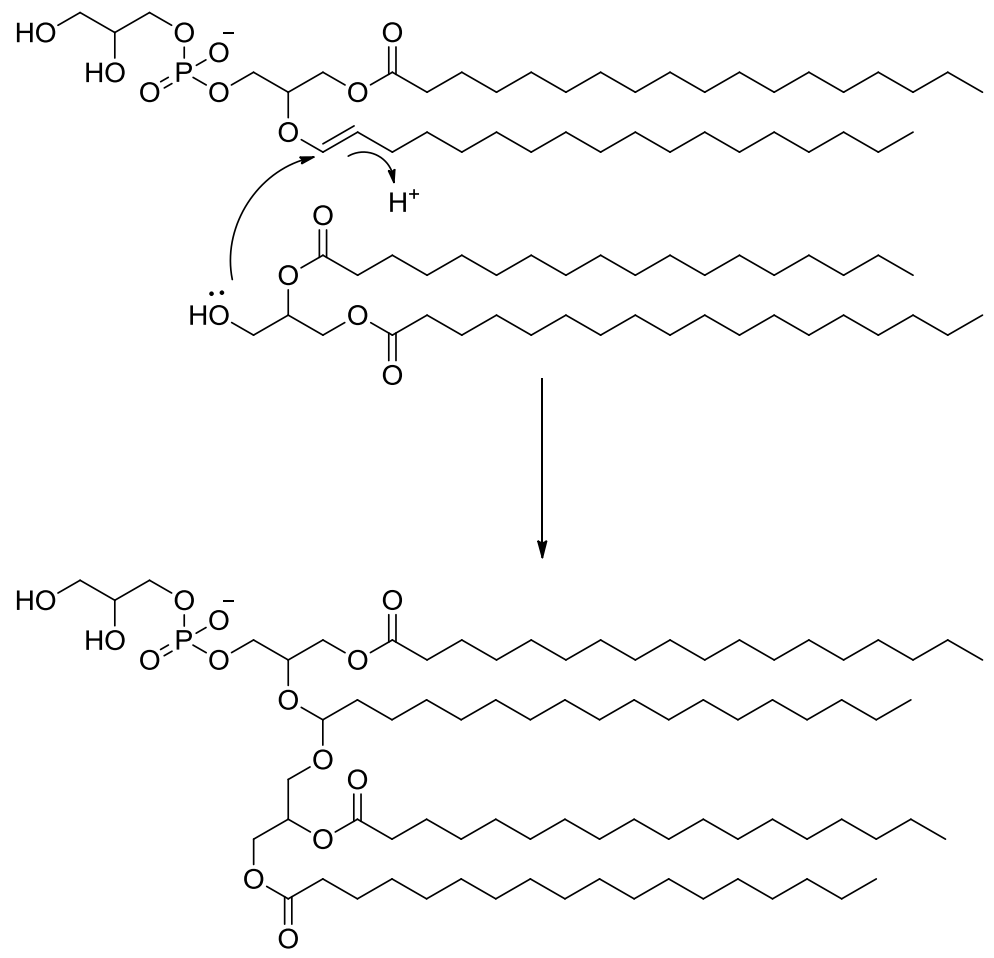

Figure 3.9 Proposed mechanism showing coupling between plasmalogen lipids and DAG. $^{261}$ 


\subsubsection{L3}

The lowest spot on TLC $\left(\mathrm{R}_{f}=0.10, \mathrm{CHCl}_{3} / \mathrm{MeOH}, 6 / 1, \mathrm{v} / \mathrm{v}\right), \mathrm{L3}$, was found to be a mixture of phospholipids. As seen in Chapter 1 (Section 1.4.4), phospholipids are common bacterial cell wall constituents. The presence of phosphorous was confirmed by TLC using a molybdate stain ${ }^{206,207}$ and through ${ }^{31} \mathrm{P}$ NMR $\left(\delta_{\mathrm{P}}=0.2 \mathrm{ppm}\right)$ although the signal was very weak and broad indicating a mixture of phospholipids. Two principal signals were detected using HRMS in negative ion mode $(\mathrm{m} / \mathrm{z}=401.2466$ for $\left[\mathrm{C}_{21} \mathrm{H}_{38} \mathrm{O}_{6} \mathrm{P}\right]^{-}$and $\left.m / z=417.2421\left[\mathrm{C}_{21} \mathrm{H}_{38} \mathrm{O}_{5} \mathrm{P}\right]^{-}\right)$which were identified as $\mathrm{C} 18: 1-$ plasmenyl-cPA and C18:1-cPA, respectively. The molecular ion of L3a $(\mathrm{m} / \mathrm{z}=401)$, dissociated into 4 fragments: $m / z=265$ (C18:1 aldehyde), 135 (cyclophosphaglyceryl), $97\left(\mathrm{PO}_{4}{ }^{-}\right)$and $79\left(\mathrm{PO}_{3}{ }^{-}\right)$(Figure 3.10). In contrast to L3a, collision induced dissociation (CID) of [L3b-H] $]^{-}(\mathrm{m} / \mathrm{z}=417)$ produced two main fragments of $m / z=281$ (oleate) and $m / z=153$ (cyclophosphaglycerol) (Figure 3.11). The difference in dissociation pattern can be attributed to the presence of an enol-ether linkage, which provides different stabilities to the fragment ions. The presence of cPA's in bifidobacteria is surprising, as this is the first time they have been isolated from bacteria. However, these cPAs have been found to be biologically active in the past, ${ }^{262}$ and evidences of plasmalogen presence has previously been identified in bifidobacteria (Chapter 1, Section 1.6.3).

Plasmalogens are usually detected in animals and although they are not often found in facultative anaerobic bacteria, ${ }^{263}$ they have been detected in such by Oberg et al. ${ }^{131}$ Plasmalogens in anaerobic bacteria have been known for $c a .50$ years, and although the plasmalogen biosynthetic pathway is well understood in animals, the biosynthetic pathway regarding their formation in anaerobic bacteria is unknown. Scheme 3.1 shows the biosynthetic pathway to form plasmenylethanolamine 6. At first, dihydroxyacetone phosphate $\mathbf{1}$ is acylated via acyl-COA. The product (Acyl-DHAP) is then transformed through the action of alkyl-DHAP synthase to form Alkyl-DHAP. At this point, NADH reduces the ketone to alcohol 2 . This compound is acylated and resulting phospholipid 3 is dephosphorylated. After this, a phosphoethanolamine group is introduced via CDPEtn resulting in glycerolipid 4. The last step involves a $1^{\prime}$-alkyl desaturase that stereospecifically abstract a proton from $\mathrm{C}-1^{\prime}$ and $\mathrm{C}-2^{\prime}$ of the $O$-alkyl chain. Compound 5 is thus transformed into a plasmalogen. As this last step involves oxygen, it is 
believed that the biosynthetic pathway differs between anaerobic bacteria and animals. $^{263}$

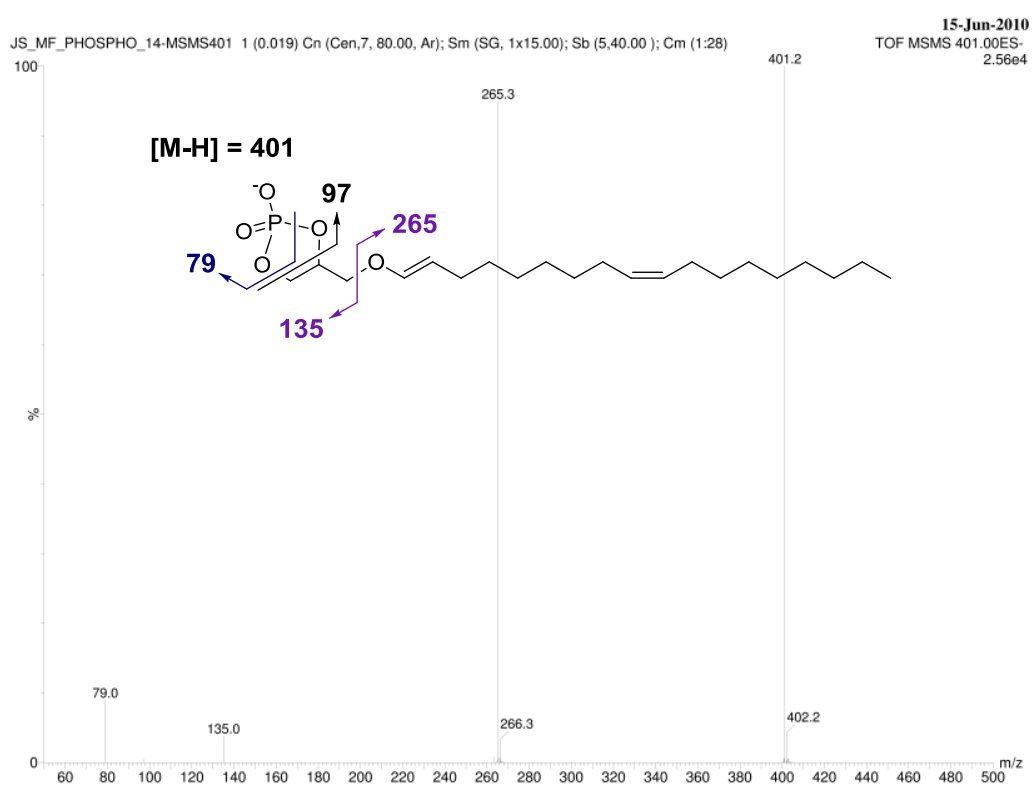

Figure 3.10 MSMS of L3a in negative ion mode.

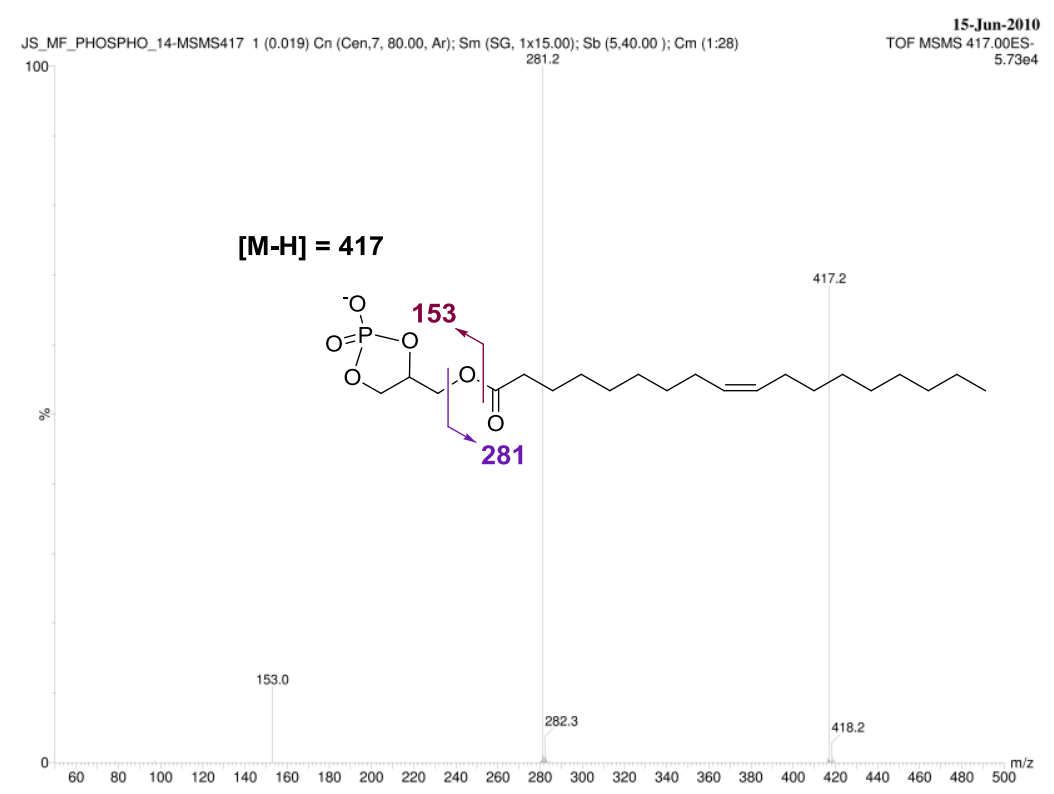

Figure 3.11 MSMS of L3b in negative ion mode. 


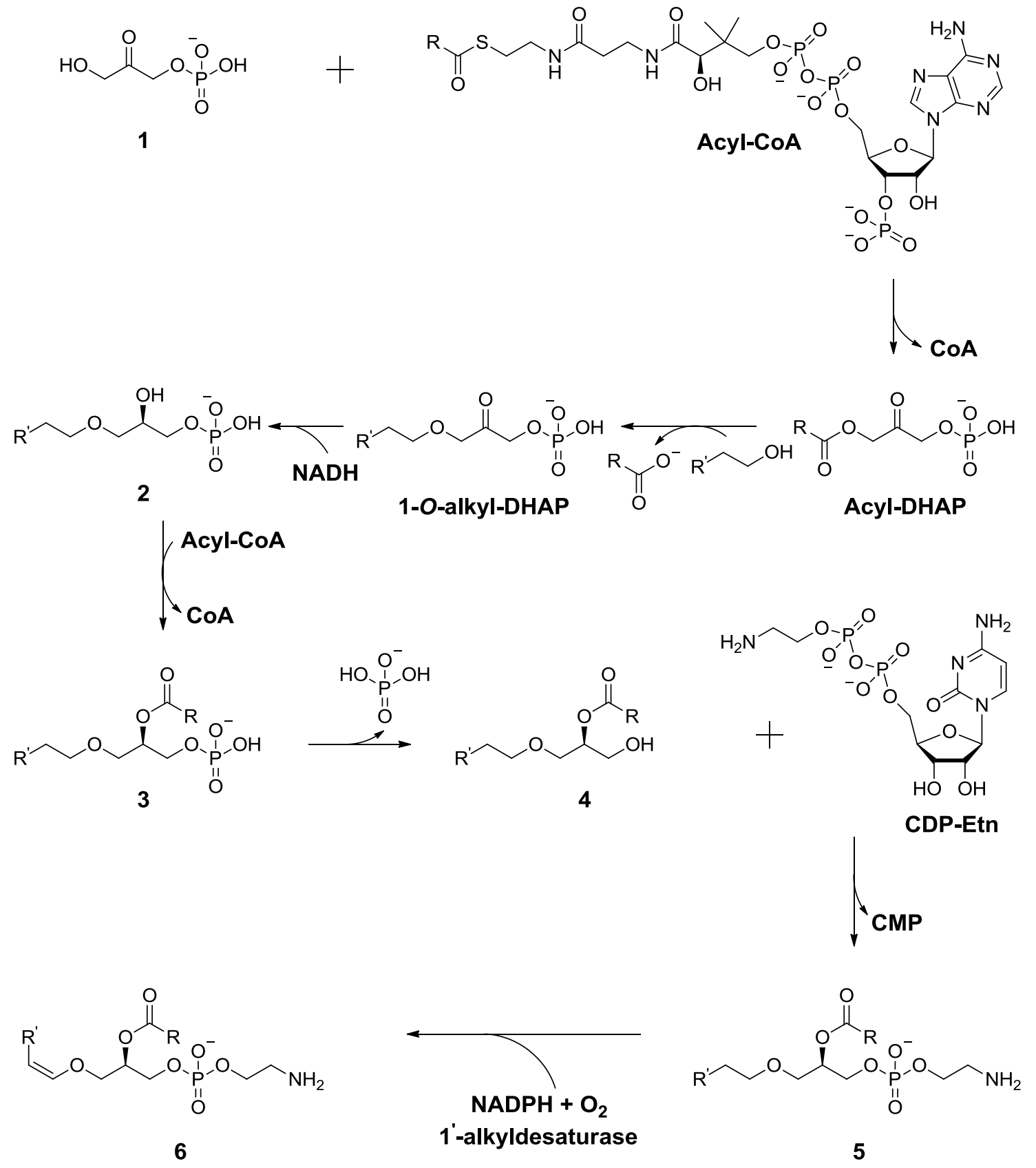

Scheme 3.1 Plasmalogen biosynthetic pathway in animals. ${ }^{263}$ 


\subsection{Biological Screening of Extracted Compounds}

As seen in Chapter 1 (Section 1.6), bifidobacteria whole cells and components were found to be biologically active. Thus, the glycolipids were tested in three different in vivo and in vitro assays. Before screening the immunological properties of these compounds, they were first confirmed to be free of endotoxin, as determined by the Limulus amebocyte lysate (LAL) assay. ${ }^{\mathrm{g}}$ It is important to note that the results obtained are only preliminary. Further assays would be required to establish the full biological assessment of those gycolipids. Firstly, the glycolipids were assessed for their allerginicity and sensitization potential. ${ }^{\mathrm{h}}$ Secondly, the ability of the glycolipids to

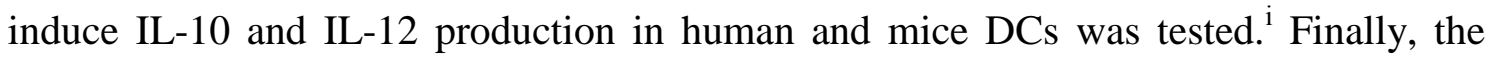
glycolipids were tested in a macrophage activation assay. ${ }^{j}$

The in vivo allerginicity and sensitization assay was deemed noteworthy due to a growing interest in the relationship between bifidobacteria and atopic disease. ${ }^{264}$ For example, research pursued by Young et al. showed that the bifidobacterial species present in the feces of infants from the United Kingdom and New Zealand, where there is a high incidence of atopic diseases, differ from those present in infants from Ghana, where incidence of atopic diseases is low. ${ }^{264,265,266,267}$ Thus, the allerginicity and sensitization assay was conducted on GL5. The results of this assay show that the glycolipids were not allergenic and additionally did not reduce the allergic response to dust mites in mice. However, in this case, the amount of glycolipid available for the assay was low and only two concentrations were tested $(10 \mu \mathrm{g} / 30 \mu \mathrm{L}$ and $100 \mu \mathrm{g} / 30$ $\mu L)$. Hence, higher amounts of material would be required for a definitive answer on the allerginicity and sensitization of these glycolipids.

A previous study, by Young et al., has indicated differential induction of IL-10 and IL12 in cord blood-derived dendritic cells (DCs) and in CD86 expression depending on

\footnotetext{
${ }^{g}$ Extracted bifidobacteria glycolipids were tested to be endotoxin-free at a sensitivity of $0.125 \mathrm{EU} / \mathrm{mL}$ with an endotoxin-kit (Pyrotell, Limulus amebocyte lysate) according to the manufacturer's instructions.

${ }^{\mathrm{h}}$ Assay conducted by Mali Cambertis.

${ }^{\mathrm{i}}$ Assay conducted by Odette Shaw and Jacquie Harper.

${ }^{\mathrm{j}}$ Assay conducted by Ashna Khan and Stephanie Chee.
} 
the bifidobacteria species. ${ }^{264}$ Thus GL5 capacity to induce IL-10 and IL-12 production in human and mice dendritic cells was tested. GL5 was found to be unable to induce pro- or anti-inflammatory cytokines in dendritic cells derived from human blood and this assay also showed negative results in mice DCs. However, to validate the results of the assays, a repeat of the assays would be required. Furthermore, additional experiments regarding cytokine induction in cells should be conducted. Here, the use of different cell type may be interesting for future assays (e.g. cord blood ${ }^{264}$ or human intestinal epithelial cells ${ }^{139}$ ).

GL4 and GL5 were tested for their ability to activate mouse macrophages as bifidobacteria species have previously been shown to induce cytokine production in macrophages. ${ }^{268}$ In this assay, the glycolipids isolated from bifidobacteria showed no significant activity. This is not unexpected as research has reported marked differences in biological activity when surveying glycolipids with different fatty acid compositions. $^{63,77,78,269,270}$

Further work concerning the immunological properties of GL4 and GL5 would be required for a broader overview of the activity of these glycolipids. Indeed, $i \mathrm{NKT}$ cells assays could be conducted as Kinjo et al. reported that glycoglycerolipids from $S$. pneumonia induced IL-2 production in $i$ NKT cells. ${ }^{78}$ 


\subsection{Concluding Remarks}

In this chapter, MALDI was first used to screen whole bifidobacteria cells for their glycolipid components. Then, the glycolipids from Bifidobacterium longum subs. infantis were characterized. The analyzes showed the presence of previously identified $\beta$-D-Gal $p$-diglyceride (GL4) and novel 1- $\beta$-D-Galf-3-glyceroplasmalo-monoglyceride (GL5). Additionally, a mixture of phospholipids containing cPAs was observed (L3). An attempt was made to screen the immunological properties of GL4 and GL5. Although the assays showed no significant activity, further research on the immunological properties of these glycolipids would be required for confirmation of the results obtained so far, and additional testing on different types of cells would be of interest to pursue.

As LTAs have been found to show greater immunological properties compared to the glycolipid anchor in specific assays, the next step in the study of these glycolipid anchors is the synthesis of LTA derivatives for further testing (Chapter 4). Other interesting subjects are the extraction of bifidobacteria LTAs and the study of GL5 stereochemistry (Chapter 5). Indeed, to date, only harsh conditions have been used for the extraction of bifidobacteria LTA and it is plausible that LTAs containing GL5 as an anchor could be extracted with milder conditions. 


\subsection{Supplementary Data-NMR and MS Spectra and Compound Characterisation}

\subsubsection{GL4}

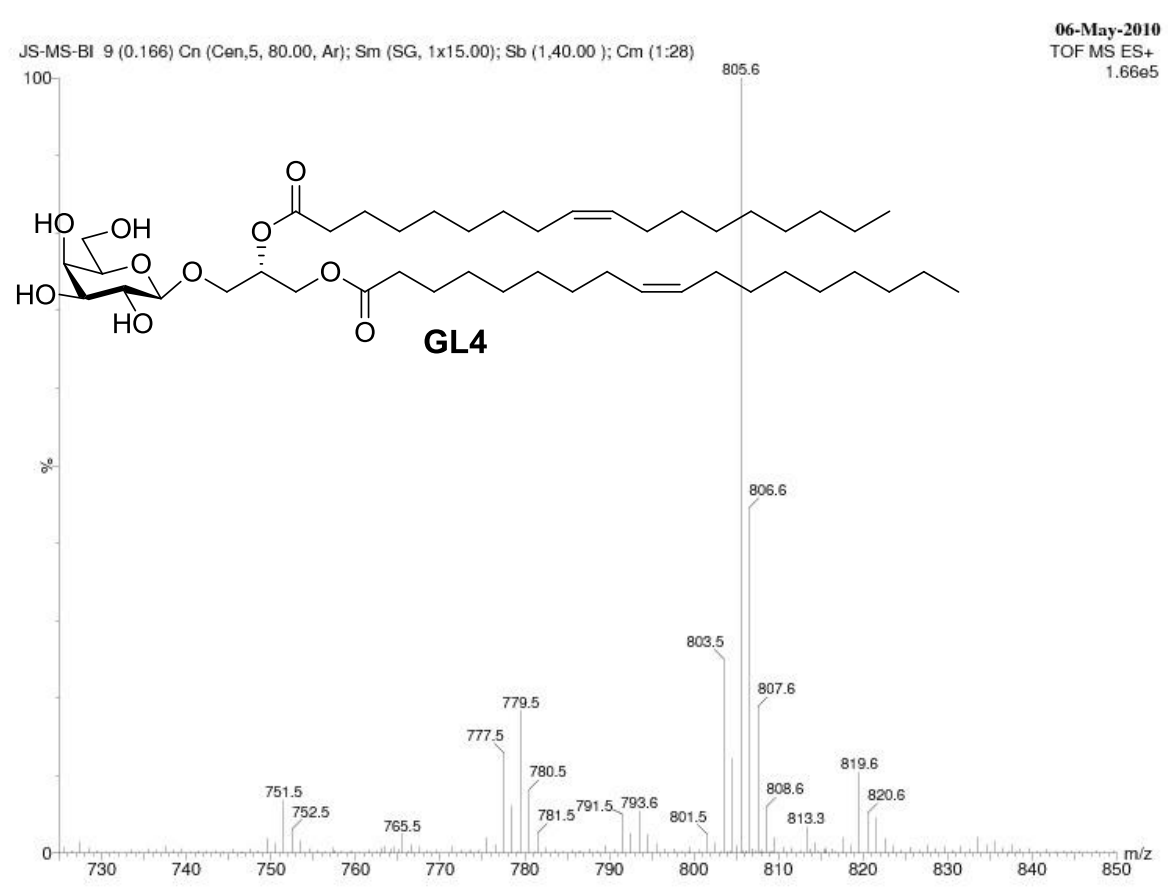

GL4: Colourless oil $(0.5 \mathrm{mg})[\alpha]_{\mathrm{D}}{ }^{20}=-1.0\left(c=0.8, \mathrm{CHCl}_{3} / \mathrm{MeOH}, 2 / 1, \mathrm{v} / \mathrm{v}\right)$; IR (film) 3348, 2922, 2853, 1738, 1366, 1216, 1164, 1073, $567 \mathrm{~cm}^{-1} ;{ }^{1} \mathrm{H},{ }^{13} \mathrm{C}$ NMR, HRMS and $\mathrm{R}_{f}$ data, see Table 3.1 and 3.2. 
GL4, ${ }^{1} \mathrm{H}$ NMR, $\mathrm{CD}_{3} \mathrm{OD} / \mathrm{CDCl}_{3}(1 / 2, \mathrm{v} / \mathrm{v}), 600 \mathrm{MHz}$

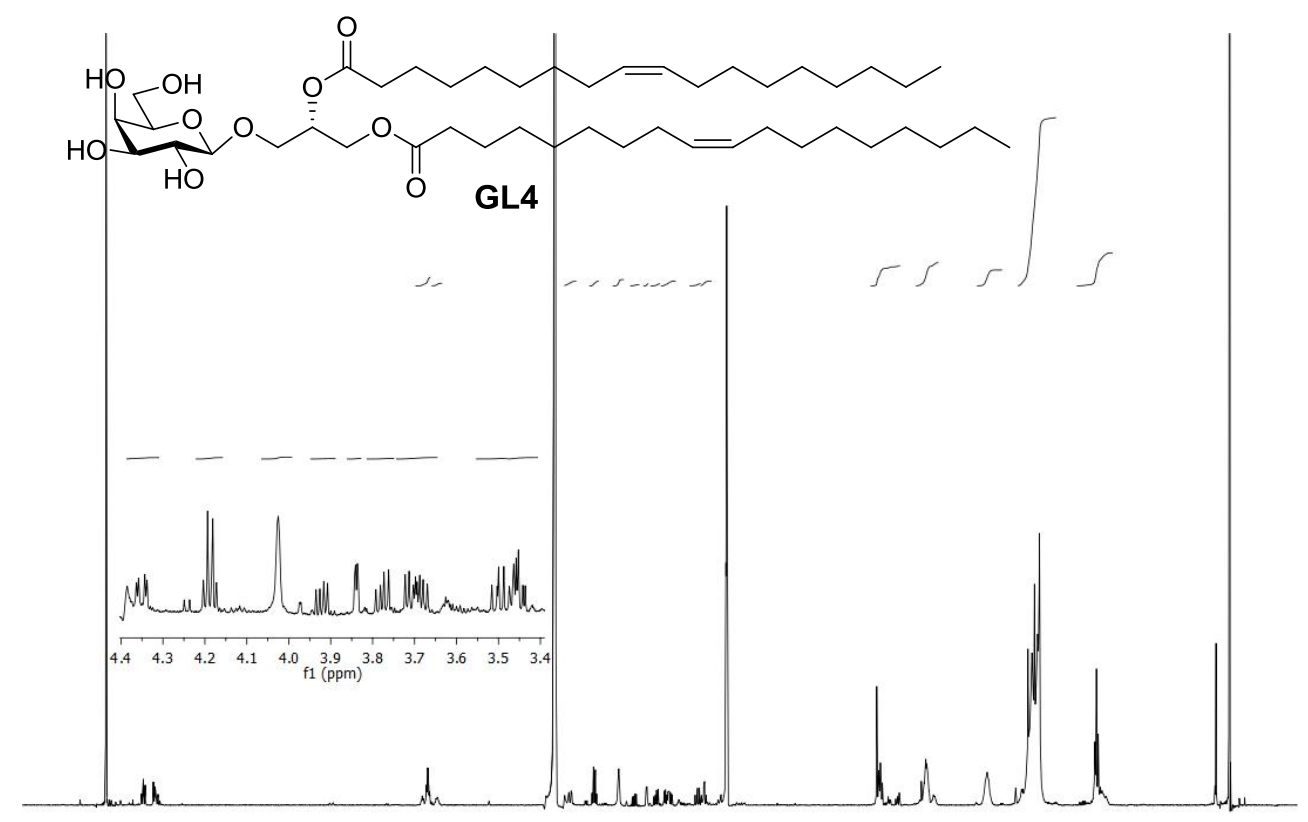

GL4 $,{ }^{1} \mathrm{H}^{1}{ }^{1} \mathrm{H} \mathrm{COSY}, \mathrm{CD}_{3} \mathrm{OD} / \mathrm{CDCl}_{3}(1 / 2, \mathrm{v} / \mathrm{v}), 600 \mathrm{MHz}$

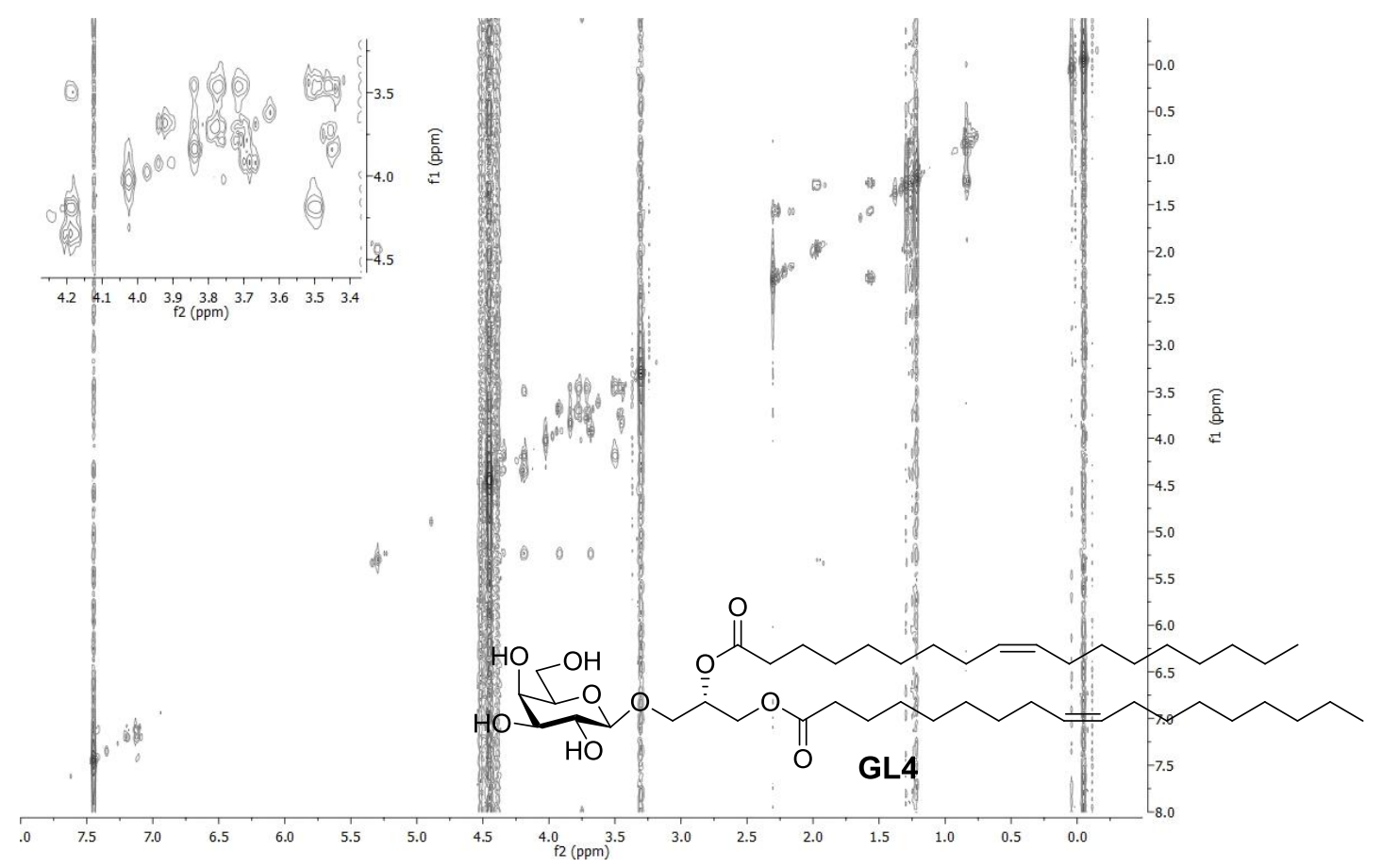


GL4, ${ }^{1} \mathrm{H}^{13} \mathrm{C}$, coupled HSQC, $\mathrm{CD}_{3} \mathrm{OD} / \mathrm{CDCl}_{3}(1 / 2, \mathrm{v} / \mathrm{v}), 600 \mathrm{MHz}$

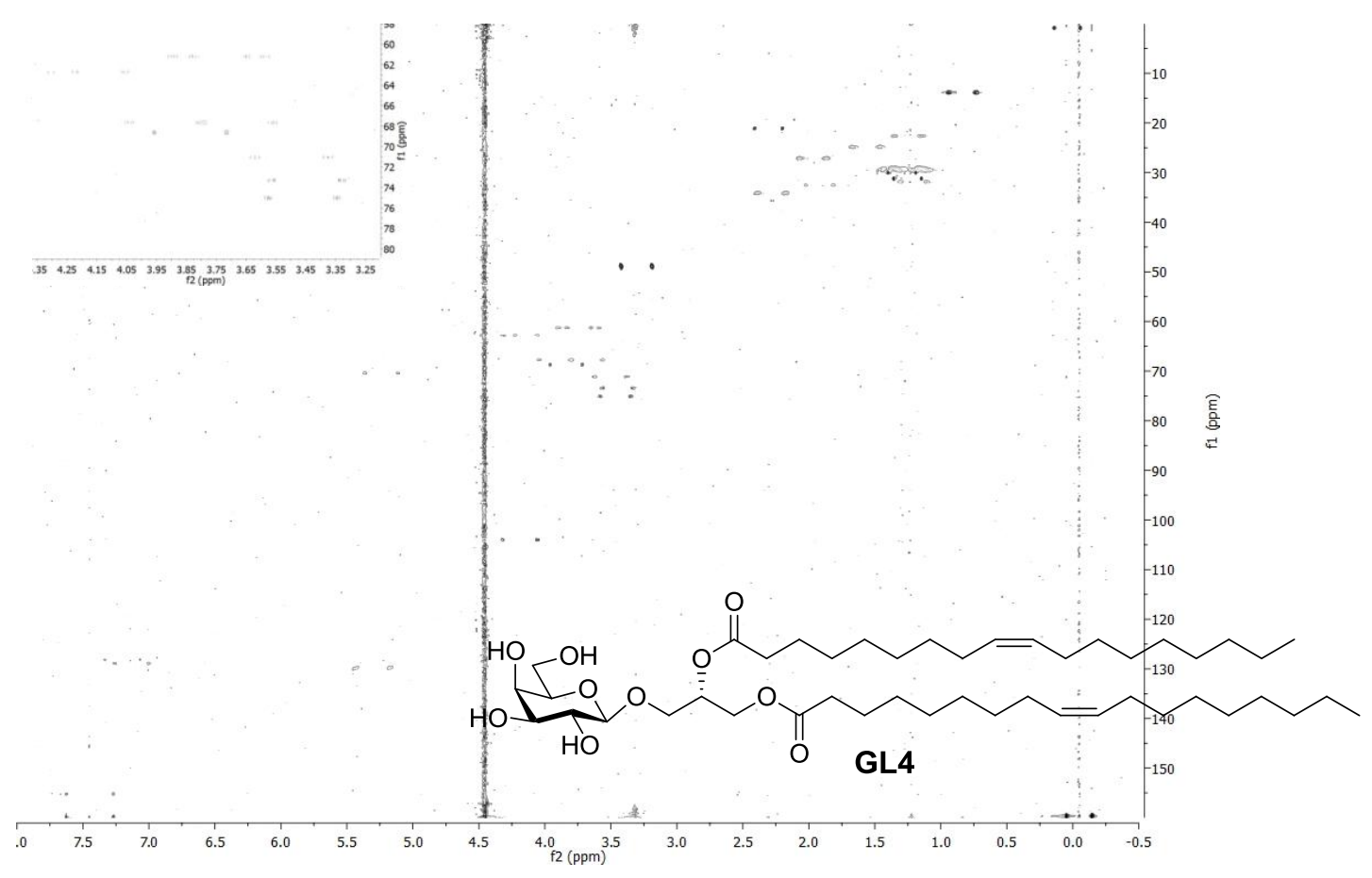

$\mathrm{GL} 4,{ }^{1} \mathrm{H},{ }^{13} \mathrm{C}, \mathrm{HMBC}, \mathrm{CD}_{3} \mathrm{OD} / \mathrm{CDCl}_{3}(1 / 2, \mathrm{v} / \mathrm{v}), 600 \mathrm{MHz}$

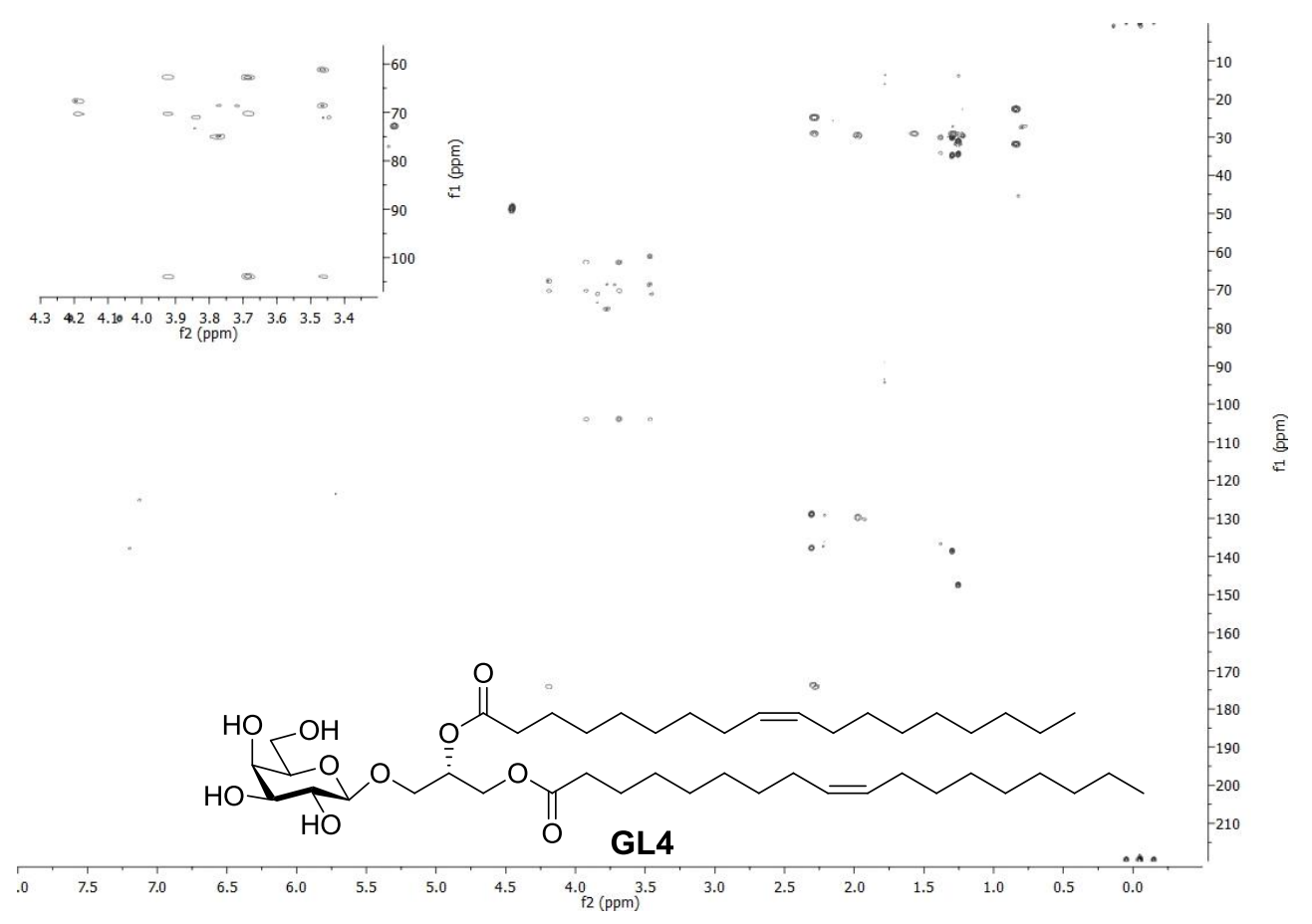




\subsubsection{GL5}

\section{ESI-MS spectrum}

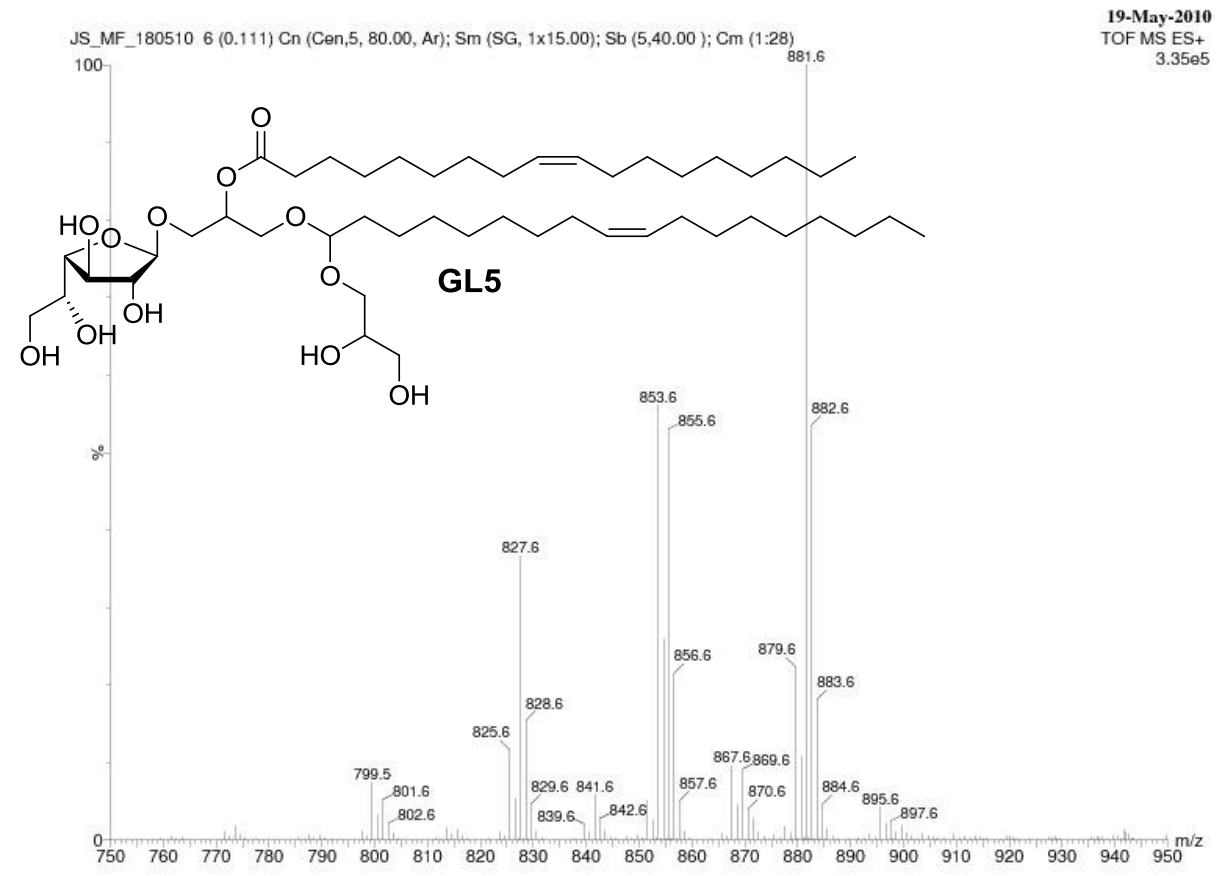

GL5: Colourless oil $(8.6 \mathrm{mg})[\alpha]_{\mathrm{D}}{ }^{20}=-29.4\left(c=0.6, \mathrm{CHCl}_{3} / \mathrm{MeOH}, 2 / 1, \mathrm{v} / \mathrm{v}\right)$; IR (film) 3361, 2922, 2852, 1465, 1249, 1028, 720, $573 \mathrm{~cm}^{-1} ;{ }^{1} \mathrm{H},{ }^{13} \mathrm{C}$ NMR, HRMS and $\mathrm{R}_{f}$ data, see Table 3.1 and 3.2. 
GL5, ${ }^{1} \mathrm{H}$ NMR, $\mathrm{CD}_{3} \mathrm{OD} / \mathrm{CDCl}_{3}(1 / 2, \mathrm{v} / \mathrm{v}), 600 \mathrm{MHz}$

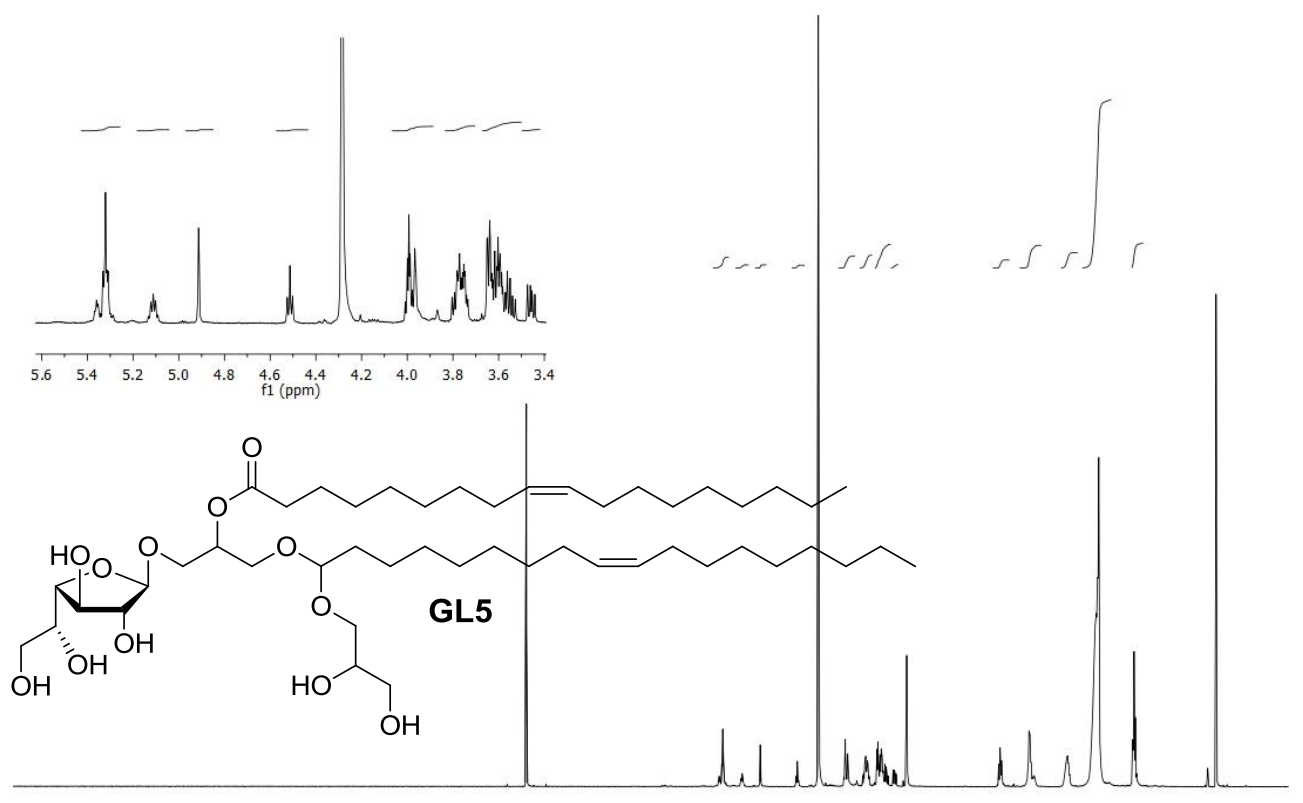

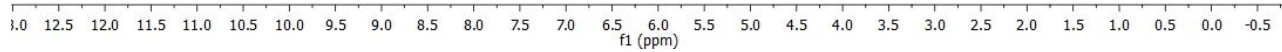

GL5, ${ }^{13} \mathrm{C}$ NMR, $\mathrm{CD}_{3} \mathrm{OD} / \mathrm{CDCl}_{3}(1 / 2, \mathrm{v} / \mathrm{v}), 151 \mathrm{MHz}$

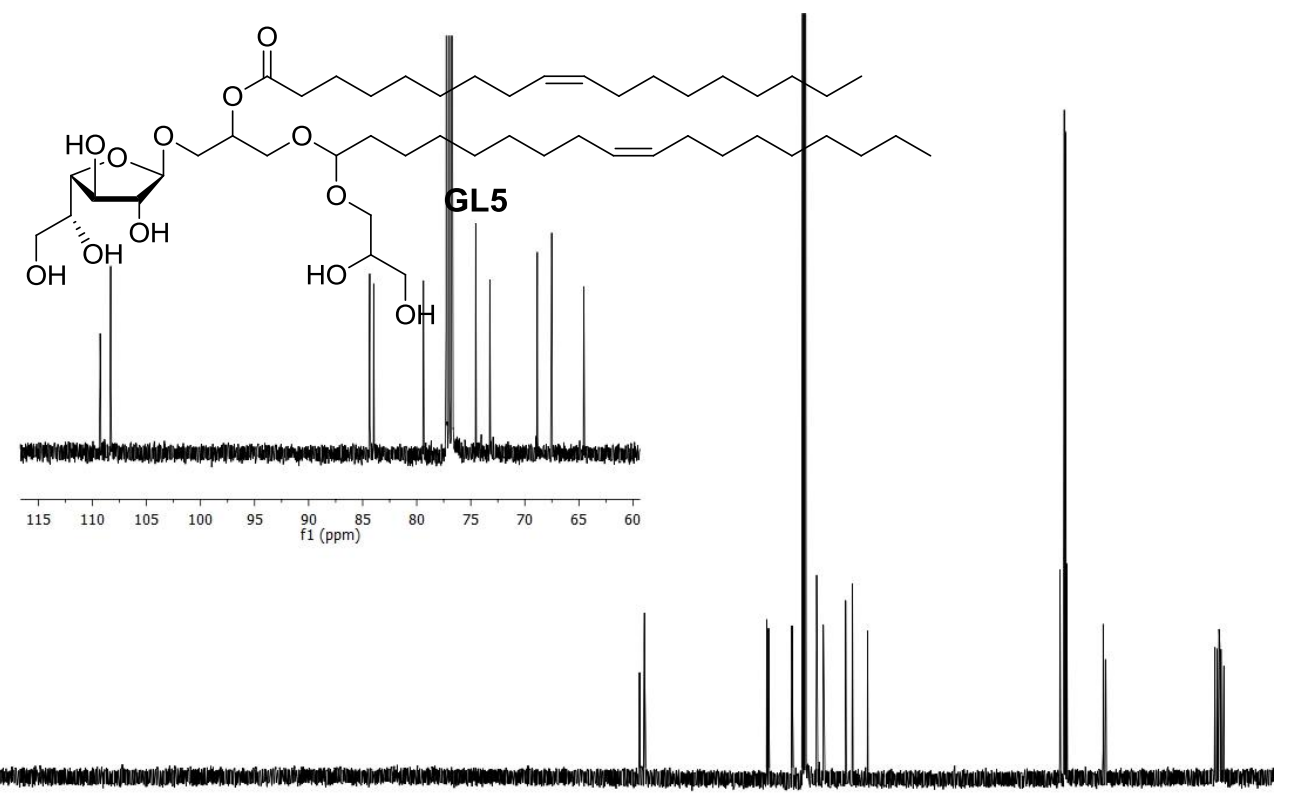

$\begin{array}{llllllllllllllllllllllllllllllll} & 1 & 230 & 220 & 210 & 200 & 190 & 180 & 170 & 160 & 150 & 140 & 130 & 120 & 110 & 100 & 9 & 80 & 70 & 60 & 50 & 40 & 30 & 20 & 10 & 0 & -10\end{array}$ 
GL5, ${ }^{1} \mathrm{H},{ }^{1} \mathrm{H}, \mathrm{COSY}, \mathrm{CD}_{3} \mathrm{OD} / \mathrm{CDCl}_{3}(1 / 2, \mathrm{v} / \mathrm{v}), 600 \mathrm{MHz}$

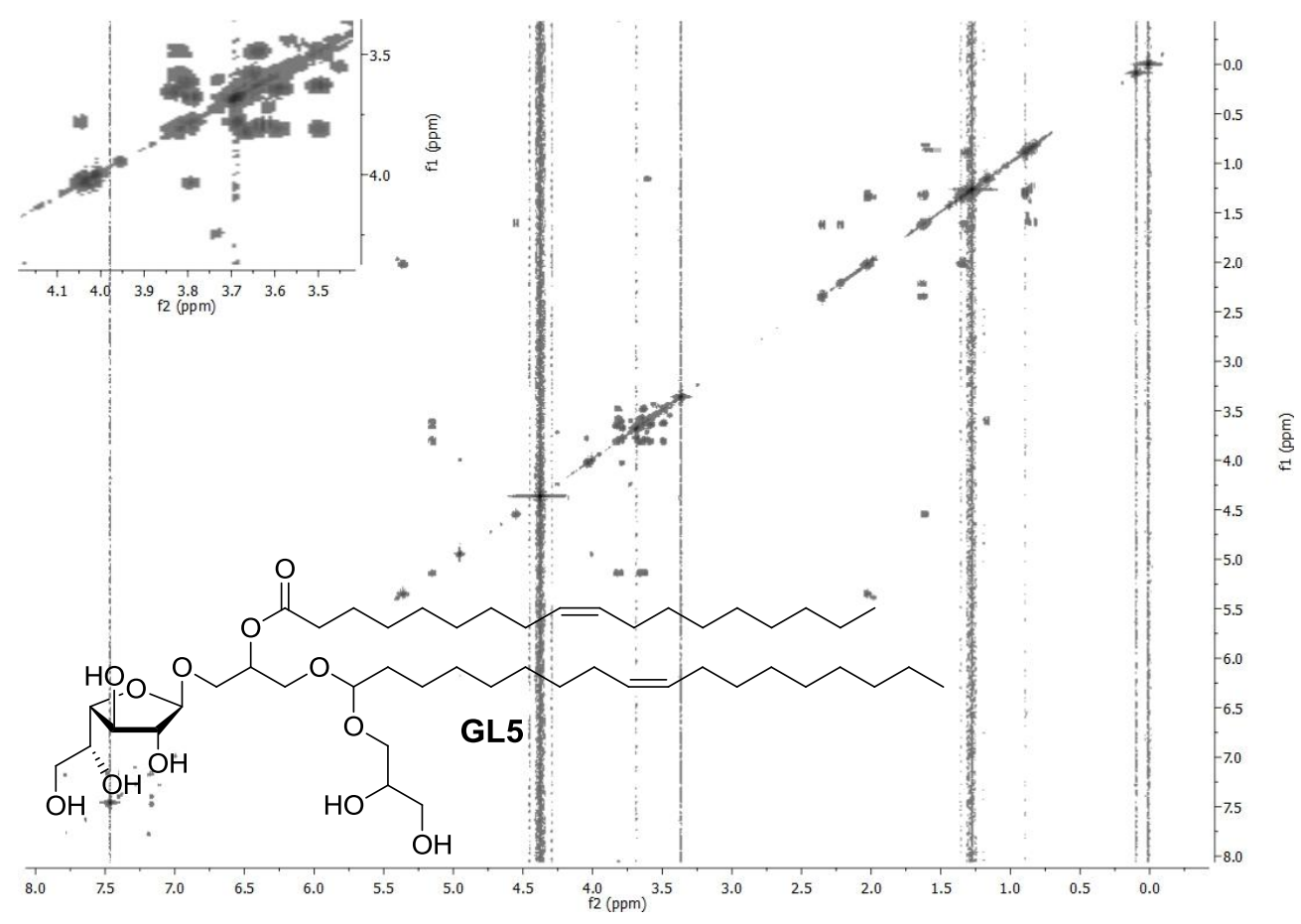

GL5, ${ }^{1} \mathrm{H},{ }^{13} \mathrm{C}$, coupled HSQC, $\mathrm{CD}_{3} \mathrm{OD} / \mathrm{CDCl}_{3}(1 / 2, \mathrm{v} / \mathrm{v}), 600 \mathrm{MHz}$

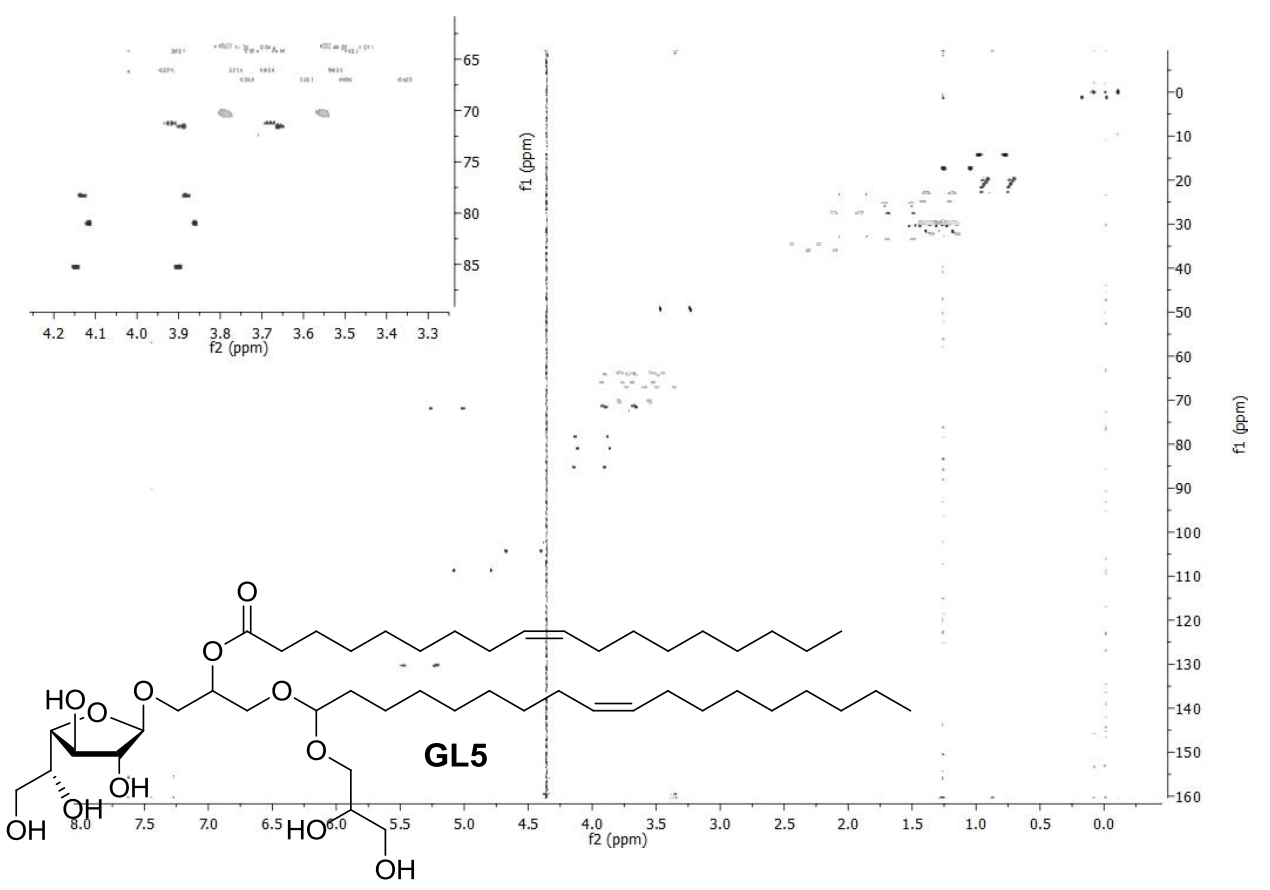


GL5 $,{ }^{1} \mathrm{H}^{13} \mathrm{C}, \mathrm{HMBC}, \mathrm{CD}_{3} \mathrm{OD} / \mathrm{CDCl}_{3}(1 / 2, \mathrm{v} / \mathrm{v}), 600 \mathrm{MHz}$

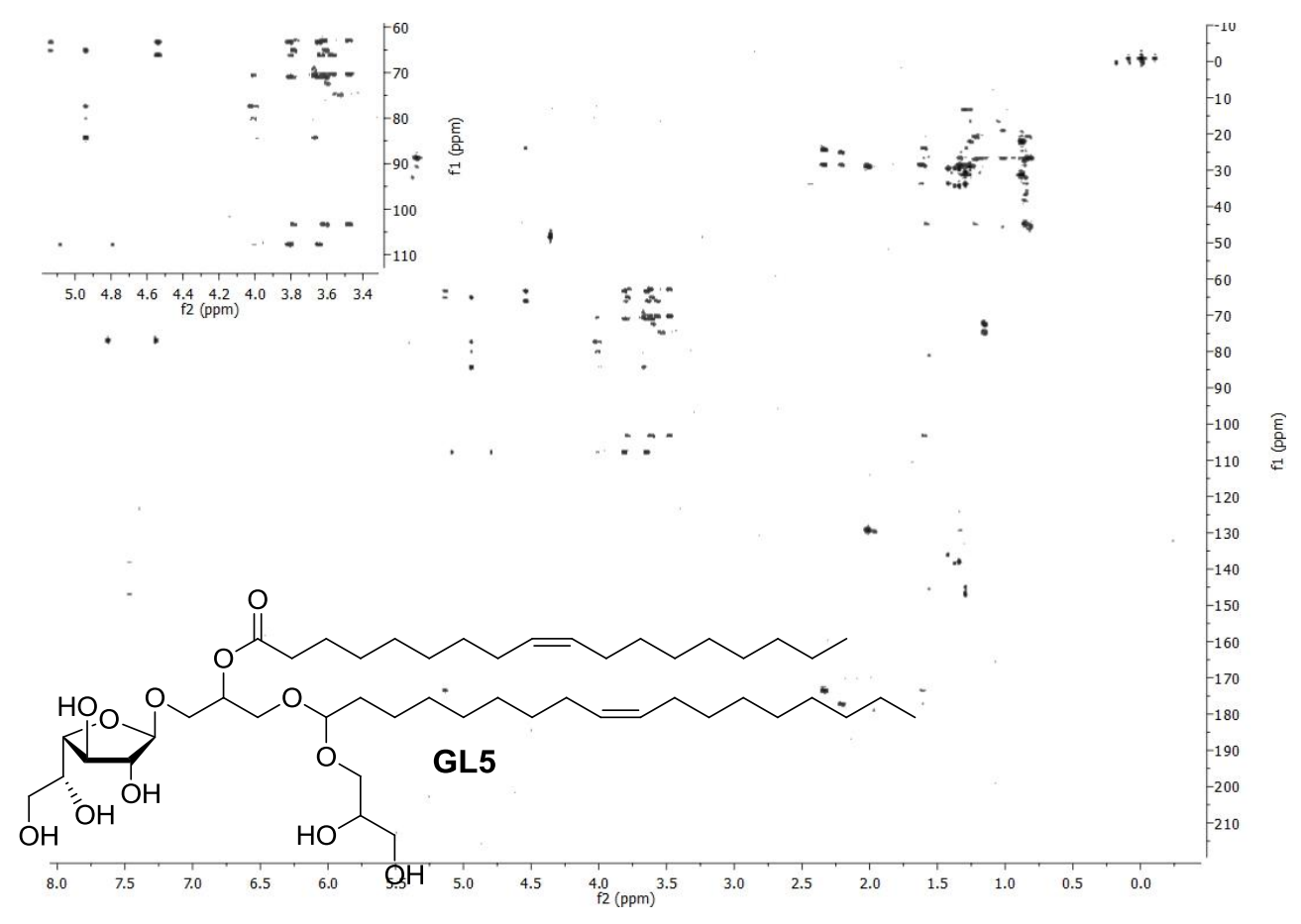




\subsubsection{L3}

ESI-MS in negative ion mode

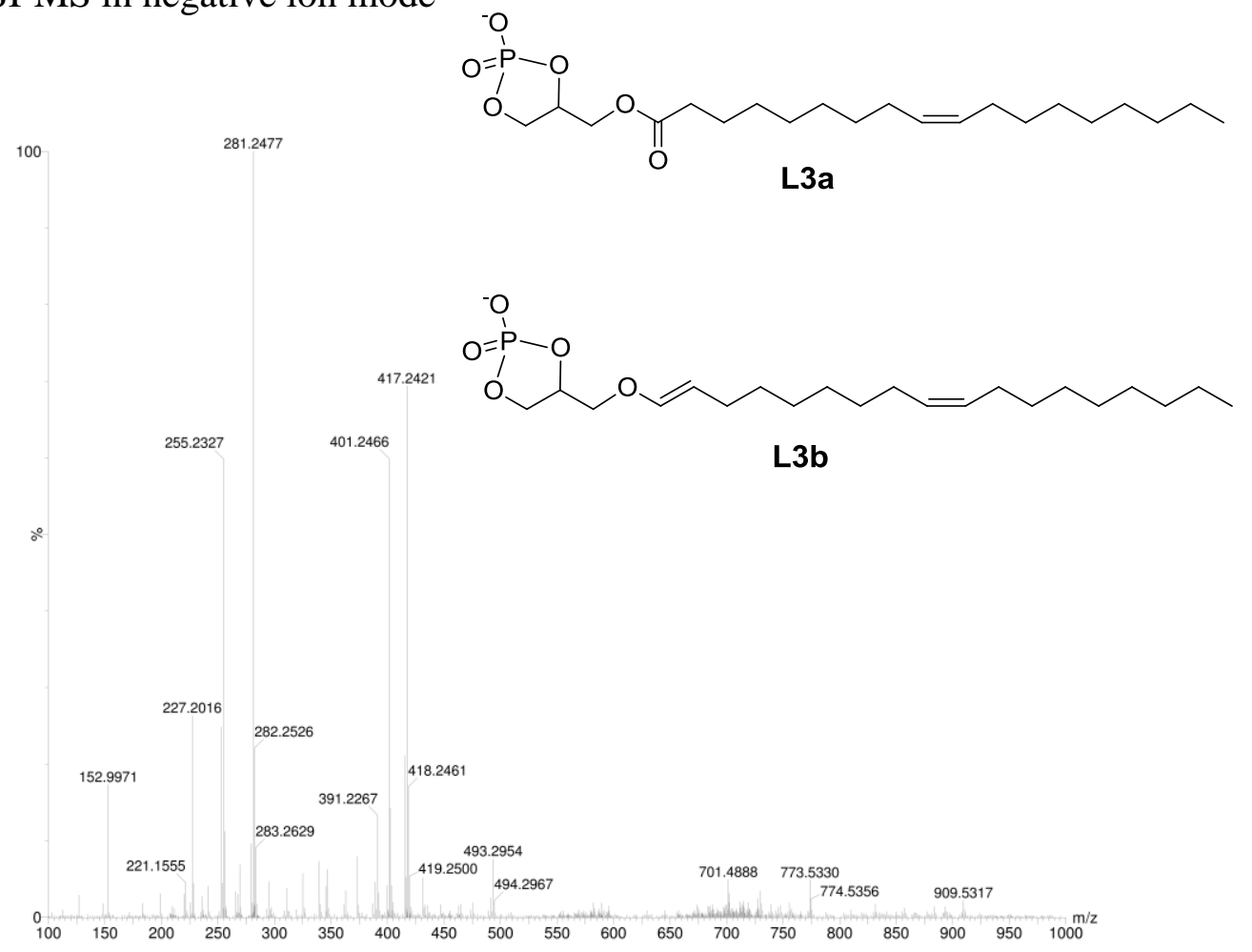

$\mathrm{L} 3,{ }^{1} \mathrm{H}_{-}{ }^{31} \mathrm{P}, \mathrm{HMBC}, \mathrm{CD}_{3} \mathrm{OD} / \mathrm{CDCl}_{3}(1 / 2, \mathrm{v} / \mathrm{v}), 500 \mathrm{MHz}$

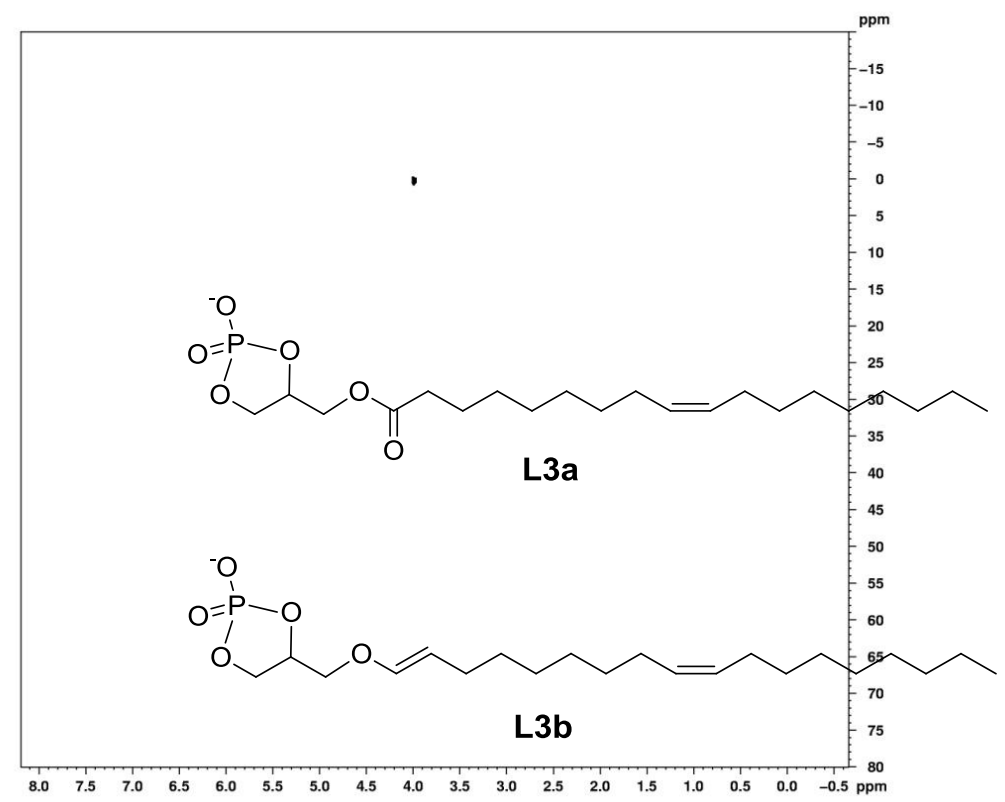




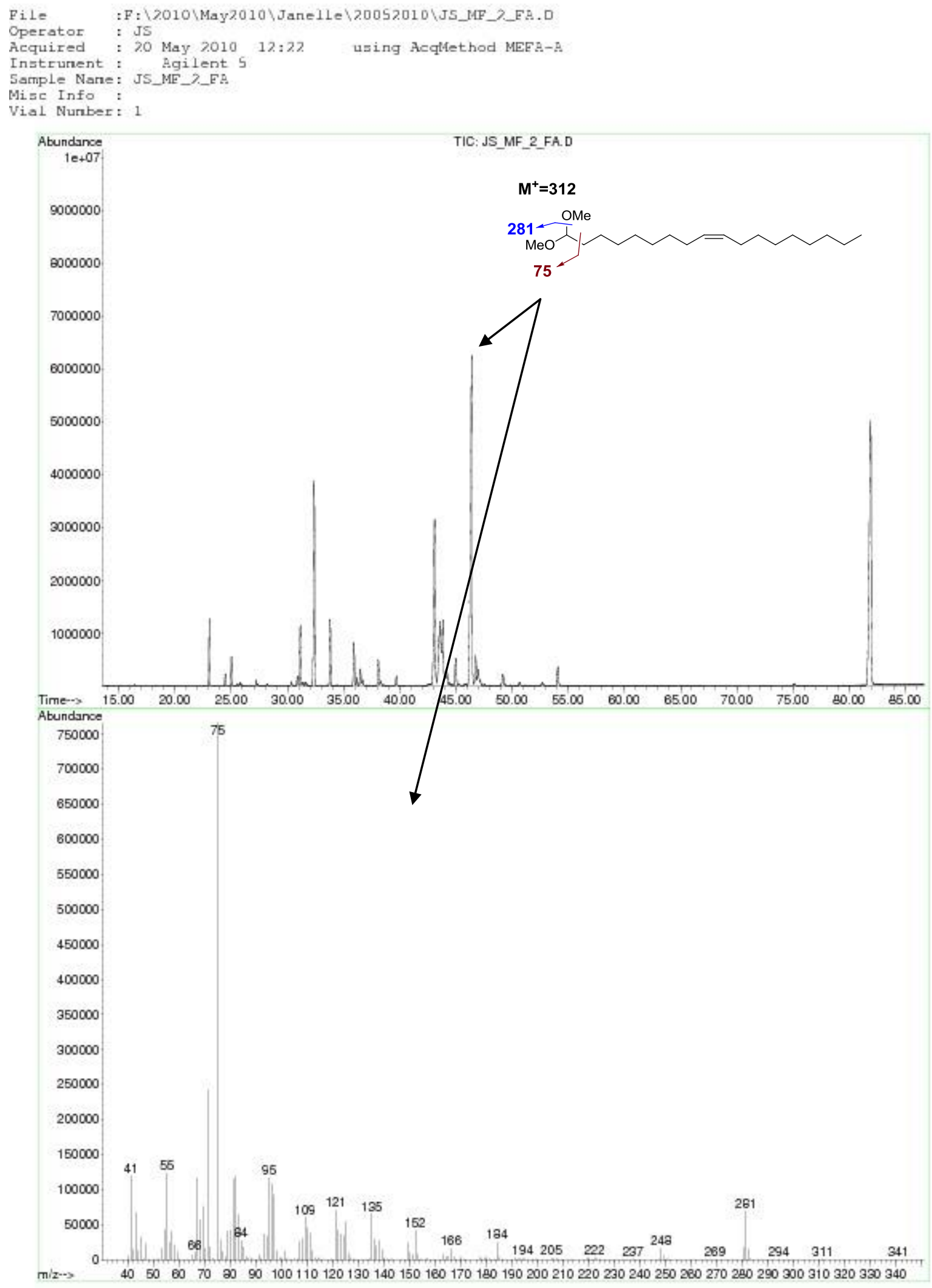




\section{Chapter 4}

\section{Synthesis and Biological Activity of the Lipoteichoic Acid Anchor from Streptococcus sp. DSM $8747^{269}$}

In this study, the role of lipoteichoic acid (LTA) anchors in the activation of the innate immune response was investigated through the chemical synthesis of a series of LTA derivatives and the determination of their ability to induce NO production in bone marrow-derived macrophages (BMM). To this end, an efficient synthesis of the $5 n-3-O$ ( $\alpha$-D-galactofuranosyl)-1,2-di-O-acylglycerol LTA core was developed, which was then used as a key structure to produce both phosphate and glycerylphosphatefunctionalised LTA anchors, as well as galactofuranosyldiglycerides with varying fatty acid chain lengths. With a series of LTA anchors in hand, the effect of these glycolipids on the innate immune response was determined by exploring their capacity to activate macrophages. Here, it is reported that several of the LTA-derivatives were able to induce NO production by BMMs. In general, the unnatural (sn-1) core glycolipid anchors showed lower levels of activity than the corresponding natural (sn-3) analogues, and the activity of the glycolipids also appears to be dependent on the length of lipid present, with an optimum lipid length of C2O for the sn-3 derivatives. Interestingly, a triacylated anchor and the 6-O-phosphorylated anchor, showed only 
modest activity, while the 6-O-glycerophosphorylated derivative was unable to induce NO production. Taken as a whole, the results of this study highlight the subtle effects that glycolipid length can have on the ability to activate BMMs.

\subsection{Background}

Poly(glycerophosphate) lipoteichoic acids (LTAs) are characteristic of Gram-positive bacteria, ${ }^{68,86,202,271}$ yet unlike their counterparts, lipopolysaccharides (LPS, endotoxin), which are found in Gram-negative bacteria, ${ }^{86,202,272,273}$ much debate still remains about the role of LTAs during bacterial infection. ${ }^{86,202,274}$ This is, in part, due to differences in the biological activities of extracted versus synthesised LTAs ${ }^{86,275}$ and highlights the need for structurally defined, non-contaminated LTAs when investigating the effect of these glycolipids on the innate immune response.

LTAs have been classified into four types (types I-IV) based on chemical structure (Chapter 1, Section 1.4.4.3.1). ${ }^{59}$ Although they represent highly challenging synthetic targets, many elegant syntheses of LTAs have been achieved, most notably by Schmidt and colleagues who have made tremendous progress towards understanding the biological relevance of these highly abundant cell wall glycolipids (see Chapter 1, Section 1.5.5). ${ }^{77,92,276}$ Indeed, as one of their synthetic endeavours, Schmidt and coworkers recently reported the first total synthesis of LTA $\mathbf{1}\left(n=4, \mathrm{R}^{1}=30 \%\right.$ D-alanine and $70 \% \mathrm{H}, \mathrm{R}^{2}=\mathrm{C}_{13} \mathrm{H}_{27}$, Figure 4.1), ${ }^{92}$ which is a unique LTA isolated from the cell wall of the Streptococcus species DSM 8747..$^{91}$ LTA 1 contains a D-alanyl substituted (1-3)-linked poly(glycerophosphate) chain linked to the 6-hydroxyl group of $s n-3-O-(\beta-$ D-galactofuranosyl)-1,2-di- $O$-acyl-glycerol, and is the first example in which a monohexosylglycerol serves as a glycolipid anchor. Additionally, furanoses are scarcely seen in LTAs. Moreover, despite the genetic similarity of the Streptococcus sp. to Streptococcus pneumoniae, LTA $\mathbf{1}$ is quite different from the LTA found in Streptococcus pneumoniae, thus further arousing people's interest in the biological function of 1. It is also interesting to note that this specific lipid anchor was thought to be present in Bifidobacterium bifidum var. pennsylvanicus, ${ }^{277}$ although subsequent studies by Fischer found an alternative lipoteichoic-acid-like amphiphile. ${ }^{124}$ 


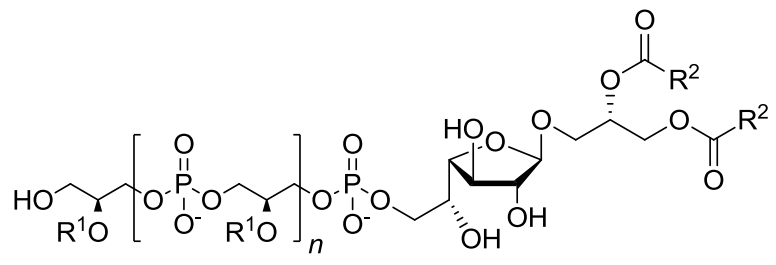

1
$\mathrm{R}^{1}=\mathrm{D}$-alanine, $\mathrm{H}$

$R^{2}=\mathrm{C}_{11} \mathrm{H}_{23}, \mathrm{C}_{13} \mathrm{H}_{27}, \mathrm{C}_{15} \mathrm{H}_{31}, \mathrm{C}_{17} \mathrm{H}_{35}$ and unsaturated fatty esters

$n=7-17$

Figure 4.1 Lipoteichoic acid from Streptococcus sp. DSM 8747.

To understand the effects LTA 1 has on the immune response, the development of an efficient synthesis of the $s n$-3- $O$-( $\beta$-D-galactofuranosyl)-1,2-di- $O$-acyl-glycerol core which could be used as a key structure to understand the effects of the different lipids on biological activity is described here. To date, no biological data have been presented for homogeneous synthetic LTA 1 or derivatives thereof, which is surprising given the marked and varied biological activities of differentially acylated bacterial glycolipids. ${ }^{63,78,80,104,278}$ To this end, here is reported a remarkably efficient synthesis of the core glycolipid anchor of LTA 1 and acylated analogues and, for the first time, insight is provided into the biological effects of these glycolipids on the innate immune response.

\subsection{Results and Discussion}

In devising the retrosynthetic analysis of the glycolipid anchor of LTA 1, the use of a regioselective deprotection strategy of a $\beta$-D-Gal $f$ building block (Scheme 4.1) was envisioned. Here, it was proposed that the core glycolipid anchors $2 \mathbf{2 a - d}\left(\mathrm{R}=\mathrm{H}, \mathrm{R}^{\prime}=\right.$ $\mathrm{C}_{17} \mathrm{H}_{35}, \mathrm{C}_{19} \mathrm{H}_{39}, \mathrm{C}_{21} \mathrm{H}_{43}$ or $\mathrm{C}_{25} \mathrm{H}_{51}$, both glycerol diastereoisomers) could be readily prepared from the per-silylated precursor 6 via global deprotection, whereas the more complex triacylated anchor 3a $\left(\mathrm{R}=\mathrm{R}^{\prime}=\mathrm{C}_{17} \mathrm{H}_{35}\right.$, sn-3-Galf), phosphorylated anchor $4 \mathbf{a}$ $\left(\mathrm{R}=\mathrm{PO}_{3} \mathrm{H}, \mathrm{R}^{\prime}=\mathrm{C}_{17} \mathrm{H}_{35}, s n-3\right)$ and the glycerophosphorylated anchor $5 \mathbf{a}(\mathrm{R}=$ glycerophosphate, $\left.\mathrm{R}^{\prime}=\mathrm{C}_{17} \mathrm{H}_{35}, s n-3\right)$ could be prepared from the selectively protected glycolipid anchor sn-3-7a containing the naturally occurring sn-3-Galf-glycerol stereochemistry. Esterification or phosphorylation of alcohol 7a followed by global 
deprotection, would provide compounds $\mathbf{3 a}$ and $\mathbf{4 a}$, respectively, whereas the more complex phosphorylated LTA anchor 5a would be available via phosphorylation of alcohol 7a with $O$-benzyl phosphoramidite $8 .^{279}$ Preparation of the key differentially protected $\beta$-D-Gal $f$ intermediate 7a was envisioned to be achieved via the selective removal of the TBS group at the 6-position of the furanoside following esterification of diol 9 with stearic (a), arachidic (b), behenic (c) or cerotic (d) acid. Finally, diol 9 could be prepared by way of a $\beta$-selective glycosylation between iodofuranoside 11, generated in situ, ${ }^{280}$ and chiral solketals $\mathbf{1 0}$, which are readily available in either enantiomeric form. ${ }^{281,282}$

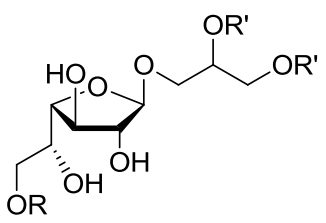

$s n-1-2 a-d, R=H$

sn-3-2a-d, R = H

sn-3-3a, $\mathrm{R}=\mathrm{C}_{17} \mathrm{H}_{35}$

sn-3-4a, $\mathrm{R}=\mathrm{PO}_{3} \mathrm{H}_{2}$

sn-3-5a, $\mathrm{R}=\mathrm{C}_{3} \mathrm{H}_{7} \mathrm{O}_{5} \mathrm{P}$
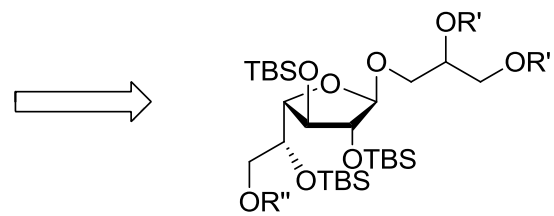

$s n-1-6 a-d, R^{\prime \prime}=$ TBS

$s n-3-6 a-d, R^{\prime \prime}=$ TBS

sn-3-7a, R" = H
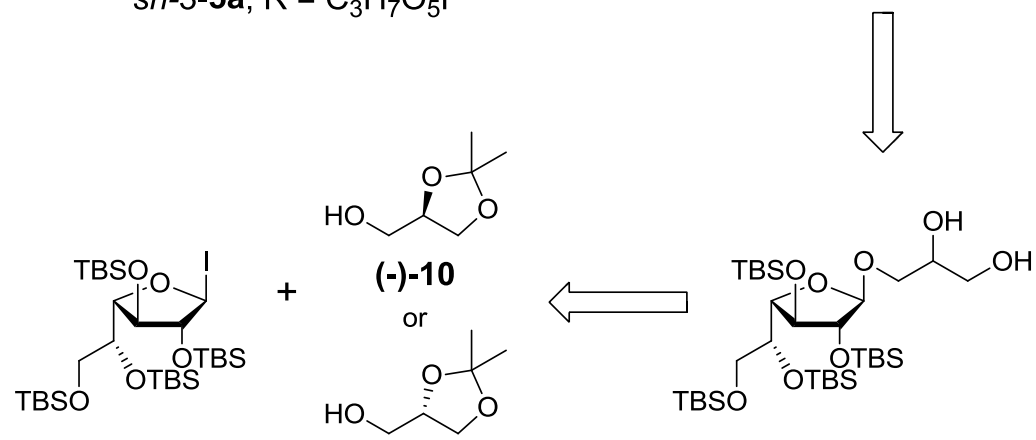

11

(+) -10

9, (sn-1- or sn-3-Galf)
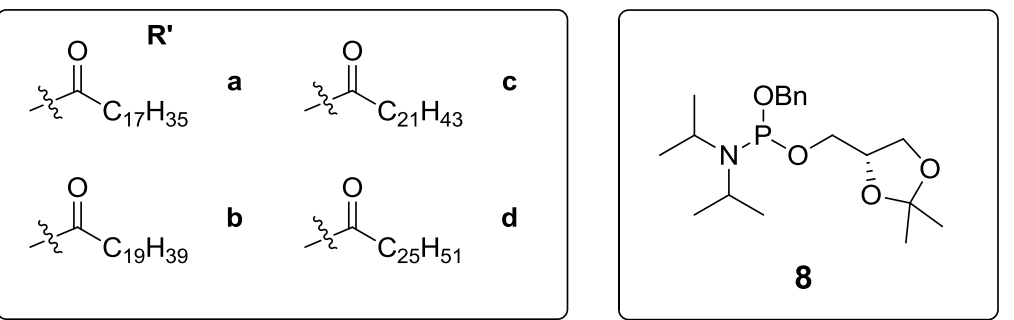

Scheme 4.1 Retrosynthesis of the LTA glycolipid anchor and lipidated derivatives. 
With the retrosynthetic strategy in place, the synthesis of core glycolipid anchor 2 was first investigated. To this end, it was decided to synthesise building blocks (-)- and (+)10. The synthesis of chiral solketals has been previously documented by different groups ${ }^{281,282}$ and chiral solketals (-)-10 and (+)-10 are commercially available (for 100 $\mathrm{mL}, \$ 2500$, Fluka). In saying this, there are various advantages to synthesising the compounds. Firstly, they are expensive to purchase although the reagents used in their synthesis are relatively cheap. Secondly, large quantities are needed for the research and the shipping times to New Zealand were lengthy. To attempt the synthesis of glycerol derivative (-)-10, Hirth's method was initially employed (Scheme 4.2). ${ }^{281}$ Here, the deamination was performed on L-serine 12 using sulfuric acid and sodium nitrite in water to obtain alcohol 13. After precipitation of sodium sulfate using methanol and acetone, the filtrate was concentrated in vacuo. Next, the appropriate amount of trimethoxymethane was added in excess with Dowex $\mathrm{H}^{+}$and methanol. However, only a small amount of the methyl ester 14 was recovered (3\%). Consequently, the approach was altered and PTSA and dimethoxypropane were employed in an attempt to form methyl ester 14. Unfortunately, this method was also low yielding despite varying the amount of PTSA, dimethoxypropane, temperature and reaction time. Following these trials, the method of Dorp and co-workers was successfully implemented (Scheme 4.3). ${ }^{283}$ Here instead of using sulfuric acid used in Hirth's method, hydrochloric acid was used for the deamination and acid $\mathbf{1 3}$ was esterified and protected with an acetonide uneventfully. Crude methyl ester $\mathbf{1 4}$ was then pure enough for reduction using $\mathrm{NaBH}_{4}$ to obtain chiral solketal (-)-10 in $40 \%$ overall yield after distillation (found: $[\alpha]_{\mathrm{D}}{ }^{19}=-$ $9.8, \mathrm{c}=1.0, \mathrm{MeOH}$, literature: $\left.[\alpha]_{\mathrm{D}}{ }^{20}=-11.1, \mathrm{c}=5.0, \mathrm{MeOH}\right){ }^{281}$

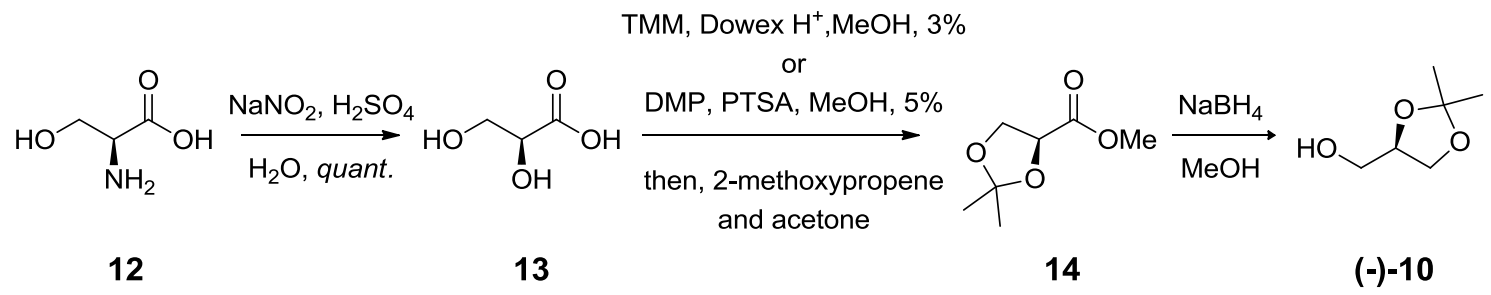

Scheme 4.2 $R$-(-)-Solketal ((-)-10) synthesis from the method of Hirth et al. ${ }^{281}$ 
With chiral solketal (-)-10 in hand, chiral solketal (+)-10 was then synthesised. In contrast to the synthesis of chiral solketal (-)-10, D-mannitol was used as a starting material. The synthesis involved converting D-mannitol 15 into diacetonide 16 using acetone and zinc chloride. The diol was then cleaved using sodium periodate to form aldehyde 17, which was reduced with sodium borohydride (Scheme 4.4). Here, the compounds were not purified between steps, the only purification utilised was a distillation of final product (+)-10. The first time this methodology was attempted it produced a racemic mixture of products. As epimerisation is more likely to occur in the oxidation step, it was concluded that shorter reaction times were essential for this step. Indeed, after a shorter periodate oxidation step of 15 minutes, the reaction mixture was immediately reduced to give chiral solketal $(+)-\mathbf{1 0}$ in $58 \%$ overall yield (found: $[\alpha]_{\mathrm{D}}{ }^{19}=$ 11.0, $\mathrm{c}=1.0, \mathrm{MeOH}$, literature: $\left.[\alpha]_{\mathrm{D}}^{20}=11.6, \mathrm{c}=5.0, \mathrm{MeOH}\right)$.

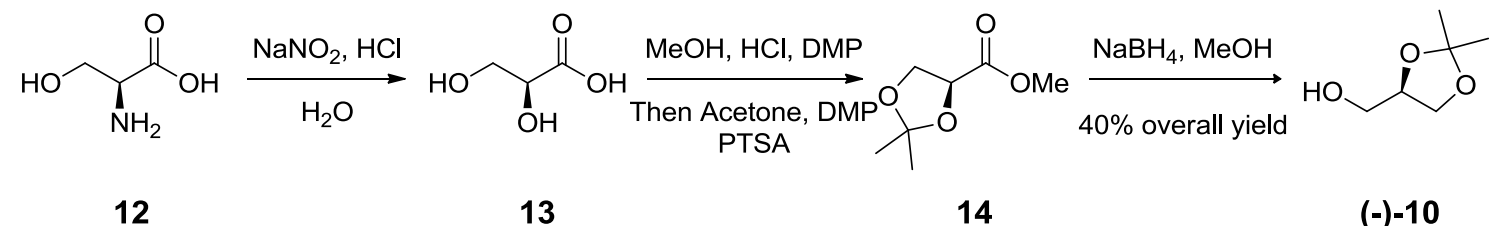

Scheme 4.3 R-(-)-Solketal ((-)-10) synthesis from the method of Lok et al. ${ }^{283}$<smiles>OC[C@@H](O)C(O)[C@H](O)CO</smiles>

15

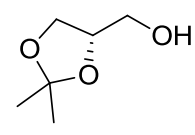

$(+)-10$<smiles>CC1(C)OC[C@H]([C@@H](O)[C@@H](O)[C@H]2COC(C)(C)O2)O1</smiles>

16

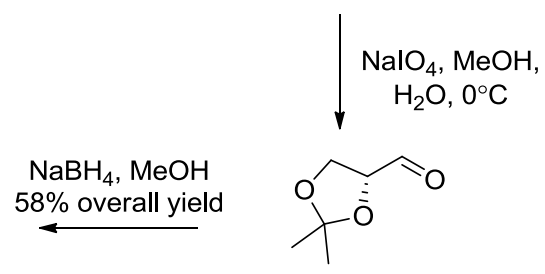

17

Scheme 4.4 $S$-(+)-Solketal ((+)-10) synthesis from the method of Eibl et al. $^{282}$ 
With the $R$ and $S$-solketals in hand, the recently developed procedure of Baldoni and Marino was then employed ${ }^{280}$ to synthesise the per-silylated furanoside 19 from Dgalactose 18 (72\% yield) (Scheme 4.5). When compared to more traditional syntheses of similar galactofuranosyl donors, which involve a intricate series of protection and deprotection steps, ${ }^{92,284-287}$ the methodology of Baldoni and Marino was remarkably efficient, with the steric bulk of the TBS-groups being thought to favour formation of the furanose isomer.

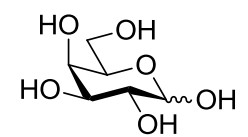

18

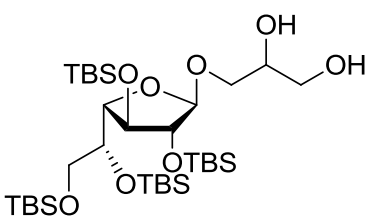

sn-1-9

sn-3-9

EDCl, acid

DMAP, Toluene

$40{ }^{\circ} \mathrm{C}$ or $70^{\circ} \mathrm{C}$

$87-97 \%(\mathbf{a}$ and $\mathbf{b})$

$65-77 \%$ (c and $\mathbf{d})$

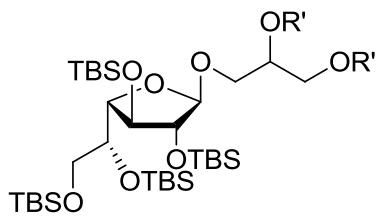

$s n-1-6 a-d$

sn-3-6a-d
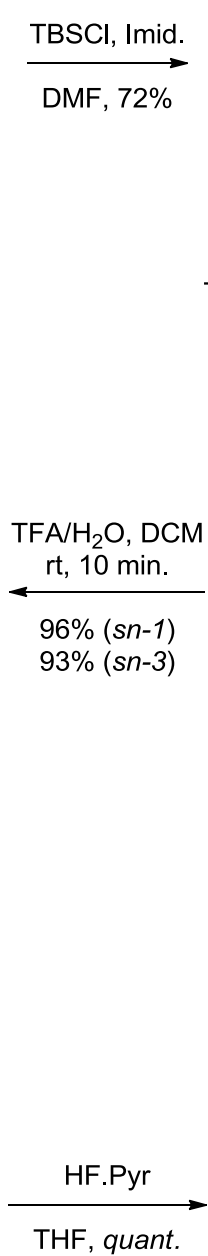

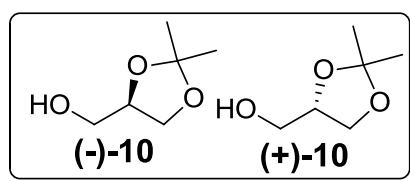

then DIPEA, 10

$90 \%(\alpha: \beta ; 1: 9)$

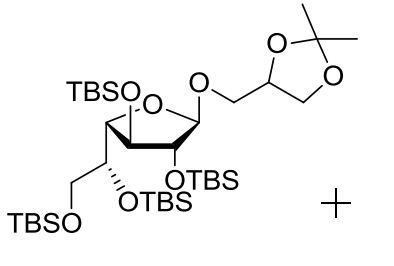

$s n-1-20$

sn-3-20

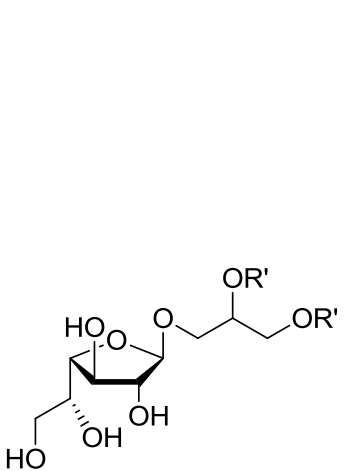

$s n-1-2 a-d$

$s n-3-2 a-d$
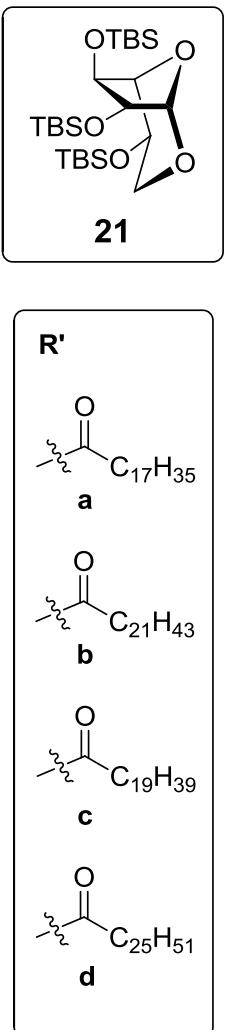

Scheme 4.5 Synthesis of core glycolipid anchor 2 . 
As seen in Chapter 1 (Section 1.5.5), a methodology that has been used to form galactofuranoses involves an anomeric $O$-alkylation step. This is followed by benzylation, deprotection of the anomeric position, and finally imidate formation for later coupling. ${ }^{92,284}$ The perbenzoylation of galactose with benzoyl chloride has also been reported to form the furanose. ${ }^{285}$ Here a $\alpha / \beta$ mixture of $56 / 44$ was obtained in $77 \%$ yield. However, with this method a 2-step 1-pot glycosylation using TMSI is not recommended as the leaving benzoyl will preferrentially recouple to the anomeric position. Such a 2-step 1-pot glycosylation strategy using tin (IV) chloride with perbenzoylated and peracetylated galactofuranose moieties was successfully attempted but afforded only modest yields (60-67\%) when compared to the silylation proposed by Baldoni. ${ }^{286,287}$ For these reasons, Baldoni’s 2-step 1-pot glycosylation strategy seemed appealing and was attempted. ${ }^{280}$ Here, protected galactofuranose building block 19 was treated with TMSI, to generate the intermediate glycosyl iodide, and to this (+)- or (-)solketal $\mathbf{1 0}^{281,282}$ was added. While this methodology led to the formation of the target glycolipids 20, the yield for these glycosylations was highly variable (44-76\%), this being attributed to the varying quality of the TMSI despite freshly distilling this reagent prior to use. Indeed, much improved and more consistent yields (90\% for both $s n-1-20$ and sn-3-20 derivatives) were observed if the TMSI was generated ex situ (via the addition of $\mathrm{I}_{2}$ to a small excess of neat TMSTMS) ${ }^{288}$ and then added to the per-silylated galactofuranose 19 to give derivative $20(\alpha / \beta, 1 / 9)$. It was also noted that the glycosylation reaction should be performed under dilute conditions $(38 \mathrm{~mL} / \mathrm{mmol})$ to limit formation of bicyclic product 21 (HRMS calc. for $\left[\mathrm{C}_{24} \mathrm{H}_{52} \mathrm{NaO}_{5} \mathrm{Si}_{3}\right]^{+}=527.0315$, found: 527.0310), which is obtained via cyclisation of the 6-position onto the iodinated anomeric centre. The $\beta$-configuration of the major product $\mathbf{2 0}$ was then confirmed by ${ }^{13} \mathrm{C}$ NMR with $\mathrm{C}-1^{\prime}$ having a characteristic NMR shift of $108.8 \mathrm{ppm}$ for the $s n-3-\mathrm{Gal} f \beta$ anomer. $^{251,280}$ In contrast, the $\alpha$-anomer had a shift of $102.3 \mathrm{ppm}$ for $\mathrm{C}-1^{-}{ }^{251,280}$ Purification of the anomers ( $\alpha$-anomer, $\mathrm{R}_{f}=0.44, \mathrm{EA} / \mathrm{PE}, 1 / 9, \mathrm{v} / \mathrm{v} ; \beta$-anomer, $\mathrm{R}_{f}=0.41$, EA/PE, 1/9, v/v) was possible via careful silica gel flash column chromatography, however separation became easier following deprotection of the acetonide (vide infra). Finally, to confirm that acceptor $\mathbf{1 0}$ had not racemised during the course of the reaction, the reaction was repeated using racemic $( \pm)-\mathbf{1 0}$. Here, extra resonances were observed in the ${ }^{13} \mathrm{C}$ NMR spectrum to account for the additional isomers that had been produced [e.g. two resonances (108.8 $\mathrm{ppm}$ and $108.6 \mathrm{ppm})$ were observed for $\mathrm{C}-1^{\prime}$ of the $\beta$ - 
anomer when the glycosylation was performed using racemic 10]. In contrast, the use of enantiomerically pure $\mathbf{1 0}$ resulted in the formation of only one $\beta$-product (Figure 4.2).

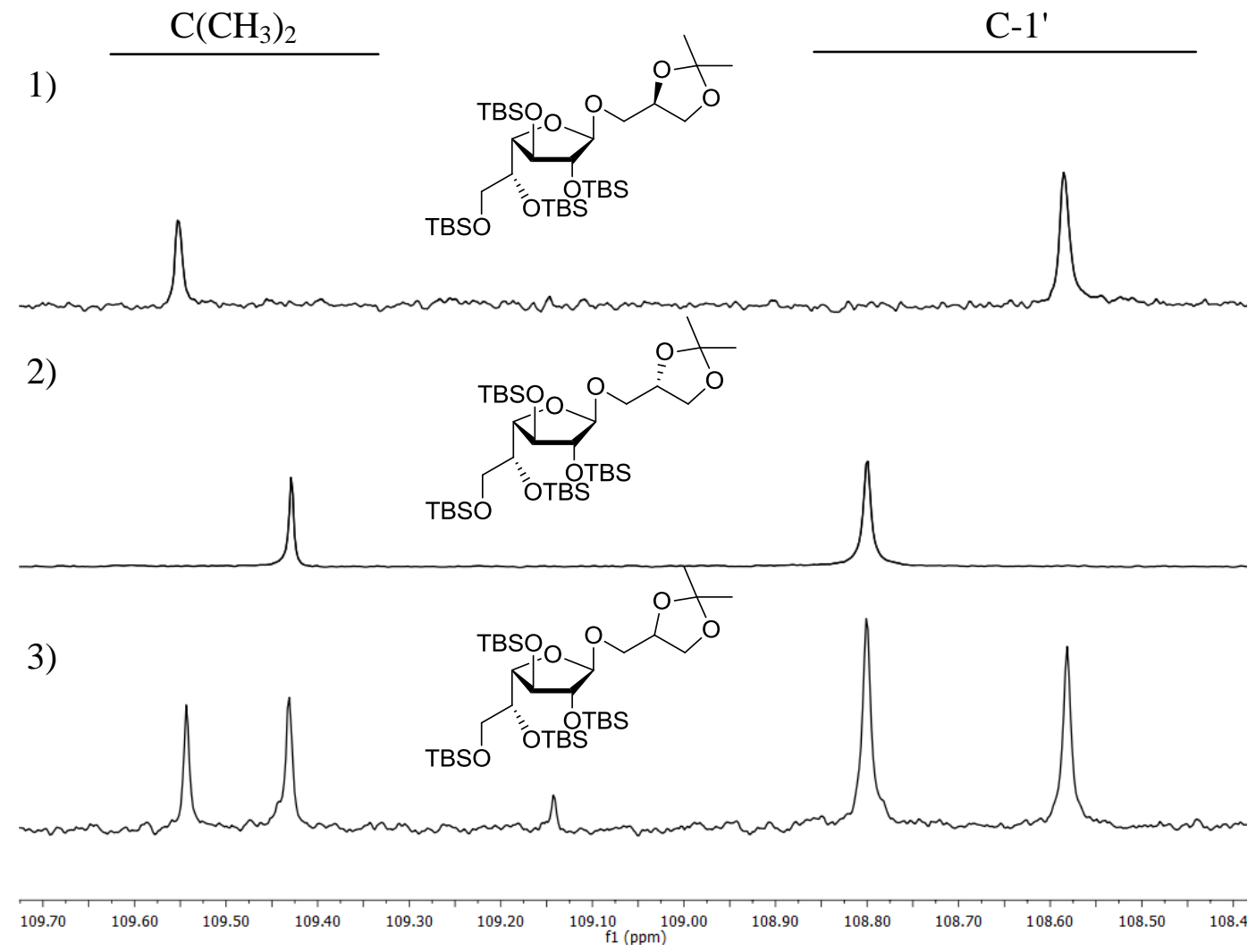

Figure 4.2 ${ }^{13} \mathrm{C}$ NMR spectrum of compounds 20 1., sn-3-20 2. and $s n-1-203$. showing the anomeric and acetal quaternary carbon region.

Having selectively prepared glycosides $\mathbf{2 0}$ in good yields, attempts were then made to remove the acetonide without concomitant removal of the TBS groups. To this end, glycolipids 20 were treated with $\mathrm{AcOH} / \mathrm{H}_{2} \mathrm{O}$ (4/1, v/v, $50{ }^{\circ} \mathrm{C}$, overnight). ${ }^{289}$ Unfortunately, this protocol resulted in $O$-desilylation as well as the desired acetonide deprotection and a complex mixture of products was observed. Other deprotection conditions were then investigated, including the use of CSA (2 equiv.) ${ }^{290}$ or $\mathrm{SnCl}_{2}$ (4 equiv.), ${ }^{291}$ though in both instances only starting material was recovered. The use of $\mathrm{TFA} / \mathrm{H}_{2} \mathrm{O}$ in DCM at room temperature ${ }^{290}$ appeared more promising, with diol 9 being obtained in a modest (20\%) yield, along with a mixture of partially desilylated products. The use of THF instead of DCM led to a reduced yield of deprotected 9, so the $\mathrm{TFA} / \mathrm{H}_{2} \mathrm{O} / \mathrm{DCM}$ strategy was revisited. Indeed, close monitoring of the reaction by TLC 
indicated that a major product $\left(\mathrm{R}_{f}=0.37, \mathrm{EA} / \mathrm{PE}, 3 / 7, \mathrm{v} / \mathrm{v}\right)$ was observed directly after the addition of TFA/ $\mathrm{H}_{2} \mathrm{O}$ and that this product quickly disappeared over time leading to baseline material. In view of this, it was determined that very short reaction times were needed to prevent desilylation and thus the reaction $(\mathbf{2 0} \rightarrow \mathbf{9})$ was quenched with a solution of $\mathrm{NaHCO}_{3}$ (sat. aq.) after only 10 minutes. Gratifyingly, this approach proved successful and both isomers of 9 were obtained in an excellent (93-96\%) yield.

Having established the selective deprotection conditions, an EDCI mediated esterification of diols 9 then followed. The esterification of diol 9 using stearic acid or arachidic acid proceeded uneventfully to yield the protected glycolipids $6 \mathbf{a}$ and $\mathbf{6 b}$ in excellent (87-97\%) yields after heating at $40{ }^{\circ} \mathrm{C}$ for $14 \mathrm{~h}$. The longer lipids, behenic or cerotic acid, however, were sluggish to react and required heating to $70{ }^{\circ} \mathrm{C}$ to give the target esters, $\mathbf{6 c}$ and $\mathbf{6 d}$, in $65 \%$ and $77 \%$ yield, respectively. The formation of the first LTA glycolipid anchor, sn-3-2a, was then attempted via the treatment of a solution of protected anchor $s n-3-6 a$ in THF with TBAF (8 equiv.) for $12 \mathrm{~h}$. These conditions resulted in the smooth transformation of the per-silylated $\mathbf{6 a}$ into anchor $\mathbf{2 a}$ as judged by TLC analysis $\left(\mathrm{R}_{f}=0.61, \mathrm{MeOH} / \mathrm{DCM}, 1 / 6, \mathrm{v} / \mathrm{v}\right)$, although the purification of anchor $\mathbf{2 a}$ proved problematic due to contamination with TBAF. Repeated silica gel column chromatography and/or dialysis could yield pure glycolipid anchor 2a, but such purification measures severely compromised the yield. Consequently, the final deprotection of per-silylated 6a was attempted using Dowex $-\mathrm{H}^{+}$resin. Although this gave a quantitative yield of desired $\mathbf{2 a}$, long reaction times were required (7 days) due to the need to use only a small amount of resin to prevent cleavage of the stearic acid esters. Accordingly, deprotection of anchor $\mathbf{2 a}$ was further attempted using HF.pyridine. Indeed, this latter strategy proved the most successful with global deprotection being achieved after $24 \mathrm{~h}$ and in quantitative yield following aqueous work-up. The other glycolipid anchors $\mathbf{2 b - d}$ ( $s n-1$ and $s n-3)$ were similarly prepared via the treatment of their corresponding per-silylated counterparts with HF.pyridine. In summary, the synthesis of glycolipid anchor $s n-3-2 \mathbf{a}$ was achieved in a remarkable six steps and in a $51 \%$ overall yield, which is the most efficient synthesis to date. ${ }^{92}$ The other derivatives (with arachidic, behenic or cerotic acid) were also synthesised in excellent overall yields. The strategy, involving the use of TBS groups, is very versatile as fatty acids with unsaturation can also be coupled. 
Having successfully prepared the core glycolipid anchors 2, the synthesis of the more complex LTA derivatives, $s n-3-3 a, s n-3-4 a$ and $s n-3-5 a$ (Scheme 4.6 - 4.10) was then attempted. Here, the greater lability of the silyl group at the 6-position of protected anchor $s n$-3-6a was exploited with the reaction conditions optimised to achieve the selective deprotection of the primary silyl group without further desilylation. Interestingly, the use of TFA/ $\mathrm{H}_{2} \mathrm{O}$ in DCM gave trace amounts of product $7 \mathbf{a}$, with various $O$-desilylation products being formed. TFA/ $\mathrm{H}_{2} \mathrm{O}$ in THF, however, resulted in a $72 \%$ yield of alcohol $7 \mathbf{a}$ after stirring for $48 \mathrm{~h}$. At this time, a small amount of starting material as well as some additional desilylation products, were observed by TLC analysis. Longer reaction times did not increase the yield and the $48 \mathrm{~h}$ time point was found to be optimum (Scheme 4.6). Confirmation of the synthesis of alcohol 7a was achieved via HRMS (HRMS calc. for $\left[\mathrm{C}_{63} \mathrm{H}_{128} \mathrm{NaO}_{10} \mathrm{Si}_{3}\right]^{+}:$1151.8708, found: 1151.8713) and also by NMR analysis, with the ${ }^{1} \mathrm{H}$ NMR spectrum clearly showing the disappearance of one TBS group and also a downfield shift of $\mathrm{H}^{-6} \mathrm{~b}$ (3.61 ppm) and $\mathrm{H}-$ 6'a (3.67 ppm) which showed COSY correlations to a hydroxyl proton at $2.41 \mathrm{ppm}$ (Figure 4.3). This new methodology allowed for an improved synthesis of the key building block $s n-3-7 \mathbf{a}$ (7 steps, $37 \%$ overall yield), which can be used for the synthesis of a number of derivatives of LTA $1 .^{92}$

To illustrate the versatility of alcohol $7 \mathbf{a}$ and prepare a number of LTA anchor derivatives for biological assessment, LTA targets $s n-3-3 \mathbf{a}$ and $s n-3-4 a$ were then synthesised (Scheme 4.6). First, alcohol 7a was esterified using stearic acid to obtain triacylated glycolipid $s n-3-22 a$ in quantitative yield. HMBC peakd between H-6'a (4.19 ppm) and the carbonyl carbon (173.3 ppm) as well as H6'b (4.02 ppm) and the same carbonyl carbon were used to confirm the position of the new lipid (Figure 4.4). 


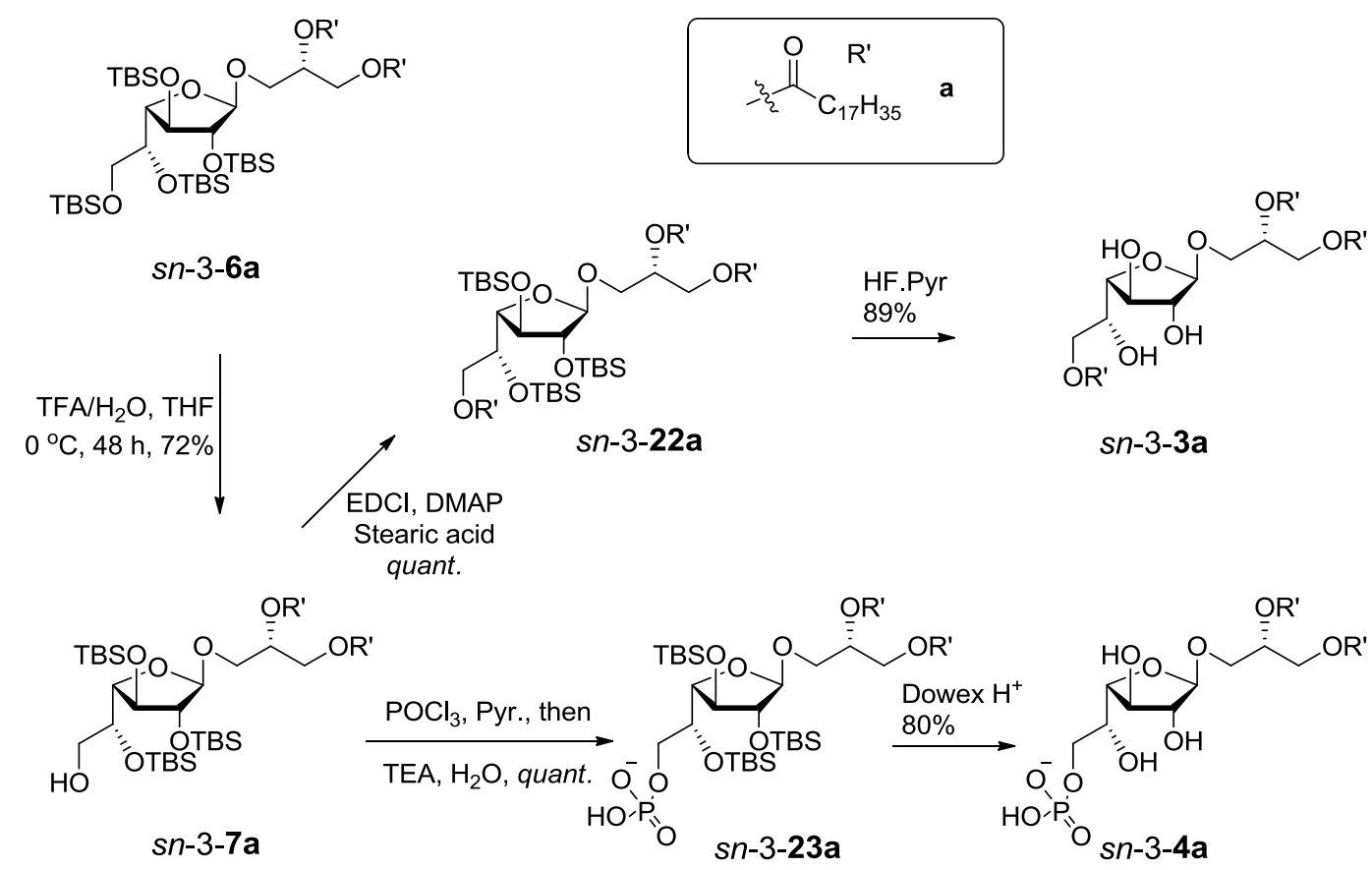

Scheme 4.6 Synthesis of $s n-3-3 \mathbf{a}$ and $s n-3-4 \mathbf{a}$.

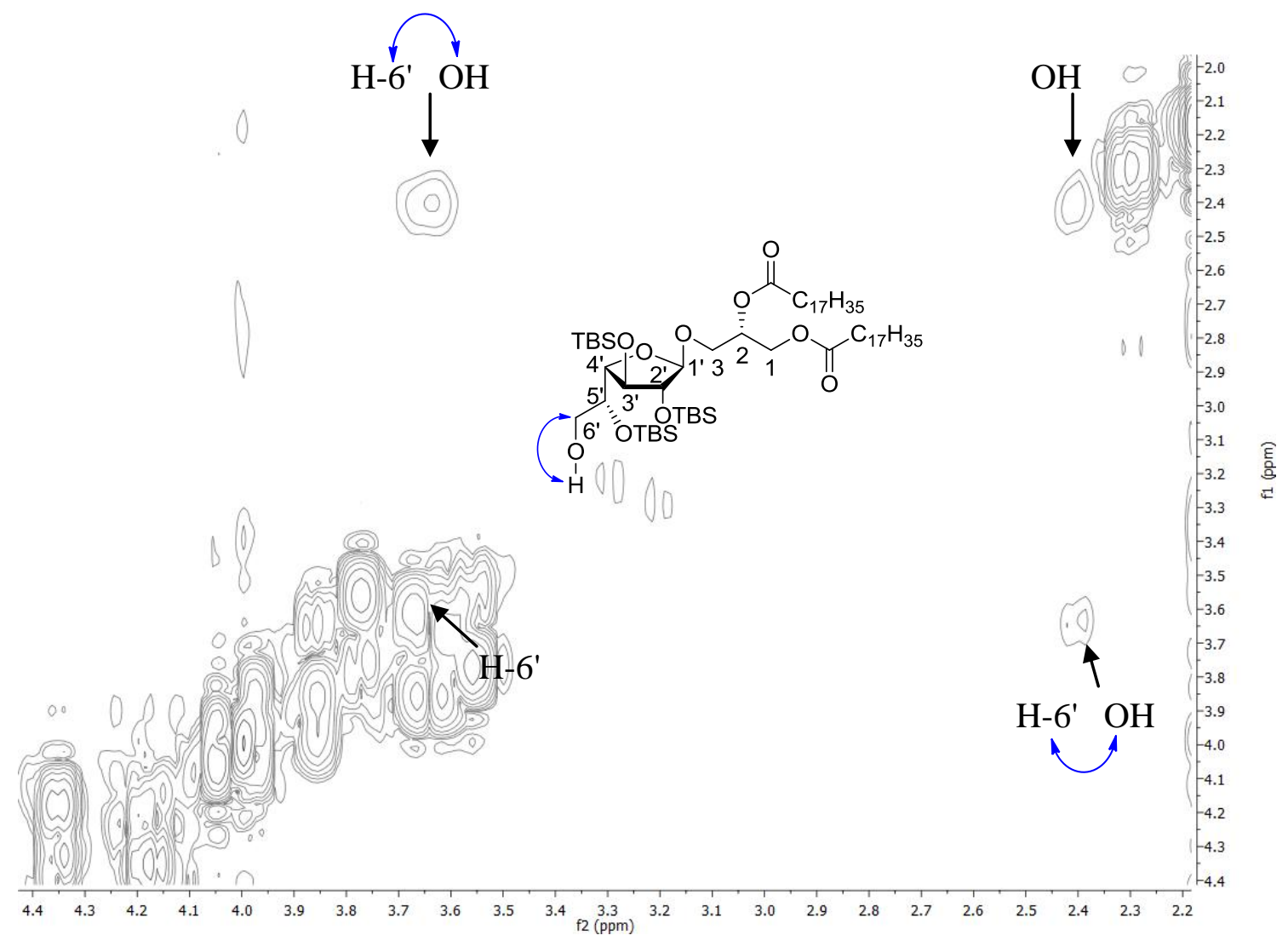

Figure 4.3 Magnification of the COSY NMR spectrum of $s n-3-7 \mathbf{a}$ showing the correlation between H-6' and the hydroxyl proton. 


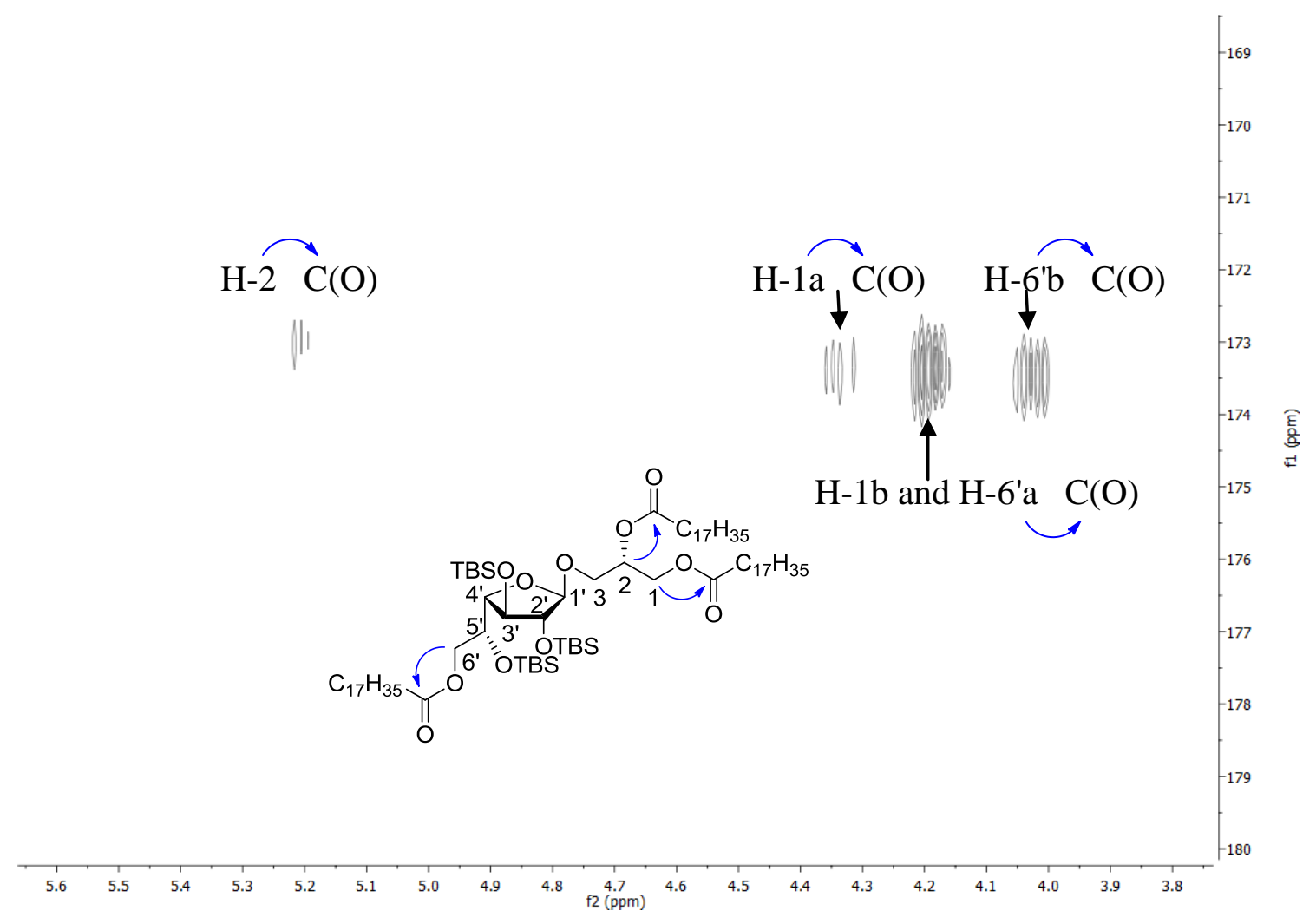

Figure 4.4 Magnification of sn-3-22a HMBC spectrum showing the correlations between H-6'a, H6'b, 1 and 2 to their respective carbonyl carbons.

Deprotection using HF.pyridine then gave lipidated target $s n-3-3 \mathbf{a}$ in $89 \%$ yield. The phosphorylated lipid anchor $s n-3-4 a$ was also readily prepared from alcohol 7a, first by treating compound 7a with freshly distilled $\mathrm{POCl}_{3}$ and then with $\mathrm{H}_{2} \mathrm{O}$ /TEA to install the corresponding phosphate to give phosphorylated $s n-3-23 a$. Key in this step was the use of an acidic work-up $(0.1 \mathrm{M} \mathrm{HCl})$, rather than Dowex $-\mathrm{Na}^{+}$, to separate the desired product from the TEA salts. ${ }^{1} \mathrm{H}-{ }^{31} \mathrm{P}$ HMBC confirmed that the phosphorus was indeed coupled at the 6-position (Figure 4.5). The TBS groups were then deprotected, using Dowex $-\mathrm{H}^{+}$, to give the target phosphorylated lipid anchor $s n-3-4 \mathbf{a}$ in excellent yield $(80 \%)$ over two-steps. 

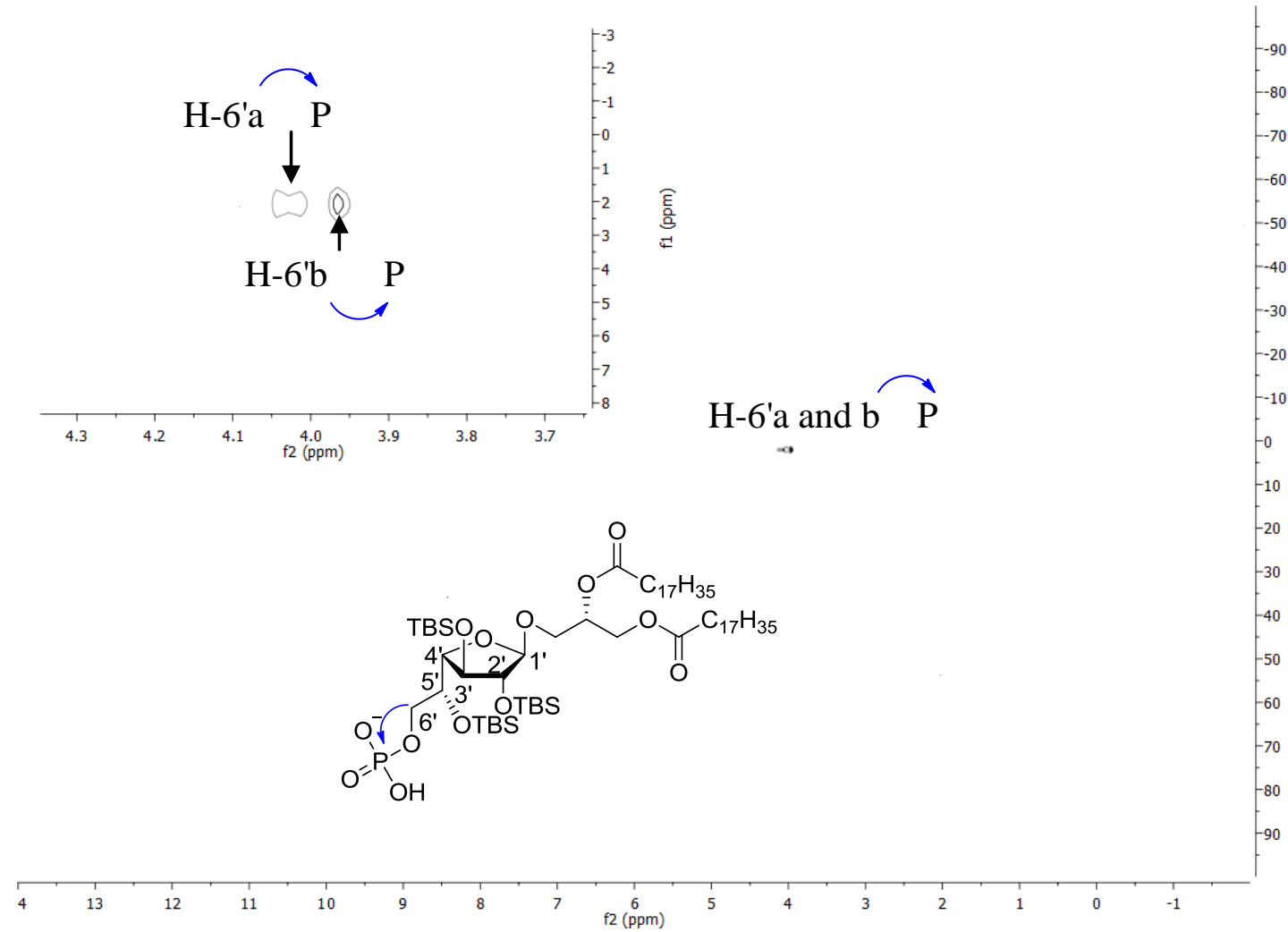

Figure 4.5 ${ }^{1} \mathrm{H},{ }^{31} \mathrm{P}$ HMBC of $s n-3-23 a$ showing the correlation between H6'a, H6'b and the phosphorous.

The final LTA lipid anchor to be prepared was the phosphate ester $s n-3-5 a$. At first, the synthesis of phosphoramidite $\mathbf{2 6}$ was attempted. As this phosphoramidite possesses a silyl group, it was hypothesised that the final LTA would be easier to deprotect than the usually used 1-O-(benzyloxybis(diisopropylamino)phosphine)-2,3-isopropylidene-snglycerol. $^{279}$ Although the conversion of phosphorous trichloride $24\left(\delta_{\mathrm{P}}=219 \mathrm{ppm}\right.$, coaxial tube, $\left.\left(\mathrm{CD}_{3}\right)_{2} \mathrm{CO}\right)$ to bis(diisopropylamino)chlorophosphine $25\left(\delta_{\mathrm{P}}=128.8 \mathrm{ppm}\right.$, $\mathrm{CDCl}_{3}$ ) was successful in reasonable yields (67\%), attempts to synthesise phosphoramidite 26 were ultimately unsuccessful and gave only 27 (HRMS calc. for $\left[\mathrm{C}_{12} \mathrm{H}_{29} \mathrm{NaOP}\right]^{+}=271.1910$, found: 271.1918$)\left(\delta_{31 \mathrm{P}}=119.0 \mathrm{ppm}, 121 \mathrm{MHz}, \mathrm{CDCl}_{3}\right)$ without any desired product (Scheme 4.7). From these results, it was hypothesised that even though the amount of water was carefully calculated and added to compound $\mathbf{2 5}$, it interfered with the formation of phosphoramidite $\mathbf{2 6}$ and thus the use of building block 29 was instead envisaged. To this end, 2-(trimethylsilyl)ethanol was treated with bis(diisopropylamino)chlorophosphine $\mathbf{2 5}$ and triethylamine to form phosphoramidite 
28 in $93 \%$ yield $\left(\delta_{\mathrm{P}}=122.8 \mathrm{ppm}, \mathrm{CDCl}_{3}\right)$ (Scheme 4.8). ${ }^{292}$ Coupling of 28 with $R$ Solketal gave phosphoramidite 29 uneventfully ( $\delta_{\mathrm{P}}=145.62$ and $145.60, \mathrm{CDCl}_{3}$ ). Next, a solution of alcohol 7a in $\mathrm{MeCN}$ was treated with phosphoramidite $\mathbf{2 9},{ }^{279}$ which, unfortunately resulted in the recovery of starting material, which was thought to be due to the poor solubility of glycolipid 7a in MeCN. A change to THF fared no better, and again, no reaction was observed. Fortunately, the use of a mixed solvent system (1/1, $\mathrm{MeCN} / \mathrm{THF}, \mathrm{v} / \mathrm{v})$ led to the formation of the protected phosphate ester $s n-3-30 \mathrm{a}(27 \%$ yield $)\left(\delta_{31 \mathrm{P}}=-1.39, \mathrm{CDCl}_{3}\right)$ and some starting material (sn-3-7a). Here attempts such as varying amounts of $1 H$-tetrazole, failed to improve the yield and starting material and small amounts of the target compound were found. With the product obtained from these investigations, the deprotection of protected LTA $s n$-3-30a using HF.Pyridine or TBAF failed to give desired compound sn-3-5a. Here small amounts of deprotected anchor and larger amounts of insoluble material were found in both the TBAF and HF.pyridine attempts.

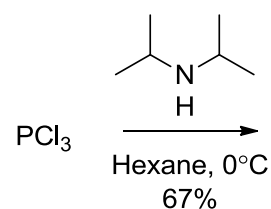

24

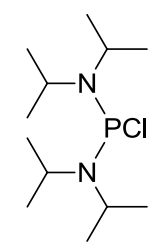

25

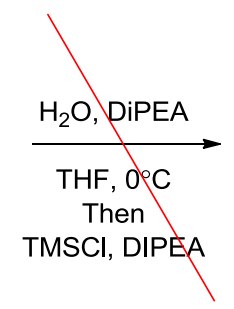

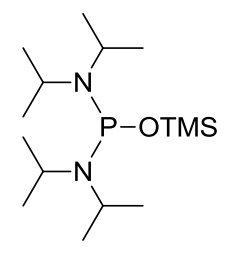

26

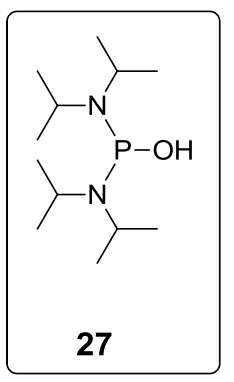

Scheme 4.7 Attempt to synthesise phosphoramidite 26.

These results suggested that to improve the yield, a more conservative approach to the synthesis of desired target $s n-3-5 a$ would be required. In the literature, 1-O(benzyloxybis(diisopropylamino)phosphine)-2,3-isopropylidene-sn-glycerol has been commonly used in various syntheses. ${ }^{293-295}$ Thus, following the procedure of Hamamoto et al. ${ }^{279}$, compound $\mathbf{8}$ was synthesised in very good yields (Scheme 4.9). Compound 25 was benzylated in excellent (97\%) yields. Then chiral solketal (-)-10 was reacted with intermediate $\mathbf{3 2}$ and $1 H$-tetrazole to give phosphoramidite $\mathbf{8}$ in $93 \%$ yield. 


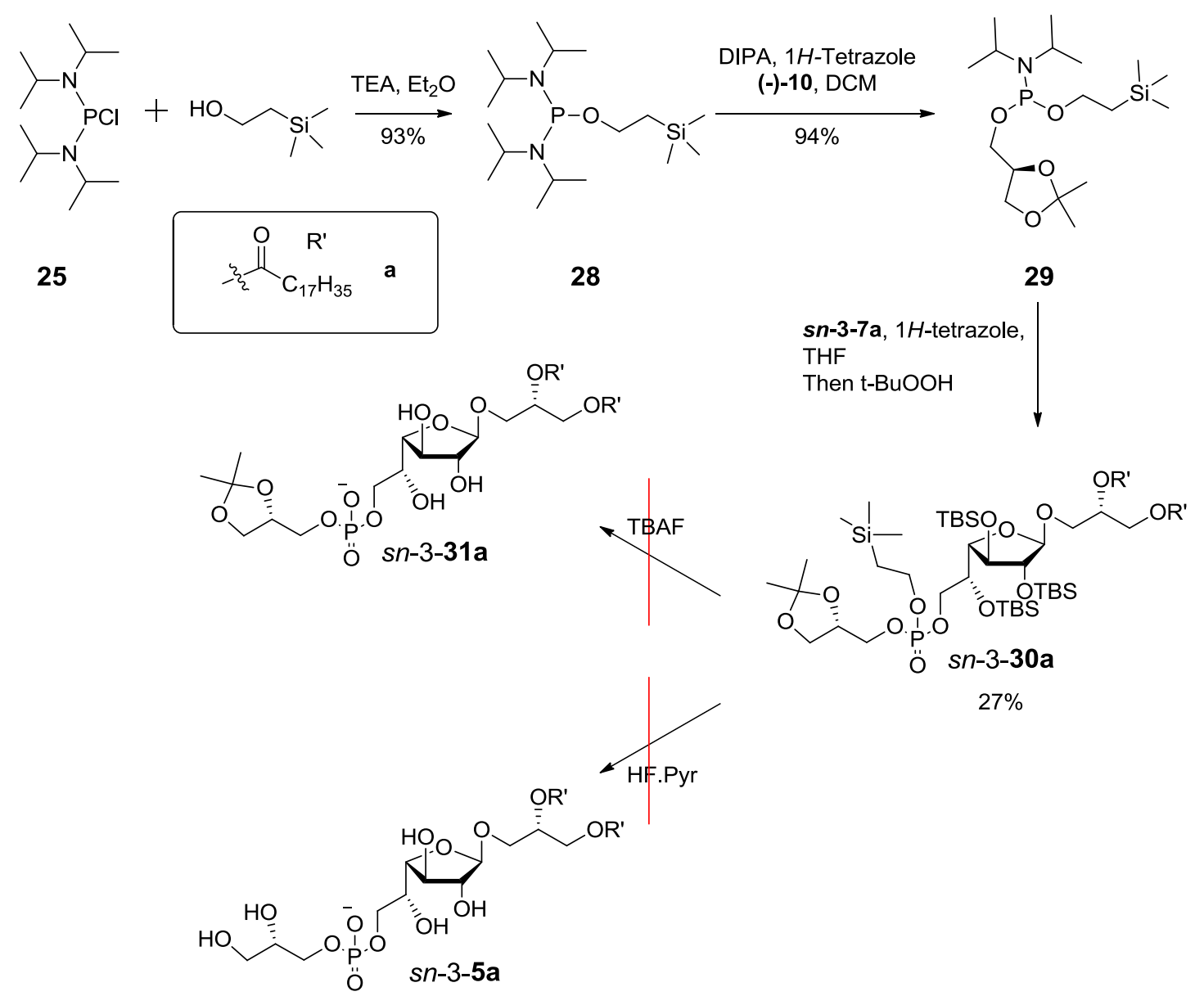

Scheme 4.8 Synthesis of compound sn-3-30a.

The synthesis of protected $s n-3-33 a$ required the use of a mixed solvent system (1/1, MeCN/THF, v/v) (Scheme 4.10). The formation of the protected phosphate ester sn-333a was synthesised in good (71\%) yield. NMR data for phosphotriester 33a included a diagnostic ${ }^{31} \mathrm{P}$ signal (-1.03 ppm). Also, ${ }^{1} \mathrm{H},{ }^{31} \mathrm{P}-\mathrm{HMBC}$ between $\mathrm{H}-6{ }^{\prime} \mathrm{b}$ (3.96 ppm), H6'a (4.12 ppm), sn-1"' (4.00 ppm), $\mathrm{CH}_{2} \mathrm{Bn}$ (5.09 ppm) and ${ }^{31} \mathrm{P}$ (-1.03 ppm) were observed (Figure 4.5). Additionally, in the ${ }^{13} \mathrm{C}$ NMR, some carbon resonances [e.g. 73.95 and $\left.73.88\left(\mathrm{C}-s n-2^{\prime \prime}\right)\right]$ showed separate signals for the two diastereomeric phosphotriesters (e.g. Two signals for $\mathrm{C}-2$ ") as well as ${ }^{31} \mathrm{P}_{-}{ }^{13} \mathrm{C}$ couplings resulting in a broad signal for C-6' (Figure 4.6). 


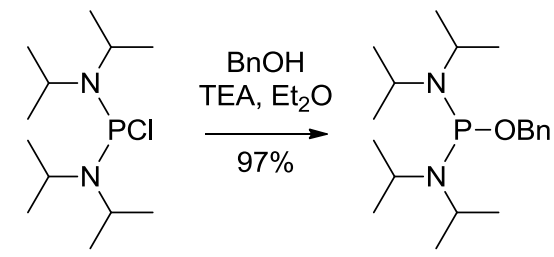

25

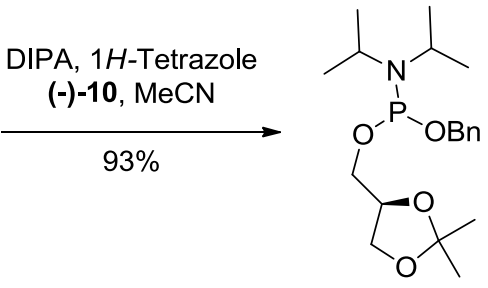

8

Scheme 4.9 Synthesis of phosphoramidite 8 .

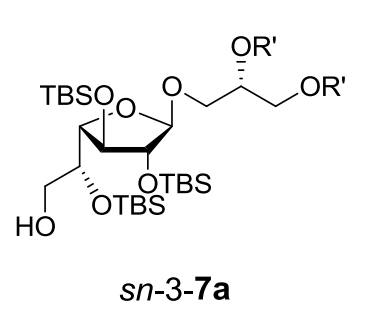

sn-3-7a
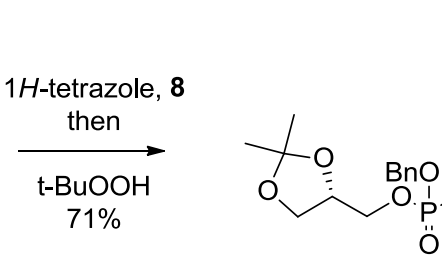

sn-3-33a

$\mathrm{Pd}(\mathrm{OH})_{2}, \mathrm{H}_{2}$, then OR'

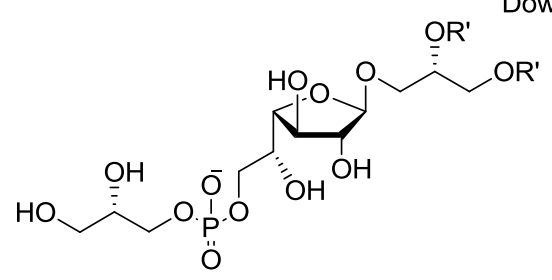

sn-3-5a

Scheme 4.10 Synthesis of target compound $s n-3-5 a$. 


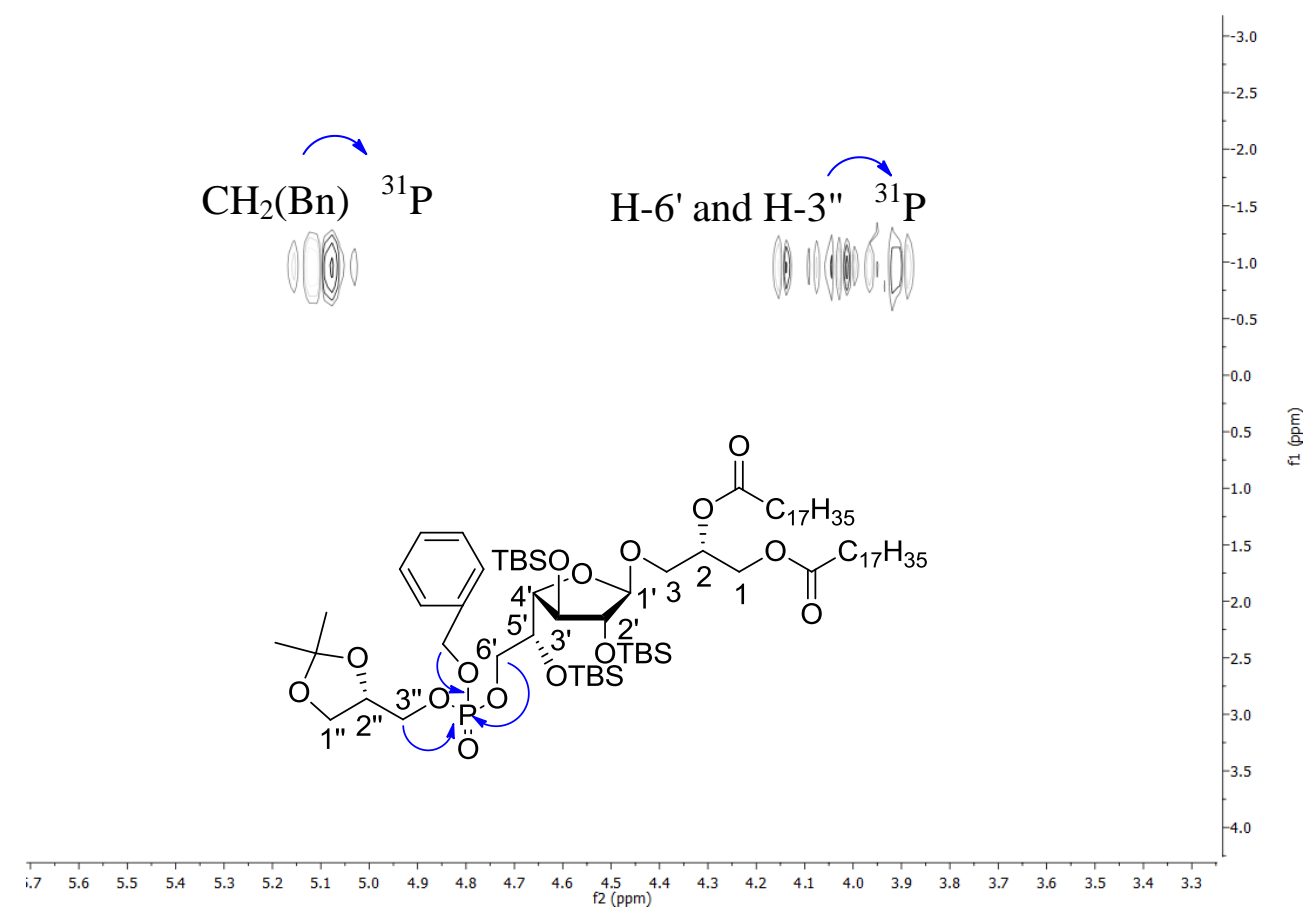

Figure 4.5 $\quad{ }^{1} \mathrm{H},{ }^{31} \mathrm{P}-\mathrm{HMBC}$ NMR spectrum of $s n$-3-33a showing HMBC correlations between $\mathrm{H}-6$ ', $\mathrm{H}-3$ " and $\mathrm{CH}_{2} \mathrm{Bn}$ with the phosphorous atom.

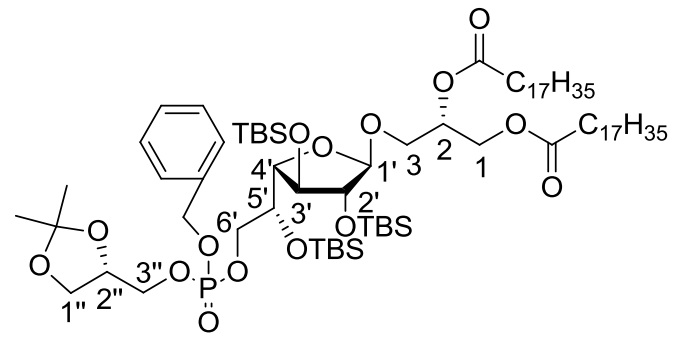

C-1"

C-2"

C-2

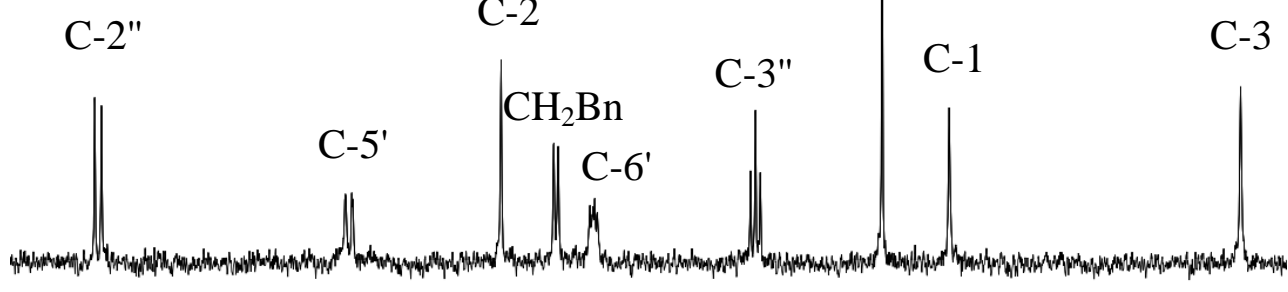

\begin{tabular}{llllllllllllllllllllllllllllllllll}
\hline 74.5 & 74.0 & 73.5 & 73.0 & 72.5 & 72.0 & 71.5 & 71.0 & 70.5 & 70.0 & 69.5 & 69.0 & 68.5 & 68.0 & 67.5 & 67.0 & 66.5 & 66.0 & 65.5 & 65.0 & 64.5 & 64.0 & 63.5 & 63.0 & 62.5 \\
\hline
\end{tabular} 
Figure 4.6 Magnification of ${ }^{13} \mathrm{C}$ NMR spectrum from $s n-3-33 a$ showing separate signals for the two phosphotriesters and coupling with phosphorous (e.g. Two signals for C-2", one broad signal for C-6').

Phosphoester 33a was hydrogenated and attempts were then made to deprotect the TBS groups using HF.pyridine at various concentrations, and using different proportions of pyridine and THF as the solvent. Unfortunately, under all conditions attempted, the hydrolysis product (glycolipid anchor 2a) was always observed. To overcome this problem, Dowex $-\mathrm{H}^{+}$resin was used to remove the TBS groups and $s n-3-5 a$ was obtained in an excellent (92\%) yield over the two steps.

With the series of the different LTA anchors in hand, the effect that these glycolipids had on the innate immune response was investigated by exploring their capacity to activate macrophages. Accordingly, all synthetic compounds were first confirmed to be free of endotoxin, as determined by the Limulus amebocyte lysate (LAL) assay, ${ }^{\mathrm{k}}$ and the compounds were then assessed for their ability to induce NO production in IFN- $\gamma$ primed bone marrow macrophages (BMMs) using the Griess assay. ${ }^{63}$ Using anchor sn1-2a, a time course was established which determined that NO production reached a plateau after $48 \mathrm{~h}$ (data not shown). This time point was then taken for all subsequent measurements, with two different concentrations of compound being tested, $20 \mu \mathrm{g} / \mathrm{mL}$ and $40 \mu \mathrm{g} / \mathrm{mL}$. LPS served as a positive control and unlipidated $s n$-3-galactofuranosylglycerol (GalfGro) as a negative control.

As illustrated in Figure 4.7, several of the LTA-derivatives were able to induce NO production by BMMs. On the whole, the unnatural $(s n-1)$ core glycolipid anchors $(\mathbf{2 a - d})$ showed lower levels of activity than the corresponding natural (sn-3) analogues with the exception of anchor sn-1-2a. The activity of the glycolipids (2a-d) also appears to be dependent on the type of lipid present, with there appearing to be an optimum lipid length (C20, sn-3-2b) for the $s n-3-2 a-d$ derivatives. Whether this is due to specific receptor-ligand interactions or the result of solubility and the formation of micelles or

\footnotetext{
${ }^{\mathrm{k}}$ Synthesised LTA-derivatives were tested to be endotoxin-free at a sensitivity of $0.125 \mathrm{EU} / \mathrm{ml}$ with an endotoxin kit (Pyrotell, Limulus amebocyte lysate) according to the manufacturer's instructions.
} 
other molecular assemblies warrants further investigation. ${ }^{296}$ Interestingly, the triacylated anchor 3a and the phosphorylated anchor 4a showed only modest activity, while the glycerophosphorylated anchor 5a was unable to induce NO production. For all substrates tested, a concentration dependence was observed with greater levels of NO being produced in response to higher concentrations of substrate being tested.

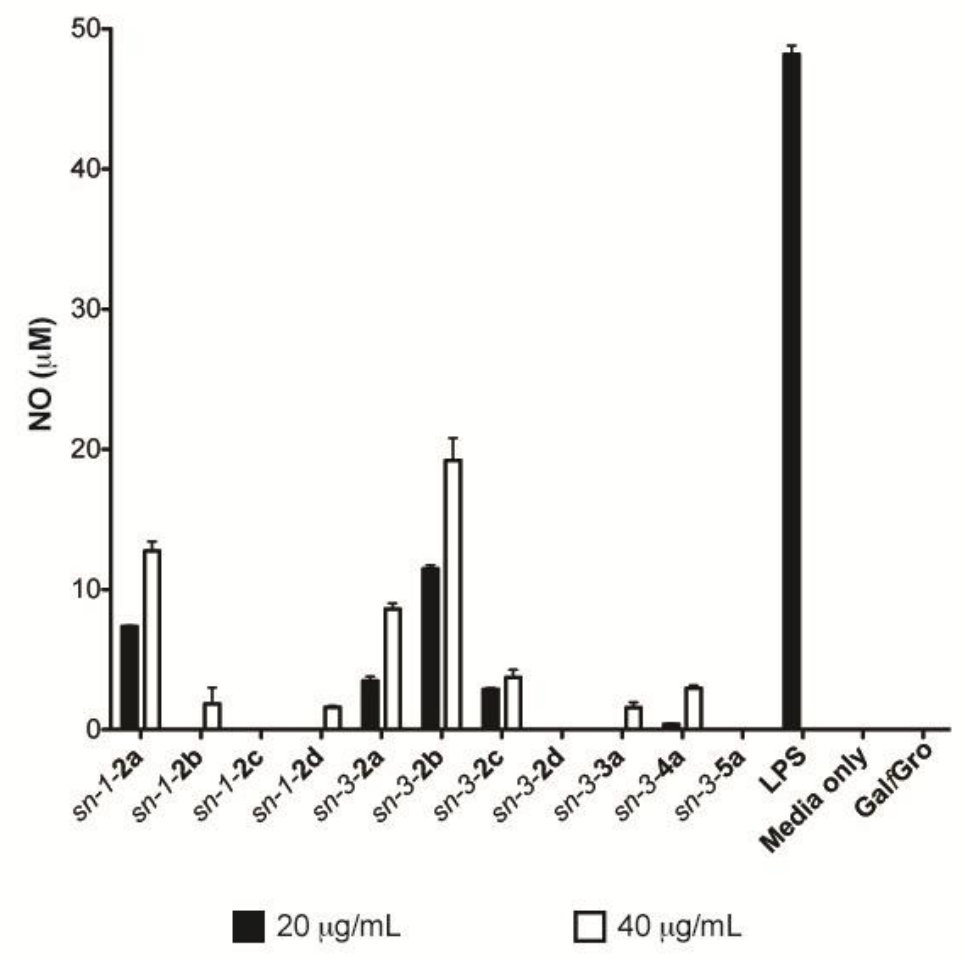

Figure 4.7 BMM were stimulated with LTA-derivatives (2a-d, 3a, 4a, 5a) (20 $\mu \mathrm{g} / \mathrm{mL}$ and $40 \mu \mathrm{g} / \mathrm{mL}$ ), or LPS (100 $\mathrm{ng} / \mathrm{mL})$, or $s n$-3-galactofuranosyl-glycerol (GalfGro, $20 \mu \mathrm{g} / \mathrm{mL}$ and $40 \mu \mathrm{g} / \mathrm{mL}$ ) and the supernatants analyzed for NO after $48 \mathrm{~h}$ using the Griess assay. Mean and SD of triplicate samples from a representative experiment of two are indicated. 


\subsection{Concluding Remarks}

Taken as a whole, the results point to the subtle effects that glycolipid length might have on the ability to activate BMMs. This phenomenon is not unexpected given that others have observed quite marked differences in biological activities when surveying glycolipids with different lipid compositions..$^{63,77,78,270}$ However, in the studies presented, absolute levels of NO production are small and further studies will be required to determine the physiological relevance of these differences. Indeed, in related work by Schmidt and co-workers, structure-activity studies concerning synthetic Staphylococcus aureus LTA anchors (which contain a gentiobiosyl diacylglyceride) revealed similar biological activities for $s n-1 / 3$ analogues and mixtures thereof. ${ }^{89}$ In addition, changing the gentiobiosyl moiety for lactose did not alter activity. From these results, it was concluded that the pattern recognition of the lipid anchor was relatively loose, allowing for variability of the lipid anchor. ${ }^{81,89,90}$ Schmidt and co-workers also observed that LTA structures including the anchor, glycerophosphate backbone and Dalanyl residues were required for optimal biological activity. Future work would include the synthesis and immunological evaluation of more complex derivatives of LTA 1 such as D-alanine derivatives and further investigation of the biological effect of LTA derivatives in a wider range of assays. 


\subsection{Experimental Section}

\subsubsection{General Methods}

Unless otherwise stated all reactions were performed under Ar. Prior to use, pyridine was dried and stored over $4 \AA$ molecular sieves (4 A MS), DCM was distilled from $\mathrm{P}_{2} \mathrm{O}_{5}$ and toluene was dried and stored under $\mathrm{Na}$ wire. Anhydrous DMF (Acros), $\mathrm{HCl}$ (Panreac), L-Serine (Sigma), Sodium nitrite (Chem Supply), dimethoxypropane (SigmaAldrich), Acetone (Pure science), PTSA (Sigma-Aldrich), $\mathrm{NaBH}_{4}$ (Aldrich), Mannitol $(\mathrm{M} \delta \mathrm{B})$, Zinc Chloride (Sigma-Aldrich), Ammonia (Fischer Scientific), Sodium periodate $(\mathrm{M} \delta \mathrm{B}), \mathrm{NaH}$ (Riedel de Haën), $\mathrm{KOH}$ (Pure Science), TBSCl (Appolo Scientific), Imidazole (Appolo Scientific), D-Galactose (J. T. Baker chemical), TMSTMS (Acros), $\mathrm{I}_{2}$ (M $\delta$ B), DIPEA (Sigma-Aldrich), $\mathrm{Na}_{2} \mathrm{~S}_{2} \mathrm{O}_{3}$ (Perkin's Reagent), THF (Panreac), HF.Pyridine (Acros), Pyridine (Fischer Scientific), $\mathrm{POCl}_{3}$ (Aldrich), TEA (Aldrich), t-BuOOH (6 M in Octane, Aldrich), MeCN (Panreac), Benzyl alcohol (Ajax Chemicals), $\mathrm{PCl}_{3}$ (Aldrich), Diisopropylamine (Sigma-Aldrich), TFA (Aldrich), EDCI (Aldrich), DMAP (Merck), arachidic acid (Sigma), Pd (OH) $)_{2} / \mathrm{C}$ (Aldrich, 20 wt\%), Dowex H+ (Supelco), anhydrous $\mathrm{Et}_{2} \mathrm{O}$ (Biolab), EtOAc (Pancreac), petroleum ether (Pure Science), anhydrous $\mathrm{MeOH}$ (Pancreac), $\mathrm{CHCl}_{3}$ (Pancreac), $\mathrm{NaHCO}_{3}$ (Pure Science), $\mathrm{MgSO}_{4}$ (Pure Science) and $\mathrm{NaCl}$ (Pancreac) were used as received. Stearic acid was recrystallised from $\mathrm{MeCN}$ (x6) and acetone (x3) until no trace of the other fatty acids was observed by ${ }^{1} \mathrm{H}$ NMR and MS. All solvents were removed under reduced pressure. Reactions were monitored by TLC analysis on Macherey-Nagel silica gel coated plastic sheets $(0.20 \mathrm{~mm}$ with fluorescent indicator UV254) via detection by UVabsorption (short wave-254 nm; long wave-366 nm) and by dipping in $10 \% \mathrm{H}_{2} \mathrm{SO}_{4}$ in $\mathrm{EtOH}$ followed by charring at $150{ }^{\circ} \mathrm{C}$. Silica gel flash column chromatography was performed using Pure Science silica gel (40-63 $\mu \mathrm{m})$. High resolution mass spectra were recorded on a Waters Q-TOF Premier ${ }^{\mathrm{TM}}$ Tandem Mass Spectrometer using positive electro-spray ionisation. Optical rotations were recorded on a Perkin-Elmer 241 polarimeter or Autopol II (Rudolph Research Analytical) at $589 \mathrm{~nm}$ (sodium D-line). Infrared spectra were recorded as thin films using a Bruker Tensor 27 FTIR spectrometer equipped with an Attenuated Total Reflectance (ATR) sampling accessory 
and are reported in wave numbers $\left(\mathrm{cm}^{-1}\right)$. Nuclear magnetic resonance spectra were recorded at $20{ }^{\circ} \mathrm{C}$ in $\mathrm{CDCl}_{3}, \mathrm{CDCl}_{3} / \mathrm{CD}_{3} \mathrm{OD}(2 / 1, \mathrm{v} / \mathrm{v})$ or $\mathrm{CDCl}_{3} / \mathrm{CD}_{3} \mathrm{OD} / \mathrm{D}_{2} \mathrm{O}(70 / 40 / 6$, $\mathrm{v} / \mathrm{v} / \mathrm{v}$ ) using a Varian INOVA operating at $500 \mathrm{MHz}$. Chemical shifts are given in ppm

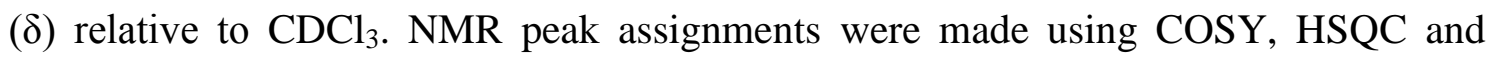
HMBC, ${ }^{1} \mathrm{H}^{31}{ }^{31} \mathrm{P}-\mathrm{HMBC}$ 2D experiments. Melting points were obtained using a Gallenkamp Melting Point Apparatus.

\subsubsection{Macrophage Assay}

\section{[Conducted by Ashna Khan and Stephanie Chee]}

\subsubsection{Materials and Methods}

C57BL/6 male mice were bred and housed in a conventional animal facility at the Malaghan Institute of Medical Research, Wellington, New Zealand. All animals used for the experiments were aged between 8-10 weeks. All experimental procedures were approved by the Victoria University Animal Ethics Committee in accordance with their guidelines for the care of animals. Extracted glycolipids were tested to be endotoxinfree $(\leq 0.125 \mathrm{EU} / \mathrm{mL})$ with an endotoxin kit (Pyrotell, LAL test, Associates of Cape Cod Inc., E. Falmouth, MA, USA).

\subsubsection{Generation of bone marrow derived macrophage (BMMs)}

Bone marrow cells were collected from the tibia and femur of C57BL/6 mice and were cultured (250000 cells per mL) in Iscove's Modified Dulbecco's Medium (IMDM, Gibco) supplemented with 5\% FBS (Gibco), 1\% Penicillin streptomycin (Gibco) and 2mercaptoethanol (55 mmol, Invitrogen) containing $10 \mathrm{ng} / \mathrm{mL}$ GM-CSF (clone X63/GMCSF murine cells). Cells were incubated at $37{ }^{\circ} \mathrm{C}\left(5 \% \mathrm{CO}_{2}\right)$ and the medium was changed on days 2, 5 and 7 . On day 10 , the medium was removed, and the BMMs 
were primed with IFN- $\gamma$ (10 ng/mL, Peprotech, Rocky Hill, NJ, USA) for $3 \mathrm{~h}$ prior to the addition of the compounds.

\subsubsection{BMM assays}

Glycolipids stock solutions were prepared $(2.5 \mathrm{mg} / \mathrm{mL}$ in PBS containing 2\% DMSO), vortexed and warmed to $50{ }^{\circ} \mathrm{C}$ for $30 \mathrm{~min}$ (x 3) to ensure suspension of the compounds prior to administration to BMM cultures. BMM cultures were then treated with 20 or 40 $\mu \mathrm{g} / \mathrm{mL}$ of glycolipid and with LPS (100 $\mathrm{ng} / \mathrm{mL}$ ) as a positive control and medium only for the times indicated. Supernatants were collected and tested immediately for NO levels using the Griess assay. ${ }^{216}$

\subsubsection{Compound Characterisation}

HO 1

2,3-O-Isopropylidene-sn-glycerol, $R$-solketal ((-)-10). (-)-10 was prepared following the procedure of Lok et al. and Hirth et al. with some variations. ${ }^{281,283}$ To a solution of L-serine $(26 \mathrm{~g}, 250 \mathrm{mmol})$ in water $(1.5 \mathrm{~L})$ and conc. $\mathrm{HCl}(37.5 \mathrm{~mL})$, was added $\mathrm{NaNO}_{2}(20 \mathrm{~g}, 290 \mathrm{mmol})$ in small portions at $0{ }^{\circ} \mathrm{C}$ for $24 \mathrm{~h}$. The solution was then stirred at RT for $24 \mathrm{~h}$ and concentrated in vacuo. Then, $\mathrm{MeOH}(75 \mathrm{~mL})$, conc. $\mathrm{HCl}$ $(7.5 \mathrm{~mL})$ and dimethoxypropane $(200 \mathrm{~mL})$ were added and the reaction mixture was stirred for $2 \mathrm{~h}$. After filtration, concentration in vacuo and the addition of acetone (200 $\mathrm{mL}), 2,2$-dimethoxypropane $(50 \mathrm{~mL})$ and PTSA $(0.25 \mathrm{~g}, 1.5 \mathrm{mmol})$ the mixture was stirred for $12 \mathrm{~h}$ at RT. The reaction mixture was then filtered, concentrated in vacuo and distilled under vacuum to give the methyl ester $(24.8 \mathrm{~g}, 142 \mathrm{mmol}, 57 \%)$. Then to a solution of the methyl ester $(12.7 \mathrm{~g}, 79.2 \mathrm{mmol})$ in $\mathrm{MeOH}(130 \mathrm{~mL})$ at $0{ }^{\circ} \mathrm{C}, \mathrm{NaBH}_{4}$ ( $2.4 \mathrm{~g}, 62.6 \mathrm{mmol}$ ) was added and the solution slowly warmed up to RT and then stirred for $1 \mathrm{~h}$. Acetone $(5 \mathrm{~mL})$ and water $(4 \mathrm{~mL})$ were added to the reaction mixture and after $5 \mathrm{~min}$, the reaction mixture was concentrated in vacuo. The residue was diluted with DCM, $\mathrm{NaCl}(4 \mathrm{~g})$ added then washed with brine $(16 \mathrm{~mL})$. The organic phase was then dried $\left(\mathrm{MgSO}_{4}\right)$, filtered, concentrated in vacuo and the residue distilled under high 
vacuum to give pure (-)-10 as a colourless oil $(7.3 \mathrm{~g}, 55.4 \mathrm{mmol}, 70 \%) ;\left(\mathrm{R}_{f}=0.40\right.$, $\mathrm{EtOAc} / \mathrm{PE}, 8 / 2, \mathrm{v} / \mathrm{v})$; $\mathrm{bp}_{20 \mathrm{mbar}}=82{ }^{\circ} \mathrm{C}$; (found: $[\alpha]_{\mathrm{D}}{ }^{19}=-9.8(\mathrm{c}=1.0, \mathrm{MeOH})$, literature: $[\alpha]_{\mathrm{D}}{ }^{20}=-11.1,(\mathrm{c}=5.0, \mathrm{MeOH}){ }^{.281} \rho=1.02 \mathrm{~g} / \mathrm{mL}$; IR (film) 3374, 2987, 2904, 2832, 1455, 1372, 1256, 1213, 1156, 1027, 844, $791 \mathrm{~cm}^{-1} ;{ }^{1} \mathrm{H}$ NMR (500 MHz, $\mathrm{CDCl}_{3}$ ) found: $\delta 4.20(\mathrm{~m}, 1 \mathrm{H}, \mathrm{H}-2), 4.08\left(\mathrm{t}, J_{\mathrm{H}-3 \mathrm{a}}, \mathrm{H}-3 \mathrm{~b}=J_{\mathrm{H}-1 \mathrm{a}}, \mathrm{H}-2=7.8 \mathrm{~Hz}, 1 \mathrm{H}, \mathrm{H}-3 \mathrm{a}\right), 3.76\left(\mathrm{ABX}, J_{\mathrm{H}-3 \mathrm{~b}}\right.$, $\mathrm{H}-3 \mathrm{a}=J_{\mathrm{H}-3 \mathrm{~b}}, \mathrm{H}-2=7.5 \mathrm{~Hz}, 1 \mathrm{H}, \mathrm{H}-3 \mathrm{~b}$ ), 3.68 (ABX, 1H, H-1a), 3.57 (ABX, 1H, H-1b), 2.42 (bs, $1 \mathrm{H}, \mathrm{OH}), 1.41$ and $1.35\left(\mathrm{~s}, 3 \mathrm{H}, \mathrm{CH}_{3}(\mathrm{iPr})\right.$ ); ${ }^{1} \mathrm{H}$ NMR (solvent and frequency unknown) literature: $\delta 4.21(\mathrm{~m}, 1 \mathrm{H}, \mathrm{H}-2), 4.06\left(\mathrm{t}, J_{\mathrm{H}-3 \mathrm{a}}, \mathrm{H}-3 \mathrm{~b}=J_{\mathrm{H}-1 \mathrm{a}}, \mathrm{H}-2=8.0 \mathrm{~Hz}, 1 \mathrm{H}, \mathrm{H}-\right.$ 3a), 3.80 (ABX, $\left.J_{\mathrm{H}-3 \mathrm{~b}, \mathrm{H}-3 \mathrm{a}}=J_{\mathrm{H}-3 \mathrm{~b}}, \mathrm{H}-2=5.2 \mathrm{~Hz}, 1 \mathrm{H}, \mathrm{H}-3 \mathrm{~b}\right), 3.75(\mathrm{~m}, 1 \mathrm{H}, \mathrm{H}-1 \mathrm{a}), 3.52$ (m, 1H, H-1b), 2.34 (bs, 1H, OH), 1.39 and 1.45 (s, 3H, $\left.\mathrm{CH}_{3}(\mathrm{iPr})\right) ;{ }^{13} \mathrm{C}$ NMR (125 MHz, $\left.\mathrm{CDCl}_{3}\right) \delta 109.4\left(\underline{\mathrm{C}}\left(\mathrm{CH}_{3}\right)_{2}\right), 76.2(\mathrm{C}-2), 65.7(\mathrm{C}-3), 62.9(\mathrm{C}-1), 26.7$ and $25.2\left(\mathrm{CH}_{3}(\mathrm{iPr})\right)$; HRMS(ESI) $m / z$ calcd. for $\left[\mathrm{C}_{6} \mathrm{H}_{12} \mathrm{NaO}_{3}\right]^{+}:$155.0679, found: 155.0677 .

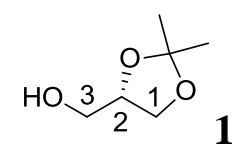

1,2-O-Isopropylidene-sn-glycerol ((+)-10). (+)-10 was prepared following the procedure of Eibl et al. ${ }^{282}$ Mannitol (20 g, $110 \mathrm{mmol}$ ) was added to a solution of zinc chloride $(20 \mathrm{~g}, 110 \mathrm{mmol})$ in acetone $(170 \mathrm{~mL})$. The reaction was stirred until full consumption of the starting material into the diacetonide product gauged $\left(\mathrm{R}_{f}=0.70\right.$, $\mathrm{MeOH} / \mathrm{DCM}, 1 / 9, \mathrm{v} / \mathrm{v})$. A minor amount of the monoacetonide was also observed $\left(\mathrm{R}_{f}=\right.$ $0.20, \mathrm{MeOH} / \mathrm{DCM}, 1 / 9, \mathrm{v} / \mathrm{v})$. Brine was added and the aqueous layer extracted three times with DCM. The combined organic layers were washed with ammonia (5\%, v/v) and concentrated in vacuo. The residue was dissolved in methanol $(20 \mathrm{~mL})$ and slowly added to a chilled $\left(0^{\circ} \mathrm{C}\right)$ solution of sodium periodate $(17.7 \mathrm{~g}, 82.5 \mathrm{mmol})$ in water $(170$ $\mathrm{mL}$ ), adjusted to $\mathrm{pH} 6$ using $\mathrm{LiOH}$. After 10 minutes and complete conversion of the diol into the aldehyde $\left(\mathrm{R}_{f}=0.21\right.$, EtOAc/PE, $\left.2 / 8, \mathrm{v} / \mathrm{v}\right)$, the $\mathrm{pH}$ was adjusted to 8 , by the addition of $\mathrm{KOH}$, methanol $(144 \mathrm{~mL})$ added and the solution filtered. The filtrate was subjected to sodium borohydride $(2.75 \mathrm{~g}, 72.6 \mathrm{mmol})$ until complete reduction of the aldehyde was observed (ca. $2 \mathrm{~h}),\left(\mathrm{R}_{f}=0.37, \mathrm{EtOAc} / \mathrm{PE}, 8 / 2, \mathrm{v} / \mathrm{v}\right) ; \mathrm{NaCl}(20 \mathrm{~g})$ and petroleum ether were added and the organic layer was discarded. The reaction mixture was extracted three times with DCM. The organic layer was washed with water, dried $\left(\mathrm{MgSO}_{4}\right)$, filtered, evaporated to dryness and distilled to give pure (+)-10 as a colourless liquid $(8.4 \mathrm{~g}, 63.8 \mathrm{mmol}, 58 \%)$. B.p.20mbar $=82{ }^{\circ} \mathrm{C}$; found: $[\alpha]_{\mathrm{D}}{ }^{19}=11.0(\mathrm{c}=1.0$, $\mathrm{MeOH})$, literature: $[\alpha]_{\mathrm{D}}{ }^{20}=11.6(\mathrm{c}=5.0, \mathrm{MeOH})^{281} ; \rho=1.01 \mathrm{~g} / \mathrm{mL} ;{ }^{1} \mathrm{H}$ NMR $(500$ 
$\mathrm{MHz}, \mathrm{CDCl}_{3}$ ) found: $\delta 4.20(\mathrm{~m}, 1 \mathrm{H}, \mathrm{H}-2), 4.08\left(\mathrm{t}, J_{\mathrm{H}-3 \mathrm{a}}, \mathrm{H}-3 \mathrm{~b}=J_{\mathrm{H}-1 \mathrm{a}}, \mathrm{H}-2=7.8 \mathrm{~Hz}, 1 \mathrm{H}, \mathrm{H}-\right.$ 3a), $3.76\left(\mathrm{ABX}, J_{\mathrm{H}-3 \mathrm{~b}}, \mathrm{H}-3 \mathrm{a}=J_{\mathrm{H}-3 \mathrm{~b}}, \mathrm{H}-2=7.5 \mathrm{~Hz}, 1 \mathrm{H}, \mathrm{H}-3 \mathrm{~b}\right.$ ), 3.68 (ABX, 1H, H-1a), 3.57 (ABX, 1H, H-1b), 2.42 (bs, 1H, OH), 1.41 and 1.35 (s, 3H, $\mathrm{CH}_{3}(\mathrm{iPr})$ ); ${ }^{1} \mathrm{H}$ NMR (solvent and frequency unknown) literature: $\delta 4.21(\mathrm{~m}, 1 \mathrm{H}, \mathrm{H}-2), 4.06\left(\mathrm{t}, J_{\mathrm{H}-3 \mathrm{a}}, \mathrm{H}-3 \mathrm{~b}=J_{\mathrm{H}-1 \mathrm{a}}, \mathrm{H}-2=\right.$ $8.0 \mathrm{~Hz}, 1 \mathrm{H}, \mathrm{H}-3 \mathrm{a}), 3.80\left(\mathrm{ABX}, J_{\mathrm{H}-3 \mathrm{~b}}, \mathrm{H}-3 \mathrm{a}=J_{\mathrm{H}-3 \mathrm{~b}}, \mathrm{H}-2=5.2 \mathrm{~Hz}, 1 \mathrm{H}, \mathrm{H}-3 \mathrm{~b}\right), 3.75(\mathrm{~m}, 1 \mathrm{H}$, $\mathrm{H}-1 \mathrm{a}), 3.52$ (m, 1H, H-1b), 2.34 (bs, $1 \mathrm{H}, \mathrm{OH}), 1.39$ and 1.45 (s, 3H, $\left.\mathrm{CH}_{3}(\mathrm{iPr})\right) ;{ }^{13} \mathrm{C}$ NMR (125 MHz, $\left.\mathrm{CDCl}_{3}\right) \delta 109.4\left(\underline{\mathrm{C}}\left(\mathrm{CH}_{3}\right)_{2}\right), 76.2$ (C-2), 65.7 (C-1), 62.9 (C-3), 26.6 and 25.2 (iPr); IR (neat): 2982, 2903, 1439, 1372, 1246, 1214, 1185, 1159, 1126, 1087, 1047, 1022, 968, 919, 880, 849, 800, $737 \mathrm{~cm}^{-1}$; HRMS(ESI) m/z calcd. for $\left[\mathrm{C}_{6} \mathrm{H}_{12} \mathrm{NaO}_{3}\right]^{+}:$155.0679, found: 155.0685 .

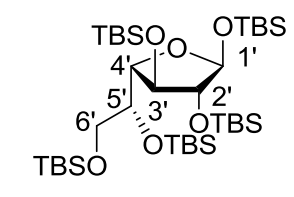

\section{1,2,3,5,6-Penta- $O$-tert-butyldimethylsilyl- $\beta$-D-galactofuranose $\quad(19)$.}

19 was prepared following the synthesis of Baldoni et al. ${ }^{280}$ Imidazole (27.4 g, 402 $\mathrm{mmol})$ and TBSCl (31.4 g, $209 \mathrm{mmol})$ were added to D-galactose $(5.0 \mathrm{~g}, 27.8 \mathrm{mmol})$ in dry DMF (140 mL), and the reaction was stirred for $72 \mathrm{~h}$, during which time the product gradually precipitated. The reaction mixture was then diluted with EtOAc and washed with water, $\mathrm{HCl}(5 \%), \mathrm{NaHCO}_{3}$ (sat. aq.) and brine. The organic layer was dried $\left(\mathrm{MgSO}_{4}\right)$, filtered and concentrated in vacuo. The residue was then crystallised from $\mathrm{MeOH}$ to obtain white needles $(15.0 \mathrm{~g}, 20.0 \mathrm{mmol}, 72 \%) .\left(\mathrm{R}_{f}=0.76\right.$, EtOAc/PE, 1/9, $\mathrm{v} / \mathrm{v}) ; \mathrm{mp}$ found $=109.4-109.6{ }^{\circ} \mathrm{C}, \mathrm{mp}$ literature $=109.0-111.0{ }^{\circ} \mathrm{C},[\alpha]_{\mathrm{D}}{ }^{20}=-25.0(c=$ 1.0, $\mathrm{CHCl}_{3}$ ); IR (film) 2955, 2858, 1472, 1251, 1109, 1005, 834, 777, $743 \mathrm{~cm}^{-1} ;{ }^{1} \mathrm{H}$ $\operatorname{NMR}\left(500 \mathrm{MHz}, \mathrm{CDCl}_{3}\right) \delta 5.13\left(\mathrm{~d}, J_{\mathrm{H}-1^{\prime}}, \mathrm{H}-2^{\prime}=2.3 \mathrm{~Hz}, 1 \mathrm{H}, \mathrm{H}-1^{\prime}\right), 4.10\left(\mathrm{~m}, 1 \mathrm{H}, \mathrm{H}-3^{\prime}\right)$, $4.01\left(\mathrm{t}, J_{\mathrm{H}-4^{\prime}}, \mathrm{H}-3^{\prime}=J_{\mathrm{H}-4^{\prime}, \mathrm{H}-5^{\prime}}=4.0 \mathrm{~Hz}, 1 \mathrm{H}, \mathrm{H}-4^{\prime}\right), 3.92\left(\mathrm{~m}, 1 \mathrm{H}, \mathrm{H}-2^{\prime}\right), 3.74(\mathrm{~m}, 1 \mathrm{H}, \mathrm{H}-$ $\left.5^{\prime}\right), 3.68\left(\mathrm{ABX}, J_{\mathrm{H}-6^{\prime} \mathrm{a}}, \mathrm{H}-6{ }^{\prime} \mathrm{b}=10.1 \mathrm{~Hz}, J_{\mathrm{H}-6^{\prime} \mathrm{a}, \mathrm{H}-5^{\prime}}=6.5 \mathrm{~Hz}, 1 \mathrm{H}, \mathrm{H}-6^{\prime} \mathrm{a}\right), 3.56\left(\mathrm{ABX}, J_{\mathrm{H}-6^{\prime} \mathrm{a}}\right.$,

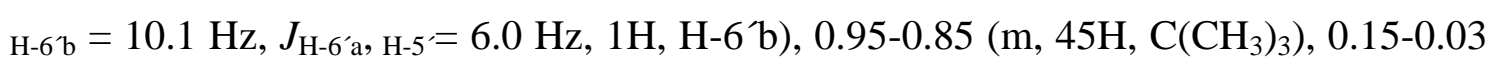
$\left(\mathrm{m}, 30 \mathrm{H}, \mathrm{Si}\left(\mathrm{CH}_{3}\right)_{2}\right) .{ }^{13} \mathrm{C} \mathrm{NMR}\left(125 \mathrm{MHz}, \mathrm{CDCl}_{3}\right) \delta 102.8\left(\mathrm{C}-1^{\prime}\right), 85.8\left(\mathrm{C}-2^{\prime}\right), 84.5(\mathrm{C}-$ 4’), 79.5 (C-3'), 74.0 (C-5'), 64.6 (C-6'), 26.0 and 25.9 and 25.8 and 25.7 and 25.7 $\left(\mathrm{C}\left(\mathrm{CH}_{3}\right)_{3}\right), 18.3\left(\underline{\mathrm{C}}\left(\mathrm{CH}_{3}\right)_{3}\right), 18.2\left(\underline{\mathrm{C}}\left(\mathrm{CH}_{3}\right)_{3}\right), 17.9\left(\underline{\mathrm{C}}\left(\mathrm{CH}_{3}\right)_{3}\right), 17.87\left(\underline{\mathrm{C}}\left(\mathrm{CH}_{3}\right)_{3}\right), 17.78$ $\left(\underline{\mathrm{C}}\left(\mathrm{CH}_{3}\right)_{3}\right),-3.87\left(\mathrm{Si}\left(\mathrm{CH}_{3}\right)_{2}\right),-4.18\left(\mathrm{Si}\left(\mathrm{CH}_{3}\right)_{2}\right),-4.23(\mathrm{x} 2)\left(\mathrm{Si}\left(\mathrm{CH}_{3}\right)_{2}\right),-4.33\left(\mathrm{Si}\left(\mathrm{CH}_{3}\right)_{2}\right),-$ $4.6\left(\mathrm{Si}\left(\mathrm{CH}_{3}\right)_{2}\right),-4.7\left(\mathrm{Si}\left(\mathrm{CH}_{3}\right)_{2}\right),-5.1\left(\mathrm{Si}\left(\mathrm{CH}_{3}\right)_{2}\right),-5.43\left(\mathrm{Si}\left(\mathrm{CH}_{3}\right)_{2}\right),-5.37\left(\mathrm{Si}\left(\mathrm{CH}_{3}\right)_{2}\right)$; HRMS(ESI) $m / z$ calcd. for $\left[\mathrm{C}_{36} \mathrm{H}_{82} \mathrm{NaO}_{6} \mathrm{Si}_{5}\right]^{+}:$773.4850, found: 773.4852 . 


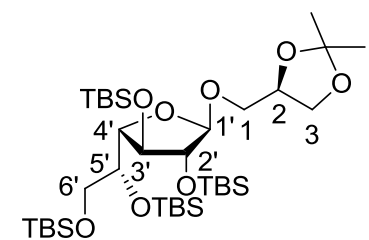

\section{1-O-(2, 3, 5, 6-Tetra- $O$-tert-butyldimethylsilyl- $\beta$-D-}

galactofuranosyl)-2,3-O-isopropylidene-sn-glycerol (sn-1-20). A solution of 19 (0.49 g, $0.66 \mathrm{mmol})$ in anhydrous DCM (70 mL) was stirred with $4 \AA$ mol. sieves for $30 \mathrm{~min}$. and then cooled to $0{ }^{\circ} \mathrm{C}$. To this was added neat TMSI $(160 \mathrm{mg}, 0.80 \mathrm{mmol})$, which was freshly prepared under Ar by the addition of TMSTMS (0.66 g, $4.5 \mathrm{mmol}$ ) to iodine $(0.76 \mathrm{~g}, 3.0 \mathrm{mmol})$ and the reaction heated at $60{ }^{\circ} \mathrm{C}$ until the mixture turned colourless. After 15 min., TLC showed full consumption of 19 into two glycosyl iodides $\left[\mathrm{R}_{f}=0.67\right.$ $\left(\right.$ EtOAc/PE, 1/9, v/v) and $\mathrm{R}_{f}=0.53($ EtOAc/PE, 1/9, v/v)], at which point DIPEA (190 $\mathrm{mg}, 1.47 \mathrm{mmol})$ and isopropylidene glycerol (-)-10 (0.19 g, $1.47 \mathrm{mmol})$ were added and the reaction stirred at $\mathrm{RT}$ for $3 \mathrm{~h}$ until full consumption of the iodide into a slightly higher running product $\left(\mathrm{R}_{f}=0.68, \mathrm{EtOAc} / \mathrm{PE}, 1 / 9, \mathrm{v} / \mathrm{v}\right)$. The reaction mixture was then diluted with EtOAc, washed with $\mathrm{NaHCO}_{3}$ (sat. aq.), $\mathrm{Na}_{2} \mathrm{~S}_{2} \mathrm{O}_{3}$ (sat. aq.) and brine, dried $\left(\mathrm{MgSO}_{4}\right)$, filtered and concentrated. The residue was then purified by silica gel flash column chromatography $(\mathrm{EA} / \mathrm{PE}, 2 / 98, \mathrm{v} / \mathrm{v})$, to give a $1 / 9$ mixture of the $\alpha$ - and $\beta$ isomers as a colourless oil $(0.45 \mathrm{~g}, 0.61 \mathrm{mmol}, 90 \%)$. Here, it should be noted that separation of the anomers could be achieved via careful silica gel column chromatograpy (DCM/PE, 1/1, v/v), however, separation of the anomers became easier following acetonide deprotection (vide infra). $\left(\mathrm{R}_{f}=0.68, \mathrm{EtOAc} / \mathrm{PE}, 1 / 9, \mathrm{v} / \mathrm{v}\right) ; \beta$ isomer; $[\alpha]_{\mathrm{D}}{ }^{26}=-34.0\left(c=1.0, \mathrm{CHCl}_{3}\right)$; IR (film) 2930, 2858, 1473, 1254, 1109, 837, $773 \mathrm{~cm}^{-1}$; ${ }^{1} \mathrm{H}$ NMR $\left(500 \mathrm{MHz}, \mathrm{CDCl}_{3}\right) \delta 4.84\left(\mathrm{~d}, J_{\mathrm{H}-1^{\prime}}, \mathrm{H}-2^{\prime}=2.1 \mathrm{~Hz}, 1 \mathrm{H}, \mathrm{H}-1^{\prime}\right), 4.26$ (m, 1H, H-2), $4.12\left(\mathrm{dd}, J_{\mathrm{H}-3^{\prime}, \mathrm{H}-2^{\prime}}=3.2 \mathrm{~Hz}, J_{\mathrm{H}-3^{\prime}, \mathrm{H}^{\prime} 4^{\prime}}=5.7 \mathrm{~Hz}, 1 \mathrm{H}, \mathrm{H}-3^{\prime}\right), 4.06\left(\mathrm{ABX}, J_{\mathrm{H}-3 \mathrm{a}, \mathrm{H}-3 \mathrm{~b}}=8.4\right.$ $\left.\mathrm{Hz}, J_{\mathrm{H}-3 \mathrm{a}}, \mathrm{H}-2=6.3 \mathrm{~Hz}, 1 \mathrm{H}, \mathrm{H}-3 \mathrm{a}\right), 4.00\left(\mathrm{dd}, J_{\mathrm{H}-2^{\prime}, \mathrm{H}-1^{\prime}}=2.2 \mathrm{~Hz}, J_{\mathrm{H}-2^{\prime}}, \mathrm{H}-3^{\prime}=3.3 \mathrm{~Hz}, 1 \mathrm{H}, \mathrm{H}-\right.$ $\left.2^{\prime}\right), 3.94\left(\mathrm{dd}, J_{\mathrm{H}-4^{\prime}, \mathrm{H}-3}=5.7 \mathrm{~Hz}, J_{\mathrm{H}-4^{\prime}}, \mathrm{H}-5^{\prime}=3.1, \mathrm{H}^{\prime} 4^{\prime}\right), 3.76\left(\mathrm{~m}, 1 \mathrm{H}, \mathrm{H}-5^{\prime}\right), 3.74(\mathrm{ABX}$, $\left.J_{\mathrm{H}-3 \mathrm{~b}, \mathrm{H}-3 \mathrm{a}}=8.4 \mathrm{~Hz}, J_{\mathrm{H}-3 \mathrm{~b}}, \mathrm{H}-2=6.0 \mathrm{~Hz}, 1 \mathrm{H}, \mathrm{H}-3 \mathrm{~b}\right), 3.66$ (m, 1H, H-6'a), 3.64 (ABX, $J_{\mathrm{H}-1 \mathrm{a}}, \mathrm{H}-$ $\left.1 \mathrm{~b}=9.8 \mathrm{~Hz}, J_{\mathrm{H}-1 \mathrm{a}}, \mathrm{H}-2=6.8,1 \mathrm{H}, \mathrm{H}-1 \mathrm{a}\right), 3.58\left(\mathrm{ABX}, J_{\mathrm{H}-6^{\prime} \mathrm{b}}, \mathrm{H}-6^{\prime} \mathrm{a}=10.1 \mathrm{~Hz}, J_{\mathrm{H}-6^{\prime} \mathrm{b}}, \mathrm{H}-5^{\prime}=6.2\right.$ $\left.\mathrm{Hz}, 1 \mathrm{H}, \mathrm{H}-6^{\prime} \mathrm{b}\right), 3.52\left(\mathrm{ABX}, J_{\mathrm{H}-1 \mathrm{~b}}, \mathrm{H}-1 \mathrm{a}=9.6 \mathrm{~Hz}, J_{\mathrm{H}-1 \mathrm{~b}}, \mathrm{H}-2=5.5 \mathrm{~Hz}, 1 \mathrm{H}, \mathrm{H}-1 \mathrm{~b}\right), 1.40$ and 1.35 (s, 3H, iPr), 1.0-0.8 (m, 36H, C $\left.\left(\mathrm{CH}_{3}\right)_{3}\right), 0.2-0.0\left(\mathrm{~m}, 24 \mathrm{H}, \mathrm{Si}\left(\mathrm{CH}_{3}\right)_{2}\right) ;{ }^{13} \mathrm{C}$ NMR $(125$ $\left.\mathrm{MHz} \mathrm{CDCl}_{3}\right) \delta 109.3(\mathrm{C}), 108.6\left(\mathrm{C}^{\prime} 1^{\prime}\right), 84.4\left(\mathrm{C}-2^{\prime}\right), 84.0$ (C-4'), 79.5 (C-3’), 74.5 (C2), 73.2 (C-5'), 68.7 (C-1); 67.5 (C-3), 64.5 (C-6’), 26.8 (iPr), $26.02\left(\mathrm{C}\left(\underline{\mathrm{C}}_{3}\right)_{3}\right), 25.96$ $\left(\mathrm{C}\left(\underline{\mathrm{CH}}_{3}\right)_{3}\right), 25.8 \quad\left(\mathrm{C}\left(\underline{\mathrm{CH}}_{3}\right)_{3}\right), 25.7 \quad\left(\mathrm{C}\left(\underline{\mathrm{CH}}_{3}\right)_{3}\right), 25.5$ (iPr), $18.33 \quad\left(\left(\underline{\mathrm{C}}\left(\mathrm{CH}_{3}\right)_{3}\right), 18.32\right.$ $\left(\left(\underline{\mathrm{C}}\left(\mathrm{CH}_{3}\right)_{3}\right), 17.9\left(\left(\underline{\mathrm{C}}\left(\mathrm{CH}_{3}\right)_{3}\right), 17.8\left(\left(\underline{\mathrm{C}}\left(\mathrm{CH}_{3}\right)_{3}\right),-3.6\left(\mathrm{Si}\left(\underline{\mathrm{CH}}_{3}\right)_{2}\right),-4.0\left(\mathrm{Si}\left(\underline{\mathrm{CH}}_{3}\right)_{2}\right),-4.40\right.\right.\right.$ 
$\left.\left(\mathrm{Si}\left(\underline{\mathrm{CH}}_{3}\right)_{2}\right),-4.41\left(\mathrm{Si}\left(\underline{\mathrm{CH}}_{3}\right)_{2}\right),-4.6\left(\mathrm{Si}\left(\underline{\mathrm{CH}}_{3}\right)_{2}\right),-4.8\left(\mathrm{Si}_{(\underline{\mathrm{CH}}}\right)_{2}\right),-5.3\left(\mathrm{Si}\left(\underline{\mathrm{CH}}_{3}\right)_{2}\right),-5.4$ $\left(\mathrm{Si}\left(\underline{\mathrm{CH}}_{3}\right)_{2}\right)$; HRMS(ESI) $m / z$ calcd. for $\left[\mathrm{C}_{36} \mathrm{H}_{78} \mathrm{NaO}_{8} \mathrm{Si}_{4}+\right]^{+}:$773.4666, found: 773.4673 .

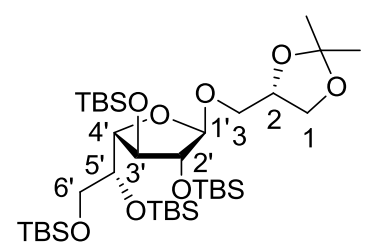

\section{3-O-(2,3,5,6-Tetra-O-tert-butyldimethylsilyl- $\beta$ -}

D-galactofuranosyl)-1,2-O-isopropylidene-sn-glycerol (sn-3-20). A solution of 19 $(1.31 \mathrm{~g}, 1.75 \mathrm{mmol})$ in anhydrous DCM $(70 \mathrm{~mL})$ was stirred with $4 \AA$ mol. sieves for 30 min. and then cooled to $0{ }^{\circ} \mathrm{C}$. To this was added neat TMSI $(0.408 \mathrm{~mL})$, which was freshly prepared under Ar by the addition of TMSTMS (0.66 g, $4.5 \mathrm{mmol}$ ) to iodine $(0.76 \mathrm{~g}, 3.0 \mathrm{mmol})$ and the reaction heated at $60{ }^{\circ} \mathrm{C}$ until the mixture turned colourless. After 15 min., TLC showed full consumption of 19 into two glycosyl iodides $\left[\mathrm{R}_{f}=0.67\right.$ $\left(\right.$ EtOAc/PE, 1/9, v/v) and $\mathrm{R}_{f}=0.53($ EtOAc/PE, 1/9, v/v)], at which point DIPEA $(0.51$ $\mathrm{g}, 3.96 \mathrm{mmol})$ and isopropylidene glycerol (+)-10 (0.52 g, $3.96 \mathrm{mmol})$ were added and the reaction stirred at RT for $3 \mathrm{~h}$ until full consumption of the iodide into a slightly higher running product $\left(\mathrm{R}_{f}=0.68, \mathrm{EtOAc} / \mathrm{PE}, 1 / 9, \mathrm{v} / \mathrm{v}\right)$. The reaction mixture was then diluted with EtOAc, washed with $\mathrm{NaHCO}_{3}$ (sat. aq.), $\mathrm{Na}_{2} \mathrm{~S}_{2} \mathrm{O}_{3}$ (sat. aq.) and brine, dried $\left(\mathrm{MgSO}_{4}\right)$, filtered and concentrated. The residue was then purified by silica gel flash column chromatography (EA/PE, 2/98, v/v), to give a $1 / 9$ mixture of the $\alpha$ - and $\beta$ isomers as a colourless oil (1.22 g, $1.62 \mathrm{mmol}$, 90\% overall yield). Here, it should be noted that separation of the anomers could be achieved via careful silica gel column chromatograpy (DCM/PE, 1/1, v/v), however, separation of the anomers became easier following acetonide deprotection (vide infra). $\beta$ isomer; $[\alpha]_{\mathrm{D}}{ }^{25}=-7.3\left(c=1.0, \mathrm{CHCl}_{3}\right)$; IR (film) 2930, 2858, 1473, 1251, 1106, 836, $777 \mathrm{~cm}^{-1} ;{ }^{1} \mathrm{H}$ NMR $\left(500 \mathrm{MHz}, \mathrm{CDCl}_{3}\right) \delta$ $4.83\left(\mathrm{~d}, J_{\mathrm{H}-1^{\prime}}, \mathrm{H}-2^{\prime}=2.2 \mathrm{~Hz}, 1 \mathrm{H}, \mathrm{H}-1^{\prime}\right), 4.29(\mathrm{~m}, 1 \mathrm{H}, \mathrm{H}-2), 4.13\left(\mathrm{dd}, J_{\mathrm{H}-3^{\prime}}, \mathrm{H}-2^{\prime}=3.3 \mathrm{~Hz}\right.$, $\left.J_{\mathrm{H}-3^{\prime}, \mathrm{H}^{\prime} 4^{\prime}}=5.8 \mathrm{~Hz}, 1 \mathrm{H}, \mathrm{H}-3^{\prime}\right), 4.05\left(\mathrm{ABX}, J_{\mathrm{H}-3 \mathrm{a}}, \mathrm{H}-2=6.3 \mathrm{~Hz}, J_{\mathrm{H}-3 \mathrm{a}, \mathrm{H}-3 \mathrm{~b}}=8.4, \mathrm{H}-3 \mathrm{a}\right), 4.02$ $\left(\mathrm{dd}, J_{\mathrm{H}-2^{\prime}, \mathrm{H}-1^{\prime}}=2.2 \mathrm{~Hz}, J_{\mathrm{H}-2^{\prime}}, \mathrm{H}-3^{\prime}=3.4 \mathrm{~Hz}, 1 \mathrm{H}, \mathrm{H}-2^{\prime}\right), 3.94\left(\mathrm{dd}, J_{\mathrm{H}-4^{\prime}}, \mathrm{H}-3^{\prime}=5.8, J_{\mathrm{H}-4^{\prime}, \mathrm{H}-}\right.$ $\left.5^{\prime}=3.0, \mathrm{H}^{\prime} 4^{\prime}\right), 3.83\left(\mathrm{ABX}, J_{\mathrm{H}-1 \mathrm{~b}, \mathrm{H}-2}=6.3 \mathrm{~Hz}, J_{\mathrm{H}-1 \mathrm{~b}}, \mathrm{H}-3 \mathrm{a}=8.4,1 \mathrm{H}, \mathrm{H}-1 \mathrm{~b}\right), 3.77\left(\mathrm{ABX}, J_{\mathrm{H}-3 \mathrm{a}}\right.$, $\left.\mathrm{H}-2=4.7 \mathrm{~Hz}, J_{\mathrm{H}-3 \mathrm{a}}, \mathrm{H}-3 \mathrm{~b}=10.0 \mathrm{~Hz}, 1 \mathrm{H}, \mathrm{H}-3 \mathrm{a}\right), 3.75\left(\mathrm{~m}, 1 \mathrm{H}, \mathrm{H}-5^{\prime}\right), 3.66\left(\mathrm{ABX}, J_{\mathrm{H}-6^{\prime} \mathrm{a}}, \mathrm{H}-5^{\prime}\right.$ $\left.=6.9 \mathrm{~Hz}, J_{\mathrm{H}-6^{\prime} \mathrm{a}, \mathrm{H}-6^{\prime} \mathrm{b}}=10.0 \mathrm{~Hz}, 1 \mathrm{H}, \mathrm{H}-6^{\prime} \mathrm{a}\right), 3.58\left(\mathrm{ABX}, J_{\mathrm{H}-6^{\prime} \mathrm{b}, \mathrm{H}-5^{\prime}}=5.9 \mathrm{~Hz}, J_{\mathrm{H}-6^{\prime} \mathrm{b}, \mathrm{H}-6^{\prime} \mathrm{a}}=\right.$ $\left.10.0 \mathrm{~Hz}, 1 \mathrm{H}, \mathrm{H}-6^{\prime} \mathrm{b}\right), 3.42\left(\mathrm{ABX}, J_{\mathrm{H}-3 \mathrm{~b}}, \mathrm{H}-2=6.7 \mathrm{~Hz}, J_{\mathrm{H}-3 \mathrm{~b}}, \mathrm{H}-3 \mathrm{a}=10.0 \mathrm{~Hz}, 1 \mathrm{H}, \mathrm{H}-3 \mathrm{~b}\right)$, 1.42 and $1.36\left(\mathrm{~s}, 3 \mathrm{H}, \mathrm{CH}_{3}(\mathrm{iPr})\right), 0.92-0.85\left(\mathrm{~m}, 36 \mathrm{H}, \mathrm{C}\left(\mathrm{CH}_{3}\right)_{3}\right), 0.13-0.03(\mathrm{~m}, 24 \mathrm{H}$, 
$\left.\mathrm{CH}_{3} \mathrm{Si}\right) ;{ }^{13} \mathrm{C}$ NMR $\left(75 \mathrm{MHz}, \mathrm{CDCl}_{3}\right) \delta 109.1\left(\underline{\mathrm{C}}\left(\mathrm{CH}_{3}\right)_{2}\right), 108.8\left(\mathrm{C}^{-1}{ }^{\prime}\right), 84.4\left(\mathrm{C}-2^{\prime}\right), 83.9$ (C-4'), 79.5 (C-3'), 74.5 (C-2), 73.1 (C-5’), 68.3 (C-3), 67.2 (C-1), 64.6 (C-6'), 26.7

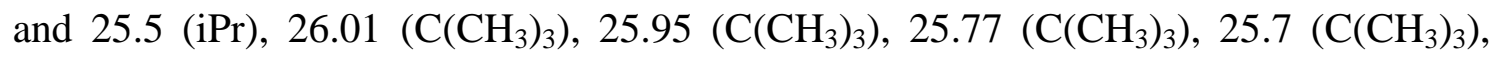
$18.32\left(\mathrm{SiC}\left(\mathrm{CH}_{3}\right)_{3}\right), 18.31 \quad\left(\mathrm{SiC}\left(\mathrm{CH}_{3}\right)_{3}\right), 17.9 \quad\left(\mathrm{Si} \underline{\mathrm{C}}\left(\mathrm{CH}_{3}\right)_{3}\right), 17.8 \quad\left(\mathrm{SiC}\left(\mathrm{CH}_{3}\right)_{3}\right),-3.6$ $\left(\mathrm{Si}\left(\underline{\mathrm{CH}}_{3}\right)_{2}\right),-4.0\left(\mathrm{Si}\left(\underline{\mathrm{CH}}_{3}\right)_{2}\right),-4.36\left(\mathrm{Si}\left(\underline{\mathrm{CH}}_{3}\right)_{2}\right),-4.41\left(\mathrm{Si}\left(\underline{\mathrm{CH}}_{3}\right)_{2}\right),-4.6\left(\mathrm{Si}\left(\underline{\mathrm{CH}}_{3}\right)_{2}\right),-4.8$

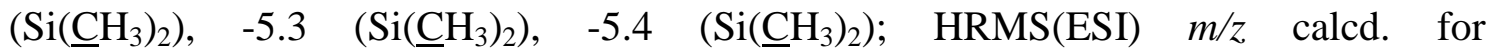
$\left[\mathrm{C}_{36} \mathrm{H}_{78} \mathrm{NaO}_{8} \mathrm{Si}_{4}+\right]^{+}$: 773.4666, found: 773.4667 .

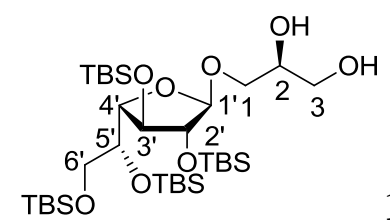

\section{1-O- $(2, \quad 3, \quad 5, \quad$ 6-Tetra-O-tert-butyldimethylsilyl- $\beta$-D-}

galactofuranosyl)-sn-glycerol (sn-1-9). A solution of $s n-1-20(48.5 \mathrm{mg}, 0.064 \mathrm{mmol})$ in TFA/ $\mathrm{H}_{2} \mathrm{O} / \mathrm{DCM}(2.5 / 2.5 / 95, \mathrm{v} / \mathrm{v} / \mathrm{v}, 3 \mathrm{~mL})$ was stirred for $10 \mathrm{~min}$. at RT, after which the reaction was quenched by the addition of $\mathrm{NaHCO}_{3}$ (sat. aq.). The mixture was then extracted with EtOAc and the combined organic layers washed with $\mathrm{NaHCO}_{3}$ (sat. aq.) and brine, dried $\left(\mathrm{MgSO}_{4}\right)$, filtered and concentrated. Purification of the residue by silica gel flash column chromatography $(\mathrm{EtOAc} / \mathrm{PE}, 1 / 9, \mathrm{v} / \mathrm{v})$, gave the pure $\beta$-isomer as a colourless oil (43.0 mg, $0.061 \mathrm{mmol}, 96 \%) .\left(\mathrm{R}_{f}=0.35\right.$, EtOAc/PE, 3/7, v/v); $[\alpha]_{\mathrm{D}}{ }^{20}=-$ $20.0\left(c=0.1, \mathrm{CHCl}_{3}\right)$; IR (film) 3463, 2954, 2928, 2857, 1463, 1255, 1103, 837, 780, $759 \mathrm{~cm}^{-1} ;{ }^{1} \mathrm{H}$ NMR $\left(500 \mathrm{MHz}, \mathrm{CDCl}_{3}\right) \delta 4.83\left(\mathrm{~s}, 1 \mathrm{H}, \mathrm{H}-1^{\prime}\right), 4.11\left(\mathrm{t}, J_{\mathrm{H}-3^{\prime}, \mathrm{H}-2^{\prime}}=J_{\mathrm{H}-3^{\prime}}, \mathrm{H}-\right.$ $\left.4^{\prime}=3.8 \mathrm{~Hz}, 1 \mathrm{H}, \mathrm{H}-3^{\prime}\right), 3.99$ (m, 1H, H-4`), 3.98 (m, 1H, H-2`), 3.77 (m, 1H, H-2), 3.72 (m, 1H, H-5'), 3.69 (m, 1H, H-1a), 3.67 (m, 1H, H-1b), 3.64 (m, 1H, H-3a), 3.62 (m, $1 \mathrm{H}, \mathrm{H}-3 \mathrm{~b}), 3.59$ (m, 1H, H-6’a), 3.57 (m, $J_{\mathrm{H}-6^{\prime} \mathrm{b}, \mathrm{H}-6^{\prime} \mathrm{a}}=10.0 \mathrm{~Hz}, J_{\mathrm{H}-6{ }^{\prime} \mathrm{b}, \mathrm{H}-5^{\prime}}=5.83 \mathrm{~Hz}, 1 \mathrm{H}$, H-6’b), 3.21 (s, 1H, OH(2)), 2.22 (s, 1H, OH (3)), 1.0-0.8 (m, 36H, C( $\left.\left.\mathrm{CH}_{3}\right)_{3}\right), 0.2-0.0$ (m, 24H, $\left.\mathrm{Si}\left(\mathrm{CH}_{3}\right)_{2}\right) ;{ }^{13} \mathrm{C}$ NMR $\left(125 \mathrm{MHz}, \mathrm{CDCl}_{3}\right) \delta 108.9\left(\mathrm{C}-1^{\prime}\right), 85.5\left(\mathrm{C}-4^{\prime}\right), 83.3(\mathrm{C}-$ 2’), 78.7 (C-3'), 73.4 (C-5'), 70.4 (C-2), 70.3 (C-1), 64.5 (C-6'), 64.0 (C-3), 25.96 $\left(\mathrm{C}\left(\underline{\mathrm{CH}}_{3}\right)_{3}\right), 25.93\left(\mathrm{C}\left(\underline{\mathrm{C}} \mathrm{H}_{3}\right)_{3}\right), 25.73\left(\mathrm{C}\left(\underline{\mathrm{CH}}_{3}\right)_{3}\right), 25.70\left(\mathrm{C}\left(\underline{\mathrm{CH}}_{3}\right)_{3}\right), 18.3\left(\mathrm{Si} \underline{\mathrm{C}}\left(\mathrm{CH}_{3}\right)_{3}\right), 18.2$ $\left(\mathrm{SiC}\left(\mathrm{CH}_{3}\right)_{3}\right), 17.9\left(\mathrm{SiC}\left(\mathrm{CH}_{3}\right)_{3}\right), 17.8\left(\mathrm{SiC}\left(\mathrm{CH}_{3}\right)_{3}\right),-3.9\left(\mathrm{Si}\left(\mathrm{CH}_{3}\right)_{2}\right),-4.2\left(\mathrm{Si}\left(\mathrm{CH}_{3}\right)_{2}\right),-4.44$ $\left(\mathrm{Si}\left(\mathrm{CH}_{3}\right)_{2}\right),-4.46\left(\mathrm{Si}\left(\mathrm{CH}_{3}\right)_{2}\right),-4.5\left(\mathrm{Si}\left(\mathrm{CH}_{3}\right)_{2}\right),-4.8\left(\mathrm{Si}\left(\mathrm{CH}_{3}\right)_{2}\right),-5.3\left(\mathrm{Si}\left(\mathrm{CH}_{3}\right)_{2}\right),-5.4$ $\left(\mathrm{Si}\left(\mathrm{CH}_{3}\right)_{2}\right)$; HRMS(ESI) $\mathrm{m} / z$ calcd. for $\left[\mathrm{C}_{33} \mathrm{H}_{74} \mathrm{NaO}_{8} \mathrm{Si}_{4}\right]^{+}:$733.4353, found: 733.4365 . 


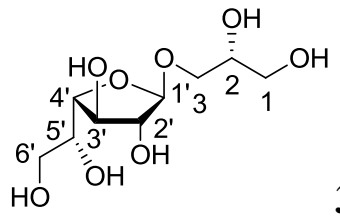

3-O-( $\beta$-D-galactofuranosyl)-sn-glycerol (GalfGro). To a solution

of $s n-3-9(68 \mathrm{mg}, 0.26 \mathrm{mmol})$ in $\mathrm{MeOH}(1 \mathrm{~mL})$ was added Dowex- $\mathrm{H}^{+}$and the reaction mixture stirred at RT for $12 \mathrm{~h}$. The solution was filtered and concentrated in vacuo. Purification of the residue by silica gel flash column chromatography (MeOH/EtOAC, $15 / 85, \mathrm{v} / \mathrm{v})$ gave GalfGro as a white solid (41.6 mg, $0.16 \mathrm{mmol}, 63 \%) .\left(\mathrm{R}_{f}=0.14\right.$, $\mathrm{MeOH} /$ EtOAc, $1 / 4, \mathrm{v} / \mathrm{v}) ;[\alpha]_{\mathrm{D}}{ }^{26}=-40.0\left(c=0.1, \mathrm{H}_{2} \mathrm{O}\right)$; IR (film) 3465, 2928, 1462, 1309, 1250, 1162, 1031, $834 \mathrm{~cm}^{-1} ;{ }^{1} \mathrm{H}$ NMR (500 MHz, $\left.\mathrm{CDCl}_{3}\right) \delta 4.84$ (s, 1H, H-1'), $3.93\left(\mathrm{dd}, J_{\mathrm{H}-2^{\prime}, \mathrm{H}-1^{\prime}}=1.92, J_{\mathrm{H}-2^{\prime}, \mathrm{H}-3^{\prime}}=3.55,1 \mathrm{H}, \mathrm{H}-2^{\prime}\right), 3.79\left(\mathrm{~m}, J_{\mathrm{H}-4^{\prime}}, \mathrm{H}-3^{\prime}=5.8 \mathrm{~Hz}, J_{\mathrm{H}-4^{\prime}}\right.$, $\left.\mathrm{H}-5^{\prime}=4.3 \mathrm{~Hz}, 1 \mathrm{H}, \mathrm{H}-4^{\prime}\right), 3.90\left(\mathrm{dd}, J_{\mathrm{H}-3^{\prime}, \mathrm{H}-2^{\prime}}=3.55, J_{\mathrm{H}-3^{\prime}},{\mathrm{H}-4^{\prime}}^{\prime}=6.15,1 \mathrm{H}, \mathrm{H}-3^{\prime}\right), 3.74(\mathrm{~m}$, 1H, H-2), 3.67 (m, 1H, H-5') 3.56 (m, 1H, H-3a), 3.46 (m, 1H, H-3b), 3.54 (m, 1H, H6’a), 3.47 (m, 1H, H-6’b), 3.44 (m, 1H, H-1a), 3.40 (m, 1H, H-1b); ${ }^{13} \mathrm{C}$ NMR (125 $\mathrm{MHz}, \mathrm{CDCl}_{3}$ ) $\delta 106.9\left(\mathrm{C}-1^{\prime}\right), 82.6\left(\mathrm{C}^{-} 4^{\prime}\right), 80.9$ (C-2'), 76.6 (C-3’), 70.7 (C-5'), $70.0(\mathrm{C}-$ 2), 68.4 (C-3), 62.5 (C-6'), 62.3 (C-1); HRMS(ESI) m/z calcd. for $\left[\mathrm{C}_{9} \mathrm{H}_{18} \mathrm{NaO}_{8}\right]^{+}$: 277.0894, found: 277.0895 .

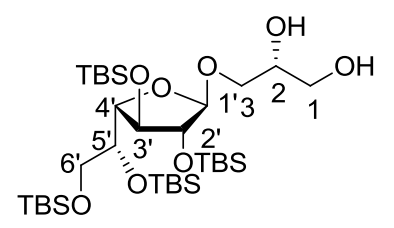

\section{3-O-(2,3,5,6-Tetra-O-tert-butyldimethylsilyl- $\beta$ -}

D-galactofuranosyl)-sn-glycerol (sn-3-9). A solution of $s n-3-20(149 \mathrm{mg}, 0.20 \mathrm{mmol})$ in TFA/ $\mathrm{H}_{2} \mathrm{O} / \mathrm{DCM}(2.5 / 2.5 / 95, \mathrm{v} / \mathrm{v} / \mathrm{v}, 10 \mathrm{~mL})$ was stirred for $10 \mathrm{~min}$. at RT, after which the reaction was quenched by the addition of $\mathrm{NaHCO}_{3}$ (sat. aq.). The mixture was then extracted with EtOAc and the combined organic layers washed with $\mathrm{NaHCO}_{3}$ (sat. aq.) and brine, dried $\left(\mathrm{MgSO}_{4}\right)$, filtered and concentrated. Purification of the residue by silica gel flash column chromatography (EtOAc/PE, 1/9, v/v), gave the pure $\beta$-isomer as a colourless oil $(131 \mathrm{mg}, 0.18 \mathrm{mmol}, 93 \%) .\left(\mathrm{R}_{f}=0.37\right.$, EtOAc/PE, 3/7, v/v); $[\alpha]_{\mathrm{D}}{ }^{24}=-$ $20.0\left(c=0.1, \mathrm{CHCl}_{3}\right)$; IR (film) 3498, 2955, 2929, 2858, 1472, 1361, 1253, 1107, 836, 776, $670 \mathrm{~cm}^{-1} ;{ }^{1} \mathrm{H} \mathrm{NMR}\left(500 \mathrm{MHz}, \mathrm{CDCl}_{3}\right) \delta 4.85\left(\mathrm{~d}, J_{\mathrm{H}-1^{\prime}, \mathrm{H}-2^{\prime}}=2.2 \mathrm{~Hz}, 1 \mathrm{H}, \mathrm{H}^{-1} 1^{\prime}\right)$, $4.14\left(\mathrm{dd}, J_{\mathrm{H}-3^{\prime}, \mathrm{H}-2^{\prime}}=3.1 \mathrm{~Hz}, J_{\mathrm{H}-3^{\prime}, \mathrm{H}-4^{\prime}}=4.5 \mathrm{~Hz}, 1 \mathrm{H}, \mathrm{H}-3^{\prime}\right), 4.0$ (m, 2H, H-2 ${ }^{\prime}$ and $\left.\mathrm{H}^{-} 4^{\prime}\right)$, 3.83 (m, 2H, H-2), 3.75 (m, 1H, H-5), 3.69 (m, 2H, H-3a and b), 3.68 (ABX, 1H, H1a), $3.63\left(\mathrm{ABX}, J_{\mathrm{H}-6^{\prime} \mathrm{a}, \mathrm{H}-2}=3.4 \mathrm{~Hz}, J_{\mathrm{H}-6^{\prime} \mathrm{a}, \mathrm{H}_{-}^{\prime} 6^{\prime}}=10.3 \mathrm{~Hz}, 1 \mathrm{H}, \mathrm{H}-6^{\prime} \mathrm{a}\right), 3.60\left(\mathrm{ABX}, J_{\mathrm{H}-3 \mathrm{~b}}\right.$, 
$\left.\mathrm{H}-2=5.6 \mathrm{~Hz}, J_{\mathrm{H}-3 \mathrm{a}}, \mathrm{H}-3 \mathrm{~b}=11.2 \mathrm{~Hz} 1 \mathrm{H}, \mathrm{H}-1 \mathrm{~b}\right), 3.59\left(\mathrm{ABX}, J_{\mathrm{H}-6^{\prime} \mathrm{b}}, \mathrm{H}_{-} 5^{\prime}=5.74 \mathrm{~Hz}, J_{\mathrm{H}-6^{\prime} \mathrm{b}},{\mathrm{H}-6^{\prime} \mathrm{a}}\right.$ $=10.0 \mathrm{~Hz} 1 \mathrm{H}, \mathrm{H}-6$ b $), 3.31\left(\mathrm{~d}, J_{\mathrm{H}-2}, \mathrm{OH}=4.3 \mathrm{~Hz}, 1 \mathrm{H}, \mathrm{OH}\right), 2.3\left(\mathrm{t}, J_{\mathrm{H}-1}, \mathrm{OH}=5.8 \mathrm{~Hz}, 1 \mathrm{H}\right.$, $\mathrm{OH})$ ), $1.0-0.8\left(\mathrm{~m}, 36 \mathrm{H}, \mathrm{C}\left(\mathrm{CH}_{3}\right)_{3}\right), 0.2-0.0\left(\mathrm{~m}, 24 \mathrm{H}, \mathrm{Si}\left(\mathrm{CH}_{3}\right)_{2}\right) ;{ }^{13} \mathrm{C}$ NMR $(75 \mathrm{MHz}$, $\left.\mathrm{CDCl}_{3}\right) \delta 108.4$ (C-1'), 85.3 (C-4'), 83.7 (C-2'), 79.0 (C-3'), 73.5 (C-5'), 70.9 (C-3), 70.6 (C-2), $\left.64.6\left(\mathrm{C}^{\prime} 6^{\prime}\right), 63.7(\mathrm{C}-1), 25.96\left(\mathrm{C}\left(\underline{\mathrm{CH}}_{3}\right)_{3}\right), 25.95\left(\mathrm{C}\left(\underline{\mathrm{C}} \mathrm{H}_{3}\right)_{3}\right), 25.8\left(\mathrm{C}^{\left(\mathrm{C}_{3}\right.}\right)_{3}\right)$, $25.7 \quad\left(\mathrm{C}\left(\underline{C}_{3}\right)_{3}\right), \quad 18.3 \quad\left(\mathrm{SiC}\left(\mathrm{CH}_{3}\right)_{3}\right), \quad 18.26 \quad\left(\mathrm{Si} \underline{\mathrm{C}}\left(\mathrm{CH}_{3}\right)_{3}\right), \quad 17.9 \quad\left(\mathrm{SiC}\left(\mathrm{CH}_{3}\right)_{3}\right), \quad 17.8$ $\left(\mathrm{SiC}\left(\mathrm{CH}_{3}\right)_{3}\right),-3.8\left(\mathrm{Si}\left(\mathrm{CH}_{3}\right)_{2}\right),-4.2\left(\mathrm{Si}\left(\mathrm{CH}_{3}\right)_{2}\right),-4.40\left(\mathrm{Si}\left(\mathrm{CH}_{3}\right)_{2}\right),-4.43\left(\mathrm{Si}\left(\mathrm{CH}_{3}\right)_{2}\right),-4.49$ $\left(\mathrm{Si}\left(\mathrm{CH}_{3}\right)_{2}\right),-4.8\left(\mathrm{Si}\left(\mathrm{CH}_{3}\right)_{2}\right),-5.3\left(\mathrm{Si}\left(\mathrm{CH}_{3}\right)_{2}\right),-5.4\left(\mathrm{Si}\left(\mathrm{CH}_{3}\right)_{2}\right)$; HRMS(ESI) $m / z$ calcd. for $\left[\mathrm{C}_{33} \mathrm{H}_{74} \mathrm{NaO}_{8} \mathrm{Si}_{4}\right]^{+}:$733.4353, found: 733.4366.

\section{General esterification procedure.}

To a solution of alcohol $(0.2 \mathrm{M})$ and the appropriate fatty acid (4.4 equiv.) in dry toluene was added EDCI (4.4 equiv.) and DMAP (0.15 equiv.). The reaction was then heated as required (see specific procedures) until TLC showed complete conversion of starting material. The reaction mixture was then diluted with EtOAc, washed with $\mathrm{NaHCO}_{3}$ (sat. aq.) and brine, dried $\left(\mathrm{MgSO}_{4}\right)$, filtered and concentrated in vacuo. Purification of the residue by silica gel flash column chromatography afforded the title compound.

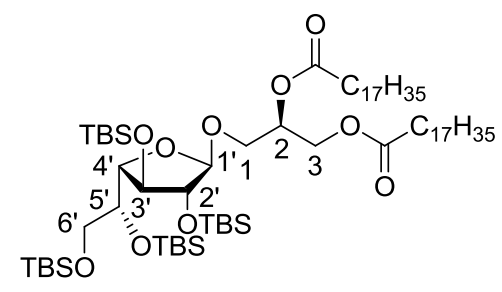

\section{1-O-(2, 3, 5, 6-Tetra- $O$-tert-butyldimethylsilyl- $\beta$-D}

galactofuranosyl)-2,3-di-O-stearoyl-sn-glycerol (sn-1-6a). Subjection of $s n-1-9$ (71 $\mathrm{mg}, 0.1 \mathrm{mmol})$, stearic acid (124 mg, $0.44 \mathrm{mmol})$, DMAP (1.8 mg, $0.015 \mathrm{mmol})$ and EDCI $(68.3 \mathrm{mg}, 0.44 \mathrm{mmol})$ to the general esterification procedure at $40{ }^{\circ} \mathrm{C}$ for $12 \mathrm{~h}$ gave the title compound sn-1-6a (108.5 mg, $0.087 \mathrm{mmol}, 87 \%)$ as a colourless oil after column chromatrography $\left(\right.$ EtOAc/PE, 1/99, v/v). $\left(\mathrm{R}_{f}=0.60\right.$, EtOAc/PE, $\left.1 / 9, \mathrm{v} / \mathrm{v}\right) ;[\alpha]_{\mathrm{D}}{ }^{25}$ $=-20.0\left(c=1, \mathrm{CHCl}_{3}\right)$; IR (film) 2924, 2854, 1745, 1463, 1252, 1111, 836, $776 \mathrm{~cm}^{-1}$; ${ }^{1} \mathrm{H}$ NMR $\left(500 \mathrm{MHz}, \mathrm{CDCl}_{3}\right) \delta 5.19(\mathrm{~m}, 1 \mathrm{H}, \mathrm{H}-2), 4.79\left(\mathrm{~d}, J_{\mathrm{H}-1^{\prime}},{\mathrm{H}-2^{\prime}}^{\prime}=2.0 \mathrm{~Hz}, 1 \mathrm{H}, \mathrm{H}-1^{\prime}\right)$, $4.36\left(\mathrm{ABX}, J_{\mathrm{H}-3 \mathrm{a}}, \mathrm{H}-2=3.7 \mathrm{~Hz}, J_{\mathrm{H}-3 \mathrm{a}}, \mathrm{H}-3 \mathrm{~b}=12.0 \mathrm{~Hz}, 1 \mathrm{H}, \mathrm{H}-3 \mathrm{a}\right), 4.13\left(\mathrm{ABX}, J_{\mathrm{H}-3 \mathrm{~b}}, \mathrm{H}-2=\right.$ 
$\left.6.3 \mathrm{~Hz}, J_{\mathrm{H}-3 \mathrm{~b}}, \mathrm{H}-3 \mathrm{a}=8.3 \mathrm{~Hz}, 1 \mathrm{H}, \mathrm{H}-3 \mathrm{~b}\right), 4.11\left(\mathrm{~m}, 1 \mathrm{H}, \mathrm{H}-3^{\prime}\right), 4.00\left(\mathrm{dd}, J_{\mathrm{H}-2^{\prime}}, \mathrm{H}-3^{\prime}=3.0 \mathrm{~Hz}\right.$, $\left.J_{\mathrm{H}-2^{\prime}, \mathrm{H}-1^{\prime}}=2.1 \mathrm{~Hz}, 1 \mathrm{H}, \mathrm{H}-2^{\prime}\right), 3.92\left(\mathrm{dd}, J_{\mathrm{H}-4^{\prime}, \mathrm{H}-3^{\prime}}=5.3 \mathrm{~Hz}, J_{\mathrm{H}-4^{\prime}, \mathrm{H}-5^{\prime}}=3.4 \mathrm{~Hz}, 1 \mathrm{H}, \mathrm{H}^{\prime} 4^{\prime}\right)$, $3.79\left(\mathrm{ABX}, J_{\mathrm{H}-1 \mathrm{a}}, \mathrm{H}-2=5.3 \mathrm{~Hz}, J_{\mathrm{H}-1 \mathrm{a}}, \mathrm{H}-3 \mathrm{~b}=10.4 \mathrm{~Hz}, 1 \mathrm{H}, \mathrm{H}-1 \mathrm{a}\right), 3.76\left(\mathrm{~m}, 1 \mathrm{H}, \mathrm{H}-5^{\prime}\right), 3.67$ $\left(\mathrm{ABX}, J_{\mathrm{H}-6^{\prime} \mathrm{a}}, \mathrm{H}-5^{\prime}=6.2 \mathrm{~Hz}, J_{\mathrm{H}-6^{\prime} \mathrm{a}, \mathrm{H}-6^{\prime} \mathrm{b}}=10.1 \mathrm{~Hz}, 1 \mathrm{H}, \mathrm{H}-6^{\prime} \mathrm{a}\right), 3.57\left(\mathrm{ABX}, J_{\mathrm{H}-66^{\prime}}, \mathrm{H}_{-} 5^{\prime}=5.9\right.$ $\left.\mathrm{Hz}, J_{\mathrm{H}-6 \mathrm{~b}}, \mathrm{H}-6^{\prime} \mathrm{a}=10.0 \mathrm{~Hz}, 1 \mathrm{H}, \mathrm{H}-6^{\prime} \mathrm{b}\right), 3.55\left(\mathrm{ABX}, J_{\mathrm{H}-1 \mathrm{~b}}, \mathrm{H}-2=5.9 \mathrm{~Hz}, J_{\mathrm{H}-1 \mathrm{~b}}, \mathrm{H}-1 \mathrm{a}=10.3\right.$ $\mathrm{Hz}, 1 \mathrm{H}, \mathrm{H}-1 \mathrm{~b}), 2.30$ (t, $\left.J_{\mathrm{H} \alpha}, \mathrm{H} \beta=7.6 \mathrm{~Hz}, 4 \mathrm{H}, \mathrm{CH}_{2} \alpha\right), 1.60\left(\mathrm{~m}, 4 \mathrm{H}, \mathrm{CH}_{2} \beta\right), 1.37-1.14$ (m, $\left.56 \mathrm{H}, \mathrm{CH}_{2}\right), 0.9-0.8\left(\mathrm{~m}, 42 \mathrm{H}, \mathrm{CH}_{3}\right.$ and $\left.\mathrm{C}\left(\mathrm{CH}_{3}\right)_{3}\right), 0.16-0.00\left(\mathrm{~m}, 24 \mathrm{H}, \mathrm{Si}\left(\mathrm{CH}_{3}\right)_{2}\right) ;{ }^{13} \mathrm{C}$ NMR (125 MHz, $\mathrm{CDCl}_{3}$ ) $\delta 173.3$ and $173.0(\mathrm{C}(\mathrm{O})), 108.3$ (C-1'), $84.5\left(\mathrm{C}-4^{\prime}\right), 84.1$ (C2’), 79.5 (C-3’), 73.4 (C-5’), 70.1 (C-2), 65.6 (C-1), 64.7 (C-6'), 62.8 (C-3); 34.3 and $\left.34.1\left(\mathrm{CH}_{2} \alpha\right), 31.9\left(\underline{\mathrm{CH}}_{2} \mathrm{CH}_{2} \mathrm{CH}_{3}\right), 29.9-29.0\left(\mathrm{CH}_{2}\right), 26.0\left(\mathrm{C}(\underline{\mathrm{CH}})_{3}\right), 26.97\left(\mathrm{C}^{\left(\mathrm{CH}_{3}\right.}\right)_{3}\right)$, $25.8\left(\mathrm{C}\left(\underline{\mathrm{CH}}_{3}\right)_{3}\right), 25.7\left(\mathrm{C}\left(\underline{\mathrm{CH}}_{3}\right)_{3}\right), 24.9\left(\mathrm{CH}_{2} \beta\right), 18.3$ (x2) $\left(\mathrm{Si} \underline{\mathrm{C}}\left(\mathrm{CH}_{3}\right)_{3}\right), 17.9\left(\mathrm{SiC}\left(\mathrm{CH}_{3}\right)_{3}\right)$, 17.8, $14.1\left(\mathrm{CH}_{3}\right)\left(\mathrm{Si} \underline{\mathrm{C}}\left(\mathrm{CH}_{3}\right)_{3}\right),-3.7\left(\mathrm{Si}\left(\mathrm{CH}_{3}\right)_{2}\right),-4.0\left(\mathrm{Si}\left(\mathrm{CH}_{3}\right)_{2}\right),-4.4\left(\mathrm{Si}\left(\mathrm{CH}_{3}\right)_{2}\right),-4.43$

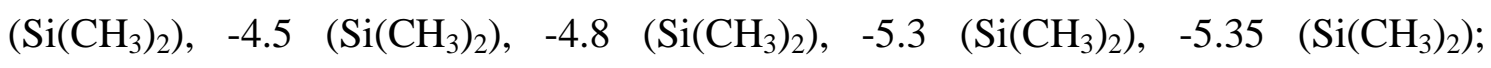
HRMS(ESI) $m / z$ calcd. for $\left[\mathrm{C}_{69} \mathrm{H}_{142} \mathrm{NaO}_{10} \mathrm{Si}_{4}\right]^{+}: 1265.9572$, found: 1265.9584 .

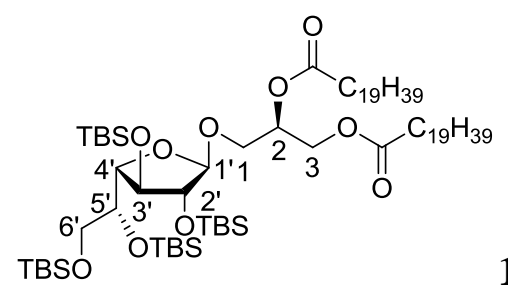

1-O-(2, 3, 5, 6-Tetra-O-tert-butyldimethylsilyl- $\beta$-D

galactofuranosyl)-2,3-di- $O$-arachidoyl-sn-glycerol (sn-1-6b). Subjection of $s n$-1-9 (19.0 mg, $0.026 \mathrm{mmol}$ ), arachidic acid (35.8 mg, $0.114 \mathrm{mmol}$ ), DMAP (0.47 mg, 0.0039 $\mathrm{mmol})$ and EDCI $(17.7 \mathrm{mg}, 0.114 \mathrm{mmol})$ to the general esterification procedure at $40{ }^{\circ} \mathrm{C}$ for $12 \mathrm{~h}$ gave the title compound $\mathrm{s} n-1-6 \mathbf{b}(33.1 \mathrm{mg}, 0.025 \mathrm{mmol}, 98 \%)$, as a colourless oil after column chromatrography (EtOAc/PE, 1/99, v/v). $\left(\mathrm{R}_{f}=0.56, \mathrm{EtOAc} / \mathrm{PE}, 1 / 9\right.$, $\mathrm{v} / \mathrm{v}) ;[\alpha]_{\mathrm{D}}{ }^{21}=-21.0\left(c=0.1, \mathrm{CHCl}_{2}\right)$; IR (film) $2925,2854,1743,1769,1555,1512$, 1463, 1401, 1275, 1258, 1108, 1005, 922, 836, 764, 750, 711, $668 \mathrm{~cm}^{-1}$; ${ }^{1} \mathrm{H}$ NMR (500 $\left.\mathrm{MHz} \mathrm{CDCl}_{3}\right) \delta 5.18(\mathrm{~m}, 1 \mathrm{H}, \mathrm{H}-2), 4.78\left(\mathrm{~d}, J_{\mathrm{H}-1^{\prime}}, \mathrm{H}-2^{\prime}=1.8 \mathrm{~Hz}, 1 \mathrm{H}, \mathrm{H}-1^{\prime}\right), 4.34(\mathrm{ABX}$, $\left.J_{\mathrm{H}-3 \mathrm{a}}, \mathrm{H}-2=3.6 \mathrm{~Hz}, J_{\mathrm{H}-3 \mathrm{a}}, \mathrm{H}-3 \mathrm{~b}=11.8 \mathrm{~Hz}, 1 \mathrm{H}, \mathrm{H}-3 \mathrm{a}\right), 4.13\left(\mathrm{ABX}, J_{\mathrm{H}-3 \mathrm{~b}}, \mathrm{H}-2=6.3 \mathrm{~Hz}, J_{\mathrm{H}-3 \mathrm{~b}}, \mathrm{H}-\right.$ $3 \mathrm{a}=8.3 \mathrm{~Hz}, 1 \mathrm{H}, \mathrm{H}-3 \mathrm{~b}), 4.10\left(\mathrm{~m}, 1 \mathrm{H}, \mathrm{H}-3^{\prime}\right), 3.98\left(\mathrm{dd}, J_{\mathrm{H}-2^{\prime}, \mathrm{H}-3}=3.3 \mathrm{~Hz}, J_{\mathrm{H}-2^{\prime}}, \mathrm{H}-1=2.3\right.$ $\left.\mathrm{Hz}, 1 \mathrm{H}, \mathrm{H}-2^{\prime}\right), 3.88$ (dd, $\left.J_{\mathrm{H}-4^{\prime}}, \mathrm{H}-3^{\prime}=5.7 \mathrm{~Hz}, J_{\mathrm{H}-4^{\prime}}, \mathrm{H}-5^{\prime}=3.4 \mathrm{~Hz}, 1 \mathrm{H}, \mathrm{H}-4^{\prime}\right), 3.75$ (ABX, $\left.J_{\mathrm{H}-1 \mathrm{a}}, \mathrm{H}-2=5.3 \mathrm{~Hz}, J_{\mathrm{H}-1 \mathrm{a}}, \mathrm{H}-3 \mathrm{~b}=10.4 \mathrm{~Hz}, 1 \mathrm{H}, \mathrm{H}-1 \mathrm{a}\right), 3.72\left(\mathrm{~m}, 1 \mathrm{H}, \mathrm{H}-5^{\prime}\right), 3.67\left(\mathrm{ABX}, J_{\mathrm{H}-6^{\prime} \mathrm{a}}\right.$, $\left.\mathrm{H}-5^{\prime}=6.3 \mathrm{~Hz}, J_{\mathrm{H}-6^{\prime} \mathrm{a}, \mathrm{H}-6^{\prime} \mathrm{b}}=9.8 \mathrm{~Hz}, 1 \mathrm{H}, \mathrm{H}-6^{\prime} \mathrm{a}\right), 3.57\left(\mathrm{ABX}, J_{\mathrm{H}-6^{\prime} \mathrm{b}, \mathrm{H}-5}=5.9 \mathrm{~Hz}, J_{\mathrm{H}-6^{\prime} \mathrm{b}, \mathrm{H}-6^{\prime} \mathrm{a}}\right.$ $=10.2 \mathrm{~Hz}, 1 \mathrm{H}, \mathrm{H}-6 \mathrm{~b}), 3.55\left(\mathrm{ABX}, J_{\mathrm{H}-1 \mathrm{~b}, \mathrm{H}-2}=5.5 \mathrm{~Hz}, J_{\mathrm{H}-1 \mathrm{~b}, \mathrm{H}-1 \mathrm{a}}=10.2 \mathrm{~Hz}, 1 \mathrm{H}, \mathrm{H}-1 \mathrm{~b}\right)$, 
$2.30\left(\mathrm{t}, J_{\mathrm{CH} 2 \alpha}, \mathrm{CH}_{2} \beta=7.6 \mathrm{~Hz}, 4 \mathrm{H}, \mathrm{CH}_{2} \alpha\right), 1.58\left(\mathrm{~m}, 4 \mathrm{H}, \mathrm{CH}_{2} \beta\right), 1.36-1.17\left(\mathrm{~m}, 64 \mathrm{H}, \mathrm{CH}_{2}\right)$, 0.9-0.8 (m, 42H, $\mathrm{CH}_{3}$ and $\left.\mathrm{C}\left(\mathrm{CH}_{3}\right)_{3}\right), 0.12-0.02\left(\mathrm{~m}, 24 \mathrm{H}, \mathrm{Si}\left(\mathrm{CH}_{3}\right)_{2}\right) ;{ }^{13} \mathrm{C}$ NMR (125 $\left.\mathrm{MHz}, \mathrm{CDCl}_{3}\right) \delta 173.3$ and $173.0(\mathrm{C}(\mathrm{O})), 108.2\left(\mathrm{C}-1^{\prime}\right), 84.4\left(\mathrm{C}^{\prime} 4^{\prime}\right), 84.2\left(\mathrm{C}-2^{\prime}\right), 79.4(\mathrm{C}-$ 3’), 73.4 (C-5'), 70.1 (C-2), 65.6 (C-1), 64.7 (C-6’), 62.8 (C-3); 34.3 and $34.1\left(\mathrm{CH}_{2} \alpha\right)$, $31.9\left(\underline{\mathrm{CH}}_{2} \mathrm{CH}_{2} \mathrm{CH}_{3}\right), \quad 29.9-29.0 \quad\left(\mathrm{CH}_{2}\right), \quad 26.0 \quad\left(\mathrm{C}\left(\underline{\mathrm{CH}}_{3}\right)_{3}\right), \quad 26.96 \quad\left(\mathrm{C}\left(\underline{\mathrm{CH}}_{3}\right)_{3}\right), \quad 25.75$ $\left(\mathrm{C}\left(\underline{\mathrm{CH}_{3}}\right)_{3}\right), 25.70\left(\mathrm{C}\left(\underline{\mathrm{C}} \mathrm{H}_{3}\right)_{3}\right), 24.9\left(\mathrm{CH}_{2} \beta\right), 18.3(\mathrm{x} 2)\left(\mathrm{SiC}\left(\mathrm{CH}_{3}\right)_{3}\right), 17.9\left(\mathrm{SiC}\left(\mathrm{CH}_{3}\right)_{3}\right)$, $17.8\left(\mathrm{SiC}\left(\mathrm{CH}_{3}\right)_{3}\right), 14.1\left(\mathrm{CH}_{3}\right),-3.7\left(\mathrm{Si}\left(\mathrm{CH}_{3}\right)_{2}\right),-4.0\left(\mathrm{Si}\left(\mathrm{CH}_{3}\right)_{2}\right),-4.4\left(\mathrm{Si}\left(\mathrm{CH}_{3}\right)_{2}\right),-4.44$

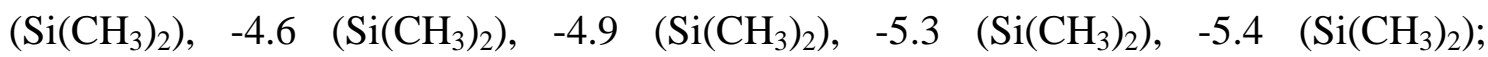
HRMS(ESI) $m / z$ calcd. for $\left[\mathrm{C}_{73} \mathrm{H}_{150} \mathrm{NaO}_{10} \mathrm{Si}_{4}\right]^{+}:$1322.0198, found: 1322.0210 .

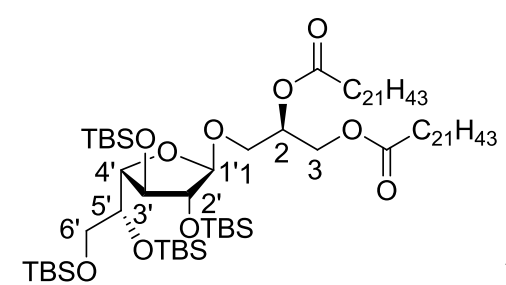

1-O-(2, 3, 5, 6-Tetra-O-tert-butyldimethylsilyl- $\beta$-D galactofuranosyl)-2,3-di-O-behenoyl-sn-glycerol (sn-1-6c). Subjection of $s n-1-9$ (16.7 $\mathrm{mg}, 0.023 \mathrm{mmol})$, behenic acid (39.0 mg, $0.10 \mathrm{mmol})$, DMAP (0.42 mg, $0.003 \mathrm{mmol})$ and EDCI $(15.7 \mathrm{mg}, 0.10 \mathrm{mmol})$ to the general esterification procedure at $70{ }^{\circ} \mathrm{C}$ for 6 days gave the title compound sn-1-6c (20.9 mg, $0.015 \mathrm{mmol}, 77 \%)$, as a colourless oil after column chromatrography (EtOAc/PE, 1/99, v/v). $\left(\mathrm{R}_{f}=0.50\right.$, EtOAc/PE, 1/9, v/v); $[\alpha]_{\mathrm{D}}^{21}=-24.5\left(c=1.0, \mathrm{CHCl}_{3}\right) ; \mathrm{IR}($ film) $2921,2852,1744,1464,1252,1108,835,776$ $\mathrm{cm}^{-1} ;{ }^{1} \mathrm{H}$ NMR $\left(500 \mathrm{MHz}, \mathrm{CDCl}_{3}\right) \delta 5.20(\mathrm{~m}, 1 \mathrm{H}, \mathrm{H}-2), 4.79\left(\mathrm{~d}, J_{\mathrm{H}-1^{\prime}}, \mathrm{H}-2^{\prime}=1.8 \mathrm{~Hz}, 1 \mathrm{H}\right.$, H-1 $\left.{ }^{\prime}\right), 4.36\left(\mathrm{ABX}, J_{\mathrm{H}-3 \mathrm{a}}, \mathrm{H}-2=3.4 \mathrm{~Hz}, J_{\mathrm{H}-3 \mathrm{a}}, \mathrm{H}-3 \mathrm{~b}=11.8 \mathrm{~Hz}, 1 \mathrm{H}, \mathrm{H}-3 \mathrm{a}\right), 4.13(\mathrm{~m}, 1 \mathrm{H}, \mathrm{H}-$ 3b), $4.11\left(\mathrm{~m}, 1 \mathrm{H}, \mathrm{H}-3^{\prime}\right), 3.99\left(\mathrm{~m}, 1 \mathrm{H}, \mathrm{H}-2^{\prime}\right), 3.92\left(\mathrm{dd}, J_{\mathrm{H}-4^{\prime}, \mathrm{H}-3^{\prime}}=5.9 \mathrm{~Hz}, J_{\mathrm{H}-4^{\prime}, \mathrm{H}-5^{\prime}}=3.5\right.$ $\left.\mathrm{Hz}, 1 \mathrm{H}, \mathrm{H}-4^{\prime}\right), 3.79$ (ABX, $\left.J_{\mathrm{H}-1 \mathrm{a}}, \mathrm{H}-2=5.6 \mathrm{~Hz}, J_{\mathrm{H}-1 \mathrm{a}}, \mathrm{H}-3 \mathrm{~b}=10.4 \mathrm{~Hz}, 1 \mathrm{H}, \mathrm{H}-1 \mathrm{a}\right), 3.76(\mathrm{~m}$, $\left.1 \mathrm{H}, \mathrm{H}-5^{\prime}\right), 3.66\left(\mathrm{ABX}, J_{\mathrm{H}-6^{\prime} \mathrm{a}}, \mathrm{H}-5^{\prime}=6.2 \mathrm{~Hz}, J_{\mathrm{H}-6^{\prime} \mathrm{a}}, \mathrm{H}-6^{\prime} \mathrm{b}=9.8 \mathrm{~Hz}, 1 \mathrm{H}, \mathrm{H}-6^{\prime} \mathrm{a}\right), 3.58(\mathrm{ABX}$, $\left.J_{\mathrm{H}-6^{\prime} \mathrm{b}}, \mathrm{H}-5^{\prime}=6.0 \mathrm{~Hz}, J_{\mathrm{H}-6^{\prime} \mathrm{b}, \mathrm{H}-6^{\prime} \mathrm{a}}=9.8 \mathrm{~Hz}, 1 \mathrm{H}, \mathrm{H}-6^{\prime} \mathrm{b}\right), 3.50\left(\mathrm{ABX}, J_{\mathrm{H}-1 \mathrm{~b}}, \mathrm{H}-2=5.8 \mathrm{~Hz}, J_{\mathrm{H}-1 \mathrm{~b}}\right.$, $\mathrm{H}-1 \mathrm{a}=10.3 \mathrm{~Hz}, 1 \mathrm{H}, \mathrm{H}-1 \mathrm{~b}$ ), 2.30 (t, $\left.J_{\mathrm{CH} 2 \alpha}, \mathrm{CH}_{2} \beta=7.4 \mathrm{~Hz}, 4 \mathrm{H}, \mathrm{CH}_{2} \alpha\right), 1.60\left(\mathrm{~m}, 4 \mathrm{H}, \mathrm{CH}_{2} \beta\right)$, 1.35-1.21 (m, 72H, $\left.\mathrm{CH}_{2}\right), 1.0-0.8\left(\mathrm{~m}, 42 \mathrm{H}, \mathrm{CH}_{3}\right.$ and $\left.\mathrm{C}\left(\mathrm{CH}_{3}\right)_{3}\right), 0.15-0.03(\mathrm{~m}, 24 \mathrm{H}$, $\left.\mathrm{Si}\left(\mathrm{CH}_{3}\right)_{2}\right) ;{ }^{13} \mathrm{C}$ NMR $\left(125 \mathrm{MHz}, \mathrm{CDCl}_{3}\right) \delta 173.3$ and $173.0(\mathrm{C}(\mathrm{O})), 108.3\left(\mathrm{C}-1^{\prime}\right), 84.4$ (C-4'), 84.2 (C-2'), 79.4 (C-3'), 73.4 (C-5'), 70.1 (C-2), 65.6 (C-1), 64.7 (C-6’), 62.7 (C-3); 34.3 and $\left.34.1\left(\mathrm{CH}_{2} \alpha\right), 31.9\left(\underline{\mathrm{CH}}_{2} \mathrm{CH}_{2} \mathrm{CH}_{3}\right), 29.9-29.0\left(\mathrm{CH}_{2}\right), 26.0\left(\mathrm{C}_{(} \underline{\mathrm{CH}}_{3}\right)_{3}\right)$, $26.96\left(\mathrm{C}\left(\underline{\mathrm{CH}}_{3}\right)_{3}\right), \quad 25.75 \quad\left(\mathrm{C}\left(\underline{\mathrm{CH}}_{3}\right)_{3}\right), \quad 25.70 \quad\left(\mathrm{C}\left(\underline{\mathrm{CH}}_{3}\right)_{3}\right), \quad 24.9 \quad\left(\mathrm{CH}_{2} \beta\right), \quad 18.3 \quad$ (x2) $\left(\mathrm{Si} \underline{\mathrm{C}}\left(\mathrm{CH}_{3}\right)_{3}\right), 17.9\left(\mathrm{Si} \underline{\mathrm{C}}\left(\mathrm{CH}_{3}\right)_{3}\right), 17.8\left(\mathrm{Si} \underline{\mathrm{C}}\left(\mathrm{CH}_{3}\right)_{3}\right), 14.1\left(\mathrm{CH}_{3}\right),-3.7\left(\mathrm{Si}\left(\mathrm{CH}_{3}\right)_{2}\right),-4.0$ 


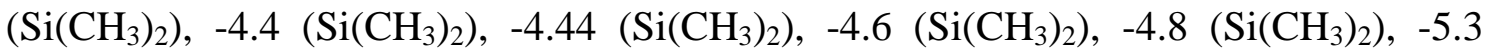
$\left(\mathrm{Si}\left(\mathrm{CH}_{3}\right)_{2}\right)$, -5.4 $\left(\mathrm{Si}\left(\mathrm{CH}_{3}\right)_{2}\right)$; HRMS(ESI) $\mathrm{m} / z$ calcd. for $\left[\mathrm{C}_{77} \mathrm{H}_{158} \mathrm{NaO}_{10} \mathrm{Si}_{4}\right]^{+}: 1378.0824$, found: 1378.0820 .

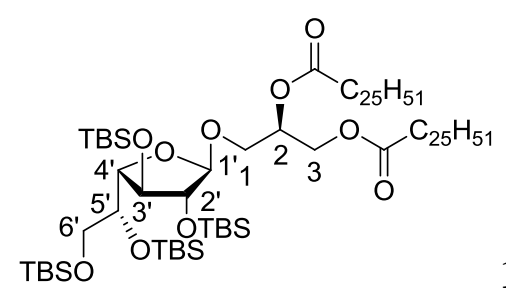

\section{1-O-(2, 3, 5, 6-Tetra-O-tert-butyldimethylsilyl- $\beta$-D}

galactofuranosyl)-2,3-di- $O$-cerotoyl-sn-glycerol (sn-1-6d). Subjection of $s n-1-9$ (24.7 $\mathrm{mg}, 0.035 \mathrm{mmol})$ cerotic acid $(61.1 \mathrm{mg}, 0.15 \mathrm{mmol})$, DMAP $(0.60 \mathrm{mg}, 0.0050 \mathrm{mmol})$ and EDCI $(23.9 \mathrm{mg}, 0.15 \mathrm{mmol})$ to the general esterification procedure at $70{ }^{\circ} \mathrm{C}$ for 6 days gave the title compound $\mathrm{s} n-1-\mathbf{6 d}(33.4 \mathrm{mg}, 0.022 \mathrm{mmol}, 65 \%)$, as a colourless oil after column chromatrography $\left(\right.$ EtOAc/PE, 1/99, v/v). $\left(\mathrm{R}_{f}=0.55\right.$, EtOAc/PE, 1/9, v/v); $[\alpha]_{\mathrm{D}}{ }^{18}=-0.19\left(c=1.0, \mathrm{CHCl}_{3}\right)$; IR (film) 2917, 2849, 1744, 1470, 1253, 1110, 836, 774 $\mathrm{cm}^{-1} ;{ }^{1} \mathrm{H} \mathrm{NMR}\left(500 \mathrm{MHz}, \mathrm{CDCl}_{3}\right) \delta 5.20(\mathrm{~m}, 1 \mathrm{H}, \mathrm{H}-2), 4.79\left(\mathrm{~d}, J_{\mathrm{H}-1^{\prime}}, \mathrm{H}-2^{\prime}=1.5 \mathrm{~Hz}, 1 \mathrm{H}\right.$, H-1'), $4.36\left(\mathrm{ABX}, J_{\mathrm{H}-3 \mathrm{a}}, \mathrm{H}-2=3.5 \mathrm{~Hz}, J_{\mathrm{H}-3 \mathrm{a}}, \mathrm{H}-3 \mathrm{~b}=11.9 \mathrm{~Hz}, 1 \mathrm{H}, \mathrm{H}-3 \mathrm{a}\right), 4.13(\mathrm{~m}, 1 \mathrm{H}, \mathrm{H}-$ 3b), 4.11 (m, 1H, H-3’ ), 3.99 (m, 1H, H-2'), 3.92 (dd, $J_{\mathrm{H}-4^{\prime}, \mathrm{H}-3^{\prime}}=5.4 \mathrm{~Hz}, J_{\mathrm{H}-4^{\prime}}, \mathrm{H}-5^{\prime}=3.5$ $\mathrm{Hz}, 1 \mathrm{H}, \mathrm{H}-4^{\prime}$ ), 3.79 (ABX, $\left.J_{\mathrm{H}-1 \mathrm{a}}, \mathrm{H}-2=5.4 \mathrm{~Hz}, J_{\mathrm{H}-1 \mathrm{a}} \mathrm{H}-3 \mathrm{~b}=10.3 \mathrm{~Hz}, 1 \mathrm{H}, \mathrm{H}-1 \mathrm{a}\right), 3.76$ (m, $\left.1 \mathrm{H}, \mathrm{H}-5^{\prime}\right), 3.66\left(\mathrm{ABX}, J_{\mathrm{H}-6^{\prime} \mathrm{a}},{\mathrm{H}-5^{\prime}}^{\prime}=6.2 \mathrm{~Hz}, J_{\mathrm{H}-6^{\prime} \mathrm{a}}, \mathrm{H}-6^{\prime} \mathrm{b}=10.2 \mathrm{~Hz}, 1 \mathrm{H}, \mathrm{H}-6^{\prime} \mathrm{a}\right), 3.58$ $\left(\mathrm{ABX}, J_{\mathrm{H}-6^{\prime} \mathrm{b}, \mathrm{H}-5^{\prime}}=6.0 \mathrm{~Hz}, J_{\mathrm{H}-6^{\prime} \mathrm{b}, \mathrm{H}-6^{\prime} \mathrm{a}}=10.0 \mathrm{~Hz}, 1 \mathrm{H}, \mathrm{H}-6^{\prime} \mathrm{b}\right), 3.50\left(\mathrm{ABX}, J_{\mathrm{H}-1 \mathrm{~b}, \mathrm{H}-2}=5.8\right.$ $\left.\mathrm{Hz}, J_{\mathrm{H}-1 \mathrm{~b}}, \mathrm{H}-1 \mathrm{a}=10.4 \mathrm{~Hz}, 1 \mathrm{H}, \mathrm{H}-1 \mathrm{~b}\right), 2.30\left(\mathrm{t}, J_{\mathrm{CH} 2 \alpha}, \mathrm{CH} 2 \beta=7.3 \mathrm{~Hz}, 4 \mathrm{H}, \mathrm{CH}_{2} \alpha\right), 1.60(\mathrm{~m}$, $\left.4 \mathrm{H}, \mathrm{CH}_{2} \beta\right), 1.35-1.18\left(\mathrm{~m}, 88 \mathrm{H}, \mathrm{CH}_{2}\right), 1.0-0.8\left(\mathrm{~m}, 42 \mathrm{H}, \mathrm{CH}_{3}\right.$ and $\left.\mathrm{C}\left(\mathrm{CH}_{3}\right)_{3}\right), 0.14-0.03$ (m, 24H, $\left.\mathrm{Si}\left(\mathrm{CH}_{3}\right)_{2}\right) ;{ }^{13} \mathrm{C}$ NMR $\left(125 \mathrm{MHz}, \mathrm{CDCl}_{3}\right) \delta 173.3$ and $173.0(\mathrm{C}(\mathrm{O})), 108.3(\mathrm{C}-$ 1’), 84.4 (C-4’), 84.2 (C-2’), 79.4 (C-3’), 73.4 (C-5’), 70.1 (C-2), 65.6 (C-1), 64.7 (C6'), $62.7(\mathrm{C}-3)$; 34.3 and $34.1\left(\mathrm{CH}_{2} \alpha\right), 31.9\left(\mathrm{CH}_{2} \mathrm{CH}_{2} \mathrm{CH}_{3}\right), 30.0-29.0\left(\mathrm{CH}_{2}\right), 26.0$ $\left(\mathrm{C}\left(\underline{\mathrm{CH}}_{3}\right)_{3}\right), 26.96\left(\mathrm{C}\left(\underline{\mathrm{CH}}_{3}\right)_{3}\right), 25.75\left(\mathrm{C}\left(\underline{\mathrm{CH}}_{3}\right)_{3}\right), 25.70\left(\mathrm{C}\left(\underline{\mathrm{CH}}_{3}\right)_{3}\right), 24.9\left(\mathrm{CH}_{2} \beta\right), 18.3(\mathrm{x} 2)$ $\left(\mathrm{SiC}\left(\mathrm{CH}_{3}\right)_{3}\right), 17.9\left(\mathrm{SiC}\left(\mathrm{CH}_{3}\right)_{3}\right), 17.8\left(\mathrm{SiC}\left(\mathrm{CH}_{3}\right)_{3}\right), 14.1\left(\mathrm{CH}_{3}\right),-3.7\left(\mathrm{Si}\left(\mathrm{CH}_{3}\right)_{2}\right),-4.0$ $\left(\mathrm{Si}\left(\mathrm{CH}_{3}\right)_{2}\right),-4.4\left(\mathrm{Si}\left(\mathrm{CH}_{3}\right)_{2}\right),-4.44\left(\mathrm{Si}\left(\mathrm{CH}_{3}\right)_{2}\right),-4.6\left(\mathrm{Si}\left(\mathrm{CH}_{3}\right)_{2}\right),-4.8\left(\mathrm{Si}\left(\mathrm{CH}_{3}\right)_{2}\right),-5.3$ $\left(\mathrm{Si}\left(\mathrm{CH}_{3}\right)_{2}\right),-5.4\left(\mathrm{Si}\left(\mathrm{CH}_{3}\right)_{2}\right)$; HRMS(ESI) $\mathrm{m} / z$ calcd. for $\left[\mathrm{C}_{85} \mathrm{H}_{174} \mathrm{NaO}_{10} \mathrm{Si}_{4}\right]^{+}:$1490.2076, found: 1490.2079. 


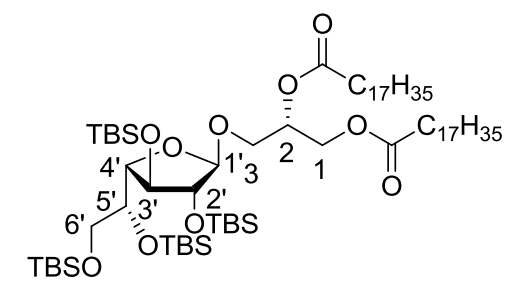

\section{3-O-(2,3,5,6-Tetra-O-tert-butyldimethylsilyl- $\beta$ -}

D-galactofuranosyl)-1,2-di- $O$-stearoyl-sn-glycerol (sn-3-6a). Subjection of $s n-3-9$ (98.1 mg, $0.14 \mathrm{mmol}$ ), stearic acid (172 mg, $0.607 \mathrm{mmol})$, DMAP (2.5 mg, $0.02 \mathrm{mmol}$ ) and EDCI (116 mg, $0.607 \mathrm{mmol})$ to the general esterification procedure at $40{ }^{\circ} \mathrm{C}$ for 12 h, gave the title compound sn-3-6a as a colourless oil (163.6 mg, $0.13 \mathrm{mmol}, 94 \%)$ after column chromatrography $\left(\right.$ EtOAc/PE, 1/99, v/v). $\left(\mathrm{R}_{f}=0.76\right.$, EtOAc/PE, 1/9, v/v); $[\alpha]_{\mathrm{D}}{ }^{26}$ $=-5.75\left(c=0.1, \mathrm{CHCl}_{3}\right)$; IR (film) 2924, 2853,1740,1463,1113,837, $759 \mathrm{~cm}^{-1} ;{ }^{1} \mathrm{H}$ NMR (500 MHz, $\left.\mathrm{CDCl}_{3}\right) \delta 5.20(\mathrm{~m}, 1 \mathrm{H}, \mathrm{H}-2), 4.79\left(\mathrm{~d}, J_{\mathrm{H}-1^{\prime}, \mathrm{H}_{2} 2^{\prime}}=1.8 \mathrm{~Hz}, 1 \mathrm{H}, \mathrm{H}-1^{\prime}\right)$, $4.35\left(\mathrm{ABX}, J_{\mathrm{H}-1 \mathrm{a}} \mathrm{H}-2=3.5 \mathrm{~Hz}, J_{\mathrm{H}-1 \mathrm{a}}, \mathrm{H}-1 \mathrm{~b}=12.0 \mathrm{~Hz}, 1 \mathrm{H}, \mathrm{H}-1 \mathrm{a}\right), 4.20\left(\mathrm{ABX}, J_{\mathrm{H}-1 \mathrm{~b}}, \mathrm{H}-2=\right.$ $\left.7.0 \mathrm{~Hz}, J_{\mathrm{H}-1 \mathrm{~b}}, \mathrm{H}-1 \mathrm{a}=11.9 \mathrm{~Hz}, 1 \mathrm{H}, \mathrm{H}-1 \mathrm{~b}\right), 4.13\left(\mathrm{~m}, J_{\mathrm{H}-3^{\prime}}, \mathrm{H}-2^{\prime}=3.1 \mathrm{~Hz}, J_{\mathrm{H}-3^{\prime}}, \mathrm{H}-4^{\prime}=5.5 \mathrm{~Hz}\right.$ $\left.1 \mathrm{H}, \mathrm{H}^{-} 3^{\prime}\right), 4.00\left(\mathrm{t}, J_{\mathrm{H}-2^{\prime}}, \mathrm{H}-3^{\prime}=J_{\mathrm{H}-2^{\prime}}, \mathrm{H}-1^{\prime}=2.5 \mathrm{~Hz}, 1 \mathrm{H}, \mathrm{H}-2^{\prime}\right), 3.91\left(\mathrm{dd}, J_{\mathrm{H}-4^{\prime}}, \mathrm{H}-3^{\prime}=6.0 \mathrm{~Hz}\right.$, $\left.J_{\mathrm{H}-4^{\prime}, \mathrm{H}-5^{\prime}}=3.2 \mathrm{~Hz}, 1 \mathrm{H}, \mathrm{H}^{-} 4^{\prime}\right), 3.79\left(\mathrm{ABX}, J_{\mathrm{H}-3 \mathrm{a}, \mathrm{H}-2}=4.8 \mathrm{~Hz}, J_{\mathrm{H}-3 \mathrm{a}}, \mathrm{H}-3 \mathrm{~b}=10.9 \mathrm{~Hz}, 1 \mathrm{H}\right.$, H-3a), 3.76 (m, 1H, H-5 $\left.{ }^{\prime}\right), 3.65\left(\mathrm{ABX}, J_{\mathrm{H}-6^{\prime} \mathrm{a}, \mathrm{H}-5^{\prime}}=6.6 \mathrm{~Hz}, J_{\mathrm{H}-6^{\prime} \mathrm{a}, \mathrm{H}-6^{\prime} \mathrm{b}}=9.9 \mathrm{~Hz}, 1 \mathrm{H}, \mathrm{H}-\right.$ $\left.6^{\prime} \mathrm{a}\right), 3.57\left(\mathrm{ABX}, J_{\mathrm{H}-66^{\prime}},{\mathrm{H}-5^{\prime}}^{\prime}=6.0 \mathrm{~Hz}, J_{\mathrm{H}-6^{\prime} \mathrm{b}, \mathrm{H}-6^{\prime} \mathrm{a}}=10.0 \mathrm{~Hz}, 1 \mathrm{H}, \mathrm{H}-6^{\prime} \mathrm{b}\right), 3.55\left(\mathrm{ABX}, J_{\mathrm{H}-}\right.$ $\left.3 \mathrm{~b}, \mathrm{H}-2=5.2 \mathrm{~Hz}, J_{\mathrm{H}-3 \mathrm{~b}}, \mathrm{H}-3 \mathrm{a}=10.9 \mathrm{~Hz}, 1 \mathrm{H}, \mathrm{H}-3 \mathrm{~b}\right), 2.28\left(\mathrm{t}, J_{\mathrm{H} \alpha}, \mathrm{H} \beta=7.7 \mathrm{~Hz}, 4 \mathrm{H}, \mathrm{H \alpha}\right), 1.59$ $(\mathrm{m}, 4 \mathrm{H}, \mathrm{H} \beta), 1.37-1.17\left(\mathrm{~m}, 56 \mathrm{H}, \mathrm{CH}_{2}\right), 0.9-0.8\left(\mathrm{t}, 42 \mathrm{H}, \mathrm{CH}_{3}\right.$ and $\left.\mathrm{C}\left(\mathrm{CH}_{3}\right)_{3}\right), 0.16-0.00$ (m, 24H, $\left.\mathrm{Si}\left(\mathrm{CH}_{3}\right)_{2}\right) ;{ }^{13} \mathrm{C} \mathrm{NMR}\left(126 \mathrm{MHz}, \mathrm{CDCl}_{3}\right) \delta 173.3(\mathrm{C}(\mathrm{O}) 1), 172.9(\mathrm{C}(\mathrm{O}) 2)$, $108.3\left(\mathrm{C}-1^{\prime}\right), 84.4\left(\mathrm{C}^{\prime} 2^{\prime}\right), 84.2\left(\mathrm{C}^{\prime} 4^{\prime}\right), 79.3$ (C-3'), 73.2 (C-5'), 70.0 (C-2), 65.6 (C-3), 64.6 (C-6'), 62.7 (C-1); 34.3 and $34.1\left(\mathrm{CH}_{2} \alpha\right), 31.9\left(\mathrm{CH}_{2} \mathrm{CH}_{2} \mathrm{CH}_{3}\right), 29.9-29.0\left(\mathrm{CH}_{2}\right)$, $\left.26.0\left(\mathrm{C}\left(\underline{\mathrm{CH}}_{3}\right)_{3}\right), 26.9\left(\mathrm{C}\left(\underline{\mathrm{CH}}_{3}\right)_{3}\right), 25.8\left(\mathrm{C}\left(\underline{\mathrm{CH}}_{3}\right)_{3}\right), 25.7\left(\mathrm{C}{ }_{\mathrm{CH}}\right)_{3}\right), 24.94$ and 24.90 $\left(\mathrm{CH}_{2} \beta\right), 18.3(\mathrm{x} 2)\left(\mathrm{SiC}\left(\mathrm{CH}_{3}\right)_{3}\right), 17.9\left(\mathrm{SiC}\left(\mathrm{CH}_{3}\right)_{3}\right), 17.2\left(\mathrm{Si} \underline{\mathrm{C}}\left(\mathrm{CH}_{3}\right)_{3}\right), 14.1\left(\mathrm{CH}_{3}\right),-3.6$ $\left(\mathrm{Si}\left(\mathrm{CH}_{3}\right)_{2}\right),-4.0\left(\mathrm{Si}\left(\mathrm{CH}_{3}\right)_{2}\right),-4.4(\mathrm{x} 2)\left(\mathrm{Si}\left(\mathrm{CH}_{3}\right)_{2}\right),-4.5\left(\mathrm{Si}\left(\mathrm{CH}_{3}\right)_{2}\right),-4.9\left(\mathrm{Si}\left(\mathrm{CH}_{3}\right)_{2}\right),-5.3$ $\left(\mathrm{Si}\left(\mathrm{CH}_{3}\right)_{2}\right),-5.4\left(\mathrm{Si}\left(\mathrm{CH}_{3}\right)_{2}\right)$; HRMS(ESI) $\mathrm{m} / z$ calcd. for $\left[\mathrm{C}_{69} \mathrm{H}_{142} \mathrm{NaO}_{10} \mathrm{Si}_{4}\right]^{+}: 1265.9572$, found: 1265.9578 . 


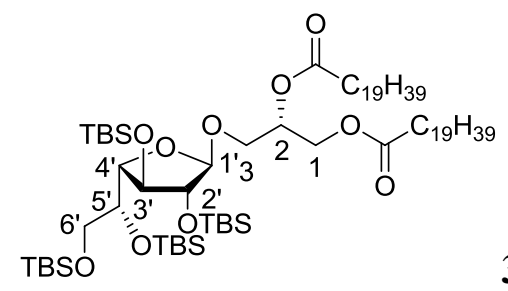

\section{3-O-(2, 3, 5, 6-Tetra-O-tert-butyldimethylsilyl- $\beta$-D}

galactofuranosyl)-1,2-di- $O$-arachidoyl-sn-glycerol (sn-3-6b). Subjection of $s n-3-9$ (70 mg, $0.098 \mathrm{mmol}$ ), arachidic acid (134 mg, $0.43 \mathrm{mmol}$ ), DMAP (1.8 mg, 0.014 $\mathrm{mmol})$ and EDCI $(66.9 \mathrm{mg}, 0.43 \mathrm{mmol})$ to the general esterification procedure at $40{ }^{\circ} \mathrm{C}$ for $12 \mathrm{~h}$ gave the title compound $\mathrm{s} n-3-6 \mathbf{b}$ as a colourless oil $(111.7 \mathrm{mg}, 0.086 \mathrm{mmol}$, $88 \%)$ after column chromatrography (EtOAc/PE, 1/99, v/v). $\left(\mathrm{R}_{f}=0.44\right.$, EtOAc/PE, 1/9, $\mathrm{v} / \mathrm{v}) ;[\alpha]_{\mathrm{D}}{ }^{26}=-13.0\left(c=1.0, \mathrm{CHCl}_{3}\right)$; IR (film) 2924, 2854, 1744, 1463, 1252, 1112, 836, $776 \mathrm{~cm}^{-1}$; ${ }^{1} \mathrm{H}$ NMR (500 MHz, $\left.\mathrm{CDCl}_{3}\right) \delta 5.21(\mathrm{~m}, 1 \mathrm{H}, \mathrm{H}-2), 4.80\left(\mathrm{~s}, 1 \mathrm{H}, \mathrm{H}^{-1}{ }^{\prime}\right)$, 4.35 (m, 1H, H-1a), $4.20\left(\mathrm{ABX}, J_{\mathrm{H}-1 \mathrm{~b}, \mathrm{H}-2}=6.6 \mathrm{~Hz}, J_{\mathrm{H}-1 \mathrm{~b}, \mathrm{H}-1 \mathrm{a}}=11.6 \mathrm{~Hz}, 1 \mathrm{H}, \mathrm{H}-1 \mathrm{~b}\right), 4.13$ (m, 1H, H-3`), 4.00 (bs, 1H, H-2'), 3.93 (bs, 1H, H-4'), 3.79 (m, $J_{\mathrm{H}-3 \mathrm{a}}, \mathrm{H}-2=4.5 \mathrm{~Hz}, J_{\mathrm{H}-3 \mathrm{a}}$, $\mathrm{H}-3 \mathrm{~b}=10.9 \mathrm{~Hz}, 1 \mathrm{H}, \mathrm{H}-3 \mathrm{a}), 3.76\left(\mathrm{~m}, 1 \mathrm{H}, \mathrm{H}-5^{\prime}\right), 3.66\left(\mathrm{ABX}, J_{\mathrm{H}-6^{\prime} \mathrm{a}}, \mathrm{H}-5^{\prime}=6.0 \mathrm{~Hz}, J_{\mathrm{H}-6^{\prime} \mathrm{a}}, \mathrm{H}-\right.$ $\left.6^{\prime} \mathrm{b}=9.3 \mathrm{~Hz}, 1 \mathrm{H}, \mathrm{H}-6^{\prime} \mathrm{a}\right), 3.59$ (m, 1H, H-6`b), $3.55\left(\mathrm{ABX}, J_{\mathrm{H}-3 \mathrm{~b}, \mathrm{H}-2}=5.9 \mathrm{~Hz}, J_{\mathrm{H}-3 \mathrm{~b}, \mathrm{H}-3 \mathrm{a}}=\right.$ $11.9 \mathrm{~Hz}, 1 \mathrm{H}, \mathrm{H}-3 \mathrm{~b}$ ), 2.29 (bs, $4 \mathrm{H}, \mathrm{H \alpha}$ ), 1.61 (bs, $4 \mathrm{H}, \mathrm{H} \beta$ ), 1.44-1.18 (m, 56H, $\mathrm{CH}_{2}$ ), 1.0$0.8\left(\mathrm{t}, 42 \mathrm{H}, \mathrm{CH}_{3}\right.$ and $\left.\mathrm{C}\left(\mathrm{CH}_{3}\right)_{3}\right), 0.24-0.00\left(\mathrm{~m}, 24 \mathrm{H}, \mathrm{Si}\left(\mathrm{CH}_{3}\right)_{2}\right) ;{ }^{13} \mathrm{C}$ NMR $(125 \mathrm{MHz}$, $\left.\mathrm{CDCl}_{3}\right) \delta 173.3(\mathrm{C}(\mathrm{O})-1), 172.9(\mathrm{C}(\mathrm{O})-2), 108.2$ (C-1'), 84.3 (C-2'), 84.2 (C-4'), 79.4 (C-3’), 73.2 (C-5'), 70.0 (C-2), 65.6 (C-3), 64.6 (C-6’), 62.7 (C-1); 34.3 and 34.1 $\left(\mathrm{CH}_{2} \alpha\right), 31.9\left(\underline{\mathrm{CH}}_{2} \mathrm{CH}_{2} \mathrm{CH}_{3}\right), 30.1-28.0\left(\mathrm{CH}_{2}\right), 26.0\left(\mathrm{C}\left(\underline{\mathrm{CH}}_{3}\right)_{3}\right), 25.96\left(\mathrm{C}\left(\underline{\mathrm{CH}}_{3}\right)_{3}\right), 25.76$ $\left(\mathrm{C}\left(\underline{\mathrm{CH}}_{3}\right)_{3}\right), 25.70\left(\mathrm{C}\left(\underline{\mathrm{CH}}_{3}\right)_{3}\right), 24.9\left(\mathrm{CH}_{2} \beta\right), 18.3(\mathrm{x} 2)\left(\mathrm{SiC}\left(\mathrm{CH}_{3}\right)_{3}\right), 17.9\left(\mathrm{SiC}\left(\mathrm{CH}_{3}\right)_{3}\right)$, $17.8\left(\mathrm{Si} \underline{\mathrm{C}}\left(\mathrm{CH}_{3}\right)_{3}\right), 14.1\left(\mathrm{CH}_{3}\right),-3.6\left(\mathrm{Si}\left(\mathrm{CH}_{3}\right)_{2}\right),-4.0\left(\mathrm{Si}\left(\mathrm{CH}_{3}\right)_{2}\right),-4.4(\mathrm{x} 2)\left(\mathrm{Si}\left(\mathrm{CH}_{3}\right)_{2}\right),-$ $4.6\left(\mathrm{Si}\left(\mathrm{CH}_{3}\right)_{2}\right),-4.8\left(\mathrm{Si}\left(\mathrm{CH}_{3}\right)_{2}\right),-5.3\left(\mathrm{Si}\left(\mathrm{CH}_{3}\right)_{2}\right),-5.4\left(\mathrm{Si}\left(\mathrm{CH}_{3}\right)_{2}\right) ; \mathrm{HRMS}(\mathrm{ESI}) \mathrm{m} / z$ calcd. for $\left[\mathrm{C}_{73} \mathrm{H}_{150} \mathrm{NaO}_{10} \mathrm{Si}_{4}\right]^{+}:$1322.0198, found: 1322.0201 .

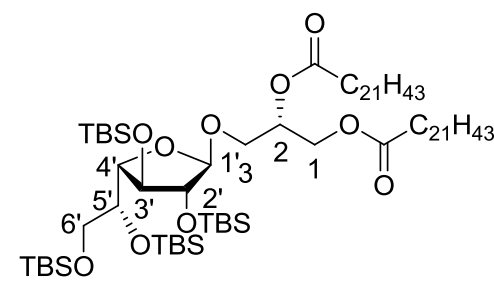

3-O-(2, 3, 5, 6-Tetra-O-tert-butyldimethylsilyl- $\beta$-D

galactofuranosyl)-1,2-di-O-behenoyl-sn-glycerol (sn-3-6c). Subjection of $s n$-3-9 (10.9 $\mathrm{mg}, 0.015 \mathrm{mmol})$ to the general esterification procedure at $70{ }^{\circ} \mathrm{C}$ for 6 days, behenic acid (22.4 mg, $0.066 \mathrm{mmol})$, DMAP (0.27 mg, $0.0022 \mathrm{mmol})$ and EDCI (10.2 mg, 
$0.066 \mathrm{mmol})$ gave the title compound $\mathrm{s} n-3-6 \mathrm{c}(15.1 \mathrm{mg}, 0.011 \mathrm{mmol}, 73 \%)$, as a colourless oil after column chromatrography (EtOAc/PE, 1/99, v/v). $\left(\mathrm{R}_{f}=0.48\right.$, EtOAc/PE, 1/9, v/v); $[\alpha]_{\mathrm{D}}{ }^{18}=-20.0\left(c=0.5, \mathrm{CHCl}_{3}\right)$; IR (film) 2923, 2853, 1745, 1252, $1112,836,776 \mathrm{~cm}^{-1} ;{ }^{1} \mathrm{H}$ NMR $\left(500 \mathrm{MHz}, \mathrm{CDCl}_{3}\right) \delta 5.21(\mathrm{~m}, 1 \mathrm{H}, \mathrm{H}-2), 4.80(\mathrm{~s}, 1 \mathrm{H}, \mathrm{H}-$ $\left.1^{\prime}\right), 4.35\left(\mathrm{ABX}, J_{\mathrm{H}-1 \mathrm{a}}, \mathrm{H}-2 \mathrm{a}=3.30 \mathrm{~Hz}, J_{\mathrm{H}-1 \mathrm{a}}, \mathrm{H}-2 \mathrm{a}=12.11 \mathrm{~Hz}, 1 \mathrm{H}, \mathrm{H}-1 \mathrm{a}\right), 4.19\left(\mathrm{ABX}, J_{\mathrm{H}-1 \mathrm{~b}}\right.$, $\left.\mathrm{H}-2=6.4 \mathrm{~Hz}, J_{\mathrm{H}-1 \mathrm{~b}}, \mathrm{H}-1 \mathrm{a}=11.4 \mathrm{~Hz}, 1 \mathrm{H}, \mathrm{H}-1 \mathrm{~b}\right), 4.13\left(\mathrm{~m}, 1 \mathrm{H}, \mathrm{H}-3^{\prime}\right), 4.00$ (bs, 1H, H-2'), $3.93\left(\mathrm{~m}, 1 \mathrm{H}, \mathrm{H}-4^{\prime}\right), 3.80\left(\mathrm{~m}, J_{\mathrm{H}-3 \mathrm{a}}, \mathrm{H}-2=5.0 \mathrm{~Hz}, J_{\mathrm{H}-3 \mathrm{a}, \mathrm{H}-3 \mathrm{~b}}=11.0 \mathrm{~Hz}, 1 \mathrm{H}, \mathrm{H}-3 \mathrm{a}\right), 3.76(\mathrm{~m}$, $\left.1 \mathrm{H}, \mathrm{H}-5^{\prime}\right), 3.66\left(\mathrm{ABX}, J_{\mathrm{H}-6^{\prime} \mathrm{a}}, \mathrm{H}-5^{\prime}=6.6 \mathrm{~Hz}, J_{\mathrm{H}-6^{\prime} \mathrm{a}}, \mathrm{H}^{\prime} \mathrm{b}=9.4 \mathrm{~Hz}, 1 \mathrm{H}, \mathrm{H}-6^{\prime} \mathrm{a}\right), 3.58(\mathrm{ABX}$, $\left.J_{\mathrm{H}-6^{\prime} \mathrm{b}, \mathrm{H}-5^{\prime}}=6.0 \mathrm{~Hz}, J_{\mathrm{H}-6^{\prime} \mathrm{b}, \mathrm{H}-6^{\prime} \mathrm{a}}=9.4 \mathrm{~Hz}, 1 \mathrm{H}, \mathrm{H}-6^{\prime} \mathrm{b}\right), 3.55\left(\mathrm{ABX}, J_{\mathrm{H}-3 \mathrm{~b}, \mathrm{H}-2}=4.9 \mathrm{~Hz}, J_{\mathrm{H}-3 \mathrm{~b}}\right.$, $\mathrm{H}-3 \mathrm{a}=10.6 \mathrm{~Hz}, 1 \mathrm{H}, \mathrm{H}-3 \mathrm{~b}), 2.29\left(\mathrm{~m}, 4 \mathrm{H}, \mathrm{CH}_{2} \alpha\right), 1.60$ (bs, 4H, Hß), 1.35-1.18 (m, 72H, $\left.\mathrm{CH}_{2}\right), 1.0-0.8\left(\mathrm{t}, 42 \mathrm{H}, \mathrm{CH}_{3}\right.$ and $\left.\mathrm{C}\left(\mathrm{CH}_{3}\right)_{3}\right), 0.17-0.01\left(\mathrm{~m}, 24 \mathrm{H}, \mathrm{Si}\left(\mathrm{CH}_{3}\right)_{2}\right) ;{ }^{13} \mathrm{C} \mathrm{NMR}(125$ $\left.\mathrm{MHz} \mathrm{CDCl}_{3}\right) \delta 173.4(\mathrm{C}(\mathrm{O})-1), 173.0(\mathrm{C}(\mathrm{O})-2), 108.2\left(\mathrm{C}^{\prime} 1^{\prime}\right), 84.4\left(\mathrm{C}-2^{\prime}\right), 84.2\left(\mathrm{C}^{\prime} 4^{\prime}\right)$, 79.4 (C-3'), 73.2 (C-5’), 70.0 (C-2), 65.6 (C-3), 64.6 (C-6’), 62.7 (C-1); 34.3 and 34.1 $\left.\left(\mathrm{CH}_{2} \alpha\right), 31.9\left(\underline{\mathrm{CH}_{2}} \mathrm{CH}_{2} \mathrm{CH}_{3}\right), 30.2-28.0\left(\mathrm{CH}_{2}\right), 26.0\left(\mathrm{C}\left(\underline{\mathrm{CH}}_{3}\right)_{3}\right), 25.96\left(\mathrm{C}_{(\mathrm{CH}}\right)_{3}\right), 25.76$ $\left(\mathrm{C}\left(\underline{\mathrm{CH}}_{3}\right)_{3}\right), 25.70\left(\mathrm{C}\left(\underline{\mathrm{CH}}_{3}\right)_{3}\right), 24.9\left(\mathrm{CH}_{2} \beta\right), 18.3(\mathrm{x} 2)\left(\mathrm{Si} \underline{\mathrm{C}}\left(\mathrm{CH}_{3}\right)_{3}\right), 17.9\left(\mathrm{SiC}\left(\mathrm{CH}_{3}\right)_{3}\right)$, $17.8\left(\mathrm{SiC}\left(\mathrm{CH}_{3}\right)_{3}\right), 14.1\left(\mathrm{CH}_{3}\right),-3.6\left(\mathrm{Si}\left(\mathrm{CH}_{3}\right)_{2}\right),-4.0\left(\mathrm{Si}\left(\mathrm{CH}_{3}\right)_{2}\right),-4.4(\mathrm{x} 2)\left(\mathrm{Si}\left(\mathrm{CH}_{3}\right)_{2}\right),-$ 4.6 $\left(\mathrm{Si}\left(\mathrm{CH}_{3}\right)_{2}\right)$, -4.8 $\left(\mathrm{Si}\left(\mathrm{CH}_{3}\right)_{2}\right)$, -5.3 $\left(\mathrm{Si}\left(\mathrm{CH}_{3}\right)_{2}\right)$, -5.4 $\left(\mathrm{Si}\left(\mathrm{CH}_{3}\right)_{2}\right) ; \mathrm{HRMS}(\mathrm{ESI}) \mathrm{m} / z$ calcd. for $\left[\mathrm{C}_{73} \mathrm{H}_{150} \mathrm{NaO}_{10} \mathrm{Si}_{4}\right]^{+}:$1378.0824, found: 1378.0820 .

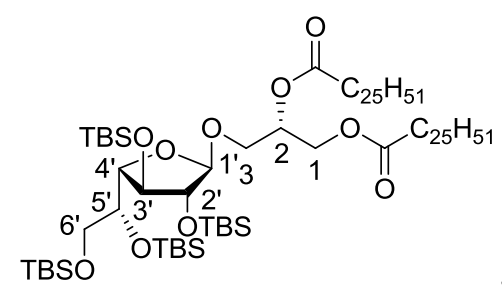

\section{3-O- $(2, \quad 3, \quad 5, \quad 6$-Tetra-O-tert-butyldimethylsilyl- $\beta$-D}

galactofuranosyl)-1,2-di-O-cerotoyl-sn-glycerol (sn-3-6d). Subjection of $s n-3-9$ (15 $\mathrm{mg}, 0.021 \mathrm{mmol})$, cerotic acid $(36.6 \mathrm{mg}, 0.092 \mathrm{mmol})$, DMAP $(0.38 \mathrm{mg}, 0.0031 \mathrm{mmol})$ and EDCI (14.3 mg, $0.092 \mathrm{mmol})$ to the general esterification procedure at $70{ }^{\circ} \mathrm{C}$ for 6 days gave the title compound sn-3-6d (23.4 mg, $0.016 \mathrm{mmol}, 76 \%)$, as a colourless oil after column chromatrography $\left(\right.$ EtOAc/PE, 1/99, v/v). $\left(\mathrm{R}_{f}=0.55\right.$, EtOAc/PE, 1/9, v/v); $[\alpha]_{\mathrm{D}}{ }^{18}=-9.0\left(c=0.1, \mathrm{CHCl}_{3}\right) ; \mathrm{IR}$ (film) $2917,2850,1744,1470,1253,1110,837,776$, $668 \mathrm{~cm}^{-1} ;{ }^{1} \mathrm{H}$ NMR $\left(500 \mathrm{MHz}, \mathrm{CDCl}_{3}\right) \delta 5.21(\mathrm{~m}, 1 \mathrm{H}, \mathrm{H}-2), 4.80\left(\mathrm{~s}, 1 \mathrm{H}, \mathrm{H}-1^{\prime}\right), 4.35$ $\left(\mathrm{ABX}, J_{\mathrm{H}-1 \mathrm{a}}, \mathrm{H}-2=3.3 \mathrm{~Hz}, J_{\mathrm{H}-1 \mathrm{a}}, \mathrm{H}-1 \mathrm{~b}=11.8 \mathrm{~Hz}, 1 \mathrm{H}, \mathrm{H}-1 \mathrm{a}\right), 4.19\left(\mathrm{ABX}, J_{\mathrm{H}-1 \mathrm{~b}}, \mathrm{H}-2=6.6 \mathrm{~Hz}\right.$, $\left.J_{\mathrm{H}-1 \mathrm{~b}, \mathrm{H}-1 \mathrm{a}}=11.8 \mathrm{~Hz}, 1 \mathrm{H}, \mathrm{H}-1 \mathrm{~b}\right), 4.13\left(\mathrm{dd}, J_{\mathrm{H}-3^{\prime}, \mathrm{H}-2^{\prime}}=2.9 \mathrm{~Hz}, J_{\mathrm{H}-3^{\prime}},{\mathrm{H}-4^{\prime}}^{\prime}=5.4 \mathrm{~Hz}, 1 \mathrm{H}, \mathrm{H}-\right.$ 
$\left.3^{\prime}\right), 4.00\left(\mathrm{bs}, 1 \mathrm{H}, \mathrm{H}-2^{\prime}\right), 3.93\left(\mathrm{dd}, J_{\mathrm{H}-4^{\prime}, \mathrm{H}-5^{\prime}}=3.3 \mathrm{~Hz}, J_{\mathrm{H}-4^{\prime}}, \mathrm{H}-3^{\prime}=5.2 \mathrm{~Hz} 1 \mathrm{H}, \mathrm{H}-4^{\prime}\right), 3.80$ $\left(\mathrm{ABX}, J_{\mathrm{H}-3 \mathrm{a}}, \mathrm{H}-2=4.8 \mathrm{~Hz}, J_{\mathrm{H}-3 \mathrm{a}}, \mathrm{H}-3 \mathrm{~b}=11.1 \mathrm{~Hz}, 1 \mathrm{H}, \mathrm{H}-3 \mathrm{a}\right), 3.76\left(\mathrm{~m}, 1 \mathrm{H}, \mathrm{H}-5^{\prime}\right), 3.66$ $\left(\mathrm{ABX}, J_{\mathrm{H}-6^{\prime} \mathrm{a}}, \mathrm{H}-5^{\prime}=6.7 \mathrm{~Hz}, J_{\mathrm{H}-6^{\prime} \mathrm{a}}, \mathrm{H}-6^{\prime} \mathrm{b}=9.6 \mathrm{~Hz}, 1 \mathrm{H}, \mathrm{H}-6^{\prime} \mathrm{a}\right), 3.59\left(\mathrm{ABX}, J_{\mathrm{H}-6^{\prime} \mathrm{b}}, \mathrm{H}-5^{\prime}=6.0\right.$ $\left.\mathrm{Hz}, J_{\mathrm{H}-5 ` \mathrm{~b}, \mathrm{H}-5^{\prime} \mathrm{a}}=9.9 \mathrm{~Hz}, 1 \mathrm{H}, \mathrm{H}-6^{\prime} \mathrm{b}\right), 3.55\left(\mathrm{ABX}, J_{\mathrm{H}-3 \mathrm{~b}}, \mathrm{H}-2=5.5 \mathrm{~Hz}, J_{\mathrm{H}-3 \mathrm{~b}}, \mathrm{H}-3 \mathrm{a}=10.4 \mathrm{~Hz}\right.$, $1 \mathrm{H}, \mathrm{H}-3 \mathrm{~b}), 2.29$ (t, $\left.J_{\mathrm{H} \alpha}, \mathrm{H} \beta=7.9,4 \mathrm{H}, \mathrm{H \alpha}\right), 1.61$ (bs, $\left.4 \mathrm{H}, \mathrm{H} \beta\right), 1.44-1.18\left(\mathrm{~m}, 88 \mathrm{H}, \mathrm{CH}_{2}\right)$, 1.0-0.8 (m, 42H, $\mathrm{CH}_{3}$ and $\left.\mathrm{C}\left(\mathrm{CH}_{3}\right)_{3}\right), 0.16-0.01\left(\mathrm{~m}, 24 \mathrm{H}, \mathrm{Si}\left(\mathrm{CH}_{3}\right)_{2}\right) ;{ }^{13} \mathrm{C} \mathrm{NMR}(125$ $\left.\mathrm{MHz}, \mathrm{CDCl}_{3}\right) \delta 173.4(\mathrm{C}(\mathrm{O})-1), 173.0(\mathrm{C}(\mathrm{O})-2), 108.2$ (C-1'), 84.3 (C-2’), $84.2\left(\mathrm{C}^{\prime} 4^{\prime}\right)$, 79.4 (C-3'), 73.2 (C-5'), 70.0 (C-2), 65.6 (C-3), 64.6 (C-6'), 62.7 (C-1); 34.3 and 34.1 $\left(\mathrm{CH}_{2} \alpha\right), 31.9\left(\underline{\mathrm{CH}}_{2} \mathrm{CH}_{2} \mathrm{CH}_{3}\right), 30.1-28.0\left(\mathrm{CH}_{2}\right), 26.0\left(\mathrm{C}\left(\underline{\mathrm{CH}}_{3}\right)_{3}\right), 25.96\left(\mathrm{C}\left(\underline{\mathrm{CH}}_{3}\right)_{3}\right), 25.76$ $\left(\mathrm{C}\left(\underline{C H}_{3}\right)_{3}\right), 25.70\left(\mathrm{C}\left(\underline{\mathrm{CH}}_{3}\right)_{3}\right), 24.94$ and $24.91\left(\mathrm{CH}_{2} \beta\right), 18.3(\mathrm{x} 2)\left(\mathrm{SiC}\left(\mathrm{CH}_{3}\right)_{3}\right), 17.9$ $\left(\mathrm{Si} \underline{\underline{C}}\left(\mathrm{CH}_{3}\right)_{3}\right), 17.8\left(\mathrm{Si} \underline{\mathrm{C}}\left(\mathrm{CH}_{3}\right)_{3}\right), 14.1\left(\mathrm{CH}_{3}\right),-3.6\left(\mathrm{Si}\left(\mathrm{CH}_{3}\right)_{2}\right),-4.0\left(\mathrm{Si}\left(\mathrm{CH}_{3}\right)_{2}\right),-4.4(\mathrm{x} 2)$

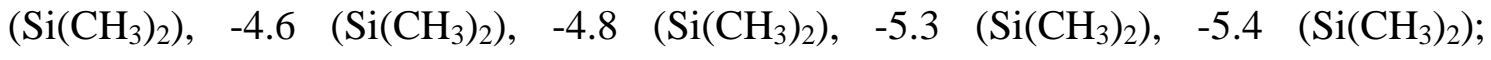
HRMS(ESI) $m / z$ calcd. for $\left[\mathrm{C}_{85} \mathrm{H}_{174} \mathrm{NaO}_{10} \mathrm{Si}_{4}\right]^{+}: 1490.2076$, found: 1490.2073 .

\section{Global desilylation procedure for glycolipids sn-1-6a-d and $s n-3-6 a-d$}

To a solution of compounds 6 in THF $(0.9 \mathrm{~mL})$ was added HF.pyridine $(0.1 \mathrm{~mL})$. After $12 \mathrm{~h}$, the reaction mixture was neutralised with $\mathrm{NaHCO}_{3}$ (sat. aq.), diluted with EtOAc, washed with $\mathrm{HCl}(0.1 \mathrm{M})$ and brine, dried $\left(\mathrm{MgSO}_{4}\right)$, filtered and concentrated in vacuo.

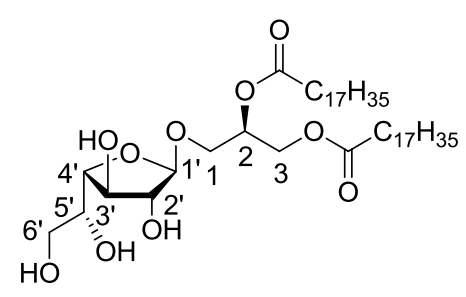

\section{1-O-( $\beta$-D-Galactofuranosyl)-2,3-di- $O$-stearoyl-sn-glycerol}

(sn-1-2a). Subjection of $s n-1-6 a(23.2 \mathrm{mg}, 0.019 \mathrm{mmol})$ to the global desilylation procedure gave the title compound sn-1-2a, as a colourless oil $(14.9 \mathrm{mg}, 0.019 \mathrm{mmol}$, quant. $),\left(\mathrm{R}_{f}=0.57, \mathrm{MeOH} / \mathrm{DCM}, 1 / 6, \mathrm{v} / \mathrm{v}\right) ;[\alpha]_{\mathrm{D}}{ }^{25}=-21.4(c=1.0$, Pyridine); IR (film) 3560, 2955, 2917, 2849, 1740, 1462, 1377, $1164 \mathrm{~cm}^{-1} ;{ }^{1} \mathrm{H}$ NMR (500 MHz, $\mathrm{CD}_{3} \mathrm{OD} / \mathrm{CDCl}_{3}, 1 / 2$, v/v) $\delta 5.18(\mathrm{~m}, 1 \mathrm{H}, \mathrm{H}-2), 4.90\left(\mathrm{~s}, 1 \mathrm{H}, \mathrm{H}-1^{\prime}\right), 4.24\left(\mathrm{ABX}, J_{\mathrm{H}-3 \mathrm{a}}, \mathrm{H}-2=\right.$ $\left.3.7 \mathrm{~Hz}, J_{\mathrm{H}-3 \mathrm{a}}, \mathrm{H}-3 \mathrm{~b}=12.1 \mathrm{~Hz}, 1 \mathrm{H}, \mathrm{H}-3 \mathrm{a}\right), 4.10\left(\mathrm{ABX}, J_{\mathrm{H}-3 \mathrm{~b}}, \mathrm{H}-2=6.3 \mathrm{~Hz}, J_{\mathrm{H}-3 \mathrm{~b}}, \mathrm{H}-3 \mathrm{a}=12.0\right.$ $\mathrm{Hz}, 1 \mathrm{H}, \mathrm{H}-3 \mathrm{~b}$ ), 4.00 (t, $\left.J_{\mathrm{H}-4^{\prime}, \mathrm{H}-3^{\prime}}=J_{\mathrm{H}-4^{\prime}}, \mathrm{H}-5^{\prime}=3.0 \mathrm{~Hz}, 1 \mathrm{H}, \mathrm{H}-4^{\prime}\right), 3.96$ (m, 1H, H-3'), 3.91

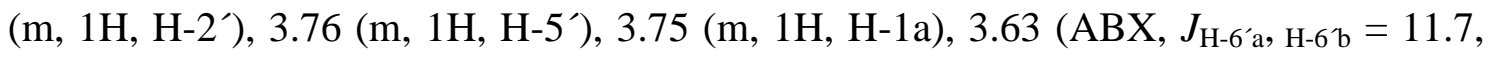


$\left.J_{\mathrm{H}-6^{\prime} \mathrm{a}, \mathrm{H}-5^{\prime}}=6.7,1 \mathrm{H}, \mathrm{H}-6^{\prime} \mathrm{a}\right), 3.61\left(\mathrm{ABX}, J_{\mathrm{H}-6^{\prime} \mathrm{b}, \mathrm{H}-6^{\prime} \mathrm{a}}=11.7, J_{\mathrm{H}-6^{\prime} \mathrm{b}, \mathrm{H}-5^{\prime}}=5.1,1 \mathrm{H}, \mathrm{H}-6^{\prime} \mathrm{b}\right)$, $3.53\left(\mathrm{ABX}, J_{\mathrm{H}-1 \mathrm{~b}, \mathrm{H}-2}=6.6 \mathrm{~Hz}, J_{\mathrm{H}-1 \mathrm{~b}}, \mathrm{H}-1 \mathrm{a}=10.5 \mathrm{~Hz}, 1 \mathrm{H}, \mathrm{H}-1 \mathrm{~b}\right), 2.27(\mathrm{~m}, 4 \mathrm{H}, \mathrm{H \alpha}), 1.55$ $(\mathrm{m}, 4 \mathrm{H}, \mathrm{H} \beta), 1.38-1.10\left(\mathrm{~m}, 56 \mathrm{H}, \mathrm{CH}_{2}\right), 0.75\left(\mathrm{t}, J_{\mathrm{CH} 3}, \mathrm{CH} 3 \mathrm{CH} 2=6.8,6 \mathrm{H}, \mathrm{CH}_{3}\right) ;{ }^{13} \mathrm{C} \mathrm{NMR}$ (151 MHz, $\mathrm{CD}_{3} \mathrm{OD} / \mathrm{CDCl}_{3}, 1 / 2$, v/v) $\delta 173.8(\mathrm{C}(\mathrm{O})-3), 173.6(\mathrm{C}(\mathrm{O})-2), 108.4\left(\mathrm{C}-1^{\prime}\right)$, 86.3 (C-4’), 79.5 (C-2’), 78.2 (C-3’), 71.0 (C-5’), 70.0 (C-2), 65.8 (C-1), 63.6 (C-6’), 62.4 (C-3), 34.2 and $34.0\left(\mathrm{CH}_{2} \alpha\right), 31.8\left(\mathrm{CH}_{2} \mathrm{CH}_{2} \mathrm{CH}_{3}\right), 29.8-28.8\left(\mathrm{CH}_{2}\right), 24.85$ and $24.79\left(\mathrm{CH}_{2} \beta\right), 22.5\left(\underline{\mathrm{CH}}_{2} \mathrm{CH}_{3}\right), 13.9\left(\mathrm{CH}_{3}\right) ; \mathrm{HRMS}(\mathrm{ESI}) \mathrm{m} / z$ calcd. for $\left[\mathrm{C}_{45} \mathrm{H}_{86} \mathrm{NaO}_{10}\right]^{+}$: 809.6113, found: 809.6116.

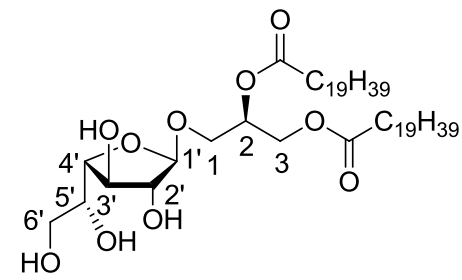

\section{1-O-( $\beta$-D-Galactofuranosyl)-2,3-di- $O$-arachidoyl-sn-}

glycerol (sn-1-2b). Subjection of $s n-1-6 b(23.2 \mathrm{mg}, 0.018 \mathrm{mmol})$ to the global desilylation procedure gave the title compound $\mathrm{sn}-1-\mathbf{2 b}$ as a colourless oil $(15.1 \mathrm{mg}$, $0.018 \mathrm{mmol}$, quant. $),\left(\mathrm{R}_{f}=0.41, \mathrm{MeOH} / \mathrm{DCM}, 1 / 6, \mathrm{v} / \mathrm{v}\right) ;[\alpha]_{\mathrm{D}}{ }^{27}=-21.0(c=1.0$, Pyridine); IR (film) 3463, 2916, 2849, 1738, 1466, 1246, 1175, 1104, 1022, 794, 720, 668, $634 \mathrm{~cm}^{-1} ;{ }^{1} \mathrm{H}$ NMR (500 MHz, $\left.\mathrm{CD}_{3} \mathrm{OD} / \mathrm{CDCl}_{3}, 1 / 2, \mathrm{v} / \mathrm{v}\right) \delta 5.18(\mathrm{~m}, 1 \mathrm{H}, \mathrm{H}-2), 4.9$ $\left(\mathrm{s}, 1 \mathrm{H}, \mathrm{H}-1^{\prime}\right), 4.25(\mathrm{~m}, 1 \mathrm{H}, \mathrm{H}-3 \mathrm{a}), 4.09\left(\mathrm{ABX}, J_{\mathrm{H}-3 \mathrm{~b}}, \mathrm{H}-2=6.2 \mathrm{~Hz}, J_{\mathrm{H}-3 \mathrm{~b}}, \mathrm{H}-3 \mathrm{a}=12.7 \mathrm{~Hz}\right.$, 1H, H-3b), 3.96 (m, 1H, H-4), 3.94 (m, 1H, H-3’), 3.91 (m, 1H, H-2'), 3.76 (m, 1H, H5’), 3.75 (m, 1H, H-1a), 3.60 (m, 1H, H-6’a), 3.61 (m, 1H, H6` b), 3.53 (ABX, J J-1b, H-2 $\left.=6.0 \mathrm{~Hz}, J_{\mathrm{H}-1 \mathrm{~b}, \mathrm{H}-1 \mathrm{a}}=11.0 \mathrm{~Hz}, 1 \mathrm{H}, \mathrm{H}-1 \mathrm{~b}\right), 2.26(\mathrm{~m}, 4 \mathrm{H}, \mathrm{H \alpha}), 1.55(\mathrm{~m}, 4 \mathrm{H}, \mathrm{H} \beta), 1.36-$ $1.08\left(\mathrm{~m}, 64 \mathrm{H}, \mathrm{CH}_{2}\right), 0.89\left(\mathrm{t}, J_{\mathrm{CH} 3}, \mathrm{CH}_{3} \mathrm{H}_{2}=5.0,6 \mathrm{H}, \mathrm{CH}_{3}\right) ;{ }^{13} \mathrm{C} \mathrm{NMR}(125 \mathrm{MHz}$, $\mathrm{CD}_{3} \mathrm{OD} / \mathrm{CDCl}_{3}, 1 / 2$, v/v) $\delta 173.9(\mathrm{C}(\mathrm{O})-3), 173.7(\mathrm{C}(\mathrm{O})-2), 108.3\left(\mathrm{C}^{-1} 1^{\prime}\right), 85.5\left(\mathrm{C}^{-} 4^{\prime}\right)$, 80.0 (C-2'), 77.9 (C-3’), 71.1 (C-5'), 70.0 (C-2), 65.6 (C-1), 63.4 (C-6'), 62.5 (C-3), 34.2 and $34.0\left(\mathrm{CH}_{2} \alpha\right), 31.8\left(\mathrm{CH}_{2} \mathrm{CH}_{2} \mathrm{CH}_{3}\right), 30.1-28.6\left(\mathrm{CH}_{2}\right), 24.8$ and $24.7\left(\mathrm{CH}_{2} \beta\right), 22.6$ $\left(\mathrm{CH}_{2} \mathrm{CH}_{3}\right), 13.9\left(\mathrm{CH}_{3}\right)$; HRMS(ESI) $\mathrm{m} / z$ calcd. for $\left[\mathrm{C}_{49} \mathrm{H}_{94} \mathrm{NaO}_{10}\right]^{+}$: 865.6739, found: 865.6750 . 


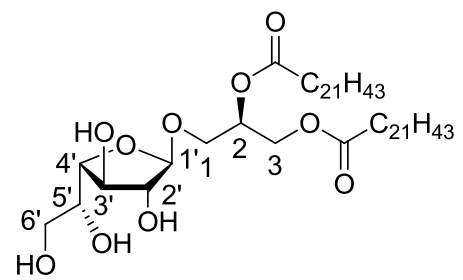

1-O-( $\beta$-D-Galactofuranosyl)-2,3-di- $O$-behenoyl-

sn-glycerol (sn-1-2c). Subjection of $s n-1-6 c(4 \mathrm{mg}, 0.0029 \mathrm{mmol})$ to the global desilylation procedure gave the title compound $\mathrm{s} n-1-2 \mathrm{c}$, as a colourless oil after aqueous workup (2.6 mg, $0.0029 \mathrm{mmol}$, quant.), $\left(\mathrm{R}_{f}=0.56, \mathrm{MeOH} / \mathrm{DCM}, 1 / 6, \mathrm{v} / \mathrm{v}\right) ;[\alpha]_{\mathrm{D}}{ }^{25}=-$ 20.0 ( $c=0.1$, Pyridine); IR (film) 3463, 2955, 2917, 2850, 1735, 1465, 1377, 1106, 783, 735, $632 \mathrm{~cm}^{-1} ;{ }^{1} \mathrm{H}$ NMR (500 MHz, $\mathrm{CD}_{3} \mathrm{OD} / \mathrm{CDCl}_{3}, 1 / 2$, v/v) $\delta 5.17$ (m, 1H, H-2), 4.90 $\left(\mathrm{s}, 1 \mathrm{H}, \mathrm{H}-1^{\prime}\right), 4.29\left(\mathrm{ABX}, J_{\mathrm{H}-3 \mathrm{a}}, \mathrm{H}-2=3.2 \mathrm{~Hz}, J_{\mathrm{H}-3 \mathrm{a}}, \mathrm{H}-3 \mathrm{~b}=12.5 \mathrm{~Hz}, 1 \mathrm{H}, \mathrm{H}-3 \mathrm{a}\right), 4.09$ $\left(\mathrm{ABX}, J_{\mathrm{H}-3 \mathrm{~b}}, \mathrm{H}-2=5.8 \mathrm{~Hz}, J_{\mathrm{H}-3 \mathrm{~b}}, \mathrm{H}-3 \mathrm{a}=12.3 \mathrm{~Hz}, 1 \mathrm{H}, \mathrm{H}-3 \mathrm{~b}\right), 3.94\left(\mathrm{~m}, 1 \mathrm{H}, \mathrm{H}-4^{\prime}\right), 3.93(\mathrm{~m}$, 1H, H-3'), 3.91 (m, 1H, H-2') 3.76 (m, 1H, H-5'), 3.71 (m, 1H, H-1a), 3.60 (m, 2H, H-

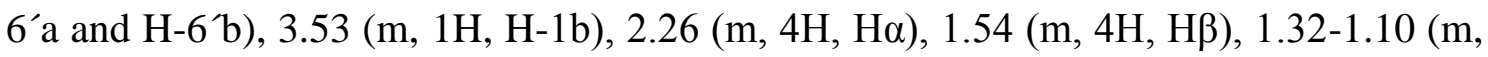
$\left.72 \mathrm{H}, \mathrm{CH}_{2}\right), 0.81\left(\mathrm{t}, J_{\mathrm{CH} 3}, \mathrm{CH} 3 \mathrm{C} \underline{\mathrm{H}} 2=7.0,6 \mathrm{H}, \mathrm{CH}_{3}\right) ;{ }^{13} \mathrm{C} \mathrm{NMR}\left(125 \mathrm{MHz}, \mathrm{CD}_{3} \mathrm{OD} / \mathrm{CDCl}_{3}\right.$, 1/2, v/v) $\delta 173.8(\mathrm{C}(\mathrm{O})-3), 173.6(\mathrm{C}(\mathrm{O})-2), 108.2\left(\mathrm{C}^{\prime}\right), 84.7\left(\mathrm{C}^{\prime}\right), 79.8\left(\mathrm{C} 2^{\prime}\right), 78.2$ (C3), 71.0 (C5'), 70.0 (C-2), 65.5 (C-1), 63.6 (C6'), 62.4 (C-3), 34.0 and $33.8\left(\mathrm{CH}_{2} \alpha\right)$, $31.7\left(\underline{\mathrm{CH}}_{2} \mathrm{CH}_{2} \mathrm{CH}_{3}\right), 29.8-28.8\left(\mathrm{CH}_{2}\right), 24.7$ and $24.6\left(\mathrm{CH}_{2} \beta\right), 22.4\left(\underline{\mathrm{CH}}_{2} \mathrm{CH}_{3}\right), 13.6$ $\left(\mathrm{CH}_{3}\right)$; HRMS(ESI) $\mathrm{m} / z$ calcd. for $\left[\mathrm{C}_{53} \mathrm{H}_{102} \mathrm{NaO}_{10}\right]^{+}: 921.7365$, found: 921.7364

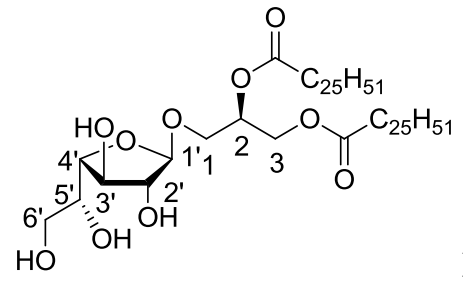

\section{1-O-( $\beta$-D-Galactofuranosyl)-2,3-di- $O$-cerotoyl-sn-glycerol}

(sn-1-2d). Subjection of $s n-1-6 d(24.7 \mathrm{mg}, 0.016 \mathrm{mmol})$ to the global desilylation procedure gave the title compound $\mathrm{s} n-1-2 \mathrm{~d}$ as a colourless oil $(16.5 \mathrm{mg}, 0.016 \mathrm{mmol}$, quant. $),\left(\mathrm{R}_{f}=0.59, \mathrm{MeOH} / \mathrm{DCM}, 1 / 6, \mathrm{v} / \mathrm{v}\right) ;[\alpha]_{\mathrm{D}}^{25}=-10.4(c=1.0$, Pyridine $) ;$ IR (film) 3464, 2955, 2920, 2849, 1731, 1460, 1378, $1024 \mathrm{~cm}^{-1} ;{ }^{1} \mathrm{H}$ NMR (500 MHz, $\mathrm{CD}_{3} \mathrm{OD} / \mathrm{CDCl}_{3}, 1 / 2$, v/v) $\delta 5.18(\mathrm{~m}, 1 \mathrm{H}, \mathrm{H}-2), 4.90\left(\mathrm{~s}, 1 \mathrm{H}, \mathrm{H}-1^{\prime}\right), 4.28\left(\mathrm{ABX}, J_{\mathrm{H}-3 \mathrm{a}}, \mathrm{H}-2=\right.$ $\left.3.8 \mathrm{~Hz}, J_{\mathrm{H}-3 \mathrm{a}}, \mathrm{H}-3 \mathrm{~b}=12.1 \mathrm{~Hz}, 1 \mathrm{H}, \mathrm{H}-3 \mathrm{a}\right), 4.09\left(\mathrm{ABX}, J_{\mathrm{H}-3 \mathrm{~b}}, \mathrm{H}-2=6.4 \mathrm{~Hz}, J_{\mathrm{H}-3 \mathrm{~b}}, \mathrm{H}-3 \mathrm{a}=11.9\right.$ Hz, 1H, H-3b), 3.95 (m, 1H, H-4'), 3.95 (m, 1H, H-3’), 3.91 (m, 1H, H-2'), 3.76 (m, 1H, H-5’), 3.71 (m, 1H, H-1a), 3.61 (m, 1H, H-6’a), 3.59 (m, 1H, H-6’b), 3.53 (ABX, $\left.J_{\mathrm{H}-1 \mathrm{~b}, \mathrm{H}-2}=6.7 \mathrm{~Hz}, J_{\mathrm{H}-1 \mathrm{~b}}, \mathrm{H}-1 \mathrm{a}=10.6 \mathrm{~Hz}, 1 \mathrm{H}, \mathrm{H}-1 \mathrm{~b}\right), 2.26(\mathrm{~m}, 4 \mathrm{H}, \mathrm{H \alpha}), 1.54(\mathrm{~m}, 4 \mathrm{H}, \mathrm{H} \beta)$, 
1.32-1.14 (m, 56H, $\left.\mathrm{CH}_{2}\right), 0.81\left(\mathrm{t}, J_{\mathrm{CH} 3}, \mathrm{CH} 3 \mathrm{CH} 2=6.8,6 \mathrm{H}, \mathrm{CH}_{3}\right) ;{ }^{13} \mathrm{C} \mathrm{NMR}(151 \mathrm{MHz}$, $\mathrm{CD}_{3} \mathrm{OD} / \mathrm{CDCl}_{3}, 1 / 2$, v/v) $\delta 174.0(\mathrm{C}(\mathrm{O})-3), 173.8$ (C(O)-2), 108.4 (C-1'), 85.0 (C-4’), 80.5 (C-2’), 77.9 (C-3’), 71.2 (C-5'), 70.2 (C-2), 65.7 (C-1), 63.5 (C-6’), 62.7 (C-3), 34.2 and $34.1\left(\mathrm{CH}_{2} \alpha\right), 31.9\left(\mathrm{CH}_{2} \mathrm{CH}_{2} \mathrm{CH}_{3}\right), 29.9-28.8\left(\mathrm{CH}_{2}\right), 24.84$ and $24.83\left(\mathrm{CH}_{2} \beta\right)$, $22.6\left(\mathrm{CH}_{2} \mathrm{CH}_{3}\right), 13.8\left(\mathrm{CH}_{3}\right)$; HRMS(ESI) $m / z$ calcd. for $\left[\mathrm{C}_{61} \mathrm{H}_{118} \mathrm{NaO}_{10}\right]^{+}: 1033.8617$, found: 1033.8629 .

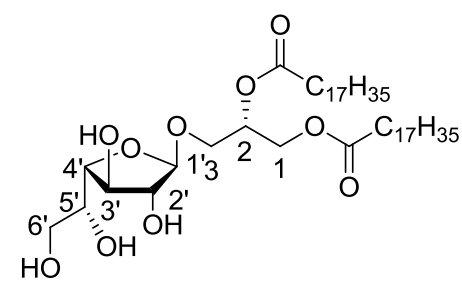

\section{3-O-( $\beta$-D-Galactofuranosyl)-1,2-di- $O$-stearoyl-sn-glycerol}

(sn-3-2a). Subjection of $s n-3-6 a(24.4 \mathrm{mg}, 0.02 \mathrm{mmol})$ to the global desilylation procedure gave $s n-3-2 \mathrm{a}$ as a colourless oil (15.7 $\mathrm{mg}, 0.02 \mathrm{mmol}$, quant.), $\left(\mathrm{R}_{f}=0.61\right.$, $\mathrm{MeOH} / \mathrm{DCM}, 1 / 6, \mathrm{v} / \mathrm{v}) ;[\alpha]_{\mathrm{D}}{ }^{25}=-35.7$ ( $c=0.1$, Pyridine); IR (film) 3446, 2917, 2849, 2361, 2341, 1734, 1451, 1119, 1023, 977, $668 \mathrm{~cm}^{-1} ;{ }^{1} \mathrm{H}$ NMR (600 MHz, $\mathrm{CD}_{3} \mathrm{OD} / \mathrm{CDCl}_{3}, 1 / 2$, v/v) $\delta 5.18(\mathrm{~m}, 1 \mathrm{H}, \mathrm{H}-2), 4.90\left(\mathrm{~s}, 1 \mathrm{H}, \mathrm{H}-1^{\prime}\right), 4.32\left(\mathrm{ABX}, J_{\mathrm{H}-1 \mathrm{a}, \mathrm{H}-2}=\right.$ $\left.3.5 \mathrm{~Hz}, J_{\mathrm{H}-1 \mathrm{a}}, \mathrm{H}-1 \mathrm{~b}=12.0 \mathrm{~Hz}, 1 \mathrm{H}, \mathrm{H}-1 \mathrm{a}\right), 4.11\left(\mathrm{ABX}, J_{\mathrm{H}-1 \mathrm{~b}}, \mathrm{H}-2=6.5 \mathrm{~Hz}, J_{\mathrm{H}-1 \mathrm{a}}, \mathrm{H}-1 \mathrm{~b}=12.0\right.$ Hz, 1H, H-1b), 3.99 (m, 3H, H-4', H-3', H-2'), 3.75 (m, 2H, H-3a, H-5'), 3.68 (m, 2H, H-6'a and b), $3.58\left(\mathrm{ABX}, J_{\mathrm{H}-3 \mathrm{~b}, \mathrm{H}-2}=5.5 \mathrm{~Hz}, J_{\mathrm{H}-3 \mathrm{~b}}, \mathrm{H}-3 \mathrm{a}=11.0 \mathrm{~Hz}, 1 \mathrm{H}, \mathrm{H}-3 \mathrm{~b}\right), 2.28$ (t, $\left.J_{\mathrm{H} \alpha}, \mathrm{H} \beta=7.3 \mathrm{~Hz}, 4 \mathrm{H}, \mathrm{H} \alpha\right), 1.57(\mathrm{~m}, 4 \mathrm{H}, \mathrm{H} \beta), 1.35-1.14\left(\mathrm{~m}, 56 \mathrm{H}, \mathrm{CH}_{2}\right), 0.84\left(\mathrm{t}, J_{\mathrm{CH} 3}, \mathrm{CH} 2\right.$ $\left.=6.9 \mathrm{~Hz} 6 \mathrm{H}, \mathrm{CH}_{3}\right) ;{ }^{13} \mathrm{C} \mathrm{NMR}\left(151 \mathrm{MHz}, \mathrm{CD}_{3} \mathrm{OD} / \mathrm{CDCl}_{3}, 1 / 2, \mathrm{v} / \mathrm{v}\right) \delta 173.9$ and 173.6 (C(O)), $108.4\left(\mathrm{C}-1^{\prime}\right), 85.3$ (C-4'), 80.3 (C-2'), 77.9 (C-3'), 71.1 (C-5'), 69.9 (C-2), 65.7 (C-3), 63.5 (C-6'), $62.6(\mathrm{C}-1) ; 34.2$ and $34.0\left(\mathrm{CH}_{2} \alpha\right), 31.8\left(\underline{\mathrm{CH}_{2}} \mathrm{CH}_{2} \mathrm{CH}_{3}\right), 29.9-28.8$ $\left(\mathrm{CH}_{2}\right), 24.81$ and $24.78\left(\mathrm{CH}_{2} \beta\right), 22.6\left(\mathrm{CH}_{2} \mathrm{CH}_{3}\right), 13.9\left(\mathrm{CH}_{3}\right) ; \mathrm{HRMS}(\mathrm{ESI}) \mathrm{m} / z$ calcd. for $\left[\mathrm{C}_{45} \mathrm{H}_{86} \mathrm{NaO}_{10}\right]^{+}:$809.6113, found: 809.6111.

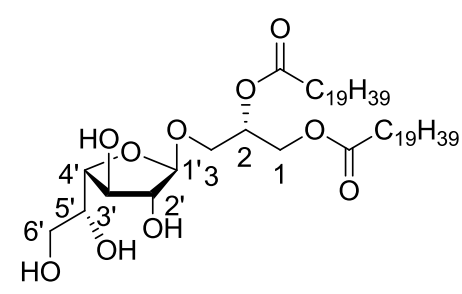

3-O-( $\beta$-D-Galactofuranosyl)-1,2-di- $O$-arachidoyl-sn-

glycerol (sn-3-2b). Subjection of $s n-3-6 b(43.3 \mathrm{mg}, 0.033 \mathrm{mmol})$ to the global desilylation procedure gave the title compound $\mathrm{s} n-\mathbf{3}-\mathbf{2 b}$ as a colourless oil $(28.0 \mathrm{mg}$, 
0.033 mmol, quant. $),\left(\mathrm{R}_{f}=0.59, \mathrm{MeOH} / \mathrm{DCM}, 1 / 6, \mathrm{v} / \mathrm{v}\right) ;[\alpha]_{\mathrm{D}}{ }^{26}=-26.0(c=0.1$, Pyridine); IR (film) 3454, 2917, 2849, 1734, 1265, 895, 758, 733, $704 \mathrm{~cm}^{-1}$; ${ }^{1} \mathrm{H}$ NMR (600 MHz, $\mathrm{CD}_{3} \mathrm{OD} / \mathrm{CDCl}_{3}, 1 / 2$, v/v) $\delta 5.15$ (m, 1H, H-2), 4.85 (s, 1H, H-1'), 4.30 (m, 1H, H-1a), 4.09 (m, 1H, H-1b), 3.96-3.87 (m, 3H, H-4’' H-3’' H-2'), 3.76-3.65 (m, 2H,

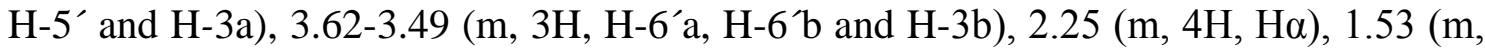
$4 \mathrm{H}, \mathrm{H} \beta), 1.30-1.09\left(\mathrm{~m}, 64 \mathrm{H}, \mathrm{CH}_{2}\right), 0.80\left(\mathrm{~m}, 6 \mathrm{H}, \mathrm{CH}_{3}\right) ;{ }^{13} \mathrm{C} \mathrm{NMR}(151 \mathrm{MHz}$, $\mathrm{CD}_{3} \mathrm{OD} / \mathrm{CDCl}_{3}, 1 / 2$, v/v) $\delta 174.0(\mathrm{C}-1), 173.6(\mathrm{C}-2), 108.4\left(\mathrm{C}-1^{\prime}\right), 84.8$ (C-4'), 80.7 (C2'), 77.7 (C-3'), 71.1 (C-5'), 70.0 (C-2), 65.6 (C-3), 63.4 (C-6’), 62.6 (C-1), 34.1 and $34.0\left(\mathrm{CH}_{2} \alpha\right), 31.8\left(\underline{\mathrm{CH}}_{2} \mathrm{CH}_{2} \mathrm{CH}_{3}\right), 30.1-28.8\left(\mathrm{CH}_{2}\right), 24.81\left(\mathrm{CH}_{2} \beta\right), 22.5\left(\underline{\mathrm{CH}}_{2} \mathrm{CH}_{3}\right), 13.8$ $\left(\mathrm{CH}_{3}\right) ; \mathrm{HRMS}(\mathrm{ESI}) \mathrm{m} / z$ calcd. for $\left[\mathrm{C}_{49} \mathrm{H}_{94} \mathrm{NaO}_{10}\right]^{+}:$865.6744, found: 865.6744.

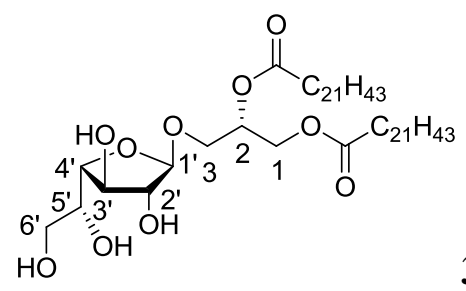

\section{3-O-( $\beta$-D-Galactofuranosyl)-1,2-di- $O$-behenyl-sn-glycerol}

(sn-3-2c). Subjection of $s n-3-6 c(20.4 \mathrm{mg}, 0.015 \mathrm{mmol})$ to the global desilylation procedure gave the title compound $\mathrm{s} n-3-2 \mathrm{c}$ as a colourless oil $(13.5 \mathrm{mg}, 0.015 \mathrm{mmol}$, quant. $),\left(\mathrm{R}_{f}=0.55, \mathrm{MeOH} / \mathrm{DCM}, 1 / 6, \mathrm{v} / \mathrm{v}\right) ;[\alpha]_{\mathrm{D}}{ }^{26}=-10.0(c=0.1$, Pyridine $) ;$ IR (film) 3450, 2917, 2849, 1736, 1466, 1265, 1108, 758, 732, 704, $634 \mathrm{~cm}^{-1} ;{ }^{1} \mathrm{H}$ NMR $(600$ $\left.\mathrm{MHz}, \mathrm{CDCl}_{3} / \mathrm{CD}_{3} \mathrm{OD}, 2 / 1, \mathrm{v} / \mathrm{v}\right) \delta 5.17$ (m, 1H, H-2), 4.88 (s, 1H, H-1'), $4.32(\mathrm{~m}, 1 \mathrm{H}, \mathrm{H}-$ 1a), 4.11 (m, 1H, H-1b), 3.98-3.91 (m, 3H, H-4', H-3' and H-2'), 3.77-3.68 (m, 2H, H-

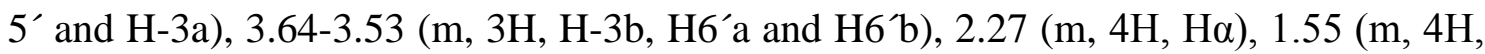
$\mathrm{H} \beta), \quad 1.35-1.12\left(\mathrm{~m}, 72 \mathrm{H}, \mathrm{CH}_{2}\right), 0.82\left(\mathrm{~m}, 6 \mathrm{H}, \mathrm{CH}_{3}\right) ;{ }^{13} \mathrm{C} \quad \mathrm{NMR}(151 \mathrm{MHz}$, $\left.\mathrm{CDCl}_{3} / \mathrm{CD}_{3} \mathrm{OD}, 2 / 1, \mathrm{v} / \mathrm{v}\right) \delta 174.0(\mathrm{C}-1), 173.6$ (C-2), 108.3 (C-1'), 84.8 (C-4'), 80.7 (C2'), 77.7 (C-3’), 71.1 (C-5’), 70.0 (C-2), 65.6 (C-3), 63.4 (C6'), 62.6 (C-1), 34.1 and $33.9\left(\mathrm{CH}_{2} \alpha\right), 31.8\left(\mathrm{CH}_{2} \mathrm{CH}_{2} \mathrm{CH}_{3}\right), 30.2-28.5\left(\mathrm{CH}_{2}\right), 24.80$ and $24.76\left(\mathrm{CH}_{2} \beta\right), 22.5$ $\left(\mathrm{CH}_{2} \mathrm{CH}_{3}\right), 13.8\left(\mathrm{CH}_{3}\right)$; HRMS(ESI) $m / z$ calcd. for $\left[\mathrm{C}_{53} \mathrm{H}_{102} \mathrm{NaO}_{10}\right]^{+}: 921.7365$, found: 921.7381 . 


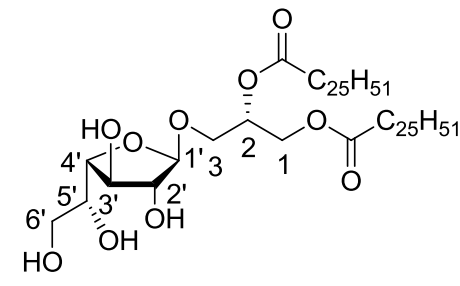

\section{3-O-( $\beta$-D-Galactofuranosyl)-1,2-di- $O$-cerotoyl-sn-glycerol}

(sn-3-2d). Subjection of $s n-3-6 d(3.0 \mathrm{mg}, 0.002 \mathrm{mmol})$ to the global desilylation procedure gave the title compound $\mathrm{sn}-3-2 \mathrm{~d}$ as a colourless oil $(2.1 \mathrm{mg}, 0.002 \mathrm{mmol}$, quant. $),\left(\mathrm{R}_{f}=0.56, \mathrm{MeOH} / \mathrm{DCM}, 1 / 6, \mathrm{v} / \mathrm{v}\right) ;[\alpha]_{\mathrm{D}}{ }^{26}=-15.0(c=0.1$, Pyridine); IR (film) 3562, 2955, 2917, 2849, 1740, 1462, 1377, 1164, 719, $633 \mathrm{~cm}^{-1} ;{ }^{1} \mathrm{H}$ NMR (600 MHz, $\left.\mathrm{CD}_{3} \mathrm{OD} / \mathrm{CDCl}_{3}, 1 / 2, \mathrm{v} / \mathrm{v}\right) \delta 5.19(\mathrm{~m}, 1 \mathrm{H}, \mathrm{H}-2), 4.89\left(\mathrm{~s}, 1 \mathrm{H}, \mathrm{H}^{-1} 1^{\prime}\right), 4.34\left(\mathrm{ABX}, J_{\mathrm{H}-1 \mathrm{a}}, \mathrm{H}-2=\right.$ $\left.3.4 \mathrm{~Hz}, J_{\mathrm{H}-1 \mathrm{a}}, \mathrm{H}-1 \mathrm{~b}=12.2 \mathrm{~Hz}, 1 \mathrm{H}, \mathrm{H}-1 \mathrm{a}\right), 4.12\left(\mathrm{ABX}, J_{\mathrm{H}-1 \mathrm{~b}}, \mathrm{H}-2=6.5 \mathrm{~Hz}, J_{\mathrm{H}-1 \mathrm{a}}, \mathrm{H}-1 \mathrm{~b}=12.2\right.$ $\mathrm{Hz}, 1 \mathrm{H}, \mathrm{H}-1 \mathrm{~b}), 3.99-3.93$ (m, 3H, H-4', H-3'and H-2'), 3.76 (ABX, $J_{\mathrm{H}-3 \mathrm{a}}, \mathrm{H}-2=5.7 \mathrm{~Hz}$, $\left.J_{\mathrm{H}-3 \mathrm{a}, \mathrm{H}-3 \mathrm{~b}}=11.0 \mathrm{~Hz}, 1 \mathrm{H}, \mathrm{H}-3 \mathrm{a}\right), 3.73\left(\mathrm{~m}, 1 \mathrm{H}, \mathrm{H}-5^{\prime}\right), 3.64-3.60$ (m, 2H, H-6'a and b), $3.58\left(\mathrm{ABX}, J_{\mathrm{H}-3 \mathrm{~b}}, \mathrm{H}-2=5.8 \mathrm{~Hz}, J_{\mathrm{H}-3 \mathrm{~b}}, \mathrm{H}-3 \mathrm{a}=11.5 \mathrm{~Hz}, 1 \mathrm{H}, \mathrm{H}-3 \mathrm{~b}\right), 2.26\left(\mathrm{~m}, 4 \mathrm{H}, \mathrm{CH}_{2} \alpha\right), 1.56$ $\left(\mathrm{m}, 4 \mathrm{H}, \mathrm{CH}_{2} \beta\right), 1.37-1.09\left(\mathrm{~m}, 88 \mathrm{H}, \mathrm{CH}_{2}\right), 0.83\left(\mathrm{~m}, 6 \mathrm{H}, \mathrm{CH}_{3}\right) ;{ }^{13} \mathrm{C} \mathrm{NMR}(151 \mathrm{MHz}$, $\left.\mathrm{CDCl}_{3} / \mathrm{CD}_{3} \mathrm{OD}, 2 / 1, \mathrm{v} / \mathrm{v}\right) \delta 174.0$ (C-1), 173.6 (C-2), 108.4 (C-1'), 84.7 (C-4'), 80.7 (C2’), 77.7 (C-3’), 71.1 (C-5'), 70.0 (C-2), 65.6 (C-3), 63.4 (C-6’), 62.7 (C-1), 34.0 and $34.2\left(\mathrm{CH}_{2} \alpha\right), 31.8\left(\mathrm{CH}_{2} \mathrm{CH}_{2} \mathrm{CH}_{3}\right), 29.9-28.8\left(\mathrm{CH}_{2}\right), 24.81$ and $24.78\left(\mathrm{CH}_{2} \beta\right), 22.5$ $\left(\mathrm{CH}_{2} \mathrm{CH}_{3}\right), 13.8\left(\mathrm{CH}_{3}\right)$; HRMS(ESI) $m / z$ calcd. for $\left[\mathrm{C}_{61} \mathrm{H}_{118} \mathrm{NaO}_{10}\right]^{+}: 1033.8617$, found: 1033.8634 .

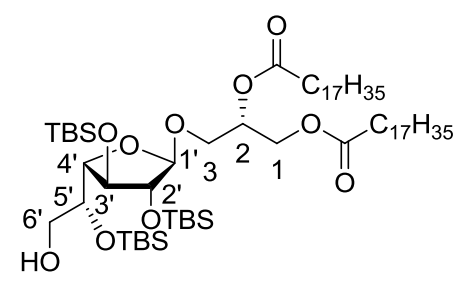

\section{3-O-(2,3,5-Tri-O-tert-butyldimethylsilyl-}

$\boldsymbol{\beta}$-D-galactofuranosyl)-1,2-di- $\boldsymbol{O}$-stearoyl-sn-glycerol ( $s \boldsymbol{n}$-3-7a). A solution of $s n$-3-6a (150 mg, $0.12 \mathrm{mmol})$ in THF/TFA/ $\mathrm{H}_{2} \mathrm{O}(95 / 2.5 / 2.5, \mathrm{v} / \mathrm{v} / \mathrm{v}, 4.2 \mathrm{~mL})$ was stirred at $0{ }^{\circ} \mathrm{C}$ for $48 \mathrm{~h}$. The reaction mixture was then diluted with EtOAc, washed with $\mathrm{NaHCO}_{3}$ (sat. aq.) and brine, dried $\left(\mathrm{MgSO}_{4}\right)$, filtered and concentrated. Following silica gel flash column chromatography (EtOAc/PE, 4/96, v/v), pure $s n-3-7 \mathbf{a}$ was obtained as a colourless oil (97 mg, $0.086 \mathrm{mmol}, 72 \%$ yield), $\left(\mathrm{R}_{f}=0.38\right.$, EtOAc/PE, $\left.1 / 9, \mathrm{v} / \mathrm{v}\right) ;[\alpha]_{\mathrm{D}}{ }^{20}$ $=-20.0\left(c=1.0, \mathrm{CHCl}_{3}\right)$; IR (film) 2924, 2854, 1743, 1463, 1361, 1252, 1113, 1054, 1006, 940, 836, 776, 739, $670 \mathrm{~cm}^{-1} .{ }^{1} \mathrm{H}$ NMR (500 MHz, $\left.\mathrm{CDCl}_{3}\right) \delta 5.20(\mathrm{~m}, 1 \mathrm{H}, \mathrm{H}-2)$, 
$4.81\left(\mathrm{~s}, 1 \mathrm{H}, \mathrm{H}-1^{\prime}\right), 4.35\left(\mathrm{ABX}, J_{\mathrm{H}-1 \mathrm{a}}, \mathrm{H}-2=3.7 \mathrm{~Hz}, J_{\mathrm{H}-1 \mathrm{a}}, \mathrm{H}-1 \mathrm{~b}=11.9 \mathrm{~Hz}, 1 \mathrm{H}, \mathrm{H}-1 \mathrm{a}\right), 4.18$ $\left(\mathrm{ABX}, J_{\mathrm{H}-1 \mathrm{~b}}, \mathrm{H}-2=6.6 \mathrm{~Hz}, J_{\mathrm{H}-1 \mathrm{a}}, \mathrm{H}-1 \mathrm{~b}=12.0 \mathrm{~Hz}, 1 \mathrm{H}, \mathrm{H}-1 \mathrm{~b}\right), 4.05\left(\mathrm{~m}, J_{\mathrm{H}-3}, \mathrm{H}-2^{\prime}=1.8 \mathrm{~Hz}\right.$, $\left.J_{\mathrm{H}-3^{\prime}},{\mathrm{H}-4^{\prime}}^{\prime}=4.5 \mathrm{~Hz} 1 \mathrm{H}, \mathrm{H}-3^{\prime}\right), 4.00\left(\mathrm{dd}, J_{\mathrm{H}-2^{\prime}, \mathrm{H}-3^{\prime}}=1.8 \mathrm{~Hz}, J_{\mathrm{H}-2^{\prime}, \mathrm{H}-1^{\prime}}=0.9 \mathrm{~Hz}, 1 \mathrm{H}, \mathrm{H}-2^{\prime}\right)$, $3.99\left(\mathrm{dd}, J_{\mathrm{H}-4^{\prime}, \mathrm{H}-3^{\prime}}=4.7 \mathrm{~Hz}, J_{\mathrm{H}-4^{\prime}, \mathrm{H}-5^{\prime}}=3.3 \mathrm{~Hz}, 1 \mathrm{H}, \mathrm{H}-4^{\prime}\right), 3.86\left(\mathrm{~m}, 1 \mathrm{H}, \mathrm{H}-5^{\prime}\right), 3.78$ $\left(\mathrm{ABX}, J_{\mathrm{H}-3 \mathrm{a}}, \mathrm{H}-2=4.6 \mathrm{~Hz}, J_{\mathrm{H}-3 \mathrm{a}}, \mathrm{H}-3 \mathrm{~b}=10.8 \mathrm{~Hz}, 1 \mathrm{H}, \mathrm{H}-3 \mathrm{a}\right), 3.67\left(\mathrm{ABX}, J_{\mathrm{H}-6 \mathrm{a}}, \mathrm{H}-5^{\prime}=5.6\right.$ $\left.\mathrm{Hz}, J_{\mathrm{H}-6^{\prime} \mathrm{a}, \mathrm{H}-6^{\prime} \mathrm{b}}=11.8 \mathrm{~Hz}, 1 \mathrm{H}, \mathrm{H}-6^{\prime} \mathrm{a}\right), 3.61\left(\mathrm{ABX}, J_{\mathrm{H}-6^{\prime} \mathrm{b}, \mathrm{H}-5^{\prime}}=3.9 \mathrm{~Hz}, J_{\mathrm{H}-6^{\prime} \mathrm{b}, \mathrm{H}-6^{\prime} \mathrm{a}}=11.8\right.$ $\mathrm{Hz}, 1 \mathrm{H}, \mathrm{H}-6$ b $), 3.56\left(\mathrm{ABX}, J_{\mathrm{H}-3 \mathrm{~b}}, \mathrm{H}-2=5.6 \mathrm{~Hz}, J_{\mathrm{H}-3 \mathrm{~b}}, \mathrm{H}-3 \mathrm{a}=10.8 \mathrm{~Hz}, 1 \mathrm{H}, \mathrm{H}-3 \mathrm{~b}\right), 2.40$ (s,

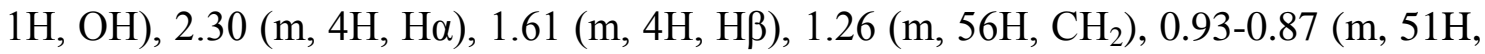
$\left.\mathrm{CH}_{3}\right)$, 0.14-0.07 (m, 18H, $\left.\mathrm{SiCH}_{3}\right) ;{ }^{13} \mathrm{C} \mathrm{NMR}\left(75 \mathrm{MHz}, \mathrm{CDCl}_{3}\right) \delta 173.4(\mathrm{C}(\mathrm{O}) 1), 173.0$ (C(O)2), 108.2 (C-1'), 87.2 (C-4'), 83.5 (C-2'), 79.1 (C-3’), 72.4 (C-5’), 65.1 (C-2), 64.1 (C-6'), 62.6 (C-1); 34.3 and $34.1\left(\mathrm{CH}_{2} \alpha\right), 31.9\left(\mathrm{CH}_{2} \mathrm{CH}_{2} \mathrm{CH}_{3}\right), 29.9-29.0\left(\mathrm{CH}_{2}\right)$, $26.0\left(\mathrm{C}\left(\underline{\mathrm{CH}}_{3}\right)_{3}\right), 25.70\left(\mathrm{C}\left(\underline{\mathrm{CH}}_{3}\right)_{3}\right), 25.66\left(\mathrm{C}\left(\underline{\mathrm{CH}}_{3}\right)_{3}\right), 24.94$ and $24.90\left(\mathrm{CH}_{2} \beta\right), 22.5\left(\mathrm{CH}_{2}\right)$, $18.4\left(\mathrm{Si} \underline{\mathrm{C}}\left(\mathrm{CH}_{3}\right)_{3}\right), 17.9\left(\mathrm{SiC}\left(\mathrm{CH}_{3}\right)_{3}\right), 17.8\left(\mathrm{SiC}\left(\mathrm{CH}_{3}\right)_{3}\right), 14.1\left(\mathrm{CH}_{3}\right),-4.2\left(\mathrm{SiCH}_{3}\right),-4.3$ $\left.\left(\mathrm{SiCH}_{3}\right),-4.4\left(\mathrm{SiCH}_{3}\right),-4.5 \mathrm{SiCH}_{3}\right)$, -4.6 $\left(\mathrm{SiCH}_{3}\right)$, -4.8 $\left(\mathrm{SiCH}_{3}\right) ; \mathrm{HRMS}(\mathrm{ESI}) \mathrm{m} / z$ calcd. for $\left[\mathrm{C}_{63} \mathrm{H}_{128} \mathrm{NaO}_{10} \mathrm{Si}_{3}\right]^{+}: 1151.8708$, found: 1151.8713 .

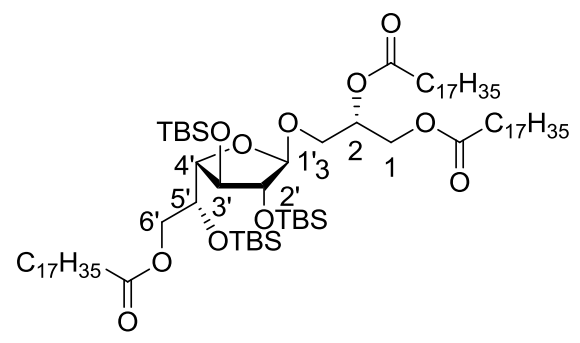

\section{3-O-(6-O-Stearoyl-2,3,5-Tri-O-}

tert-butyldimethylsilyl- $\beta$-D-galactofuranosyl)-1,2-di- $O$-stearoyl-sn-glycerol $\quad$ (sn-322a). To a solution of $s n-3-7 \mathbf{a}(16 \mathrm{mg}, 0.014 \mathrm{mmol})$ and stearic acid $(173 \mathrm{mg}, 0.61$ mmol, recrystalised from $\mathrm{MeCN}$ and acetone) in dry toluene $(1.0 \mathrm{~mL})$ at $40{ }^{\circ} \mathrm{C}$ was added EDCI (116 mg, $0.61 \mathrm{mmol})$ and DMAP (2.5 mg, $0.020 \mathrm{mmol})$. The reaction mixture was stirred for $12 \mathrm{~h}$ and then diluted with EtOAc, washed with $\mathrm{NaHCO}_{3}$ (sat. aq.) and brine, dried $\left(\mathrm{MgSO}_{4}\right)$, filtered and concentrated. Following purification by silica gel flash column chromatography (EtOAc/PE, 2/98, v/v), pure $s n-3-22 a$ was obtained as a colourless oil (19 mg, $0.014 \mathrm{mmol}$, quant.), $\left(\mathrm{R}_{f}=0.76\right.$, EtOAc/PE, 1/9, $\mathrm{v} / \mathrm{v}) ;[\alpha]_{\mathrm{D}}{ }^{25}=-11.8\left(c=0.1, \mathrm{CHCl}_{3}\right)$; IR (film) 3500, 2920, 2851, 1742, 1463, 1219, $1115,837,773,668 \mathrm{~cm}^{-1} .{ }^{1} \mathrm{H}$ NMR $\left(500 \mathrm{MHz}, \mathrm{CDCl}_{3}\right) \delta 5.21(\mathrm{~m}, 1 \mathrm{H}, \mathrm{H}-2), 4.81(\mathrm{~s}, 1 \mathrm{H}$, H-1'), $4.34\left(\mathrm{ABX}, J_{\mathrm{H}-1 \mathrm{a}}, \mathrm{H}-1 \mathrm{~b}=11.9 \mathrm{~Hz}, J_{\mathrm{H}-1 \mathrm{~b}}, \mathrm{H}-2=3.6 \mathrm{~Hz}, 1 \mathrm{H}, \mathrm{H}-1 \mathrm{a}\right), 4.19$ (m, 2H, H-1b and H-6’a), 4.02 (ABX, $J_{\mathrm{H}-6^{\prime} \mathrm{a}}, \mathrm{H}-6^{\prime} \mathrm{b}=11.4 \mathrm{~Hz}, J_{\mathrm{H}-6^{\prime} \mathrm{b}, \mathrm{H}-2}=7.0 \mathrm{~Hz} 1 \mathrm{H}, \mathrm{H}-6^{\prime} \mathrm{b}$ ), 4.06 (dd, 
$\left.J_{\mathrm{H}-4^{\prime}, \mathrm{H}-3^{\prime}}=4.7 \mathrm{~Hz}, J_{\mathrm{H}-4^{\prime}, \mathrm{H}-5^{\prime}}=2.3 \mathrm{~Hz}, 1 \mathrm{H}, \mathrm{H}^{-} 4^{\prime}\right), 3.99$ (m, 1H, H-2 $\left.{ }^{\prime}\right), 3.95$ (m, 1H, H-5 $)$, $3.86\left(\mathrm{t}, J_{\mathrm{H}-3^{\prime}, \mathrm{H}-4^{\prime}}=J_{\mathrm{H}-3^{\prime}}, \mathrm{H}-2^{\prime}=4.8 \mathrm{~Hz}, 1 \mathrm{H}, \mathrm{H}-3^{\prime}\right), 3.79\left(\mathrm{ABX}, J_{\mathrm{H}-3 \mathrm{a}}, \mathrm{H}-2=4.6 \mathrm{~Hz}, J_{\mathrm{H}-3 \mathrm{a}}, \mathrm{H}-3 \mathrm{~b}\right.$ $=10.9 \mathrm{~Hz}, 1 \mathrm{H}, \mathrm{H}-3 \mathrm{a}), 3.54\left(\mathrm{ABX}, J_{\mathrm{H}-3 \mathrm{~b}}, \mathrm{H}-2=5.20 \mathrm{~Hz}, J_{\mathrm{H}-3 \mathrm{~b}}, \mathrm{H}-3 \mathrm{a}=11.0 \mathrm{~Hz}, 1 \mathrm{H}, \mathrm{H}-3 \mathrm{~b}\right)$, $2.30(\mathrm{~m}, 4 \mathrm{H}, \mathrm{H \alpha}), 1.61(\mathrm{~m}, 4 \mathrm{H}, \mathrm{H} \beta), 1.17-1.37\left(\mathrm{~m}, 56 \mathrm{H}, \mathrm{CH}_{2}\right), 0.96-0.81(\mathrm{~m}, 51 \mathrm{H}$, $\mathrm{C}_{3} \mathrm{C}$ and $\left.\mathrm{CH}_{3}\right), 0.15-0.00\left(\mathrm{~m}, 18 \mathrm{H}, \mathrm{SiCH}_{3}\right) ;{ }^{13} \mathrm{C}$ NMR $\left(126 \mathrm{MHz}, \mathrm{CDCl}_{3}\right) \delta 173.5$ (C(O)6'), $173.3(\mathrm{C}(\mathrm{O}) 1), 172.9$ (C(O)2), 108.2 (C-1'), 85.9 (C-4'), 83.8 (C-2'), 79.2 (C3'), 70.8 (C-5'), 65.2 (C-2), 66.0 (C-6’), 62.5 (C-1); 34.4-34.0 ( $\left.\mathrm{CH}_{2} \alpha\right), 31.9$ $\left.\left(\mathrm{C}_{2} \mathrm{CH}_{2} \mathrm{CH}_{3}\right), 29.9-29.0\left(\mathrm{CH}_{2}\right), 25.9\left(\mathrm{C}\left(\underline{\mathrm{CH}}_{3}\right)_{3}\right), 25.72\left(\mathrm{C}\left(\underline{\mathrm{CH}}_{3}\right)_{3}\right), 25.68\left(\mathrm{C}_{(} \underline{\mathrm{CH}}_{3}\right)_{3}\right)$, 24.94 and $24.90\left(\mathrm{CH}_{2} \beta\right), 22.7\left(\mathrm{CH}_{2}\right), 18.3\left(\mathrm{Si} \underline{\mathrm{C}}\left(\mathrm{CH}_{3}\right)_{3}\right), 17.9\left(\mathrm{Si} \underline{\mathrm{C}}\left(\mathrm{CH}_{3}\right)_{3}\right), 17.8$ $\left(\mathrm{SiC}\left(\mathrm{CH}_{3}\right)_{3}\right), 14.1\left(\mathrm{CH}_{3}\right),-4.10\left(\mathrm{SiCH}_{3}\right),-4.2\left(\mathrm{SiCH}_{3}\right),-4.44\left(\mathrm{SiCH}_{3}\right),-4.47\left(\mathrm{SiCH}_{3}\right),-$ $4.6\left(\mathrm{SiCH}_{3}\right),-4.8\left(\mathrm{SiCH}_{3}\right) ; \mathrm{HRMS}(\mathrm{ESI}) \mathrm{m} / z$ calcd. for $\left[\mathrm{C}_{81} \mathrm{H}_{162} \mathrm{NaO}_{11} \mathrm{Si}_{3}\right]^{+}:$: 1418.1317, found: 1418.1324 .

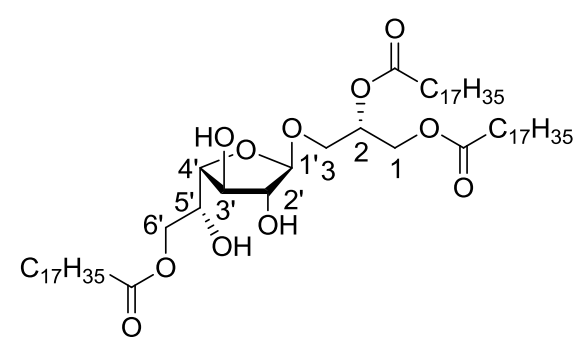

\section{3-O-(6-O-Stearoyl- $\beta$-D-galactofuranosyl)-1,2-di- $O$ -}

stearoyl-sn-glycerol (sn-3-3a). To a solution of $s n-3-22 a(11 \mathrm{mg}, 0.0080 \mathrm{mmol})$ in THF $(900 \mu \mathrm{L})$ was added HF.Pyridine $(100 \mu \mathrm{L})$ and the reaction stirred at RT for $12 \mathrm{~h}$. After this time, the $\mathrm{HF}$ was quenched with $\mathrm{NaHCO}_{3}$ (sat aq.) and the compound extracted using EtOAc. The organic layer was washed with $\mathrm{HCl}(0.1 \mathrm{M})$, brine, and then dried $\left(\mathrm{MgSO}_{4}\right)$, filtered and concentrated to give pure sn-3-3a as a colourless oil (8.4 mg, $0.008 \mathrm{mmol}$, 90\%) after purification by silica gel flash column chromatography $\left(\mathrm{R}_{f}=0.42, \mathrm{MeOH} / \mathrm{DCM}, 1 / 9, \mathrm{v} / \mathrm{v}\right) ;[\alpha]_{\mathrm{D}}{ }^{20}=-36.0\left(c=0.1, \mathrm{CHCl}_{3}\right) ; \mathrm{IR}$ (film) 3461, 2919, 2850, 1733, 1466, 1215, 757, $668 \mathrm{~cm}^{-1} .{ }^{1} \mathrm{H} \mathrm{NMR}\left(500 \mathrm{MHz}, \mathrm{CDCl}_{3}\right)$ $\delta 5.20(\mathrm{~m}, 1 \mathrm{H}, \mathrm{H}-2), 5.02\left(\mathrm{~s}, 1 \mathrm{H}, \mathrm{H}-1^{\prime}\right), 4.35\left(\mathrm{ABX}, J_{\mathrm{H}-1 \mathrm{a}}, \mathrm{H}-1 \mathrm{~b}=11.7 \mathrm{~Hz}, J_{\mathrm{H}-1 \mathrm{~b}}, \mathrm{H}-2=3.9\right.$ $\mathrm{Hz}, 1 \mathrm{H}, \mathrm{H}-1 \mathrm{a}), 4.32$ (ABX, $\left.J_{\mathrm{H}-6^{\prime} \mathrm{a}}, \mathrm{H}-6^{\prime} \mathrm{b}=11.9 \mathrm{~Hz}, J_{\mathrm{H}-6^{\prime} \mathrm{a}}, \mathrm{H}-5^{\prime}=8.8 \mathrm{~Hz}, 1 \mathrm{H}, \mathrm{H}-6^{\prime} \mathrm{a}\right), 4.21$

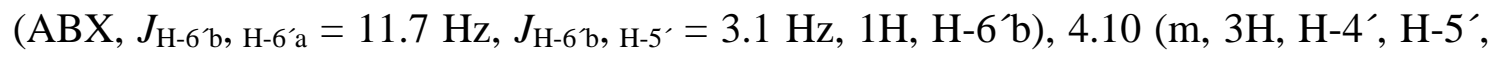
H-1b), 4.07 (bs, 1H, H-2'), 4.01 (bs, 1H, H-3'), 3.82 (ABX, $J_{\mathrm{H}-3 \mathrm{a}, \mathrm{H}-3 \mathrm{~b}}=10.8 \mathrm{~Hz}, J_{\mathrm{H}-3 \mathrm{a}}, \mathrm{H}-$ $2=5.8 \mathrm{~Hz}, 1 \mathrm{H}, \mathrm{H}-3 \mathrm{a}), 3.61\left(\mathrm{ABX}, J_{\mathrm{H}-3 \mathrm{~b}}, \mathrm{H}-3 \mathrm{a}=10.8 \mathrm{~Hz}, J_{\mathrm{H}-3 \mathrm{~b}}, \mathrm{H}-2=4.5 \mathrm{~Hz}, 1 \mathrm{H}, \mathrm{H}-3 \mathrm{~b}\right)$, $2.83\left(\mathrm{~s}, 1 \mathrm{H}, \mathrm{OH}-\mathrm{H}^{-} 5^{\prime}\right), 2.81\left(\mathrm{~s}, 1 \mathrm{H}, \mathrm{O} \underline{\mathrm{H}}-\mathrm{H}-2^{\prime}\right), 2.36\left(\mathrm{t}, J_{\mathrm{H \alpha}}, \mathrm{H \beta}=7.7 \mathrm{~Hz}, 2 \mathrm{H}\right.$, $\left.\mathrm{RC}_{2} \underline{\underline{\alpha}} \underline{\alpha} \mathrm{COOCH}_{2}-6^{\prime}\right), 2.32\left(\mathrm{~m}, 4 \mathrm{H}, J_{\mathrm{H} \alpha}, \mathrm{H \beta}=7.9 \mathrm{~Hz}, \mathrm{RC}_{2} \underline{{ }_{2}} \underline{\alpha C O O C H}{ }_{2}-1\right.$ and 3), 1.62 (m, 
$6 \mathrm{H}, \mathrm{H} \beta), 1.26\left(\mathrm{~m}, 84 \mathrm{H}, \mathrm{CH}_{2}\right), 0.89\left(\mathrm{t}, J_{\mathrm{CH} 3}, \mathrm{CH} 2=7.0 \mathrm{~Hz}, 9 \mathrm{H}, \mathrm{CH}_{3}\right){ }^{13} \mathrm{C} \mathrm{NMR}(126 \mathrm{MHz}$, $\left.\mathrm{CD}_{3} \mathrm{OD} / \mathrm{CDCl}_{3}, 1 / 2, \mathrm{v} / \mathrm{v}\right) \delta 174.6\left(\mathrm{C}(\mathrm{O}) 6^{\prime}\right), 173.4$ and $173.1(\mathrm{C}(\mathrm{O})), 108.7\left(\mathrm{C}-1^{\prime}\right), 87.5$ (C-4'), 78.2 (C-2'), 78.6 (C-3’), 70.3 (C-5'), 69.6 (C-2), 66.5 (C-6’), 65.8 (C-3), 62.1 (C-1), $34.2\left(\mathrm{RCH}_{2} \alpha \mathrm{COOCH}_{2}-6^{\prime}\right), 34.12$ and $34.08\left(\mathrm{RCH}_{2} \alpha \mathrm{COOCH}_{2}-1\right.$ and 3), 29.5 and $31.9\left(\mathrm{CH}_{2}\right), 24.8\left(\mathrm{CH}_{2} \beta\right), 22.7\left(\mathrm{CH}_{3}\right)$; HRMS(ESI) $m / z$ calcd. for $\left[\mathrm{C}_{81} \mathrm{H}_{162} \mathrm{NaO}_{11} \mathrm{Si}_{3}\right]^{+}$: 1075.8723, found: 1075.8730 .

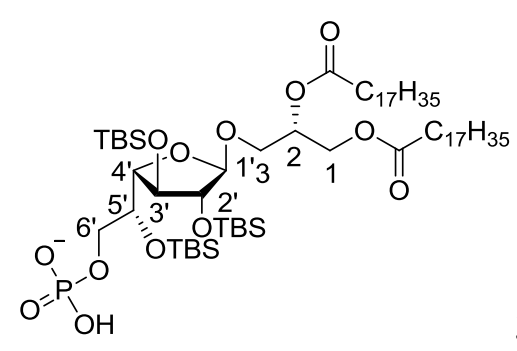

\section{3-O-(6-O-[Phosphate]- $\beta$-D-galactofuranosyl)-1,2-di- $O$ -}

stearoyl-sn-glycerol (sn-3-23a). To a solution of $s n-3-7 \mathbf{a}(16.1 \mathrm{mg}, 0.014 \mathrm{mmol})$ in dry pyridine $(1 \mathrm{~mL})$ was added $\mathrm{POCl}_{3}(3.7 \mathrm{mg}, 0.028 \mathrm{mmol})$. After $1 \mathrm{~h}, \mathrm{H}_{2} \mathrm{O} / \mathrm{TEA}(1 / 1, \mathrm{v} / \mathrm{v}$, $2 \mathrm{~mL}$ ) was added and the reaction stirred for $15 \mathrm{~min}$. The reaction mixture was then diluted with EtOAc, washed with $\mathrm{NaHCO}_{3}, \mathrm{HCl}(0.1 \mathrm{M})$ and brine, dried $\left(\mathrm{MgSO}_{4}\right)$, filtered and concentrated. Following purification by silica gel flash column chromatography (DCM/MeOH, 24/1, v/v), pure $s n$-3-23a was obtained as a colourless oil (16.9 mg, $0.014 \mathrm{mmol}$ quant. $),\left(\mathrm{R}_{f}=0.57, \mathrm{MeOH} / \mathrm{DCM}, 1 / 9, \mathrm{v} / \mathrm{v}\right) ;[\alpha]_{\mathrm{D}}{ }^{22}=-16.0(c$ $=0.5, \mathrm{CHCl}_{3}$ ); IR (film) 3540, 2923, 2853, 1742, 1464, 1361, 1251, 1114, 1046, 837, 777, $\mathrm{cm}^{-1} .{ }^{1} \mathrm{H}$ NMR (500 MHz, $\left.\mathrm{CDCl}_{3}\right) \delta 5.19$ (m, 1H, H-2), $4.80\left(\mathrm{~s}, 1 \mathrm{H}, \mathrm{H}-1^{\prime}\right), 4.35$

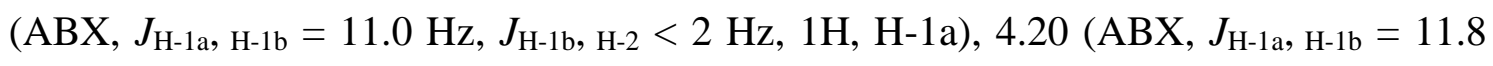
$\left.\mathrm{Hz}, J_{\mathrm{H}-1 \mathrm{~b}, \mathrm{H}-2}=6.8 \mathrm{~Hz}, 1 \mathrm{H}, \mathrm{H}-1 \mathrm{~b}\right), 4.14$ (bs, 1H, H-3'), 3.98 (m, 2H, H-2' and H-5'), 4.00 (m, 1H, H-6’a), 3.93 (m, 1H, H-6’ b), 3.90, (m, 1H, H-4'), $3.80\left(\mathrm{ABX}, J_{\mathrm{H}-3 \mathrm{a}, \mathrm{H}-2}=\right.$ $\left.3.6 \mathrm{~Hz}, J_{\mathrm{H}-3 \mathrm{a}}, \mathrm{H}-3 \mathrm{~b}=10.4 \mathrm{~Hz}, 1 \mathrm{H}, \mathrm{H}-3 \mathrm{a}\right), 3.54\left(\mathrm{ABX}, J_{\mathrm{H}-3 \mathrm{~b}}, \mathrm{H}-2=5.2 \mathrm{~Hz}, J_{\mathrm{H}-3 \mathrm{~b}}, \mathrm{H}-3 \mathrm{a}=10.4\right.$ $\mathrm{Hz}, 1 \mathrm{H}, \mathrm{H}-3 \mathrm{~b}$ ), 2.29 (m, 4H, $\left.\mathrm{CH}_{2} \alpha\right), 1.60$ (m, 4H, $\mathrm{CH}_{2} \beta$ ), 1.17-1.48 (m, 56H, $\mathrm{CH}_{2}$ ), 1.02-0.78 (m, 51H, $\left(\mathrm{CH}_{3}\right)_{3} \mathrm{C}$ and $\left.\mathrm{CH}_{3}\right), 0.21-0.00\left(\mathrm{~m}, 24 \mathrm{H}, \mathrm{Si}\left(\mathrm{CH}_{3}\right)_{2}\right) ;{ }^{13} \mathrm{C}$ NMR $(126$ $\left.\mathrm{MHz}, \mathrm{CDCl}_{3}\right) \delta 173.8$ and $173.3(\mathrm{C}(\mathrm{O}) 1$ and $\mathrm{C}(\mathrm{O}) 2), 108.2\left(\mathrm{C}-1^{\prime}\right), 84.3\left(\mathrm{C}-4^{\prime}\right), 84.1(\mathrm{C}-$

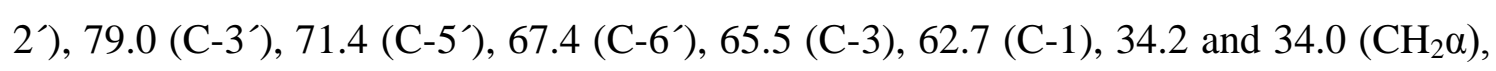
$31.8\left(\mathrm{CH}_{2} \mathrm{CH}_{2} \mathrm{CH}_{3}\right), 30.5-28.7\left(\mathrm{CH}_{2}\right), 26.0\left(\mathrm{C}\left(\underline{\mathrm{CH}}_{3}\right)_{3}\right), 25.8(\mathrm{x} 2)\left(\mathrm{C}\left(\underline{\mathrm{CH}}_{3}\right)_{3}\right), 24.93$ and $24.90\left(\mathrm{CH}_{2} \beta\right), 22.7\left(\underline{\mathrm{CH}_{2}} \mathrm{CH}_{3}\right), 18.3\left(\mathrm{Si} \underline{\mathrm{C}}\left(\mathrm{CH}_{3}\right)_{3}\right), 17.9\left(\mathrm{SiC}\left(\mathrm{CH}_{3}\right)_{3}\right), 17.8\left(\mathrm{SiC}\left(\mathrm{CH}_{3}\right)_{3}\right)$, $14.0\left(\mathrm{CH}_{3}\right),-3.73\left(\mathrm{Si}\left(\mathrm{CH}_{3}\right)_{2}\right),-4.17\left(\mathrm{Si}\left(\mathrm{CH}_{3}\right)_{2}\right),-4.37\left(\mathrm{Si}\left(\mathrm{CH}_{3}\right)_{2}\right),-4.43\left(\mathrm{Si}\left(\mathrm{CH}_{3}\right)_{2}\right),-4.7$ 
$\left(\mathrm{Si}\left(\mathrm{CH}_{3}\right)_{2}\right),-4.8\left(\mathrm{Si}\left(\mathrm{CH}_{3}\right)_{2}\right) ;{ }^{31} \mathrm{P}$ NMR $\left(121 \mathrm{MHz}, \mathrm{CDCl}_{3}\right) \delta 0.59 \mathrm{HRMS}(\mathrm{ESI}) \mathrm{m} / z$ calcd. for $\left[\mathrm{C}_{63} \mathrm{H}_{128} \mathrm{O}_{13} \mathrm{PSi}_{3}\right]^{-}:$1207.8406, found: 1207.8384 .

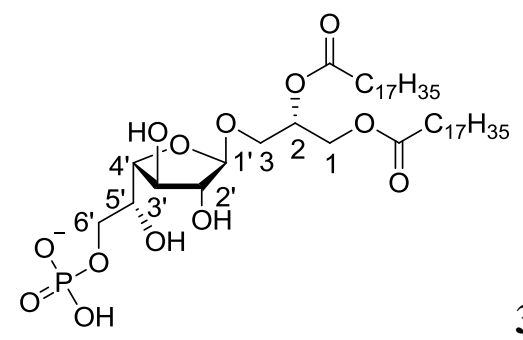

3-O-(6-O-[Phosphate]- $\beta$-D-galactofuranosyl)-1,2-di- $O$ -

stearoyl-sn-glycerol (sn-3-4a). To a solution of $s n-3-23 a(16.3 \mathrm{mg}, 0.013 \mathrm{mmol})$ in $\mathrm{DCM} / \mathrm{MeOH}(2 / 1, \mathrm{v} / \mathrm{v})$ was added Dowex $\mathrm{H}^{+}$and the reaction mixture stirred at $\mathrm{RT}$ for two weeks. The solution was then filtered and concentrated in vacuo to give sn-3-4a as a colourless oil $(9.3 \mathrm{mg}, 0.011 \mathrm{mmol}, 80 \%),\left(\mathrm{R}_{f}=0.15, \mathrm{DCM} / \mathrm{MeOH}, 8 / 2, \mathrm{v} / \mathrm{v}\right) ;[\alpha]_{\mathrm{D}}{ }^{23}=$ -18.0 ( $c=0.5$, Pyridine); IR (film) 3413, 2917, 2849, 1740, 1463, 1176, $1034 \mathrm{~cm}^{-1} .{ }^{1} \mathrm{H}$ NMR (500 MHz, $\mathrm{CDCl}_{3} / \mathrm{CD}_{3} \mathrm{OD} / \mathrm{D}_{2} \mathrm{O}, 70 / 40 / 6$, v/v/v) $\delta 5.20(\mathrm{~m}, 1 \mathrm{H}, \mathrm{H}-2), 4.92(\mathrm{~s}, 1 \mathrm{H}$, H-1'), 4.35 (m, 1H, H-1a), 4.17 (m, 1H, H-1b), 4.08 (m, 2H, H-6’a and b), 4.03 (m, 1H, H-3'), 4.01 (m, 1H, H-4'), 3.97 (m, 1H, H-2'), 3.77 (m, 1H, H-3a), 3.60 (m, 1H, H-3b),

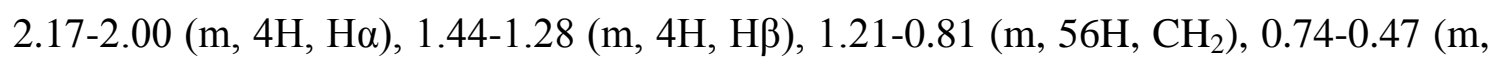
$\left.6 \mathrm{H}, \mathrm{CH}_{3}\right) ;{ }^{13} \mathrm{C} \mathrm{NMR}\left(126 \mathrm{MHz}, \mathrm{CDCl}_{3}\right) \delta 174.1$ and $173.7(\mathrm{C}(\mathrm{O})), 108.6\left(\mathrm{C}-1^{\prime}\right), 84.2$ (C-4'), 80.8 (C-2'), 77.6 (C-3'), 70.2 (C-2), 69.5 (C-5’), 66.4 (C-6’), 65.6 (C-3); 34.10$34.09\left(\mathrm{CH}_{2} \alpha\right), 31.9\left(\mathrm{C}_{2}{ }_{2} \mathrm{CH}_{2} \mathrm{CH}_{3}\right), 29.9-29.0\left(\mathrm{CH}_{2}\right), 24.95\left(\mathrm{CH}_{2} \beta\right), 22.6\left(\mathrm{CH}_{2}\right), 13.9$ $\left(\mathrm{CH}_{3}\right),{ }^{31} \mathrm{P} \quad \mathrm{NMR} \quad\left(121 \mathrm{MHz}, \mathrm{CDCl}_{3}\right) \quad \delta \quad 4.09 \quad \mathrm{HRMS}(\mathrm{ESI}) \mathrm{m} / \mathrm{z}$ calcd. for $\left[\mathrm{C}_{63} \mathrm{H}_{128} \mathrm{O}_{13} \mathrm{PSi}_{3}\right]$ : 865.5812, found: 865.5809. ${ }^{31} \mathrm{P} \mathrm{NMR}\left(121 \mathrm{MHz}, \mathrm{CDCl}_{3}\right) \delta 4.09$ HRMS(ESI) $m / z$ calcd. for $\left[\mathrm{C}_{63} \mathrm{H}_{128} \mathrm{O}_{13} \mathrm{PSi}_{3}\right]^{-}: 865.5812$, found: 865.5809.

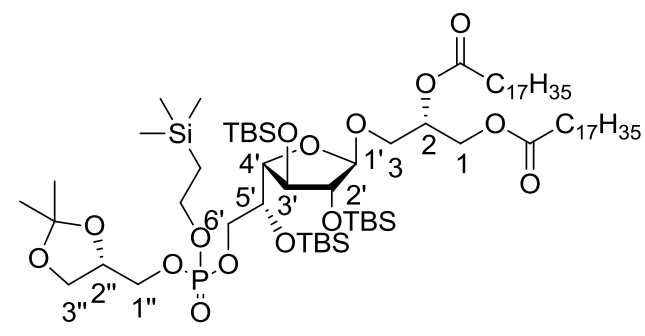

3-O-(6-O-[2,3-O-isopropylidene-sn-

glyceroloxy(trimethylsilylethyloxy)phosphoryl]-2, 3, 5-Tetra-O-tertbutyldimethylsilyl- $\beta$-D galactofuranosyl)-1,2-di- $O$-stearyl-sn-glycerol (sn-3-30a). To a solution of $s n-3-7 \mathbf{a}(56.7 \mathrm{mg}, 0.050 \mathrm{mmol})$ in THF $(0.5 \mathrm{~mL})$ was added a solution of 
29 (28.0 mg, $0.11 \mathrm{mmol})$ in anhydrous $\mathrm{MeCN}(0.5 \mathrm{~mL})$ followed by $1 H$-tetrazole $(10.6$ $\mathrm{mg}, 0.15 \mathrm{mmol})$. The reaction mixture was stirred for $4 \mathrm{~h}$ and then $t-\mathrm{BuOOH}(6 \mathrm{M}$ in octane, $42 \mu \mathrm{L}$ ) was added and the solution stirred for another $15 \mathrm{~min}$. The reaction mixture was then diluted with EtOAc, washed with $\mathrm{NaHCO}_{3}$ (sat. aq.) and brine, dried $\left(\mathrm{MgSO}_{4}\right)$, filtered and concentrated. Purification of the residue by silica gel flash column chromatography (EtOAc/PE, 3/7, v/v) gave sn-3-30a as colourless oil (14.6 mg, $0.013 \mathrm{mmol}, 27 \%),\left(\mathrm{R}_{f}=0.65, \mathrm{EtOAc} / \mathrm{PE}, 3 / 7, \mathrm{v} / \mathrm{v}\right) ;[\alpha]_{\mathrm{D}}{ }^{21}=-12.0\left(c=0.1, \mathrm{CHCl}_{3}\right) ; \mathrm{IR}$ (film) 2925, 2855, 1743, 1464, 1371, 1251, 1114, 1005, 838, 778, 668, $636 \mathrm{~cm}^{1}$; ${ }^{1} \mathrm{H}$ $\operatorname{NMR}\left(500 \mathrm{MHz}, \mathrm{CDCl}_{3}\right) \delta 5.20(\mathrm{~m}, 1 \mathrm{H}, \mathrm{H}-2), 4.80\left(\mathrm{~d}, J_{\mathrm{H}-1^{\prime}}, \mathrm{H}-2^{\prime}=1.5 \mathrm{~Hz}, 1 \mathrm{H}, \mathrm{H}^{\prime} 1^{\prime}\right)$, $4.33\left(\mathrm{ABX}, J_{\mathrm{H}-1 \mathrm{a}}, \mathrm{H}-1 \mathrm{~b}=11.9 \mathrm{~Hz}, J_{\mathrm{H}-1 \mathrm{a}}, \mathrm{H}-2=3.8 \mathrm{~Hz}, 1 \mathrm{H}, \mathrm{H}-1 \mathrm{a}\right), 4.30$ (m, 1H, H-2”) 4.18 $\left(\mathrm{ABX}, J_{\mathrm{H}-1 \mathrm{~b}, \mathrm{H}-1 \mathrm{a}}=11.9 \mathrm{~Hz}, J_{\mathrm{H}-1 \mathrm{~b}, \mathrm{H}-2}=6.2 \mathrm{~Hz}, 1 \mathrm{H}, \mathrm{H}-1 \mathrm{~b}\right), 4.17\left(\mathrm{~m}, 2 \mathrm{H}, \mathrm{POC}_{2} \mathrm{CH}_{2} \mathrm{Si}\right)$, 4.12 (m, 1H, H-6’a), 4.07 (m, 1H, H-3“a), 4.06 (m, 1H, H-3’), 4.04 (m, 1H, H-1“a), 4.00 (m, 1H, H-1’b), 3.99 (m, 1H, H-2'), 3.98 (m, 1H, H-5’), 3.94 (m, 1H, H-6’b), 3.87 $\left(\mathrm{m}, 1 \mathrm{H}, \mathrm{H}-4^{\prime}\right), 3.83$ (m, 1H, H-3’’b), 3.79 (ABX, $J_{\mathrm{H}-3 \mathrm{a}}, \mathrm{H}-3 \mathrm{~b}=10.9 \mathrm{~Hz}, J_{\mathrm{H}-3 \mathrm{a}}, \mathrm{H}-2=4.9 \mathrm{~Hz}$, $1 \mathrm{H}, \mathrm{H}-3 \mathrm{a}), 3.55\left(\mathrm{ABX}, J_{\mathrm{H}-3 \mathrm{~b}}, \mathrm{H}-3 \mathrm{a}=10.9 \mathrm{~Hz}, J_{\mathrm{H}-3 \mathrm{~b}}, \mathrm{H}-2=5.2 \mathrm{~Hz}, 1 \mathrm{H}, \mathrm{H}-3 \mathrm{~b}\right), 2.29$ (t, $J_{\mathrm{H \alpha}}$, $\left.\mathrm{H} \beta=7.7 \mathrm{~Hz}, 4 \mathrm{H}, \mathrm{CH}_{2} \alpha\right), 1.61\left(\mathrm{~m}, 4 \mathrm{H}, \mathrm{CH}_{2} \beta\right), 1.41\left(\mathrm{~s}, 3 \mathrm{H}, \mathrm{C}\left(\mathrm{CH}_{3} \mathrm{a}\right)\right), 1.36(\mathrm{~s}, 3 \mathrm{H}$, $\left.\mathrm{C}\left(\mathrm{CH}_{3} \mathrm{~b}\right)\right), 1.26\left(\mathrm{~m}, 56 \mathrm{H}, \mathrm{CH}_{2}\right), 1.12\left(\mathrm{~m}, 2 \mathrm{H}, \mathrm{CH}_{2} \mathrm{Si}\right), 0.93-0.66\left(\mathrm{~m}, 24 \mathrm{H}, \mathrm{CH}_{3}\right), 0.14-$ $0.03\left(\mathrm{~m}, 36 \mathrm{H}, \mathrm{CH}_{3} \mathrm{Si}\right) ;{ }^{13} \mathrm{C} \mathrm{NMR}\left(125 \mathrm{MHz}, \mathrm{CDCl}_{3}\right) \delta 173.3(\mathrm{C}(\mathrm{O}) 1), 172.9(\mathrm{C}(\mathrm{O}) 2)$, 109.8 (C), $108.3\left(\mathrm{C}-1^{\prime}\right), 85.29$ and 85.26 (C-4'), 83.8 (C-2'), 79.1 (C-3'), 74.07 and 74.02 (C-2’'), 71.62 and 71.55 (C-5'), 69.9 (C-2), 68.95 and 68.89 (C-6'), 67.31 and 67.26 (C-3”), 66.67 and $66.62\left(\mathrm{POCH}_{2} \mathrm{CH}_{2} \mathrm{Si}\right), 66.26$ and 66.27 (C-1”), 65.5 (C-3), $62.6(\mathrm{C}-1), 34.3$ and $34.1\left(\mathrm{CH}_{2} \alpha\right), 31.9\left(\mathrm{CH}_{2} \mathrm{CH}_{2} \mathrm{CH}_{3}\right), 29.8-29.6\left(\mathrm{CH}_{2}\right), 26.7(i-\operatorname{Pr}(\mathrm{a}))$, $25.8\left(\mathrm{C}\left(\underline{\mathrm{CH}}_{3}\right)_{3}\right), 25.2(i-\operatorname{Pr}(\mathrm{b})), 24.92$ and $24.90\left(\mathrm{CH}_{2} \beta\right), 22.7\left(\mathrm{CH}_{2}\right), 19.67$ and 19.63 $\left(\mathrm{CH}_{2} \mathrm{Si}\right), 18.3\left(\mathrm{SiC}\left(\mathrm{CH}_{3}\right)_{3}\right), 17.9\left(\mathrm{SiC}\left(\mathrm{CH}_{3}\right)_{3}\right), 17.8\left(\mathrm{SiC}\left(\mathrm{CH}_{3}\right)_{3}\right), 14.1\left(\mathrm{CH}_{3}\right),-1.52$ $\left(\mathrm{CH}_{2} \mathrm{Si}\right),-4.02\left(\mathrm{SiCH}_{3}\right),-4.08\left(\mathrm{SiCH}_{3}\right),-4.43\left(\mathrm{SiCH}_{3}\right),-4.48\left(\mathrm{SiCH}_{3}\right),-4.50\left(\mathrm{SiCH}_{3}\right)$, $4.83\left(\mathrm{SiCH}_{3}\right) ;{ }^{31} \mathrm{P}$ NMR $\left(121 \mathrm{MHz}, \mathrm{CDCl}_{3}\right) \delta-1.39 ; \mathrm{HRMS}(\mathrm{ESI}) \mathrm{m} / \mathrm{z}$ calcd. for $\left[\mathrm{C}_{74} \mathrm{H}_{151} \mathrm{NaO}_{15} \mathrm{PSi}_{4}\right]^{+}:$1445.9760, obsd.: 1445.9765 .

\section{Synthesis of 1-O-(Benzyloxybis[diisopropylamino]phosphine)-2,3-isopropylidene-}

sn-glycerol (8). Was performed according to the procedure of Hamamoto et al. ${ }^{279}$ with the following modifications. 
<smiles>CC(C)N(C(C)C)P(Cl)N(C(C)C)C(C)C</smiles>

Bis(diisopropylamino)chlorophosphine (25). To a 3 neck $1 \mathrm{~L}$ flask equipped with a mechanical stirrer and a condenser was added anhydrous diisopropylamine $(80 \mathrm{~mL}, 0.56 \mathrm{~mol})$ and hexanes $(60 \mathrm{~mL})$. The solution was cooled to 0 ${ }^{\circ} \mathrm{C}$ and $\mathrm{PCl}_{3}(12.2 \mathrm{~mL}, 0.14 \mathrm{~mol})$ was cannulated dropwise into the mixture. As gauged by ${ }^{31} \mathrm{P}$ NMR [coaxial tube, $\left(\mathrm{CD}_{3}\right)_{2} \mathrm{CO}$ ] $\mathrm{PCl}_{3}(219 \mathrm{ppm})$ reacted almost immediately with diisopropyamine to form the diisopropylamino-dichlorophosphine (166 ppm) and the bis(diisopropylamino)chlorophosphine (128.8 ppm) ${ }^{31} \mathrm{P}$ signal slowly increased over time. The reaction was found to be complete after $12 \mathrm{~h}$ at which point it was filtered and washed with hexanes. Concentration and crystallisation from hexanes then afforded the aminochlorophosphine $(25.0 \mathrm{~g}, 9.4 \mathrm{mmol}, 67 \%),\left(\mathrm{R}_{f}=0.4\right.$, EtOAc/PE, 8/2, v/v, cospotted using TEA); ${ }^{1} \mathrm{H}$ NMR $\left(500 \mathrm{MHz}, \mathrm{CDCl}_{3}\right) \delta 3.66(\mathrm{~m}, 4 \mathrm{H}, \mathrm{CH}), 1.3-1.1(\mathrm{~m}, 24 \mathrm{H}$, $\left.\mathrm{CH}_{3}\right) ;{ }^{13} \mathrm{C}$ NMR $\left(125 \mathrm{MHz}, \mathrm{CDCl}_{3}\right) \delta 47.6-47.2(\mathrm{CH}), 24.9-22.1\left(\mathrm{CH}_{3}\right) ;{ }^{31} \mathrm{P}$ NMR $(121$ $\left.\mathrm{MHz}, \mathrm{CDCl}_{3}\right) \delta 128.8$.<smiles>CC(C)N(C(C)C)C(C)I</smiles>

Benzyloxybis(diisopropylamino)phosphine (32). To a solution of bisisopropylaminochlorophosphine $(4.0 \mathrm{~g}, 15 \mathrm{mmol})$ in dry $\mathrm{Et}_{2} \mathrm{O}(80 \mathrm{~mL})$ was added benzyl alcohol $(1.65 \mathrm{~mL}, 16 \mathrm{mmol})$ and triethylamine $(2.37 \mathrm{~mL}, 17 \mathrm{mmol})$. The reaction was monitored using ${ }^{31} \mathrm{P}$ NMR and upon complete consumtion of the starting material was filtered and concentrated in vacuo. Silica gel flash column chromatography (TEA/PE, 0.5/99.5, v/v) then gave the benzyloxybis(diisopropylamino)phosphine (4.91 g, $14.5 \mathrm{mmol}, 97 \%) ;\left(\mathrm{R}_{f}=0.88\right.$, EtOAc/PE, 3/7, v/v, co-spotted with TEA); IR (film) 2996, 1496, 1454, 1362, 1184, 1156, 1126, 1079, 1023, 974, 953, 759, 730, 694, 642 $\mathrm{cm}^{-1} ;{ }^{1} \mathrm{H} \mathrm{NMR}\left(500 \mathrm{MHz}, \mathrm{CDCl}_{3}\right) \delta$ 7.47-7.20 (m, 5H, CHBn), $4.66\left(\mathrm{~s}, 2 \mathrm{H}, \mathrm{CH}_{2}\right), 3.58$ (s, 4H, CH), $1.19\left(\mathrm{~m}, 24 \mathrm{H}, \mathrm{CH}_{3}\right) ;{ }^{13} \mathrm{C} \mathrm{NMR}\left(125 \mathrm{MHz}, \mathrm{CDCl}_{3}\right) \delta$ 128.5-126.5 (CHBn), 140.5 and $140.4(\mathrm{CBn}), 66.2$ and $66.0\left(\mathrm{CH}_{2}\right), 44.5$ and $44.4(\mathrm{CH}), 24.8-23.6\left(\mathrm{CH}_{3}\right) ;{ }^{31} \mathrm{P}$ NMR (121 MHz, $\left.\mathrm{CDCl}_{3}\right) \delta$ 123.4; $\mathrm{HRMS}(\mathrm{ESI}) \mathrm{m} / z$ calcd. for $\left[\mathrm{C}_{19} \mathrm{H}_{36} \mathrm{~N}_{2} \mathrm{OP}\right]^{+}$: 339.2560, Found: 339.2557. 


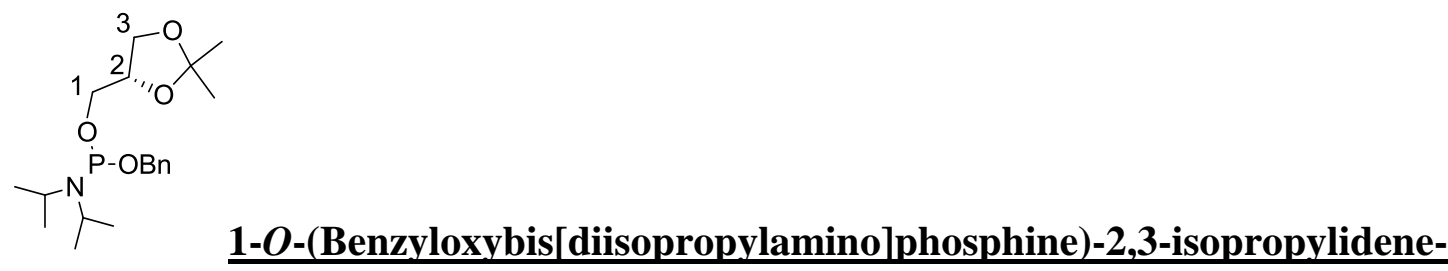

sn-glycerol (8). A solution of (-)-10 (264 mg, $2.0 \mathrm{mmol})$ in anhydrous MeCN (20 mL) was stirred with $3 \AA$ mol. sieves for $30 \mathrm{~min}$. and then cooled to $0{ }^{\circ} \mathrm{C}$. To this was added diisopropylamine $(400 \mathrm{mg}, 4.0 \mathrm{mmol})$, tetrazole $(280 \mathrm{mg}, 4.0 \mathrm{mmol})$ and benzyloxybis(diisopropylamino)phosphine $(1.0 \mathrm{~g}, 3.0 \mathrm{mmol})$ and the reaction monitored using ${ }^{31} \mathrm{P}$ NMR. After $3 \mathrm{~h}$, the reaction mixture was diluted with EtOAc, washed with brine and dried $\left(\mathrm{MgSO}_{4}\right)$. Silica gel flash column chromatography (TEA/PE, 0.5/99.5, $\mathrm{v} / \mathrm{v})$ then gave $8(686.7 \mathrm{mg}, 1.86 \mathrm{mmol}, 93 \%)$ as a colourless liquid, $\left(\mathrm{R}_{f}=0.81\right.$, EtOAc/PE, 3/7, v/v); $[\alpha]_{\mathrm{D}}{ }^{16}=-7.5\left(c=0.1, \mathrm{CHCl}_{3}\right)$; IR (film) 2967, 1455, 1370, 1201, 1184, 1156, 1126, 1053, 1022, 974, 846, 770, 732, $695 \mathrm{~cm}^{-1} ;{ }^{1} \mathrm{H}$ NMR $(500 \mathrm{MHz}$, $\left.\mathrm{CDCl}_{3}\right) \delta$ 7.44-7.21 (m, 5H, CHBn), 4.76 and $4.79\left(\mathrm{~m}, 1 \mathrm{H}, \mathrm{CH}_{2} \mathrm{Bnb}\right), 4.26(\mathrm{~m}, 1 \mathrm{H}, \mathrm{H}-$ 2), 4.06 (m, 1H, H-3a), 3.87 (m, 1H, H-3b), 3.80 (m, 1H, H-1a), 3.71 (m, 1H, H-1a), $3.64(\mathrm{~m}, 2 \mathrm{H}, \mathrm{CH}), 1.43$ and $1.37\left(\mathrm{~m}, 6 \mathrm{H}, \mathrm{C}\left(\mathrm{CH}_{3}\right)_{2}\right), 1.20\left(\mathrm{~m}, 12 \mathrm{H}, \mathrm{CH}_{3}\right) ;{ }^{13} \mathrm{C} \mathrm{NMR}(125$ $\left.\mathrm{MHz}, \mathrm{CDCl}_{3}\right) \delta 139.3(\underline{\mathrm{CCHBn}}), 128.8-126.4(\mathrm{CHBn}), 109.3$ and $109.2\left(\mathrm{C}_{\left.\left(\mathrm{CH}_{3}\right)_{2}\right), 75.2}\right.$ and $75.10(\mathrm{~m}, \mathrm{C}-2), 67.21$ and $67.16(\mathrm{C}-3), 65.45$ and $65.32\left(\mathrm{~m}, \mathrm{CH}_{2} \mathrm{Bn}\right), 64.1\left(\mathrm{~d}, J_{\mathrm{H}-1}\right.$, $31 \mathrm{P}=3.44 \mathrm{~Hz}, \mathrm{C}-1)$ and $64.0(\mathrm{~d}, 2.0 \mathrm{~Hz}, \mathrm{C}-1), 43.0(\mathrm{CH}), 26.82,26.8125 .47$ and 25.44 $\left(\mathrm{C}\left(\mathrm{CH}_{3}\right)_{2}\right), 24.8-24.5\left(\mathrm{CH}_{3}\right) ;{ }^{31} \mathrm{P}$ NMR $\left(121 \mathrm{MHz}, \mathrm{CDCl}_{3}\right) \delta 148.1 ; \mathrm{HRMS}(\mathrm{ESI}) \mathrm{m} / \mathrm{z}$ calcd. for $\left[\mathrm{C}_{19} \mathrm{H}_{33} \mathrm{NO}_{4} \mathrm{P}\right]^{+}: 370.2142$, Found: 370.2149 .

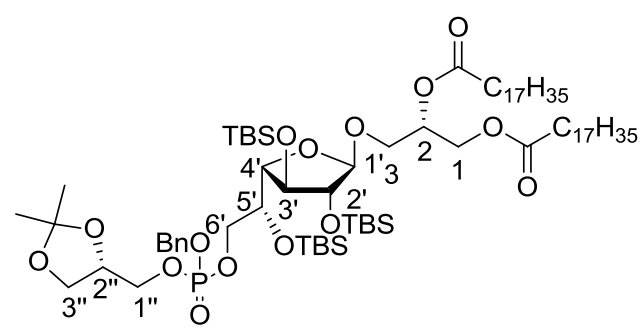

3-O-(6-O-[2,3-O-Isopropylidene-sn-glycerol-1-

$O$-phospho- $O$-benzyl]-2,3,5-Tetra- $O$-tert-butyldimethylsilyl- $\beta$-D-galactofuranosyl)-

1,2-di- $\boldsymbol{O}$-stearoyl-sn-glycerol (sn-3-33a). To a solution of $s n-3-7 \mathbf{a}(58.9 \mathrm{mg}, 0.052$ $\mathrm{mmol})$ in THF $(0.5 \mathrm{~mL})$ was added a solution of $8(20.5 \mathrm{mg}, 0.078 \mathrm{mmol})$ in anhydrous $\operatorname{MeCN}(0.5 \mathrm{~mL})$ followed by tetrazole $(10.9 \mathrm{mg}, 0.16 \mathrm{mmol})$. The reaction mixture was stirred for $4 \mathrm{~h}$ and then $t$ - $\mathrm{BuOOH}(6 \mathrm{M}$ in octane, $70 \mu \mathrm{L})$ was added and the solution stirred for another $15 \mathrm{~min}$. The reaction mixture was then diluted with EtOAc, washed 
with $\mathrm{NaHCO}_{3}$ (sat. aq.) and brine, dried $\left(\mathrm{MgSO}_{4}\right)$, filtered and concentrated. Purification of the residue by silica gel flash column chromatography (EtOAc/PE, 2/25, v/v) gave 33a as a colourless oil $(52.5 \mathrm{mg}, 0.037 \mathrm{mmol}, 72 \%),\left(\mathrm{R}_{f}=0.53, \mathrm{EtOAc} / \mathrm{PE}\right.$, $3 / 7, \mathrm{v} / \mathrm{v}) ;[\alpha]_{\mathrm{D}}{ }^{18}=-6.0\left(c=0.1, \mathrm{CHCl}_{3}\right) ; \mathrm{IR}($ film) $2925,2854,1743,1463,1253,1113$, 1016, 837, $777 \mathrm{~cm}^{1}$; ${ }^{1} \mathrm{H}$ NMR (600 MHz, $\mathrm{CDCl}_{3}$ ) $\delta$ 7.40-7.30 (m, 5H, CHBn), 5.19 (m, $1 \mathrm{H}, \mathrm{H}-2), 5.09\left(\mathrm{~m}, 2 \mathrm{H}, \mathrm{CH}_{2} \mathrm{Bn}\right), 4.79\left(\mathrm{~d}, J_{\mathrm{H}-1^{\prime}, \mathrm{H}-2^{\prime}}=1.3 \mathrm{~Hz}, 1 \mathrm{H}, \mathrm{H}-1^{\prime}\right), 4.34(\mathrm{~m}, 1 \mathrm{H}, \mathrm{H}-$ 1a), 4.23 (m, 1H, H-2”'), 4.18 (m, 1H, H-1b), 4.12 (m, 1H, H-6’a), 4.05 (dd, $J_{\mathrm{H}-33^{\prime}, \mathrm{H}^{\prime}}=$

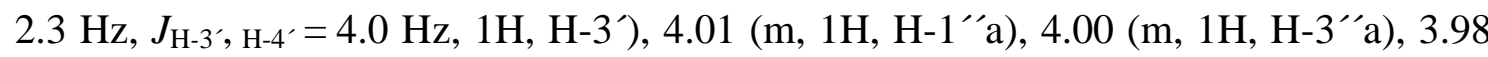
(m, 1H, H-2`), 3.97 (m, 1H, H-5') 3.96 (m, 1H, H-6’b), 3.93 (m, 1H, H-1’ b), 3.87 (m, 1H, H-4’), 3.77 (m, 1H, H-3a), 3.74 (m, 1H, H-3``b), 3.53 (ABX, $J_{\mathrm{H}-3 \mathrm{a}}, \mathrm{H}-3 \mathrm{~b}=10.9 \mathrm{~Hz}$, $\left.J_{\mathrm{H}-3 \mathrm{a}}, \mathrm{H}-2=5.2 \mathrm{~Hz}, 1 \mathrm{H}, \mathrm{H}-3 \mathrm{~b}\right), 2.28\left(\mathrm{t}, J_{\mathrm{H} \alpha}, \mathrm{H} \beta=7.3 \mathrm{~Hz}, 4 \mathrm{H}, \mathrm{CH}_{2} \alpha\right), 1.60\left(\mathrm{~m}, 4 \mathrm{H}, \mathrm{CH}_{2} \beta\right)$, 1.39 (s, 3H, C( $\left.\left.\mathrm{CH}_{3} \mathrm{a}\right)\right), 1.33$ (s, 3H, $\left.\mathrm{C}\left(\mathrm{CH}_{3} \mathrm{~b}\right)\right), 1.26$ (m, 56H, $\left.\mathrm{CH}_{2}\right), 0.95-0.80(\mathrm{~m}, 33 \mathrm{H}$, $\left.\mathrm{CH}_{3}\right), 0.15-0.00\left(\mathrm{~m}, 18 \mathrm{H}, \mathrm{CH}_{3} \mathrm{Si}\right) ;{ }^{13} \mathrm{C} \mathrm{NMR}\left(151 \mathrm{MHz}, \mathrm{CDCl}_{3}\right) \delta 173.3(\mathrm{C}(\mathrm{O}) 1), 173.0$ (C(O)2), 109.8 (C), 108.3 (C-1'), 85.2 (C-4’), 83.8 (C-2’), 79.0 (C-3’), 73.95 and 73.88 (C-2”), 71.47 and 71.41 (C-5'), 69.9 (C-2), 69.42 and $69.38\left(\mathrm{CH}_{2} \mathrm{Bn}\right), 69.0$ (C-6'), 67.46 and $67.41\left(\mathrm{~d}, J_{\mathrm{H}-3{ }^{\prime \prime}, \mathrm{P}}=6.0 \mathrm{~Hz}, \mathrm{C}-1^{\prime \prime}\right), 66.2\left(\mathrm{C}-3^{\prime \prime}\right), 65.5(\mathrm{C}-3), 62.6(\mathrm{C}-1), 34.3$ and $\left.34.1\left(\mathrm{CH}_{2} \alpha\right), 31.9\left(\underline{\mathrm{CH}_{2}} \mathrm{CH}_{2} \mathrm{CH}_{3}\right), 30.0-29.0\left(\mathrm{CH}_{2}\right), 26.7(i-\operatorname{Pr}(\mathrm{a})), 26.0\left(\mathrm{C}_{(\mathrm{CH}}\right)_{3}\right)$, $25.75\left(\mathrm{C}\left(\underline{\mathrm{CH}}_{3}\right)_{3}\right), 25.70\left(\mathrm{C}\left(\underline{\mathrm{CH}}_{3}\right)_{3}\right), 25.2(i-\operatorname{Pr}(\mathrm{b})), 24.92$ and $24.90\left(\mathrm{CH}_{2} \beta\right), 22.7$

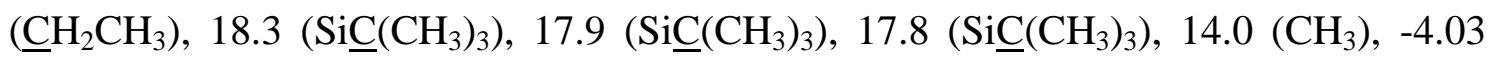
$\left(\mathrm{SiCH}_{3}\right),-4.08\left(\mathrm{SiCH}_{3}\right),-4.44\left(\mathrm{SiCH}_{3}\right),-4.50\left(\mathrm{SiCH}_{3}\right),-4.52\left(\mathrm{SiCH}_{3}\right),-4.8\left(\mathrm{SiCH}_{3}\right) ;{ }^{31} \mathrm{P}$ NMR (121 $\left.\mathrm{MHz}, \mathrm{CDCl}_{3}\right) \quad \delta \quad-0.95$ and -0.97 ; HRMS(ESI) $\mathrm{m} / \mathrm{z}$ calcd. for $\left[\mathrm{C}_{76} \mathrm{H}_{145} \mathrm{NaO}_{15} \mathrm{PSi}_{3}\right]^{+}:$1435.9521, found: 1435.9523 .

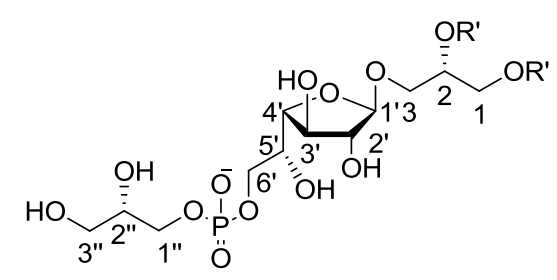

\section{3-O-(6-O-[1-O-phospho-sn-glycerol]- $\beta$-D-}

galactofuranosyl)-1,2-di- $O$-stearoyl-sn-glycerol ( $s n-3-5 a)$. To a solution of $s n$-3-33a (8.5 mg, $0.0060 \mathrm{mmol}$ ) in $\mathrm{CHCl}_{3} / \mathrm{MeOH} / \mathrm{H}_{2} \mathrm{O}(70 / 40 / 6$, v/v/v, $1 \mathrm{~mL}$ ) was added $\mathrm{Pd}(\mathrm{OH})_{2} \mathrm{C}(5 \mathrm{mg})$ and the mixture stirred under an $\mathrm{H}_{2}$ atmosphere (using a balloon) for $12 \mathrm{~h}$ at RT. The solution was then filtered through a glass fiber filter, Dowex $-\mathrm{H}^{+}(10 \%$, $0.8 \mathrm{mg}$ ) was added, and the reaction mixture stirred for two weeks at RT. The reaction mixture was then filtered and concentrated in vacuo to give pure $s n-3-5 \mathbf{a}$ as a colourless 
oil (5.2 mg, $0.0055 \mathrm{mmol}, 92 \%$ yield), $\left(\mathrm{R}_{f}=0.14, \mathrm{DCM} / \mathrm{MeOH}, 4 / 1, \mathrm{v} / \mathrm{v}\right) ;[\alpha]_{\mathrm{D}}{ }^{26}=64.0$ ( $c=0.5$, Pyridine); IR (film) 3450, 2918, 2850, 1733, 1558, 1466, 1258, 1033, 804, 771, 720, 668, $656 \mathrm{~cm}^{1} ;{ }^{1} \mathrm{H}$ NMR (600 MHz, $\left.\mathrm{CDCl}_{3} / \mathrm{CD}_{3} \mathrm{OD} / \mathrm{D}_{2} \mathrm{O}, 70 / 40 / 6, \mathrm{v} / \mathrm{v} / \mathrm{v}\right) \delta 5.21(\mathrm{~m}$, $1 \mathrm{H}, \mathrm{H}-2), 4.90$ (s, 1H, H-1'), 4.34 (m, 1H, H-1a), 4.17 (ABX, $J_{\mathrm{H}-1 \mathrm{a}}, \mathrm{H}-1 \mathrm{~b}=12.1 \mathrm{~Hz}, J_{\mathrm{H}-1 \mathrm{~b}}$, $\mathrm{H}-2=7.3 \mathrm{~Hz} 1 \mathrm{H}, \mathrm{H}-1 \mathrm{~b}), 4.01$ (m, 1H, H-3’), 3.96 (m, 1H, H-2'), 3.94 (m, 1H, H-4’), 3.92 (m, 1H, H-6’a), 3.91 (m, 1H, H-6’b) 3.90 (m, 1H, H-5’), 3.88 (m, 1H, H-3“`a), 3.86 (m, 1H, H-3”a), 3.79 (m, 1H, H-3a), 3.59 (m, 1H, H-1“a), 3.58 (m, 1H, H-3”b), $3.55\left(\mathrm{~m}, 1 \mathrm{H}, \mathrm{H}-1^{\prime} ` \mathrm{~b}\right), 3.53\left(\mathrm{ABX}, J_{\mathrm{H}-3 \mathrm{a}}, \mathrm{H}-3 \mathrm{~b}=10.9 \mathrm{~Hz}, J_{\mathrm{H}-3 \mathrm{a}}, \mathrm{H}-2=5.2 \mathrm{~Hz}, 1 \mathrm{H}, \mathrm{H}-3 \mathrm{~b}\right)$, $2.30\left(\mathrm{~m}, 4 \mathrm{H}, \mathrm{CH}_{2} \alpha\right), 1.58\left(\mathrm{~m}, 4 \mathrm{H}, \mathrm{CH}_{2} \beta\right), 1.24\left(\mathrm{~m}, 56 \mathrm{H}, \mathrm{CH}_{2}\right), 0.85\left(\mathrm{t}, J_{\mathrm{CH} 3}, \mathrm{CH} 2=7.0\right.$ $\left.\mathrm{Hz}, 6 \mathrm{H}, \mathrm{CH}_{3}\right) ;{ }^{13} \mathrm{C} \mathrm{NMR}\left(151 \mathrm{MHz}, \mathrm{CDCl}_{3} / \mathrm{CD}_{3} \mathrm{OD} / \mathrm{D}_{2} \mathrm{O}, 70 / 40 / 6, \mathrm{v} / \mathrm{v} / \mathrm{v}\right) \delta 174.2$ (C(O)1), 173.8(C(O)2), $108.2\left(\mathrm{C}-1^{\prime}\right), 84.4\left(\mathrm{C}^{\prime} 4^{\prime}\right), 81.1$ (C-2’), 77.5 (C-3’), 71.01 and 70.96 (C-2”), 70.24 (C-5’a), 70.19 (C-5’b), 70.0 (C-2), 66.82 (C-6’a), 66.79 (C-6’b), 66.26 and $66.22\left(\mathrm{C}-3^{\prime \prime}\right), 65.5(\mathrm{C}-3), 62.8(\mathrm{C}-1), 62.2\left(\mathrm{C}-1^{\prime \prime}\right), 34.2$ and $34.0\left(\mathrm{CH}_{2} \alpha\right)$, $31.8\left(\mathrm{CH}_{3} \mathrm{CH}_{2} \underline{\mathrm{CH}}_{2}\right), 24.85$ and $24.79\left(\mathrm{CH}_{2} \beta\right), 13.8\left(\mathrm{CH}_{3}\right) ;{ }^{31} \mathrm{P} \mathrm{NMR}\left(121 \mathrm{MHz}, \mathrm{CDCl}_{3}\right)$ $\delta$ 3.98; HRMS(ESI) $m / z$ calcd. for $\left[\mathrm{C}_{48} \mathrm{H}_{93} \mathrm{NaO}_{15} \mathrm{P}\right]^{+}: 963.6149$, found: 963.6144 . 


\subsection{Supplementary Data-NMR Spectra}

${ }^{1} \mathrm{H} \mathrm{NMR}, \mathrm{CDCl}_{3}, 500 \mathrm{MHz}$

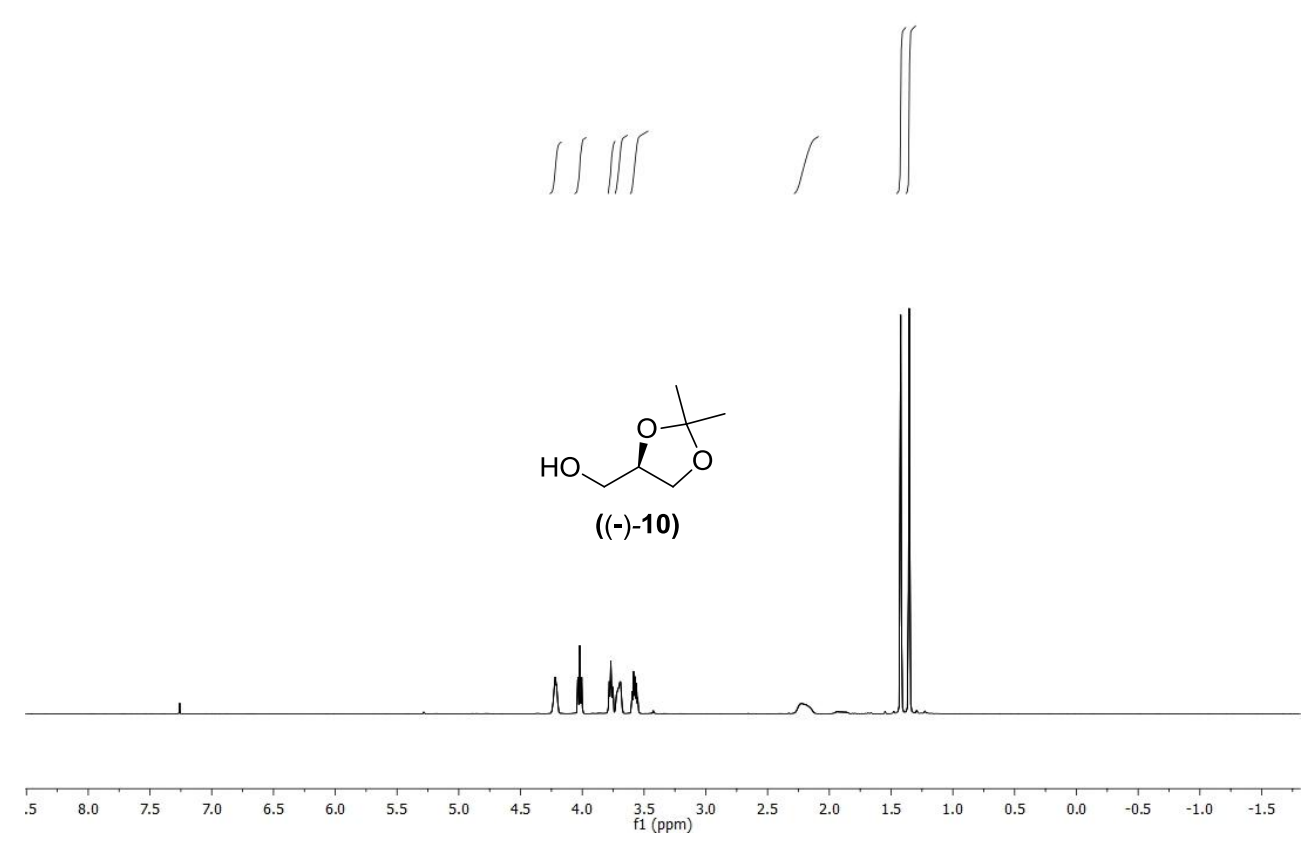

${ }^{13} \mathrm{C} \mathrm{NMR}, \mathrm{CDCl}_{3}, 125 \mathrm{MHz}$

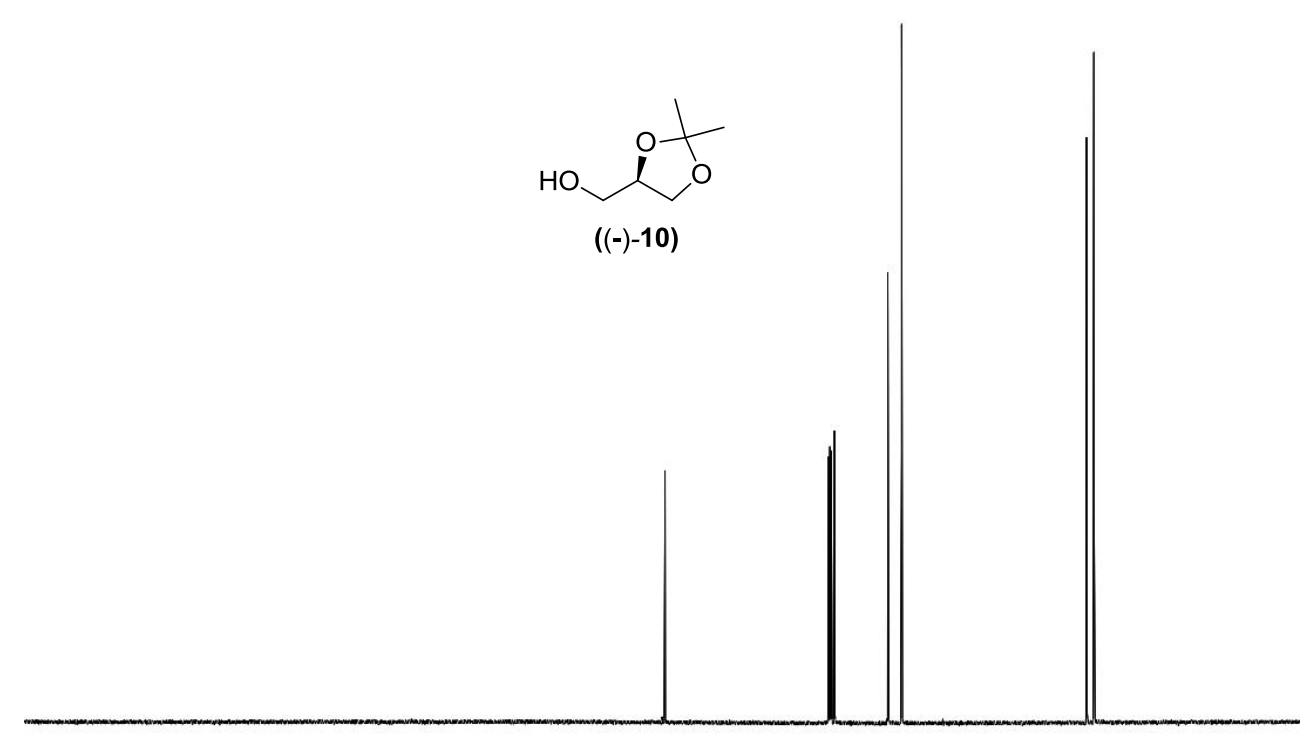

\begin{tabular}{lllllllllllllllllllllllllllll}
\hline & 1 & 1 & 220 & 210 & 200 & 190 & 180 & 170 & 160 & 150 & 140 & 130 & 120 & 110 & 100 & 90 & 80 & 70 & 60 & 50 & 40 & 30 & 20 & 10 & 0 & -10 \\
\hline
\end{tabular} 
${ }^{1} \mathrm{H}$ NMR, $\mathrm{CDCl}_{3}, 500 \mathrm{MHz}$

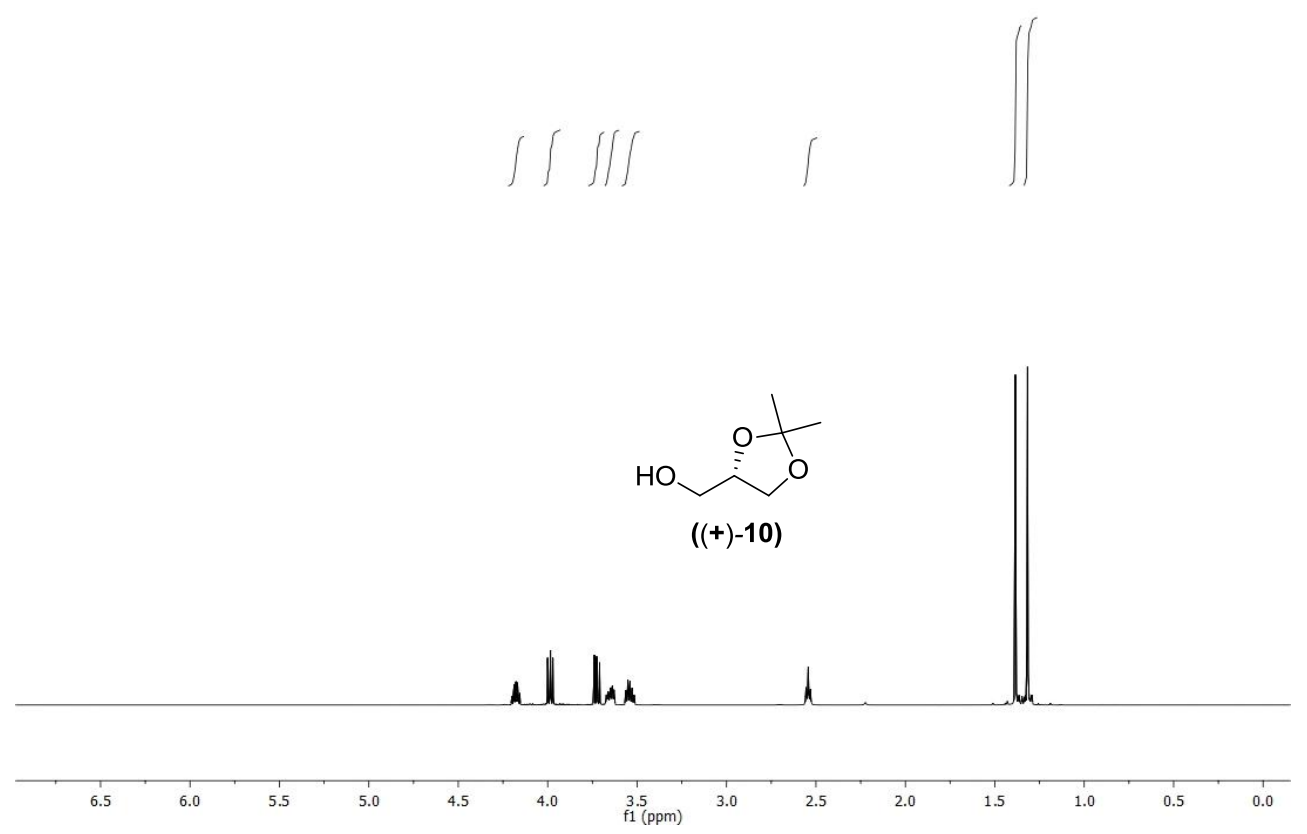

${ }^{13} \mathrm{CNMR}_{\mathrm{N}} \mathrm{CDCl}_{3}, 125 \mathrm{MHz}$

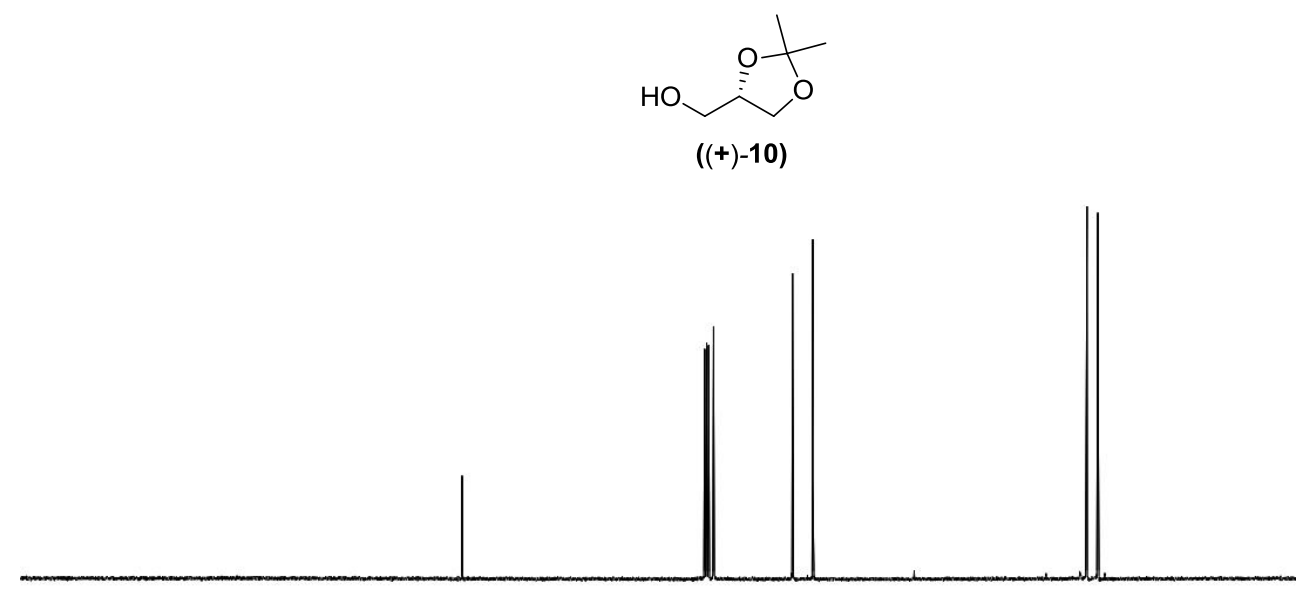


${ }^{1} \mathrm{H} \mathrm{NMR}, \mathrm{CDCl}_{3}, 500 \mathrm{MHz}$
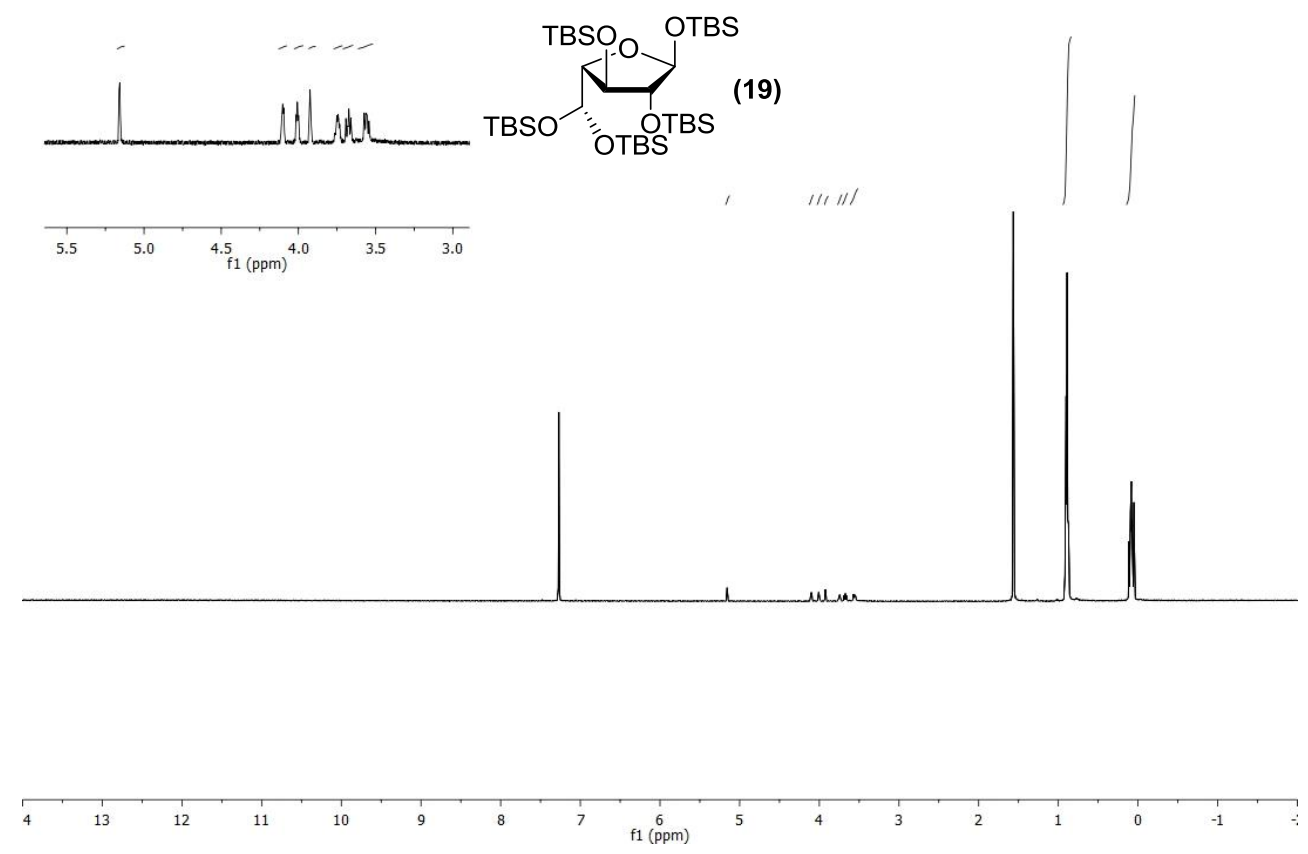

${ }^{13} \mathrm{C} \mathrm{NMR}, \mathrm{CDCl}_{3}, 125 \mathrm{MHz}$

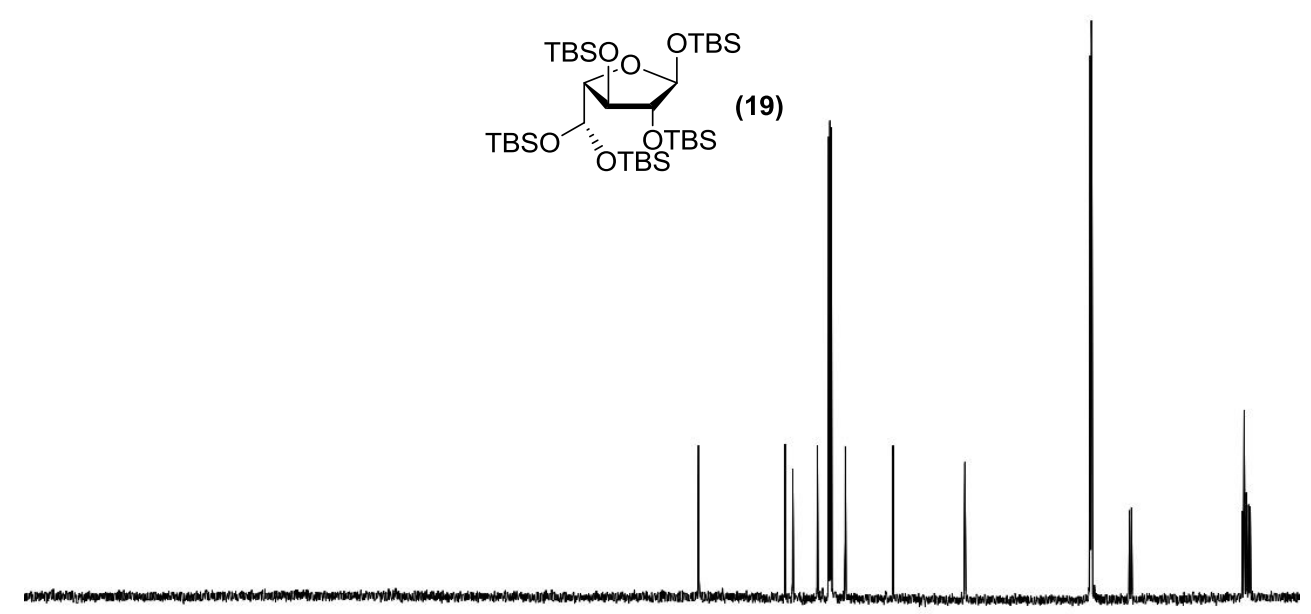

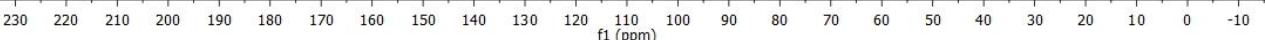


${ }^{1} \mathrm{H} \mathrm{NMR}, \mathrm{CDCl}_{3}, 500 \mathrm{MHz}$

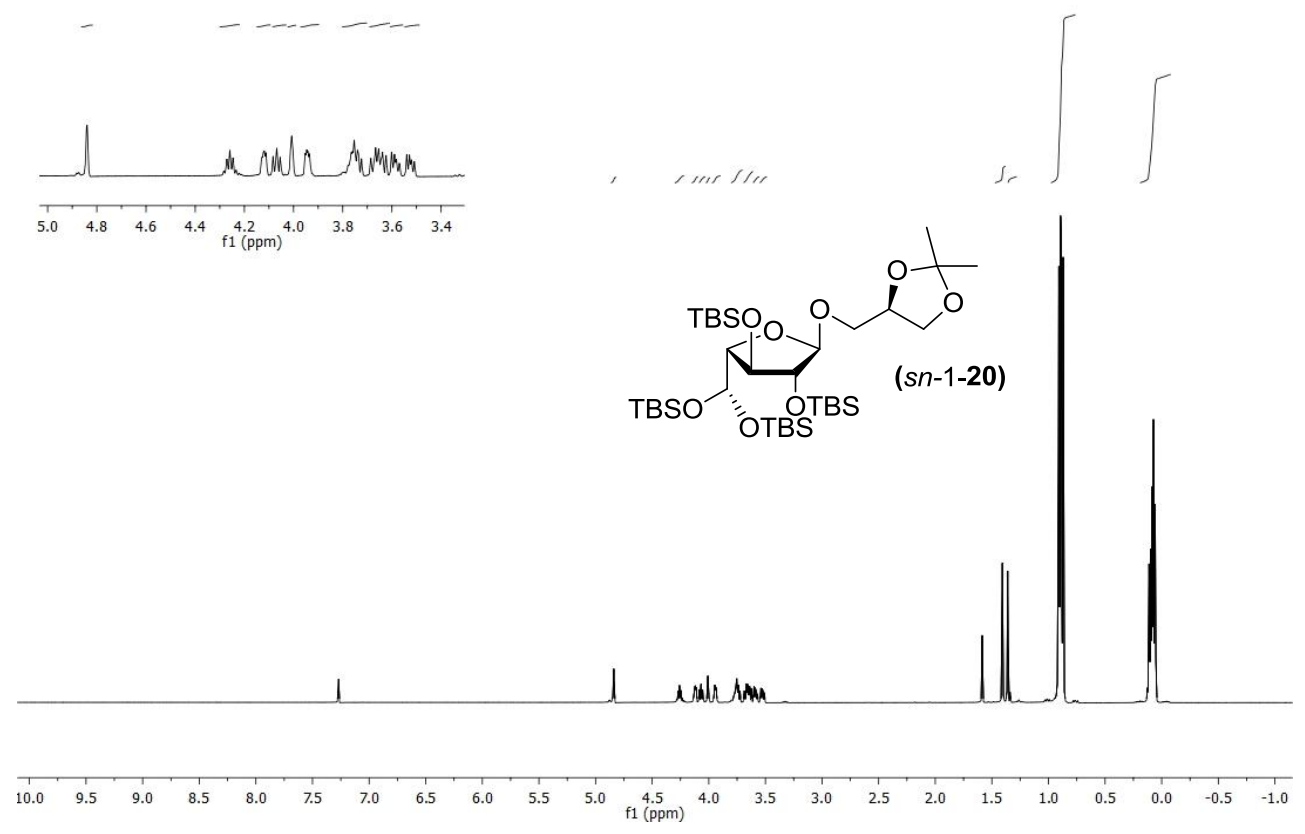

${ }^{13} \mathrm{C}$ NMR, $\mathrm{CDCl}_{3}, 125 \mathrm{MHzC}$

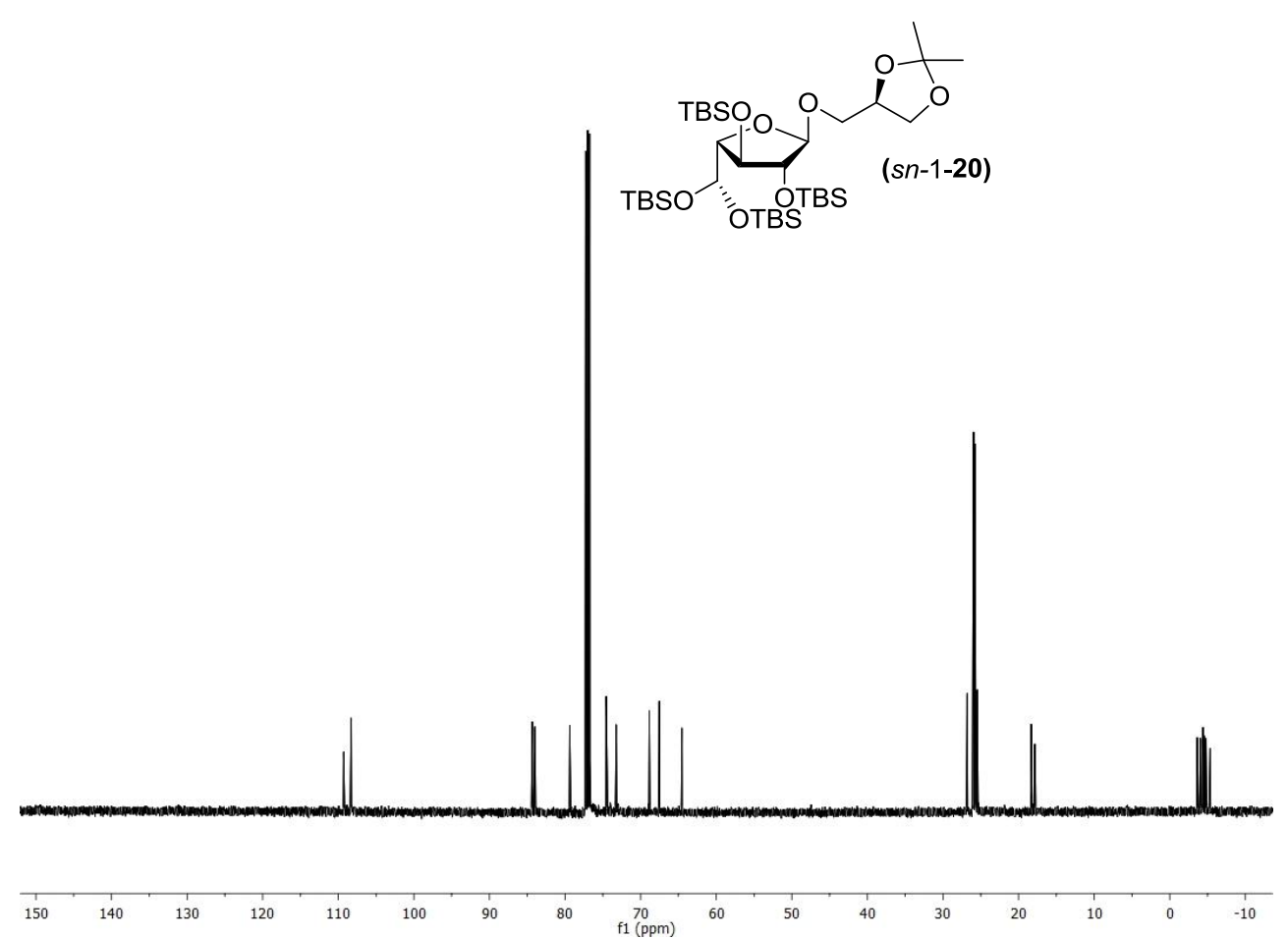


${ }^{1} \mathrm{H} \mathrm{NMR}, \mathrm{CDCl}_{3}, 500 \mathrm{MHz}$

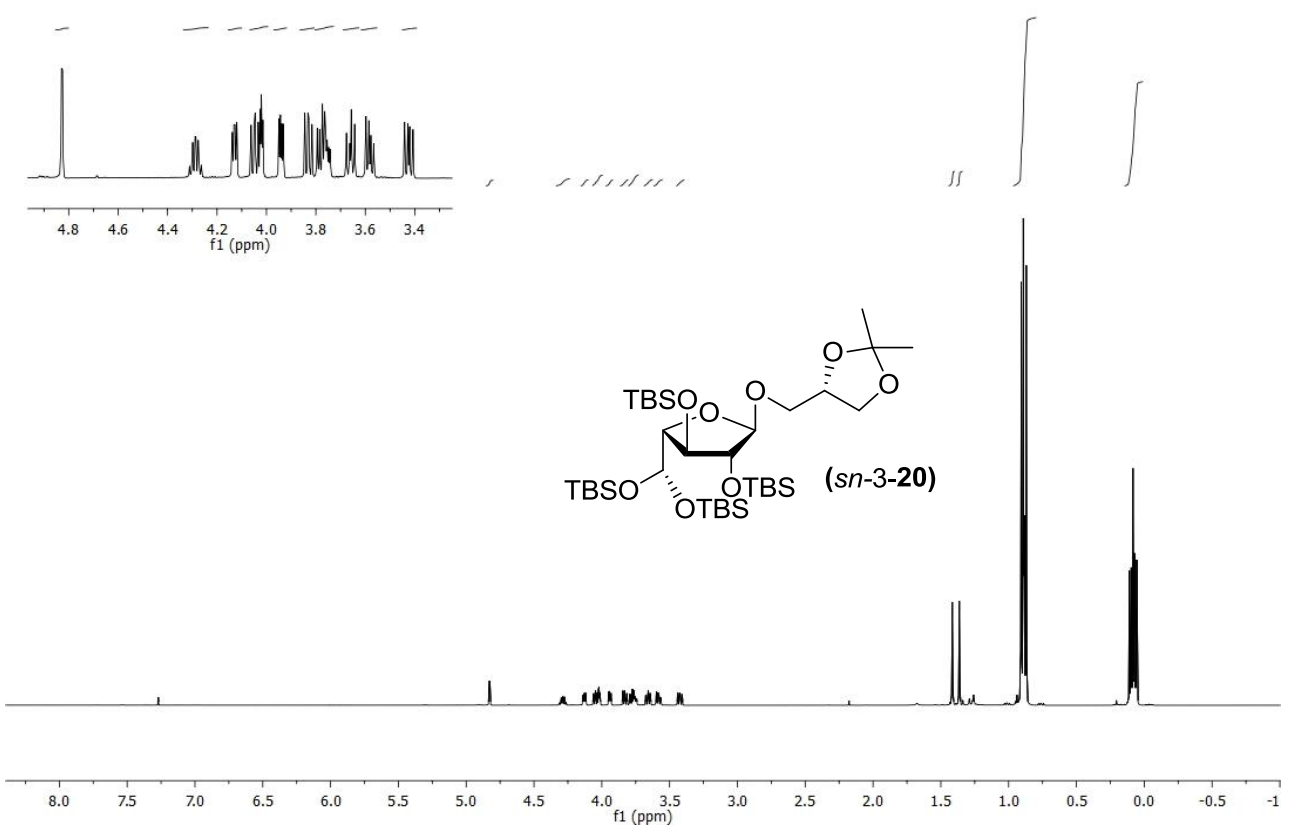

${ }^{13} \mathrm{C} \mathrm{NMR} \mathrm{CDCl}_{3}, 125 \mathrm{MHz}$

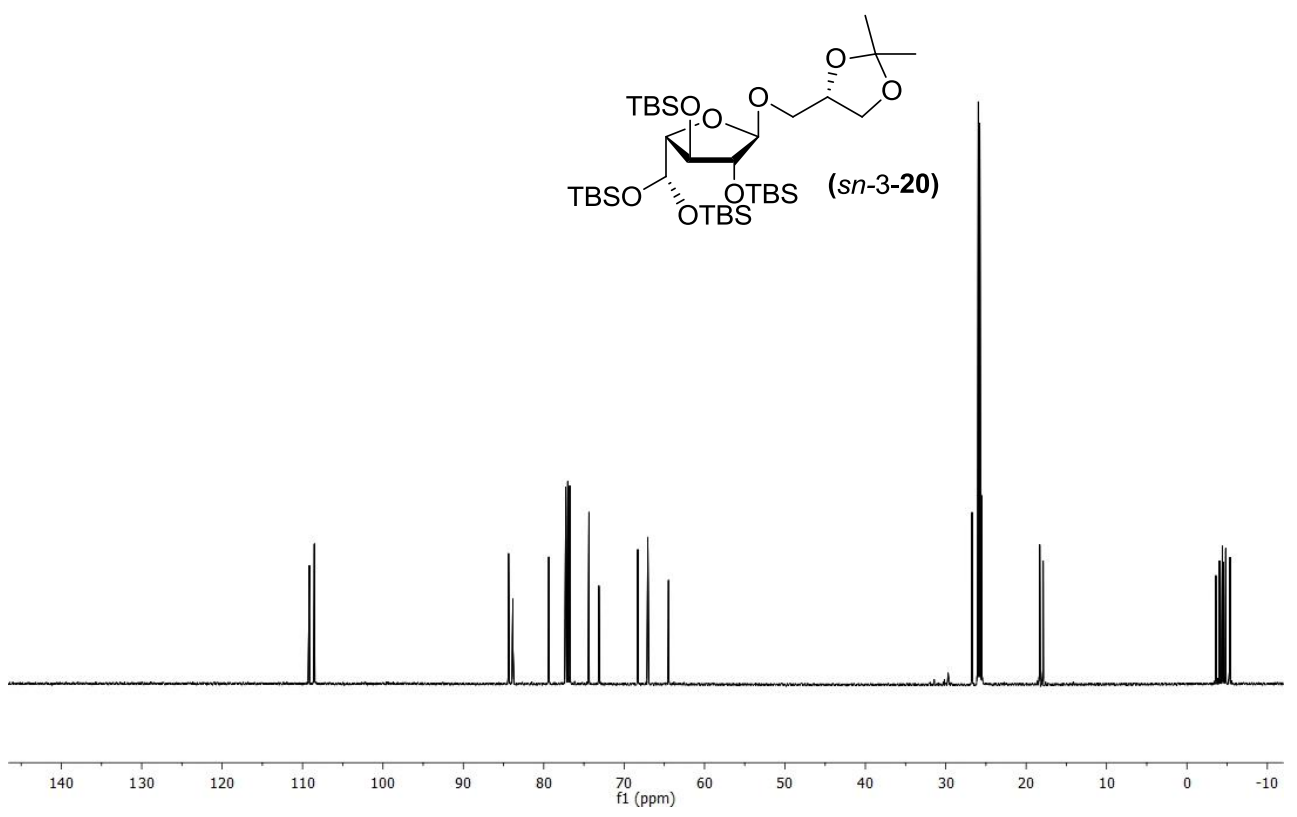


${ }^{1} \mathrm{H}$ NMR, $\mathrm{CDCl}_{3}, 500 \mathrm{MHz}$

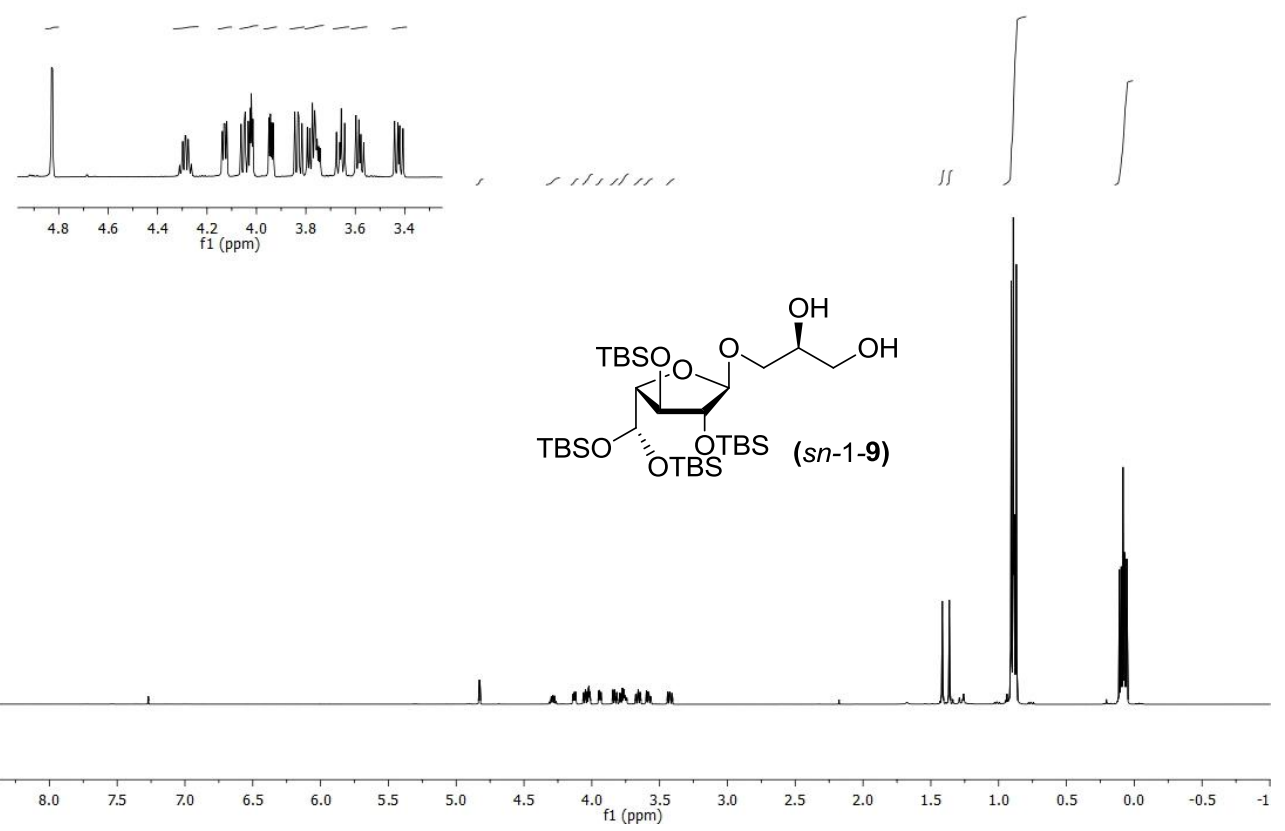

${ }^{13} \mathrm{CNMR}_{\mathrm{N}} \mathrm{CDCl}_{3}, 125 \mathrm{MHz}$
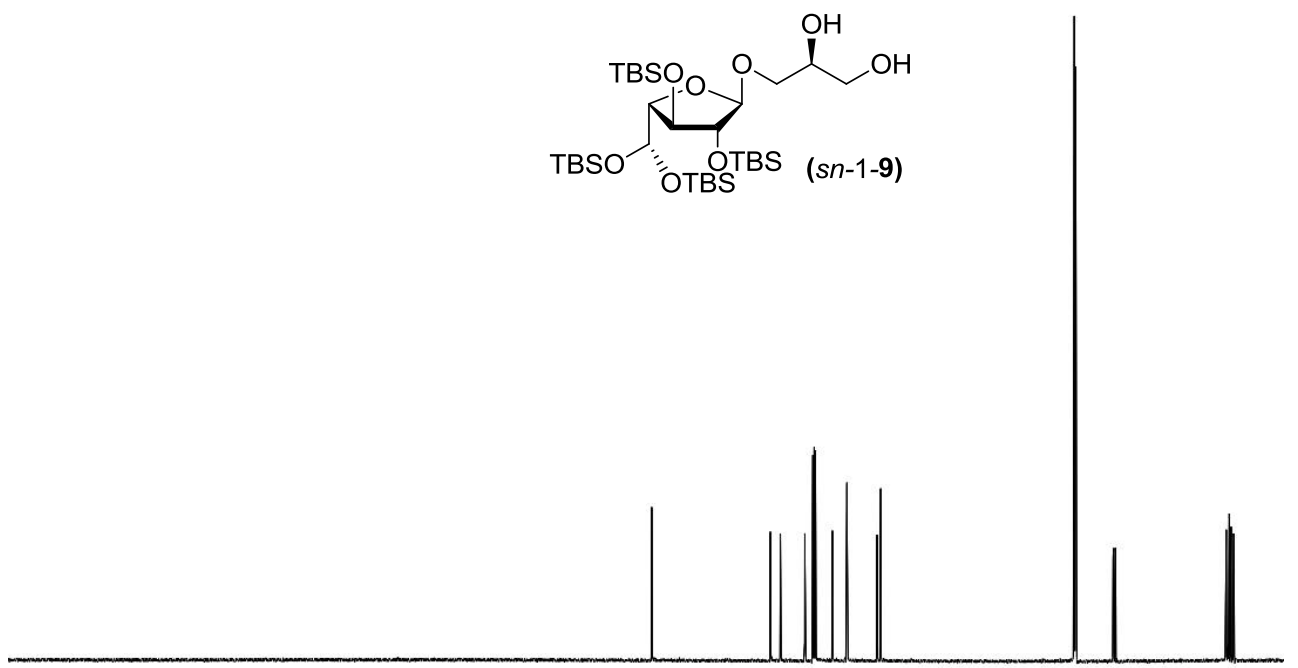

$\begin{array}{llllllllllllllllllllllllllll}230 & 220 & 210 & 200 & 190 & 180 & 170 & 160 & 150 & 140 & 130 & 120 & 110 & 100 & 90 & 80 & 70 & 60 & 50 & 40 & 30 & 20 & 10 & 0 & -10\end{array}$ 
${ }^{1} \mathrm{H} \mathrm{NMR}, \mathrm{CDCl}_{3}, 500 \mathrm{MHz}$

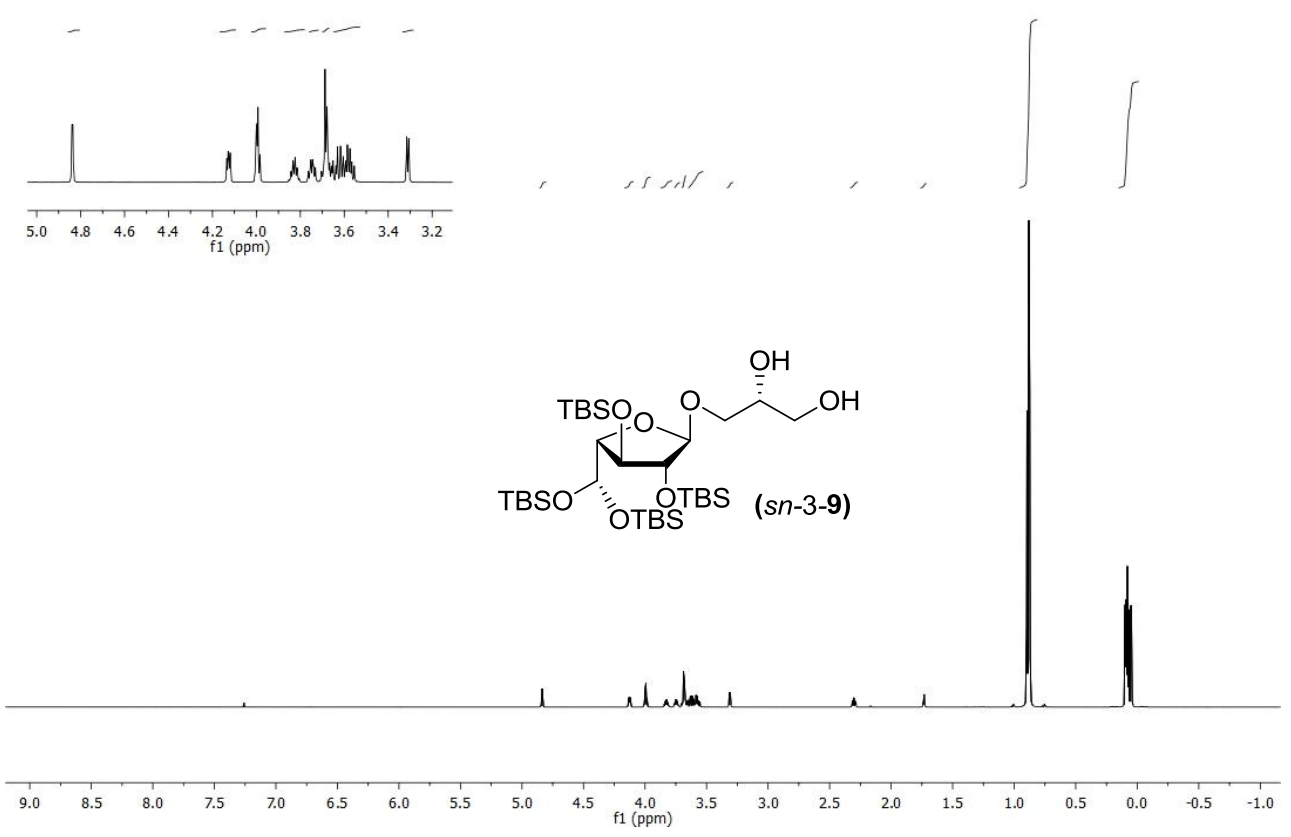

${ }^{13} \mathrm{C} \mathrm{NMR}, \mathrm{CDCl}_{3}, 125 \mathrm{MHz}$
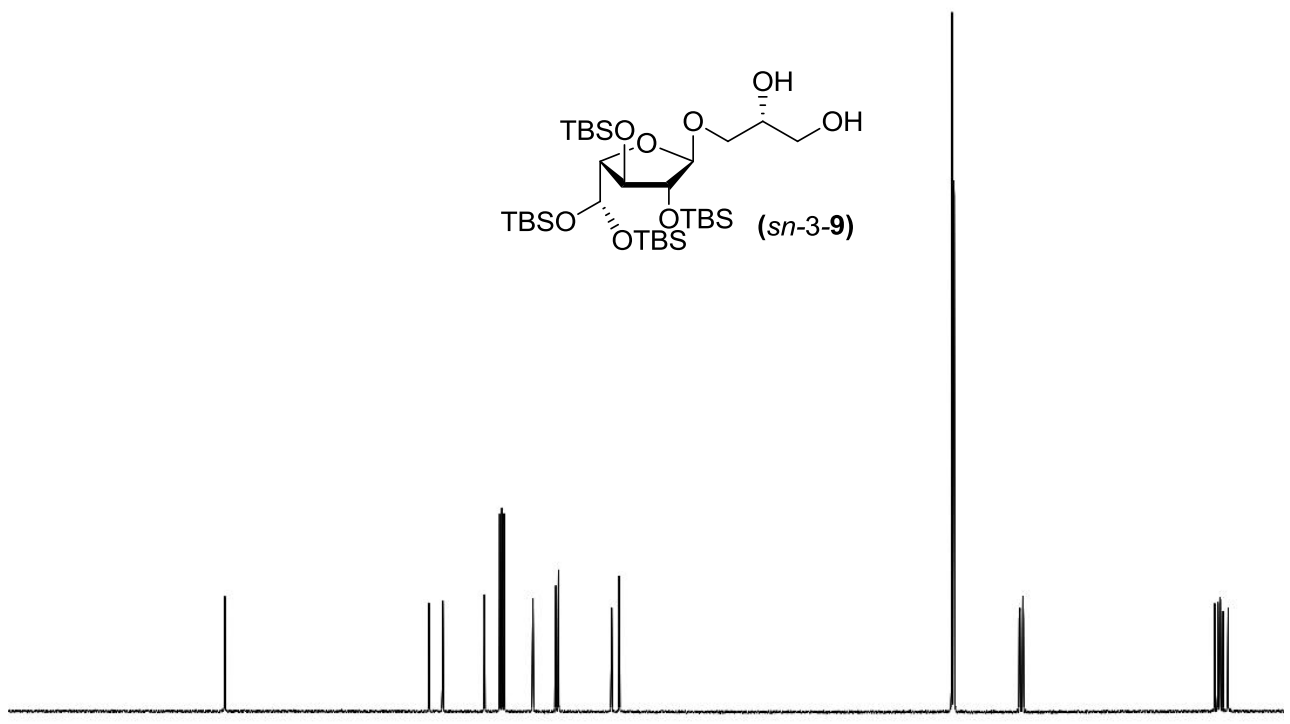

\begin{tabular}{lllllllllllllllllllllllllllllllllllll}
\hline 130 & 125 & 120 & 115 & 110 & 105 & 100 & 95 & 90 & 85 & 80 & 75 & 70 & 65 & 60 & 55 & 50 & 45 & 40 & 35 & 30 & 25 & 20 & 1 & 1 & 1 & 1 & 1 & 1 \\
\hline
\end{tabular} 
${ }^{1} \mathrm{H}$ NMR, $\mathrm{CD}_{3} \mathrm{OD}, 500 \mathrm{MHz}$
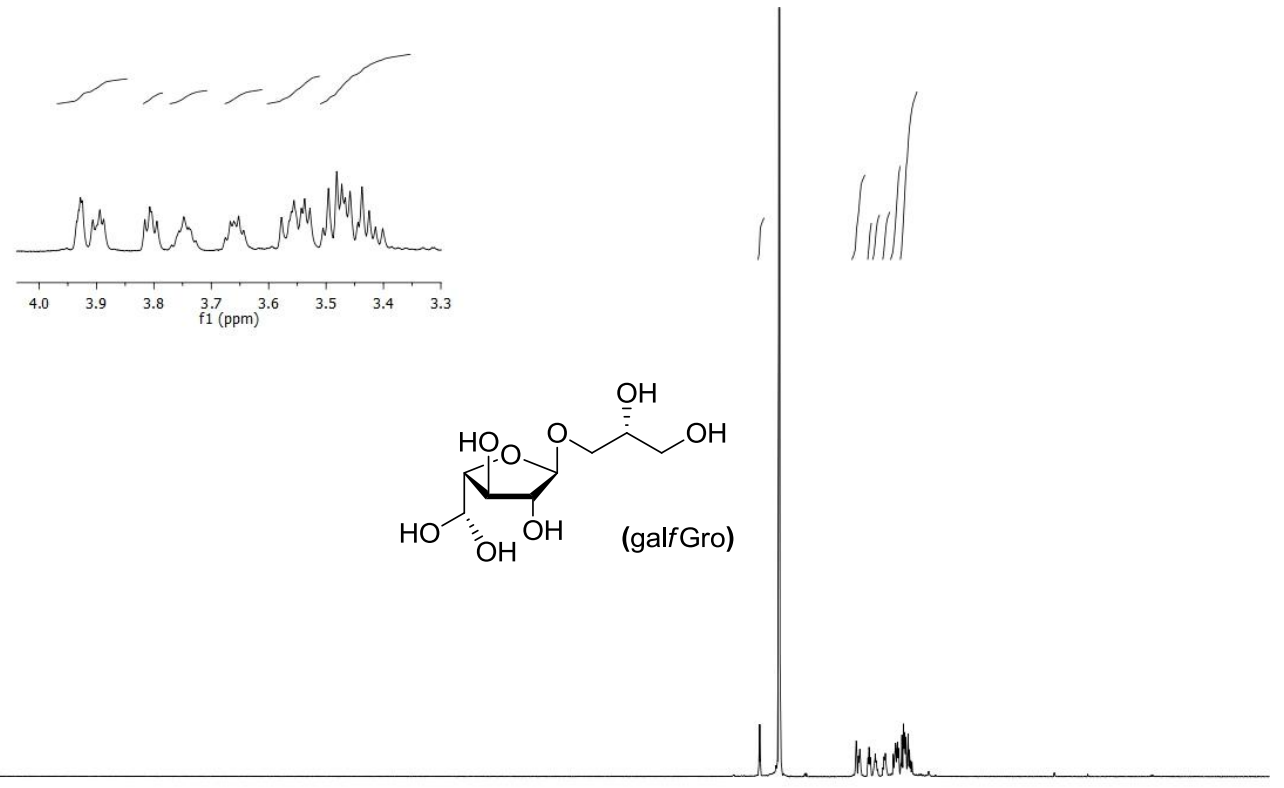

\begin{tabular}{lllllllllllllllllllllllllllllll}
\hline 2.0 & 11.5 & 11.0 & 10.5 & 10.0 & 9.5 & 9.0 & 8.5 & 8.0 & 7.5 & 7.0 & 6.5 & 6.0 & 5.5 & 5.0 & 4.5 & 4.0 & 3.5 & 3.0 & 2.5 & 2.0 & 1.5 & 1.0 & 0.5
\end{tabular}

${ }^{13} \mathrm{C}$ NMR, $\mathrm{CD}_{3} \mathrm{OD}, 125 \mathrm{MHz}$
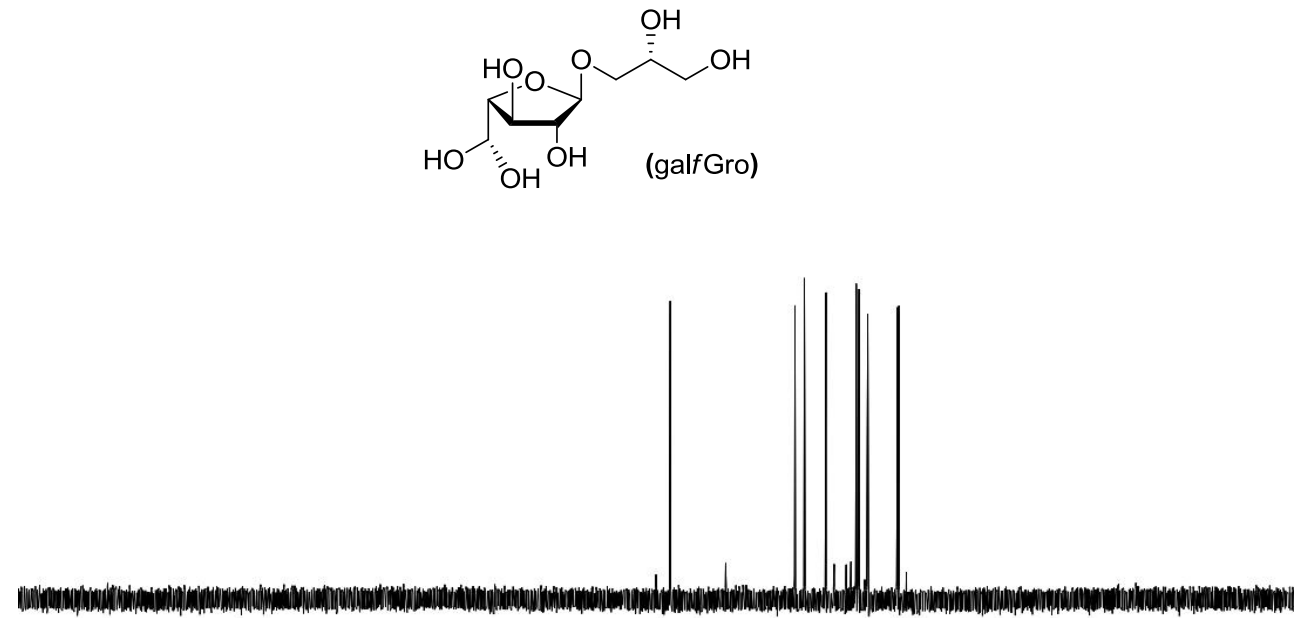

$\begin{array}{llllllllllllllllllllllllllll}230 & 220 & 210 & 200 & 190 & 180 & 170 & 160 & 150 & 140 & 130 & 120 & 110 & 100 & 90 & 80 & 70 & 60 & 50 & 40 & 30 & 20 & 10 & 0 & -10\end{array}$ 
${ }^{1} \mathrm{H} \mathrm{NMR}, \mathrm{CDCl}_{3}, 500 \mathrm{MHz}$
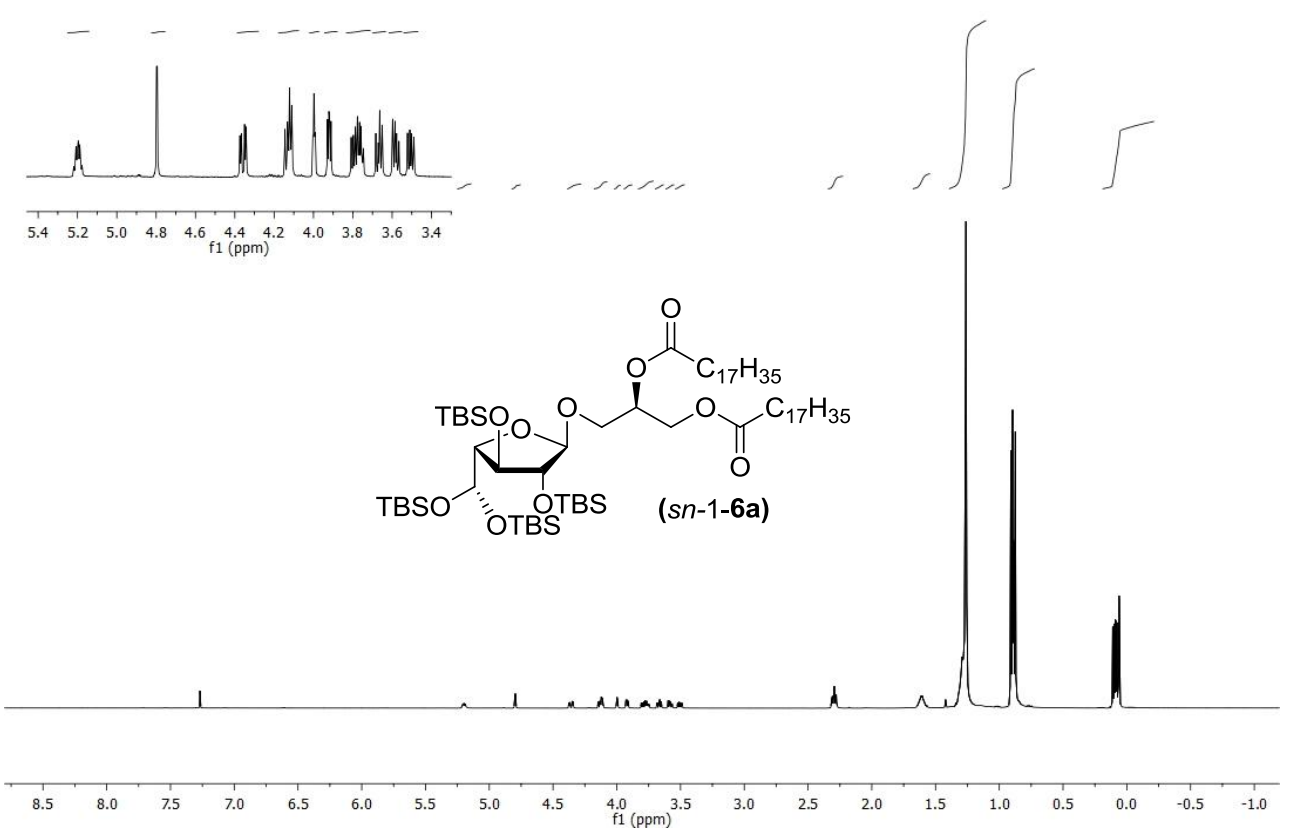

${ }^{13} \mathrm{C}$ NMR, $\mathrm{CDCl}_{3}, 125 \mathrm{MHz}$

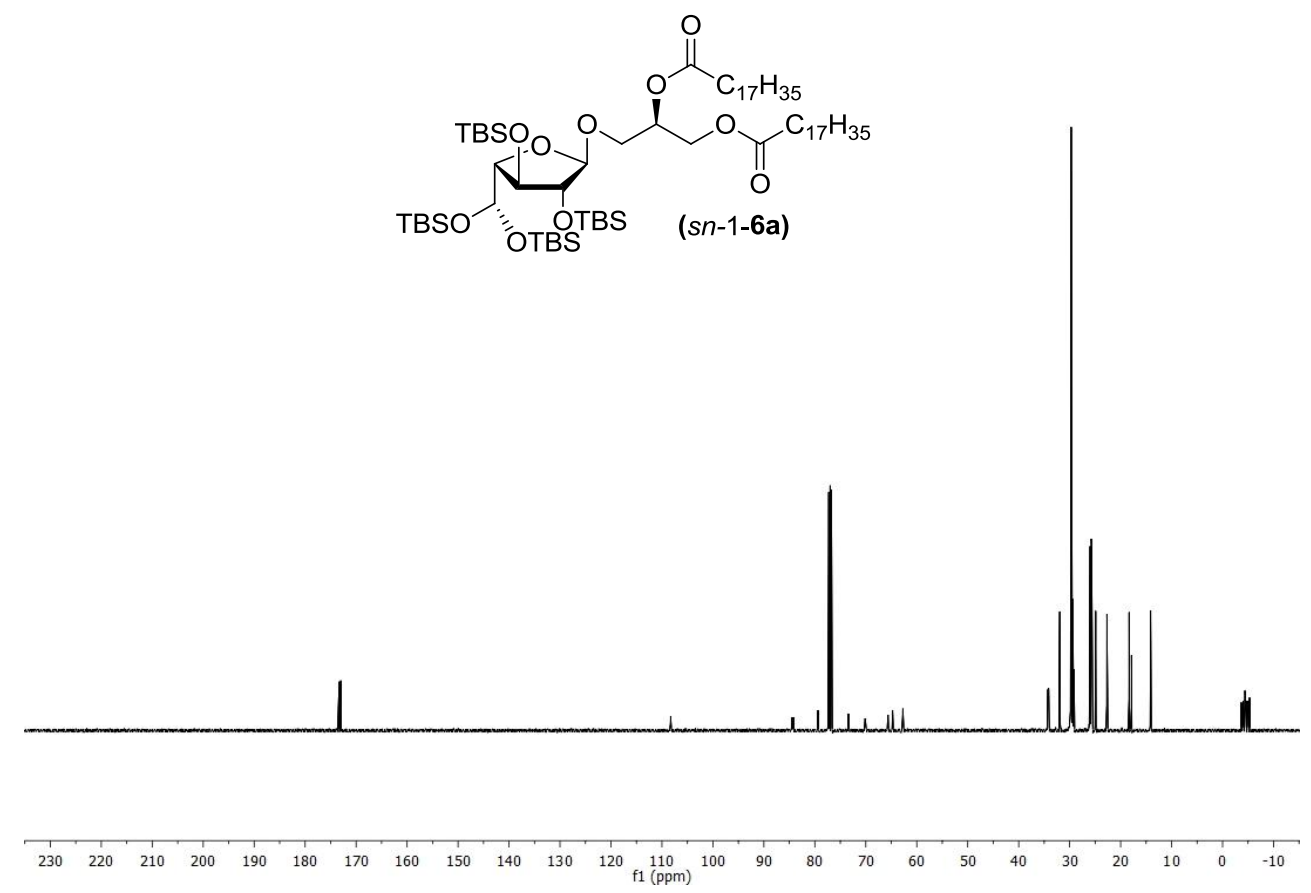


${ }^{1} \mathrm{H}$ NMR, $\mathrm{CDCl}_{3}, 500 \mathrm{MHz}$

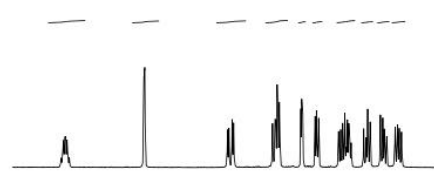

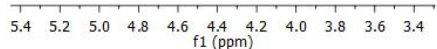

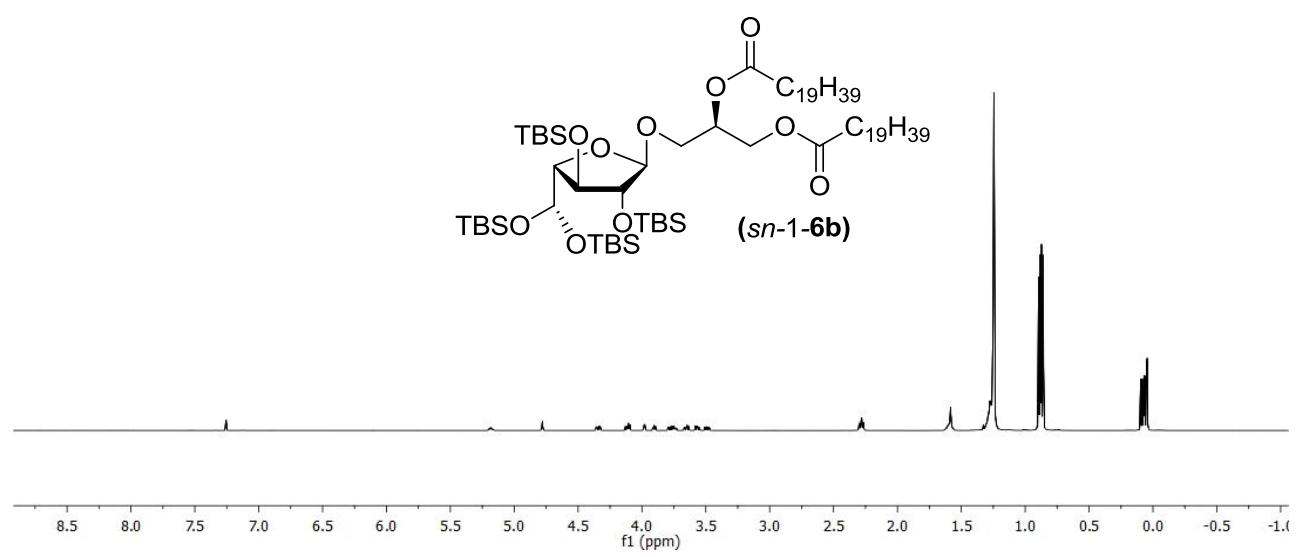

${ }^{13} \mathrm{C}_{\mathrm{NMR}}, \mathrm{CDCl}_{3}, 125 \mathrm{MHz}$

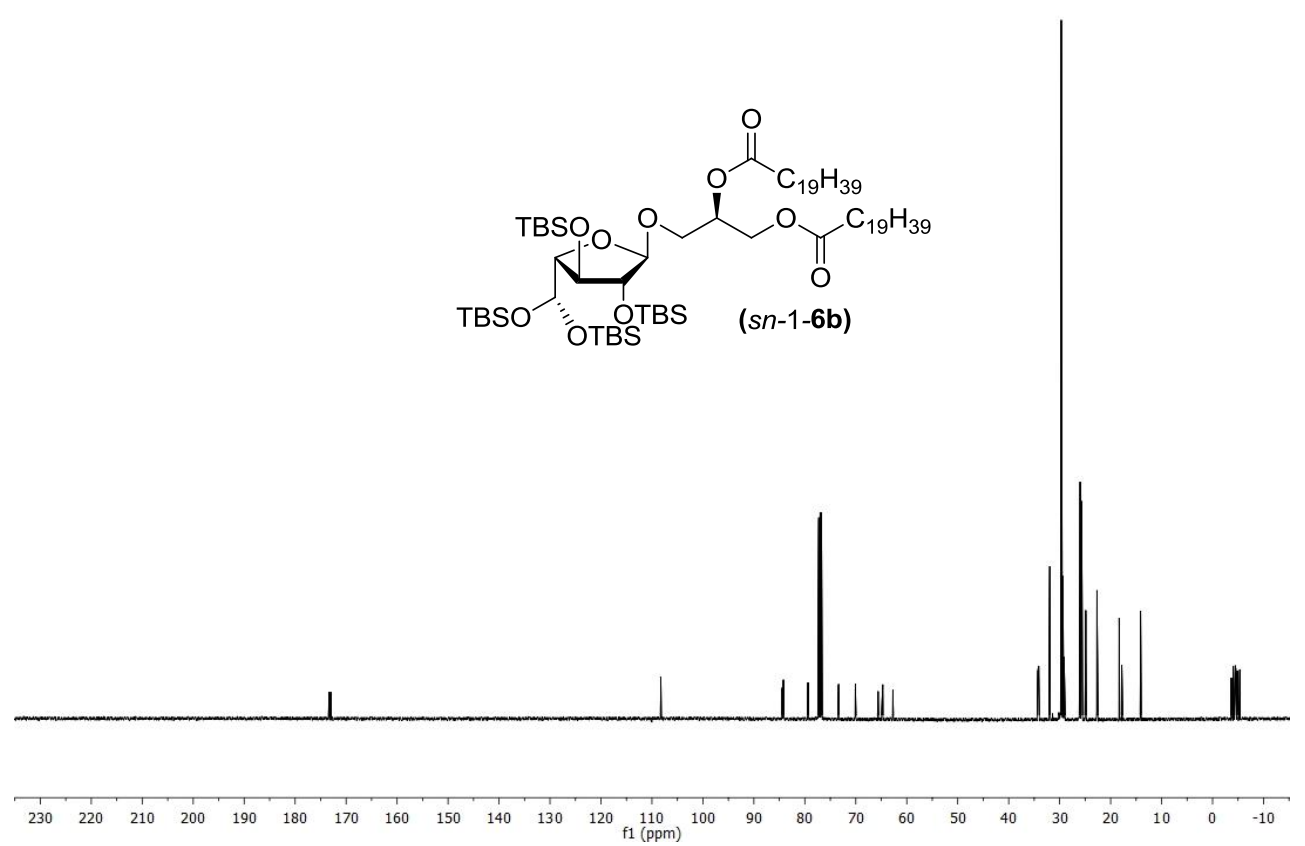


${ }^{1} \mathrm{H} \mathrm{NMR}, \mathrm{CDCl}_{3}, 500 \mathrm{MHz}$
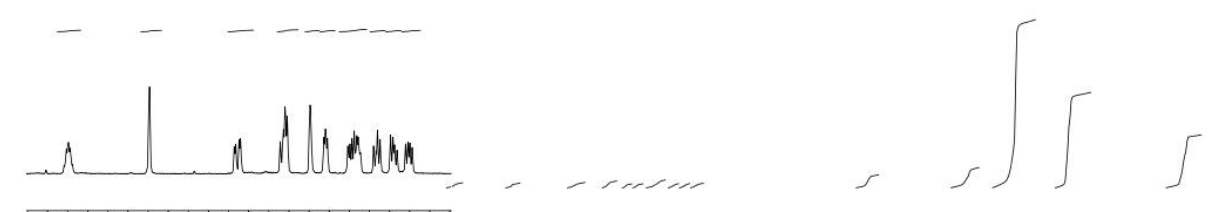

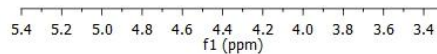
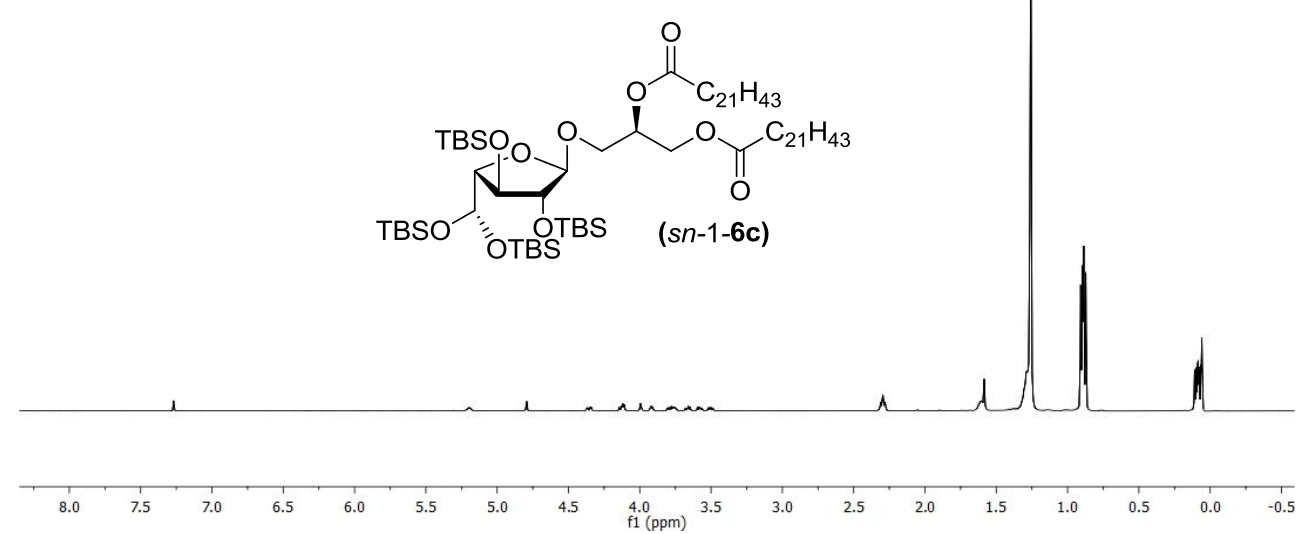

${ }^{13} \mathrm{C}$ NMR, $\mathrm{CDCl}_{3}, 125 \mathrm{MHz}$

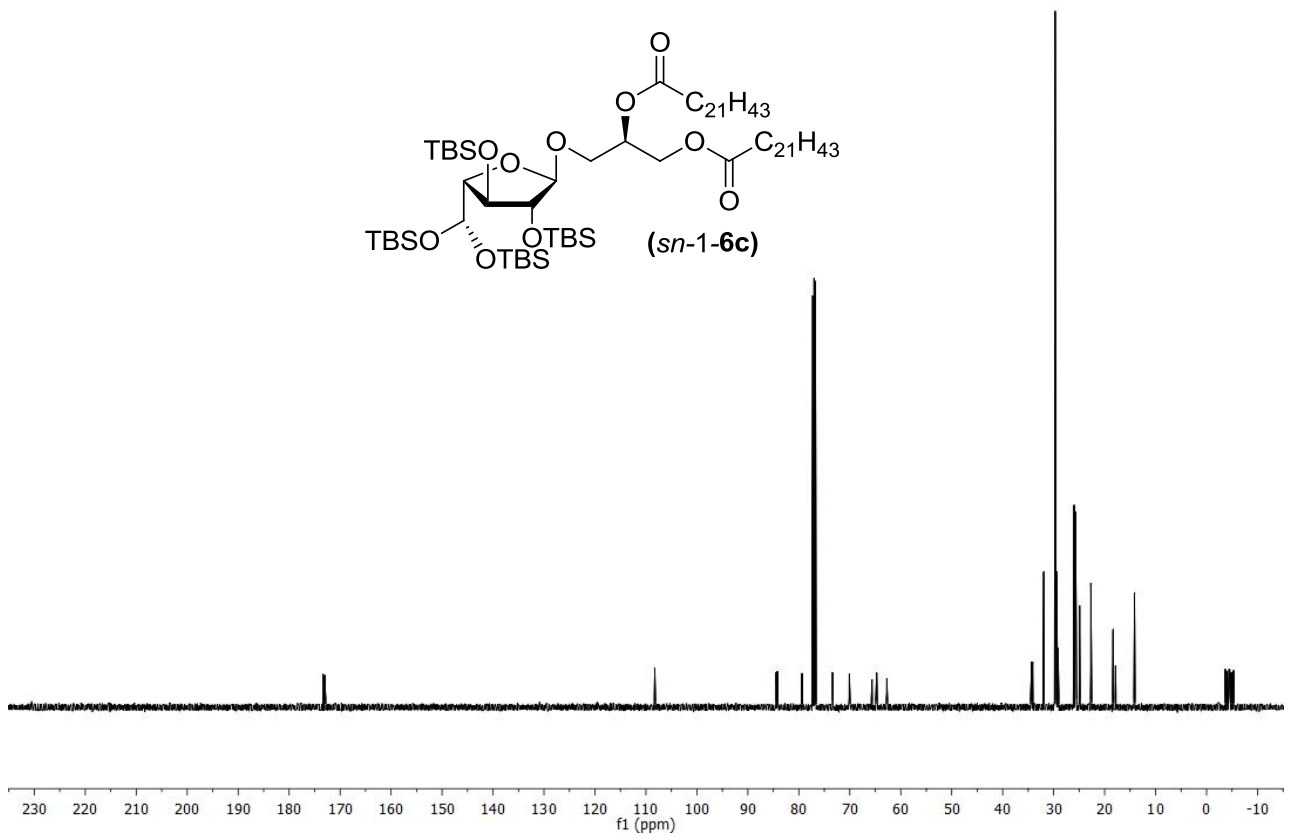


${ }^{1} \mathrm{H}$ NMR, $\mathrm{CDCl}_{3}, 500 \mathrm{MHz}$

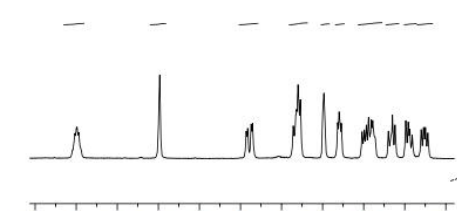

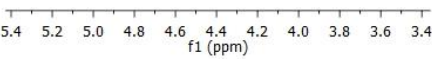
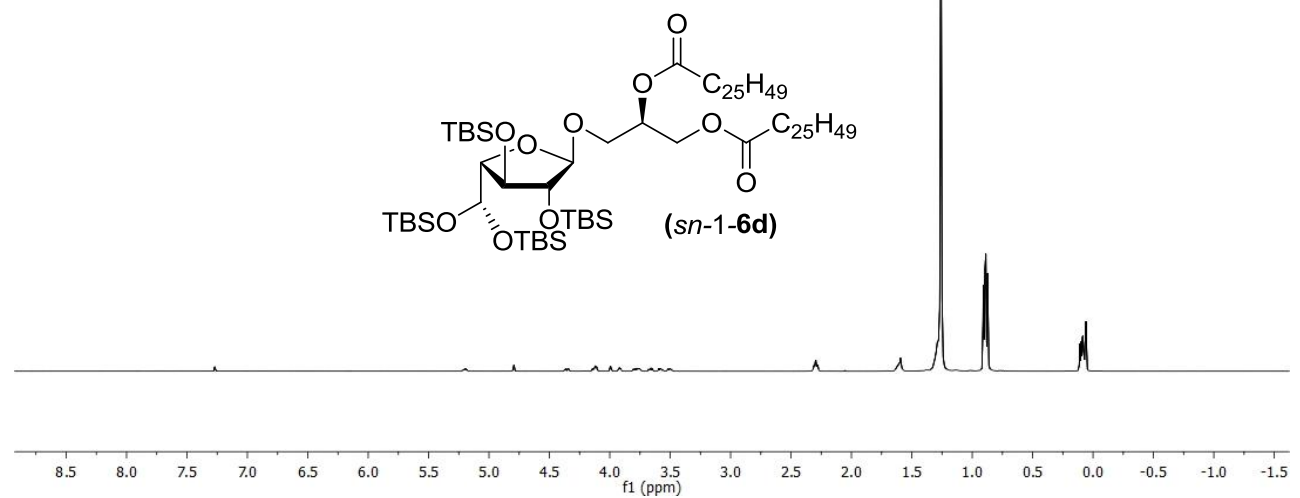

${ }^{13} \mathrm{C} \mathrm{NMR}, \mathrm{CDCl}_{3}, 125 \mathrm{MHz}$
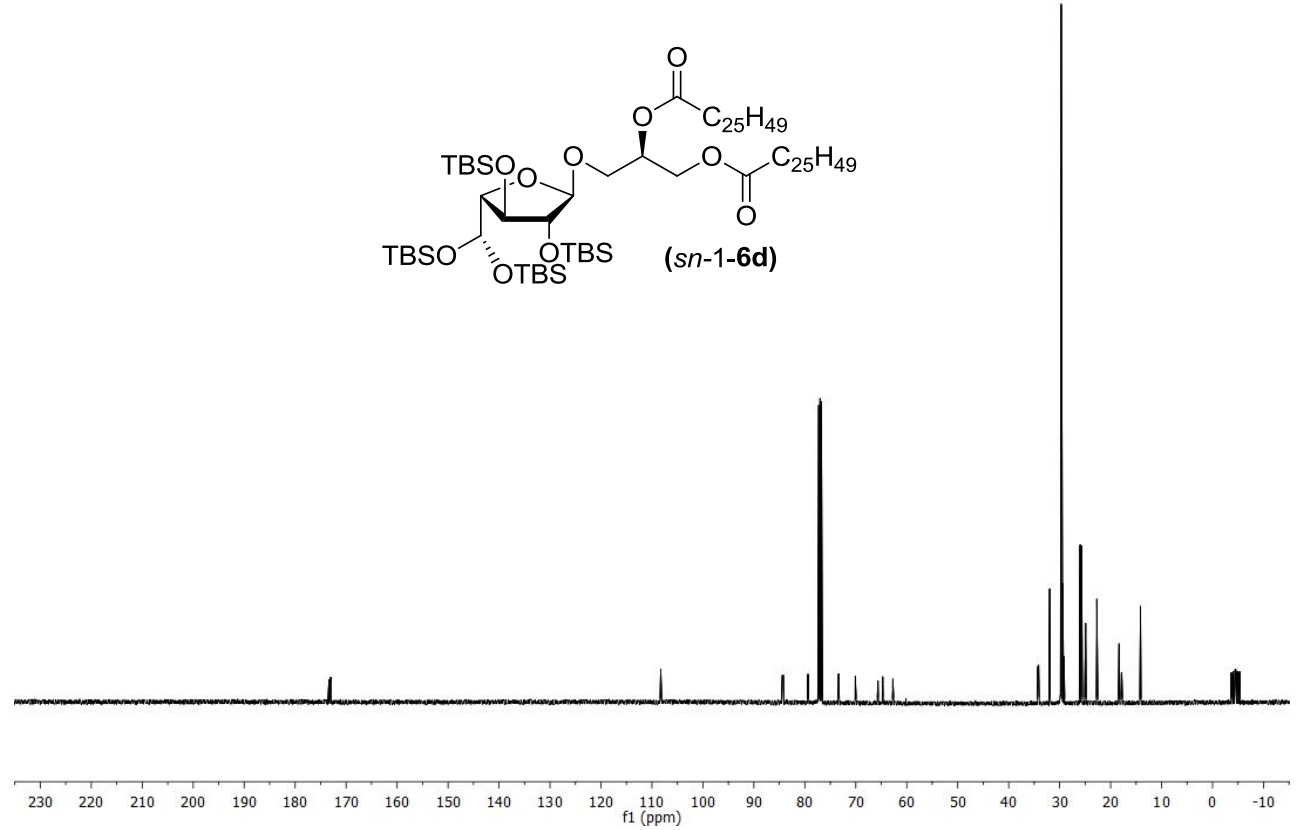
${ }^{1} \mathrm{H} \mathrm{NMR}, \mathrm{CDCl}_{3}, 500 \mathrm{MHz}$

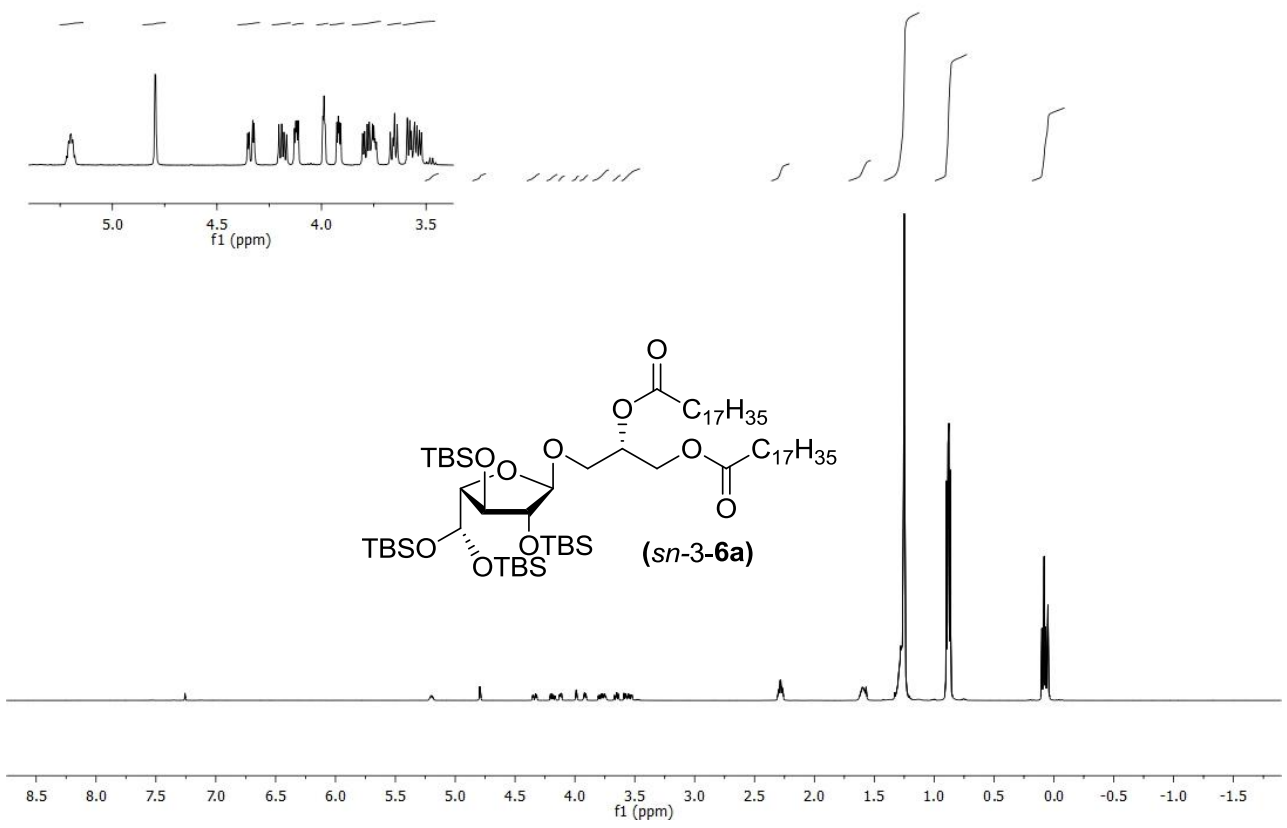

${ }^{13} \mathrm{C}$ NMR, $\mathrm{CDCl}_{3}, 125 \mathrm{MHz}$

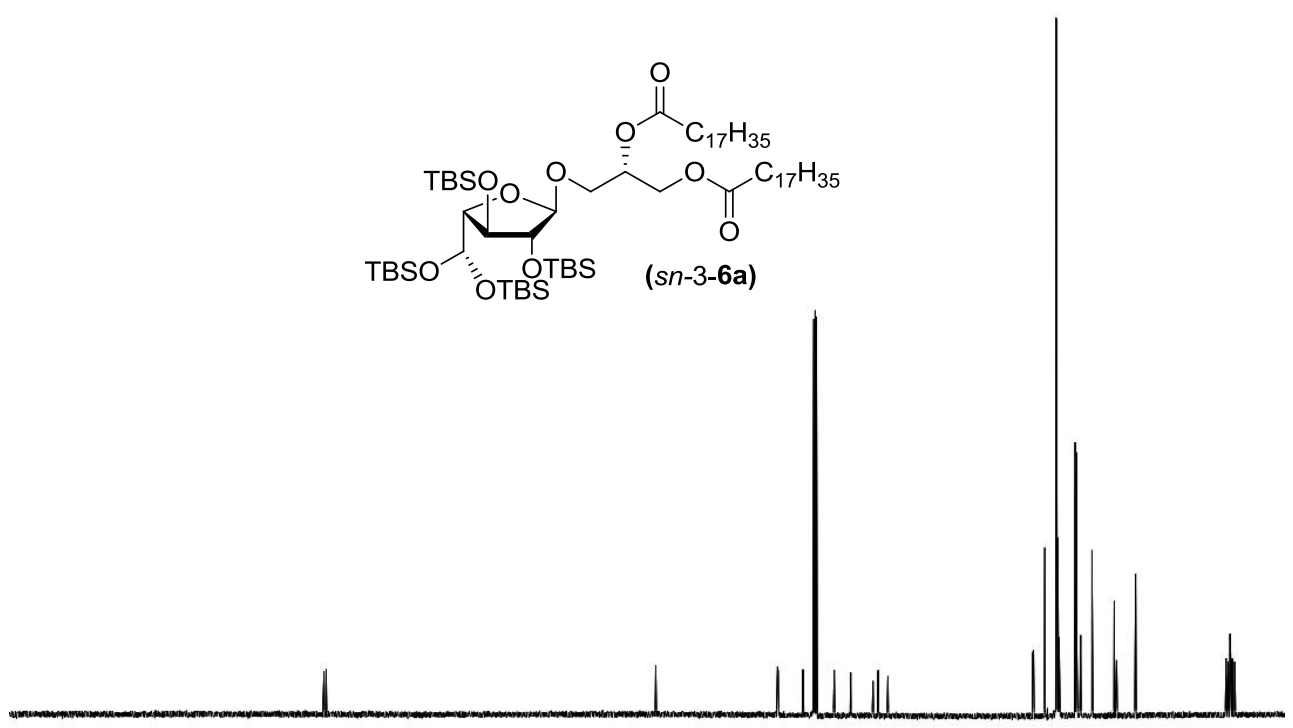

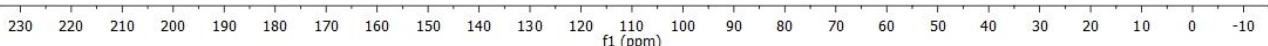


${ }^{1} \mathrm{H}$ NMR, $\mathrm{CDCl}_{3}, 500 \mathrm{MHz}$

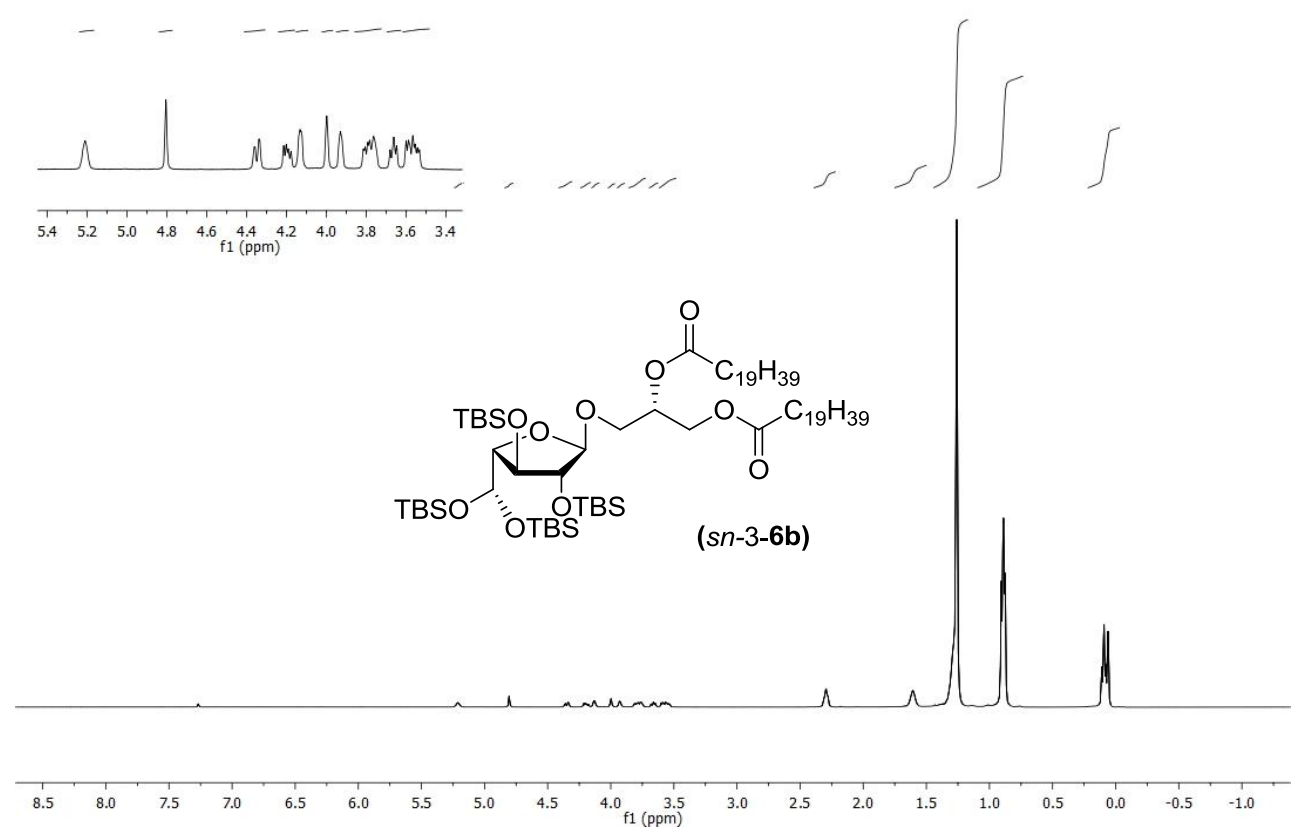

${ }^{13} \mathrm{C}$ NMR, $\mathrm{CDCl}_{3}, 125 \mathrm{MHz}$
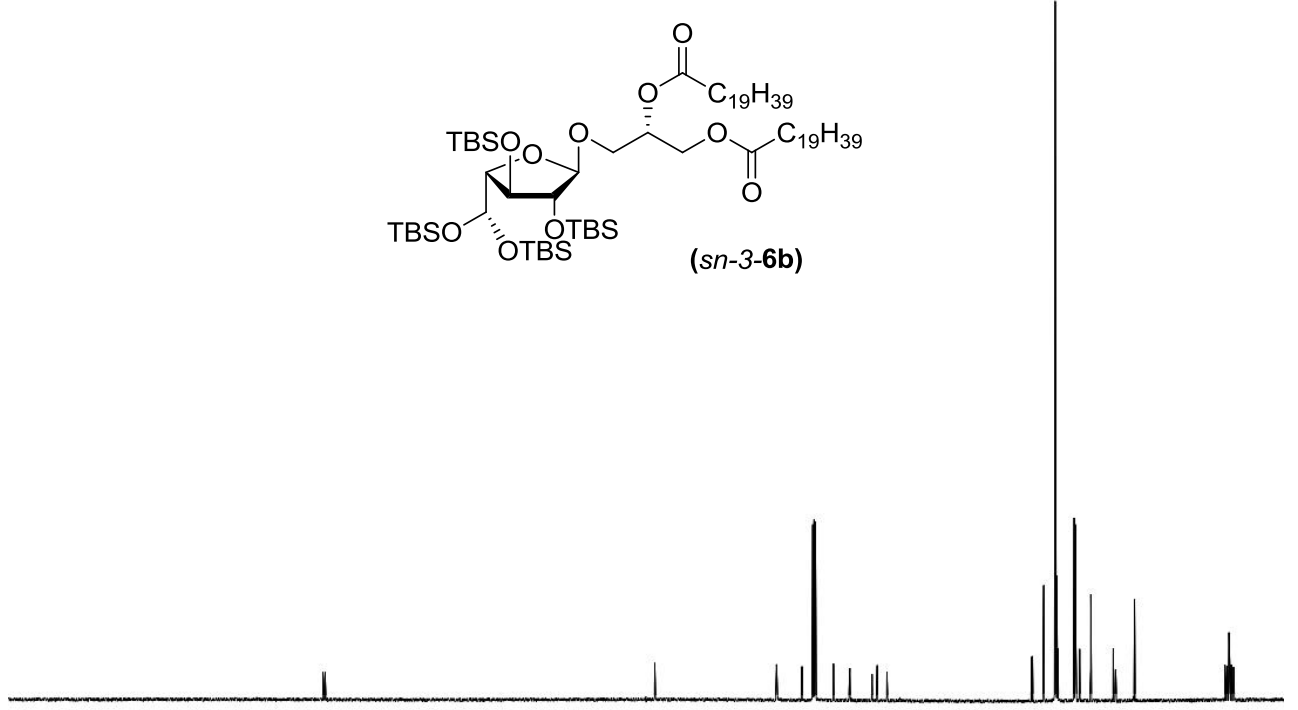

$\begin{array}{llllllllllllllllllllllllllllllllllllllllll}230 & 220 & 210 & 200 & 190 & 180 & 170 & 160 & 150 & 140 & 130 & 120 & 110 & 100 & 90 & 80 & 70 & 60 & 50 & 40 & 30 & 20 & 10 & 0 & -10\end{array}$ 
${ }^{1} \mathrm{H} \mathrm{NMR}, \mathrm{CDCl}_{3}, 500 \mathrm{MHz}$

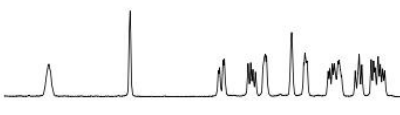

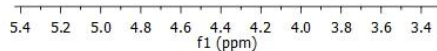
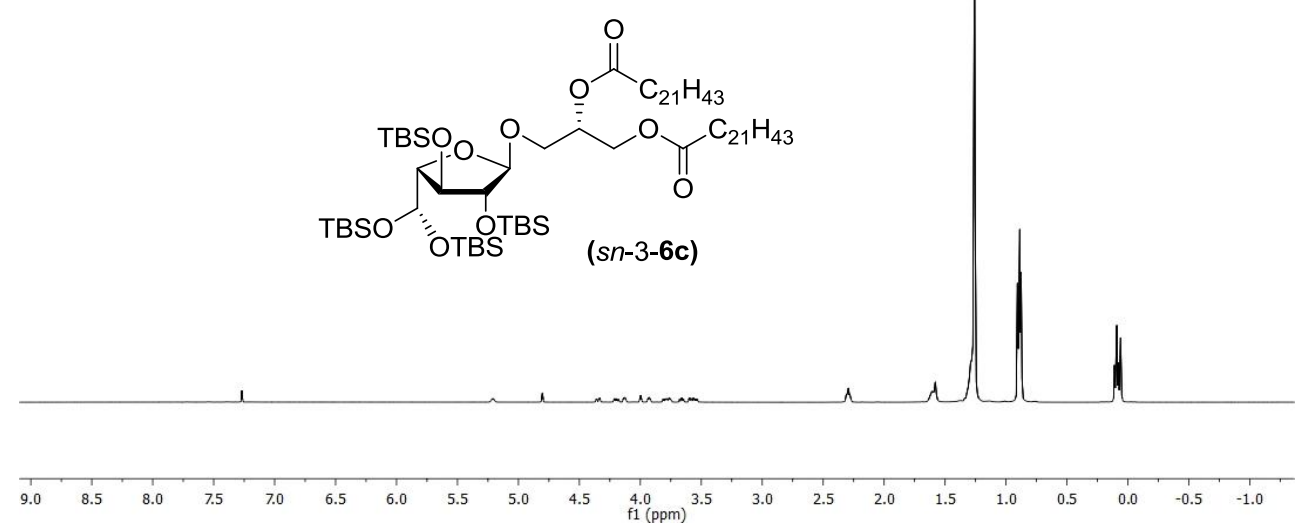

${ }^{13} \mathrm{C}$ NMR, $\mathrm{CDCl}_{3}, 125 \mathrm{MHz}$

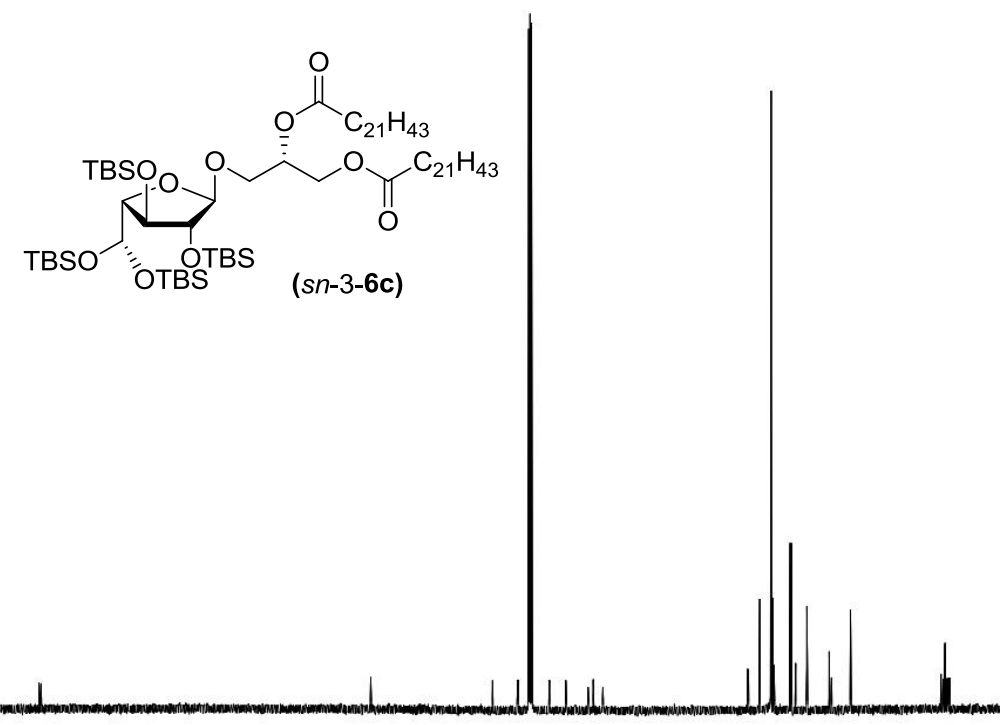

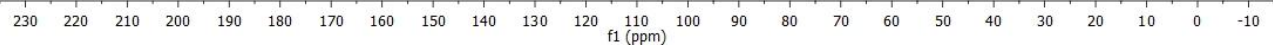


${ }^{1} \mathrm{H}$ NMR, $\mathrm{CDCl}_{3}, 500 \mathrm{MHz}$

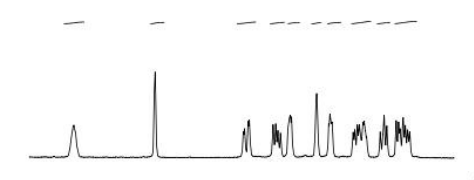

$\begin{array}{llllllllllllll}5.4 & 5.2 & 5.0 & 4.8 & 4.6 & 4 & & 4.4 & 4.2 & 4.0 & 3.8 & 3.6 & 3.4\end{array}$
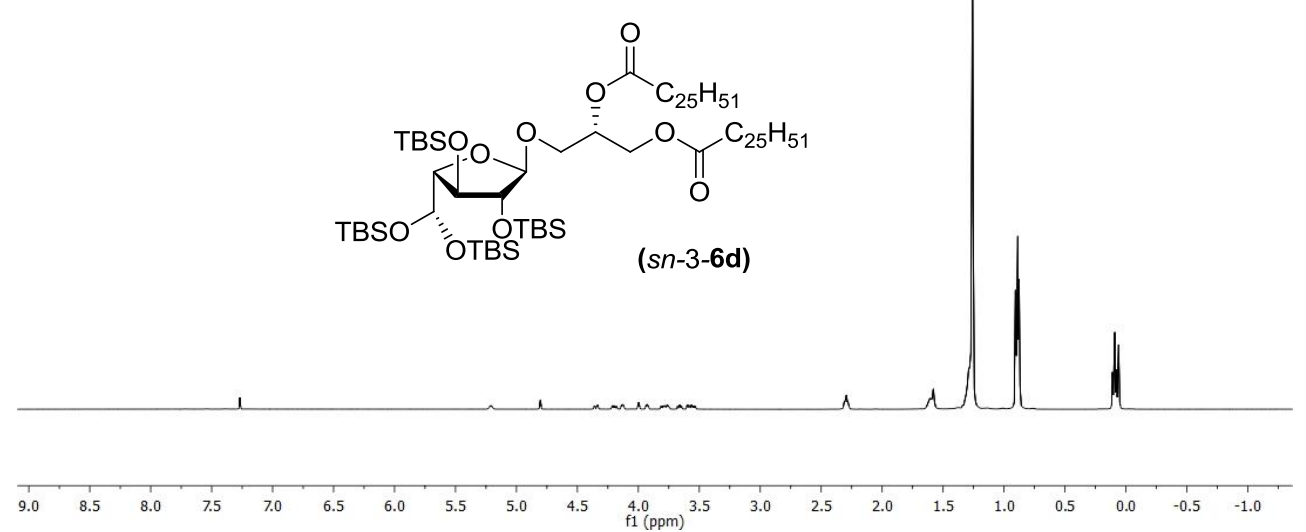

${ }^{13} \mathrm{C}$ NMR, $\mathrm{CDCl}_{3}, 125 \mathrm{MHz}$

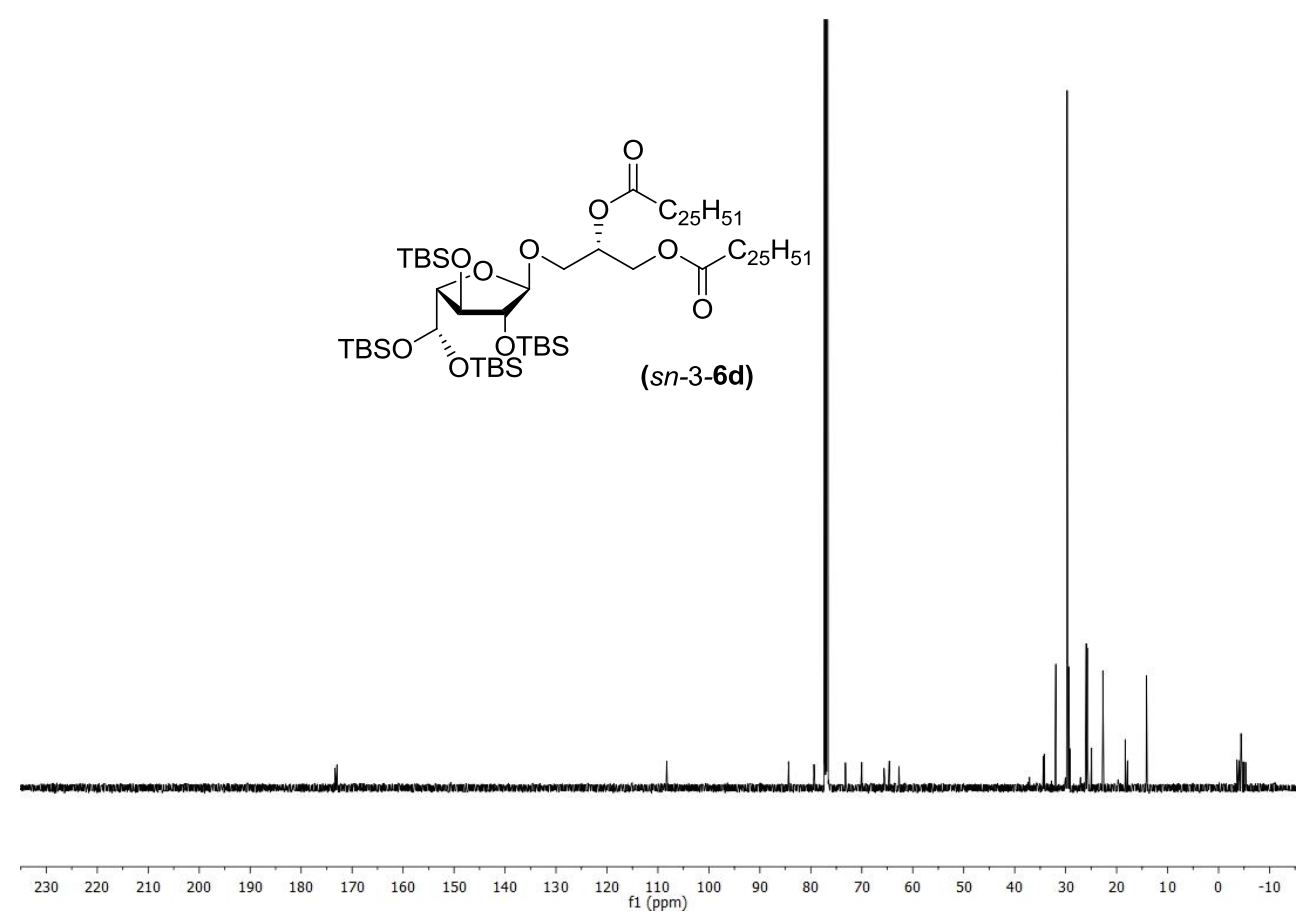


${ }^{1} \mathrm{H} \mathrm{NMR}, \mathrm{CD}_{3} \mathrm{OD} / \mathrm{CDCl}_{3}(1 / 2, \mathrm{v} / \mathrm{v}), 600 \mathrm{MHz}$

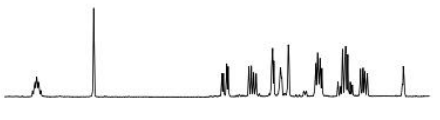

$\begin{array}{lllllllllllll}5.2 & 5.0 & 4.8 & 4.6 & 4.4 & 4.2 & 4 & 1 & 1 & 1 & 1 \\ \text { f1 (ppm) } & 4.0 & 3.8 & 3.6 & 3.4\end{array}$

$\underbrace{(s n-1-2 \mathrm{a})}_{\mathrm{OH}}$

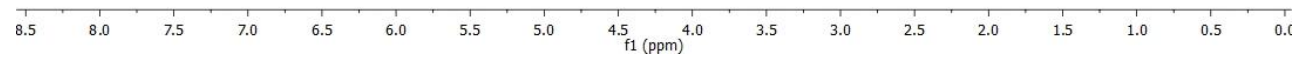

${ }^{13} \mathrm{C}$ NMR, $\mathrm{CD}_{3} \mathrm{OD} / \mathrm{CDCl}_{3}(1 / 2, \mathrm{v} / \mathrm{v}), 151 \mathrm{MHz}$

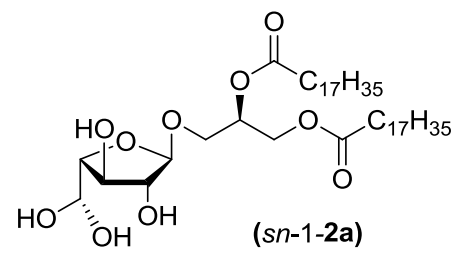

$\begin{array}{lllllllllllllllllllllllll}230 & 220 & 210 & 200 & 190 & 180 & 170 & 160 & 150 & 140 & 130 & 120 & 110 & 100 & 90 & 80 & 70 & 60 & 50 & 40 & 30 & 20 & 10 & 0 & -10\end{array}$ 
${ }^{1} \mathrm{H} \mathrm{NMR}, \mathrm{CD}_{3} \mathrm{OD} / \mathrm{CDCl}_{3}(1 / 2, \mathrm{v} / \mathrm{v}), 600 \mathrm{MHz}$
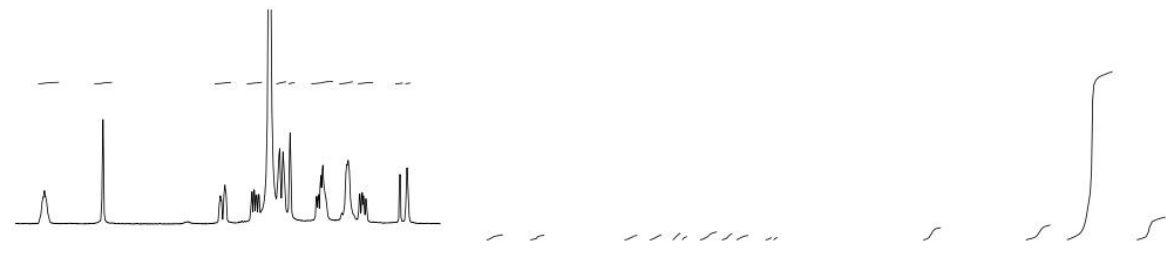

\begin{tabular}{llllllllllll}
\hline 5.2 & 5.0 & 4.8 & 4.6 & 4.4 & 4.2 & 4.0 & 3.8 & 3.6 & 3.4 & 3.2
\end{tabular}
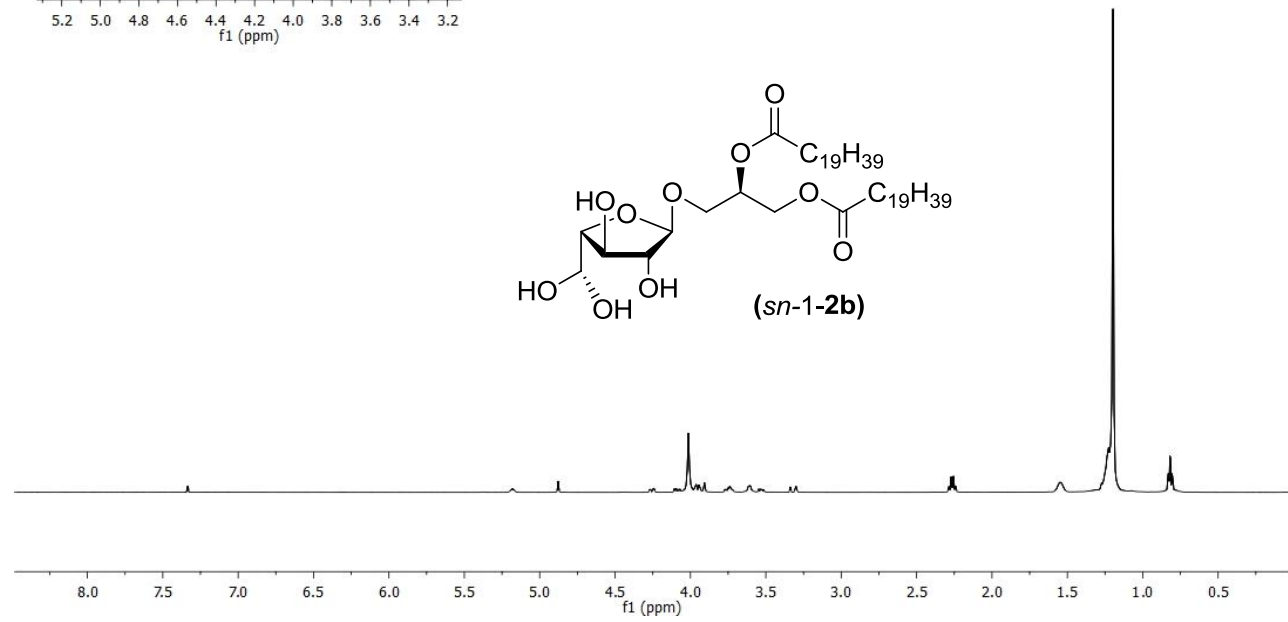

${ }^{13} \mathrm{C} \mathrm{NMR}, \mathrm{CD}_{3} \mathrm{OD} / \mathrm{CDCl}_{3}(1 / 2, \mathrm{v} / \mathrm{v}), 151 \mathrm{MHz}$

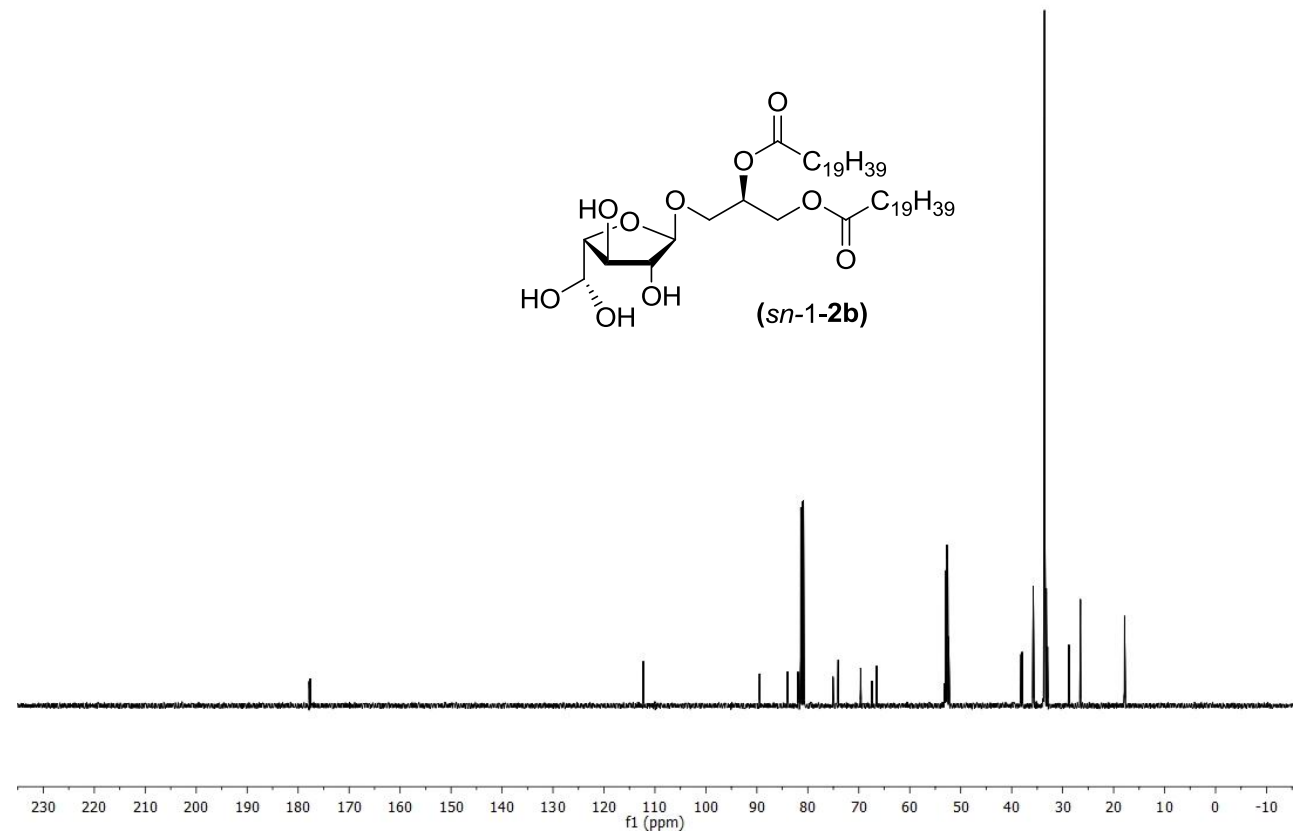


${ }^{1} \mathrm{H} \mathrm{NMR}, \mathrm{CD}_{3} \mathrm{OD} / \mathrm{CDCl}_{3}(1 / 2, \mathrm{v} / \mathrm{v}), 600 \mathrm{MHz}$
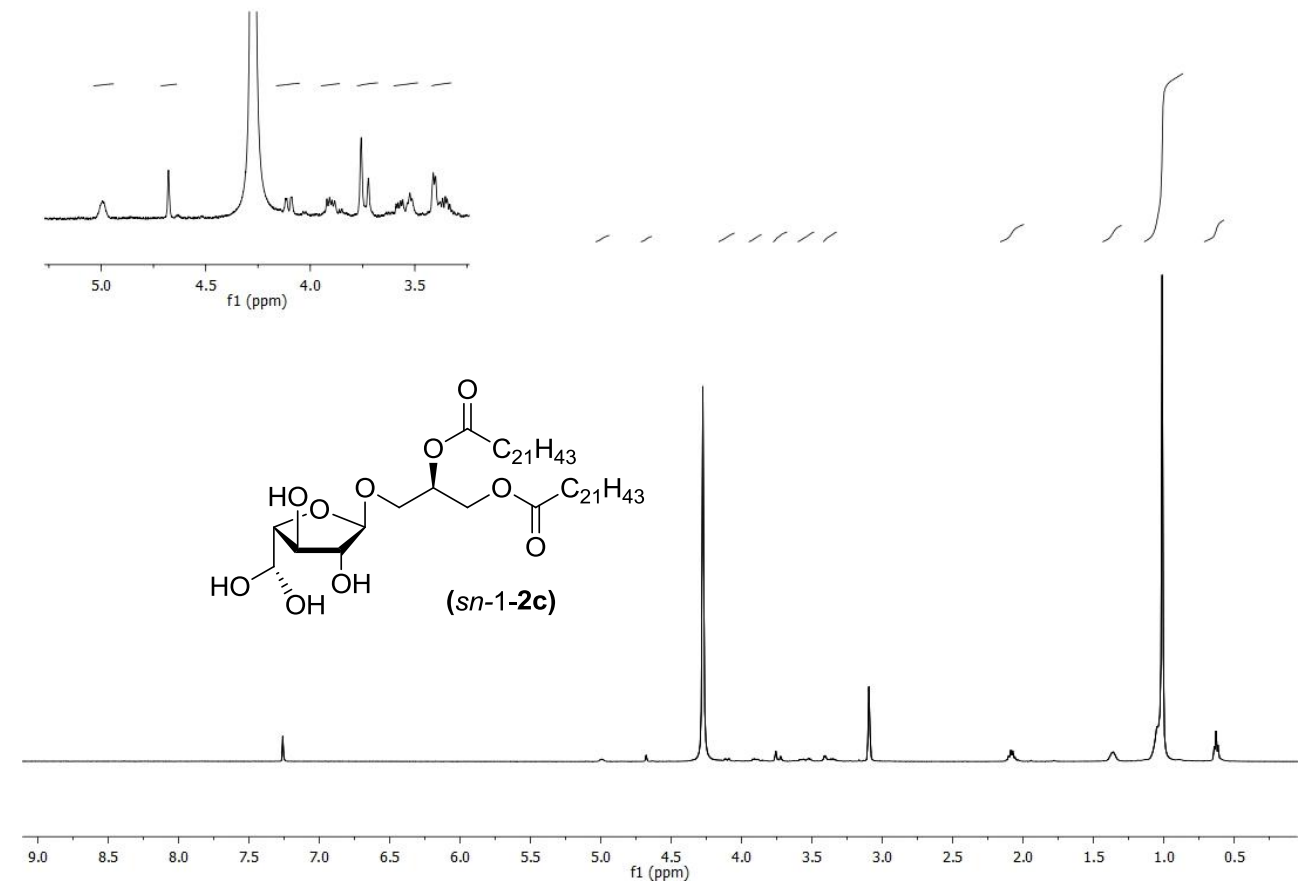

${ }^{13} \mathrm{C}$ NMR, $\mathrm{CD}_{3} \mathrm{OD} / \mathrm{CDCl}_{3}(1 / 2, \mathrm{v} / \mathrm{v}), 151 \mathrm{MHz}$
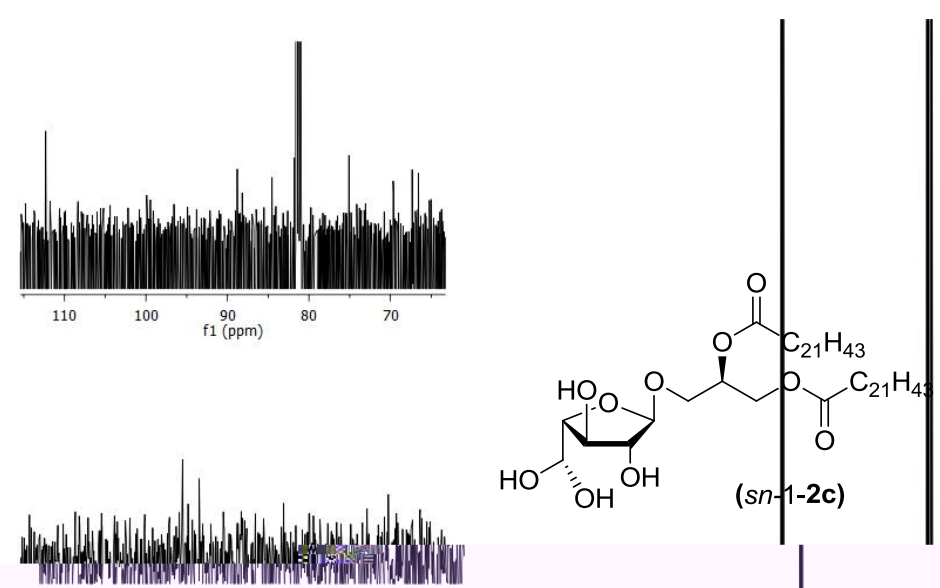

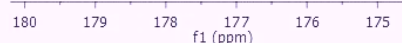


${ }^{1} \mathrm{H} \mathrm{NMR}, \mathrm{CD}_{3} \mathrm{OD} / \mathrm{CDCl}_{3}(1 / 2, \mathrm{v} / \mathrm{v}), 600 \mathrm{MHz}$
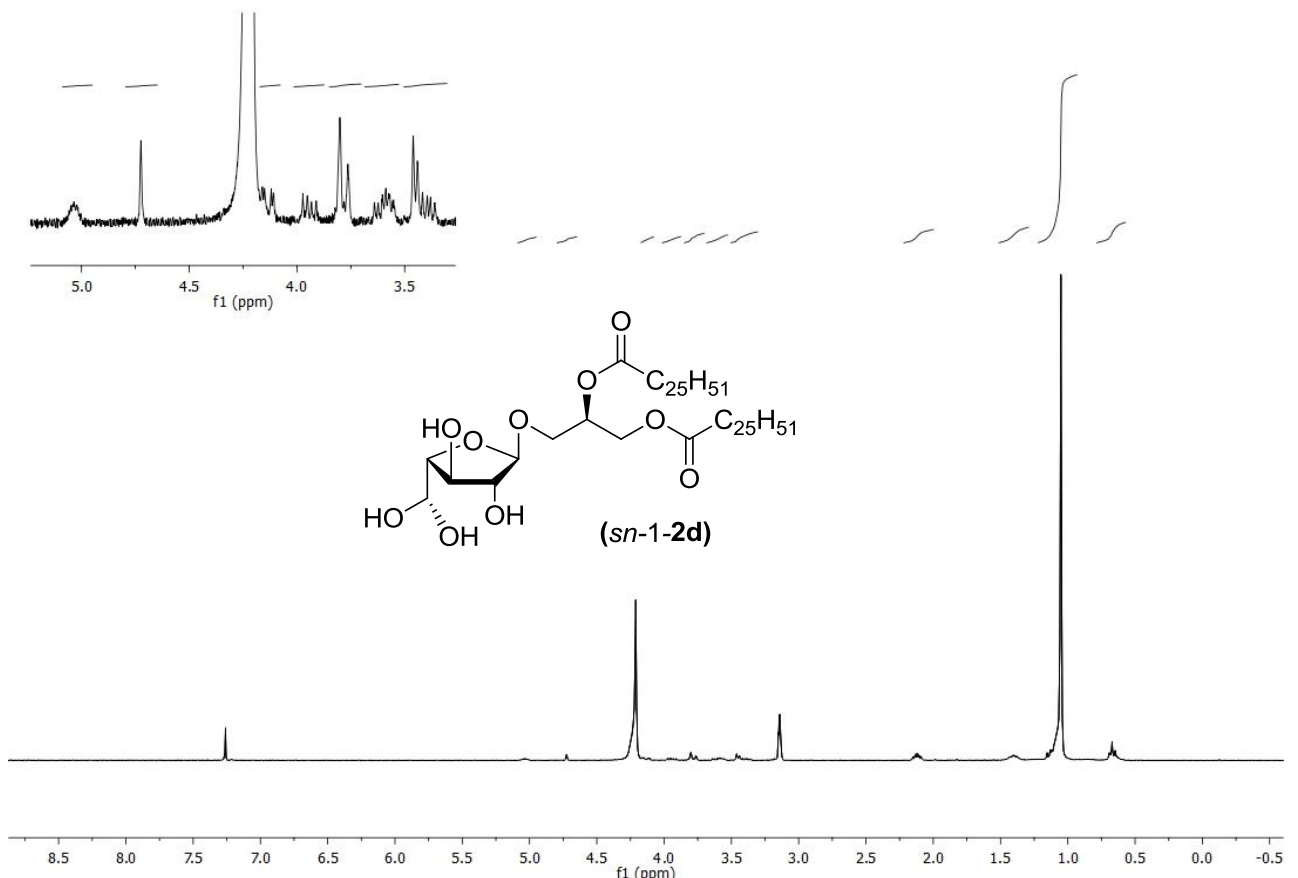

${ }^{13} \mathrm{C} \mathrm{NMR}, \mathrm{CD}_{3} \mathrm{OD} / \mathrm{CDCl}_{3}(1 / 2, \mathrm{v} / \mathrm{v}), 151 \mathrm{MHz}$

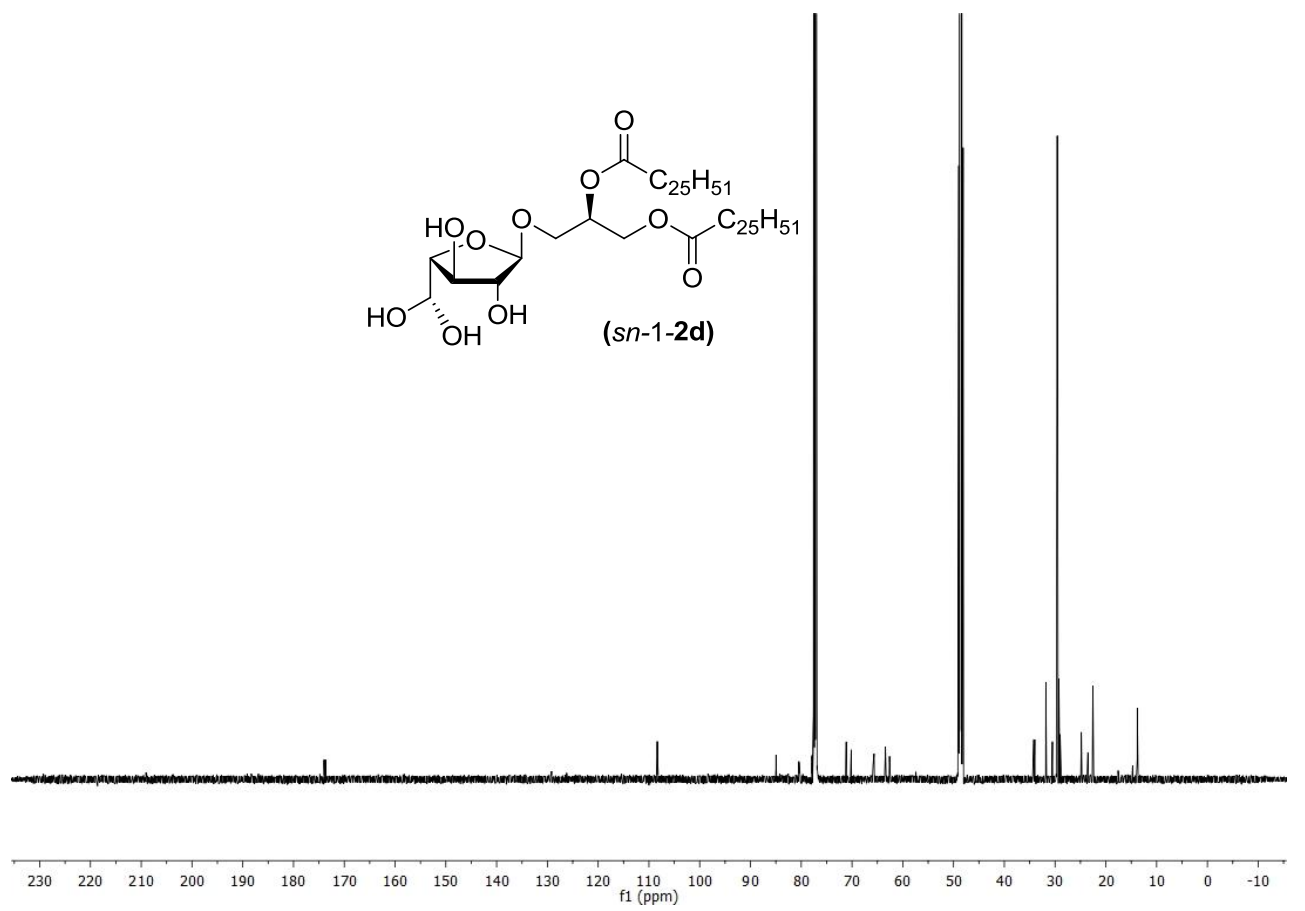


${ }^{1} \mathrm{H} \mathrm{NMR}, \mathrm{CD}_{3} \mathrm{OD} / \mathrm{CDCl}_{3}(1 / 2, \mathrm{v} / \mathrm{v}), 600 \mathrm{MHz}$
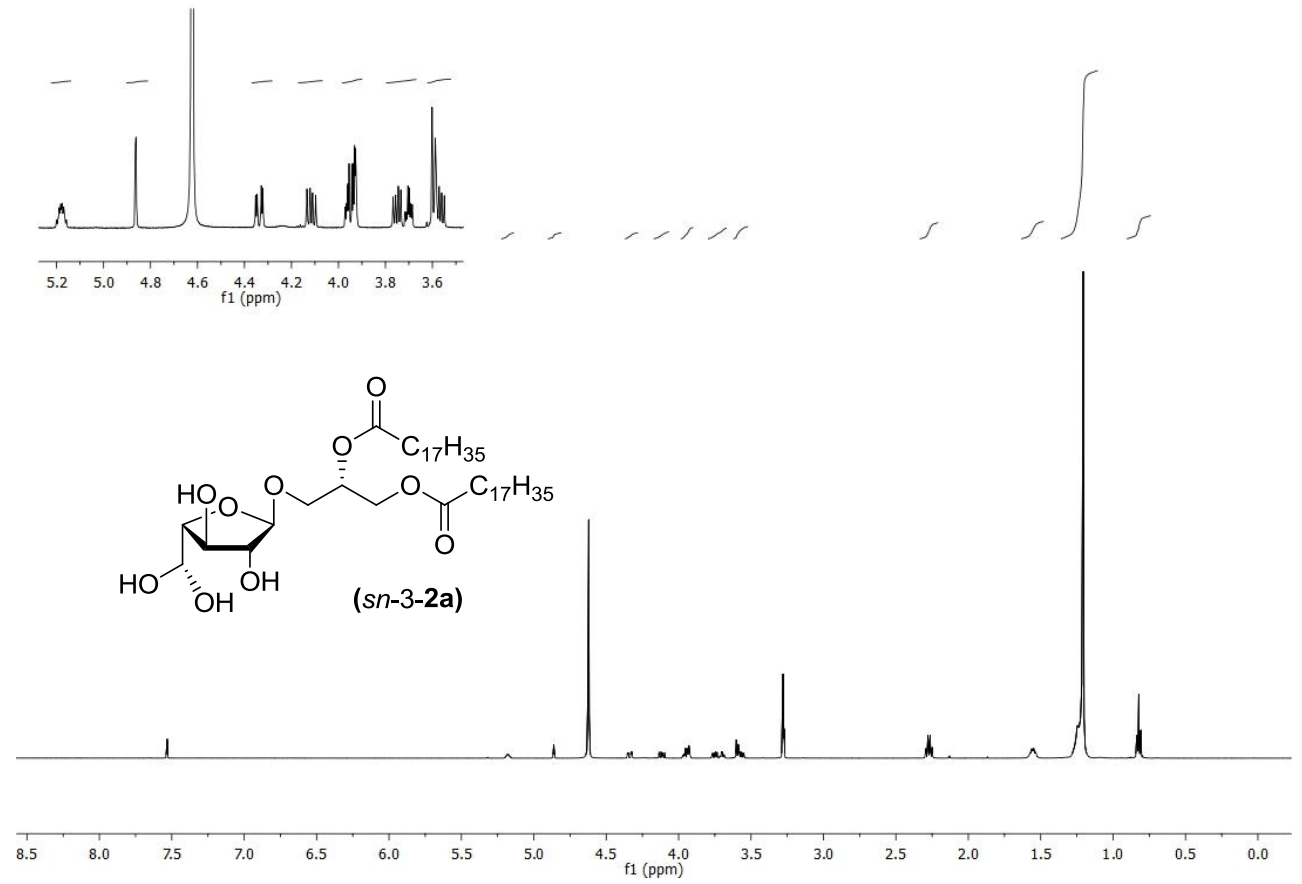

${ }^{13} \mathrm{C} \mathrm{NMR}, \mathrm{CD}_{3} \mathrm{OD} / \mathrm{CDCl}_{3}(1 / 2, \mathrm{v} / \mathrm{v}), 151 \mathrm{MHz}$

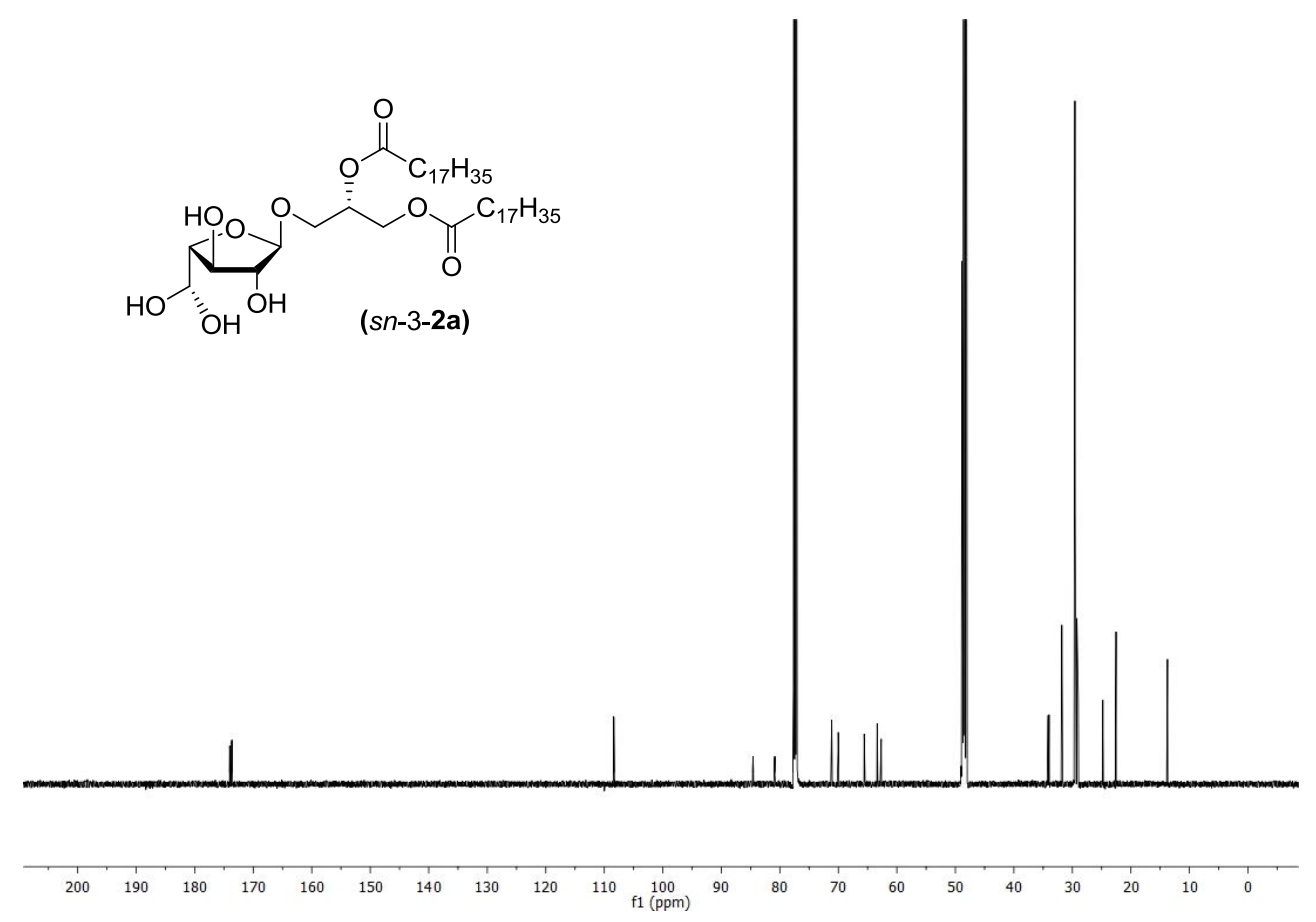


${ }^{1} \mathrm{H} \mathrm{NMR}, \mathrm{CD}_{3} \mathrm{OD} / \mathrm{CDCl}_{3}(1 / 2, \mathrm{v} / \mathrm{v}), 500 \mathrm{MHz}$

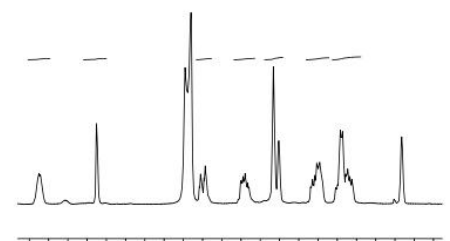

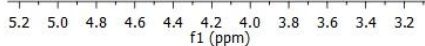

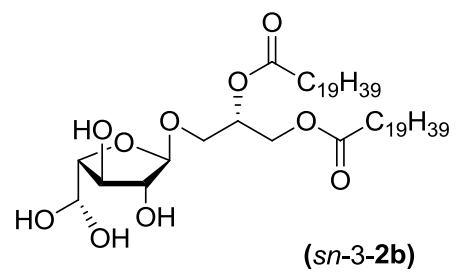

(sn-3-2b)

${ }^{13} \mathrm{C} \mathrm{NMR}, \mathrm{CD}_{3} \mathrm{OD} / \mathrm{CDCl}_{3}(1 / 2, \mathrm{v} / \mathrm{v}), 125 \mathrm{MHz}$

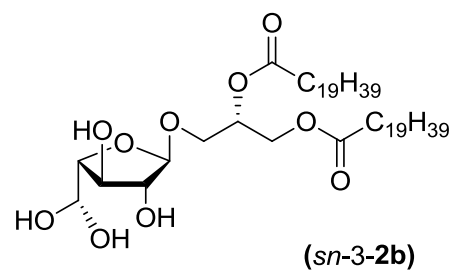

$(s n-3-2 b)$

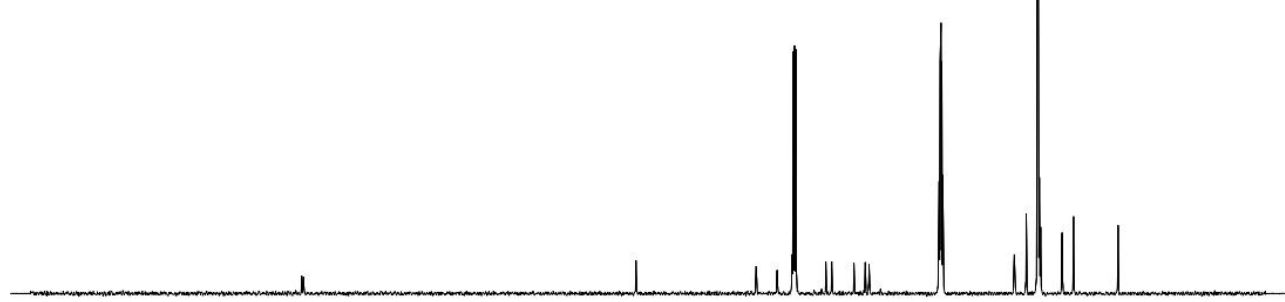

$\begin{array}{lllllllllllllllllllllllllllll}30 & 220 & 210 & 200 & 190 & 180 & 170 & 160 & 150 & 140 & 130 & 120 & 110 & 100 & 9 & 80 & 70 & 60 & 50 & 40 & 30 & 20 & 10 & 0 & -10\end{array}$ 
${ }^{1} \mathrm{H} \mathrm{NMR}, \mathrm{CD}_{3} \mathrm{OD} / \mathrm{CDCl}_{3}(1 / 2, \mathrm{v} / \mathrm{v}), 500 \mathrm{MHz}$
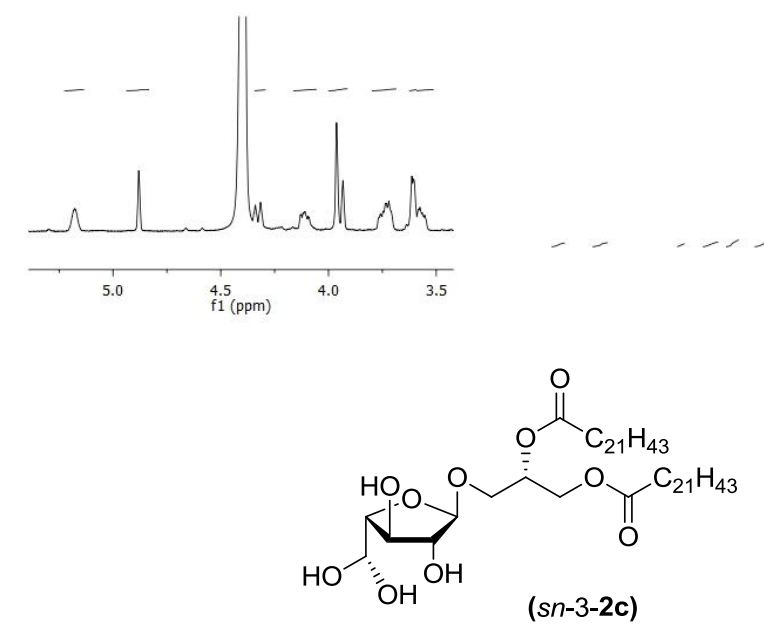

${ }^{13} \mathrm{C}$ NMR, $\mathrm{CD}_{3} \mathrm{OD} / \mathrm{CDCl}_{3}(1 / 2, \mathrm{v} / \mathrm{v}), 125 \mathrm{MHz}$

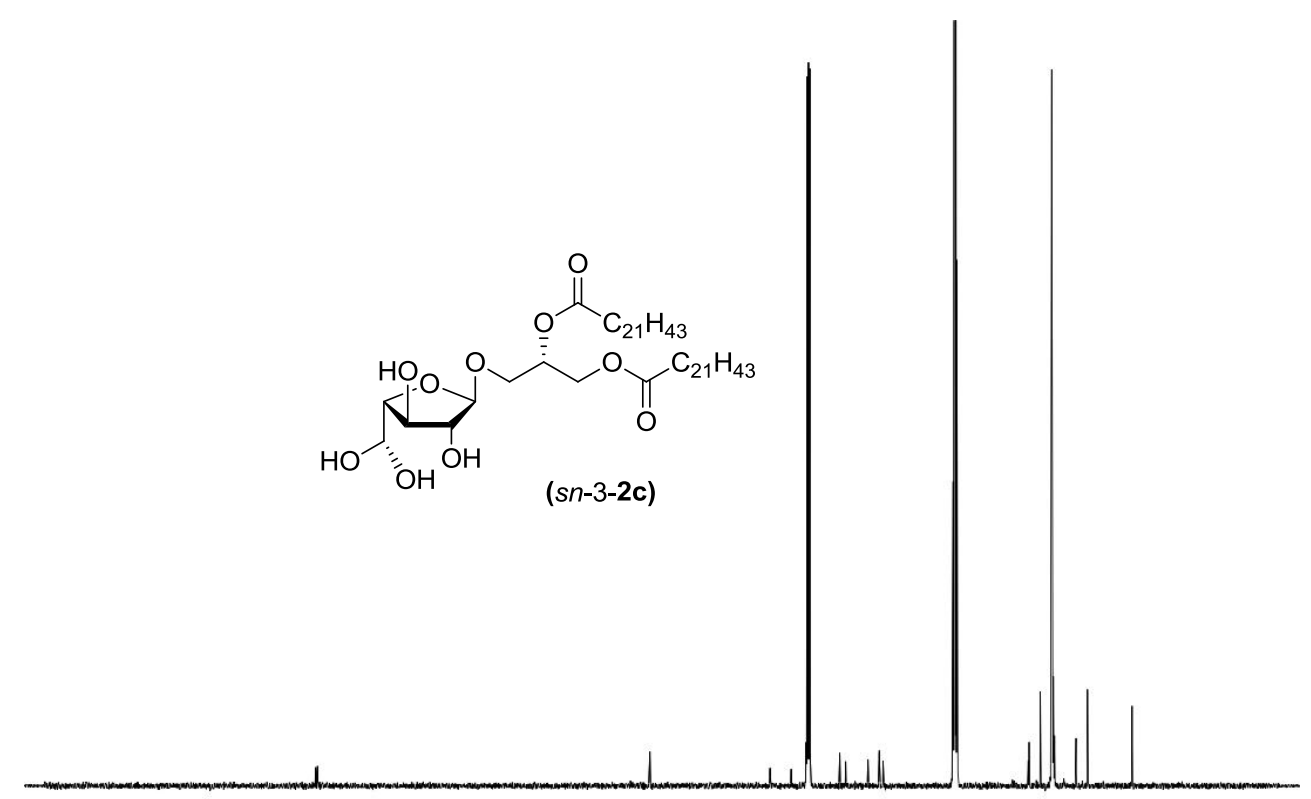

$\begin{array}{llllllllllllllllllllllllllllllll}130 & 220 & 210 & 200 & 190 & 180 & 170 & 160 & 150 & 140 & 130 & 120 & 110 & 100 & 90 & 1 & 1 & 70 & 60 & 50 & 40 & 30 & 20 & 10 & 0 & -10\end{array}$ 
${ }^{1} \mathrm{H} \mathrm{NMR}, \mathrm{CD}_{3} \mathrm{OD} / \mathrm{CDCl}_{3}(1 / 2, \mathrm{v} / \mathrm{v}), 500 \mathrm{MHz}$
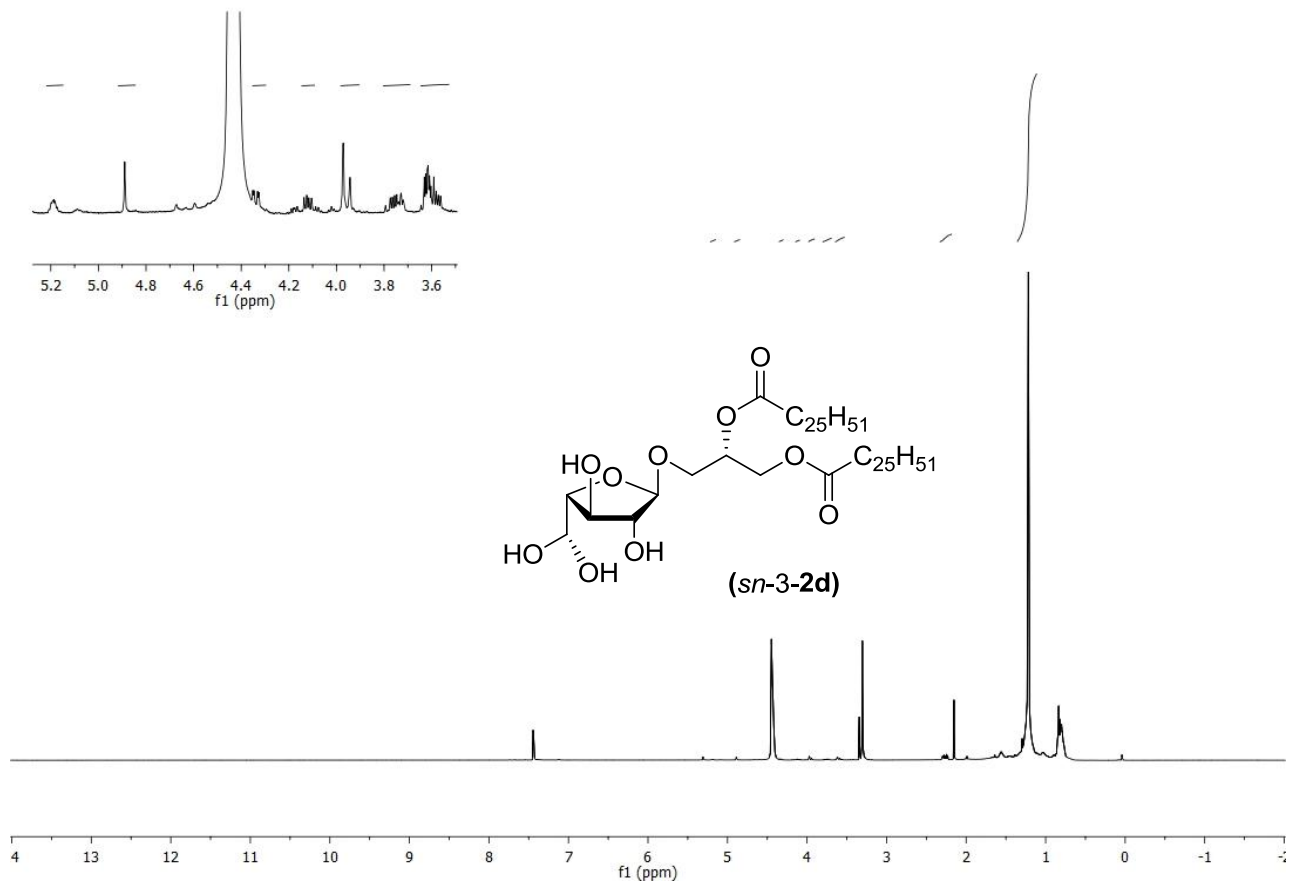

${ }^{13} \mathrm{C} \mathrm{NMR}, \mathrm{CD}_{3} \mathrm{OD} / \mathrm{CDCl}_{3}(1 / 2, \mathrm{v} / \mathrm{v}), 151 \mathrm{MHz}$
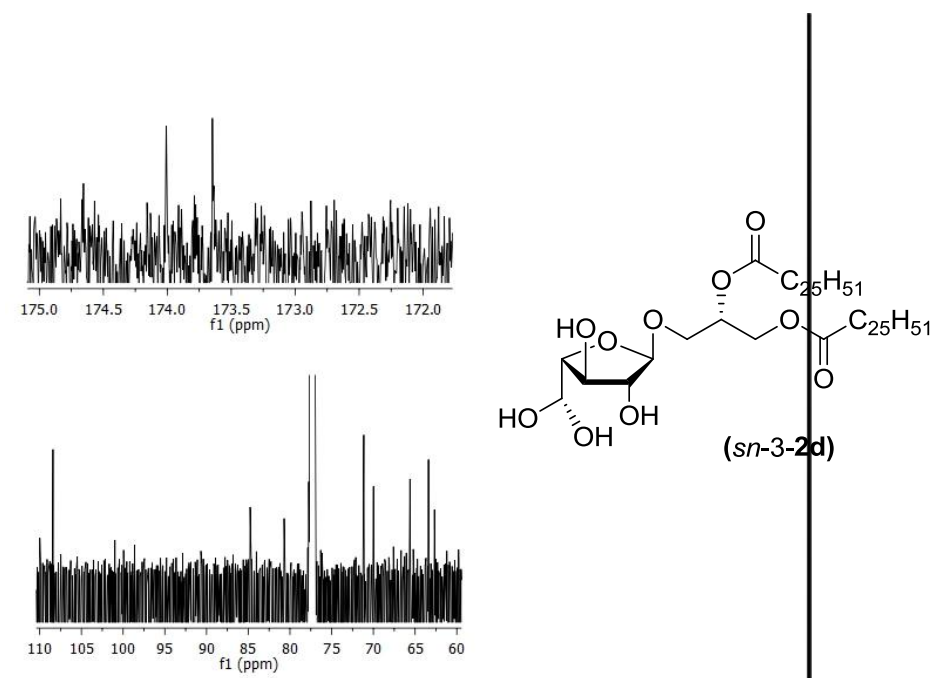

$\begin{array}{llllllllll}210 & 200 & 190 & 180 & 170 & 160 & 150 & 140 & 130 & 120 \\ \mathrm{f} 1(\mathrm{ppm}) & 100\end{array}$ 
${ }^{1} \mathrm{H} \mathrm{NMR}, \mathrm{CDCl}_{3}, 500 \mathrm{MHz}$
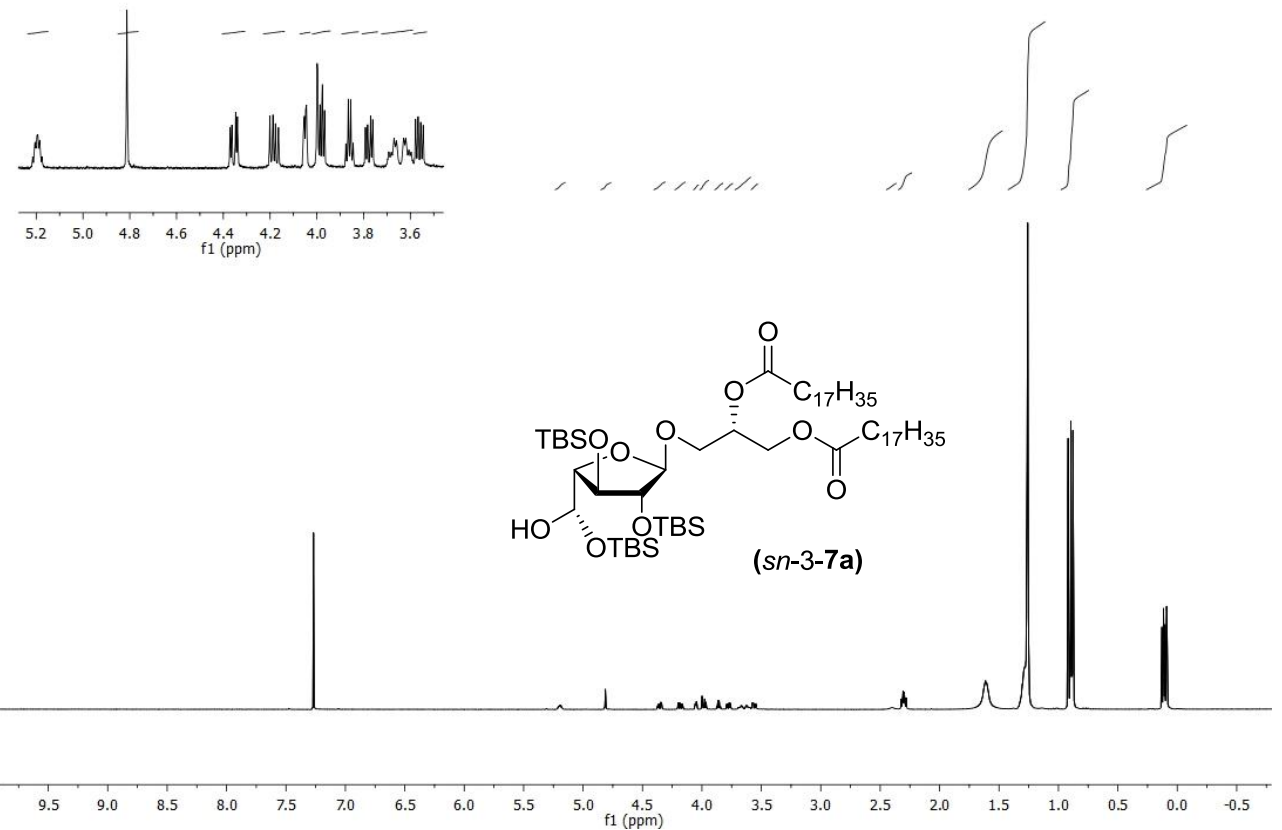

${ }^{13} \mathrm{C}$ NMR, $\mathrm{CDCl}_{3}, 125 \mathrm{MHz}$

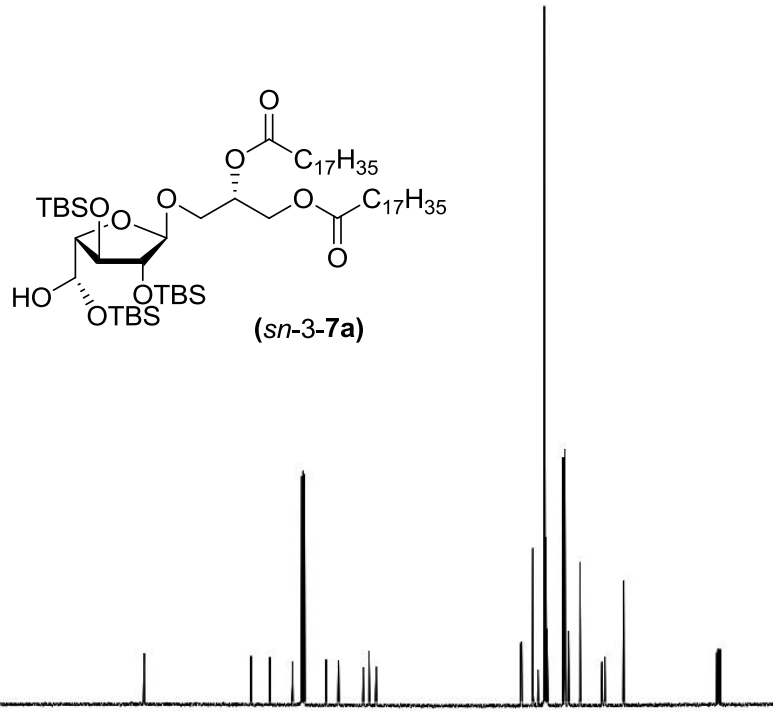

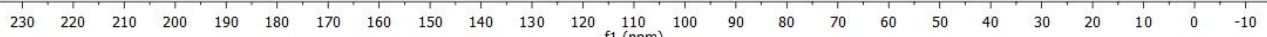


${ }^{1} \mathrm{H}$ NMR, $\mathrm{CDCl}_{3}, 500 \mathrm{MHz}$
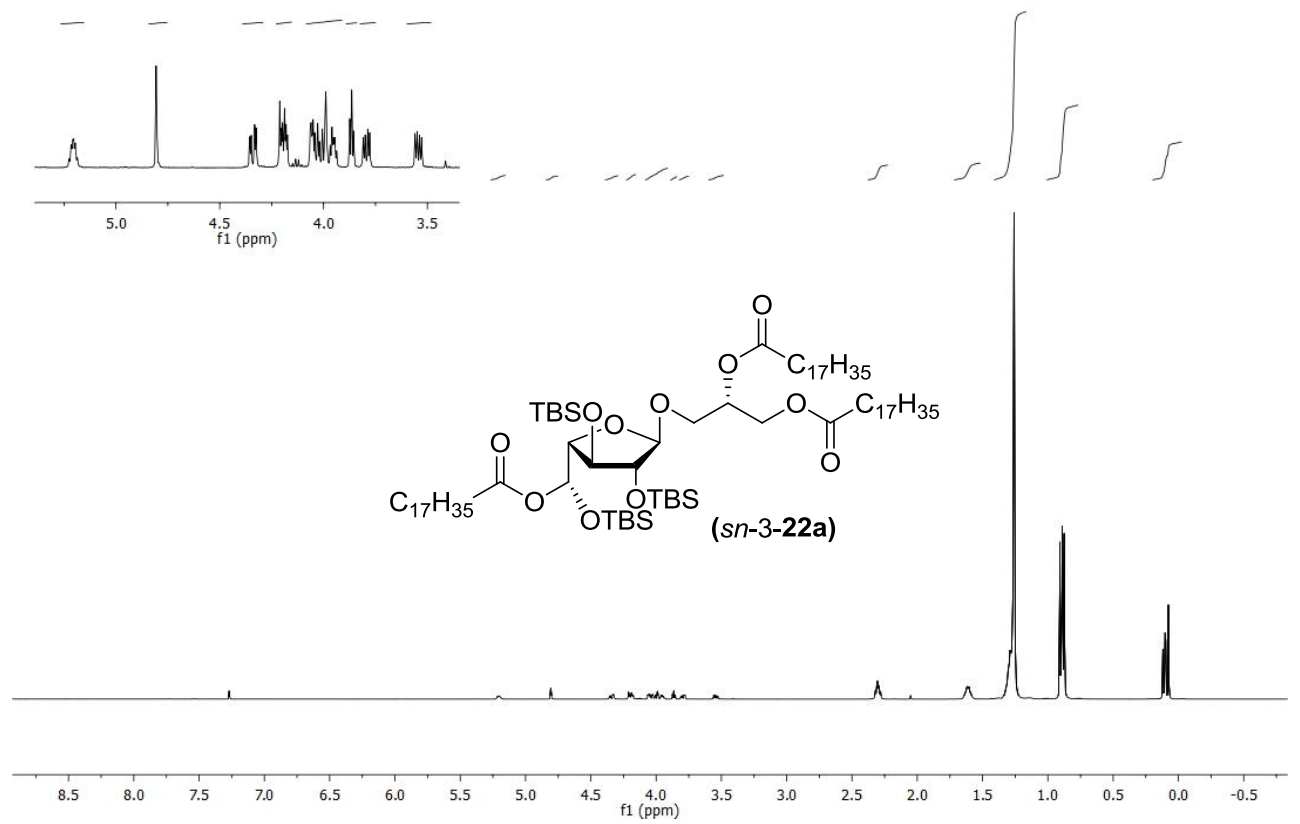

${ }^{13} \mathrm{C}$ NMR, $\mathrm{CDCl}_{3}, 125 \mathrm{MHz}$
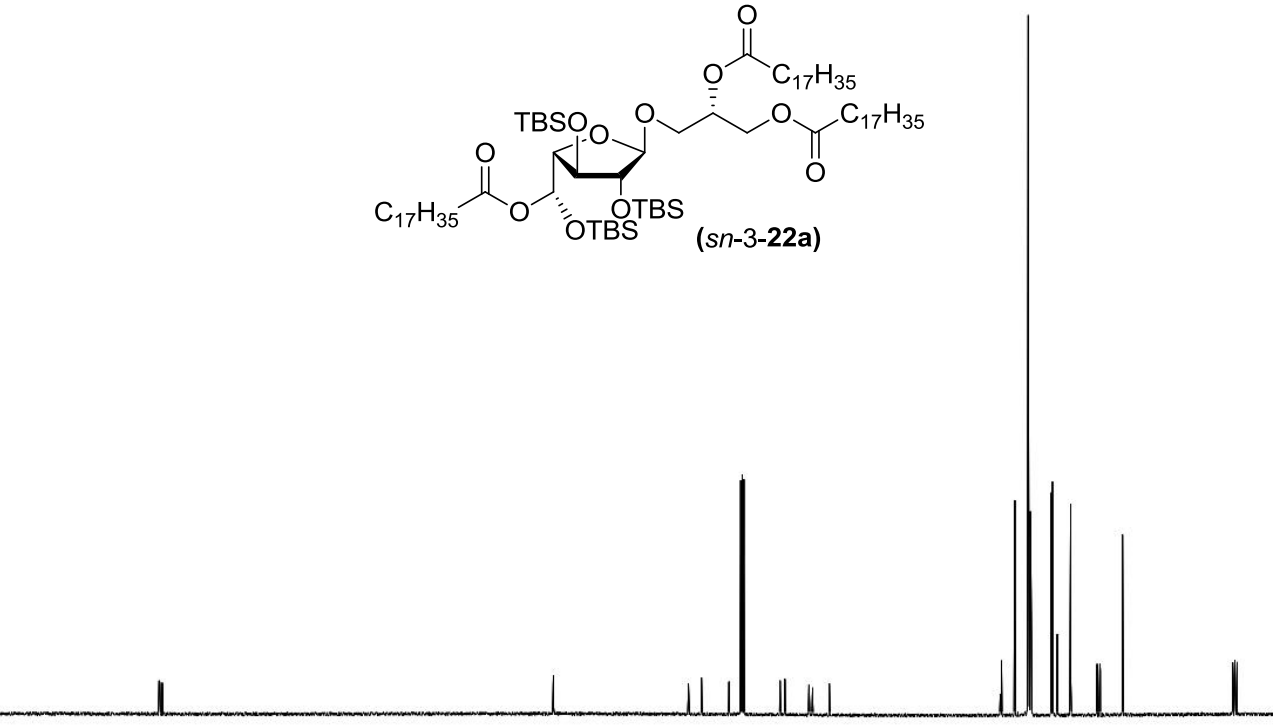

$\begin{array}{lllllllllll}30 & 190 & 180 & 170 & 160 & 150 & 140 & 130 & 120 & 110 & \substack{1 \\ \mathrm{f} 1(\mathrm{ppm})}\end{array}$ 
${ }^{1} \mathrm{H} \mathrm{NMR}, \mathrm{CD}_{3} \mathrm{OD} / \mathrm{CDCl}_{3}(1 / 2, \mathrm{v} / \mathrm{v}), 600 \mathrm{MHz}$

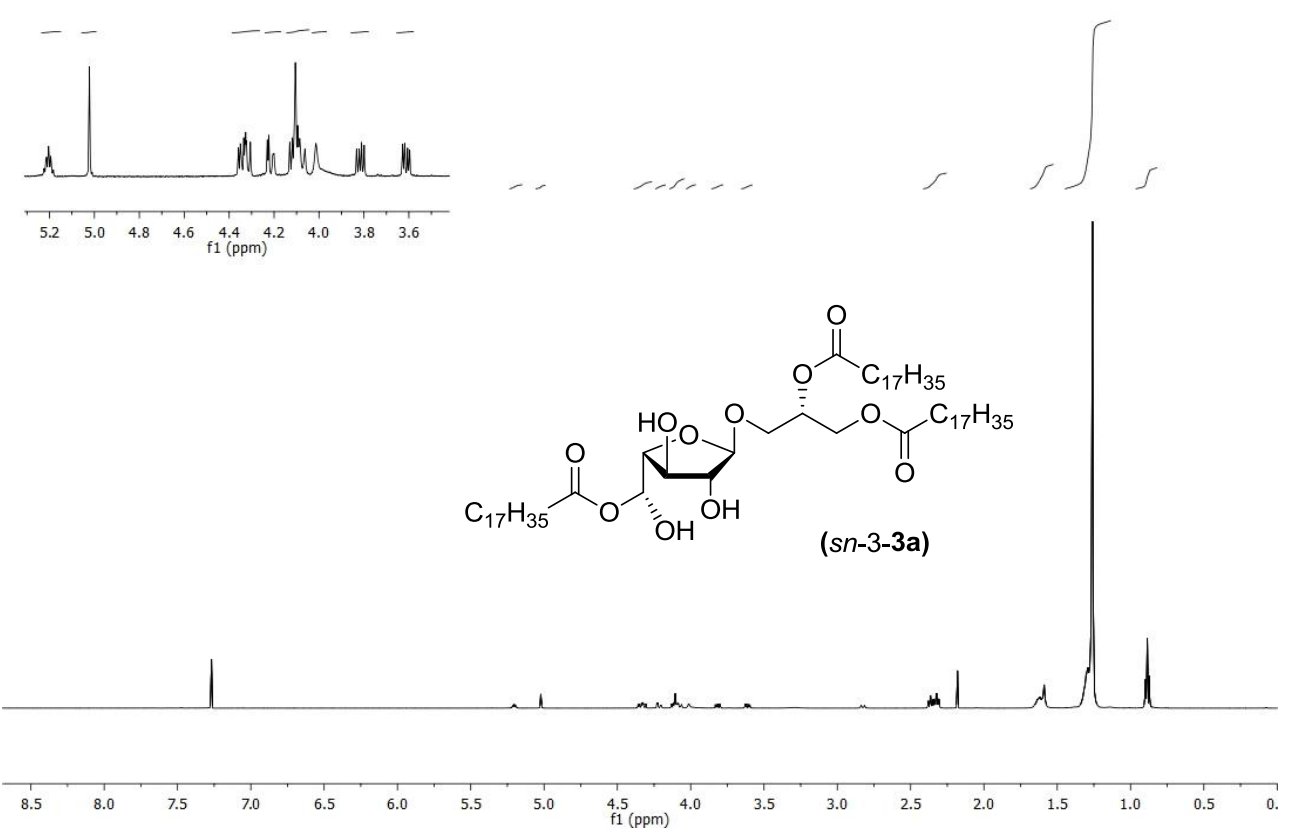

${ }^{13} \mathrm{C} \mathrm{NMR,} \mathrm{CD}_{3} \mathrm{OD} / \mathrm{CDCl}_{3}(1 / 2, \mathrm{v} / \mathrm{v}), 151 \mathrm{MHz}$
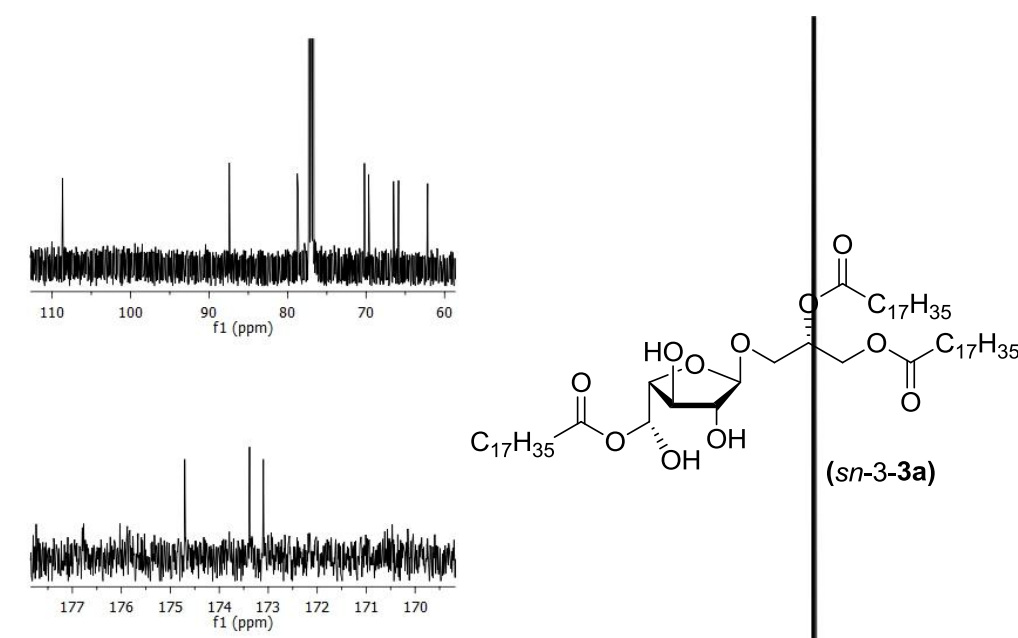

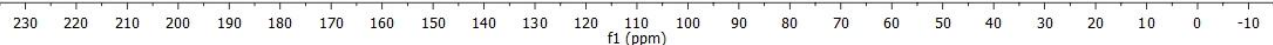


${ }^{1} \mathrm{H}$ NMR, $\mathrm{CDCl}_{3}, 500 \mathrm{MHz}$
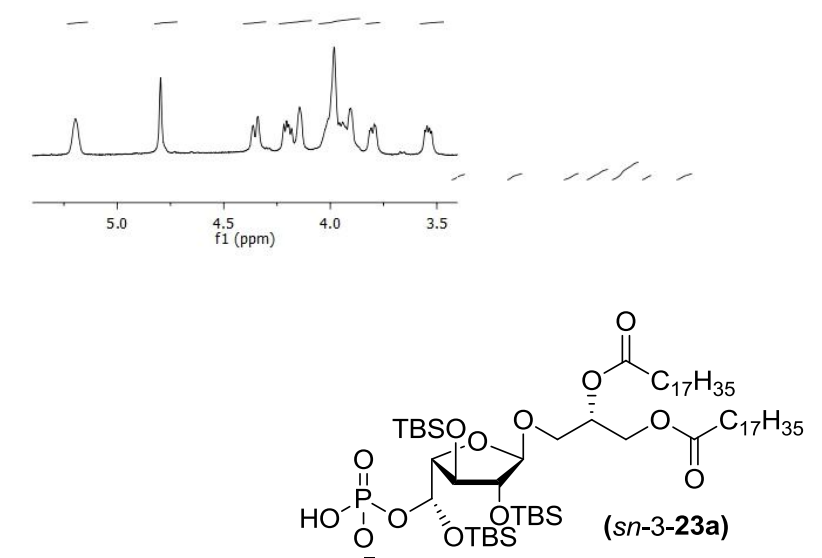

${ }^{13} \mathrm{C}$ NMR, $\mathrm{CDCl}_{3}, 125 \mathrm{MHz}$

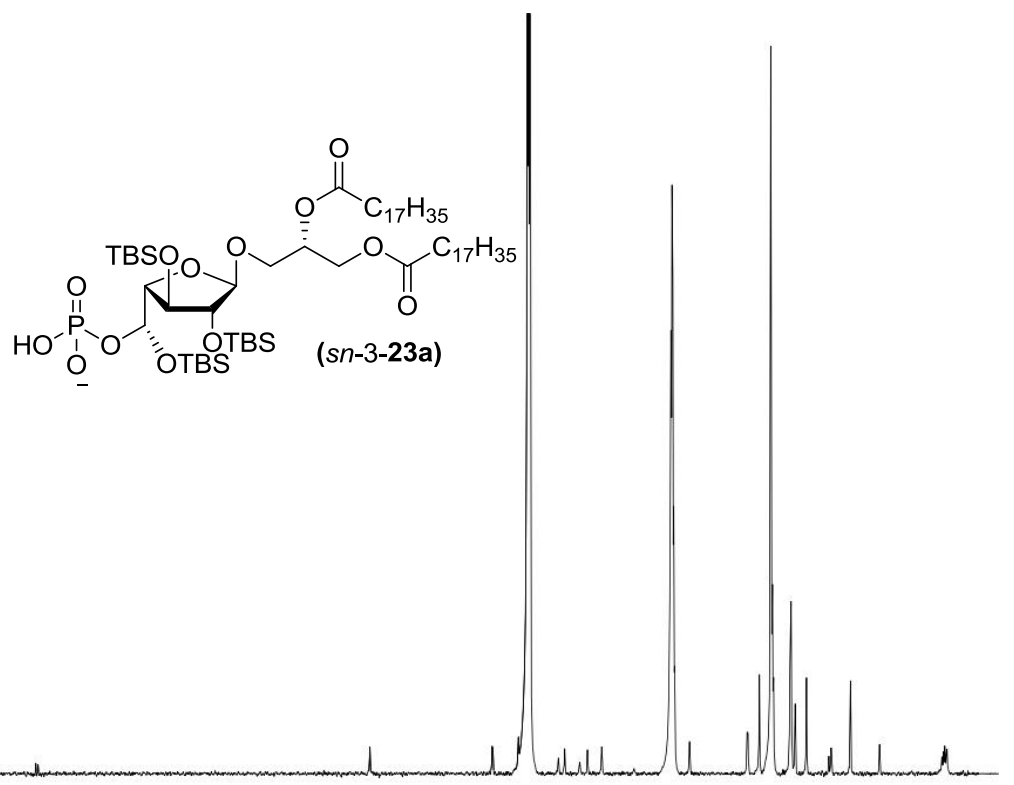

$\begin{array}{llllllllllllllllllllllllll}230 & 220 & 210 & 200 & 190 & 180 & 170 & 160 & 150 & 140 & 130 & 120 & \begin{array}{l}110 \\ \mathrm{f} 1(\mathrm{ppm})\end{array} & 100 & 90 & 80 & 70 & 60 & 50 & 40 & 30 & 20 & 10 & 0 & -10\end{array}$ 
${ }^{31} \mathrm{P} \mathrm{NMR}, \mathrm{CDCl}_{3}, 121 \mathrm{MHz}$

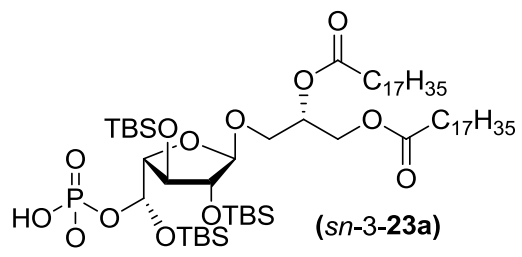

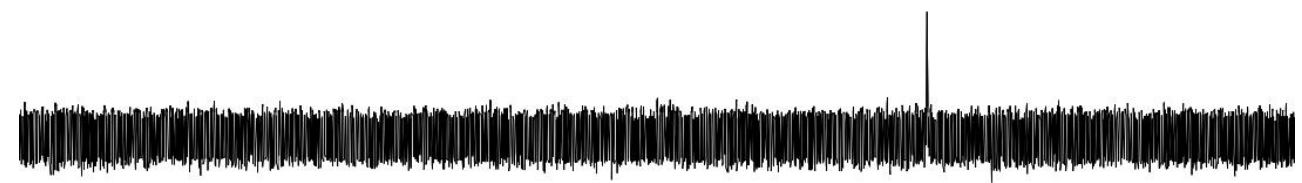

$50240230220210200190180170160150140130120110100 \quad 90 \begin{aligned} & 80 \\ & \mathrm{f1}(\mathrm{ppm})\end{aligned}$

${ }^{1} \mathrm{H},{ }^{31} \mathrm{P}, \mathrm{HMBC}, \mathrm{CDCl} 3,300 \mathrm{MHz}\left({ }^{1} \mathrm{H}\right), 121 \mathrm{MHz}\left({ }^{31} \mathrm{P}\right)$

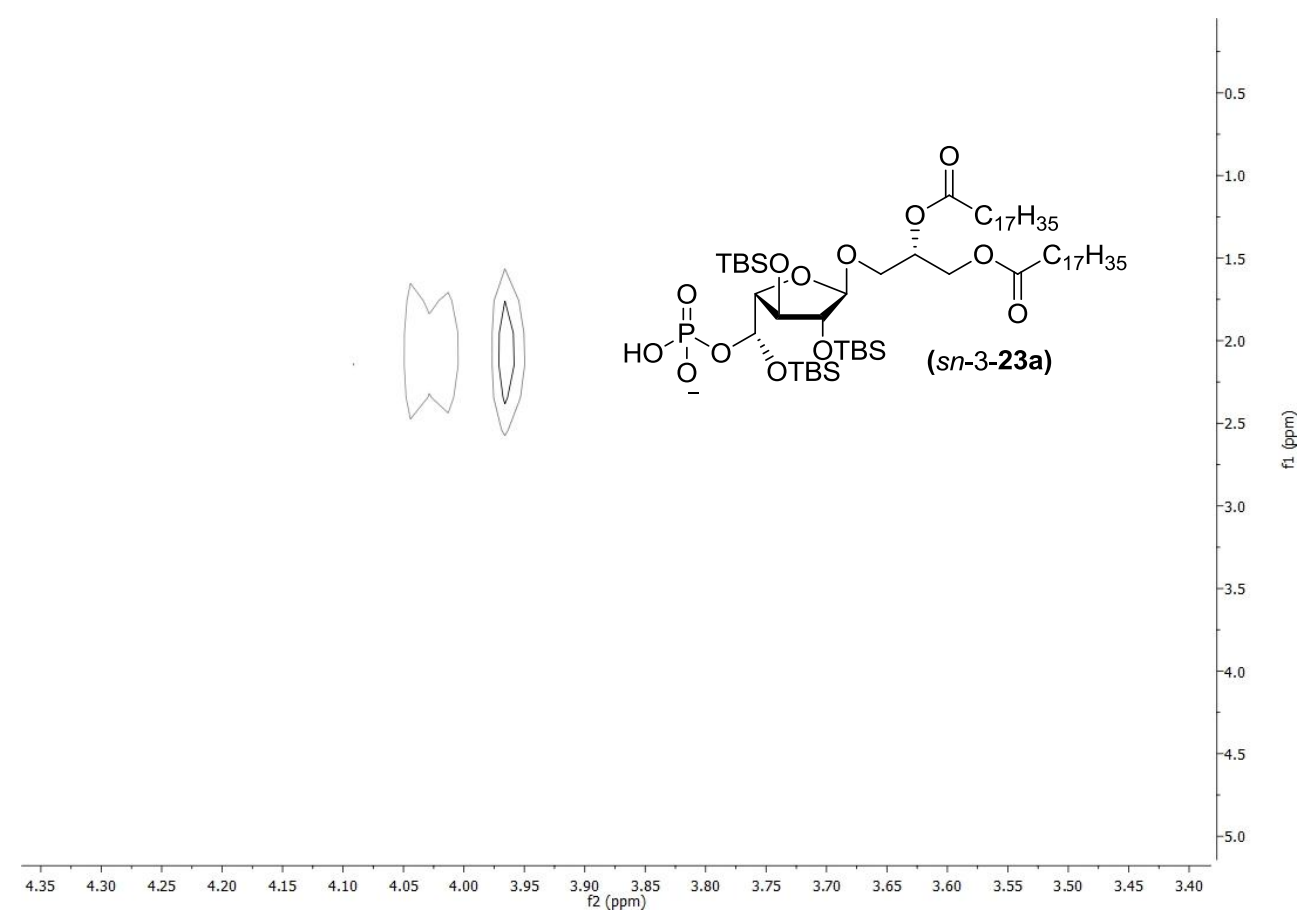


${ }^{1} \mathrm{H} \mathrm{NMR}, \mathrm{CDCl}_{3} / \mathrm{CD}_{3} \mathrm{OD} / \mathrm{D}_{2} \mathrm{O}(70 / 40 / 6, \mathrm{v} / \mathrm{v} / \mathrm{v}), 500 \mathrm{MHz}$

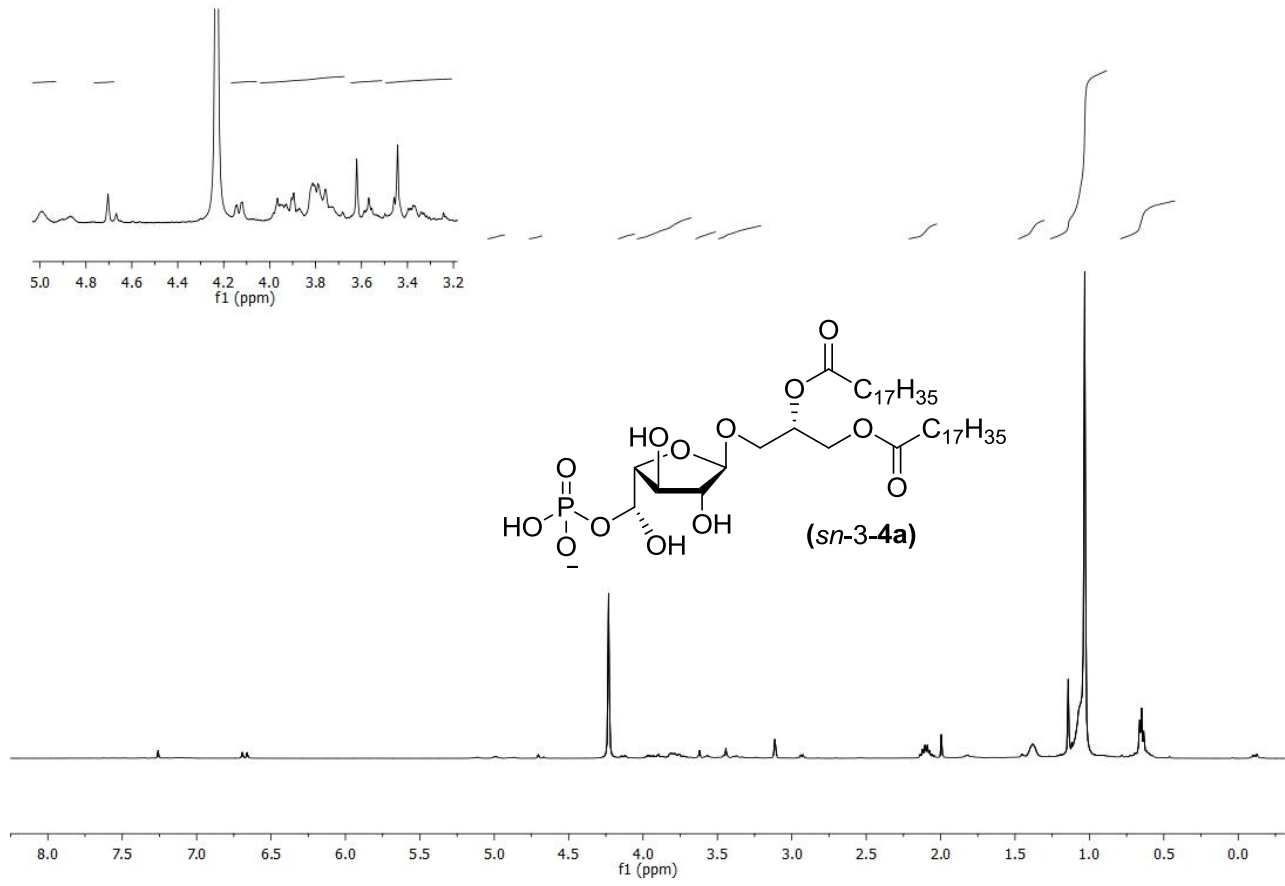

${ }^{13} \mathrm{C} \mathrm{NMR}_{\mathrm{CDCl}} / \mathrm{CD}_{3} \mathrm{OD} / \mathrm{D}_{2} \mathrm{O}(70 / 40 / 6, \mathrm{v} / \mathrm{v} / \mathrm{v}), 125 \mathrm{MHz}$

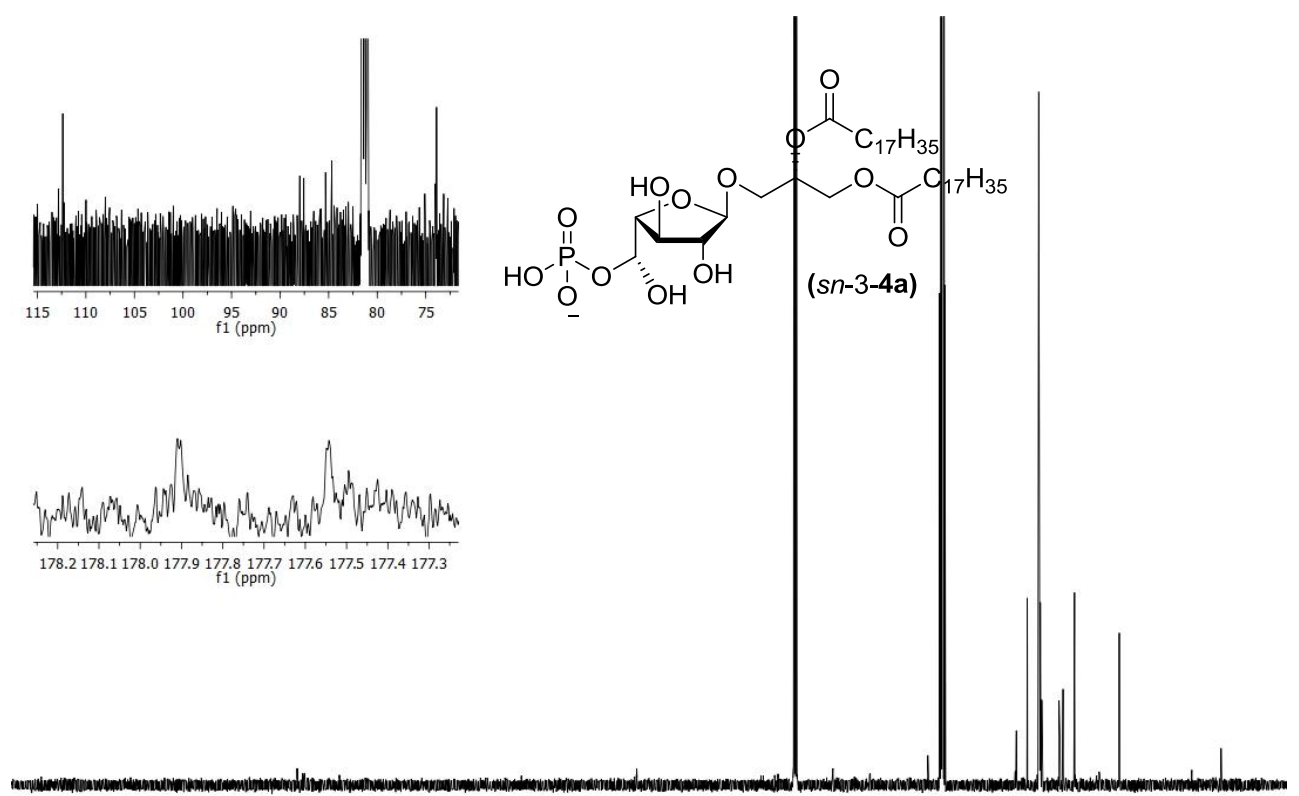

$\begin{array}{llllllllllllllllllllllllllllllll}1 & 230 & 220 & 210 & 200 & 190 & 180 & 170 & 160 & 150 & 140 & 130 & 120 & 110 & 10 & 1 & 1 & 1 & 1 & 1 & 1 & 1 & 1 & 1 & 1 & 1 & 1 & 1 & 1 & 1 & 1 & 1\end{array}$ 


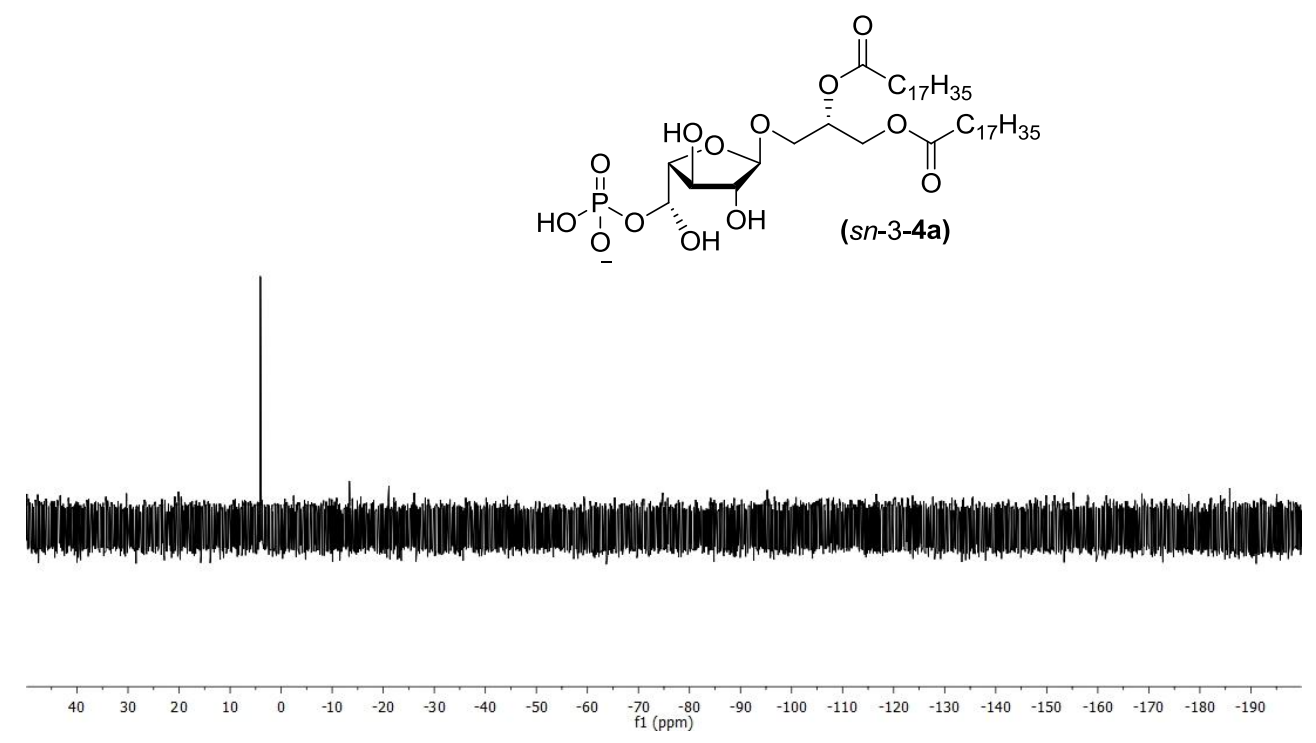

${ }^{1} \mathrm{H} \mathrm{NMR}, \mathrm{CDCl}_{3}, 500 \mathrm{MHz}$

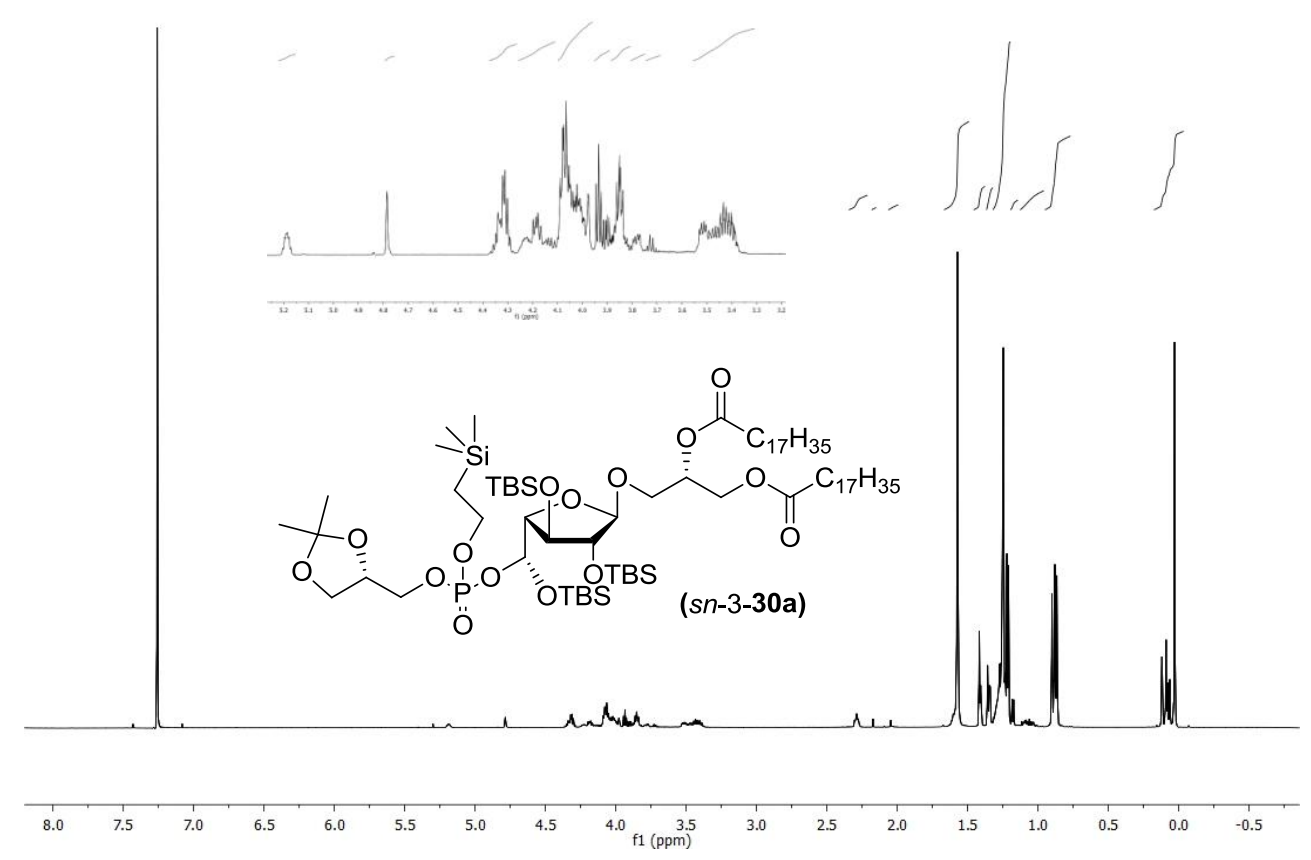


${ }^{13} \mathrm{C} \mathrm{NMR}, \mathrm{CDCl}_{3}, 500 \mathrm{MHz}$

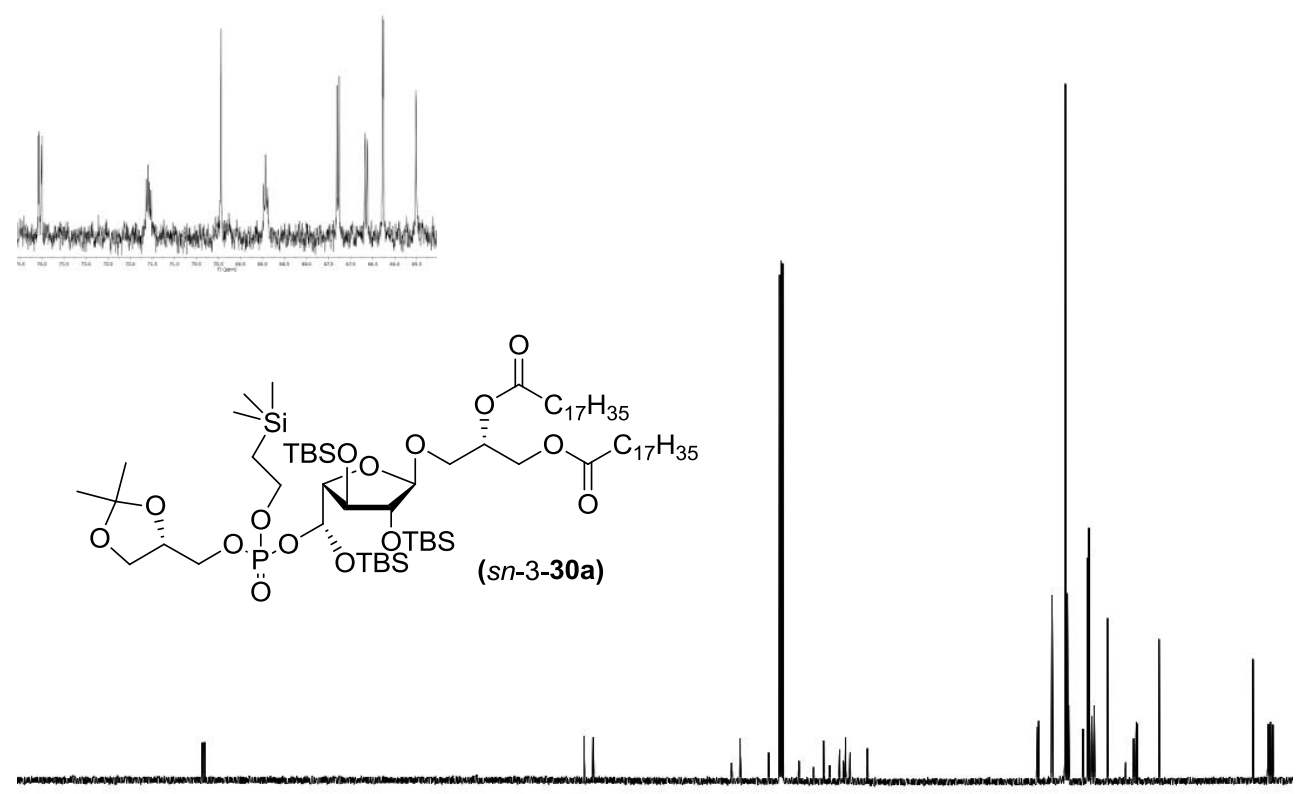

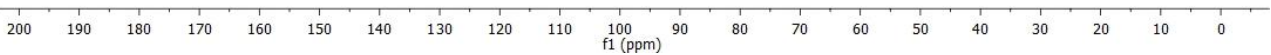

${ }^{13} \mathrm{C} \mathrm{NMR}, \mathrm{CDCl}_{3}, 125 \mathrm{MHz}$

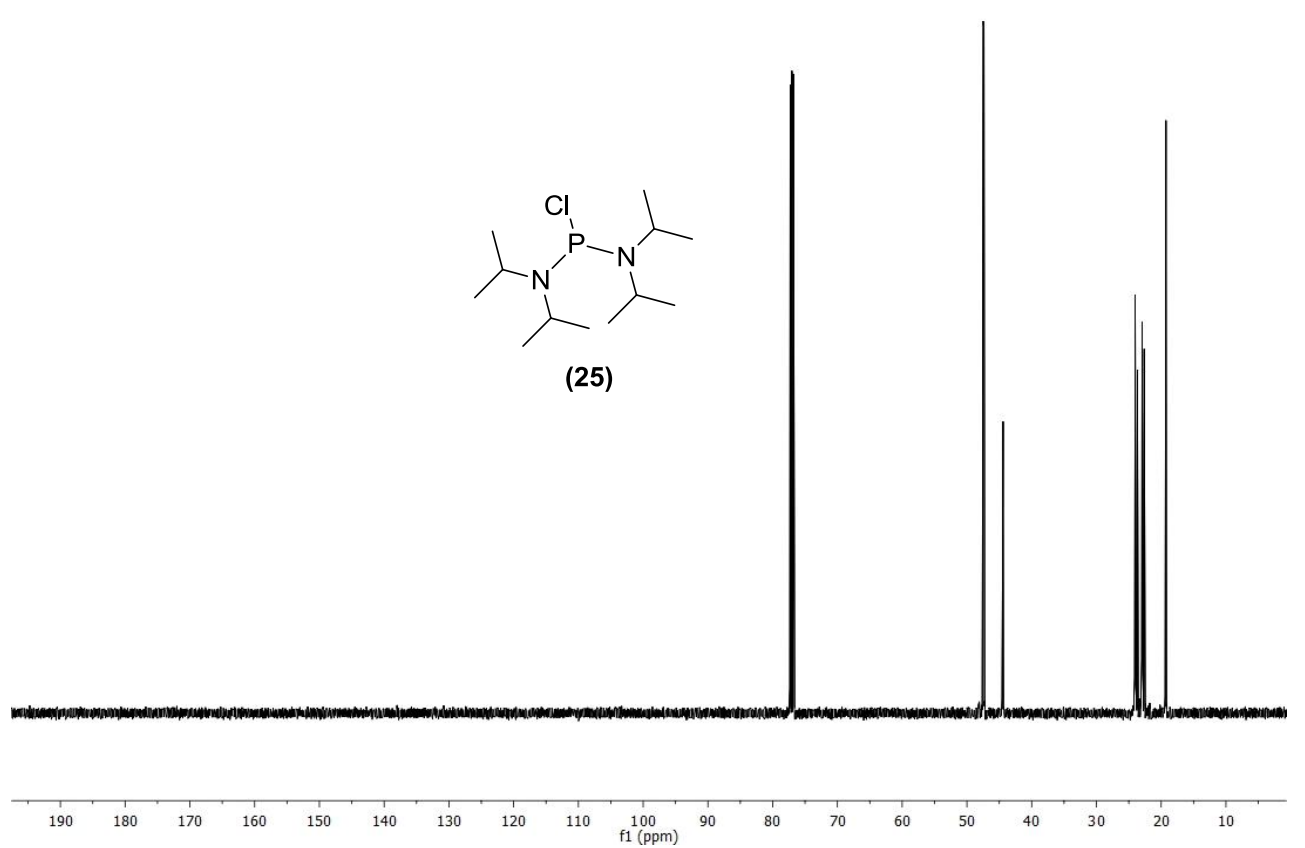


${ }^{31} \mathrm{P} \mathrm{NMR}, \mathrm{CDCl}_{3}, 121 \mathrm{MHz}$

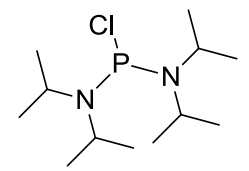

(25)

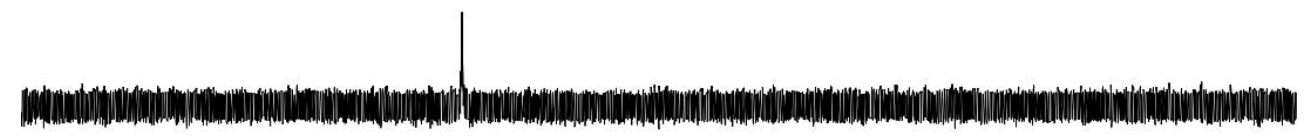

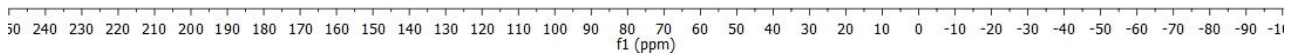

${ }^{1} \mathrm{H}$ NMR, $\mathrm{CDCl}_{3}, 500 \mathrm{MHz}$
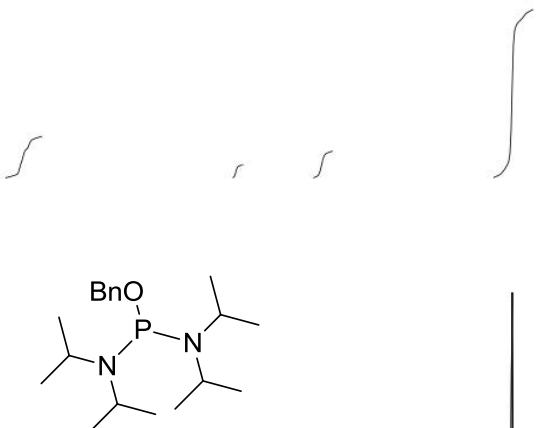

(32)

$\mu$

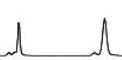
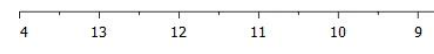

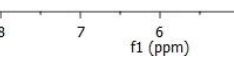


${ }^{13} \mathrm{C} \mathrm{NMR} \mathrm{CDCl}_{3}, 125 \mathrm{MHz}$

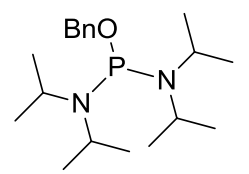

(32)

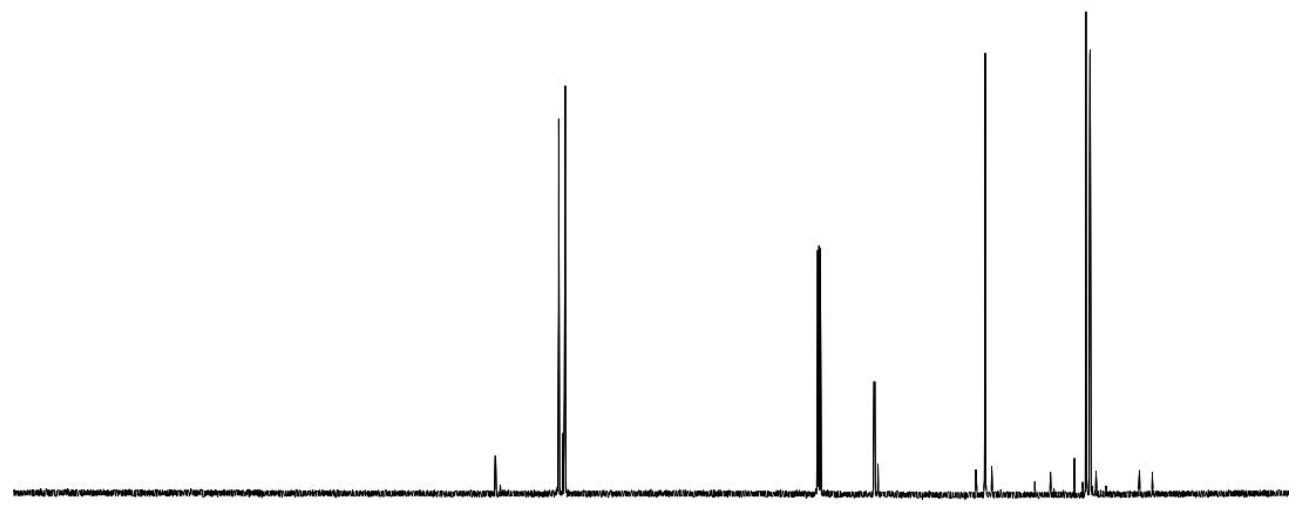

$\begin{array}{lllllllllllllllllllllllllllllll}230 & 220 & 210 & 200 & 190 & 180 & 170 & 160 & 150 & 140 & 130 & 120 & 110 & 100 & 90 & 80 & 70 & 60 & 50 & 40 & 30 & 20 & 10 & 0 & -10\end{array}$

${ }^{31} \mathrm{P} \mathrm{NMR}, \mathrm{CDCl}_{3}, 121 \mathrm{MHz}$

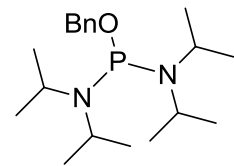

(32)

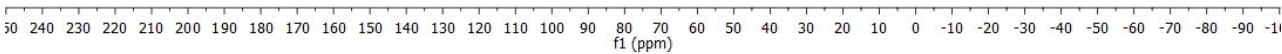


${ }^{1} \mathrm{H} \mathrm{NMR}, \mathrm{CDCl}_{3}, 500 \mathrm{MHz}$

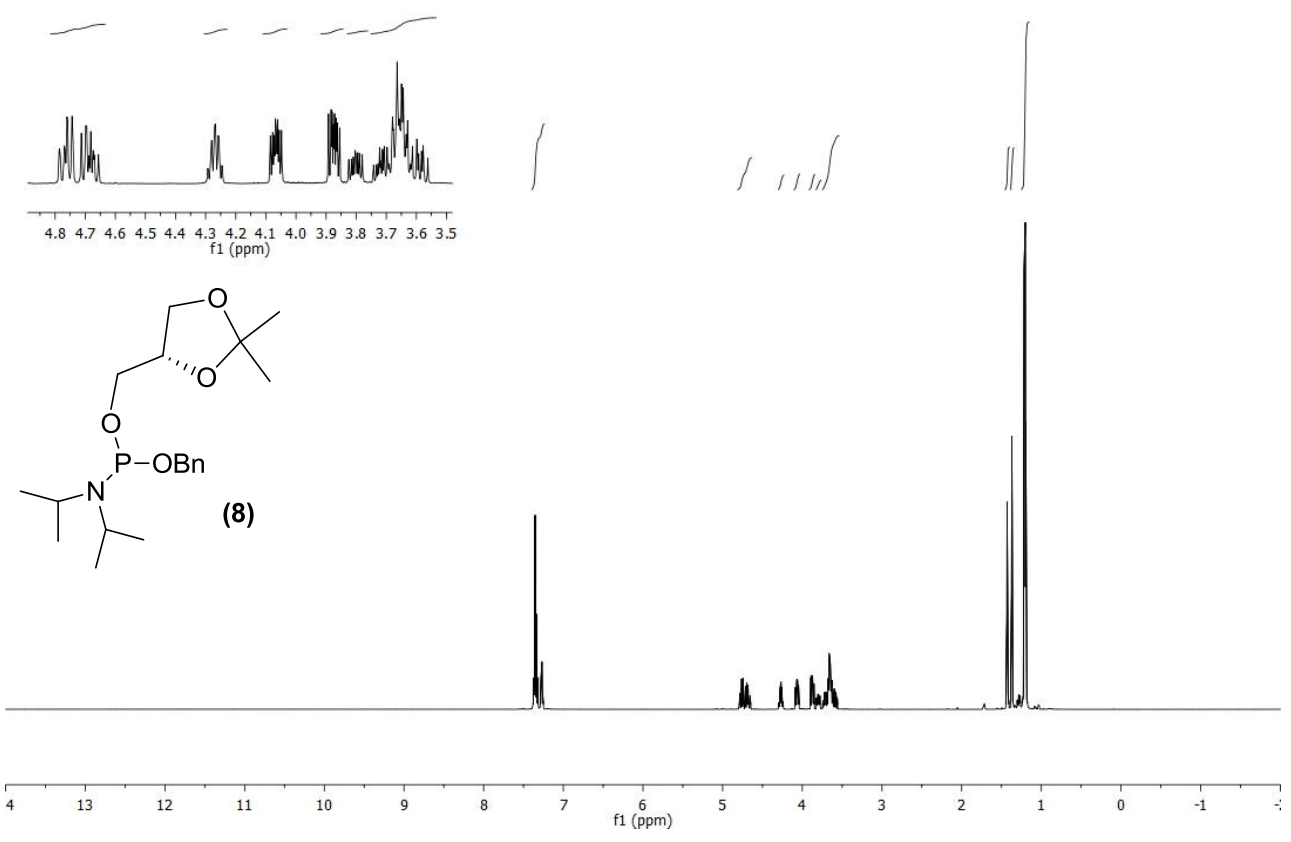

${ }^{13} \mathrm{C}$ NMR, $\mathrm{CDCl}_{3}, 125 \mathrm{MHz}$

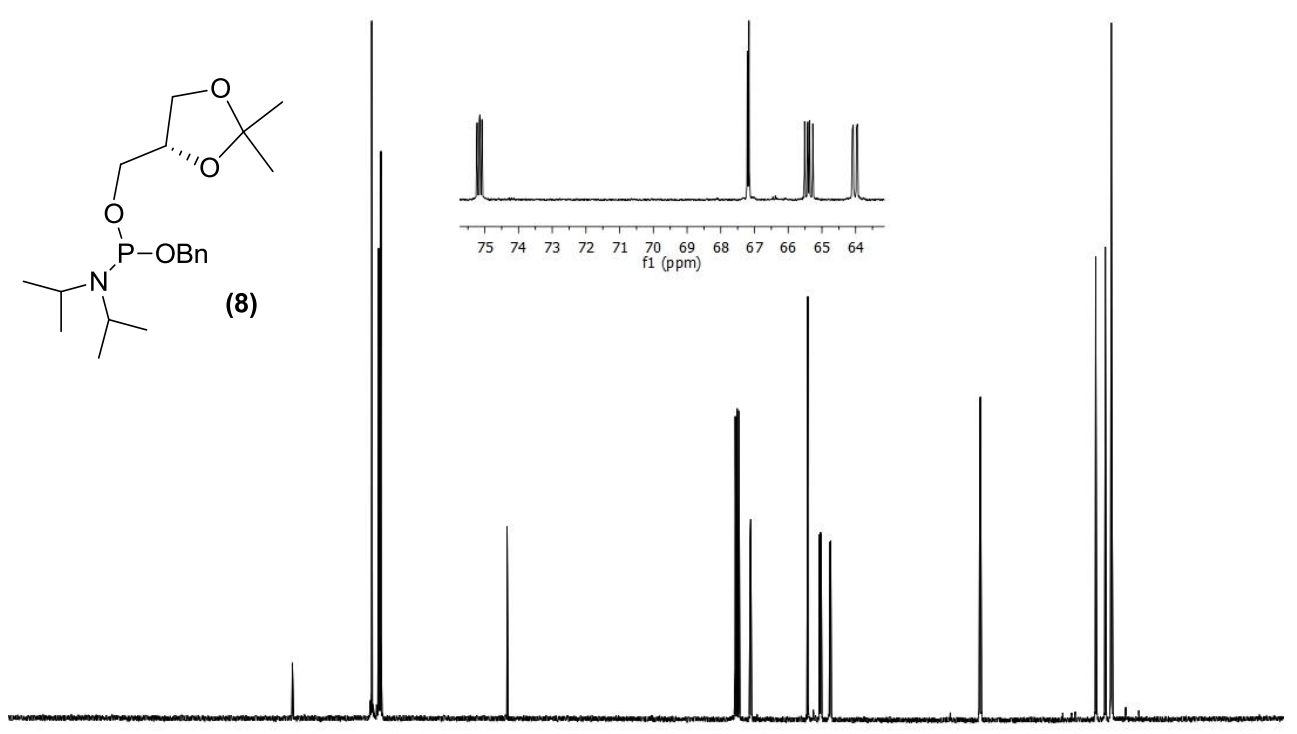


${ }^{31} \mathrm{P} \mathrm{NMR}, \mathrm{CDCl}_{3}, 121 \mathrm{MHz}$
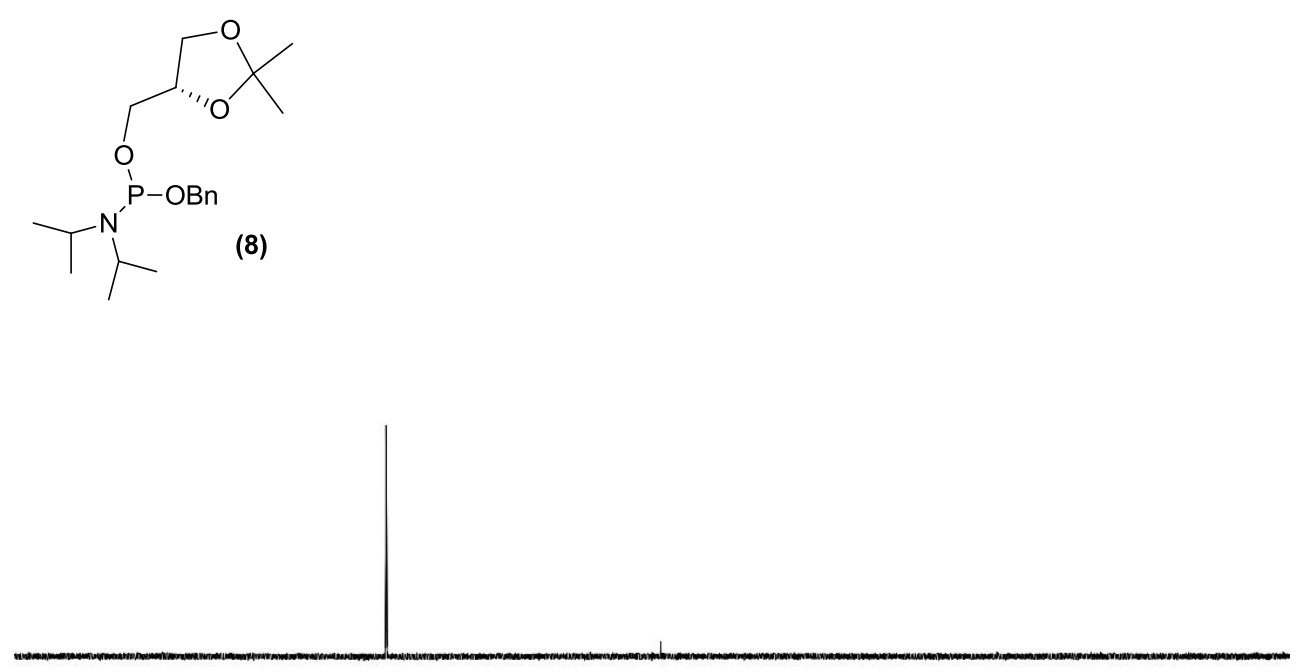

$\begin{array}{llllllllllllllllllllllllllllllllllllllll}50 & 240 & 230 & 220 & 210 & 200 & 190 & 180 & 170 & 160 & 150 & 140 & 130 & 120 & 110 & 100 & 90 & 80 & 70 & 60 & 50 & 40 & 30 & 20 & 10 & 0 & -10 & -20 & -30 & -40 & -50 & -60 & -70 & -80 & -90 & -1\end{array}$

${ }^{1} \mathrm{H} \mathrm{NMR}, \mathrm{CDCl}_{3}, 600 \mathrm{MHz}$

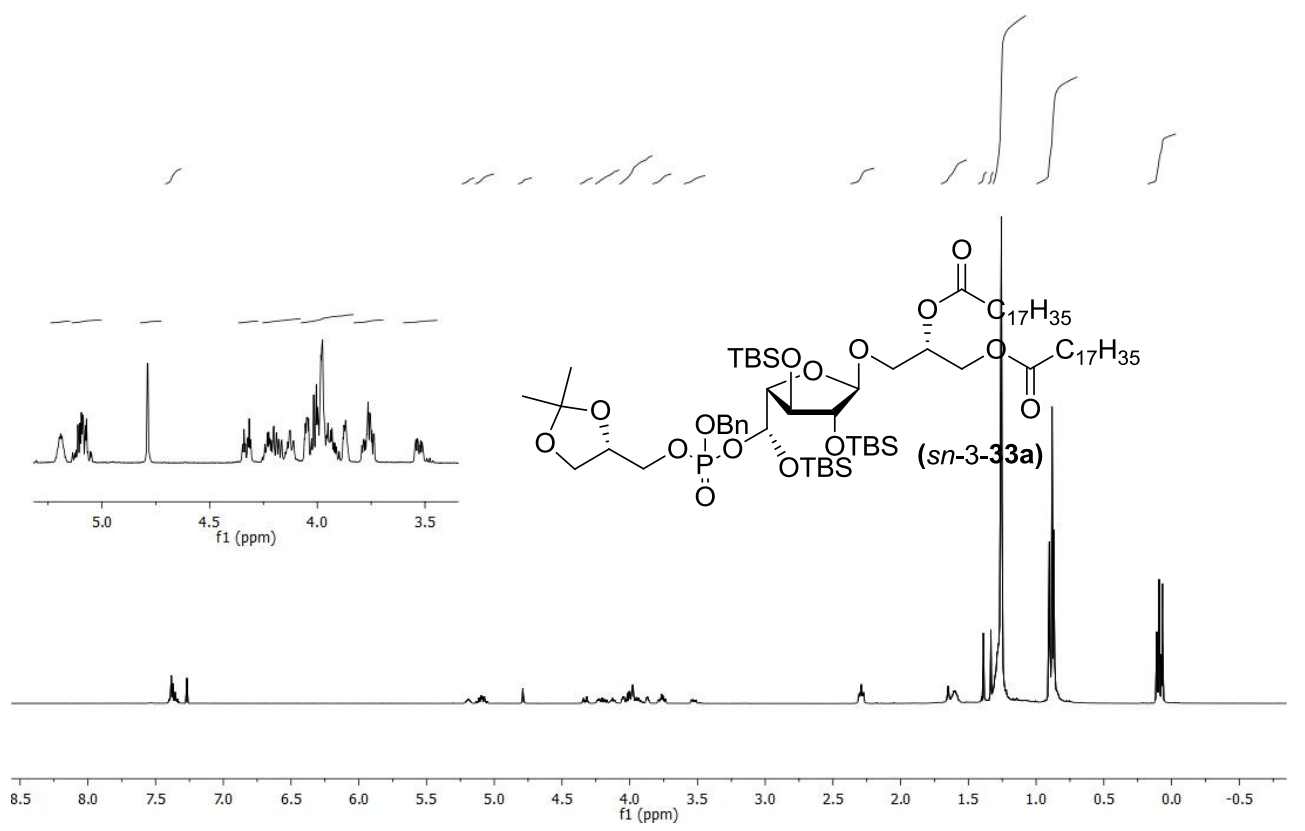


${ }^{13} \mathrm{C} \mathrm{NMR}, \mathrm{CDCl}_{3}, 151 \mathrm{MHz}$
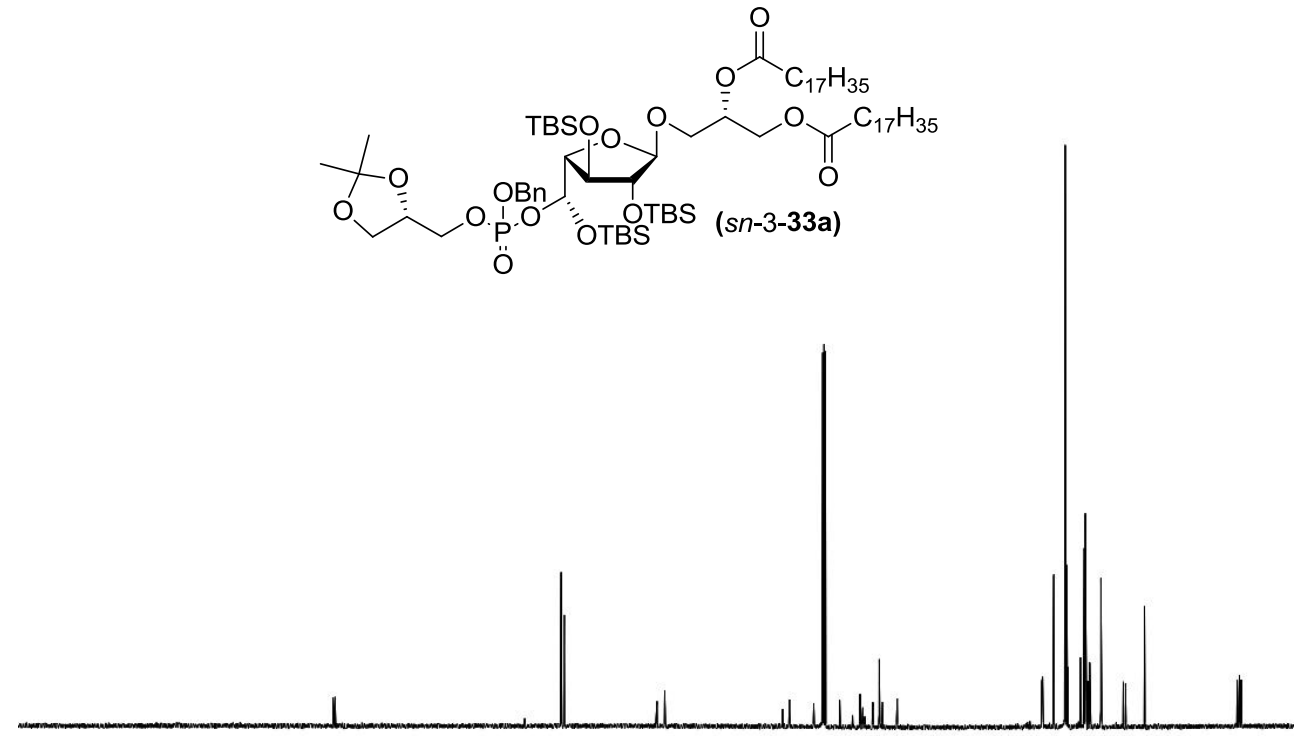

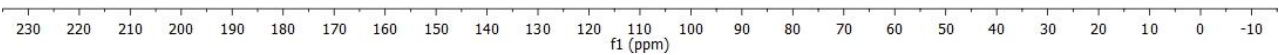

${ }^{31} \mathrm{P} \mathrm{NMR}, \mathrm{CDCl}_{3}, 121 \mathrm{MHz}$
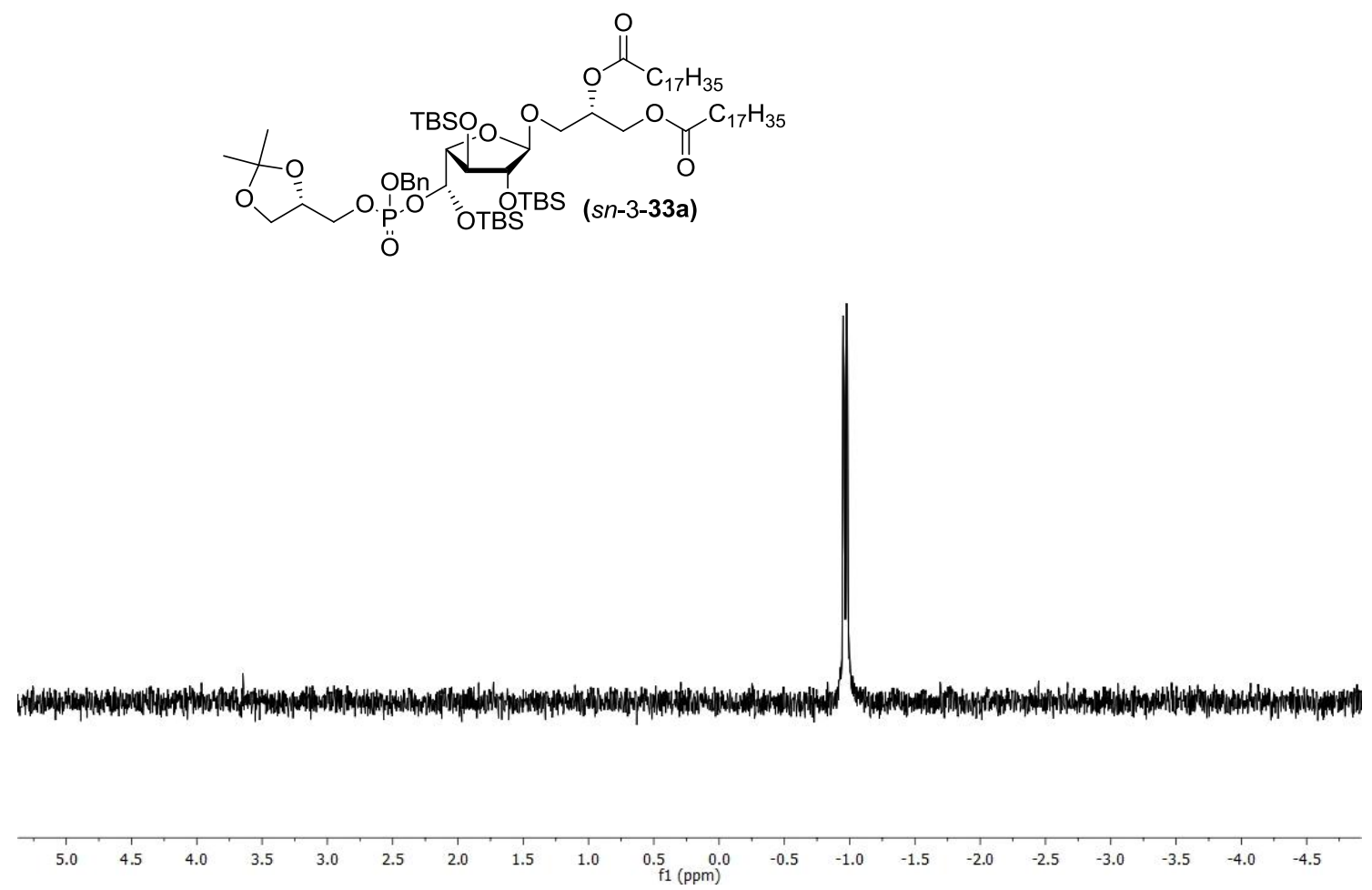
${ }^{1} \mathrm{H},{ }^{31} \mathrm{P}$ NMR HMBC, $\mathrm{CDCl}_{3}, 300 \mathrm{MHz}\left({ }^{1} \mathrm{H}\right), 121 \mathrm{MHz}\left({ }^{31} \mathrm{P}\right)$

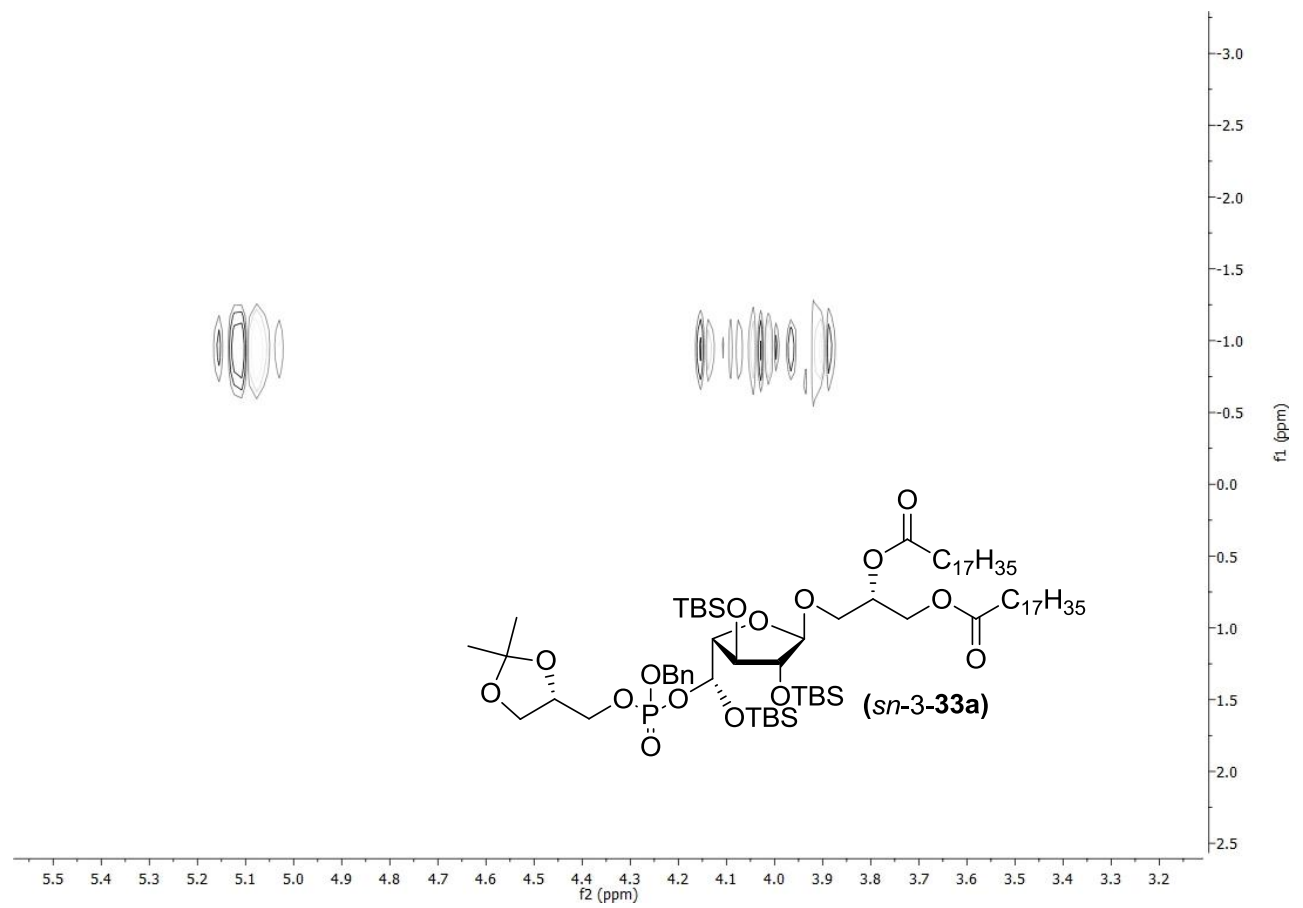

${ }^{1} \mathrm{H} \mathrm{NMR}, \mathrm{CDCl}_{3} / \mathrm{CD}_{3} \mathrm{OD} / \mathrm{D}_{2} \mathrm{O}(70 / 40 / 6, \mathrm{v} / \mathrm{v} / \mathrm{v}), 600 \mathrm{MHz}$
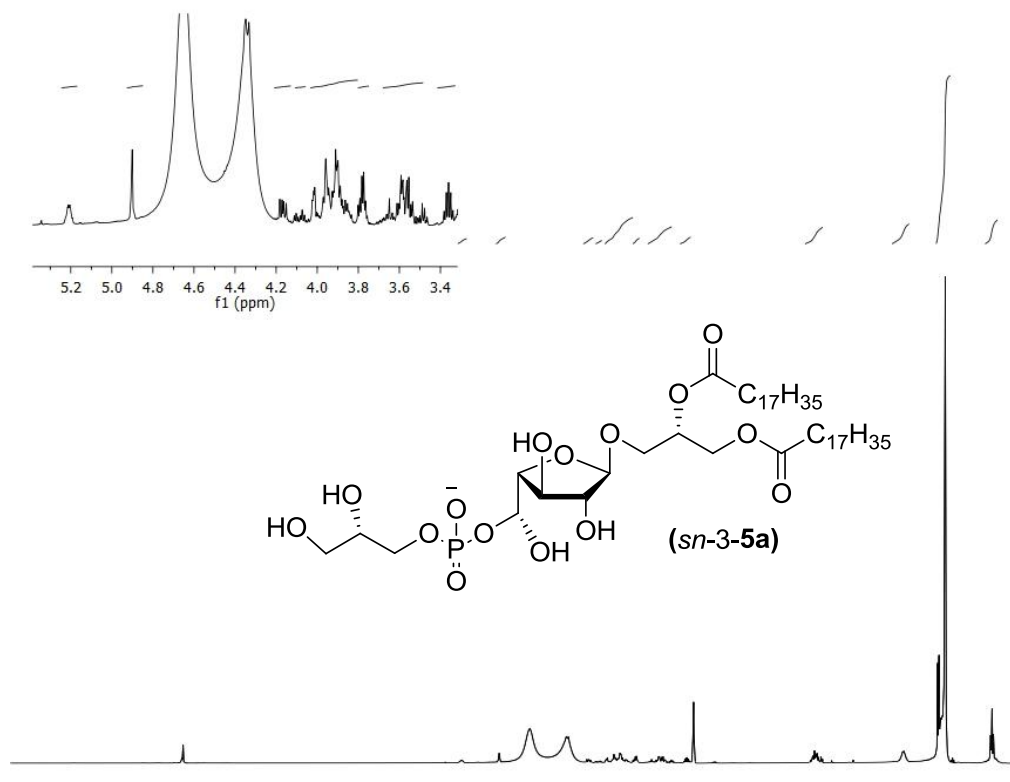

$\begin{array}{llllllllllll}8.5 & 8.0 & 7.5 & 7.0 & 6.5 & 6.0 & 5.5 & 5.0 & 4.5 & 4.0 & 3 \\ & & & & & & & & & & & \end{array}$ 
${ }^{13} \mathrm{C} \mathrm{NMR}, \mathrm{CDCl}_{3} / \mathrm{CD}_{3} \mathrm{OD} / \mathrm{D}_{2} \mathrm{O}(70 / 40 / 6, \mathrm{v} / \mathrm{v} / \mathrm{v}), 151 \mathrm{MHz}$

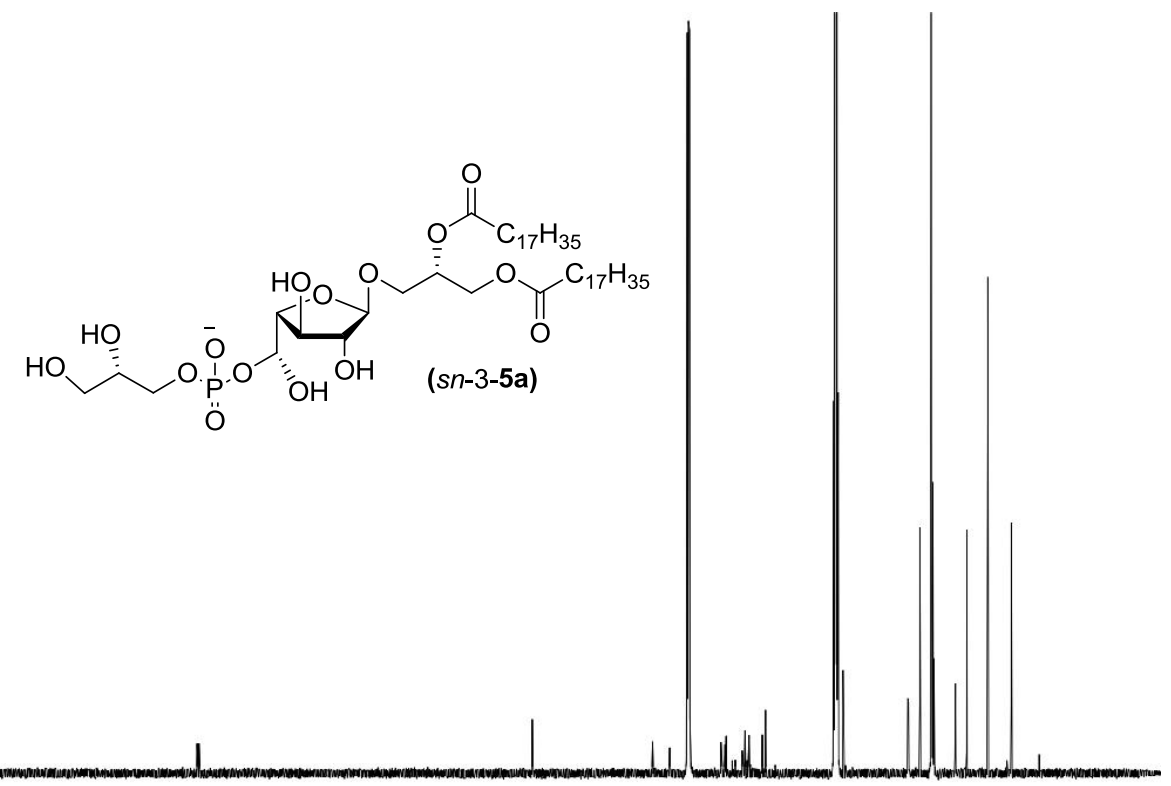

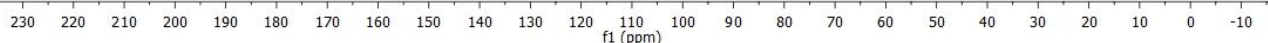

${ }^{31} \mathrm{P} \mathrm{NMR}, \mathrm{CDCl}_{3} / \mathrm{CD}_{3} \mathrm{OD} / \mathrm{D}_{2} \mathrm{O}(70 / 40 / 6, \mathrm{v} / \mathrm{v} / \mathrm{v}), 121 \mathrm{MHz}$

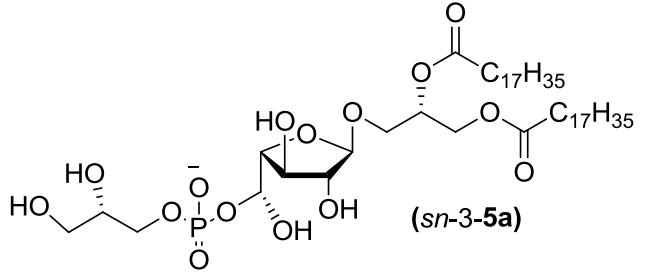

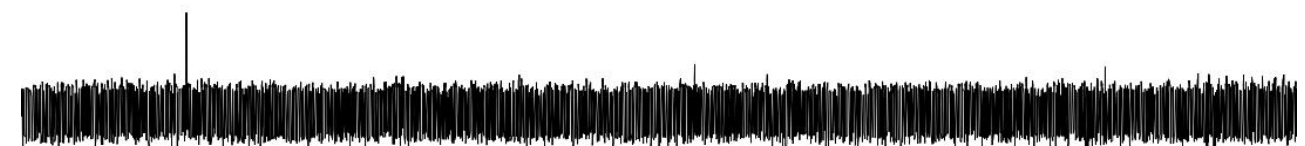

$\begin{array}{lllllllllllllllllllllllll}30 & 20 & 10 & 0 & -10 & -20 & -30 & -40 & -50 & -60 & -70 & -80 & -90 & -100 & -110 & -120 & -130 & -140 & -150 & -160 & -170 & -180 & -190 & -200 & -210\end{array}$ 
${ }^{1} \mathrm{H},{ }^{31} \mathrm{P}, \mathrm{HMBC}, \mathrm{CDCl}_{3} \mathrm{CD}_{3} \mathrm{OD} / \mathrm{D}_{2} \mathrm{O}(70 / 40 / 6, \mathrm{v} / \mathrm{v} / \mathrm{v}), 300 \mathrm{MHz}\left({ }^{1} \mathrm{H}\right) 121 \mathrm{MHz}\left({ }^{31} \mathrm{P}\right)$

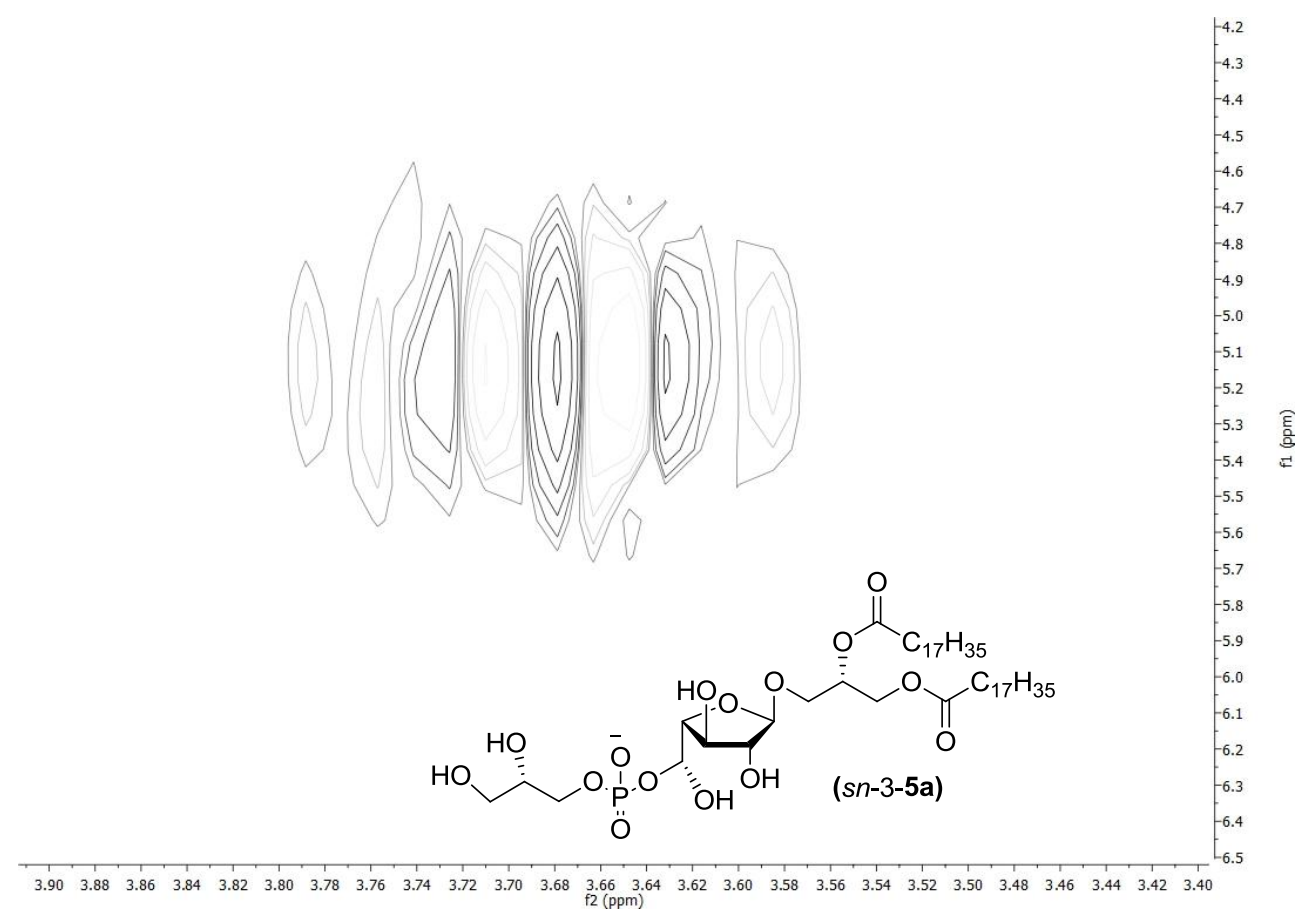




\section{Chapter 5}

\section{Future Prospects and Conclusion}

Reports concerning bacterial glycosyldiglycerides claim that glycosyldiglycerides have immunological properties ${ }^{104}$ while other publications reveal that synthesised glycolipid anchors show only weak activity. For example, Deiniger synthesised the LTA anchor 3$O$-( $\beta$-D-gentiobiosyl)-1,2-di- $O$-myristoyl-sn-glycerol and found that it only weakly activated whole blood cells compared to its synthesised LTAs. ${ }^{81}$ Furthermore, glycoglycerolipids with differing fatty acid chains seem to exhibit different immunological properties. ${ }^{78}$ Indeed, Kinjo et al. synthesised a number of $\alpha$-D-Gal $p$ diglyceride analogues by varying the type of fatty acid on $s n-1$ and $s n$-2-position of the glycerol. They found that 3-O-( $\alpha$-D-galactopyranosyl)-1- $O$-oleoyl-2- $O$-palmitoyl-snglycerol was the most potent antigen as it induced the highest level of IL-2 production in $i$ NKT cells compared to the other glycolipids (Chapter 1, Section 1.5.4). These seemingly contradictory results were intriguing and thus the aim of this thesis was to shed light on the confusion regarding glycosyldiglyceride activity by providing a detailed structural analysis of the glycolipids tested from gut commensal bacteria and synthesising defined glycolipid structures. First, glycolipids were extracted from a beneficial commensal present in the human gut (L. plantarum). Here, the anomeric 
configuration of the residues was clarified and the compounds tested for their ability to activate macrophages. Although whole L. plantarum cells have been reported, in the literature, to activate macrophages, the purified glycolipids in this research demonstrated no significant activity (Chapter 2). After elucidating the structure of the glycolipids from $L$. plantarum the next step was to study the glycolipids from bifidobacteria to determine if glycolipids from different commensals activated macrophages to the same extent. Thus, the glycolipids from $B$. longum subs. infantis were purified and their structure elucidated. Interestingly, a novel glycolipid structure was found (GL5). Again, the glycolipids showed no significant macrophage activity (Chapter 3). Figure 5.1 shows the ability of glycolipids extracted from L. plantarum (GL1-GL3) to activate macrophages compared and the glycolipids extracted from $B$. longum subs. infantis (GL4 and GL5) compared to LPS. None of the isolated glycolipids induced significant NO production in BMM. ${ }^{1}$

As glycolipids are generally considered as forming the anchor for LTAs, with LTAs shown to be active (Chapter 1, Section 1.5.5), the next logical step was to analyze LTAs for their biological activity. However, due to the difficulties of LTA purification, most of the activation observed in LTAs produced from extracted and purified LTAs preparations was attributed to lipoprotein contamination (Chapter 1, Section 1.4.4.3). ${ }^{55}$ Thus the best approach was to synthesise LTA analogues for comparison. Therefore, Chapter 4 illustrated the synthesis of LTA derivatives from Streptococcus 8747 which are also analogues of the novel glycolipid (GL5) found in Chapter 3. In Chapter 4, the synthesis of glycolipid anchors containing different fatty acid chain lengths with phosphated derivatives was achieved. The glycolipids were tested for their ability to activate BMM. The analyzes showed that most of the glycosyldiglycerides activated bone marrow macrophages only to a small extent. However, some compounds such as sn-3-2b (Figure 5.2), compared to other glycosyldiglycerides, significantly activated macrophages. The activity seem to be related to the type of fatty acid present on the sugar moiety, which is not surprising as the activity of other glycolipids have been found to correspond to lipid length. ${ }^{82}$

\footnotetext{
${ }^{1}$ Assay conducted by Ashna Khan and Stephanie Chee
} 


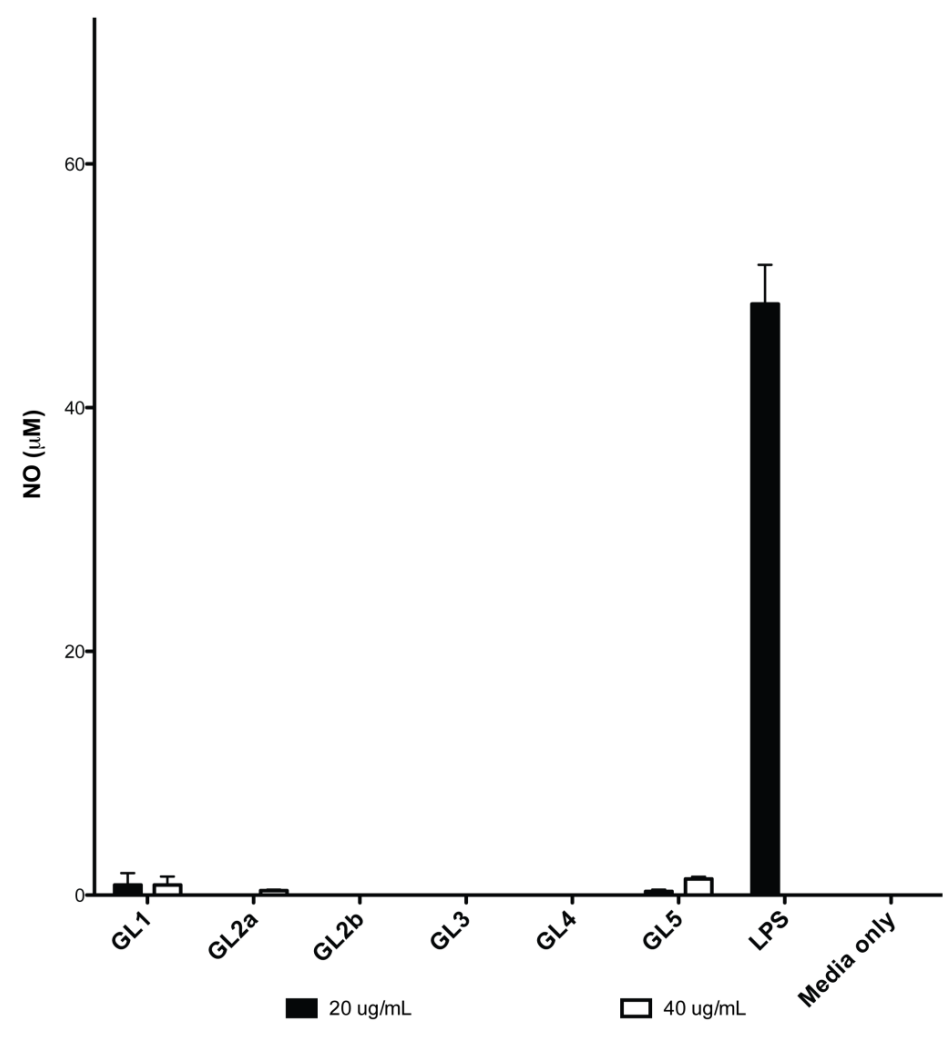

Figure 5.1 Glycoglycerolipids NO production by BMMs. BMMs were stimulated with glycoglycerolipids (GL1, GL2a, GL2b, GL3, GL4 and GL5) (20 $\mu \mathrm{g} / \mathrm{mL}$ and 40 $\mu \mathrm{g} / \mathrm{mL})$, or LPS (100 $\mathrm{ng} / \mathrm{mL})$ and the supernatants were analyzed for NO after $48 \mathrm{~h}$ using the Griess assay. Mean and SD of triplicate samples from a representative experiment of two performed are indicated.

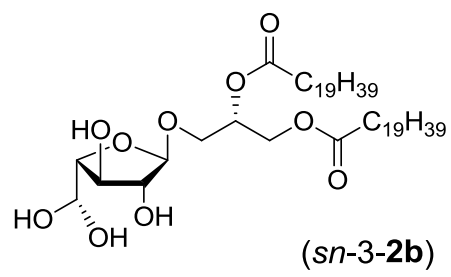

Figure 5.2 sn-3-2b activates BMM.

The biological studies conducted in this work are only a preliminary screening and additional testing is needed to assess the full biological potential of these glycolipids. At 
first, as described in Chapter 2, the glycolipids are dissolved in a small amount of DMSO before being diluted in a buffer to obtain a suspension after multiple cooling down and heating up iterations. Due to the varying extents of solubility of these glycolipids, different amounts of glycolipid are dissolved using this standard solubilisation protocol. Thus, solubility studies need to be conducted using higher amounts of DMSO. Also, as Kinjo et al. found that glycolipids derived from $B$. burgdorferi, with varying chain lengths, induced IL-2 production in $i$ NKT cells (Chapter 1, Section 1.5.4), such an assay might be interesting to pursue. ${ }^{82}$

From the work presented in this thesis, preliminary studies were undertaken and a number of avenues arise for possible future research. Firstly, it would be of interest to analyze structurally and biologically the LTAs from bifidobacteria. Indeed, the presence of putative bifidobacterial LTAs responsible for the beneficial effects of bifidobacteria is a noteworthy subject. Secondly, identification of the stereochemistry of the three unknown stereocenters of GL5 would be required for further synthesis of analogues. Finally, synthetic analogues with defined stereocenters need to be made so that they can be compared with the extracted glycolipids. This will allow for the determination of all the stereocenters. Furthermore, the development of methodology for such a synthesis could lead to the synthesis of analogues with varying lipid chain lengths. These glycolipids analogues could then be tested for their biological activity (e.g. BMM assay) in order to better understand their structural activity relationship. In this chapter, preliminary studies and future work concerning these points will be discussed in more detail.

\subsection{Bifidobacteria LTA Extraction}

As alluded to in Chapter 1 (Section 1.4.4), LTAs are usually formed after multiple condensation steps with glycosyldiglycerides that elongate the phosphoglycerol chain. The glycosyldiglyceride is thus the anchor for this long chain and as they were found to be active in some cases, the bifidobacterial glycolipid structures were characterised in Chapter 3. In fact, a novel glycolipid (GL5) was extracted from bifidobacteria and is described in Chapter 3. At this point, it seemed likely that an LTA containing GL5 as an 
anchor might exist. However, as isolated LTAs are contaminated with active lipoproteins, the most reliable way to assess their biological activity is via synthesis as synthetic LTAs have been shown to be immunologically active in multiple reports. ${ }^{12,86}$ Thus in Chapter 4, analogues of GL5 were synthesised to assess their biological activity and the phosphated LTA analogues ( $s n-3-4 a$ and $s n-3-5 a)$ showed no significant activity (Figure 5.3).

To date, study of the biological activity of natural LTAs has been hampered by difficulties in obtaining pure LTAs (free from active lipoproteins) and by harsh extraction methods (hot-phenol) forming less active LTAs. ${ }^{56}$ The hot-phenol extraction method has been used by Fischer et al. and Iwasaki et al. to characterise the LTAs from bifidobacteria (Chapter 1, Section 1.6.6). ${ }^{62,124,146}$ However, under the harsh extraction conditions, ${ }^{62}$ a LTA possessing GL5 as an anchor might not have been detected due to degradation. Thus, to compare the LTA activity and structure produced by three different extraction methods, the bifidobacterial LTA was extracted using hot phenol, water or butanol. These crude extracts were compared in a macrophage assay with synthesised LTA analogues from Chapter $4 .{ }^{\mathrm{m}}$ However, all three crude extracts were able to activate macrophages strongly and to a similar extent (Figure 5.3) and thus no differences were observed. These extraction methods thus do not seem to give different glycolipid activity, however as the LTAs were not purified, the presence of lipoprotein might explain this high activity.

As the biological activity seemed similar in a BMM assay, another quick screening to observe the differences in the structures was performed. As MALDI has been successfully used as a screening method for glycoglycerolipid analysis in whole cells (Chapter 3), the crude extracts were subjected to MALDI analysis and the results were compared to obtain insights on the structures. Both positive and negative ion mode, as well as different matrices (e.g. DHB, $\alpha$-cyano-4-hydroxycinnamic acid, proton sponge $)^{184,235,297}$ were investigated and the results were inconclusive. In some cases, no signal was visible and in other cases already known compounds, such as glycoglycerolipids, DAG or impurities (such as polyethylene glycol), were detected. It

\footnotetext{
${ }^{\mathrm{m}}$ This assay was conducted by Ashna Khan and Stephanie Chee.
} 
was then concluded that the sample was not pure enough for analysis and would first need to be purified before subsequent MALDI analyzes.

In order to effectively compare the LTAs extracted with the three methods, the LTA would need to be purified using Octyl-sepharose and DEAE (See Chapter 1, Section 1.4.4.3). Also, another mild extraction method that could be investigated involves the use of Triton-X-114, a detergent, to dissolve the LTAs. ${ }^{298}$ From these extractions and purifications, it is expected that more conventional analytical methods might have to be used to determine the structure. Such methods would include NMR, constituent sugar analysis, linkage analysis and fatty acid analysis.

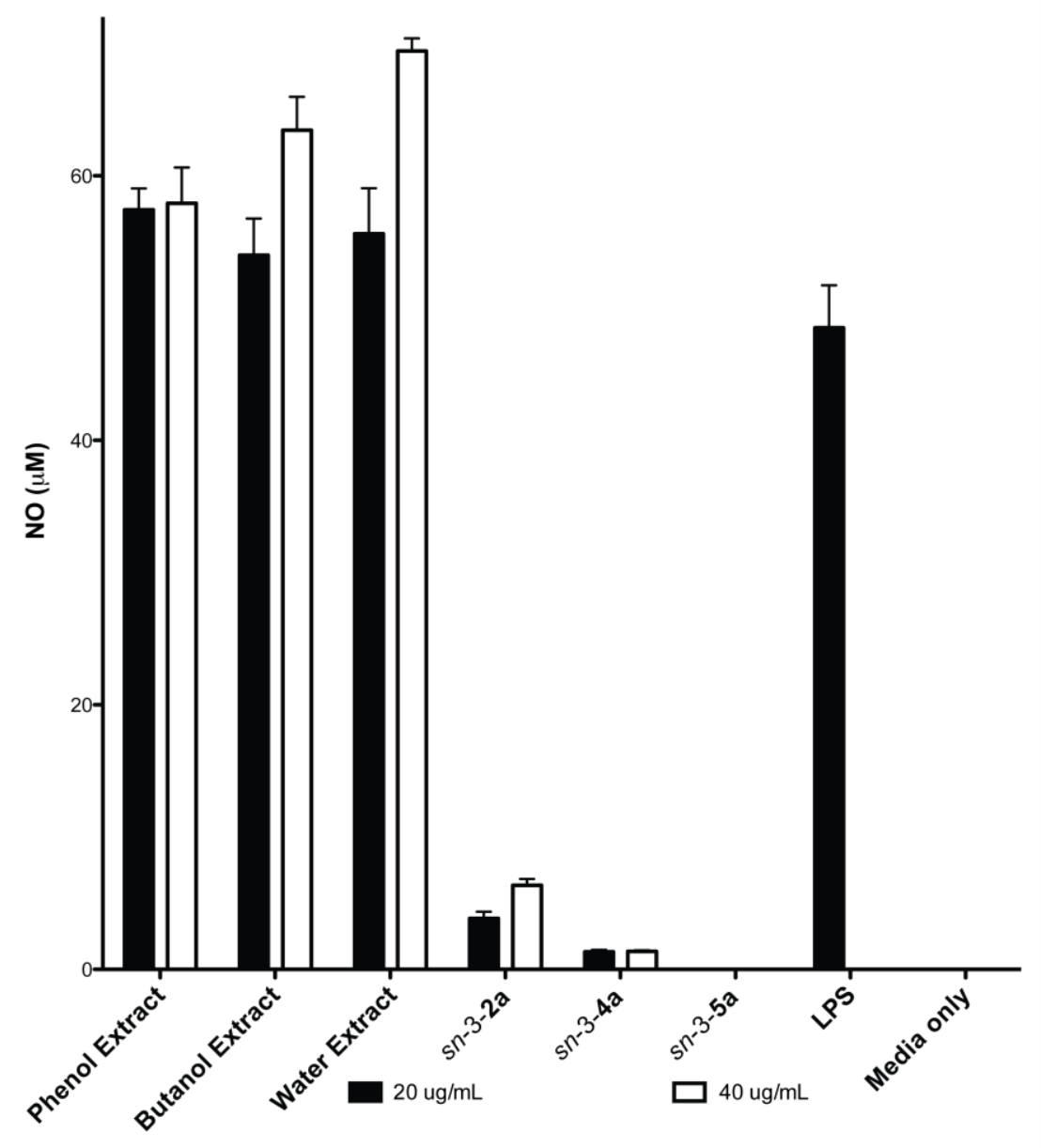

Figure 5.3 BMM were stimulated with a LTA extract from Bifidobacteria, (20 $\mu \mathrm{g} / \mathrm{mL}$ and $40 \mu \mathrm{g} / \mathrm{mL})$, or LPS (100 ng/mL) and the supernatants analyzed for NO using 
the Griess assay after $48 \mathrm{~h}$. Mean and SD of triplicate samples from a representative experiment of two performed are indicated.

\subsection{Stereochemistry of Novel Glycolipid (GL5)}

In Chapter 3, a novel glycolipid has been described (Chapter 3, Section 3.3.4). Figure 5.4 depicts the novel glycolipid showing the three unknown stereocenters found in the molecule. In order to determine the stereochemistry of these three stereocenters, a number of methods were attempted. A first method that has been conducted to define the stereochemistry of some of the stereocenters is described in the seminal work of Rundlöf et al. ${ }^{299}$

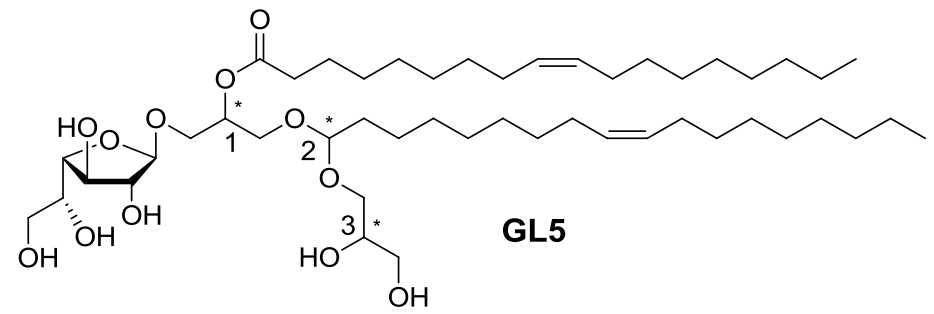

Figure 5.4 Structure of GL5 showing the 3 unknown stereocenters.

In this work, Rundlöf et al. described a degradation method for the determination of the absolute configuration of the glycerol moiety. In Scheme 5.1, the polysaccharide containing glycerol moiety $\mathbf{1}$ is first oxidised using TEMPO to form acid $\mathbf{2}$. After this, the resulting mixture is hydrolysed to give the glyceric acid $\mathbf{3}$ and other products. Then the glyceric acid is esterified with $(S)-(+)$-2-butanol to give ester 4. A final acetylation leads to derivative 5. After this, the reaction mixture was analyzed by GC-MS and the traces obtained compared with derivatives of D- and L-glyceric acid. Even though this method is very elegant, it can be applied to find out the stereochemistry of only one stereocenter (\#3). Therefore, the method was adapted to elucidate the stereochemistry of stereocenter \#1. Scheme 5.2 depicts three different derivatisation methods that were employed to determine the stereochemistry of stereocenters \#1 and \#3. Due to small 
amounts of extracted GL5, the three strategies were employed without monitoring and purification of the intermediates and every step was assumed to be sucessful. Derivatisation method 1 , the same as Rundlöf et al., was expected to identify the stereochemistry of stereocenter \#3. In this method, GL5 was first oxidised with TEMPO and it was assumed that diacid 6 was formed. Then hydrolysis of diacid 6 using TFA was intended to lead to glyceric acid $\mathbf{3}$ and other degradation products. Esterification of $(S)$-(+)-2-butanol with glyceric acid $\mathbf{3}$ was assumed to give diastereomer $\mathbf{4}$. Derivative $\mathbf{5}$ was finally assumed to be obtained after acetylation.

Derivatisation method 2 was intended to determine the configuration of stereocenter \#1. Similarly to derivatisation method 1 , it started with the oxidation of GL5 to form diacid 6. Then the acetal was presumably cleaved using acetic acid to give alcohol 7. This compound was then subjected to an oxidation that was intended to yield to diacid 8 . Finally, glyceric acid 3 was supposedly released using TFA and esterification of $(S)-(+)$ 2-butanol followed by an acetylation was intended to give derivative $\mathbf{5}$.

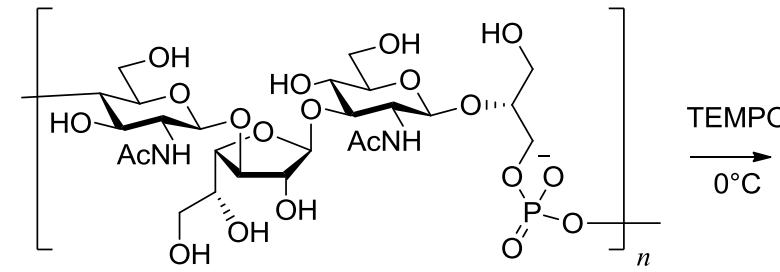

1<smiles>CC[C@H](C)OC(=O)[C@H](COC(C)=O)OC(C)=O</smiles>

5<smiles>CC[C@H](C)OC(=O)[C@H](O)CO</smiles>

4

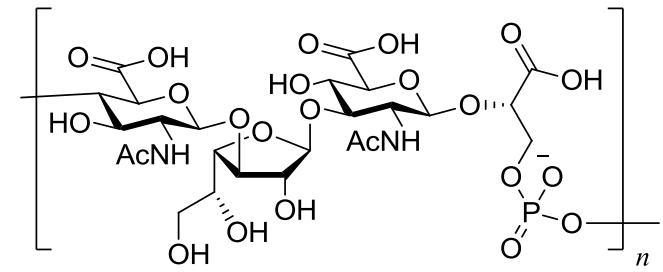

2

$\downarrow \begin{aligned} & \mathrm{H}_{2} \mathrm{O}, \mathrm{H}^{+} \\ & 100^{\circ} \mathrm{C}\end{aligned}$<smiles>CCC(C)O</smiles>
3

Scheme 5.1 Degradation method for absolute configuration determination by Rundlöf et al. ${ }^{299}$ 
The derivatisation method \#3 was designed to test if the other derivatisation methods worked properly. Indeed, the stereochemistry of stereocenter \#3 should be inverted compared to the result obtained for derivatisation method \#1. This is due to the fact that the TEMPO oxidation occurs at different primary alcohols on the glycerol bearing stereocenter \#3 depending on the derivatisation method. Here, in contrast to derivatisation methods 1 and 2, an attempt was made to first acetylate GL5. Presumed peracetylated compound $\mathbf{9}$ was then subjected to hydrolysis using acetic acid and supposedly released alcohols 10 and 11. Acid 12 and 13 were then assumed to be obtained after TEMPO oxidation. Glyceric acid was then intended to be released from acid 12 using TFA. Esterification of $(S)-(+)-2$-butanol with the mixture of acid 12 and $\mathbf{1 3}$ and acetylation was intended to give derivative $\mathbf{5}$.

Having completed the three derivatisation protocols, the derivatisation products were analyzed by GC-MS and the results were compared with the GC-MS traces of D and Lglyceric acid derivatives. The results obtained were very puzzling. Indeed, all three derivatisation methods produced D-glyceric acid. From these results, it seemed likely that the esterification with $(S)-(+)-2$-butanol was successful as well as the acetylation. This was assumed as D- and L-glyceric acids successfully gave the acetylated derivatives $\mathbf{5}$ as expected. It was then suspected that some of the intermediate reactions did not occur as planned. As a very small amount of material was used, it was not possible to follow the reactions using TLC, NMR and MS. Thus, the implementation of a simpler method which does not include oxidation might be more successful. 

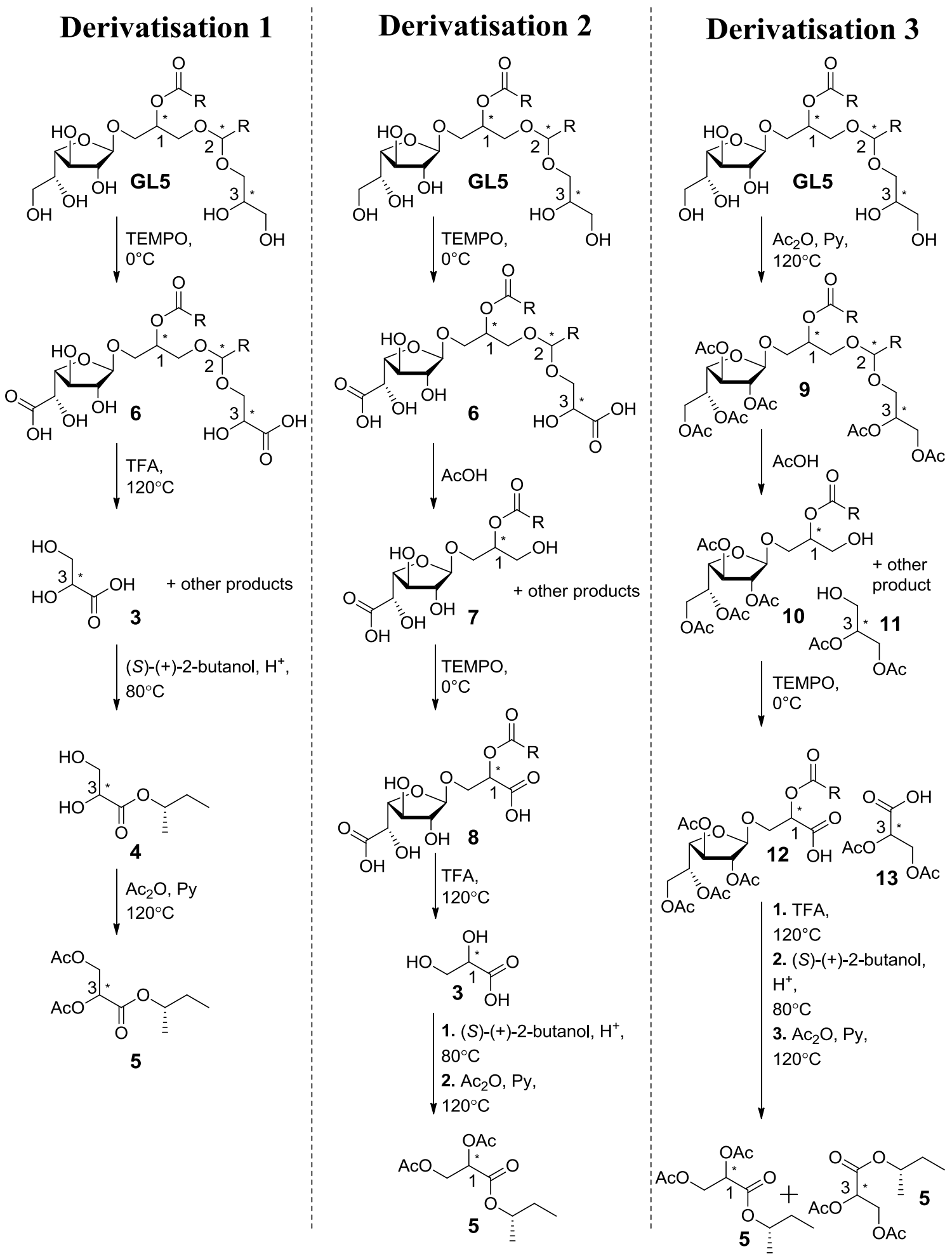

Scheme 5.2 Derivatisation methods adapted from Rundlöf $e t$ al. 
A simpler method described by Meguro and co-workers involves the synthesis of a chiral derivatising reagent for HPLC. ${ }^{300,301}$ Scheme 5.3 depicts the degradation methodology from a glycosyldiglyceride containing an unknown stereocenter to form di-O-methyl-sn-glycerol. Here, the esters from glycosyldiglyceride $\mathbf{1 4}$ are first removed using sodium methoxide and the hydroxyls methylated. Finally the sugar residue is cleaved to give glycerol derivative 15. In Scheme 5.4, the condensation of pinacolone with 2,3-hydroxytoluene (16) to form acetal $\mathbf{1 7}$ is first achieved. Then oxidation of $\mathbf{1 7}$ with $\mathrm{KMnO}_{4}$ gives acid 18. The $S$ enantiomer 19 is next optically resolved using cinchonidine. After this, acid 19 is subjected to thionyl chloride to obtain the acyl chloride 20. Finally, the di-O-methyl-sn-glycerol $\mathbf{1 5}$ is esterified with the chiral reagent 20 to form ester 21.

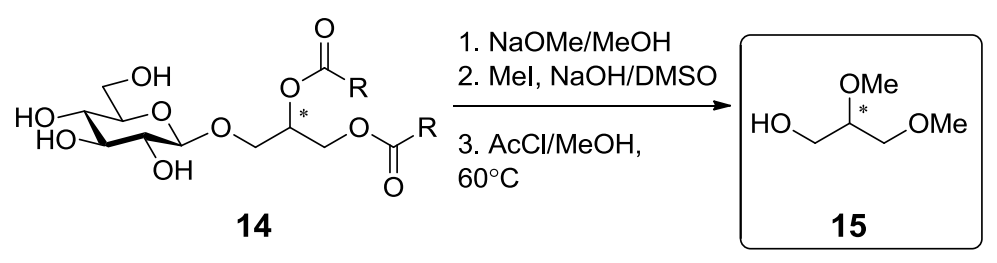

Scheme 5.3 Degradation method to form di- $O$-methyl-sn-glycerol. ${ }^{300}$

As 2-octanone was available in the lab, its use was preferred over pinacolone. Here, following the method of Nishida et al. 2,3-hydroxytoluene 16 was condensed with 2octanone to form acetal 22. Acid 23 was then obtained in good yields (60\%) after the oxidation of acetal 22 (Scheme 5.5). ${ }^{301}$ However, the crystallisation attempts using cinchonidine were unsuccessful and failed to produce 24. Here, either no crystallisation occurred or only the cinchonidine precipitated out of solution even though many solvents and solvent mixtures were investigated (e.g. hexane, ethanol, acetone, methanol, water, acetone/methanol). It appeared that the long chain on the acetal moiety might have hindered the crystallisation and the use of pinacolone was then considered as the chain is shorter and it is more bulky. 


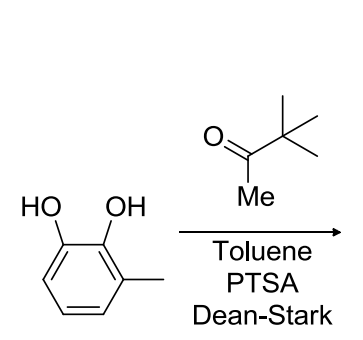

16

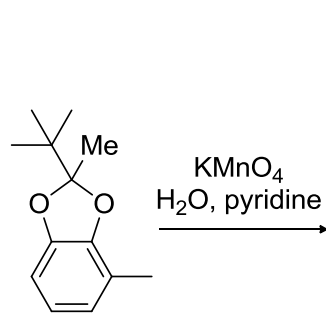

17

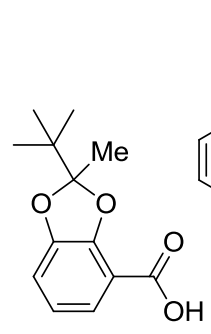

18

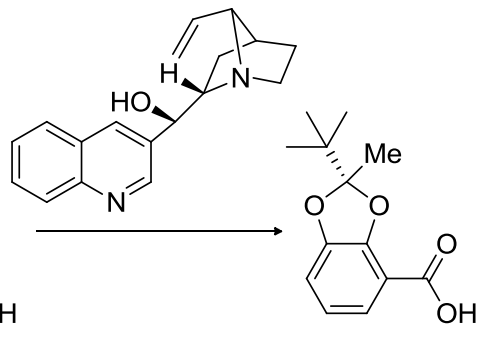

$(+)-19$

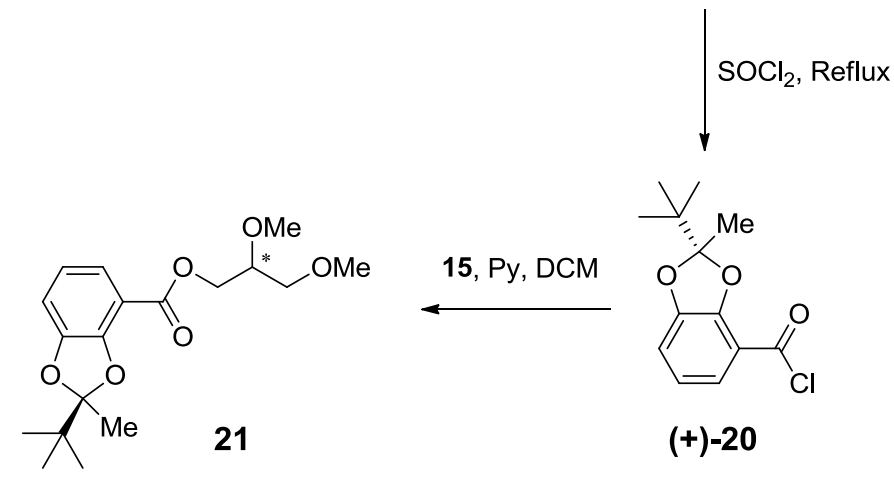

Scheme 5.4 Synthesis of derivatisating agent for HPLC. ${ }^{301}$

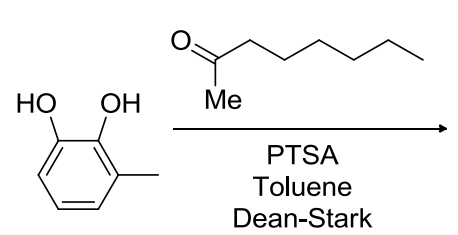

16

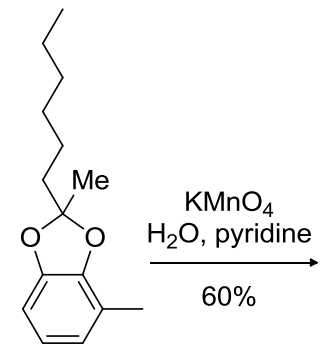

22

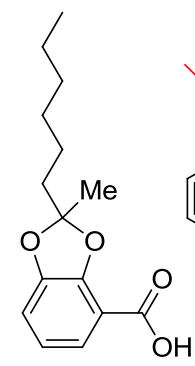

23

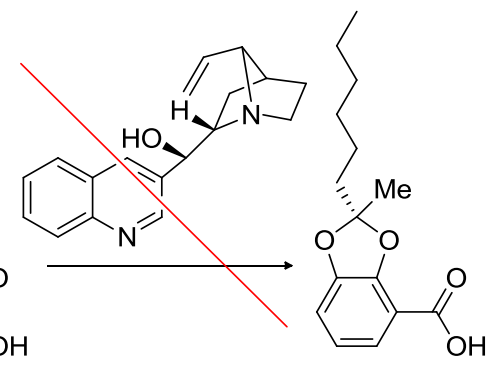

24

Scheme 5.5 Attempt to synthesise enantiomerically pure 24 .

The use of pinacolone was indeed successful. Here, following again the procedure of Nishida et al. (Scheme 5.4), derivatised glycerol 21 was obtained uneventfully. After the investigation of a few solvents (e.g. ethanol, methanol, acetone) a mixture of methanol/acetone $(1 / 1, v / v)$ proved fruitful and after six subsequent crystallisations, an enantiomeric excess of $66 \%$ was obtained. With these encouraging results, the need for glycerol standards to compare the unknown glycerol derivatives arose. Scheme 5.6 shows the synthesis of the glycerol standard. Here, the isopropylidene protected racemic 
glycerol $\mathbf{\pm 2 5}$ was first acetylated. Deprotection of acetonide $\pm \mathbf{2 6}$ using $\mathrm{AcOH} / \mathrm{H}_{2} \mathrm{O}$ followed by methylation of diol $\pm \mathbf{2 7}$ then gave glycerol derivative $\mathbf{\pm 2 8}$. This compound was deacetylated to give alcohol $\mathbf{\pm 2 9}$ and esterified with the derivatising agent $\pm \mathbf{2 0}$, and a mixture of enantiomers and diastereoisomers $\mathbf{\pm 2 1}$ was obtained in $\mathbf{3 1 \%}$ overall yield.

Preliminary HPLC investigations on ester $\mathbf{\pm 2 1}$ failed to separate the diastereoisomers present in the mixture even though different type of columns (e.g. silica, two silica columns in series, C-18, Hilic) and solvent systems (e.g. $n$-hexane/tert-BuOH, different gradients) were used. Following these unsuccessful attempts, GC was then assayed. The diastereomeric mixture of ester $\mathbf{\pm 2 1}$ was injected in a GC-MS, but here, again, even though different columns (HP-5ms and BPX70) and different temperature programs were used, only one signal was observed (Figure 5.5). From these results, NMR seemed to be the best option for the analysis of the diastereoisomers. As shown in Figure 5.6, the duplication of ${ }^{13} \mathrm{C}$ NMR signals for $\mathrm{C}-2$ indicates the presence of two diastereomers. However, greater quantity of GL5 is needed for NMR analysis. Moreover, a single diastereoisomer of $\mathbf{\pm 2 1}$ starting from pure $R$ or $S$-solketal $\mathbf{2 5}$ needs to be synthesised for comparison with derivatives of the isolated mixture of diastereoisomers.

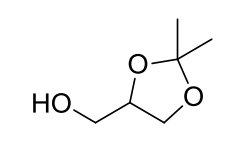

$\pm 25$

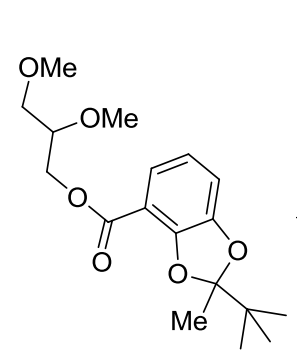

$\pm 21$
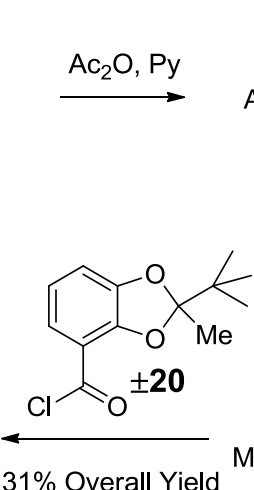

$31 \%$ Overall Yield

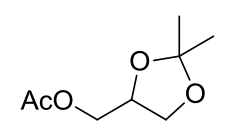

$\pm 26$

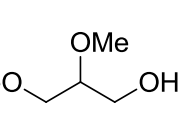

$\pm 29$

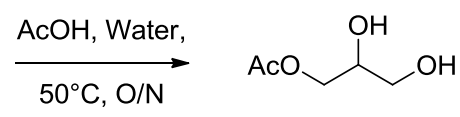

$\pm 27$<smiles>O=[W]O</smiles>

$\frac{\mathrm{NaOMe}}{\mathrm{MeOH}}$<smiles>COCC(COC)OC</smiles>

$\pm 28$

Scheme 5.6 Synthesis of glycerol standard. 


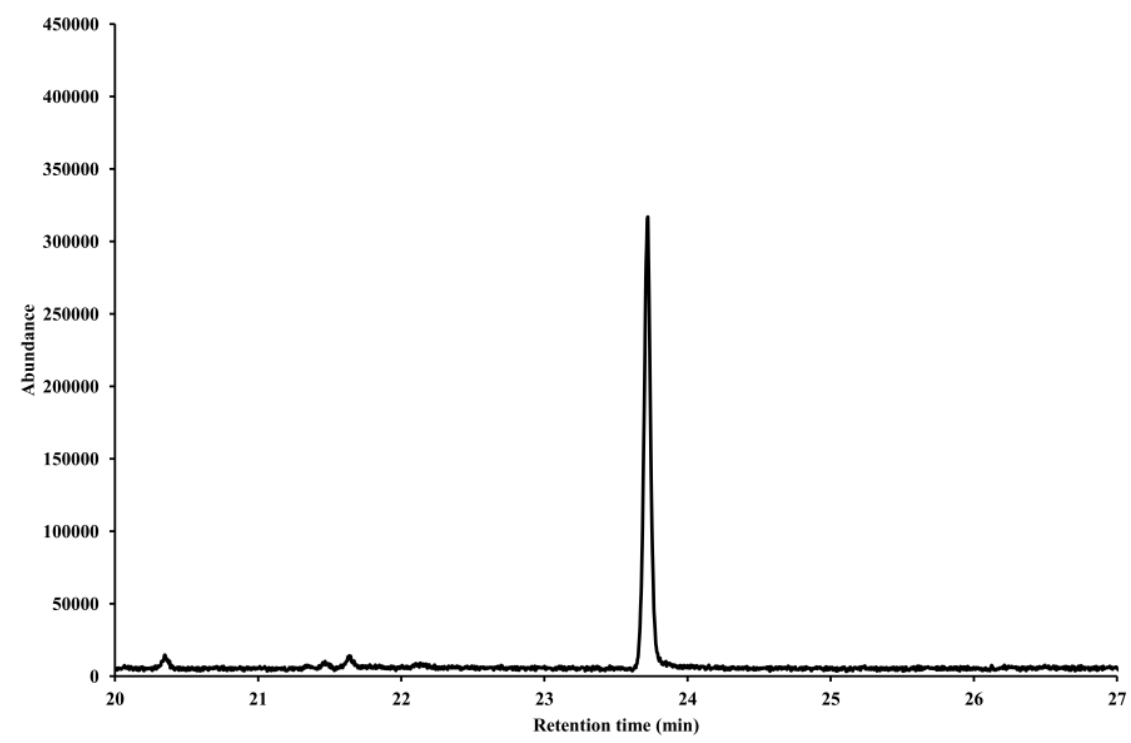

Figure 5.5 GC-MS chromatogram of derivatised glycerol 21.

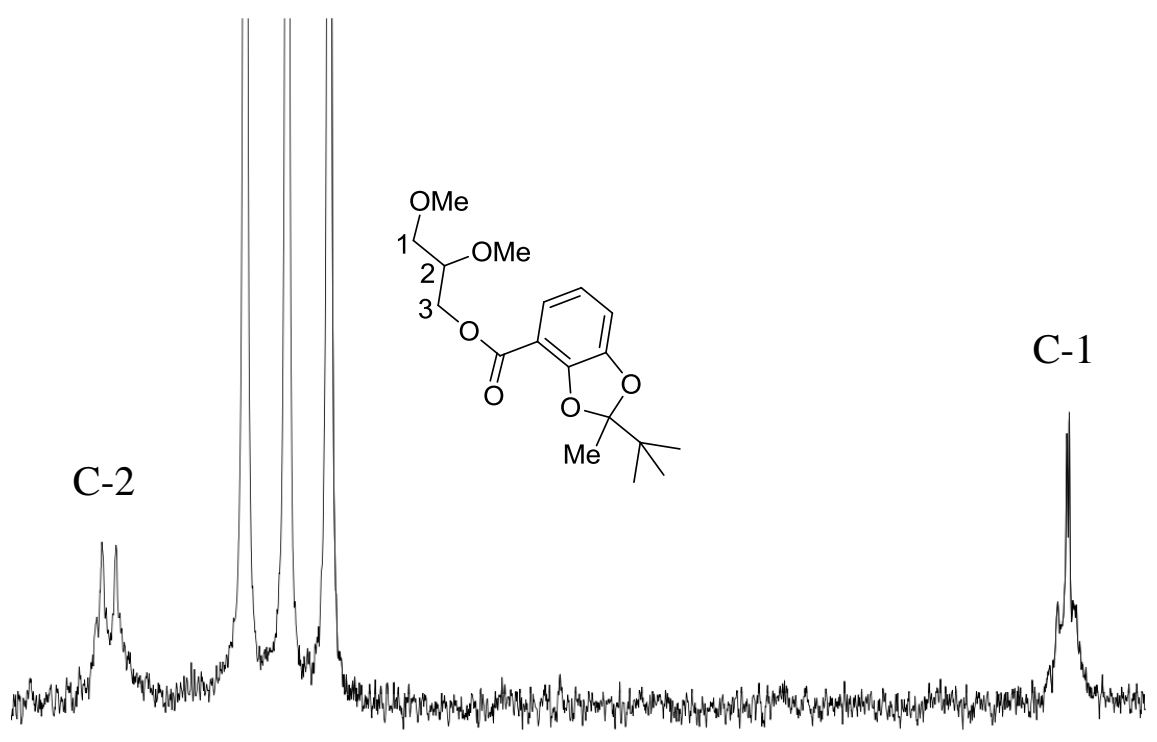

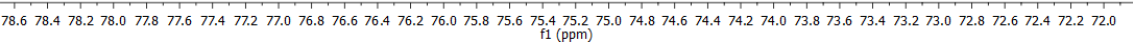

Figure 5.6 $\quad{ }^{13} \mathrm{C}$ NMR spectrum expansion of derivatised glycerol 21 showing the presence of diastreomers. 
Scheme 5.7 shows the design for a degradation method to find the stereochemistry of stereocenters \#1 and \#3 preparatively. In derivatisation method 1 (to determine the stereochemistry of stereocenter \#3), it is proposed that the methylation of GL5 would lead to permethylated compound 30. Hydrolysis of $\mathbf{3 0}$ with $\mathrm{TFA} / \mathrm{H}_{2} \mathrm{O}$ would then give partially methylated glycerol 29. Finally, glycerol derivative 21 could be obtained by a final esterification using the derivatisating agent $((+)-20)$. The second derivatisation method (determine the stereochemistry of stereocenter \#1) would involve first an acetal hydrolysis step to obtain pentaol $\mathbf{3 1}$ from GL5. Then permethylation of $\mathbf{3 1}$ would lead to methylated compound 32. After this, glycerol 29 could be obtained by cleaving the acetal. Then esterification with derivatising agent (+)-20 would give derivatised glycerol 21. 


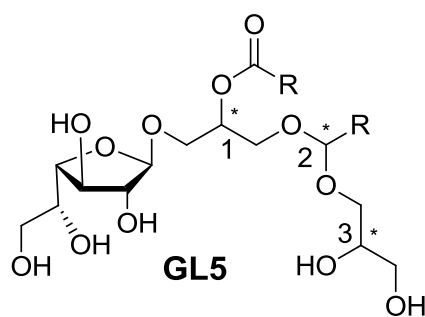

Mel, $\mathrm{NaOH}$

$\checkmark$

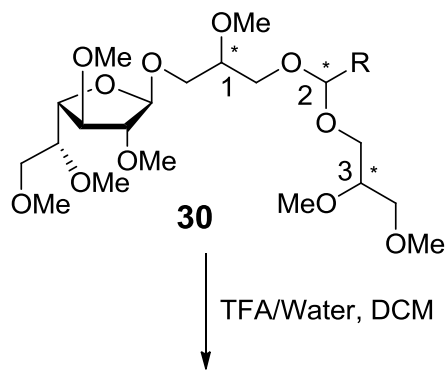

$\mathrm{HO}$

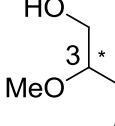

29 OMe
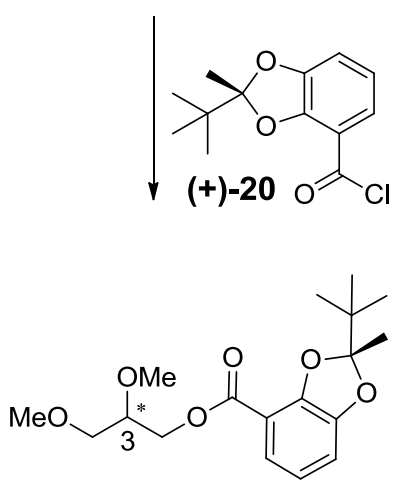

21

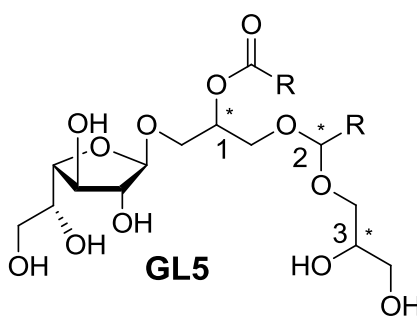

TFA/Water, DCM

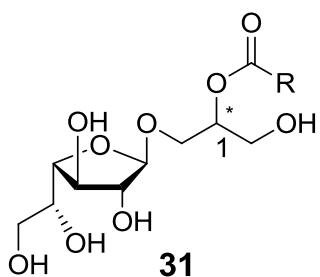

Mel, NaOH/DMSO

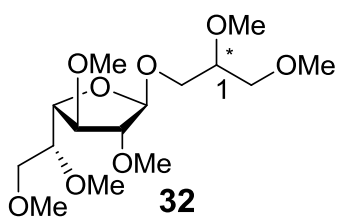

$\mathrm{AcCl}, \mathrm{MeOH}$
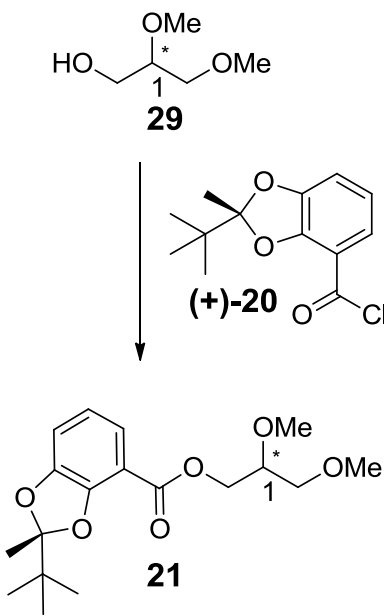

Scheme 5.7 Degradation method to form glycerol derivatives. 


\subsection{Synthesis of Novel Glycolipid (GL5)}

Attempts to unmask the relative stereochemistry of stereocenter \#2 using NOE measurements were also unsuccessful as no NOE correlations were observed. In this case, it seemed likely that this part of the molecule is too flexible for any NOE observation. It is anticipated that the comparison of synthetic samples to extracted GL5 will next be necessary for the determination of stereocenter \#2. Furthermore, while it was shown in Chapter 4 that a chain length of C20 was optimal for macrophage activity, it will be interesting to probe the activity of analogues of GL5 with varying chain length.

Scheme 5.8 shows the retrosynthetic pathway for the synthesis of GL5. Here, a convergent strategy is proposed whereby the three key intermediates, the protected galactofuranose derivative $\mathbf{3 3}$, fatty acid $\mathbf{3 4}$ and sugar derivative $\mathbf{3 5}$ could be coupled to obtain a synthetic intermediate which would need to undergo further modifications in order to form the acetal function. Then, it is envisioned that the coupling of solketal (+)25 to galactofuranoside iodide $\mathbf{3 6}$ followed by deprotection of the acetonide will proceed as covered in Chapter 4 to form galactofuranose intermediate 33. For the sake of simplification, it is assumed that stereocenter \#1 has an $R$ configuration which is the same as DAG found in nature (see Chapter 1, Section 1.4.4).

For the determination of stereocenter \#2, isomers with known stereochemistry are needed to compare the structural data of the synthesised compounds with the structural data of the extracted compound. Here, depending on if sugar derivative $\mathbf{3 5}$ is D- or Land if it is linked through an $\alpha$ - or $\beta$-linkage to the glycerol, the stereochemistry of the stereocenters can be manipulated (Scheme 5.9).

For example, for both stereocenter $\# 2$ and \#3 to be $S$, a D-sugar linked in a $\beta$-fashion to the glycerol is required for the synthesis. Scheme 5.10 shows the synthetic plan to form the $R, S, S$ isomer of GL5. In order to synthesise vic-diol 38, the galactofuranose donor $\mathbf{3 3}$ would need to be coupled with the arabinopyranose acceptor $\mathbf{3 5}$ in order to form 
coupled product 37, followed by deprotection of the benzoyl groups in order to liberate vic-diol 38. Periodate cleavage of the intermediate diol $\mathbf{3 8}$ and reduction of the resulting dialdehyde would lead to triol 39. Subjection of 39 to DDQ would form the $p$ methoxybenzylidene acetal $\mathbf{4 0}$ in an intramolecular protection step. Ketone $\mathbf{4 1}$ would then be obtained via oxidation of the primary alcohol in $\mathbf{4 0}$ using mild conditions. The elongation of the fatty acetal using Wittig methodology would lead to alkene $\mathbf{4 2}$. Esterification of the secondary alcohol in $\mathbf{4 2}$ with stearic acid would lead to ester $\mathbf{4 3}$, followed by hydrogenation to yield to saturated diol 44. A final removal of the TBS groups using HF.pyridine would lead to target compound R,S,S-GL5 (Scheme 5.10).

An attempt was made to form the D-arabinose building block for the synthesis of $R, S, S$,GL5 (Scheme 5.11). D-Arabinose was partially benzoylated to form alcohol $\mathbf{4 5}$ in $43 \%$ yield. As mono, di, tri and tetrabenzoylated products were obtained, repetitive column chromatography was needed to obtain pure 45, and hence the poor yield was not unexpected. Then, the next step, consisting of protecting the 4-position of the arabinose using PMBCl proved unsuccessful. Even with the use of good quality, distilled DMF, addition of TBAI as catalyst and varying reaction temperature, the reaction was unsuccessful and starting material degradation was observed. From these failed attempts, it was concluded that the reaction mixture was too basic and that another strategy should be used. Indeed, the use of a PMB imidate to couple the PMB group at the 4-position was successful and formed derivative $\mathbf{4 6}$ in $65 \%$ yield. After this, the deprotection of the anomeric benzoyl group using hydrazine failed, even though different temperature and reaction times were assayed. In this case, the starting material 46 was always recovered. Other reagents were also investigated such as hydrazine acetate and diethylamine and, again, only the starting material was recovered. Finally, using different quantities of $\mathrm{NH}_{3}$, the anomeric benzoyl group was removed, but with poor selectivity. Both the anomeric benzoyl and the benzoyls at C-2 and C-3 were removed. From these results, it seemed likely that the activation of the anomeric benzoyl group might work better under acid conditions. 


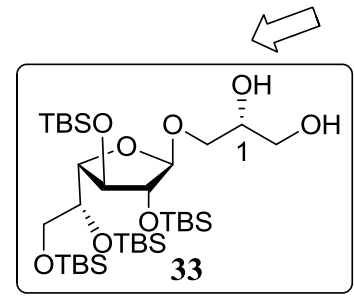

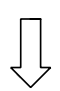

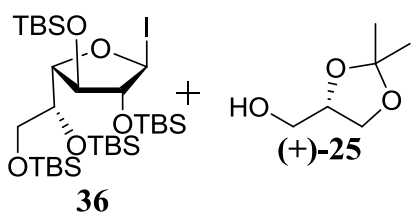

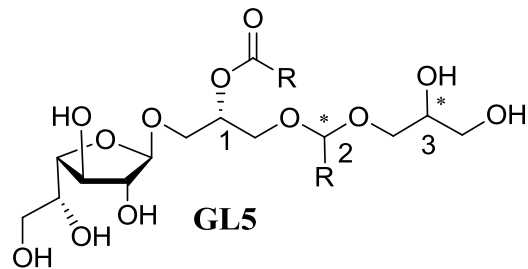

$+\mathrm{HO}_{\mathrm{R}}^{\mathrm{O}}$

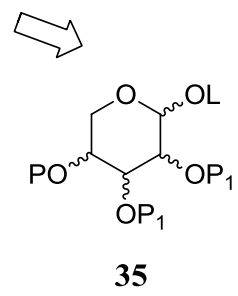

34

$\mathrm{R}=$ Alkane chain

$\mathrm{L}=$ Leaving group

$\mathrm{P}=$ Protecting group

Scheme 5.8 Retrosynthetic pathway for four different diastereomers of GL5.

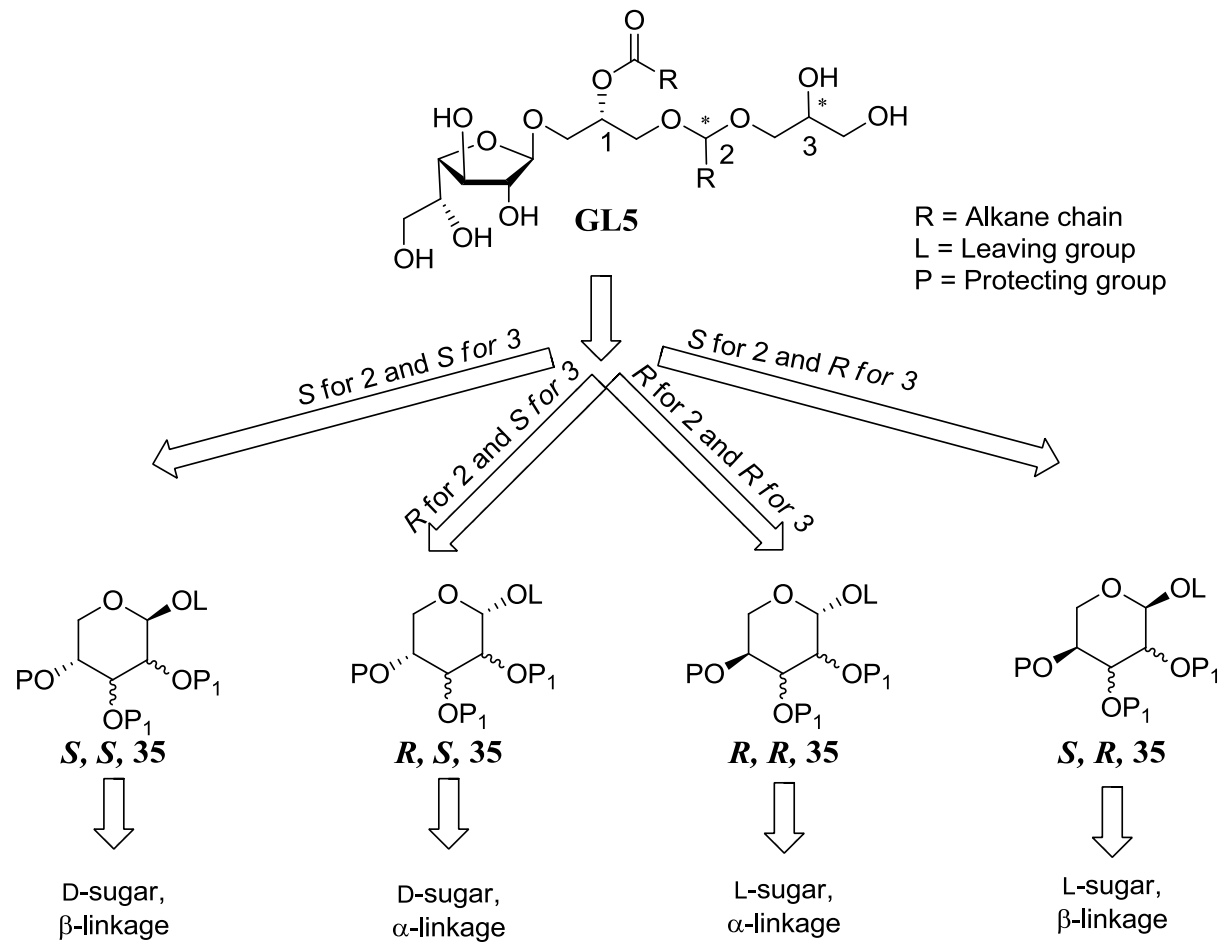

Scheme 5.9 Analogues for varying the stereochemistry of stereocenter 2 and 3 . 


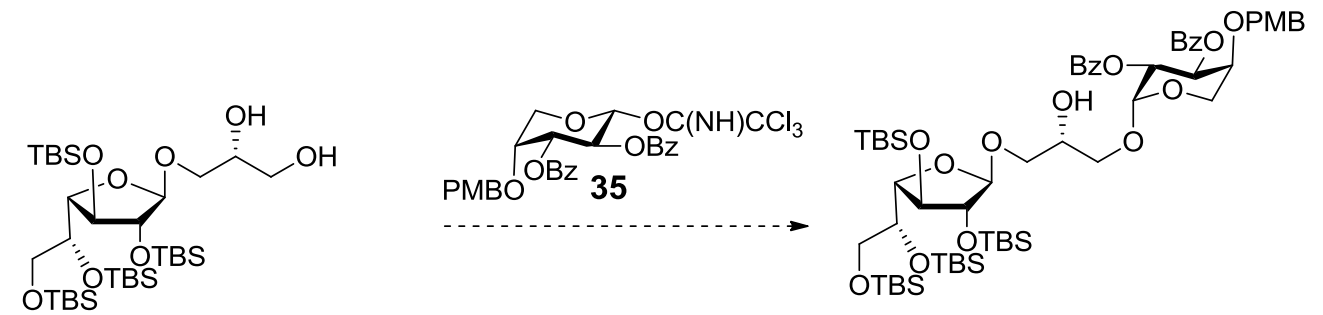

33

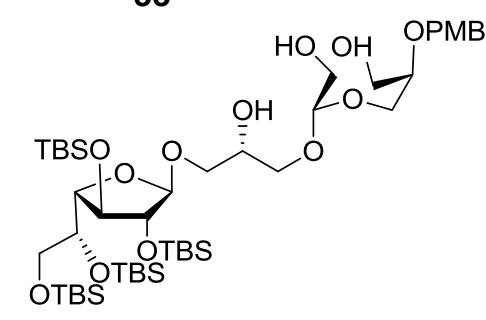

39

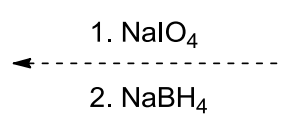

DDQ
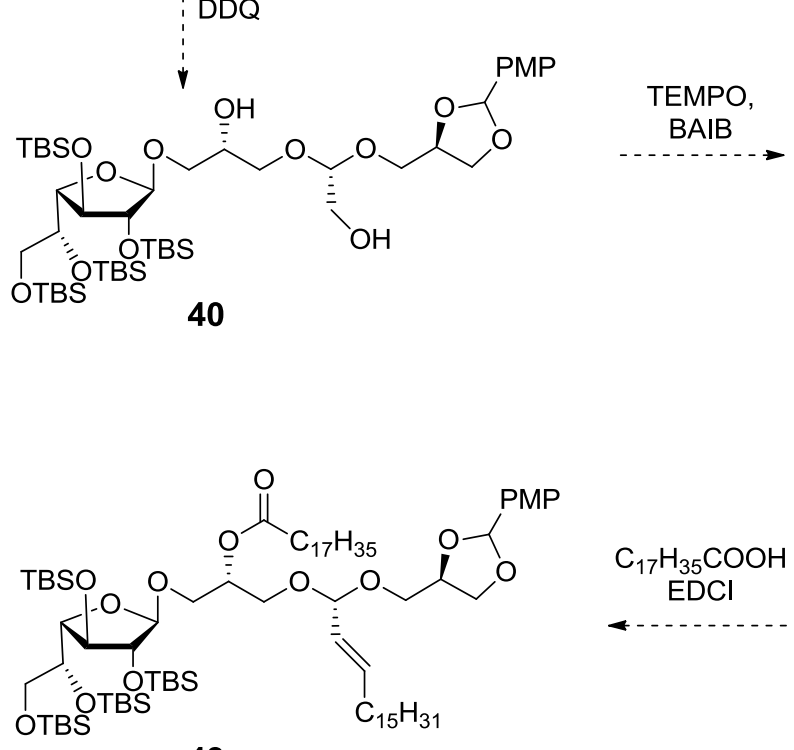

43
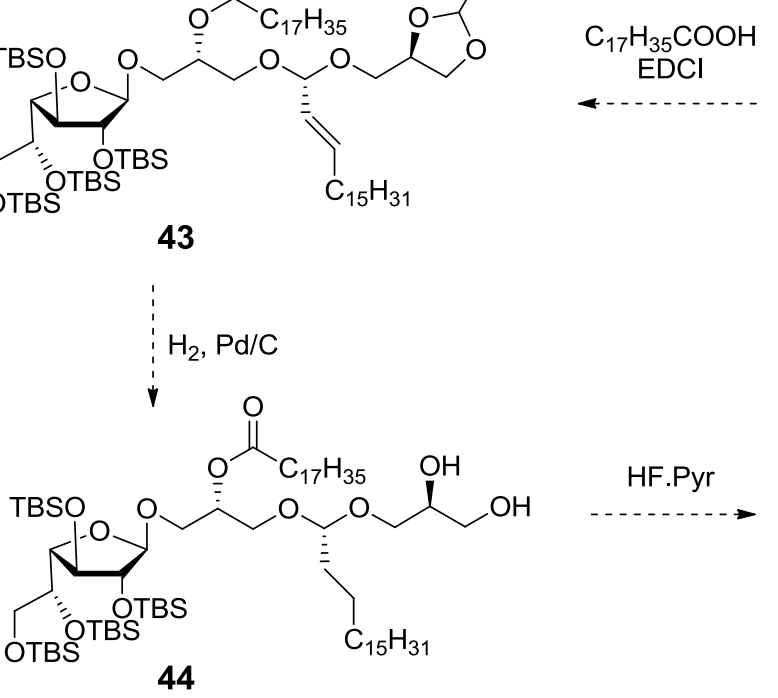
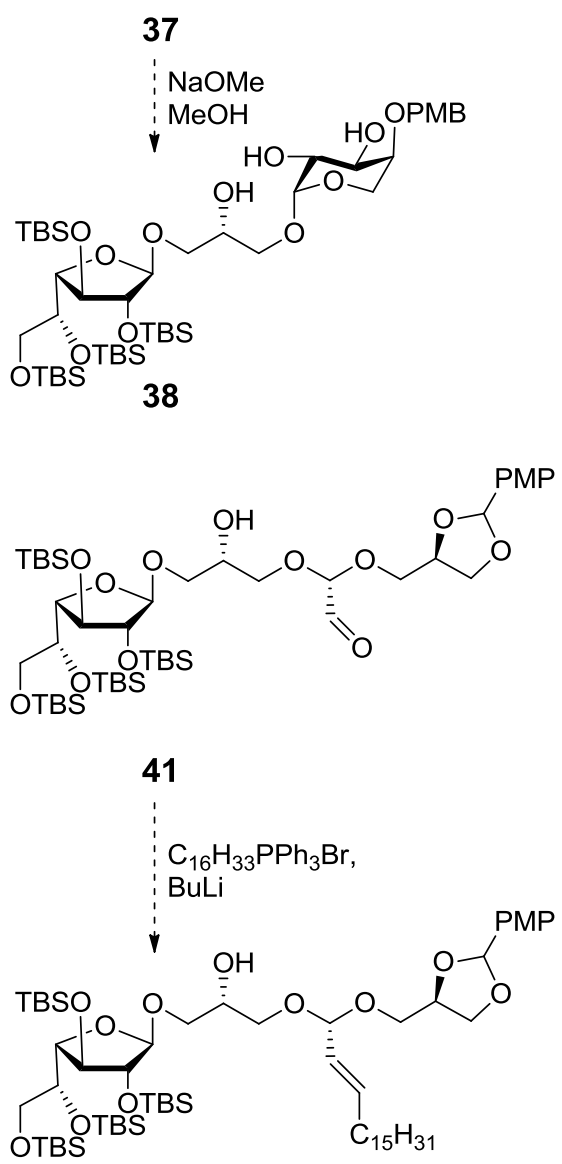

42

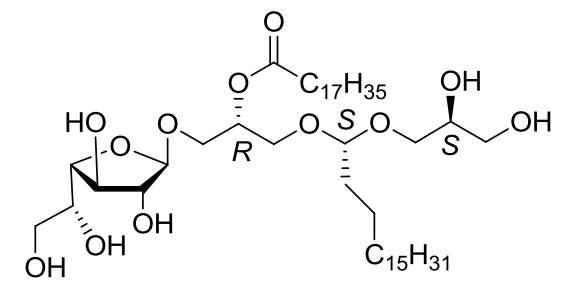

$R, S, S,-G L 5$

Scheme 5.10 Synthetic plan to form $R, S, S$ isomer of GL5. 


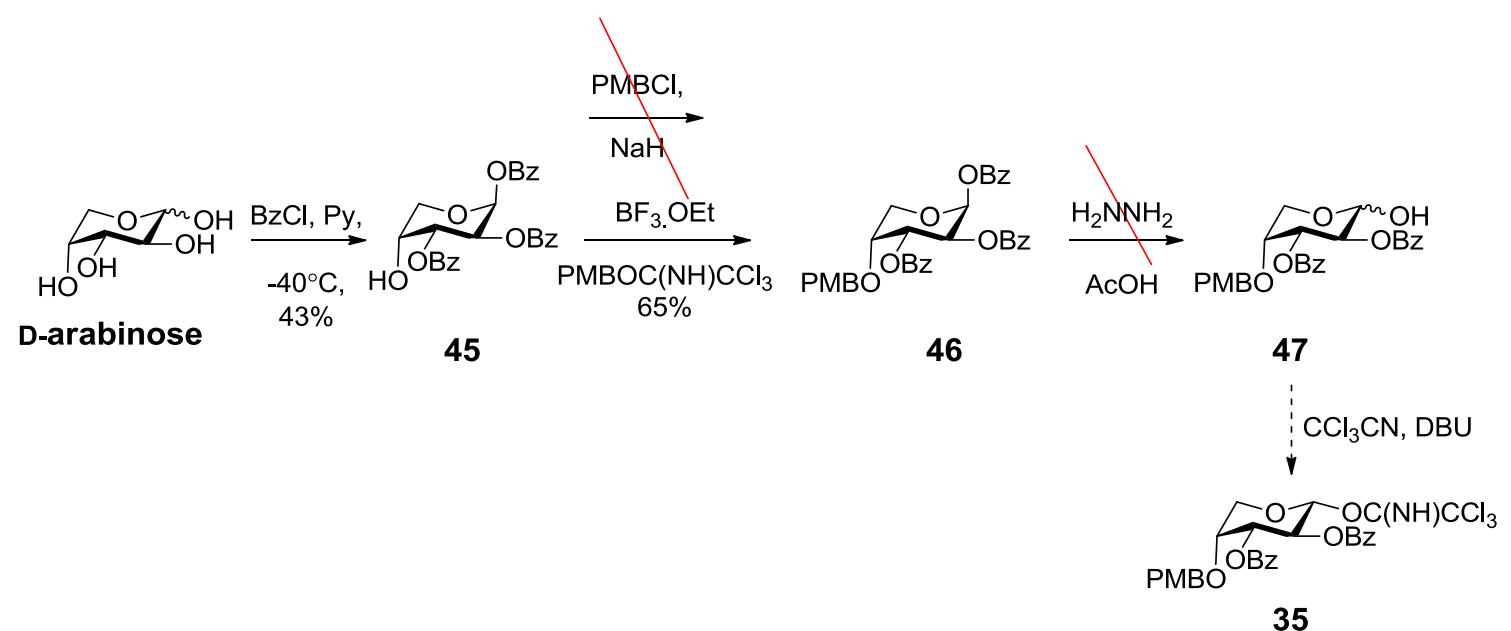

Scheme 5.11 Attempts to synthesise building block 34 to obtain a $S, S$ configuration for GL5.

A test reaction was next conducted using tetrabenzoyl arabinose $\mathbf{4 8}$ formed from the previous efforts. Here, using TMSTMS and $\mathrm{I}_{2}$ to form TMSI in situ, iodide 49 was obtained in excellent yields. Then, the coupling was attempted using NIS and zinc iodide and coupled compound $\mathbf{5 0}$ was successfully obtained in $40 \%$ yield without further attempts to improve the yield (Scheme 5.12). As TMSI is known to deprotect PMB groups, ${ }^{302}$ another protecting group was needed that would play the same role as PMB and protect the diol intramolecularly to form a compound analogous to $\mathbf{4 0}$. To this end, the use of a naphthalene ether was investigated, ${ }^{303}$ however, the coupling of the NAP imidate did not proceed as smoothly as the PMB imidate and even though different promoters $\left(\mathrm{BF}_{3}\right.$.OEt, TMSOTf and $\left.\mathrm{TfOH}\right)$ were used, the very stable NAP imidate was recovered after the reaction. At this point in time, it could be assumed that the PMB imidate could be installed later on in the synthesis and a per-acetylated lyxose building block 51 was investigated for coupling to galactofuranose derivative 33 . This coupling however resulted in orthoester 53 (Scheme 5.13) with no desired product 52 formed.

Alternatively, it is envisioned that the use of benzoyls as protecting groups could reduce the amount of orthoester formed during the coupling of galactofuranose derivative $\mathbf{3 3}$ and lyxose building block 54 and thus benzoylated compound $\mathbf{5 4}$ is proposed as a 
potential building block for the synthesis of intermediate 39. Subsequent removal of benzoyl groups on coupled product 55 would give tetraol 56 and the selective protection of the 2- and 3-positions of the lyxose with an isopropylidene would give diol 57. Next, the protection of the 4-position with a PMB group would lead to alcohol 58, as described by Parhi et al. ${ }^{304}$ This compound could finally lead to the desired triol $\mathbf{3 9}$ after isopropylidene cleavage, oxidative cleavage of the diol and reduction of the formed diketone (Scheme 5.14).

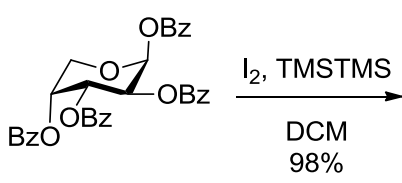

48

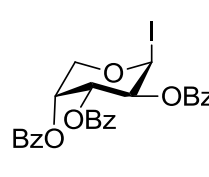

49

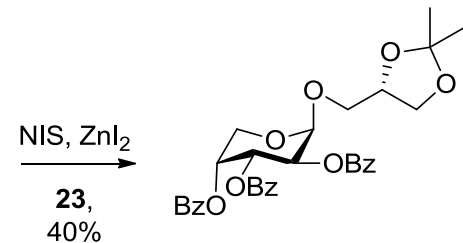

50

Scheme 5.12 Test reaction to form coupled product $\mathbf{5 0}$.

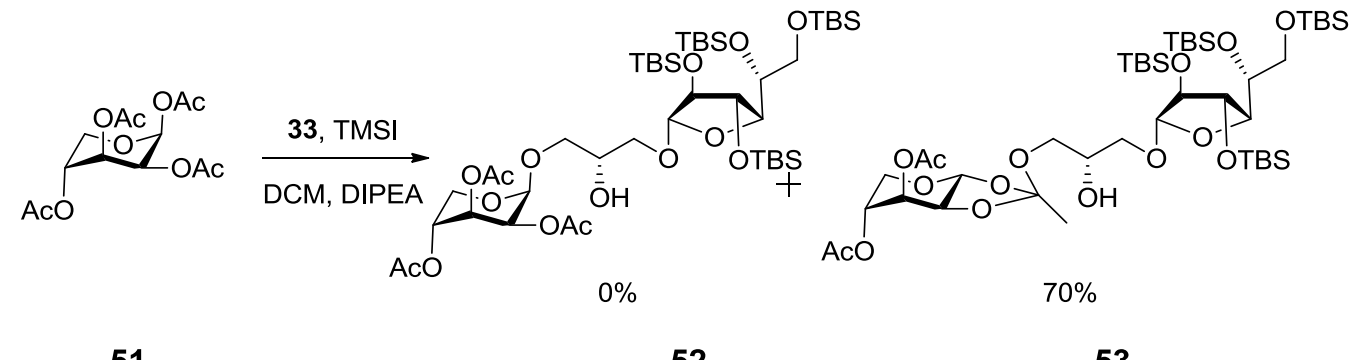

51

52

53

Scheme 5.13 Attempted coupling to form coupled product $\mathbf{5 2}$. 


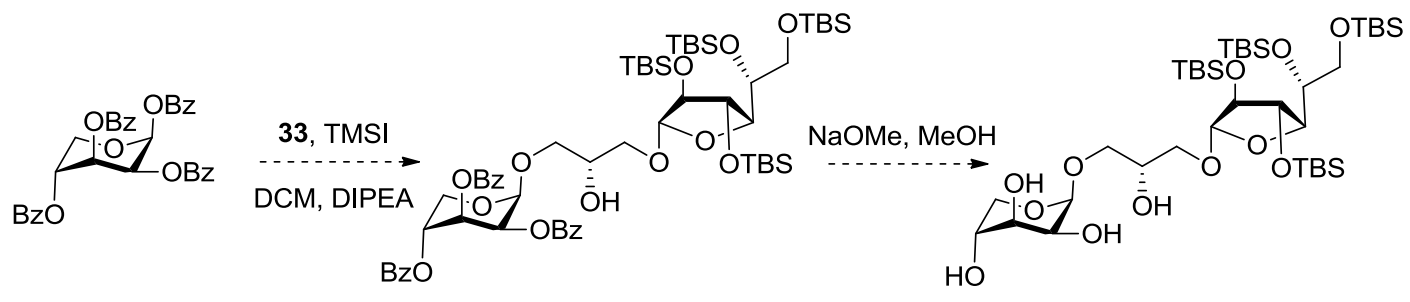

54

55

56

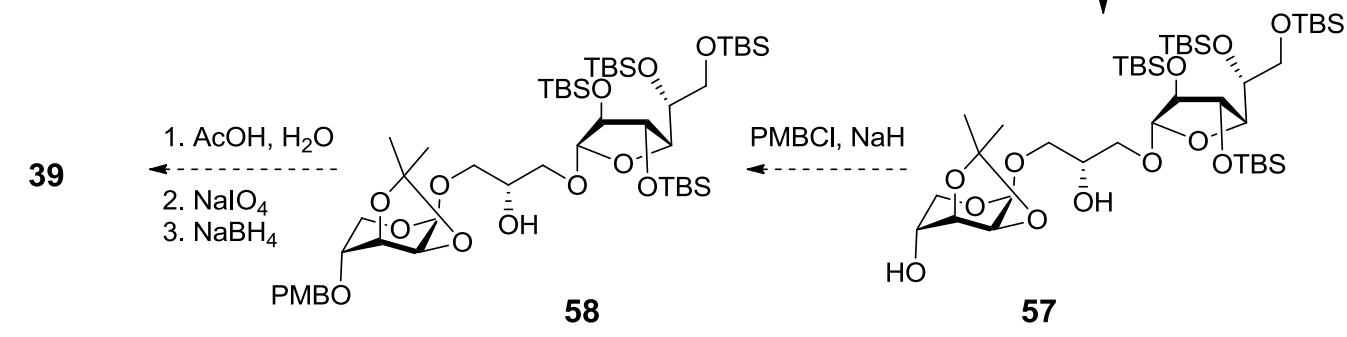

Scheme 5.14 Plan for synthesising intermediate 39. 


\subsection{Concluding Remarks}

In this thesis, the characterisation, synthesis and biological testing of L. plantarum and Bifidobacterium longum subs. infantis glycolipids was achieved. Hence, for the first time, the full characterisation of the four principal glycolipids of the L. plantarum cell wall was reported and tested in a BMM assay. The major glycolipids were identified as: $\alpha$-D-Glc $p$-diglyceride, $\quad \alpha$-D-Gal $p$ - $(1 \rightarrow 2)-\alpha$-D-Glc $p$-diglyceride, $\quad \beta$-D-Glc $p$ - $(1 \rightarrow 6)-\alpha$-DGal $p$ - $(1 \rightarrow 2)-6-O$-acyl- $\alpha$-D-Glcp-diglyceride and $\beta$-D-Glcp-( $1 \rightarrow 6)-\alpha-\mathrm{D}-\mathrm{Gal} p-(1 \rightarrow 2)-\alpha$ D-Glcp-diglyceride and showed no significant activity in BMM (Chapter 2). Then, a novel glycolipid, containing an unprecedented mixed acetal moiety and a galactofuranose moiety as a head group was reported in Bifidobacterium longum subs. infantis (Chapter 3). Finally, analogues of GL5 were then synthesised and led to macrophage activation (Chapter 4).

The results of these different studies led to preliminary work and future prospects that were covered in this present chapter (Chapter 5). Interesting future work that would be conducted would be the extraction of bifidobacteria LTAs and the stereochemical determination of GL5. The extraction of bifidobacteria LTA could shed light on the beneficial properties of bifidobacteria by using less harsh conditions for the extraction. As a LTA anchor consisting of GL5 is plausible and given that synthetic LTAs without active contamination are needed, the knowledge of the stereochemistry of the three unknown centers of GL5 would help in future bifidobacterial glycolipids syntheses for further biological studies. 


\section{Bibliography}

1. Nomenclature of Lipids. In Biochemical Nomenclature and Related Documents, 2nd Edition, Liébecq, C., Ed. Portland Press: London, 1992; p 10.

2. Microbial Glycobiology. Moran, A. P.; Holst, O.; Brennan, P. J.; von Itzstein, M., Eds. Academic Press: London, 2009.

3. Lebeer, S.; Vanderleyden, J.; De Keersmaecker, S. C. J., Nat. Rev. Microbiol. 2010, $8,171-184$.

4. Bacterial Infections of Humans. Brachman, P. S.; Abrutyn, E., Eds. Springer Science: New York, 2009.

5. Gram, H. C., Fortschr. Med. 1884, 2, 185-189.

6. Costerton, J. W.; Ingram, J. M.; Cheng, K. J., Bacteriol. Rev. 1974, 38, 87-110.

7. Holst, O.; Moran, A. P.; Brennan, P. J., Overview of the Glycosylated Components of the Bacterial Cell Envelope. In Microbial Glycobiology, Moran, A. P.; Holst, O.; Brennan, P. J.; von Itzstein, M., Eds. Elsevier: London, U.K., 2009; pp 3-13.

8. Decad, G. M.; Nikaido, H., J. Bacteriol. 1976, 128, 325-336.

9. Lugtenberg, B.; van Alphen, L., Biochim. Biophys. Acta-Rev biomembranes 1983, 737, 51-115.

10. Typas, A.; Banzhaf, M.; Gross, C. A.; Vollmer, W., Nat. Rev. Microbiol. 2012, 10, 123-136.

11. Hanson, B. R.; Neely, M. N., Curr. Opin. Microbiol. 2012, 15, 204-210.

12. Weidenmaier, C.; Peschel, A., Nat. Rev. Microbiol. 2008, 6, 276-287.

13. Ruas-Madiedo, P.; Hugenholtz, J.; Zoon, P., Int. Dairy J. 2002, 12, 163-171.

14. Seltmann, G.; Holst, O., The Bacterial Cell Wall. Springer: Berlin, Germany, 2002.

15. Yother, J., Annu. Rev. Microbiol. 2011, 65, 563-581.

16. Looijesteijn, P. J.; Trapet, L.; de Vries, E.; Abee, T.; Hugenholtz, J., Int. J. Food Microbiol. 2001, 64, 71-80.

17. Roberts, I. S., Annu. Rev. Microbiol. 1996, 50, 285-315.

18. Ruas-Madiedo, P.; Salazar, N.; de los Reyes-Gavilán, C. G.; Schmidt, R. R., Exopolysaccharides produced by lactic acid bacteria in food and probiotic 
applications. In Microbial Glycobiology, Moran, A. P.; Holst, O.; Brennan, P. J.; von Itzstein, M., Eds. Academic Press: London, 2009; pp 887-902.

19. Wang, Y.; Li, C.; Liu, P.; Ahmed, Z.; Xiao, P.; Bai, X., Carbohydr. Polym. 2010, 82, 895-903.

20. Vanhaverbeke, C.; Bosso, C.; Colin-Morel, P.; Gey, C.; Gamar-Nourani, L.; Blondeau, K.; Simonet, J.-M.; Heyraud, A., Carbohydr. Res. 1998, 314, 211220.

21. Xia, G.; Peschel, A., Chem. Biol. 2008, 15, 95-96.

22. Brown, S.; Zhang, Y.-H.; Walker, S., Chem. Biol. 2008, 15, 12-21.

23. Bhavsar, A. P.; Brown, E. D., Mol. Microbiol. 2006, 60, 1077-1090.

24. Fischer, W.; Behr, T.; Hartmann, R.; Peter-Katalinić, J.; Egge, H., Eur. J. Biochem. 1993, 215, 851-857.

25. Brinster, S.; Lamberet, G.; Staels, B.; Trieu-Cuot, P.; Gruss, A.; Poyart, C., Nature 2009, 458, 83-86.

26. Chan, D. I.; Vogel, H. J., Biochem. J. 2010, 430, 1-19.

27. Schujman, G. E.; de Mendoza, D., Curr. Opin. Microbiol. 2008, 11, 148-152.

28. Svetashev, V. I.; Vysotskii, M. V.; Ivanova, E. P.; Mikhailov, V. V., Syst. Appl. Microbiol. 1995, 18, 37-43.

29. The Lipid Handbook with CD-ROM. Gunstone, F. D.; Harwood, J. L.; Dijkstra, A. J., Eds. CRC press: Boca Raton, FL, 2009.

30. Fischer, W., Lipoteichoic Acids and Lipoglycans. In Bacterial Cell Wall, Hakenbeck, J.-M. G. a. R., Ed. Elsevier: Amsterdam, Netherlands, 1994; pp 199-215.

31. Delcour, J.; Ferain, T.; Deghorain, M.; Palumbo, E.; Hols, P., Anton. Leeuw. Int. J. G. 1999, 76, 159-184.

32. Xia, G.; Kohler, T.; Peschel, A., Int. J. Med. Microbiol. 2010, 300, 148-154.

33. Lu, D.; Woermann, M. E.; Zhang, X.; Schneewind, O.; Gruendling, A.; Freemont, P. S., PNAS 2009, 106, 1584-1589.

34. Kohler, T.; Xia, G.; Kulauzovic, E.; Peschel, A., Teichoic Acids, Lipoteichoic Acids and Related Cell Wall Glycopolymers of Gram-Positive Bacteria. In Microbial Glycobiology, Moran, A. P.; Holst, O.; Brennan, P. J.; von Itzstein, M., Eds. Academic Press: London, 2009; pp 75-91.

35. Kiriukhin, M. Y.; Neuhaus, F. C., J. Bacteriol. 2001, 183, 2051-2058. 
36. Huijbregts, R. P. H.; de Kroon, A. I. P. M.; de Kruijff, B., Biochim. Biophys. Acta 2000, 1469, 43-61.

37. Dowhan, W., Annu. Rev. Biochem. 1997, 66, 199-232.

38. Goldfine, H., Curr.Top. in Membr. and Trans. 1982, 17, 1-43.

39. Larsen, Å; Hvattum, E., Analysis of Phospholipids by Liquid Chromatography Coupled with On-Line Electrospray Ionization Mass Spectrometry and Tandem Mass Spectrometry. In Modern Methods for Lipid Analysis by Liquid Chromatography/Mass Spectrometry and Related Techniques, Byrdwell, W. C., Ed. AOCS Press: Champaign, Ill, 2005; pp 19-60.

40. Hölzl, G.; Dörmann, P., Prog. Lipid Res. 2007, 46, 225-243.

41. Morrison, I. M., Carbohydr. Chem. 1986, 15, 578-617.

42. Bligh, E. G.; Dyer, W. J., Can. J. Biochem. Physiol. 1959, 37, 911-917.

43. Brundish, D. E.; Shaw, N.; Baddiley, J., Biochem. J. 1966, 99, 546-549.

44. Veerkamp, J. H., Biochim. Biophys. Acta, Gen. Subj. 1972, 273, 359-367.

45. Thompson, B. G.; Anderson, R.; Murray, R. G. E., Can. J. Microbiol. 1980, 26, $1408-1411$.

46. Shaw, N., Bacteriol. Rev. 1970, 34, 365-377.

47. Heinz, E., Biochim. Biophys. Acta 1967, 144, 333-343.

48. Heinz, E., Z. Pflanzenphysiol. 1973, 69, 359-376.

49. Blakeney, A. B.; Harris, P. J.; Henry, R. J.; Stone, B. A., Carbohydr. Res. 1983, 113, 291-299.

50. Ciucanu, I.; Kerek, F., Carbohydr. Res. 1984, 131, 209-217.

51. Paściak, M.; Holst, O.; Lindner, B.; Mordarska, H.; Gamian, A., J. Biol. Chem. 2003, 278, 3948-3956.

52. Huang, Y.; Anderson, R., J. Bacteriol. 1995, 177, 2567-2571.

53. Paściak, M.; Sanchez-Carballo, P.; Duda-Madej, A.; Lindner, B.; Gamian, A.; Holst, O., Carbohydr. Res. 2010, 345, 1497-1503.

54. Rahman, O.; Dover, L. G.; Sutcliffe, I. C., Trends Microbiol. 2009, 17, 219-225.

55. Deininger, S.; Traub, S.; Aichele, D.; Rupp, T.; Baris, T.; Moeller, H. M.; Hartung, T.; von Aulock, S., Immunobiology 2008, 213, 519-529.

56. Morath, S.; Geyer, A.; Hartung, T., J. Exp. Med. 2001, 193, 393-397.

57. Jang, K.-S.; Baik, J. E.; Han, S. H.; Chung, D. K.; Kim, B.-G., Biochem. Biophys. Res. Commun. 2011, 407, 823-830.

58. Fischer, W.; Koch, H. U.; Haas, R., Eur. J. Biochem. 1983, 133, 523-530. 
59. Greenberg, J. W.; Fischer, W.; Joiner, K. A., Infect. Immun. 1996, 64, 33183325 .

60. Koch, H. U.; Haas, R.; Fischer, W., Eur. J. Biochem. 1984, 138, 357-363.

61. Fischer, W., Microb. Drug Resist. 1997, 3, 309-325.

62. Iwasaki, H.; Araki, Y.; Ito, E.; Nagaoka, M.; Yokokura, T., J. Bacteriol. 1990, $172,845-852$.

63. Khan, A. A.; Chee, S. H.; McLaughlin, R. J.; Harper, J. L.; Kamena, F.; Timmer, M. S. M.; Stocker, B. L., ChemBioChem 2011, 12, 2572-2576.

64. Miles, E. A.; Calder, P. C., Br. J. Nutr. 2012, 107, S171-S184.

65. Yudina, O. N.; Gening, M. L.; Tsvetkov, Y. E.; Grachev, A. A.; Pier, G. B.; Nifantiev, N. E., Carbohydr. Res. 2011, 346, 905-913.

66. Srivastava, J.; Khare, A.; Khare, N. K., ARKIVOC 2009, 180-188.

67. Knox, K. K.; Wicken, A. J., Bacteriol. Rev. 1973, 37, 215-257.

68. Neuhaus, F. C.; Baddiley, J., Microbiol. Mol. Biol. Rev. 2003, 67, 686-723.

69. Weidenmaier, C.; Kokai-Kun, J. F.; Kristian, S. A.; Chanturiya, T.; Kalbacher, H.; Gross, M.; Nicholson, G.; Neumeister, B.; Mond, J. J.; Peschel, A., Nat. Med. 2004, 10, 243-245.

70. Hogendorf, W. F. J.; Van den Bos, L. J.; Overkleeft, H. S.; Codée, J. D. C.; Van der Marel, G. A., Bioorg. Med. Chem. 2010, 18, 3668-3678.

71. Fogler, W., Immunologic Properties and Activities of Phospholipids. In Phospholipids Handbook, Cevc, G., Ed. Dekker: New York, 1993; pp 817-832.

72. Dieudé, M.; Striegl, H.; Tyznik, A. J.; Wang, J.; Behar, S. M.; Piccirillo, C. A.; Levine, J. S.; Zajonc, D. M.; Rauch, J., J. Immunol. 2011, 186, 4771-4781.

73. Torchilin, V. P., Nat. Rev. Drug Discovery 2005, 4, 145-160.

74. Lie Ken Jie, M. S. F.; Harwood, J. L.; Gunstone, F. D.; Cheung, W. H.; Lam, C. N. W., Synthesis. In The Lipid Handbook with CD-ROM, Gunstone, F. D.; Harwood, J. L.; Dijkstra, A. J., Eds. CRC press: Boca Raton, FL, 2009.

75. Cheng, J. M. H.; Chee, S. H.; Knight, D. A.; Acha-Orbea, H.; Hermans, I. F.; Timmer, M. S. M.; Stocker, B. L., Carbohydr. Res. 2011, 346, 914-926.

76. de la Salle, H.; Mariotti, S.; Angenieux, C.; Gilleron, M.; Garcia-Alles, L.-F.; Malm, D.; Berg, T.; Paoletti, S.; Maître, B.; Mourey, L.; Salamero, J.; Cazenave, J. P.; Hanau, D.; Mori, L.; Puzo, G.; De Libero, G., Science 2005, 310, 13211324. 
77. Stadelmaier, A.; Figueroa-Perez, I.; Deininger, S.; von Aulock, S.; Hartung, T.; Schmidt, R. R., Bioorg. Med. Chem. 2006, 14, 6239-6254.

78. Kinjo, Y.; Illarionov, P.; Vela, J. L.; Pei, B.; Girardi, E.; Li, X.; Li, Y.; Imamura, M.; Kaneko, Y.; Okawara, A.; Miyazaki, Y.; Gómez-Velasco, A.; Rogers, P.; Dahesh, S.; Uchiyama, S.; Khurana, A.; Kawahara, K.; Yesilkaya, H.; Andrew, P. W.; Wong, C.-H.; Kawakami, K.; Nizet, V.; Besra, G. S.; Tsuji, M.; Zajonc, D. M.; Kronenberg, M., Nat. Immunol. 2011, 12, 966-974.

79. Kinjo, Y.; Ueno, K., Microbiol. Immunol. 2011, 55, 472-482.

80. Naito, M.; Kudo, I.; Mukai-Sato, Y.; Tsushima, S.; Nomura, H.; Nojima, S.; Inoue, K., Cancer Immunol. Immunother. 1987, 24, 158-164.

81. Deininger, S.; Stadelmaier, A.; von Aulock, S.; Morath, S.; Schmidt, R. R.; Hartung, T., J. Immunol. 2003, 170, 4134-4138.

82. Kinjo, Y.; Tupin, E.; Wu, D.; Fujio, M.; Garcia-Navarro, R.; Benhnia, M. R.-E.I.; Zajonc, D. M.; Ben-Menachem, G.; Ainge, G. D.; Painter, G. F.; Khurana, A.; Hoebe, K.; Behar, S. M.; Beutler, B.; Wilson, I. A.; Tsuji, M.; Sellati, T. J.; Wong, C.-H.; Kronenberg, M., Nat. Immunol. 2006, 7, 978-986.

83. Girardi, E.; Yu, E. D.; Li, Y.; Tarumoto, N.; Pei, B.; Wang, J.; Illarionov, P.; Kinjo, Y.; Kronenberg, M.; Zajonc, D. M., PLoS Biol. 2011, 9, e1001189.

84. Amara, S.; Lafont, D.; Fiorentino, B.; Boullanger, P.; Carrière, F.; De Caro, A., Biochim. Biophys. Acta 2009, 1791, 983-990.

85. Schombs, M.; Park, F. E.; Du, W.; Kulkarni, S. S.; Gervay-Hague, J., J. Org. Chem. 2010, 75, 4891-4898.

86. Schmidt, R. R.; Pedersen, C. M.; Qiao, Y.; Zaehringer, U., Org. Biomol. Chem. 2011, 9, 2040-2052.

87. Keller, R.; Fischer, W.; Keist, R.; Bassetti, S., Infect. Immun. 1992, 60, 36643672 .

88. Morath, S.; Geyer, A.; Spreitzer, I.; Hermann, C.; Hartung, T., Infect. Immun. 2002, 70, 938-944.

89. Stadelmaier, A.; Morath, S.; Hartung, T.; Schmidt, R. R., Angew. Chem. Int. Ed. 2003, 42, 916-920.

90. Morath, S.; Stadelmaier, A.; Geyer, A.; Schmidt, R. R.; Hartung, T., J. Exp. Med. 2002, 195, 1635-1640.

91. Roethlisberger, P.; lida-Tanaka, N.; Hollemeyer, K.; Heinzle, E.; Ishizuka, I.; Fischer, W., Eur. J. Biochem. 2000, 267, 5520-5530. 
92. Qiao, Y.; Lindner, B.; Zäringer, U.; Truog, P.; Schmidt, R. R., Bioorg. Med. Chem. 2010, 18, 3696-3702.

93. Tissier, H. Recherches sur la flore intestinale des nourrissons (Etat normal et pathologique). University of Paris (France), Paris, 1906.

94. Weiss, J. E.; Rettger, L. F., J. Infect. Dis. 1938, 62, 115-120.

95. Orla-Jensen, S., Lait 1924, 4, 468-474.

96. Leahy, S. C.; Higgins, D. G.; Fitzgerald, G. F.; van Sinderen, D., J. Appl. Microbiol. 2005, 98, 1303-1315.

97. Mayo, B.; van Sinderen, D., Bifidobacteria: Genomics and Molecular Aspects. Caister Academic Press: Norfolk, UK, 2010; p 259.

98. Picard, C.; Fioramonti, J.; Francois, A.; Robinson, T.; Neant, F.; Matuchansky, C., Aliment Pharmacol. Ther. 2005, 22, 495-512.

99. Zhu, J.; Zhao, L.; Guo, H.; Jiang, L.; Ren, F., Afr. J. Microbiol. Res. 2011, 5, 815.

100. López, P.; Gueimonde, M.; Margolles, A.; Suárez, A., Int. J. Food Microbiol. 2010, 138, 157-165.

101. Biavati, B.; Mattarelli, P., The family bifidobacteriaceae. In Prokaryotes, Dworkin, M.; Falkow, S.; Rosenberg, E.; Schleifer, K. H.; Stackebrandt, E., Eds. Springer: Singapore, 2006; pp 322-382.

102. Op den Camp, H. J. M.; Peeters, P. A. M.; Oosterhof, A.; Veerkamp, J. H., J. Gen. Microbiol. 1985, 131, 661-668.

103. Amrouche, T.; Boutin, Y.; Prioult, G.; Fliss, I., Int. Dairy J. 2006, 16, 70-80.

104. Novik, G. I.; Astapovich, N. I.; Pastsyak, M.; Gamian, A., Microbiology 2005, $74,678-683$.

105. Tone-Shimokawa, Y.; Toida, T.; Kawashima, T., J. Bacteriol. 1996, 178, $317-$ 320.

106. Habu, Y.; Nagaoka, M.; Yokokura, T.; Azuma, I., J. Biochem. 1987, 102, 1423 1432.

107. Nagaoka, M.; Muto, M.; Yokokura, T.; Mutai, M., J. Biochem. 1988, 103, 618621.

108. Nagaoka, M.; Shibata, H.; Kimura, I.; Hashimoto, S.; Kimura, K.; Sawada, H.; Yokokura, T., Carbohydr. Res. 1995, 274, 245-249.

109. Nagaoka, M.; Hashimoto, S.; Shibata, H.; Kimura, I.; Kimura, K.; Sawada, H.; Yokokura, T., Carbohydr. Res. 1996, 281, 285-291. 
110. Zdorovenko, E. L.; Kachala, V. V.; Sidarenka, A. V.; Izhik, A. V.; Kisileva, E. P.; Shashkov, A. S.; Novik, G. I.; Knirel, Y. A., Carbohydr. Res. 2009, 344, 2417-2420.

111. Hosono, A.; Lee, J.; Ametani, A.; Natsume, M.; Hirayama, M.; Adachi, T.; Kaminogawa, S., Biosci. Biotechnol. Biochem. 1997, 61, 312-316.

112. Hosono, A.; Lee, J.; Ametani, A.; Natsume, M.; Hirayama, M.; Adachi, T.; Kaminogawa, S., Biosci. Microflora 1998, 17, 97-104.

113. Zhang, G.; Fu, M.; Ning, J., Tetrahedron Asymmetr. 2005, 16, 733-738.

114. Veerkamp, J. H.; Hoelen, G. E. J. M.; Op Den Camp, H. J. M., Biochim. Biophys. Acta, Gen. Subj. 1983, 755, 439-451.

115. Xu, R.; Shen, Q.; Ding, X.; Gao, W.; Li, P., Eur. Food Res. Technol. 2011, 232, 231-240.

116. Fanning, S.; Hall, L. J.; Cronin, M.; Zomer, A.; MacSharry, J.; Goulding, D.; Motherway, M. O. C.; Shanahan, F.; Nally, K.; Dougan, G.; van Sinderen, D., PNAS 2012, 109, 2108-2113.

117. MacFarlane, S.; Blackett, K. E.; MacFarlane, G. T., Synthesis and utilization of exopolysaccharides and prebiotics. In Bifidobacteria, Mayo, B.; van Sinderen, D., Eds. Caister Academic Press: Norwich, UK, 2010; pp 175-193.

118. Andaloussi, S. A.; Talbaoui, H.; Marczak, R.; Bonaly, R., Appl. Microbiol. Biotechnol. 1995, 43, 995-1000.

119. Leivers, S.; Hidalgo-Cantabrana, C.; Robinson, G.; Margolles, A.; RuasMadiedo, P.; Laws, A. P., Carbohydr. Res. 2011, 346, 2710-2717.

120. You, H. J.; Oh, D.-K.; Ji, G. E., FEMS Microbiol. Lett. 2004, 240, 131-136.

121. Kohno, M.; Suzuki, S.; Kanaya, T.; Yoshino, T.; Matsuura, Y.; Asada, M.; Kitamura, S., Carbohydr. Polym. 2009, 77, 351-357.

122. Seltmann, G.; Holst, O., The outer membrane of the Gram-negative bacteria and their components. In The Bacterial Cell Wall, Seltmann, G.; Holst, O., Eds. Springer: Berlin, 2002; pp 9-102.

123. Veerkamp, J. H., J. Bacteriol. 1971, 108, 861-867.

124. Fischer, W., Eur. J. Biochem. 1987, 165, 639-646.

125. Veerkamp, J. H.; Van Schaik, F. W., Biochim. Biophys. Acta, Lipids Lipid Metab. 1974, 348, 370-387.

126. Veerkamp, J. H., Biochim. Biophys. Acta 1974, 348, 23-34. 
127. Jun Bae, A.; Hwang, H.-J.; Park, J.-H., J. Microbiol. Biotechnol. 2001, 11, $443-$ 451.

128. Ruiz, L.; Sánchez, B.; Ruas-Madiedo, P.; de los Reyes-Gavilan, C. G.; Margolles, A., FEMS Microbiol. Lett. 2007, 274, 316-322.

129. Jung, H.-K.; Kim, E.-R.; Ji, G.-E.; Park, J.-H.; Cha, S.-K.; Juhn, S.-L., J. Microbiol. Biotechnol. 2000, 10, 143-146.

130. Ryhage, R.; Stenhagen, E., Ark. Kemi 1959, 13, 523-542.

131. Oberg, T. S.; Ward, R. E.; Steele, J. L.; Broadbent, J. R., Appl. Environ. Microbiol. 2012, 78, 880-884.

132. Exterkate, F. A.; Veerkamp, J. H., Biochim. Biophys. Acta 1969, 176, 65-77.

133. Exterkate, F. A.; Otten, B. J.; Wassenberg, H. W.; Veerkamp, J. H., J. Bacteriol. 1971, 106, 824-829.

134. Novik, G. I.; Astapovich, N. I.; Grzegorzewicz, A.; Gamian, A., Microbiology 2005, 74, 670-677.

135. Novik, G. I.; Astapovich, N. I.; Grzegorzewicz, A.; Gamian, A., Microbiology 2006, 75, 29-34.

136. Exterkate, F. A.; Veerkamp, J. H., Biochimica et Biophysica Acta (BBA)/Lipids and Lipid Metabolism 1971, 231, 545-549.

137. Novik, G.; Gamian, A.; Francisco, J. D. C.; Dey, E. S., J. Biotechnol. 2006, 121, $555-562$.

138. Izhyk, A.; Novik, G.; Szwajcer Dey, E., J. Supercrit. Fluids 2012, 62, 149-154.

139. Op den Camp, H. J. M.; Oosterhof, A.; Veerkamp, J. H., Infect. Immun. 1985, 47, 332-334.

140. Jiang, H.; Hu, H.; Wei, Q., Mianyixue Zazhi 2000, 16, 429-431, 441.

141. Wang, Y.; Zhang, J.; Liu, M., Zhongguo Weishengtaixue Zazhi 2008, 20, 454456.

142. Chu, Q.; Zhang, D., Zhongguo Weishengtaixue Zazhi 2008, 20, 521-523.

143. Miyauchi, E.; Tanabe, S., Kagaku to Seibutsu 2008, 46, 590-592.

144. Op Den Camp, H. J. M.; Oosterhof, A.; Veerkamp, J. H., Biochem. J. 1985, 228, 683-688.

145. Veerkamp, J. H., Biochim. Biophys. Acta, Lipids Lipid Metab. 1976, 441, 403411.

146. Fischer, W.; Bauer, W.; Feigel, M., Eur. J. Biochem. 1987, 165, 647-652. 
147. Lukjancenko, O.; Ussery, D. W.; Wassenaar, T. M., Microb. Ecol. 2012, 63, 651-673.

148. Kleerebezem, M.; Vaughan, E. E., Annu. Rev. Microbiol. 2009, 63, 269-290.

149. Mayo, B.; Aleksandrzak-Piekarczyk, T.; Fernández, M.; Kowalczyk, M.; Alvarez-Martin, P.; Bardowski, J., Updates in the metabolism of lactic acid bacteria. In Biotechnology Of Lactic Acid Bacteria: Novel Applications, Mozzi, F.; Raya, R. R.; Vignolo, G. M., Eds. Wiley-Blackwell: Ames, 2010; pp 3-32.

150. Felis, G. E.; Dellaglio, F., Curr. Iss. Intest. Microbiol. 2007, 8, 44-61.

151. Kleerebezem, M.; Hols, P.; Bernard, E.; Rolain, T.; Zhou, M.; Siezen, R. J.; Bron, P. A., FEMS Microbiol. Rev. 2010, 34, 199-230.

152. Remus, D. M.; Kleerebezem, M.; Bron, P. A., Eur. J. Pharmacol. 2011, 668, S33-S42.

153. Fred, E. B.; Peterson, W. H.; Anderson, J. A., J. Biol. Chem. 1921, 48, 385-412.

154. Tallon, R.; Bressollier, P.; Urdaci, M. C., Res. Microbiol. 2003, 154, 705-712.

155. Dong, H.; Rowland, I.; Yaqoob, P., Br. J. Nutr. 2012, 108, 459-470.

156. Chiang, S.-S.; Liu, C.-F.; Tseng, K.-C.; Mau, J.-L.; Pan, T.-M., Food Agric. Immunol. 2012, 23, 183-202.

157. Duary, R. K.; Bhausaheb, M. A.; Batish, V. K.; Grover, S., Mol. Biol. Rep. 2012, 39, 4765-4775.

158. Tomita, S.; Irisawa, T.; Tanaka, N.; Nukada, T.; Satoh, E.; Uchimura, T.; Okada, S., Biosci., Biotechnol., Biochem. 2010, 74, 928-933.

159. Bron, P. A.; Tomita, S.; van Swam, I. I.; Remus, D. M.; Meijerink, M.; Wels, M.; Okada, S.; Wells, J. M.; Kleerebezem, M., Microb. Cell. Fact. 2012, 11, 123.

160. Wicken, A. J.; Evans, J. D.; Campbell, L. K.; Knox, K. W., Infect. Immun. 1982, $38,1-7$.

161. Archibald, A. R.; Baddiley, J.; Buchanan, J. G., Biochem. J. 1961, 81, 124-134.

162. Archibald, A. R.; Armstrong, J. J.; Baddiley, J.; Hay, J. B., Nature 1961, 191, 570-572.

163. Adams, J. B.; Archibald, A. R.; Baddiley, J.; Coapes, H. E.; Davison, A. L., Biochem. J. 1969, 113, 191-193.

164. Archibald, A. R.; Coapes, H. E., Biochem. J. 1971, 124, 449-460.

165. Tomita, S.; Furihata, K.; Nukada, T.; Satoh, E.; Uchimura, T.; Okada, S., Biosci., Biotechnol., Biochem. 2009, 73, 530-535. 
166. Tomita, S.; Furihata, K.; Tanaka, N.; Satoh, E.; Nukada, T.; Okada, S., Microbiology 2012, In press.

167. Douglas, L. J.; Wolin, M. J., Biochemistry 1971, 10, 1551-1555.

168. Ikawa, M.; Snell, E. E., J. Biol. Chem. 1960, 235, 1376-1382.

169. Critchley, P.; Archibald, A. R.; Baddiley, J., Biochem. J. 1962, 85, 420-431.

170. Sargent, L. J.; Buchanan, J. G.; Baddiley, J., J. Chem. Soc. 1962, 2184-2187.

171. Feng, M.; Zhang, Q.; Mayinuo, M.; Huang, L.; Cao, L.; Dong, M., Shipin Kexue 2011, 32, 107-111.

172. Ismail, B.; Nampoothiri, K. M., Arch. Microbiol. 2010, 192, 1049-1057.

173. Tsuda, H.; Miyamoto, T., Food Sci. Technol. Res. 2010, 16, 87-92.

174. Liu, C.-F.; Tseng, K.-C.; Chiang, S.-S.; Lee, B.-H.; Hsu, W.-H.; Pan, T.-M., J. Sci. Food Agric. 2011, 91, 2284-2291.

175. Blank, G.; Adejumo, A. A.; Zawistowski, J., Lebensm.-Wiss. Technol. 1991, 24, 231-235.

176. Henderson, T. O.; McNeill, J. J., Biochem. Biophys. Res. Commun. 1966, 25, 662-669.

177. Turujman, N.; Jabr, I.; Durr, I. F., Int. J. Biochem. 1974, 5, 791-793.

178. Weeks, G.; Wakil, S. J., J. Biol. Chem. 1970, 245, 1913-1921.

179. Smith, D. D., Jr.; Norton, S. J., Arch. Biochem. Biophys. 1980, 205, 564-570.

180. Grogan, D. W.; Cronan, J. E., Jr., Microbiol. Mol. Biol. Rev. 1997, 61, 429-441.

181. Buist, P. H.; MacLean, D. B., Can. J. Chem. 1981, 59, 828-838.

182. Tocanne, J. F., Tetrahedron 1972, 28, 363-371.

183. Drucker, D. B.; Megson, G.; Harty, D. W.; Riba, I.; Gaskell, S. J., J. Bacteriol. 1995, 177, 6304-6308.

184. Calvano, C. D.; Zambonin, C. G.; Palmisano, F., Rapid Commun. Mass Spectrom. 2011, 25, 1757-1764.

185. Arbogast, L. Y.; Henderson, T. O., J. Bacteriol. 1975, 123, 962-971.

186. Burritt, M. F.; Henderson, T. O., J. Bacteriol. 1975, 123, 972-977.

187. Ikawa, M., J. Bacteriol. 1963, 85, 772-781.

188. Nakano, M.; Fischer, W., Hoppe-Seyler's Z. Physiol. Chem. 1977, 358, 14391453.

189. Fischer, W.; Nakano, M.; Laine, R. A.; Bohrer, W., Biochim. Biophys. Acta 1978, 528, 288-297. 
190. Fischer, W.; Laine, R. A.; Nakano, M., Biochim. Biophys. Acta 1978, 528, 298318.

191. Zeng, R.-Z.; Kim, H. G.; Kim, N. R.; Gim, M. G.; Ko, M. Y.; Lee, S. Y.; Kim, C. M.; Chung, D. K., J. Korean Soc. Appl. Biol. Chem. 2011, 54, 763-770.

192. Kim, H.; Jung, B. J.; Jung, J. H.; Kim, J. Y.; Chung, S. K.; Chung, D. K., Mol. Cells 2012, 33, 479-486.

193. Kang, S.-S.; Ryu, Y. H.; Baik, J. E.; Yun, C.-H.; Lee, K.; Chung, D. K.; Han, S. H., Mol. Immunol. 2011, 48, 2170-2177.

194. Sauvageau, J.; Ryan, J.; Lagutin, K.; Sims, I. M.; Stocker, B. L.; Timmer, M. S. M., Carbohydr. Res. 2012, 357, 151-156.

195. Molin, G., Am. J. Clin. Nutr. 2001, 73, 380S-385S.

196. Ahrné, S.; Nobaek, S.; Jeppsson, B.; Adlerberth, I.; Wold, A. E.; Molin, G., J. Appl. Microbiol. 1998, 85, 88-94.

197. Rigaux, P.; Daniel, C.; Hisbergues, M.; Muraille, E.; Hols, P.; Pot, B.; Pestel, J.; Jacquet, A., Allergy 2009, 64, 406-414.

198. Ishikawa, E.; Ishikawa, T.; Morita, Y. S.; Toyonaga, K.; Yamada, H.; Takeuchi, O.; Kinoshita, T.; Akira, S.; Yoshikai, Y.; Yamasaki, S., J. Exp. Med. 2009, 206, 2879-2888.

199. Cheng, J. M. H.; Khan, A. A.; Timmer, M. S. M.; Stocker, B. L., Int. J. Carbohydr. Chem. 2011, Article ID 749591, 13.

200. Iwamori, M.; Shibagaki, T.; Nakata, Y.; Adachi, S.; Nomura, T., J. Biochem. 2009, 146, 185-191.

201. Iwamori, M.; Sakai, A.; Minamimoto, N.; Iwamori, Y.; Tanaka, K.; Aioki, D.; Adachi, S.; Nomura, T., J. Biochem. 2011, 150, 515-523.

202. Zähringer, U.; Lindner, B.; Inamura, S.; Heine, H.; Alexander, C., Immunobiology 2008, 213, 205-224.

203. Shaw, N.; Heatherington, K.; Baddiley, J., Biochem. J. 1968, 107, 491-496.

204. Wiegandt, H., Glycolipids. Eselvier: London, 1985.

205. Dubois, M.; Gilles, K. A.; Hamilton, J. K.; Rebers, P. A.; Smith, F., Anal. Chem. 1956, $28,350-356$.

206. Dittmer, J. C.; Lester, R. L., J. Lipid Res. 1964, 5, 126-127.

207. Vaskovsky, V. E.; Kostetsky, E. Y.; Vasendin, I. M., J. Chromatogr. 1975, 114, $129-141$. 
208. Doares, S. H.; Albersheim, P.; Darvill, A. G., Carbohydr. Res. 1991, 210, 311317.

209. Cases, M. R.; Cerezo, A. S.; Stortz, C. A., Carbohydr. Res. 1995, 269, 333-341.

210. Stránský, K.; Jursík, T.; Vítek, A.; Skořepa, J., J. High Resolut. Chromatogr. 1992, 15, 730-740.

211. Miwa, T. K.; Mikolajczak, K. L.; Earle, F. R.; Wolff, I. A., Anal. Chem. 1960, $32,1739-1742$.

212. Jiang, H.; McGiff, J. C.; Quilley, J.; Sacerdoti, D.; Reddy, L. M.; Falck, J. R.; Zhang, F.; Lerea, K. M.; Wong, P. Y.-K., J. Biol. Chem. 2004, 279, 3641236418.

213. McCloskey, J. A.; Law, J. H., Lipids 1967, 2, 225-230.

214. Carballeira, N. M.; Shalabi, F., J. Nat. Prod. 1994, 57, 1152-1159.

215. Andersson, B. ̊̊.; Holman, R. T., Lipids 1974, 9, 185-190.

216. Stuehr, D. J.; Nathan, C. F., J. Exp. Med. 1989, 169, 1543-1555.

217. Gunstone, F. D.; Harwood, J. L.; Dijkstra, A. J., Nuclear Magnetic Resonnance Spectroscopy. In The Lipid Handbook with CD-ROM, CRC press: Boca Raton, FL, 2009; pp 455-465.

218. Kerwin, J. L.; Torvik, J. J., Anal. Biochem. 1996, 237, 56-64.

219. Kerwin, J. L.; Wiens, A. M.; Ericsson, L. H., J. Mass Spectrom. 1996, 31, 184192.

220. Knothe, G., Lipids 2006, 41, 393-396.

221. Christie, W. W.; Holman, R. T., Lipids 1966, 1, 176-182.

222. Guella, G.; Frassanito, R.; Mancini, I., Rapid Commun. Mass Spectrom. 2003, 17, 1982-1994.

223. Grimmecke, H. D.; Knirel, Y. A.; Shashkov, A. S.; Kiesel, B.; Lauk, W.; Voges, M., Carbohydr. Res. 1994, 253, 277-282.

224. van Leeuwen, S. S.; Leeflang, B. R.; Gerwig, G. J.; Kamerling, J. P., Carbohydr. Res. 2008, 343, 1114-1119.

225. Bhunia, S. K.; Dey, B.; Maity, K. K.; Patra, S.; Mandal, S.; Maiti, S.; Maiti, T. K.; Sikdar, S. R.; Islam, S. S., Carbohydr. Res. 2010, 345, 2542-2549.

226. Bogle, R. G.; Baydoun, A. R.; Pearson, J. D.; Moncada, S.; Mann, G. E., Biochem. J. 1992, 284, 15-18.

227. Fang, F. C.; Vazquez-Torres, A., Am. J. Physiol. Lung. Mol. Physiol. 2002, 282, L941-L943. 
228. Utgaard, J. O.; Jahnsen, F. L.; Bakka, A.; Brandtzaeg, P.; Haraldsen, G., J. Exp. Med. 1998, 188, 1751-1756.

229. Reddy, B. S.; Rivenson, A., Cancer Res. 1993, 53, 3914-3418.

230. Lee, J.-H.; O'Sullivan, D. J., Microbiol. Mol. Biol. Rev. 2010, 74, 378-416.

231. Tupin, E.; Kinjo, Y.; Kronenberg, M., Nat. Rev. Micro. 2007, 5, 405-417.

232. Moran, A. P.; Prendergast, M. M.; Appelmelk, B. J., FEMS Immunol. Med. Microbiol. 1996, 16, 105-115.

233. Weintraub, A., Carbohydr. Res. 2003, 338, 2539-2547.

234. Ho, Y.-P.; Reddy, P. M., Mass Spectrom. Rev. 2010, 30, 1203-1224.

235. Evason, D. J.; Claydon, M. A.; Gordon, D. B., Rapid Commun. Mass Spectrom. 2000, 14, 669-672.

236. Madonna, A. J.; Basile, F.; Ferrer, I.; Meetani, M. A.; Rees, J. C.; Voorhees, K. J., Rapid Commun. Mass Spectrom. 2000, 14, 2220-2229.

237. Claydon, M. A.; Davey, S. N.; Edwards-Jones, V.; Gordon, D. B., Nat. Biotech. 1996, 14, 1584-1586.

238. Gidden, J.; Denson, J.; Liyanage, R.; Ivey, D. M.; Lay Jr, J. O., Int. J. Mass spectrom. 2009, 283, 178-184.

239. Fuchs, B.; Schiller, J., Eur. J. Lipid Sci. Technol. 2009, 111, 83-98.

240. Shu, X.; Li, Y.; Liang, M.; Yang, B.; Liu, C.; Wang, Y.; Shu, J., Int. J. Mass Spectrom. 2012, 321-322, 71-76.

241. Ishida, Y.; Kitagawa, K.; Nakayama, A.; Ohtani, H., Appl. Environ. Microbiol. 2005, 71, 7539-7541.

242. Ishida, Y.; Madonna, A. J.; Rees, J. C.; Meetani, M. A.; Voorhees, K. J., Rapid Commun. Mass Spectrom. 2002, 16, 1877-1882.

243. Jones, J. J.; Stump, M. J.; Fleming, R. C.; Lay, J. O.; Wilkins, C. L., J. Am. Soc. Mass Spectrom. 2004, 15, 1665-1674.

244. Jones, J. J.; Stump, M. J.; Fleming, R. C.; Lay, J. O. J.; Wilkins, C. L., Anal. Chem. 2003, 75, 1340-1347.

245. Schumaker, S.; Borror, C. M.; Sandrin, T. R., Rapid Commun. in Mass Spectrom. 2011, 26, 243-253.

246. Gheysen, K.; Mihai, C.; Conrath, K.; Martins, J. C., Chem. Eur. J. 2008, 14, 8869-8878.

247. Hoelzl, G.; Doermann, P., Prog. in Lipid Res. 2007, 46, 225-243. 
248. Huang, Y.; Zhao, N.; He, L.; Wang, L.; Liu, Z.; You, M.; Guan, F., J. Clin. Microbiol. 2005, 43, 1451-1455.

249. Marlow, A. L.; Kiessling, L. L., Org. Lett. 2001, 3, 2517-2519.

250. McConville, M. J.; Thomas-Oates, J. E.; Ferguson, M. A. J.; Homans, S. W., J. Biol. Chem. 1990, 265, 19611-19623.

251. Gorin, P. A. J.; Mazurek, M., Can. J. Chem. 1975, 53, 1212-1223.

252. Sassaki, G. L.; Iacomini, M.; Gorin, P. A. J., An. Ac. Br. Cien. 2005, 77, $223-$ 234.

253. Wieneke, R.; Klein, S.; Geyer, A.; Loos, E., Carbohydr. Res. 2007, 342, 27572765.

254. Caruso, U., Rapid Commun. Mass Spectrom. 1996, 10, 1283-1285.

255. Yachida, Y.; Kashiwagi, M.; Mikami, T.; Tsuchihashi, K.; Daino, T.; Akino, T.; Gasa, S., J. Lipid Res. 1998, 39, 1039-1045.

256. Yachida, Y.; Kashiwagi, M.; Mikami, T.; Tsuchihashi, K.; Daino, T.; Akino, T.; Gasa, S., J. Lipid Res. 1999, 40, 2271-2278.

257. Hikita, T.; Tadano-Aritomi, K.; Iida-Tanaka, N.; Anand, J. K.; Ishizuka, I.; Hakomori, S. I., J. Biol. Chem. 2001, 276, 23084-23091.

258. Matsumoto, M.; Tamiya, K.; Koizumi, K., J. Biochem. 1971, 69, 617-620.

259. Johnston, N. C.; Goldfine, H., Eur. J. Biochem. 1994, 223, 957-963.

260. Johnston, N. C.; Goldfine, H., Biochim. Biophys. Acta 1988, 961, 1-12.

261. Lee, J.; Jung, S.; Lowe, S.; Zeikus, J. G.; Hollingsworth, R. I., J. Am. Chem. Soc. 1998, 120, 5855-5863.

262. Fujiwara, Y., Biochim. Biophys. Acta 2008, 1781, 519-524.

263. Goldfine, H., Prog. Lipid Res. 2010, 49, 493-498.

264. Young, S. L.; Simon, M. A.; Baird, M. A.; Tannock, G. W.; Bibiloni, R.; Spencely, K.; Lane, J. M.; Fitzharris, P.; Crane, J.; Town, I.; Addo-Yobo, E.; Murray, C. S.; Woodcock, A., Clin. Diagn. Lab. Immunol. 2004, 11, 686-690.

265. Gore, C.; Munro, K.; Lay, C.; Bibiloni, R.; Morris, J.; Woodcock, A.; Custovic, A.; Tannock, G. W., J. Allergy Clin. Immunol. 2008, 121, 135-140.

266. Ouwehand, A. C., J. Nutr. 2007, 137, 794S-797S.

267. Suzuki, S.; Shimojo, N.; Tajiri, Y.; Kumemura, M.; Kohno, Y., Clin. Exp. Allergy 2007, 37, 506-511.

268. He, F.; Morita, H.; Ouwehand, A. C.; Hosoda, M.; Hiramatsu, M.; Kurisaki, J.I.; Isolauri, E.; Benno, Y.; Salminen, S., Microbiol. Immunol. 2002, 46, 781-785. 
269. Sauvageau, J.; Foster, A. J.; Khan, A. A.; Chee, S. H.; Lagutin, K.; Ryan, J.; Sims, I. M.; Timmer, M. S. M.; Stocker, B. L., ChemBioChem 2012, 13, 24162424.

270. Dangerfield, E. M.; Cheng, J. M. H.; Knight, D. A.; Weinkove, R.; Dunbar, P. R.; Hermans, I. F.; Timmer, M. S. M.; Stocker, B. L., ChemBioChem 2012, 13, 1349-1356.

271. Morath, S.; von Aulock, S.; Hartung, T., J. Endotoxin Res. 2005, 11, 348-356.

272. Westphal, O.; Luederitz, O.; Keiderling, W., Zentralbl. Bakteriol. Parasitenkd. Infektionskr. Hyg. 1952, 158, 152-160.

273. Cuthbertson, L., Lipopolysaccharide biosynthesis. In Bacterial Glycomics: Current Research, Technology and Applications:, Reid, C. W.; Twine, S. M.; Reid, A. N., Eds. Caister Academic Press: Norwich, UK, 2012; pp 1-25.

274. Jang, K.-S.; Baik, J. E.; Kang, S.-S.; Jeon, J. H.; Choi, S.; Yang, Y.-H.; Kim, B.G.; Yun, C.-H.; Han, S. H., Mol. Immunol. 2012, 50, 177-183.

275. Kusumoto, S.; Fukase, K.; Suda, Y.; Oikawa, M., J. Synth. Org. Chem., Jpn. 1996, 54, 976-987.

276. Pedersen, C. M.; Figueroa-Perez, I.; Lindner, B.; Ulmer, A. J.; Zähringer, U.; Schmidt, R. R., Angew. Chem., Int. Ed. 2010, 49, 2585-2590.

277. Op den Camp, H. J. M.; Veerkamp, J. H.; Oosterhof, A.; Van Halbeek, H., Biochim. Biophys. Acta 1984, 795, 301-313.

278. Gein, S. V.; Kuyukina, M. S.; Ivshina, I. B.; Baeva, T. A.; Chereshnev, V. A., Cytotechnology 2011, 63, 559-566.

279. Hamamoto, S.; Takaku, H., Chem. Lett. 1986, 15, 1401-1404.

280. Baldoni, L.; Marino, C., J. Org. Chem. 2009, 74, 1994-2003.

281. Hirth, G.; Walther, W., Helv. Chim. Acta. 1985, 68, 1863-1871.

282. Eibl, H., Chem. Phys. Lipids 1981, 28, 1-5.

283. Lok, C. M.; Ward, J. P.; van Dorp, D. A., Chem. Phys. Lipids 1976, 16, 115122.

284. Gola, G.; Libenson, P.; Gandolfí-Donadio, L.; Gallo-Rodriguez, C., ARKIVOC 2005, 12, 234-242.

285. D'Accorso, N. B.; Thiel, I. M. E.; Schueller, M., Carbohydr. res. 1983, 124, 177-184.

286. Pathak, A. K.; El-Kattan, Y. A.; Bansal, N.; Maddry, J. A.; Reynolds, R. C., Tetrahedron Lett. 1998, 39, 1497-1500. 
287. Marino, C.; Cancio, M. J.; Varela, O.; de Lederkremer, R. M., Carbohydr. res. 1995, 276, 209-213.

288. Olah, G. A.; Narang, S. C., Tetrahedron 1982, 38, 2225-2277.

289. Bartolmäs, T.; Heyn, T.; Mickeleit, M.; Fischer, A.; Reutter, W.; Danker, K., J. Med. Chem. 2005, 48, 6750-6755.

290. Petri, A. F.; Bayer, A.; Maier, M. E., Angew. Chem. Int. Ed. 2004, 43, 58215823.

291. Yang, W.-B.; Patil, S. S.; Tsai, C.-H.; Lin, C.-H.; Fang, J.-M., Tetrahedron 2002, 58, 253-259.

292. Krotz, A. H.; Wheeler, P.; Ravikumar, V. T., Angew. Chem., Int. Ed. 1995, 34, 2406-2409.

293. Guanti, G.; Banfi, L.; Basso, A.; Bondanza, L.; Guglieri, G.; Powles, K.; Riva, R., Amino Acids 2010, 39, 367-373.

294. Sureshan, K. M.; Riley, A. M.; Potter, B. V. L., Tetrahedron Lett. 2007, 48, 1923-1926.

295. Chen, J.; Feng, L.; Prestwich, G. D., J. Org. Chem. 1998, 63, 6511-6522.

296. Sen, A.; Williams, W. P.; Brain, A. P. R.; Dickens, M. J.; Quinn, P. J., Nature 1981, 293, 488-490.

297. Gibson, B. W.; Engstrom, J. J.; John, C. M.; Hines, W.; Falick, A. M., J. Am. Soc. Mass Spectrom. 1997, 8, 645-658.

298. Sutcliffe, I. C.; Hogg, S. D., J. Microbiol. Methods 1993, 17, 215-225.

299. Rundlöf, T.; Widmalm, G., Anal. Biochem. 1996, 243, 228-233.

300. Kim, J.-H.; Nishida, Y.; Ohrui, H.; Meguro, H., J. Carbohydr. Chem. 1995, 14, 889-893.

301. Nishida, Y.; Ohrui, H.; Meguro, H., Tetrahedron Lett. 1989, 30, 5277-5280.

302. Kadota, I.; Yamagami, Y.; Fujita, N.; Takamura, H., Tetrahedron Lett. 2009, 50, $4552-4553$.

303. Borbás, A.; Szabó, Z. B.; Szilágyi, L.; Bényei, A.; Liptàk, A., Carbohydr. Res. 2002, 337, 1941-1951.

304. Parhi, A. K.; Mootoo, D. R.; Franck, R. W., Tetrahedron 2008, 64, 9821-9827. 